\title{
Abstracts from ATTD 2016 9th International Conference on Advanced Technologies \& Treatments for Diabetes
}

Milan, Italy-February 3-6, 2016

ATTD 2016 Invited Speakers

ATTD 2016 E-Poster Presentations 


\section{ATTD 2016 Invited Speakers}

\section{1 \\ DEVELOPING DISEASE-MODIFYING THERAPIES IN CHILDREN WITH TYPE 1 DIABETES}

D. Schatz

$U S A$

Type 1a (autoimmune) diabetes remains a tremendous burden both to individuals and society. Glycemic targets both in children (and adolescents especially) as well as adults are met only by a minority of patients. Changing the course of the disease by preventing or slowing down beta cell destruction rather than managing hyperglycemia would significantly impact the burden.

Studies aimed at both preventing the disease in at-risk preType 1 subjects as well as intervening not only in new-onset cases, but also in established disease have been conducted over the past 30 years. Success has been limited.

Progress in our understanding of the genetics and natural history of the disease, incidence and prevalence, complications, quality of life, neurocognitive impact and response to therapy among others are critical to the design of disease modifying therapies.Clear data have emerged there are differences between adult-onset and pediatric-onset Type 1 disease.

Advances in our understanding of the natural history of Type 1 Diabetes throughout the lifespan have led to recent publications related to broader acceptance of a classification system for the presymptomatic stages of Type 1 diabetes and, importantly, more defined pathways for the development of disease modifying therapies in children.

\section{2}

\section{CSII AND OTHER TECHNOLOGIES FOR PREVENTING BETA CELL FAILURE IN TYPE 2 DIABETES}

P. Pozzilli

Italy

The value and the utility of applying technology in the management of patients with poorly controlled insulin-treated type 2 diabetes (T2D) remain controversial. The OpT2mise randomized trial was designed to compare the effects of continuous subcutaneous insulin infusion (CSII) and multiple daily injection (MDI) on glucose profiles in patients with T2D. The results showed that CSII significantly improved selected glucometrics, compared with MDI, without increasing the risk of hypoglycemia. Another recent study looking at newly diagnosed T2D patients via the use of either CSII or CSII + sitagliptin therapy in controlling glucose variability and to prevent secondary complications of T2D showed that this treatment is superior to CSII monotherapy in terms of glucose variability. Oxidative stress is a detrimental feature of diabetes implicated in the progression of the disease and its complications. A study tested the hypothesis that improved glucose control, rather than insulin dose, is central to reduced oxidative stress in patients with T2D following CSII. CSII induces reduction of plasma ox-LDL in T2D patients as compared to patients on MDI suggesting that CSII may have an impact on factors associated with progression to complications. Finally, protection of beta cell function in T2D using CSII has been reported. Regarding glucose monitoring, recent studies utilizing self monitoring blood glucose (SMBG) as an integral component of diabetes care showed improvement in mean glucose, glycemic variability, metabolic risk factors, depression and diabetes-related distress, and health behaviors. The PRISMA study (Prospective, Randomized Trial on Intensive Self-Monitoring Blood Glucose Management Added Value in Noninsulin-Treated Type 2 Diabetes Mellitus Patients), to our knowledge the largest study of the effects of SMBG in patients with T2D, confirms the clinical usefulness and overall safety of using structured SMBG to provide guidance in the prescription of diabetes medications and lifestyle changes in non insulin-treated T2D. The effectiveness of continuous glucose monitoring (CGM) in the management of type 1 diabetic patients is well known. Differently in T2D its use is still a matter of debate because clinical trials in well-selected patient groups are lacking and less convincing evidence is available. Although many studies have evaluated glucose variability through different indices, MAGE, assessed by CGM, may be considered the best method to quantify glycaemic excursion. An improvement on glycaemic excursion in insulin-treated T2D patients by using RT-CGM has been reported, showing a significant reduction of the time spent in hypoglycaemic and hyperglycaemic ranges. Short-term intermittent use of RT-CGM appears effective in improving glycaemic control over a 12-week period without increasing the risk of hypoglycaemia. The effectiveness of RT-CGM is sustained during follow-up period compared with SMBG and without a greater intensification of medication over the course of the study. Larger clinical trials with longer follow-up are needed to evaluate not only the effectiveness of CGM in terms of improving glycaemic control but also the impact on quality of life and the adherence to the use of CGM as well as related cost issues. Even if it is too early to reach a general conclusion on the specific clinical indications, we believe that the RT-CGM can be used as a powerful motivational device to change patients lifestyle. We also suggest that short-term retrospective CGM use may be beneficial in certain clinical situations such as to detect nocturnal hypoglycaemia, to assist in the management of hypoglycaemia in T2D unawareness and when significant therapeutic changes are performed. In conclusion, modern technological devices including short message service reminder, online educational programs and clinician-patient electronic communication supporting coping and 
management are increasingly popular and represent very useful healthcare tools for T2D patients.

003

\section{IS TYPE 1 DIABETES MELLITUS AN AUTOIMMUNE DISEASE?}

M. Sperling

$U S A$

Type 1 diabetes mellitus (T1DM) is considered to be an autoimmune disease based on genetic and clinical associations. Several of the most common associated genes including HLA, Insulin VNTR, CTLA4, AIRE, PTPN22, are immune regulators. Circulating islet and $\beta$-cell antibodies are hallmarks of autoimmunity present in $\sim 85 \%$ of patients considered T1DM.Yet more people harbor the same alleles and/or mutations without clinical diabetes. For example, inactivating mutations in the AIRE gene are responsible for APS1 in which Addison disease and hypoparathyroidism are common, yet T1DM is not. In APS2, Addison disease and Hashimoto thyroiditis accompany T1DM, yet co-existence of Addison with T1DM is rare. Markers of autoimmunity and various $\beta$ cell $\mathrm{Ab}$ may exist for variable periods without clinical abnormalities. Autoimmune disease is more common in women, but the male: female of T1DM is equal. To initiate autoimmunity, an "environmental trigger" is proposed; to study genetics and autoimmune processes, the NOD mouse (female predominance of DM) has been extensively investigated. Whereas almost all immune regulatory approaches succeeded in the NOD mouse, almost 40 years of clinical trials with immune interventions failed to prevent, arrest, or reverse DM for extended periods. This dilemma has raised the question as to whether the $\beta$-cell is the "target of homicide", i.e. autoimmune destruction by $\mathrm{T}$ cells, or has characteristics predisposing to its own demise as the "target of suicide". This presentation discusses these competing narratives and proposes that self-destruct mechanism(s) explain the transitory success of modulating the autoimmune "cleanup" of a primary insult to the $\beta$-cells.

004

\section{MULTI-CENTER RANDOMIZED CROSS-OVER ITALIAN PEDIATRIC SUMMER CAMP: AP VS SAP IN 5-9 YEAR OLD CHILDREN}

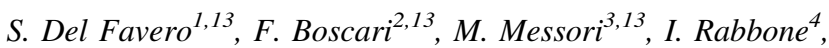
$\overline{R . B_{\text {Bonfanti }}^{5} \text {, A. Sabbion }}{ }^{6}$, D. Iafusco ${ }^{7}$, R. Schiaffini ${ }^{8}$, R. Visentin ${ }^{1}$, R. Calore $^{1}$, Y. Leal ${ }^{1}$, S. Galasso ${ }^{2}$, A. Galderisi ${ }^{9}$, V. Vallone ${ }^{2}$, F. Di Palma ${ }^{3}$, E. Losiouk ${ }^{10}$, G. Lanzola ${ }^{10}$, T. Tinti ${ }^{4}$, A. Rigamonti ${ }^{5}$, M. Marigliano ${ }^{6}$, A. Zanfardino $^{7}$, N. Rapini $^{11}$, A. Avogaro ${ }^{2}$, D. Chernavvsky ${ }^{12}$, L. Magni ${ }^{3,13}$, C. Cobelli, ${ }^{1,13}$, D. Bruttomesso 2,13

${ }^{1}$ Department of Information Engineering, University of Padova, Padova, Italy

${ }^{2}$ Unit of Metabolic Diseases, Department of Internal MedicineDIM, University of Padova, Padova, Italy

${ }^{3}$ Department of Civil Engineering and Architecture, University of Pavia, Pavia, Italy

${ }^{4}$ Department of Pediatrics, University of Turin, Turin, Italy

${ }^{5}$ Pediatric Department and Diabetes Research Institute, Scientific Institute, Hospital San Raffaele, Milan, Italy

${ }^{6}$ Regional Center for Pediatric Diabetes, Clinical Nutrition \& Obesity, Department of Life \& Reproduction Sciences, University of Verona, Verona, Italy
${ }^{7}$ Department of Pediatrics, Second University of Naples, Naples, Italy

${ }^{8}$ Unit of Endocrinology and Diabetes, Bambino Gesu, Children's Hospital, Rome, Italy

${ }^{9}$ Department of Woman's and Child's Health, University of Padua, Padua, Italy

${ }^{10}$ Department of Industrial and Information Engineering,

University of Pavia, Pavia, Italy

${ }^{11}$ Pediatric Diabetology Unit, Policlinico di TorVergata,

University of Rome TorVergata, Rome, Italy

${ }^{12}$ Center for Diabetes Technology, University of Virginia,

Charlottesville, VA, USA

${ }^{13}$ equally contributed

Background and Aims: The Artificial Pancreas (AP) system based on the Modular Model Predictive Control algorithm (MMPC) running on the wearable platform Diabetes Assistant (DiAs, University of Virginia) has been successfully used for 2 months in adults patients reducing time-in-hypo (CGM <70 mg/ $\mathrm{dL})$ and increasing time-in-target $(70-180 \mathrm{mg} / \mathrm{dL})$ with respect to Sensor Augmented Pump therapy (SAP).

Here we report the first outpatient AP trial in 5-8 year old children, using MMPC on DiAs in the PedArPan (PEDiatric ARtificial PANcreas) project.

Methods: Thirty children with type 1 diabetes, 5-8 years old, and their parents were recruited in 5 Italian pediatric centers and completed a randomized cross-over trial in a summer camp.

Glucose control achieved in 3 days of AP use was compared against 3 days of parents-managed SAP.

Results: Overnight (00:00-07:30)

The AP significantly reduced time-in-hypo with respect to SAP, $0.00 \%[0.00-2.22]$ vs $2.19 \%[0-12.3]$, p-value $=0.002$, without significant worsening of time-in-target, $55.97 \%(22.53)$ vs $59.69 \%$ (21.24), p-value $=0.422$, nor of time-in-tight-target $(80-140 \mathrm{mg} / \mathrm{dl})$, $31.27 \%(20.23)$ vs $33.02 \%(19.80)$, p-value $=0.668$.

Overall (00:00-24:00)

A statistically significant 3-fold reduction of time-in-hypo, $1.93 \%[1.17-4.54]$ vs $6.67 \%$ [2.26-11.54], p-value $<0.001$, was recorded with the AP at the expenses of a statistically significant deterioration of time-in-target, $56.79 \%(13.47)$ vs $63.09 \%(10.98)$, $\mathrm{p}$-value $=0.015$.

Parents initiated manual interventions (via correction boluses or temporary basal rate settings) occurred $0[0-0]$ times with the AP vs 3.5[1-7] times in SAP. Closed-loop functioned for $96.97 \%[93.47-98.43]$ of the time.

Conclusions: This study shows feasibility and safety of a wearable MMPC in young children. Next steps will include overnight testing at-home and re-tuning of the algorithm to improve efficacy during daytime.

\section{5}

\section{JDRF MULTI-CENTER 6-MONTH TRIAL OF 24/7 CLOSED-LOOP CONTROL}

\section{B. Kovatchev}

USA

In the summer of 2014, we initiated a two-phase long-term (6 months) trial evaluating at home a control-to-range closed-loop system - the wireless portable Diabetes Assistant (DiAs) developed at the University of Virginia. Phase 1 (1 month) recruited 30 patients with T1DM at 6 centers: the Universities of Virginia, Padova, Montpellier, Santa Barbara, Stanford University, and the Schneider Children's Medical Center of Israel. Phase 2 (5 months) 
continued with $\mathrm{N}=14$ patients at 5 sites; the study was coordinated by the Jaeb Center for Health Research.

Median subject characteristics: age $=45$ years; duration of diabetes $=27$ years; total daily insulin $=0.54(\mathrm{U} / \mathrm{kg} /$ day $)$; basal daily insulin $=0.22(\mathrm{U} / \mathrm{kg} /$ day $) ; 10 / 4$ male/female.

$\mathrm{HbA} 1 \mathrm{c}$ was reduced from $7.22 \%$ at the baseline to $7.03 \%$ $(\mathrm{p}=0.25)$ at the end of the study. This was accompanied by a significant 3-fold reduction in the frequency of hypoglycemia from baseline to the last three months of CGM monitoring $4.1 \%$ vs. $1.3 \%$ ( $\mathrm{p}<0.001)$. Improvement in $\mathrm{HbA1c}$ was highly correlated with the percent time of system use, $r=0.59$; in particular those with above-median system use $(>70 \%$ of the time) achieved $\mathrm{HbA} 1 \mathrm{c}$ reduction of $0.44 \%$, from $7.19 \%$ at the baseline to $6.74 \%$ at the end of study.

We conclude that DiAs technology has matured enough, and is safe and effective for prolonged use at patient's homes. Excellent baseline glucose control (HbA1c) was preserved and further improved in the majority of patients. This was accompanied by a very significant reduction in the frequency and extent of hypoglycemia. Improvement in glucose control was tightly related to the degree of system use.

\section{6}

\section{FLAT-SUGAR (GLYCEMIC VARIABILITY)}

\section{Hirsch ${ }^{1}$}

${ }^{1}$ University of Washington, Seattle, USA

In short-duration diabetes, the DCCT and UKPDS have clearly shown that in glucose control defined as HbA1c can predict microvascular complications and longer term macrovascular disease. However, a closer look at the data suggests there is more than $\mathrm{HbA1c}$, or mean glucose responsible for the complications of diabetes. The "glycemic variability (GV) hypothesis" notes that glucose fluctuation is at least one other etiologic factor. At a cellular level, GV has been shown to result in both reactive oxygen species accumulation and inflammatory activation. There are also several clinical trials suggesting GV is an important mechanism resulting in vascular complications. The problem is to date definitive data for the GV hypothesis is lacking. The study FLATSUGAR (FLuctuATion reduction with inSUlin and Glp-1 Added together) was designed as a feasibility study to prove it is possible to randomize two groups of patients while keeping HbA1c levels equivalent, yet show differences in GV. This was accomplished by using basal bolus insulin (BBI) in one group of "ACCORD-like" patients with type 2 diabetes and basal insulin with the GLP-1 RA exenatide (GLIPULIN) in the other group. The study was successful by showing similar HbA1c levels with more variability with the BBI therapy group. Many secondary endpoints were examined, and both ALT and SAA levels were shown to be lower with GLIPULIN. There was surprisingly little hypoglycemia and no severe hypoglycemia, so not surprisingly there were no differences between the groups in cardiac arrhythmias. FLATSUGAR was a successful study and we now feel it is possible to move forward to perform a definitive outcomes study to examine the role of $\mathrm{GV}$ in the complications of diabetes.

\section{7}

\section{ADJUNCTIVE THERAPY WITH INSULIN IN TYPE 1 DIABETES}

$\underline{\text { S. } \text { Garg }^{1}}$

${ }^{1}$ Pediatrics, University of Colorado Denver, USA
The majority of patients with T1D do not meet A1C goals established by major diabetes organizations. Hypoglycemia risks especially that of severe and a rising incidence of obesity along with associated morbidity in the T1D population limit intensification of insulin therapy in several cases. Noninsulin antihyperglycemic agents may enable T1D patients to achieve target A1C levels while using lower insulin dosages, which may reduce the risk of hypoglycemia. Several recently introduced antihyperglycemic classes hold promise as additional adjunctive therapy options that may help patients overcome barriers to optimal glucose control.

In the recent study from T1D Exchange participants, only $2 \%$ of adults reported taking pramlintide, the only agent approved by the U.S. Food and Drug Administration (FDA) for this purpose. The studies have reported significant improvement in A1c and weight loss with adjunctive pramlintide use. However most patients report significant upper GI side effects and increase in hypoglycemic episodes and thus limiting its use in clinical practice. Gradually increasing the dose of pramlintide may facilitate the use of this drug in T1D.

Studies of metformin have shown significant reductions in weight, insulin dose, and A1C, although in a meta-analysis, A1C reductions were not statistically significant.

Meanwhile, other older agents, such as colesevelam, a-glucosidase inhibitors, and thiazolidinediones (TZDs), have shown little or no promise in terms of glycemic control for patients with T1D.

In pilot studies, the SGLT2 inhibitor dapagliflozin and the GLP-1 receptor agonist liraglutide reduced blood glucose, weight, and insulin dose in patients with T1D. Phase 2 studies with the SGLT2 inhibitor empagliflozin and the dual SGLT1 and SGLT2 inhibitor sotagliflozin, which acts in the gut and the kidney, have demonstrated reductions in A1C, weight, and glucose variability without an increased incidence of hypoglycemia.

The sodium-glucose cotransporter (SGLT) inhibitors and the glucagon-like peptide 1 (GLP-1) receptor agonists may provide an effective approach to reducing some of the risks associated with intensive insulin therapy for T1D. Recent data on liragutide in T1D showed only modest effect and Novo-Nordisk has decided not to file with the FDA for its indication in T1D. Recent reports on DKA with SGLT 2 inhibitors and the FDA warning have cautioned the use of such medications in T1D. The drugs having combo SGLT $1 \& 2$ inhibitor effects may eliminate the DKA risk but are being currently evaluated. The role of many of these medications will be discussed during my talk.

\section{8}

\section{TOWARDS PERSONALIZED MEDICINE: THE IMPACT OF BLACK ETHNICITY ON METABOLIC DYSREGULATION IN EARLY TYPE 2 DIABETES}

\section{$\underline{\text { S. Amiel }}{ }^{1}$ \\ ${ }^{1}$ London, United Kingdom}

The International Diabetes Federation predicts that the fastest rise in prevalence of type 2 diabetes over the next decade will be in its African region. Although current rates of type 2 diabetes are relatively low in sub-Saharan African countries, people of African origin living abroad have significantly higher rates of diabetes than people of white ethnicity in the same environment. In on study in North East London, England, rates of diabetes were as high in the black communities as in the South Asians. In a recent study in South London, where approximately $20 \%$ of local 
residents are of black ethnicity, about $40 \%$ of people with new onset diabetes came from that group. They were younger at diagnosis and had higher HbA1c. By two years, HbA1c was not different between groups, but people of Black West African and Caribbean background were prescribed more medication to achieve this. Till now, relative cardio-protection has been noted in Black people with diabetes, associated with differences in lipid profile, but newer studies from the US suggest that this has changed. A greater prevalence of hypertension is thought to be associated with an observed higher risk of stroke. There is also a higher risk of atypical ketosis prone diabetes.

Differences in metabolic phenotype should help us tailor therapeutic and preventive interventions so it is important to understand them. There is evidence for hyperinsulinaemia in young people at high risk for diabetes in the black populations but the mechanisms of this are not fully understood. Whether early hyperinsulinism drives earlier diabetes through earlier beta cell exhaustion is also not proven. Studies are on-going to clarify the role of insulin resistance/sensitivity, both for carbohydrate and fat metabolism, and intra-organ fat deposition in the ethnic differences in metabolic phenotype. It is expected that such studies will underpin more focussed interventions to reduce the burden of type 2 diabetes in this high risk population.

009

\section{ARTIFICIAL PANCREAS PSYCHOSOCIAL MEASURES PROJECT}

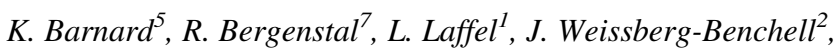

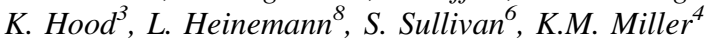

${ }^{1}$ Genetics and Epidemiology Section, Harvard Medical School, Joslin Diabetes Centerl

${ }^{2}$ Northwestern University's Feinberg School of Medicine, Ann and Robert H. Lurie Children's Hospital of Chicago, Chicago, Illinois 60611, USA

${ }^{3}$ Psychiatry \& Behavioral Sciences, Stanford University School of Medicine, USA

${ }^{4}$ Jaeb Center for Health Research, 15310 Amberly Drive,

Tampa FL. 33647

${ }^{5}$ Bournemouth University, United Kingdom

${ }^{6}$ The Leona M. and Harry B. Helmsley Charitable Trust, 230

Park Avenue, Suite 659, New York, NY 10169

${ }^{7}$ International Diabetes Center, 3800 Park Nicollet Blvd., Minneapolis, MN 55416

${ }^{8}$ Partner and Scientific Consultant

Background: Addressing and improving psychosocial outcomes complement biomedical improvements, and looking to the future, are crucial to enhance patient acceptance of automated insulin delivery systems.

Methods: Development of novel psychosocial patient reported outcome measures for children with T1D, adolescents with T1D, parents, adults with T1D and partners to assess the impact of automated insulin delivery systems. Focus groups and interviews with PWD, as well as surveys of HCPs and collaboration with the research community are informing content with piloting as the next phase. We will present preliminary qualitative data and HCP survey data at the workshop and invite delegates to discuss these in the context of device development and future use.

Results: Data will be used to achieve the primary outcome of developing five novel psychosocial patient reported outcome (PRO) measures to enable regulatory approvals bodies and reimbursement agencies to critically appraise PROs alongside medical efficacy and safety.

Conclusions: Input from collective stakeholders are needed to inform the PROs in order to maximize their future utility in appraising psychosocial outcomes of automated insulin delivery systems.

\section{0}

\section{BIOSIMILARS IN PUMPS AND PENS, DO THE DEVICES MATTER?}

\section{Kuhlmann ${ }^{1}$}

${ }^{1}$ Nephrologe, Diabetologe, Hypertensiologe Ärztlicher Direktor Chefarzt Innere Medizin - Nephrologie Vivantes Klinikum im Friedrichshain Landsberger Allee 4910249 Berlin

With the expiry of patent protection for several originator insulin analogue molecules, the availability of biosimilar insulin analogues will increase in the future. Current EMA guidelines for the approval of biosimilar insulin analogues specifically address the requirements for demonstration of comparability in structure, pharmacokinetics and pharmacodynamics, efficacy, safety, and immunogenicity of the active ingredient as well as the final formulated biosimilar product. However, insulin application devices are not addressed in these guidelines, although these devices, be it a pre-filled syringe, pen, or pump, are of particular importance for dosing accuracy and reproducibility as well as long-term patient compliance and adherence. Since the combinations of insulin and device may differ widely in their dosing characteristics, it cannot be assumed that an insulin biosimilar will be compatible with an existing administration device. In general, the EMA Medical Devices Directive (MDD) applies to all general medical devices. As a minimum requirement for an insulin biosimilar it is plausible that the device through which a biosimilar is administered must at least be able to match the reference medicine's device for convenience and comfort. Inferior usability could reduce treatment adherence and product uptake by the patients. On the other hand, current guidelines for biosimilars may be interpreted as to leave freedom to improve on the reference product's delivery devices. Therefore the design and user experience of the delivery device may serve as a key market differentiator to the reference or competing products even if they are clinically equivalent.

\section{1}

\section{INSULIN ACCESS IN THE DEVELOPING WORLD}

\section{J. Saldanha ${ }^{1}$}

${ }^{1}$ Saldanha and Santamore Pharma Consultants, Private Practice, Bel Air, USA

Much of the developing world has seen a significant rise in the treatment of diabetes primarily due to increasing awareness, which has led to larger rates of diagnosis and more willingness to seek treatment. Access to insulin is therefore more important than ever. The three major insulin manufacturers continue to dominate the landscape despite the availability of biosimilars in many countries. The market share they enjoy is similar to the more developed world. Many parties play a role in the acceptance and use of biosimilar insulins, from the respective Ministries of Health who approve the products and often provide them 
free of charge to patients, to the health care providers, and the ultimate consumers.

There are several factors that have contributed towards this situation. Barriers to entry and sustainability in the insulin business are significant. Pharmaceutical companies use a different business model and market segmentation when it comes to insulins in the developing world. Some of the factors that will be addressed include intellectual property, financial, clinical/regulatory, pricing, distribution, local partnerships and local manufacturing.

\section{2}

\section{EVIDENCE BASE FOR CGM}

\section{H. De Vries}

Netherlands

Some 15 years after its introduction in clinical practice, Continuous Glucose Monitoring (CGM) has become an established modality for diabetes treatment. There is sound evidence that patients can lower their HbA1c when using this technology, and spend less time in hypoglycaemia. Emerging evidence supports the notion that CGM can decrease the incidence of severe hypoglycemia, although the evidence for this indication is less abundant and has been criticized. The added value of CGM during pregnancy is unclear, but larger trials are under way. All this evidence has translated into a wide range of reimbursement status in various countries. In many countries reimbursement is still on a case-by-case basis. In those countries where there are formal reimbursement criteria, the interpretation and translation of the evidence base varies. An overview of the evidence supporting the use of CGM and its reimbursement will be given.

\section{3}

\section{A BREAKTHROUGH IN GERMANY?}

\section{N. Hermanns $^{I}$ \\ ${ }^{1}$ Research Instiute Diabetes FIDAM, Bad Mergentheim, Germany}

In Germany, the Federal Joint Committee (G-BA) is responsible for legally binding decisions regarding the reimbursement of medical procedures (e.g., drugs, non-drug interventions, medical devices, etc.). Since 2004, the German Institute for Quality and Efficiency in Health Care (IQWiG) assesses the advantages and disadvantages of medical procedures on behalf of the G-BA. The IQWiG uses methods of evidence-based medicine for conducting systematic searches for studies which provide sufficiently reliable results, and for selecting and assessing those studies. Based on this research IQWiG produces evidence-based reports. IQWiG reports usually have a large impact on reimbursement decisions made by the Federal Joint Committee.

In May 2015, the IQWiG released a report about potential benefits of "continuous interstitial glucose monitoring (CGM) with real-time measurement devices in insulin-dependent diabetes mellitus". The IQWiG included a total of 15 studies. The IQWiG report concluded that while there was evidence that CGM is beneficial with regard to HbAlc improvement in adults with type 1 diabetes, there was less clear evidence for the avoidance of severe hypoglycemia in this patient group. Furthermore, the IQWiG found an indication that CGM might be beneficial with regard to hypoglycemia avoidance and $\mathrm{HbA} 1 \mathrm{c}$ improvement in children with type 1 diabetes.
Given the rather positive conclusion of the IQWIG report regarding the advantages of CGM there is a guarded optimism in Germany that reimbursement of decision will significantly improve. But the final assessment by the Federal Joint Committee regarding reimbursement of CGM use is still pending. In this presentation an overview about key results of the IQWIG Report will be presented along with the current development of the reimbursement situation of CGM in Germany.

\section{4}

\section{A NICE REASSESSMENT}

\section{P. Hammond}

Harrogate, United Kingdom

Continuous glucose monitoring (CGM) is currently not funded routinely in the UK. The National Institute for Health and Care Excellence (NICE) has previously published technology appraisals (TA) for insulin pump therapy, but has not carried out a TA for CGM. Commissioners have a legal mandate to fund NICE TA recommendations, but not other NICE recommendations. NICE are due to publish a diagnostic assessment review of integrated sensor augmented pump systems in early 2016 but it is unlikely to have a significant impact on funding of these technologies. In August 2015 NICE published updated clinical guidelines for the management of children and young people with diabetes (NG18), and adults with type 1 diabetes (NG17). In NG18 NICE advised that children and young people with hypoglycaemic problems should be offered CGM, which is the strongest form of recommendation. In NG17 the advice was that CGM should be considered for adults with hypoglycaemic problems, a weaker recommendation. NICE performed a number of health economic analyses to define the cost-effectiveness of CGM for optimising control in those with an elevated HbA1c. Following these analyses it was recommended that CGM be considered if $\mathrm{HbA} 1 \mathrm{c}>75 \mathrm{mmol} / \mathrm{mol}$ [9\%] despite testing at least 10 times a day, and continued only if $\mathrm{HbA} 1 \mathrm{c}$ can be sustained at or below $53 \mathrm{mmol} / \mathrm{mol}(7 \%)$ and/or there has been a fall in $\mathrm{HbA} 1 \mathrm{c}$ of $27 \mathrm{mmol} / \mathrm{mol}$ (2.5\%) or more. This guidance will form the basis for ongoing discussions about funding CGM for small groups of patients with type 1 diabetes.

\section{5}

\section{WILL MEDICARE DELIVER?}

\author{
C. Graham
}

${ }^{1}$ Dexcom, Global Access, San Diego, USA

Medicare is the largest insurance program in the US, with over $50,000,000$ beneficiaries; it is intended for people over the age of 65 or are disabled.

Despite the recognized clinical benefits, and the fact that nearly all commercial payors in the US have positive coverage policies for Type 1 patients and CGM, the fact that Medicare does not have provide coverage is perplexing. The coverage process is dictated by Federal Law, and is therefore bound by statute, and to a certain degree by interpretation by the Centers for Medicaid and Medicare Services (CMS), who administers the program.

All real time CGM devices available in the US require SMBG confirmation according to FDA labeling. CMS has determined that CGM devices do not meet the statutory definition of "durable 
medical equipment benefit category" and has labeled real time CGM a "precautionary" device since it requires SMBG confirmation. Thus, without a "benefit category" determination, CGM cannot be considered for coverage.

There are multiple efforts to resolve this Medicare issue for CGM:

1. Congressional legislation that will create a new benefit category and mandate coverage for CGM.

2. Individual Medicare beneficiaries that are appealing their denials thru the judicial process.

3. Manufacturers may independently pursue administrative action or non-adjunctive status.

The efforts to get Medicare coverage, the role of large national stakeholders/coalitions and expected timelines will be reviewed in this presentation. Data supporting the clinical value of CGM in the elderly population will also be reviewed.

016

\section{CGM IN CHILDREN AND ADOLESCENTS USING MULTIPLE DAILY INJECTIONS: QUALITY OF LIFE AND METABOLIC CONTROL}

\section{Cherubini}

Italy

Checking blood glucose levels on a daily basis is one of the landmarks for diabetes care. The use of real time continuous glucose monitoring (CGM) has been widely available for use by people with type 1 diabetes for more than 10 years. Because of imprecision of historical models and resulting safety concerns, CGM system to date is approved for use adjunctive to SMBG in patients with type 1 diabetes.

Newest products have shown a great improvement in accuracy and portability. When used on near-daily basis they are associated with lower HbA1c, a greater percentage of blood glucose values in the target range, and reduced time spent in hypoglycemia if compared with those who use traditional self-monitoring blood glucose (SMBG). Nevertheless, recent studies on large clinical databases have shown that only a small proportion of children and adolescents are using CGM in clinical practice, either if they are treated with multiple daily injections or insulin pumps.

Relatively few studies considered the use of CGM in subjects treated with Multiple Daily Injections (MDI), mainly in children and adolescents. Moreover little evidence about the impact of CGM on Health-Related Quality of Life (HRQOL) and treatment satisfaction of children with type 1 diabetes treated with MDI and their parents exists. New studies are needed to investigate if in the future CGM can replace SMBG in children and adolescents treated with MDI.

017

\section{NIGHTSCOUT EXPERIENCE IN ITALIAN CHILDREN WITH TYPE 1 DIABETES}

\author{
R. Bonfanti ${ }^{1}$, G. Frontino ${ }^{1}$, A. Rigamonti ${ }^{1}$, \\ $\overline{\text { C. Bonura }}^{\text {, }}$ V. Favalli ${ }^{1}$ \\ ${ }^{1}$ ospedale san raffaele, pediatric diabetology, milano, Italy
}

Aim: To evaluate glucose control and rate of acute complications in a small cohort of of very young children with type 1 diabetes mellitus (T1DM) using sensor-augmented pumps (SAP) linked to a Nightscout system.

Methods: Eight children (3 males, 5 females). Mean age: $6.4 \pm 1.72$ years. Age at diabetes onset: $4.19 \pm 1.36$ years. BMI z-score: $0.28 \pm 1.06$ (mean BMI $17.1 \pm 2.26 \mathrm{~kg} / \mathrm{m} 2$ ). All children used an Animas Vibe and Dexcom G4 platinum SAP linked to a Nightscout system for 15 days. Mean HbA1c $6.6 \%$.

Results: During Nightscout use, approximately 4500 glucose values were available. Mean sensore glucose was $154 \mathrm{mg} / \mathrm{dL}$ $( \pm 54)$. Distribution analysis showed $59.75 \%, 4 \%$, and $36 \%$ of values in the target $(80-180 \mathrm{mg} / \mathrm{dL})$, below the target, and above the target respectively. In this brief period there were no episodes of severe hypoglycemias or diabetic chetoacidosis.

Conclusions: School management of young children with type 1 diabetes mellitus may often represent an obstacle in maintaining the glucose control that is achieved at home under parental supervision. The opportunity to remotely access a child's continuous glucose monitor by using Nightscout may allow a more pro-active management even when the child and caregiver are apart. By providing continuity to domestic glucose control, glucose excursions and consequent interruptions of daily school activities may be significantly reduced, thereby allowing the child to participate and perform as his/her peers. Long-term studies with larger cohorts are warranted to assess the potential of Nightscout to improve the quality of life for the child and caregivers and reduce the number of acute complications.

\section{8}

\section{NON-CONVENTIONAL USE OF CGM}

G. Grassi

Italy

Menstual cyclicity, sleep efficiency and unplanned physical activity: glycemic response in women suffering from T1DM.

Scientific evidences available in literature regarding the correlation between female menstrual cycle, sleep quality and glycemic response are heterogeneous.

Our study aim is to point out further correlations between the variables mentioned in a sample of type 1 diabetic patients, in order to find which ones deserve further investigations to become clinically relevant.

Endpoints: statistically significant differences in mean blood glucose (MBG), glycemic variability (GV), insulin intake (II) during menstrual cycle phases; correlation between sleep efficiency (SE), unplanned physical activity (PA) and glycemic response throughout the menstrual cycle.

10 T1DM women followed up at the A.O.U. Città della Salute e della Scienza or the A.O. Ordine Mauriziano in Turin, in CSII or MDI therapy, were recruited.

RT-CGM data, activity and sleep tracker (iHealth $\left.{ }^{\circledR}\right)$ to monitor unplanned PA and sleep were collected

To establish ovulation, a digital test was used in order to notice the urinary $\mathrm{LH}$ peak.

During the 120 days continuous evaluation, we analyzed: glucose values, Insulin intake, SE, nocturnal awakenings, Steps/ day, PSQI test (Pittsburg Sleep Quality Index).

In the studied population, we confirm: Increase of MBG in early luteal phase (ELP) or periovulatory phase (PP), Increase of GV in ELP or PP, Increased number of hypoglycemic episodes $(<70 \mathrm{mg} / \mathrm{dl})$ in early or late follicular phase and increased number of hyperglycemic episodes $(>180 \mathrm{mg} / \mathrm{dl}$ ) in early luteal phase, Not significant variations of II, Minimal variations 
regarding insulin intake, No significant intra-individual variations in SE throughout the menstrual cycle, Coherent results in a compared valuation of PSQI scores and SE, decrease of the number of steps/day in PP and luteal phase.

\section{9}

\section{TIDEPOOL: AN OPEN SOURCE, EXTENSIBLE PLATFORM FOR DIABETES MANAGEMENT AND RESEARCH}

\section{H. Look $^{1}$ \\ ${ }^{1}$ Tidepool, Corporate Research and Development, Palo Alto, USA}

Tidepool is a non-profit, open source organization building a platform and applications for patients, providers and researchers. Founded as a Silicon Valley startup, Tidepool's mission is to deliver technology that helps reduce the burden of managing Type 1 diabetes.

Tidepool has built a modern, extensible software platform that is freely available in source code form. The secure Tidepool Platform exposes modern application programming interfaces, enabling application developers, researchers and device makers to build custom application that take advantage of diabetes device data.

Tidepool has also built applications that leverage the Tidepool Platform. The Tidepool Uploader is a Chrome extension that enables uploading of data from blood glucose meters, continuous glucose monitors and insulin pumps via Mac or PC to the Tidepool cloud. Blip is a web-based application that integrates data from many different diabetes and fitness devices and presents it in intuitive, actionable form. Blip Notes is a companion mobile application that allows users to enter life context relevant to their diabetes therapy. Nutshell is a mobile application that keeps track of meal and exercise events and enables contextual recall and therapy optimization.

Tidepool uses "lean startup" and agile software development methodologies. Tidepool engages early and often with many end users, iterating quickly through functionality and user experience concepts with the goal of achieving the most usable and intuitive designs. Tidepool has built a regulatory quality system that leverages modern software development practices while meeting regulatory requirements.

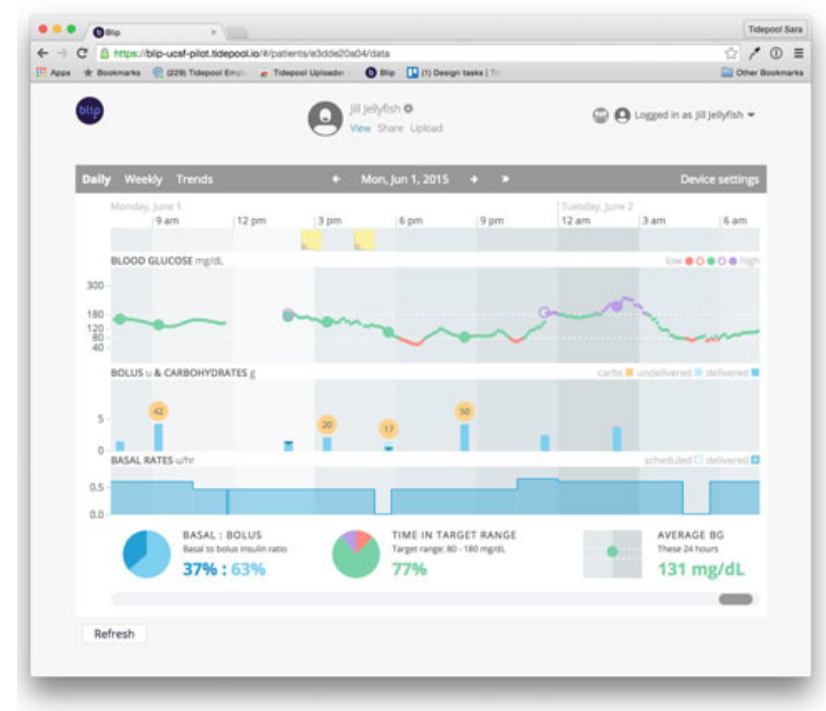

020

\section{ADVISORS: GADGETS OR REAL HELP IN DIABETES CARE?}

$\underline{\text { S. Franc }}$

Paris, France

What is the real interest for patients of "digital advisors" downloaded to smartphones in the form of "apps" or already incorporated in certain glucose meters or insulin pumps?

Diabetic patients currently have access to over 1100 Apps, both free and paid; in most cases, these apps are not required to meet any quality criteria since they are not in fact declared as "medical mobile apps". They vary greatly in terms of type and quality, and very few have so far been evaluated. The majority of these apps provide patients with support and/or advice functions. They collate certain data (capillary BG values, insulin doses, physical activity and levels of carbohydrates currently being consumed) and present them in legible fashion in the form of an electronic notebook. In addition, some apps allow sharing of this data with HCPs, either in real time, or subsequently, and allow feed-back to be received from the latter (usually via text message or automatic reminders). The aim of this first class of apps is to assist patients with their day-to-day choices but without necessarily seeking to impact glycaemic control.

The second class of apps, to which are attached more expectations but also more concerns, proposes adjustment of treatment and thus of insulin doses in particular. Most of these higher-risk apps, which must comply with the FDA definition of "medical devices", currently provide no protection against - and may in fact actively contribute to - incorrect or inappropriate dose recommendations. In all, only 20 or so such downloadable "digital advisors" have undergone robust evaluation and have for the most part demonstrated significant impact on the primary endpoint. Among these systems, those enabling interaction between patient and HCP appear the most effective. Of the dozen or so systems targeting HbA1c, 8 showed a significant improvement of close to $1 \%$ vs. controls. These studies were nevertheless marked by considerable drop-out rates and there is currently little information available on the continued use of these advisors beyond completion of the evaluation phase. One of the most sophisticated of these systems is the Diabéo system, which has already demonstrated a $0.9 \%$ reduction in HbA1c at 6 months in DT1 patients with chronic imbalance and is currently undergoing clinical study with medico-economic evaluation over 2 years in 700 patients on MDI or insulin pump.

Bolus calculators incorporating medical devices, and which may also be classified as "digital advisors", are required to meet quality criteria imposed in this sector by the regulatory authorities. Such bolus calculators have already demonstrated their value in compliance with both standard insulin treatment and pump therapy, particularly in patients with poor numeracy skills, and appear to have a favourable effect on glycaemic control.

This new category of "digital advisor" apps in the widest sense may, subject to robust clinical evaluation, prove extremely useful in diabetes management.

\section{1}

BEYOND THE ARTIFICIAL PANCREAS: TRANSLATING CLOSED-LOOP TECHNOLOGY INTO ADVISORY SYSTEMS AND CLOUD ANALYTICS

$\underline{\text { S. Patek }}$

Charlottesville, USA 
With research groups around world reporting encouraging results in outpatient clinical trials, the technical feasibility of the Artificial Pancreas (AP) is now firmly established. In the process, the research community has validated a number of key models and component technologies, including mathematical/simulation models of human physiology and sensors and drug delivery devices that facilitate automated signal processing, both leading to broadly applicable procedures for real-time and retrospective estimation of the patient's metabolic state. Indeed, the stage is now set to positively impact the lives of patients in the form of "decision support" technologies that make the best possible use of available data and are agnostic to the mode of insulin delivery. Examples include: (i) smart bolus/treatment wizards, including treatment advice in response to acknowledged physical activity, informed by continuous state estimation and real-time assessment of insulin sensitivity, (ii) meal and exercise detection algorithms that provide cues to the patient to consider treatment options, (iii) fault detection algorithms that increase patient situational awareness, (iv) risk profiling and stratification procedures that provide insights into patterns of hyper- and hypoglycemia in relation to self-treatment behavior and activities of daily living, and even (v) algorithms for individualized treatment optimization based on retrospective analysis of patient data in the cloud. This talk will argue that AP and decision support research activities are highly synergistic, highlighting recent technology developments at the University of Virginia (UVA) and featuring preliminary results from an ongoing feasibility trial with decision support components implemented atop the UVA Diabetes Assistant (DiAs) platform.

022

EXERCISE AND THE ARTIFICIAL PANCREAS: IS IT THE MOST DIFFICULT "HURDLE"?

M. Riddell

Canada

Exercise promotes health in people with diabetes; however, it can complicate blood glucose control. Factors affecting blood glucose fluctuations include exercise type, intensity and duration of the activity as well as the amount of insulin and food in the body when the exercise is performed. This lecture will discuss the main regulators of the blood glucose responses to different types of exercise in health and diabetes and highlight some of the main "hurdles" that exercise will pose for the development of the artificial pancreas.

023

\section{AUXILIARY SIGNALS FOR THE CONTROL OF PHYSICAL ACTIVITY}

M. Breton ${ }^{1}$

${ }^{1}$ UVA, Center for Diabete Technology, Charlottesville, USA

People with type 1 diabetes (T1D) are at continual risk for hypoglycemia, which is recognized as one of the principal impediments to optimal glycemic control. Physical activity in T1D has been associated with many health benefits such as reduced cardiovascular risks and improved psychological well-being, but also leads to imbalance between hepatic glucose production and muscle glucose disposal, increased insulin sensitivity and impaired counterregulatory response. As a probable consequence most adult with T1D partake in less PA than their non-diabetic counterparts.

Automated closed-loop control (CLC) could be a very significant tool in improving this situation; and CLC systems have been tested against a variety of glycemic disturbances with few proving as challenging as exercise: e.g. in a multisite study (adolescents and adults), we showed a 3 fold reduction of hypoglycemia overall and up to 6 fold post exercise and during the night afterwards, but no change during exercise. Using heart rate and accelerometers to inform the CLC system we showed a significantly reduced glycemic drop during exercise (adolescents and adults, inpatient and outpatient). Furthermore, analysis of this data allowed the development of mathematical models capable of predicting exercise induced glucose drop using recent history, leading to exercise advisory systems.

In conclusion, the advent of easy to use, cheap, devices capable of tracking physical activity, and the emergence of smart phone based medical devices to combine these signals with glucose and insulin records, are likely to enable patient specific automated and advisory systems dedicated to enabling safe exercise in T1D.

\section{4}

\section{CONTROLLING GLUCOSE LEVELS BY PERSONALIZED NUTRITION TAILORED TO THE MICROBIOME}

$\frac{\text { E. Segal }}{\text { Israel }}$

Elevated postprandial blood glucose levels constitute a global epidemic and a major risk factor for prediabetes and type-II diabetes, but existing dietary methods for controlling them have limited efficacy. Here, we continuously monitored week-long glucose levels in a 800-person cohort, measured responses to 46,898 meals, and found high variability in the response to identical meals, suggesting that universal dietary recommendations may have limited utility. We devised a machine-learning algorithm that integrates blood parameters, dietary habits, anthropometrics, physical activity, and gut microbiota measured in this cohort and showed that it accurately predicts personalized postprandial glycemic response to real-life meals. We validated these predictions in an independent 100-person cohort. Finally, a blinded randomized controlled dietary intervention based on this algorithm resulted in significantly lower postprandial responses and consistent alterations to gut microbiota configuration. Together, our results suggest that personalized diets may successfully modify elevated postprandial blood glucose and its metabolic consequences.

025

WHAT APPS CAN DO TO SUPPORT CARBS COUNTING?

M. Gillon-Keren

Israel

Smartphone applications (apps) are widely available, increasingly used by patients and clinicians, and have the potential to improve diabetes care and self-management. Diabetes apps can facilitate self-monitoring by tracking and logging health information and reporting graphs and statistics based on patient input. The main nutrition features are food journals and databases of food 
composition, including information from restaurants and fast food chains and barcode scanners. The potential benefit of these apps is their ability to help people with diabetes to evaluate the amount of carbohydrates in the food they consume (carb counting).

Carb counting is crucial for diabetes management, particularly when using rapid-acting insulin, as the dose depends on the amount of carbohydrates consumed in a meal. Yet, accuracy in carb counting is not necessarily sufficient to determine the correct dose of insulin, as other factors influence the postprandial blood level, including glycemic load, dietary fiber, fat and/or protein content and personal glycemic index. Few apps address adjustment of insulin doses according to personal past experience or crowd wisdom instead of carb counting alone. Several of the carb counting and insulin bolus calculation apps provide no protection against, and may actively contribute to, incorrect or inappropriate dose recommendations that place patients at risk of hypoglycemia/hyperglycemia.

The Diabetes team should exercise caution in recommending unregulated insulin dose calculators to patients and address app safety as part of self-management education. The prevalence of errors attributable to incorrect estimation of carbs underlines the importance of clinical input during app design.

\section{6}

\section{EPIDEMIOLOGY OF INSULIN PUMP THERAPY IN ITALY: THE IMITAS STUDIES}

D. Bruttomesso $^{1}$, G. Lepore $^{2}$, R. Bonfanti ${ }^{3}$, L. Bozzetto ${ }^{4}$,

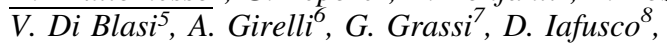
I. Rabbone ${ }^{9}$, R. Schiaffini ${ }^{10}$, L. Laviola ${ }^{11}$

${ }^{1}$ University of Padova, Department of Medicine- DIMEDMetabolic Diseases, Padova, Italy

${ }^{2}$ A.O. Papa Giovanni XXIII, Unit of Endocrine Disease and Diabetology, Bergamo, Italy

${ }^{3}$ IRCCS Ospedale San Raffaele, Pediatric Department and Diabetes Research Institute OSR-DRI, Milano, Italy

${ }^{4}$ Federico II University, Department of Clinical Medicine and Surgery, Naples, Italy

${ }^{5}$ ASL Salerno, Department of Endocrinology and Diabetology, Salerno, Italy

${ }^{6}$ A.O. Spedali Civili, Unit of Diabetology, Brescia, Italy

${ }^{7}$ A.O. Citta' della Salute e della Scienza, Division of Endocrinology and Metabolism, Turin, Italy

${ }^{8}$ Second University of Naples, Department of Pediatrics, Naples, Italy

${ }^{9}$ University of Turin, Department of Pediatrics, Turin, Italy

${ }^{10}$ IRCCS Bambino Gesu' Children's Hospital, Unit

of Endocrinology and Diabetes, Rome, Italy

${ }^{11}$ University of Aldo Moro- Bari, Internal MedicineEndocrinology- Andrology- Metabolic Diseases- Department of Emergency and Organ Transplantation, Bari, Italy

Background: continuous subcutaneous insulin infusion (CSII) is increasing due to effectiveness and improved technology.

Aim: to evaluate the current status of CSII in Italy.

Materials and Methods: clinical features, pump technology, patient management and metabolic control of CSII patients were investigated in two questionnaire-based surveys to 272 Italian diabetes centers.

Results: 217 centers $(79,8 \%)$ answered the first questionnaire giving information on 10,152 patients, $98.2 \%$ with type 1 diabetes mellitus, $81,4 \%$ adults, $57 \%$ female, $61 \%$ with a conventional pump and $39 \%$ with a sensor-augmented pump. CSII advanced functions were used by $68 \%$ of patients, and glucose sensors were used 12 days/month on average. Fifty-eight percent of centers had > 20 CSII patients. Main indication for CSII was poor glucose control. Dropout was mostly due to pump wearability or bad glucose control. A complete dedicated team was available in $23 \%$ of adult centers and in $53 \%$ of pediatric units. In the second survey, data were obtained from 114 centers on 6,886 patients with overall characteristics comparable to the previous one. Mean HbA1c was $60.1 \pm 10.7 \mathrm{mM} / \mathrm{M}$. Lower HbA1c levels were associated to age 6-13, pregnancy in the last year, use of advanced functions, sensor use $\geq 10$ days/month, availability of a dedicated team. $6,1 \%$ of patients had $\geq 1$ severe hypoglycemic event over the previous year.

Conclusions: Advanced patient skills, compliance to sensor use and an integrated team approach are associated to better metabolic outcomes in Italian CSII patients.

\section{7}

\section{PUMP FAILURE AND MALFUNCTIONS IN CHILDREN AND ADOLESCENTS WITH TYPE 1 DIABETES IN INSULIN PUMP THERAPY: AN ITALIAN PROSPECTIVE STUDY}

I. Rabbone $^{1}$, N. Minuto $^{2}$, G. Ignaccolo ${ }^{1}$, R. Bonfanti ${ }^{3}$,

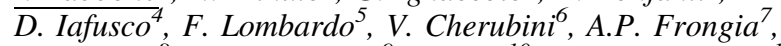
${\text { A. } \text { Pistorio }^{8}, \text { M. Marigliano }^{9}, \text { S. Toni }^{10} \text {, A.E. Scaramuzza }}^{11}$, P.F. Diabetes Study Group of ISPED ${ }^{1}$

${ }^{1}$ University of Turin, Pediatrics, Turin, Italy

${ }^{2}$ IRCCS, Pediatrics, Genova, Italy

${ }^{3}$ HSR, Pediatrics, Milan, Italy

${ }^{4}$ SUN, Pediatrics, Napoli, Italy

${ }^{5}$ University of Messina, Pediatrics, Messina, Italy

${ }^{6}$ Salesi Hospital, Pediatrics, Ancona, Italy

${ }^{7}$ Brotzu Hospital, Pediatrics, Cagliari, Italy

${ }^{8}$ IRCCS, Epidemiologia e Biostatistica, Genova, Italy

${ }^{9}$ University of Verona, Pediatrics, Verona, Italy

${ }^{10}$ Meyer Hospital, Pediatrics, Firenze, Italy

${ }^{11}$ Sacco Hospital, Pediatrics, Milan, Italy

Background and Aim: Current literature regarding insulin pump-associated adverse events including pump malfunction, infusion set/site issues is discussed. We evaluated metabolic and nonmetabolic adverse events in a large cohort of children and adolescents with type 1 diabetes (T1D), using insulin pump therapy.

Methods: Data have been collected on patients younger than 19 years, starting insulin pump therapy before December $31^{\text {st }} 2013$. For each patient age, disease duration, date of insulin pump therapy initiation, insulin pump model, breakdown/malfunction/pump replacement yes/no and reason, catheter/infusion set failures have been considered prospectively for the calendar year 2014.

Results: Data have been returned by 20 pediatric Centers belonging to the Italian Diabetes Study Group about 916 T1D children and adolescents using insulin pump. During 2014, the most frequent infusion set and site problems were bubbles (38.2\%), kinking (13\%), leakage (10.3\%), tunneling (10.2\%), blockage $(8.8 \%)$, bleeding $(11 \%)$, lipohypertrophy $(7.1 \%)$, infection $(1.4 \%)$. Pump device has been replaced in $19.2 \%$ of patients: $82 \%$ for pump breakdown/malfunctions and 18\% for 'physiologic' replacement after warranty. HbA1C mean value was $7.6 \%$ for the whole population. No relationship between pump replacement and $\mathrm{HbA} 1 \mathrm{C}$ value was found. Only $1 \mathrm{DKA}$ has been recorded due to pump failure. No severe hypoglycemia has been recorded due to pump or infusion set malfunction. 
Conclusions: Despite frequent infusion set problems, pump breakdown/malfunction and consequent replacement and metabolic adverse events in a large cohort of pediatric Italian patients with T1D are not as frequent as previously reported. Continuous educational programs are necessary for pump therapy management.

028

\section{USE OF PLGS IN ITALIAN CHILDREN WITH TYPE 1 DIABETES: THE CAMPUS STUDY}

A. Scaramuzza ${ }^{1}$, on behalf of CampUs Study Group ${ }^{2}$

${ }^{1}$ Luigi Sacco, Pediatrics, Milano, Italy

${ }^{2}$ SIEDP, Italy, Milano, Italy

MiniMed 640G has a predictive algorythm (SmartGuard) that suspends insulin infusion when patient glucose levels are still in normal range to avoid reaching a pre-set low limit. MiniMed 640G safety and efficacy in adolescents with T1 diabetes was evaluated by analyzing CareLink data. Thirty-eight type 1 diabetes $>6$ months adolescents (14-20 years) on Minimed 640G, have been evaluated during a 5 days camp. Mean meter BGs, mean SGs, sensor SD, sensor MARD, \% time spent in hypo $(<70 \mathrm{mg} / \mathrm{dl})$, \% time spent in hyper $(>160 \mathrm{mg} / \mathrm{dl})$, episodes of moderate to severe hypo, episodes of hyper or DKA have been analyzed. SmartGuard has been disabled (SG-OFF) on the $2^{\text {nd }}$ day from 9.30 am to $10 \mathrm{pm}$ and the data have been compared with those of the 3rd day with SG-ON. The level of exercise and type of food intake was comparable between the 2 days.

During the 5 days patients carried out all activities without suffering episods of severe hypo or DKA. The average BG (Bayer Contour Next Link 2.4 meter) was $152.5 \pm 25.3 \mathrm{mg} / \mathrm{dl}$, while average SG was $147.7 \pm 1.1$ (Pearson $=0,75$, MARD $9.2 \pm 7.3 \%$ ). Time spent in hypo was $0.4 \pm 0.5 \%$, while time in hyper was $17.0 \pm 8.8 \%$.

Thirty patients have turned SG off when requested and the comparison between the day SG-OFF vs day SG-ON showed comparable mean SG $(149.7 \pm 21.5 \mathrm{mg} / \mathrm{dl}$ vs $143.1 \pm 18.0 \mathrm{mg} /$ $\mathrm{dl}$ ), while glucose variability (SD) was significantly lower with SG-ON (51.4 \pm 11.9 vs $45.6 \pm 10.1, p=0.044)$.

No difference was observed regarding time spent in hypo $(0.5 \pm 0.9 \%$ vs $0.4 \pm 0.4 \%)$, though with less events per day $(1.9 \pm 1.8$ vs $1.3 \pm 1.0, p=0.084)$ and on average $5.0 \pm 1.8 \mathrm{SG}$ activations/patient/day occurred. Hyperglycemic events were $7.0 \pm 3.4$ vs $6.1 \pm 3.3$, whilst time spent in hyperglycemia was significantly reduced with SG-ON $(19.2 \pm 11.9 \%$ vs $13.5 \pm$ $10.0 \%, \mathrm{p}=0.048$ ). MiniMed $640 \mathrm{G}$ in adolescents with $\mathrm{T} 1$ diabetes was safe and effective. SmartGuard contributed to reduce both hypo events and time spent in hyper.

CampUs Study Group: Andrea Scaramuzza, Claudia Arnaldi, Gliberto Candela, Valentino Cherubini, Lucia Ferrito, Lorenzo Lenzi, Fortunato Lombardo, Elvira Piccinno, Ivana Rabbone, Sonia Toni, Davide Tinti, Stefano Tumini, Ohad Cohen.

029

\section{ULTRA-FAST ACTING INSULIN}

\section{T. Danne}

Germany

Shortening the time to peak action of fast-acting insulins may have advantages for prandial insulin coverage and may better allow postprandial insulin administration. For fast-acting insulin aspart (FiAsp, NovoNordisk) the well-characterized excipients nicotinamide and L-arginine (to stabilize the molecule) are added to the established rapid-acting insulin analog insulin aspart. The ultra-rapid effect of adding nicotinamide causes a more rapid monomer formation leading to a quick uptake into the blood stream. Studies indicated an approximately twice as fast onset of appearance in the bloodstream and 2 fold higher early exposure within the first 30 minutes. We look forward to the full publication of the Phase 3 trials of this ultra-fast insulin insulin which is likely to be the first of its kind to be approved possibly as early as 2017. Also a citrate/zinc-ion chelator combination to human regular insulin employed by Biodel, Inc. showed ultra-rapid insulin properties. Issues regarding injection pain and stability of this compound are currently under investigation. Another approach to ultra-rapid mealtime insulin action is BioChaperone ${ }^{\circledR}$ Lispro, an ultra-rapid formulation of insulin lispro. BioChaperone ${ }^{\circledR}$ is a library of polysaccherides mimicking the properties of heparin. This insulin is developed in cooperation between Lilly and Adocia and is reported to have a 30 per cent earlier onset of action and a 69 per cent stronger early metabolic effect. Thus, ultra-fast acting insulins may not only been considered for use in closed-loop approaches and pump therapy but may have therapeutic advantages in the prandial insulin treatment type 1 and type 2 diabetes.

\section{0}

\section{WEAK POINTS IN CLOSED-LOOP TECHNOLOGY: FAULT DETECTION AND MITIGATION}

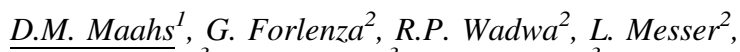
B.W. Bequette ${ }^{3}$, F. Cameron ${ }^{3}$, D. Howsmon ${ }^{3}$, L. Huyett ${ }^{4}$, E. Dassau, F.J. Doyle III ${ }^{5}$, S. Patek ${ }^{6}$, E. Schertz, E. Mauritzen ${ }^{7}$, A. Mandell ${ }^{7}$, D. DeSalvo ${ }^{8}$, T.T. Ly', B.A. Buckingham

${ }^{1}$ University of Colorado Denver, Barbara Davis Center for Childhood Diabetes, Aurora, USA

${ }^{2}$ University of Colorado, USA

${ }^{3}$ Rensselaer Polytechnic Institute, USA

${ }^{4} U C S B, U S A$

${ }^{5}$ Harvard, USA

${ }^{6}$ University Virginia, USA (7)UCSD, USA

${ }^{8}$ Baylor, USA

${ }^{9}$ Stanford, USA

Current closed-loop technology combines an insulin pump, continuous glucose monitor (CGM), and an algorithm to dose insulin. Numerous weak points in the system exist. Detection and mitigation of these faults will improve the safety of artificial pancreas systems and benefit current open-loop systems.

A 4 week multicenter outpatient trial was performed in 40 patients with type 1 diabetes (age $=29 \pm 8$ years, $43 \%$ male, A1c $=7.3 \pm 0.8 \%$ ). Specific investigations targeted: 1) the effect of acetaminophen on CGM sensor glucose compared to blood glucose meter values; 2) the effect of lipohypertrophy on infusion set duration and failure, CGM sensor performance, and glucose values; 3 ) the effect of hyaluronidase on glucose values and infusion set failure; 4) development of algorithms to detect infusion site and CGM sensor failures in advance of hyperglycemia and DKA.

As expected, acetaminophen falsely elevated CGM sensor glucose values with implications for insulin dosing in open or closed-loop. Unexpectedly, the median ARD for CGM sensor 
glucose values was improved in lipohypertrophy compared to normal tissue. Hyaluronidase did not effect the duration of infusion sets, average daily glucose, or total daily insulin, however postprandial glucose levels were improved for a day after hyaluronidase infusion.

From the data generated on infusion set and sensor failures, fault detection algorithms were developed which are now being tested in real-time closed-loop studies with the goal of making open and closed-loop technology safer. More research is needed to improve the safety and performance of diabetes technology to enhance glucose control and reduce the burden for people with diabetes.

Acknowledgments: JDRF funding, medical device support from Dexcom and Roche, technical support from the University of Virginia using the DiAs platform. Patek acknowledges equity interest in TypeZero Technologies, LLC.

\section{1}

\section{FEEDBACK CONTROL TRADEOFFS WITH FASTER INSULIN ROUTES}

\section{F.J. Doyle}

Cambridge, USA

A significant challenge in the design of algorithms for the artificial pancreas is overcoming the latencies introduced by measuring glucose subcutaneously and by delivering insulin subcutaneously. These are artificial delays introduced by an external device and limit the ability of an engineered design to emulate the behavior of the natural pancreas endogenous glucose regulation including first phase insulin secretion and proximity to the portal circulatory system. These limitations are particularly challenging when one attempts to compensate for unmeasured disturbances, such as unannounced meals. In this talk, I will discuss some of the closed-loop performance measures that are impacted by delays in the feedback loop, and will review clinical work conducted in our group to address those limitations, including inhalable insulin and IP delivery of insulin. I will also introduce some of our latest work on the design of a fully implantable version of the artificial pancreas.

\section{2}

\section{COMPOSITE MEASURES OF GLYCEMIC CONTROL}

\section{Rodbard}

USA

$\mathrm{HbA1c}$ is not a sufficient measure of response to therapy. One must consider other factors, especially risk of hypoglycemia and weight gain. HbA1c provides a measure of average blood glucose over the preceding 3 months, subject to inter-individual variability due to erythrocyte lifetime, hemoglobinopathies and the Hemoglobin Glycation Index. DCCT established relationships between HbA1c, risk of hypoglycemia, and risk of complications in T1DM. Based on these relationships, the present author proposed to use this relationship between risk of hypoglycemia and $\mathrm{HbA} 1 \mathrm{c}$ to evaluate alternative forms of therapy, physicians, clinics and other interventions (1). This curvilinear relationship has subsequently been utilized by others to demonstrate superiority of glargine and detemir relative to NPH (2), and separately, superiority of other therapies (degludec, hyaluronidase pretreatment of insulin infusion sites) compared to controls. Superiority should be tested by evaluating non-identity of the relationships between risk of hypoglycemia and $\mathrm{HbA} 1 \mathrm{c}$ for different forms of therapy, rather than by testing either HbAlc or risk of hypoglycemia alone $(1,2)$.

I recently proposed several additional methods for simultaneous analysis of hypoglycemia and mean glucose or HbA1c: 1) Risk of Hypoglycemia vs HbA1c or change in $\mathrm{HbA} 1 \mathrm{c}$ following intervention (1);2) relationship between \%Hyperglycemia and $\%$ Hypoglycemia (3); 3) variations of the foregoing approach, using \% Time in Range combined with \% Time in Hyperglycemia and/or \%time in Hypoglycemia (3); 4) Relationship between 'Change in \% Hyperglycemia' vs 'Change in \%Hypoglycemia' (3); 5) A triangular graph showing \%Hyperglycemia, \% Hypoglycemia, and \% Target Range simultaneously (3); 6) Display of hyper- vs. hypoglycemia (using High Blood Glucose Index

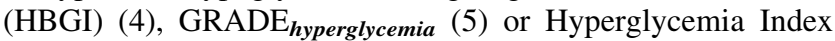
$(6,7)$ vs Low Blood Glucose Index (LBGI) (4), GRADE hypoglycemia $_{\text {in }}$ (5) or Hypoglycemia Index $(6,7) ; 7)$ As in the previous item using percentiles scores for hypo- and hyperglycemia derived from a defined reference population $(8,9) ; 8$ ) Risk of hypoglycemia vs mean glucose and the changes in one or both metrics following a therapeutic intervention (3).

One can avoid the need for an arbitrary choice of threshold for hypoglycemia by repeating the analyses with multiple thresholds (e.g. 40 to $80 \mathrm{mg} / \mathrm{dL}$ ) and evaluating robustness of findings. A threshold of $80 \mathrm{mg} / \mathrm{dL}$ can be used as a surrogate for the much less frequent occurrence of hypoglycemia at $50 \mathrm{mg} / \mathrm{dL}$; a threshold of $180 \mathrm{mg} / \mathrm{dL}$ can be used as a surrogate for hyperglycemia at a much higher threshold, e.g. $250 \mathrm{mg} / \mathrm{dL}$ (9). Alternatively, one can use penalty scores such as BGRI, GRADE or IGC (4-7).

Vigersky has proposed a composite analysis of HbA1c, risk of hypoglycemia and weight gain (10) and clearly elucidated the need to go beyond HbA1c. Thomas et al. proposed composite markers combining information from HbAlc, overall glycemic variability, and hyperglycemia (11-12). Augstein et al (13) proposed a Q-Score which adds measures of hypoglycemia, within-day, and between day variability (13). Augstein combined parameters using z-scores (13), an approach closely related to use of percentile scores $(3,8)$. An Index of Glycemic Variability (IGV) has been proposed to combine results from several types of variability (total variability, within-, and between-day variability, and variability between daily means) using a weighted average of percentile scores (8).

Conclusion: Regulatory agencies should utilize multiple criteria to simultaneously evaluate safety and efficacy of therapeutic agents and devices (1-13).

\section{References}

1) Rodbard, D., Berger, M., and Pernick, N. Computer, networking, and information systems to facilitate delivery of health care to patients with diabetes. In: Baba, S., and Kaneko, T. (Eds.), Diabetes 1994, Proceedings of the 15th International Diabetes Federation Congress, Kobe, 6 - 11 November 1994. Elsevier, Amsterdam, pp. 800-803, 1995.

2) Little S1, Shaw J, Home P. Hypoglycemia rates with basal insulin analogs. Diabetes Technol Ther. 2011 Jun;13 Suppl 1:S53-64. doi: 10.1089/dia.2011.0022.

3) Rodbard D. Evaluating quality of glycemic control: graphical displays of hypo- and hyperglycemia, time in target range, and mean glucose. J Diabetes Sci Technol. 2015 Jan; 9(1):56-62. doi: 10.1177/1932296814551046

4) Clarke W, Kovatchev B. Statistical tools to analyze continuous glucose monitor data. Diabetes Technol Ther. 2009 Jun; 11 Suppl 1:S45-54. doi: 10.1089/dia.2008.0138 
5) Hill NR, Hindmarsh PC, Stevens RJ, Stratton IM, Levy JC, Matthews DR. A method for assessing quality of control from glucose profiles. Diabet Med. 2007 Jul;24(7):753-8. Epub 2007 Apr 19.

6) Rodbard D. Interpretation of continuous glucose monitoring data: glycemic variability and quality of glycemic control. Diabetes Technol Ther. 2009 Jun;11 Suppl 1:S5567. doi: 10.1089/dia.2008.0132

7) Rodbard D. New and improved methods to characterize glycemic variability using continuous glucose monitoring. Diabetes Technol Ther. 2009 Sep;11(9):551-65. doi: 10.1089/dia.2009.0015.

8) Rodbard D. Clinical interpretation of indices of quality of glycemic control and glycemic variability. Postgrad Med. 2011 Jul;123(4):107-18. doi: 10.3810/pgm.2011.07.2310

9) Rodbard D. Hypo- and hyperglycemia in relation to the mean, standard deviation, coefficient of variation, and nature of the glucose distribution. Diabetes Technol Ther. 2012 Oct;14(10):868-76. Epub 2012 Sep 6.

10) Vigersky RA. Escaping the Hemoglobin A1c-Centric World in Evaluating Diabetes Mellitus Interventions. J Diabetes Sci Technol. 2015 Feb 19;9(5):1148-51. doi: $10.1177 / 1932296815576363$

11) Thomas A, Schönauer M, Achermann F, Schnell O, Hanefeld M, Ziegelasch HJ, Mastrototaro J, Heinemann L. The "glucose pentagon": assessing glycemic control of patients with diabetes mellitus by a model integrating different parameters from glucose profiles. Diabetes Technol Ther. 2009 Jun;11(6):399-409. doi: 10.1089/dia.2008.011

12) Thomas A, Heinemann L. Prediction of the risk to develop diabetes-related late complications by means of the glucose pentagon model: analysis of data from the Juvenile Diabetes Research Foundation continuous glucose monitoring study. J Diabetes Sci Technol. 2012 May 1;6(3):572-80.

13) Augstein P, Heinke P, Vogt L, Vogt R, Rackow C, Kohnert KD, Salzsieder E. Q-Score: development of a new metric for continuous glucose monitoring that enables stratification of antihyperglycaemic therapies. BMC Endocr Disord. 2015 May 1;15:22. doi: 10.1186/s12902-015-0019-0

033

ESCAPING THE A1C-CENTRIC ROLE OF ASSESSING GLYCEMIC CONTROL IN DIABETES

R. Vigersky

USA

Most diabetologists recognize the hemoglobin A1C (A1C) insufficiently captures the quality of glycemic control because it gives no information about the prevalence and severity of hypoand hyperglycemia both of which are important for patient care and research. Indeed, both the human and financial costs of hypoglycemia (from Emergency Room visits, hospitalizations, to the induction of adverse cardiac events like ischemia and arrhythmias) provides a compelling case for finding a way to simultaneously include hypoglycemia and possibly other relevant metrics with $\mathrm{A} 1 \mathrm{C}$ as a single composite outcome measure to assess effectiveness of a therapy whether it be pharmacologic, technologic, or educational. Such a composite metric can also be used to assess ongoing patient management. Yet, most interventions are rated by regulatory bodies, insurers, and professional organizations primarily if not exclusively on the effect on A1C. Thus, an intervention with a neutral effect on A1C but a reduction in the rate of hypoglycemia may not be considered very effective by regulatory bodies and payers. A1C, rat/severity of hypoglycemia, time-in-range, weight change, cost, patient satisfaction/quality of life, and blood pressure are among the clinically relevant variables that could be included in a composite score. To this end, there have been several attempts to devise composite endpoints and display them in graphic and/or tabular form. These include the glucose pentagon, the Q-score, and hypoglycemia-A1C score. The advantages and disadvantages of these composite metrics will be discussed.

\section{4}

\section{CSII IN TYPE 1 DIABETIC PREGNANT WOMEN: ITS EFFECTIVENESS AND SAFETY DURING GESTATION AND DELIVERY}

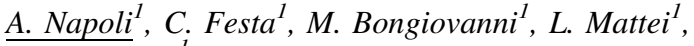 \\ A. Colatrella \\ ${ }^{1}$ Dipartimento di Medicina Clinica e Molecolare, \\ FACOLTA` DI MEDICINA E PSICOLOGIA, SAPIENZA, \\ Università di Roma
}

Insulin pump is a flexible option for people with diabetes.

However, CSII does not bring to a significant improvement of clinical outcome in type 1 diabetic pregnant women when compared with multiple daily injections.

Timely information on how carbohydrate to insulin ratio and basal insulin infusion change throughout pregnancy, during and after delivery might lead to a more appropriate pump management particularly in women under 'SAP therapy'.

We confirmed a progressive $\mathrm{CHO}$ to insulin ratio decline throughout pregnancy at each meal time with an inverse trend in very late pregnancy (after the 36th week). At breakfast, the $\mathrm{CHO} /$ I ratio was often lower than at lunch and/or dinner.

We also showed that insulin pump is feasible and safe during delivery when a protocol is given to selected and well educated women from the beginning of the third trimester.

This strategy obtains good and stable maternal 'peri-partum' capillary blood glucose profiles.

In conclusion, recommendations guiding therapeutic decisions improve patients' metabolic control and quality of life.

\section{References}

Angela Napoli*, Camilla Festa, Marzia Bongiovanni, Luca Mattei and Antonietta Colatrella.

Continuous Subcutaneous Insulin Infusion (CSII) in Diabetic Pregnancy:A Review.

Recent Patents on Endocrine, Metabolic \& Immune Drug Discovery 2009, 31872-2148/09 2009.

Raffaella Fresa, 1Natalia Visalli, 2Vincenzo Di Blasi, 1Vincenzo Cavallaro, 1Egle Ansaldi, 3Oria Trifoglio, 4Santina Abbruzzese, 2Marzia Bongiovanni, 5Mariano Agrusta and Angela Napoli5.

Experiences of Continuous Subcutaneous Insulin Infusion in Pregnant Women with Type 1 Diabetes During Delivery from Four Italian Centers: A Retrospective Observational Study.

Diabetes technology \& therapeutics volume 15 , number $4,1-8$, 2013.

\section{5}

\section{VS CSII IN PREGNANCY WITH TYPE1 DIABETES}

\section{E. Torlone}

Italy 
CSII in flexible option for people with diabetes.

However, CSII does not bring a significant improvement of clinical outcome in type 1 diabetic pregnant women when compared with multiple daily injections.

Timely information on how the carbohydrate to insulin ratio and basal insulin infusion change throughout pregnancy, during and after delivery might lead to a more appropriate pump management particularly in women under 'SAP therapy'.

We observed a progressive $\mathrm{CHO} / \mathrm{I}$ ratio decline throughout pregnancy at each meal time with an inverse trend in very late pregnancy (after the 36th week). At breakfast, the $\mathrm{CHO} / \mathrm{I}$ ratio was often lower than at lunch and/or dinner.

We also showed that insulin pump is feasible and safe during delivery by delivering a protocol for the management of metabolic control during partum of type 1 diabetic pregnant women, in selected women who have been appropriately instructed.

This strategy obtained a good and stable maternal peripartum capillary blood glucose profiles.

In conclusion, recommendations guiding therapeutic decisions could improve patients' quality of life by reducing their anxiety.

References

Angela Napoli*, Camilla Festa, Marzia Bongiovanni, Luca Mattei and Antonietta Colatrella.

Continuous Subcutaneous Insulin Infusion (CSII) in Diabetic Pregnancy:A Review.

Recent Patents on Endocrine, Metabolic \& Immune Drug Discovery 2009, 31872-2148/09 2009.

Raffaella Fresa, 1Natalia Visalli, 2Vincenzo Di Blasi, 1Vincenzo Cavallaro, 1Egle Ansaldi, 3Oria Trifoglio, 4Santina Abbruzzese, 2Marzia Bongiovanni, 5Mariano Agrusta and Angela Napoli5.

Experiences of Continuous Subcutaneous Insulin Infusion in Pregnant Women with Type 1 Diabetes During Delivery from Four Italian Centers: A Retrospective Observational Study.

Diabetes technology \& therapeutics volume 15 , number $4,1-$ 8, 2013.

\section{6}

\section{GAMES FOR HEALTH}

\section{E. Vitacolonna $^{1}$}

${ }^{1}$ University G. d'Annunzio, Department of Medicine and Aging, Chieti, Italy

Recent studies suggest that Serious Games (SG) are interesting and innovative tools useful to influence attitudes, beliefs and behaviors. SG use entertainment technology to teach, train, or change the behavior, encouraging active engagement and processing of information from the users. Games for health are games with a focus on health care, physical and mental fitness, and their popular application areas are nutrition, physical training, education, and prevention. The game-based learning principles target intrinsic motivation, learning through fun, authenticity, self- reliance/autonomy, and experiential learning. The mechanisms adopted include rules, clear but challenging goals, fantasy, progressive levels of difficulty, interactivity, player control, uncertainty, feedback and a social element. Active videogames seem to be effective in increasing energy expenditure and promoting physical activity. It has been shown that playing video games can promote extrinsic motivation and foster positive emotion with possible very important effects on health behavior. Therefore, SG can be promising tools that seek to entertain the user while attempting to elicit some form of change in behavior. Our recent studies show that an original Web Game called "Gustavo in Gnam's Planet" increases knowledge about healthy food and improves healthy lifestyle habits; our SG "could be an important useful mean and an auspicious tool for prevention programs within a multidimensional educational program".

Video games seem to be persuasive instruments for education in different interventions, and it is known the potential of using video games and gamification to promote healthy habits and better self-management of chronic diseases and diabetes in pregnancy.

\section{7}

\section{IMPACT OF SLEEP ON MATERNAL GLUCOSE CONTROL IN PREGNANCY}

\section{E. Scott ${ }^{1}$}

${ }^{1}$ Leeds Institute of Cardiovascular and Metabolic Medicine, Biostatistics and Epidemiology, University of Leeds Leeds, United Kingdom

It is increasingly well recognised that sleep duration and sleep quality are factors involved in glucose homeostasis. Recent technology, using continuous glucose monitoring combined with continuous actigraphy, allows us to explore the relationship between glucose and sleep in real-time, in a free-living rather than lab-based situation. This intensive longitudinal data allows us unprecedented insight into how sleep and glucose interact. Glucose control is particularly important in the context of pregnancy, where tight glucose control is associated with a significantly better outcome for mother and baby. Despite available treatments, this is often not achieved and women are particularly cautious about taking drugs during pregnancy. Understanding the relationship between sleep and glucose may enable us to develop novel lifestyle based interventions to optimise glucose control. I will present data from our recent studies of sleep in pregnant women with diabetes.

Follow me on Twitter: EleanorScott@TIME_research

\section{8}

\section{EFFECTIVENESS OF CONTINUOUS GLUCOSE MONITORING DURING DIABETIC PREGNANCY (GLUCOMOMS TRIAL); A RANDOMISED CONTROLLED TRIAL}

\section{Evers}

Netherlands

Objective Hyperglycemia in pregnancy is associated with poor perinatal outcome. Even if pregnant women with diabetes are monitored according to current guidelines, they do much worse than their normoglycemic counterparts. The Continuous Glucose Monitoring System (CGMS) is an efficacious new method to optimize glucose control in pregnant women with diabetes. In the present proposal, we aim to assess the effectiveness, costs and cost-effectiveness of the use of the CGMS to optimize glycemic control during diabetic pregnancies and reduce macrosomia, relative to standard control methods.

Study design: Multicenter open label randomized clinical trial (RCT) with a decision and cost-effectiveness study alongside it. 


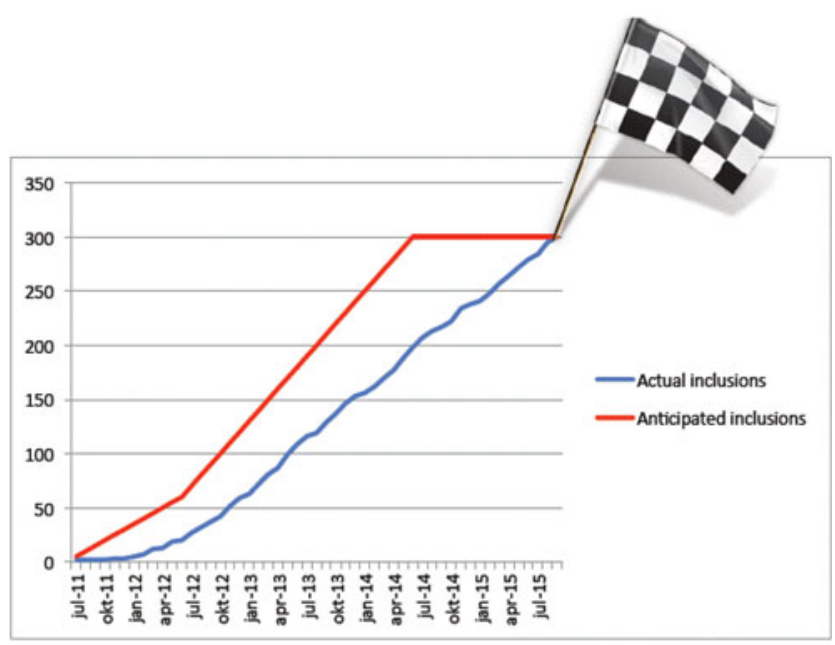

Study population: Pregnant women with type 1 and 2 diabetes or with gestational diabetes $(n=300)$

Inclusion Criteria: Pregnant women with pre-existing diabetes (type 1 or type 2) on insulin treatment regimen or use of insulinpump, before 16 weeks of pregnancy. - Pregnant women with gestational diabetes on insulin treatment, before 30 weeks of pregnancy.

Exclusion Criteria: Severe medical or psychological comorbidity, multiple pregnancies

Intervention: Consenting women will be randomly allocated to either additional use of CGMS or usual care. All women will determine their glycemic control by self-monitoring of blood glucose levels and HbA1c. In addition, women allocated to CGMS will use CGMS every month and adjust their insulin regimen based on their CGMS profile.

Outcome measures of the RCT: Primary outcome of the RCT will be macrosomia rate, defined as a birth weight above the 90th centile. Secondary outcomes will be birth weight, composite neonatal morbidity, maternal outcome and costs. The analyses will be according to the intention to treat principle.

Inclusions september 2015: pregnant women $n=300$

Type $1 \mathrm{DM} n=109$

Type 2 DM $n=83$

Gestational diabetes $n=108$

\section{9}

\section{WHO NEEDS AN ARTIFICIAL PANCREAS IN PREGNANCY?}

H. Murphy ${ }^{1}$, Z. Stewart ${ }^{2}$

${ }^{1}$ University of East Anglia, Medicine, Norwich, United Kingdom

${ }^{2}$ University of Cambridge, IMS, Cambridge, United Kingdom

Continuous glucose monitoring (CGM) has highlighted the gap that exists between our expectations of tight metabolic control and the realities of actually achieving this, particularly during type 1 diabetes pregnancy. Longitudinal measurements indicate that despite overall "good" HbA1c levels, pregnant women with type 1 diabetes spend 8 hours per day with blood glucose levels above the recommended targets. New closed-loop (CL) or artificial pancreas approaches integrate insulin pump delivery (CSII) with continuous glucose monitoring (CGM) via computerized algorithms.

The vital component of a CL system for use during pregnancy is a control algorithm which can function safely despite the physiological changes in glucose turnover, endogenous glucose production and insulin kinetics. We have defined these changes in type 1 diabetes pregnancy and completed proof-of-concept studies evaluating overnight CL, in early (12-16 weeks) and late (28-32 weeks) gestation. Pilot feasibility studies demonstrated near-normal overnight glucose control (85-100\% time within the target range of $3.5-7.8 \mathrm{mmol} / \mathrm{l}$ ), during early and late gestation. In a subsequent 24-hour crossover study comparing CL vs. conventional CSII, CL achieved excellent overnight control (95$100 \%$ time in target), with $80 \%$ overall time in target, during meals, snacks and physical activity. Home studies evaluating the feasibility, safety and efficacy of CL in real-life home settings are underway. Ongoing studies also suggest that CL can be safely used during in-patient admissions to hospital maternity units, to maintain glucose control following antenatal steroids for fetal lung maturation as well as before, during and after delivery.

\section{0}

\section{PSYCHOSOCIAL OUTCOMES OF CGM USE IN CHILDREN AND TEENS WITH TYPE 1 DIABETES}

L. Laffel $^{1}$

${ }^{1}$ Joslin Diabetes Center, Pediatrics, Boston, USA

\section{Psychosocial Outcomes of CGM Use in Children} and Teens with Type 1 Diabetes

Intensive insulin therapy and use of advanced diabetes technologies for type 1 diabetes (T1D) management place substantial burdens upon children, adolescents, and young adults. Despite these treatment tools, the majority of young patients do not achieve the recommended $\mathrm{HbA} 1 \mathrm{c}$ targets of $<7.5 \%(58 \mathrm{mmol} /$ $\mathrm{mol})$ for patients $<18$ years old and $<7 \%(53 \mathrm{mmol} / \mathrm{mol})$ for those 18 and older; only $\sim 1 / 4$ youth $<18$ reach target and only $\sim 1 / 5$ aged 18-25 achieve target. The combination of the demanding treatment program and lack of goal attainment can lead to substantial diabetes and psychosocial distress. There is likely a bidirectional interaction between psychosocial factors and CGM use as CGM adds to the burdens of care while CGM also offers opportunity to mitigate distress by providing useful real-time glucose data to guide management as well as alert patients (and family members connected remotely) of out-of-range glucose levels.

Pediatric patients are particularly burdened by CGM; although many may initiate CGM, most fail to sustain use. In turn, most of these young patients do not receive glycemic benefits from CGM. Discontinuation has been related to perceived burdens due to the device's size, pain associated with insertion, frequent alerts/alarms, and inaccuracies in early systems. Additionally, psychosocial distress has been more apparent in pediatric compared to adult patients using CGM (Diab Tech Ther 2012; 14:523-6). Youth using CGM reported more anxiety while adults reported less; youth's parents reported continued fear of hypoglycemia; youth and parents reported more negative affect around BG results; and parent-proxy report of youth depression was higher. In contrast to the youth CGM experience, adults using CGM reported less diabetes-related burden. Thus, young patients with T1D continue to demonstrate significant challenges in sustaining CGM use. Newer CGM devices with improved performance may help to overcome these behavioral barriers and 
improve psychosocial outcomes to sustain CGM use so that pediatric patients can achieve CGM benefits related to improved glycemic control without severe hypoglycemia.

\section{1}

\section{PARENTAL BURDEN, ANXIETY AND SUBJECTIVE WELL-BEING IN TYPE 1 DIABETES}

\author{
N. Bratina ${ }^{1}$, T. Pate ${ }^{2}$ \\ ${ }^{1}$ University Medical Centre Ljubljana- University Children's \\ Hospital, Department of Pediatric Endocrinology-Diabetes \\ and Metabolic Diseases, Ljubljana, Slovenia \\ ${ }^{2}$ University of Ljubljana- Faculty of Theology, Department \\ of Marital and Family Therapy, Ljubljana, Slovenia
}

Parents have a very important role in the diabetes management and emotional coping for their child with diabetes. They become responsible for the disease management in order to avoid excursions of blood glucose and enable a better quality of their child's life. Parents often experience a great burden in their life that groves with this responsibility. Therefore, they must face their fears, sense of guilt, sadness, anger, despair and the fact that they've lost their healthy child and, consequently, the previous way of life.

On the other hand they are driven by parental instinct to protect their child with tendency to over control the child and his diabetes or the outside influences. Parents' concerns and overprotection can also be associated with the fear of hypoglycemia. This fear can create a great tension and conflicts in family relationships. In 2013-2014 a study was performed at University Children's Hospital. A group of 231 parents (140 mothers and 91 fathers) participated. The results showed that mothers were experiencing greater stress, fear of hypoglycemia, feelings of anxiety, and had worse subjective well-being, whereas fathers were generally less functionally involved in the diabetes management. Parents have a central role in coping with the child's illness and its management. They are not only important as a child's health experts, but they have an important role in the regulation of child's and their own emotions created by the new life with diabetes. Therefore it is important to help and assist parents to recognize and identify emotions and debilitating affects that will likely influence family, their child and themselves.

\section{2}

\section{THE PSYCHOLOGICAL IMPACT OF USING AN AP SYSTEM}

\section{Ziegler $^{1}$, T. Danne ${ }^{1}$, O. Kordonouri ${ }^{1}$, K. Lange ${ }^{2}$}

${ }^{1}$ AUF DER BULT- Kinder- und Jugendkrankenhaus, Diabetes Centre for Children and Adolescents, Hannover, Germany

${ }^{2}$ Hannover Medical School, Department of Medical Psychology, Germany

Introduction: Different artificial pancreas (AP) systems support the superiority of the AP compared to standard CSII therapy regarding overall glucose control and risk of nocturnal hypoglycaemia. But little is known on the psychological impact of these systems and the role of human factors in the adoption and continuous use.

Method: All manuscripts identified in PubMed until November 10th 2015 that contained the words 'closed loop' or 'artificial pancreas', 'type 1 diabetes', 'fear*' or 'acceptance', 'satisfaction', 'quality of life' were reviewed. Criteria for inclusion were AP-specific studies with psychological outcomes.

Results: Three studies assessed attitudes and expectations of potential users of AP technology, without having real-life access to the system. The majority reported secure feelings, indicated positive attitudes and a high intention to use it. Size, visibility, lack of effectiveness were main concerns. Clinical trial participants with direct access to AP technology expressed high future acceptance. Two studies evaluated the psychological impact of using an AP system at home. Reduced worries of hypoglycaemia, high level of satisfaction with and increased acceptance of an AP were reported by the DREAM Group. Psychological (e.g. reassurance), physical benefits (e.g. improved diabetes control) and practical challenges (e.g. alarms) were reported by the Cambridge Group.

Conclusion: The majority of patients reported positive attitudes and psychological benefits of using an AP system. Key concerns focus on practical challenges. Future studies should focus on psychological effects of AP during long-term overnight and day-and-night use to provide closer information on benefits and barriers of using an AP technology continuously.

\section{3}

\section{ONLINE PEER-TO-PEER DIABETES SELF- MANAGEMENT SUPPORT: AN IMPORTANT ADDITION TO TRADITIONAL DIABETES EDUCATION}

\section{N. Kaufman ${ }^{1}$}

${ }^{1}$ Canary Health, Medical, Los Angeles, USA

Digital health self-management is a new category of behavior change interventions, designed to improve health and reduce costs of care for individuals with one or more chronic conditions. Patients with diabetes must master a myriad of behaviors to improve their outcomes. In addition to the medical aspects of diabetes management they need to master, they also need to be able to live their lives while managing their disease. This is made all the more difficult since nearly all patients with type 2 diabetes have one of more other chronic conditions requiring attention. Putting the patient in the center of their diabetes journey requires a consumer-centric approach to education and support.

One proven-effective approach to this requirement is the peerto-peer Chronic Disease Self-management Program (CDSMP). Developed in 1992 and continuously updated by Dr. Kate Lorig and her colleagues at Stanford University, CDSMP, and its digital transformation (Better Choices Better Health (BCBH)) teach participants how to manage their chronic conditions, adhere to medication regimens, and maintain functional ability. CDSMP is premised on certain assumptions: that patients can learn to take responsibility for the day-to-day management of their conditions; that knowledgeable patients who practice selfmanagement can improve their health status and use fewer health care resources; and that patient self-management programs should be inexpensive and widely available. In numerous published studies of CDSMP and BCBH, participants were shown to have improved their health behavior, such as exercise, symptom management, medication adherence and communication with physicians, and maintained or improved their perception of their health status. Participation in CDSMP was also shown to reduce AIC, hospitalizations and hospital days.

This presentation will focus on the digital version of the CDSMP by giving an overview of the program and a summary of the outcomes which have been demonstrated in patients with diabetes. 
044

THE QUANTIFICATION OF OBESITY WITH THREE

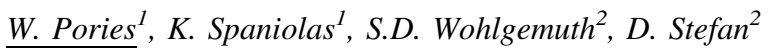

${ }^{1}$ Department of Surgery, Brody School of Medicine, East

Carolina University, Greenville, NC, USA

${ }^{2}$ Wohlgemuth, Stefan: Sentara Health System, Norfolk, VA, USA

Background: There are still no accurate methodologies to quantify obesity even though it is one of the world's most serious and growing epidemics. The Body Mass Index $\left(B M I=K g / M^{2}\right)$, the traditional formula invented by Adolphe Quetelet 200 years ago, based merely on height and weight, is not the answer. It fails on four counts:

- it is unigender even though there are sharp differences in fat distribution between men and women;

- it does not account for fitness and would, for example, accept one of our recent subjects for bariatric surgery at a weight of $307 \mathrm{lbs}$., 5'8" and a BMI of 47 even though he was our fastest running back at our university;

- it does not allow for the changes with aging and the fat infiltration of muscle and

- most seriously, it prevents access to bariatric surgery to African Americans and Asians who develop diabetes and other co-morbidities of the metabolic syndrome. Three-dimensional scanning with white light or infra-red technology, finally offers an approach to quantify the dimensions of the human body with the potential to measure and predict physical performance and health. The technology, similar to but far more accurate than that used daily at airports for the screening of travelers, is rapid, safe and inexpensive.

Methodology: We developed new software that allows us to slice the images, similar to the approach used for CT scans which, in turn, supports the calculation of body surface area, trunk and limb volumes as well as body shapes and relationship of trunk to limbs.

Note that the two individuals are the same height and weight, yet an adiposity index, calculated from the derived measurements of surface area, trunk volume and sagittal measure at levels of the pelvis can sort patient physiques into "apple" vs."pears" and allow prediction of the likelihood of the metabolic syndrome.

Results: Early clinical data indicate that 3D scanning can indicate the presence of the metabolic syndrome due to accurate and reproducible body and sagittal measurement that allow the

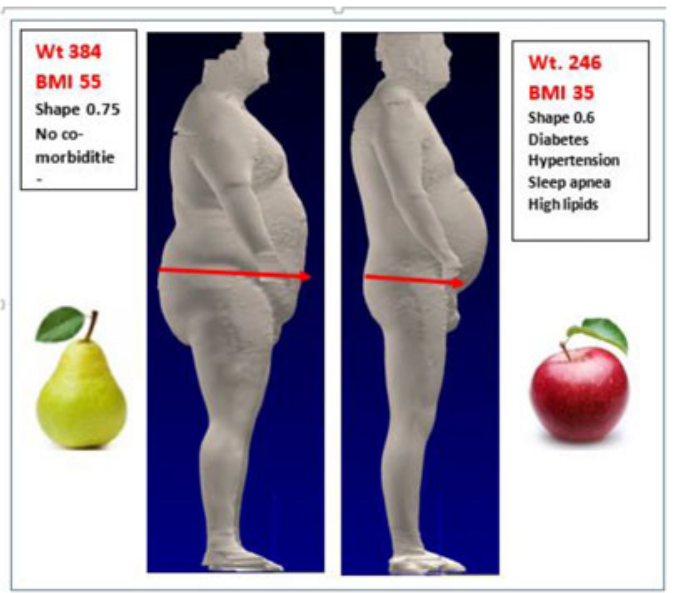

calculations of an obesity index through the determinations of trunk volumes and surface areas.

Conclusion: Three dimensional body scanning offers a new, rapid and safe approach to the quantification of obesity.

045

THE EFFICACY OF ENDOSCOPIC DUODENALJEJUNAL BYPASS LINER IN SEVERELY OBESE ADOLESCENTS

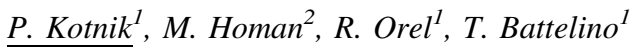

${ }^{1}$ University Children's Hospital- University Medical Centre

Ljubljana, Department of Endocrinology-Diabetes

and Metabolism, Ljubljana, Slovenia

${ }^{2}$ University Children's Hospital- UMC Ljubljana, Department

of Endocrinology- Diabetes and Metabolism, Ljubljana,

Slovenia

Background: Treatment options for severely obese adolescents are limited. Endoscopically placed and removable duodenaljejunal bypass liner (DJBL) is a bariatric procedure that has been successfully used in adults for weight loss and diabetes treatment. Efficacy and safety of this procedure in severely obese adolescent with obesity complications will be presented.

Subjects and Methods: Inclusion criteria for the use of DJBL were; age $>15$ years, BMI $>35 \mathrm{~kg} / \mathrm{m} 2$, unsuccessful conservative therapy for more than 6 months (less than $10 \%$ decrease in body weight), girls were not pregnant or planning pregnancy within next 12 months. In addition all had impaired glucose tolerance with hyperinsulinism or type 2 diabetes and were receiving metformin prior to DJBL use. None had exclusion criteria described in detail at www.ClinicalTrials.gov (NCT02183935). DJBL was inserted under general anaesthesia. There were no serious complications during the procedure however placement was not possible in a minority of subjects due to anatomical variations. DJBL was explanted after 1 year.

Results: Significant decrease in body weight, waist circumference and body composition was determined in most subjects. Metabolic improvements were determined in all subjects. Use of DJBL, as a motivational tool will be discussed. Main procedure related side effects were nausea and abdominal pain, which were most pronounced immediately following the procedure and disappeared thereafter. No serious side effects, including gastrointestinal bleeding and liver abscess were determined.

Conclusion: DJBL seems to be an efficient and safe endoscopic and completely reversible bariatric procedure in most severely obese adolescents with obesity complications.

\section{6}

\section{TIME IN RANGE - THE UNIFYING METRIC OF GLYCEMIC CONTROL IN THE CRITICALLY ILL}

J. Krinsley

$U S A$

A robust literature has demonstrated that hyperglycemia, hypoglycemia and increased glucose variability are independently associated with mortality in diverse cohorts of critically ill patients, and that diabetic status modulates these relationships. Time in targeted blood glucose range (TIR) may be a suitable descriptor of the overall efficacy and safety of glycemic control, and could be considered as marker of the severity of dysglycemia 
and as an index of the quality of care. Moreover, high TIR may be the key element of glycemic control needed to effect optimal outcome and may, in fact, blunt the deleterious impact of transient excursions into the hypoglycemic and hyperglycemic ranges.

A retrospective study involving a cohort of 3,297 patients with intensive care unit (ICU) length of stay (LOS) at least 1.0 day admitted between 2009-2013 to a single mixed medical surgical ICU demonstrated that TIR 70-140 mg/dL > 80\% was independently associated with survival in critically ill non-diabetic patients, independently of ICU LOS and severity of illness (Crit Care 2015; 19:179). For non-diabetic patients, the observed:expected mortality ratios for TIR above and below the median value of $80.6 \%$, using Acute Physiology and Chronic Health Evaluation IV methodology, were 0.53 and 0.78 . In contrast, among DM there was no clear relationship between TIR $70-140 \mathrm{mg} / \mathrm{dL}$ and survival. A growing literature corroborates the significance of TIR as a "unifying" metric of glycemic control in the critically ill and has important implications for the design of clinical protocols for glycemic control in the critically ill as well for the design of future interventional trials of intensive insulin therapy.

\section{7}

\section{PREVENTING READMISSIONS IN HIGH RISK DIABETES PATIENTS: PLANNING \& IMPLEMENTING A TRANSITIONAL CARE PROGRAM}

\section{$\underline{\text { J.J. Seley }}^{1}$, S. Sinha-Gregory ${ }^{2}$, L. Gerber ${ }^{3}$}

${ }^{1}$ New York Presbyterian Hospital/ Weill Cornell Medicine, Division of Endocrinology-Diabetes \& Metabolism, New York, USA

${ }^{2}$ Weill Cornell Medicine, Division of Endocrinology-Diabetes \& Metabolism, New York, USA

${ }^{3}$ Weill Cornell Medicine, Healthcare Policy, New York, USA

Background: Patients with diabetes in the USA have higher 30-day readmission rates (14.4-21.0\%) compared to the general inpatient population (8.5-13.5\%). Some reasons for this higher rate include: number of co-morbidities, race, ethnicity, health insurance and recent inpatient stay. One problem identified at New-York Presbyterian Hospital Weill Cornell campus is the lack of a standardized transitional care program for these highrisk diabetes patients. An evidence-based systematic approach to transitional care for this challenging population has great potential to improve health outcomes and reduce cost by lowering readmission rates.

Methods: An effective hospital discharge should include individualized self-management education, clear instructions, achievable short-term goals and an agreed upon follow-up plan. We developed a program of the following four strategies meant

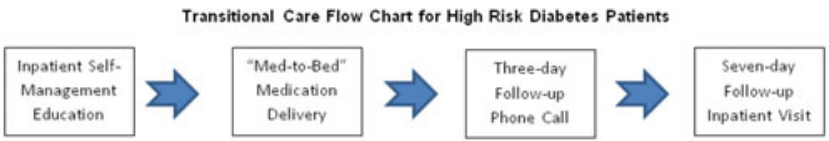

to improve the transition from inpatient to outpatient and decrease the 30-day readmission rate: 1) inpatient diabetes selfmanagement education 2) "Med-to-Bed" medication reconciliation and delivery 3) Three-day post discharge follow-up call and 4) Seven-day in-person visit to review and adjust diabetes medications.

Results: Although we continue to enroll patients, we have been fraught with unexpected obstacles. Recruitment has been hampered by the lack of timely A1c results to identify high risk patients. Discharge prescriptions were often not submitted to the "med-to-bed" pharmacy well in advance to allow for ample time to reconcile possible substitutions based on availability and cost. The most troubling barrier was the high number of patients that did not answer the phone for the follow up call and/or show up for the seven day visit. Some program modification is needed.

\section{8}

\section{READING AND MISREADING THE EVIDENCE-BASE FOR DIABETES TECHNOLOGY}

\section{J. Pickup \\ United Kingdom}

High quality evidence is crucial for establishing the clinical benefit of new technologies, which patient groups benefit the most, cost-effectiveness, short and long-term safety, and patient usability. But often diabetes technology is introduced into practice before all but the slimmest evidence base has been established.

Often there is a paucity of evidence in diabetes technology because many studies are inappropriately designed, e.g. inadequately powered or involve the wrong patient groups.

Although meta-analysis of randomized controlled trials is considered the cornerstone methodology for evidence-based medicine, it is frequently misused and quoted inappropriately, with misleading conclusions for decision-making. Examples include selecting trials that do not match intended use (e.g. in CSII vs. MDI trials, subjects have only modestly elevated HbA1c at baseline, or subjects with severe hypoglycaemia have been excluded); including short-duration trials where outcome cannot be accurately assessed (e.g. trials $<6$ months when measuring severe hypoglycaemia); and including trials using obsolete technology (e.g. first-generation insulin pumps). Individual patient data meta-analysis, linking patient-level characteristics to outcome, is useful for establishing those likely to benefit most from technologies.

Guidelines and consensus statements can be surprisingly lacking in evidence and, based usually on average trial responses without context and clinical judgement, can ill-serve the individual patient.

Stakeholders - clinicians, manufacturers, patients, funders, regulators, publishers - need to cooperate in setting, regulating, monitoring and interpreting best evidence for diabetes technology, be it garnered from trials, registers, surveys, expert opinion, patient responses or other means. 


\section{ATTD 2016 Oral Presentations}

049

\section{ELASTARGENE 3C HELPS TO IMPROVE GLYCATED HAEMOGLOBIN IN CHILDREN AND ADOLESCENTS WITH TYPE 1 DIABETES USING INSULIN PUMP THERAPY}

A. Scaramuzza ${ }^{1}$, M. Ferrari ${ }^{1}$, G. Ramponi ${ }^{2}$, M. Macedoni ${ }^{2}$, L. De Angelis $^{2}$, F. Redaelli ${ }^{2}$, G.V. Zuccotti ${ }^{2}$

${ }^{1}$ Luigi Sacco, Pediatrics, Milano, Italy

${ }^{2}$ Ospedale dei Bambini 'V. Buzzi', Pediatrics, Milano, Italy

Background and Aims: To test the efficacy of elastargene 3C (E3C), a specifically designed cream to improve lipoatrophy in patients with diabetes and composed among many ingredients by elastin, arnica, collagen, caffeine, and L-carnitine, a 6-month, double-blind, randomized trial was started.

Method: Forty children with type 1 diabetes (T1D), using insulin pumps (CSII), were randomized into 2 arms: a) $n=20$ E3C once a day on the skin of abdomen or other sites where infusion sets have been placed (EG); b) $n=20$ placebo once a day on the skin of abdomen or other sites where infusion sets have been placed (PG). BMI, HbA1c, insulin requirement, were evaulated in each child at baseline and after 6 months.

Results: Five patients dropped using the E3C or placebo. In EG, 18 patients with T1D were evaluated: age $15.2 \pm 4.8 \mathrm{yrs}$, diabetes duration $8.0 \pm 5.3$, CSII since $4.1 \pm 3.0$; in PG, 17 patients with T1D were evaluated: age $15.1 \pm 5.7$ yrs, diabetes duration $8.3 \pm 5.8$, CSII since $4.7 \pm 3.0$. HbA1c significantly improved in EG (baseline $8.08 \pm 0.80 \%$, after 6 month $7.51 \pm 0.53 \%$, $\mathrm{p}=0.005$, delta $-0.53 \%)$, but not in PG $(7.98 \pm 0.74 \%$ vs. $7.76 \pm 0.79 \%, p=0.19$, delta $-0.22 \%)$. In EG, withe scars completely disappeared in 8 patients and improved in 10; in the $\mathrm{PG}$, withe scars did not change in any of the patients.

Conclusion: This is the first time that a direct effect of E3C have been shown in improving little withe scars appeared on the skin after infusion set removal in children with T1D using CSII. Interestingly, HbA1c significantly improved only in the EG, probably because improved insulin absorption.

050

\section{HUMAN FACTORS ASSOCIATED WITH INSULIN PUMP AND CONTINUOUS GLUCOSE MONITOR DISCONTINUATION AMONG ADULTS WITH TYPE 1 DIABETES}

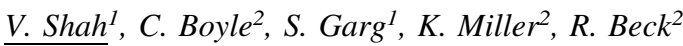

${ }^{1}$ Barbara Davis Center for Diabetes, University of Colorado, Aurora, USA

${ }^{2}$ Jaeb Center for Health Research, T1D Exchange Clinic Network, Tampa, USA
Background and Aims: Despite evidences that the use of insulin pump (CSII) and continuous glucose monitors (CGM) improve glycemic control, only a small percentage of patients with type 1 diabetes (T1D) utilize the benefits of these technologies. Therefore, the study of demographic and human factors involved in the discontinuation of these devices could assist with designing diabetes devices that will increase patient acceptance and continued use

Method: We analyzed factors associated with the device discontinuation from 2,452 adults with T1D $\geq 1$ year enrolled in the T1D Exchange clinic registry (median age 33 years; median T1D duration 18 years; $56 \%$ female, $85 \%$ white).

Results: Discontinuation rates of CSII and CGM within 1 year were $3 \%$ (54 of 1,608) and $27 \%$ (273 of 1,006), respectively. Participants who discontinued pump were more likely to be younger than $50(\mathrm{p}<0.001)$, earn over $\$ 75,000$ annually $(\mathrm{p}=0.002)$, have an HbA1c over 9.0\% ( $<0.001)$, self-monitor their blood sugar less than 6 times per day $(p=0.004)$, and experience a DKA event in the past year $(\mathrm{p}=0.01)$. Participants who discontinued CGM were also more likely to earn over $\$ 75,000$ annually $(\mathrm{p}<0.001)$, but were more likely to have an HbA1c less than $7.0 \%(\mathrm{p}<0.001)$. The most common human factors for discontinuation are shown in the Table.

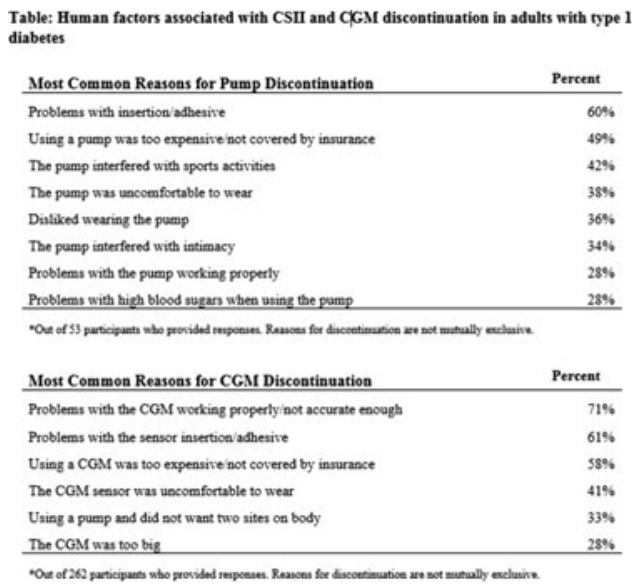

Conclusion: Rate of discontinuation of CGM was higher than CSII in adults with T1D. When designing devices, consideration of human factors associated with discontinuation may help to improve acceptance and adherence to the technologies.

051 EASE OF USE OF THE NEW INSULIN GLARGINE
300 U/ML PEN INJECTOR IN INSULIN-NAIVE PEOPLE WITH TYPE 2 DIABETES

H. Pohlmeier ${ }^{\text {, D. Klonoff }}{ }^{2}$, L. Berard $^{3}$, C. Brulle-Wohlhueter ${ }^{4}$, J. Wu ${ }^{5}$, R. Dahmen, I. Nowotny ${ }^{6}$ 
${ }^{1}$ Center for Diabetes and Vascular Disease, 48145, Muenster, Germany

${ }^{2}$ Mills-Peninsula Health Services, Diabetes Research Institute, San Mateo, USA

${ }^{3}$ Winnipeg Regional Health Authority Health Sciences Centre, Diabetes Research Group, Winnipeg, Canada

${ }^{4}$ Sanofi, Diabetes Division, Paris, France

${ }^{5}$ Sanofi China, Diabetes Division, Beijing, China

${ }^{6}$ Sanofi-Aventis Deutschland GmbH, Diabetes Division,

Frankfurt am Main, Germany

Background and Aims: New insulin glargine $300 \mathrm{U} / \mathrm{mL}$ (Gla-300) contains the same active ingredient as glargine $100 \mathrm{U} /$ $\mathrm{mL}$ (Gla-100). An improved disposable (prefilled) injector pen will be used to administer Gla-300. This study assessed ease of use/ease of learning, safety, satisfaction, glycemic control and reliability with the Gla-300 pen.

Method: The Gla-300 pen was assessed in 40 insulin- and pennaive people with T2DM. Participants received Gla-300 once daily in a 4-week, multi-center, open-label, single-arm study. The primary endpoint was evaluated by the Ease-of-Use/Ease-of-Learning questionnaire (scores range from 1 [excellent] to 5 [very poor]).

Results: At week 4, 95.0\% of participants assessed the pen as excellent/good and none as poor/very poor; $97.5 \%$ would recommend it to others (Figure). Incidences of hypoglycemic events (5.2/participant-year, none severe or serious) and adverse events (AEs) were low. Total Diabetes Treatment Satisfaction Questionnaire scores were stable from start of pen use. Mean (SD) FPG levels decreased from 166.1 (35.0) $\mathrm{mg} / \mathrm{dL}$ at baseline to 124.2 (41.1) $\mathrm{mg} / \mathrm{dL}$ at week 4. Regarding reliability, no Product Technical Complaints (PTCs) or AEs related to PTCs were reported.

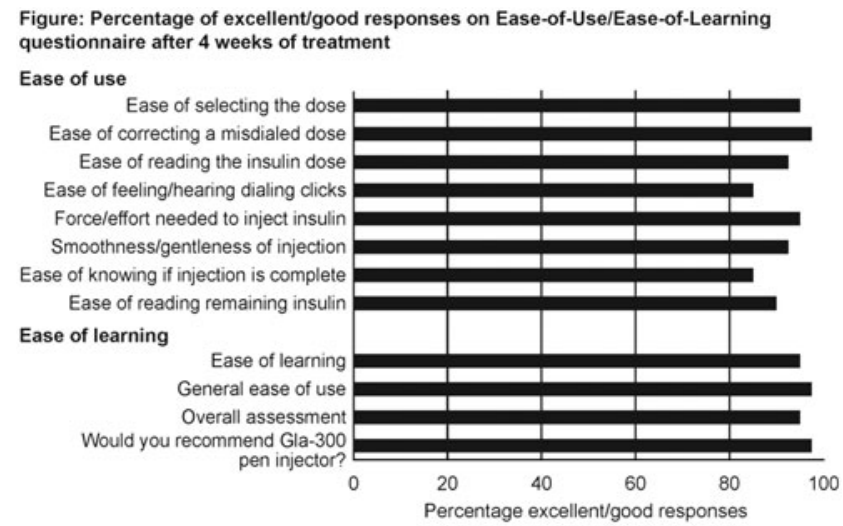

Percentage of participants responding with an excellent (1) or good (2) assessment (from possible scores: 1 [excellent], 2 [good], 3 [acceptable], 4 [poor], 5 [very poor]) or percentage of participants responding Yes (for 'Would you recommend Gla-300 pen injector?')

Conclusion: Over 4 weeks of once-daily dosing with Gla-300, pen-naive and insulin-naive people with T2DM considered the Gla-300 pen to be easy to use and easy to learn. The pen was reliable and Gla-300 basal insulin treatment was well tolerated, with no new safety concerns arising from this 4-week study.

Study sponsored by Sanofi (NCT02227212)

\section{2}

\section{IMPACT OF CONTINUOUS SUBCUTANEOUS INSULIN INFUSION ON DIABETES RELATED COMPLICATIONS AND ASSOCIATED COSTS IN TYPE 2 DIABETES PATIENTS IN SWEDEN}

$\underline{\text { S. Roze }}{ }^{1}$, E. Duteil ${ }^{1}$, A. Delbaere ${ }^{2}$, S. De Portu ${ }^{2}$, A.S. Brandt ${ }^{3}$, J. Hellman ${ }^{4}$, A. Frid ${ }^{5}$
${ }^{1}$ HEVA HEOR, Health economics, Lyon, France

${ }^{2}$ Medtronic, Health economics, Tolochenaz, Switzerland

${ }^{3}$ Medtronic Danmark, Health economics, Kobenhavn, Denmark

${ }^{4}$ Uppsala University Hospital, medical sciences, Uppsala, Sweden

${ }^{5}$ Lund University, Endocrinology, Malmö, Sweden

Background and Aims: Assessing the reduction of complications and associated costs with continuous subcutaneous insulin infusion (CSII) versus multiple daily injections (MDI) in uncontrolled type 2 diabetes patients (T2D) in Sweden.

Method: The incidence of diabetes-related complications was calculated with the Core Diabetes Model. The population characteristics, the reduction of $\mathrm{HbAlc}$, and insulin dose were based on the Opt2mise study (Reznik et al., Lancet 2014). For a baseline $\mathrm{HbA} 1 \mathrm{c}$ of $9.0 \%$, the reduction in $\mathrm{HbA} 1 \mathrm{c}$ was $-1.1 \%$ for CSII versus $-0.4 \%$ for MDI. Costs were specific to Sweden and expressed in 2015 SEK.

Results: According to the Core Diabetes Model, after 5 years of CSII therapy diabetes related complications would be reduced. The cumulative incidence for complications associated with renal disease, eye disease, ulcer/amputation and cardiovascular disease would be reduced by $31 \%, 25 \%, 6 \%$ and $5 \%$ respectively. Based on the simulation this is leading to a reduction in cost of complication of $15 \%$ per patient over 5 years. CSII over life-time would delay the onset of end-stage renal disease, amputation, myocardial infarction and severe vision loss by 0.88 years, 0.69 years, 0.66 years and 0.96 years respectively. Mortality rate would also be reduced by $5 \%$ with CSII. The insulin dose was lower for CSII patients than MDI patients (96.5UI versus 122.1UI per day), equating to a cost saving of $28^{\prime} 025$ SEK per patients over 5 years.

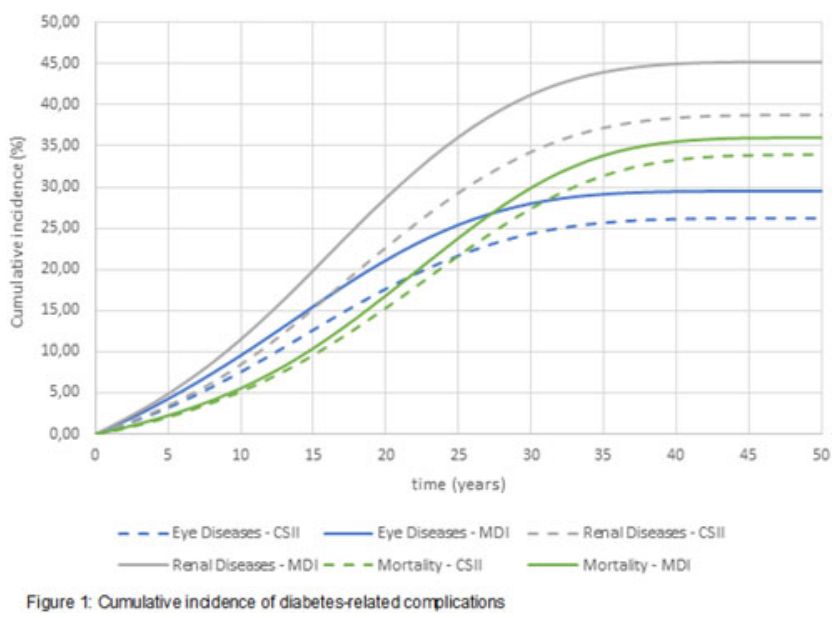

Conclusion: CSII in uncontrolled T2D in Sweden results in significant improvements in HbA1c compared to MDI, which may give important reductions in diabetes-related complications and associated costs.

053

\section{SYSTEMATIC STUDY TO OPTIMIZE INSULIN DELIVERY THROUGH A CONTINUOUS SUBCUTANEOUS INSULIN INFUSION (CSII) CATHETER}

J.R. Hauzenberger ${ }^{1}$, M.C. Torjman ${ }^{2}$, P.A. McCue ${ }^{3}$, T.R. Pieber ${ }^{\text {, J.I. Joseph }}$ 
${ }^{1}$ Medical University of Graz, Internal Medicine, Graz, Austria

${ }^{2}$ Thomas Jefferson University, Anesthesiology, Philadelphia, USA

${ }^{3}$ Thomas Jefferson University, Pathology, Philadelphia, USA

Background and Aims: A pilot study is being performed in ambulatory humans scheduled for a surgical abdominoplasty to compare histology and cytokine expression profiles of tissue surrounding Teflon CSII catheters and help formulate a hypothesis, why insulin absorption into the circulation becomes more variable 2-4 days after catheter insertion.

Method: 2 catheters (Quick-set ${ }^{\circledR}$ ) were inserted into the abdominal tissue 4, 3, 2 days, and 2 hours prior to surgery. The surrounding tissue was removed and stained histochemically to determine morphological changes and number/location of capillaries, lymph vessels and inflammatory cells. RNA was isolated and qPCR carried out to determine changes in cytokine gene expression.

Results: Tissue from the first 3 subjects has been analyzed. Histology revealed mechanical impact of catheter insertion, minimal thrombus, neutrophil recruitment as well as reticulin fiber disruption. Capillaries and lymphatic vessels remained at a normal density over 4 days. IHC showed very little macrophage recruitment, while qPCR showed a 3-fold increase in CD68 gene expression over 4 days. IL-1b expression was $13 x$ higher than in control tissue in the first 48 hours and decreased on day 3. IL-6 expression increased 32-fold within 2 hours. IL-8 mRNA levels were highest $(123 \mathrm{x} / 50 \mathrm{x})$ on day 3 in 2 out of 3 subjects. IL-10 production increased 8-fold and TNF-a 4-fold within 2 days.

Conclusion: CSII catheter insertion damaged adipose cells, connective tissue, capillaries and lymph vessels and triggered the foreign body response immediately after insertion, leading to an upregulation of cytokine gene expression. qPCR was an adequate method to evaluate tissue inflammation surrounding the CSII catheters.

\section{4}

\section{EARLIER ONSET OF EXPOSURE AND GREATER EARLY PHARMACOKINETIC AND PHARMACODYNAMIC EFFECTS FOR FASTER-ACTING INSULIN ASPART VERSUS INSULIN ASPART USING CONTINUOUS SUBCUTANEOUS INSULIN INFUSION}

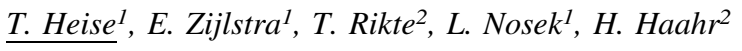

${ }^{1}$ Profil, Neuss, Germany

${ }^{2}$ Novo Nordisk A/S, Sфborg, Denmark

Background and Aims: Faster-acting insulin aspart (faster aspart) is insulin aspart (IAsp) set in a new formulation with a faster initial absorption following subcutaneous injection. This trial evaluated the pharmacokinetic $(\mathrm{PK})$ and pharmacodynamic (PD) properties of faster aspart during continuous subcutaneous insulin infusion (CSII) in a randomised crossover design.

Method: Patients $(\mathrm{N}=48)$ with type 1 diabetes (mean $\pm \mathrm{SD}$ age: $46.3 \pm 8.6 \mathrm{yrs} ; \mathrm{HbA}_{1 \mathrm{c}}: 7.4 \pm 0.6 \%$ ) received faster aspart or IAsp as a CSII bolus dose $(0.15 \mathrm{U} / \mathrm{kg})$ on top of basal CSII $(0.02 \mathrm{U} / \mathrm{kg} / \mathrm{h})$ under glucose clamp conditions (blood glucose target $5.5 \mathrm{mmol} / \mathrm{L}$ [100 mg/dL]; total duration $27 \mathrm{~h}$ : $13 \mathrm{~h}$ run-in and $14 \mathrm{~h}$ post-bolus dosing).

Results: Following a bolus dose, $\mathrm{t} 50 \% \mathrm{C}_{\max }$ and $\mathrm{t}_{\max }$ occurred $36 \%(12 \mathrm{~min})$ and $31 \%$ (26 min) earlier. Early insulin exposure (within the first $2 \mathrm{~h}$ ) was greater for faster aspart than IAsp, with a near 3 -fold greater exposure in the first $30 \mathrm{~min}$ (Table); total exposure was similar. Faster aspart had an earlier $\mathrm{t}^{50 \%} \mathrm{GIR}_{\max }$ $(21 \% ; 11 \mathrm{~min}), \mathrm{tGIR}_{\max }(14 \% ; 19 \mathrm{~min})$ and a greater glucoselowering effect during the first $2 \mathrm{~h}$ following bolus dosing versus IAsp; total glucose-lowering effect was similar (Table). Both treatments were well tolerated.

Conclusion: Faster aspart showed an enhanced early exposure and action compared with IAsp in CSII. Improvements in onset of exposure and action were more pronounced than those previously reported for subcutaneous injection of faster aspart (Heise T, et al. Diabetes Obes Metab 2015;17:682-8).

Table: PK and PD results for faster aspart versus IAsp.

\begin{tabular}{|c|c|c|c|}
\hline $\begin{array}{l}\text { PK endpoints } \\
\text { (insulin exposure") }\end{array}$ & $\begin{array}{c}\text { Treatment ratio } \\
\text { faster aspart//Asp } \\
{[95 \% \mathrm{Cl}]^{\dagger}}\end{array}$ & $\begin{array}{l}\text { PD endpoints } \\
\text { (glucose-lowering } \\
\text { effect) }\end{array}$ & $\begin{array}{c}\text { Treatment ratio } \\
\text { faster aspart/1Asp } \\
{[95 \% \mathrm{Cl}]}\end{array}$ \\
\hline $\begin{array}{l}\text { Onset } \\
t 50 \% \mathrm{C}_{\max } \\
\mathrm{t}_{\max }\end{array}$ & $\begin{array}{l}0.64[0.57 ; 0.71]^{\dagger} \\
0.69[0.60 ; 0.78]^{\dagger}\end{array}$ & $\begin{array}{l}\text { Onset } \\
\text { t50\%GIR max } \\
\text { tGIR }\end{array}$ & $\begin{array}{l}0.79[0.72 ; 0.86]^{\dagger} \\
0.86[0.75 ; 0.97]^{\dagger}\end{array}$ \\
\hline Early & & Early & \\
\hline AUC $0-15 \mathrm{~min}$ & $7.05[3.73 ; 136.57]^{t}$ & NA & NA \\
\hline AUC $-30 \mathrm{~min}$ & $2.95[2.32 ; 3.73]$ & AUCGIR,0-30min & $2.18[1.33,5.04]^{t}$ \\
\hline AUC $0-1 \mathrm{n}^{ \pm}$ & $1.52[1.37 ; 1.69]$ & AUCGIR.Q-1h & $1.52[1.29: 1.83]^{\dagger}$ \\
\hline$A \cup C_{0-2 h}$ & $1.18[1.10 ; 1.26]$ & AUCGIR,a-2n & $1.21[1.07 ; 1.36]$ \\
\hline Total & & Total & \\
\hline AUClasp, Total & $0.97[0.90 ; 1.05]$ & AUCGR,Total & $1.04[0.95 ; 1.13]$ \\
\hline $\mathrm{C}_{\max }$ & $1.11[1.03 ; 1.19]$ & GIR $\max$ & $1.04[0.94 ; 1.14]$ \\
\hline
\end{tabular}

using Fieller's method. $\mathrm{AUC}=$ area under the curve; $\mathrm{Cl}=$ confidence interval; $\mathrm{GIR}=$ glucose infusion rate; NA=not available.

055

METABOLIC CONTROL AND PERINATAL OUTCOME IN TYPE 1 PRE-GESTATIONAL DIABETES TREATED WITH CONTINUOUS SUBCUTANEOUS INSULIN INFUSION (CSII) OR SENSOR-AUGMENTED PUMP (SAP)

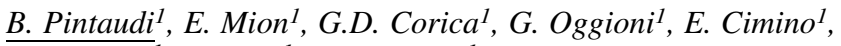

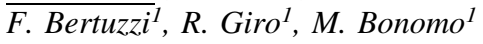

${ }^{1}$ A.O. Ospedale Niguarda Ca' Granda, Interdisciplinary Diabetes and Pregnancy Center, Milano, Italy

Background and Aims: Persistence of adverse pregnancy outcomes has recently led to a growing use of insulin pumps (CSII) in pre-gestational diabetes. Published results, however, failed to demonstrate clear-cut benefits of CSII on maternal glycemic control and obstetrical/perinatal outcomes. New perspectives could be opened by continuous glucose monitoring (CGM)-enabled insulin pumps (SAP), allowing a more aggressive therapeutic approach compared to "stand-alone" pumps. This study aimed to evaluate outcomes of pregnancies with T1D treated with CSII or SAP at our Centre in recent years.

Method: From 2010 on, 165 pregnancies with T1D were followed: 40 used CSII (age $34.9 \pm 3.9$ years, BMI $24.6 \pm 4.6$ ), 20 SAP (age $33.6 \pm 3.9$ years, BMI $22.8 \pm 7.1$ ), 105 multiple daily injections (MDI). The same outpatient protocol, with glycemic targets $<90 \mathrm{mg} / \mathrm{dl}$ fasting, $<130 \mathrm{mg} / \mathrm{dl} 1$-h post-prandial, was applied in all groups. SAP-treated patients used the sensor continuously; no structured algorithms were provided, therapy was adjusted at each visit after discussion with the medical team.

Results: No significant differences between CSII and SAP in third trimester $\mathrm{HbA} 1 \mathrm{c}(6.4 \pm 0.7 \%$ vs. $6.5 \pm 0.6 \%)$ and perinatal outcome (pre-term delivery, macrosomia, neonatal hypoglycemia, NICU admission) were found. All parameters were similar in CSII/SAP and MDI patients. Linear regression analysis did not 
show any association between the system in use and variations in HbA1c, maternal weight gain and birth weight.

Conclusion: In our experience an essentially "professional" use of Real-Time CGM-based SAP in diabetic pregnancies did not lead to better results than "stand-alone" CSII. A thorough evaluation of this treatment option requires larger controlled studies, adopting a true "patient-oriented" use of SAP.

\section{6}

LONG TERM FOLLOW-UP OF SENSOR - AUGMENTED PUMP THERAPY WITH LOW GLUCOSE SUSPEND FUNCTION IN TYPE 1 DIABETES PATIENTS WITH HIGH RISK OF HYPOGLYCEMIA

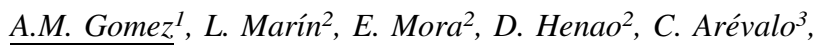
O. Muñoz ${ }^{4}$ M. Rondön ${ }^{5}$

${ }^{1}$ Hospital Universitario San Ignacio, Endocrinology, Bogotá D.C, Colombia

${ }^{2}$ Hospital Universitario San Ignacio, Endocrinology, Bogotá, Colombia

${ }^{3}$ Hospital Universitario San Ignacio, Nutrition, Bogotá, Colombia

${ }^{4}$ Hospital Universitario San Ignacio, Epidemiology, Bogotá, Colombia

${ }^{5}$ Hospital Universitario San Ignacio, Epidemiology and statistics, Bogotá, Colombia

Background and Aims: To assess the efficacy and safety of long term use of Sensor-augmented Pump Therapy with low glucose suspend function (SAP + LGS) in Type 1 Diabetes (T1D) patients who initiated this therapy because of hypoglycemia and poor metabolic control.

Method: The study was an observational prospective cohort study from August 2010 until september 2015 of T1D patients with hypoglycemia, older than 14 years, who started SAP + LGS at Hospital Universitario San Ignacio, Bogotá, Colombia. The main indication was hypoglycemia and poor metabolic control. Demographic and clinic variables were registered as well as A1c levels at the beginning and during follow-up.

Results: 94 T1D patients with hypoglycemia were included. We found a decreased incidence of severe hypoglycemia in the last year from 4 to 0.02 episodes/patient/year $(p=0.0032)$. Only $2 \%$ of the patients presented severe hypoglycemia, which is

\section{Efficacy of long term HbA1c reduction on SAP + LGS}

Figure 1. HbA1c Reduction

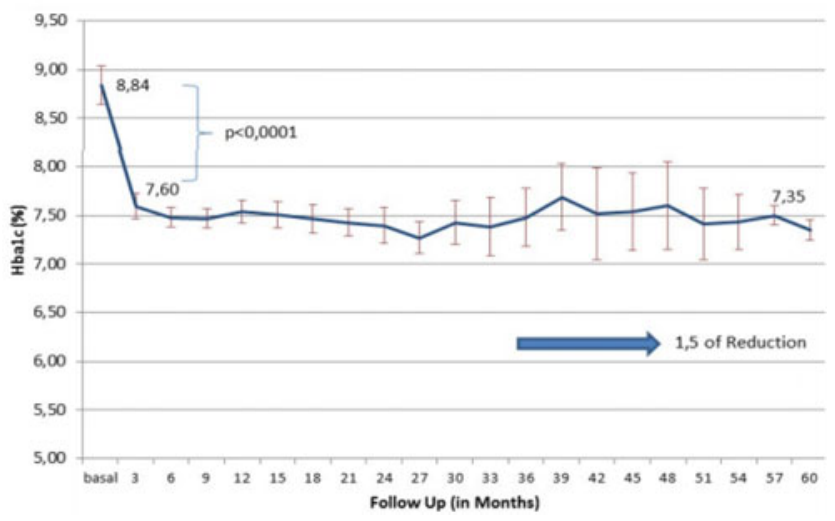

different from the baseline of $71.3 \%$ and only $12 \%$ of patients remained with hypoglycemia unawareness $(p<0.0001)$ after therapy. The patient proportion at the beginning of the study with A1C less than $7 \%$ was $16 \%$, of these patients $87 \%$ had severe hypoglycemia. At the end of the follow-up, $44.7 \%$ of patients reached an A1c less than $7 \%$ and only $4.8 \%$ had severe hypoglycemia ( $\mathrm{p}<0.0001)$.

Conclusion: SAP + LGS therapy reduces in a statistically significant quantity the severe hypoglycemic events and hypoglycemia unawareness in T1D patients who failed multiple dosis of insulin and had a high risk of severe hypoglycemia. SAP+ LGS therapy allows better metabolic control in a safer way. This effect was observed early and was maintained during the followup period.

057

\section{EVALUATION OF A NOVEL TOOL TO ADJUST INSULIN BOLUSES BASED ON CGM TREND ARROWS AND INSULIN SENSITIVITY IN CHILDREN AND YOUTH USING INSULIN PUMP THERAPY}

\author{
M.L. Lawson ${ }^{1}$, E. Heffernan ${ }^{1}$, C. Richardson ${ }^{1}$, J. Courtney ${ }^{1}$, \\ B. Bradley ${ }^{1}$ \\ ${ }^{1}$ Children's Hospital of Eastern Ontario, \\ Endocrinology and Metabolism, Ottawa, Canada
}

Background and Aims: Effective strategies for adjusting insulin for CGM trend arrows are lacking. We developed a novel tool based on patient's insulin sensitivity factor.

To compare the effect of the Trend Arrow Adjustment Tool (TAAT), JDRF CGM Study Group 10/20\% adjustment tool, and no adjustment for arrows, on postprandial glucose.

Method: Single-blinded counterbalance treatment assignment crossover study of 20 children/youth (age 5-18 years) with type 1 diabetes using Medtronic Veo pump and Enlite sensors. During a day hospital assessment, trend arrows were induced through standardized exercise or oral carbohydrate. Subjects consumed a standardized meal with insulin adjusted for trend arrows using assigned method. Subjects used this method at home in week 1, made no adjustment for arrows in week 2, and used the alternative method in week 3 . CGM data were used to analyse postprandial glucose. Child/youth and parent satisfaction and ease of use were evaluated after week 1 and 3 .

Results: Time with postprandial glucose in target range was equivalent with TAAT and $10 / 20 \%$. There was a trend towards more time in target range and less hypoglycemia with both tools compared to ignoring arrows. Significantly more errors by children/youth and parents were made with the $10 / 20 \%$ adjustment $(17 / 70$ uses vs. $1 / 84, p<0.001)$. Satisfaction and ease of tool use was greatest with TAAT. This tool was the preferred method for future use among $77 \%$ of children/youth and $80 \%$ of parents.

Conclusion: TAAT is a simple, successful, and well-received method of adjusting insulin boluses for CGM trend arrows in children and youth on insulin pump therapy.

\section{8}

HOME USE OF DAY-AND-NIGHT CLOSED-LOOP INSULIN DELIVERY IN ADOLESCENTS WITH TYPE 1 DIABETES: A RANDOMISED CLINICAL TRIAL

M. Tauschmann $^{1}$, J.A. Allen ${ }^{1}$, M.E. Wilinska ${ }^{1}$, H. Thabit ${ }^{1}$, C.L. Acerini ${ }^{2}$, D.B. Dunger ${ }^{1}$, R. Hovorka ${ }^{1}$ 
${ }^{1}$ University of Cambridge Metabolic Research Laboratories, Institute of Metabolic Science, Cambridge, United Kingdom ${ }^{2}$ University of Cambridge, Paediatrics, Cambridge, United Kingdom

Background and Aims: To evaluate feasibility, safety and efficacy of day-and-night closed-loop insulin delivery in adolescents with type 1 diabetes under free-living conditions without remote monitoring or supervision.

Method: In an open-label randomised crossover study design, 12 adolescents on insulin pump therapy (age 14.6 \pm 3.1years; HbAlc $69 \pm 8 \mathrm{mmol} / \mathrm{mol}$ [8.5 $\pm 0.7 \%$ ]; duration of diabetes $7.8 \pm 3.5$ years; mean \pm SD) underwent a period of closed-loop insulin delivery and a period of sensor-augmented insulin pump therapy, each period lasting three weeks without remote monitoring. During closed-loop, a model predictive algorithm automatically directed insulin delivery between meals and overnight; prandial boluses were administered by participants using a bolus calculator.

Results: The proportion of time that sensor glucose was in the target range (3.9 to $10 \mathrm{mmol} / 1$; primary endpoint) was greater during the closed-loop phase than with sensor-augmented pump therapy ( $66.6 \pm 7.9 \%$ vs. $47.7 \pm 14.4 \%$, mean $\pm S D, p<0.001)$. The mean glucose level was lower during closed-loop (8.7 \pm 0.9 vs. $10.5 \pm 1.8 \mathrm{mmol} / \mathrm{l}, \mathrm{p}=0.001)$, as was the time spent above target $(29.7 \pm 9.2 \%$ vs. $49.1 \pm 16.5 \%, \mathrm{p}<0.001)$. The time spent with glucose levels below $3.9 \mathrm{mmol} / 1$ was low and comparable between interventions (4.3 [1.4 to 5.2] vs 2.4 [0.3 to $5.7] \%$, median [IQR], $\mathrm{p}>0.20)$. Improved glucose control during closed-loop related to increased variability of basal insulin delivery $(\mathrm{p}<0.001)$ and an increase in total daily insulin (53.5 [39.5 to 72.1$]$ vs. 51.5 [37.6 to 64.3$] \mathrm{U} /$ day; $\mathrm{p}=0.006$ ) compared to control intervention.

Conclusion: Free-living unsupervised home use of day-andnight closed-loop in adolescents with type 1 diabetes is safe and feasible. Compared to sensor-augmented insulin pump therapy, closed-loop may improve glucose control without increasing the risk of hypoglycaemia.

059

\section{EVALUATION OF CLOSED LOOP PERFORMANCE WITH ANAEROBIC VERSUS AEROBIC EXERCISE}

D. Jayawardene $^{1}$, S. McAuley ${ }^{1,2}$, J. Horsburgh ${ }^{2}$, A. La Gerche ${ }^{3}$, A.J. Jenkins ${ }^{1}$, G.M. Ward ${ }^{1}$, R. MacIsaac ${ }^{1,2}$, T.J. Roberts ${ }^{3}$, A. Roy $^{4}$, B. Grosman ${ }^{4}$, N. Kurtz ${ }^{4}$, D. O'Neal ${ }^{1,2}$

${ }^{1}$ St Vincent's Hospital, Department of Endocrinology and Diabetes, Melbourne, Australia

${ }^{2}$ The University of Melbourne, St Vincent's Hospital, Department of Medicine, Melbourne, Australia

${ }^{3}$ St Vincent's Hospital, Department of Cardiology, Melbourne, Australia

${ }^{4}$ Medtronic Diabetes, Closed Loop Division, Northridge, USA

Background: Anaerobic (AnE) and aerobic exercise (AeE) challenge closed loop (CL) systems.

Aim: To compare CL glucose homeostasis for adults with type 1 diabetes (T1D) undertaking AnE versus AeE.

Method: Eight adults with T1D (7 females, 1 male; mean \pm SD diabetes duration $25 \pm 8$ years; age $40 \pm 4$ years; HbA1c $7.7 \pm 0.8 \%$ ) undertook 45 minutes of $\mathrm{AnE}$ and $\mathrm{AeE}$ in random

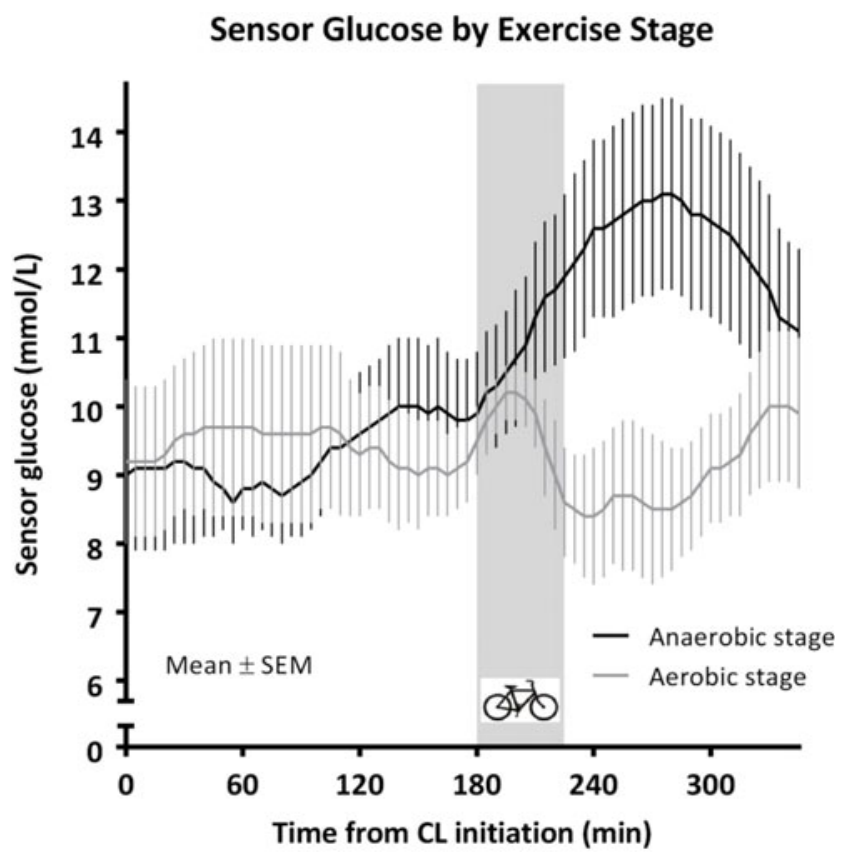

order one week apart. CL (PID algorithm) was activated 180 minutes pre-exercise. CL glucose target was increased at 120 minutes pre-exercise until 15 minutes post-exercise. Venous samples were collected for glucose, ketones, lactate, insulin and counter-regulatory hormones hourly for the first 120 minutes post-CL activation, then at 15 minute intervals until two hours post-exercise completion.

Results: Plasma lactate (mean \pm SD) was greater after AnE than AeE $(6.4 \pm 1.5$ vs. $3.2 \pm 1.5 \mathrm{mmol} / \mathrm{L} ; \mathrm{p}=0.0001)$. Plasma glucose was equivalent for $\mathrm{AnE}$ and $\mathrm{AeE}$ pre-exercise (9.2 \pm 2.7 vs. $9.5 \pm 2.1 \mathrm{mmol} / \mathrm{L} ; \mathrm{p}=0.59)$; was greater for AnE than AeE during exercise $(11.6 \pm 1.8$ vs. $9.0 \pm 2.8 \mathrm{mmol} / \mathrm{L} ; \mathrm{p}=0.005)$ and $60 \mathrm{~min}-$ utes post-exercise $(13.2 \pm 4.0 \mathrm{vs} .8 .8 \pm 2.9 \mathrm{mmol} / \mathrm{L} ; \mathrm{p}=0.003)$ but not $60-120$ minutes post-exercise $(12.2 \pm 4.4 \mathrm{vs} .9 .7 \pm 2.6 \mathrm{mmol} / \mathrm{L}$; $\mathrm{p}=0.20)$. AnE sensor glucose $(\mathrm{SG}) \mathrm{AUC}>10 \mathrm{mmol} / \mathrm{L}$ was greater than AeE $(62.4 \pm 69.8 \mathrm{mmol} / \mathrm{L}$ vs. $19.6 \pm 26.1 \mathrm{mmol} / \mathrm{L} ; \mathrm{p}=0.03$; see figure). During AnE, two participants had glucose $>14 \mathrm{mmol} / \mathrm{L}$ and ketones $>0.6 \mathrm{mmol} / \mathrm{L}$. There was no $\mathrm{SG}<4 \mathrm{mmol} / \mathrm{L}$ during either AnE or AeE. Insulin and counter-regulatory hormone levels are pending.

Conclusion: CL minimised hypoglycaemia during both AnE and AeE. During AnE mean glucose levels increased, and ketosis occurred in $25 \%$ of participants. These observations may relate to counter-regulatory hormone release. Tailored AnE and AeE protocols may improve CL performance.

060

\section{SIPPING FOR SAFETY: A SIMPLE SNACKING STRATEGY TO MITIGATE HYPOGLYCEMIA DURING EXERCISE WHILE ON CLOSED LOOP INSULIN DELIVERY}

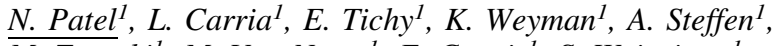
M. Zgorski ${ }^{1}$, M. Van Name ${ }^{1}$, E. Cengiz ${ }^{1}$, S. Weinzimer ${ }^{1}$, W. Tamborlane ${ }^{1}$, J. Sherr ${ }^{1}$

${ }^{1}$ Yale School of Medicine, Pediatric Endocrinology, New Haven, USA 


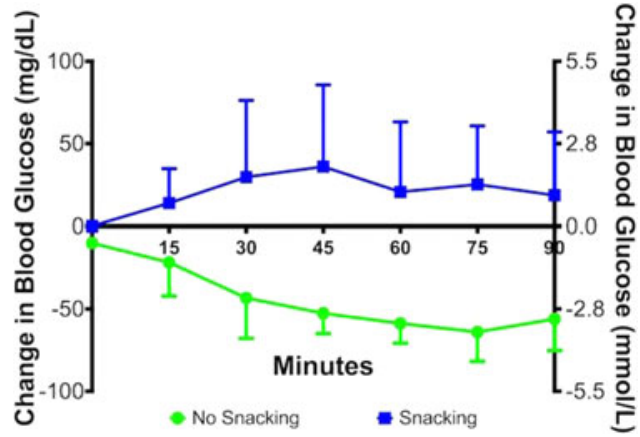

Background and Aims: While closed loop (CL) insulin delivery systems are approaching commercialization, avoidance of hypoglycemia during and after exercise remains problematic. Dual hormone systems and use of activity monitors to inform algorithm parameters increase the complexity of potential commercial devices. Simple strategies frequently recommended in clinical practice include consuming a snack prior to exercise. Therefore, our objective was to assess whether proactive snacking prior to exercise could be used in conjunction with CL insulin delivery to avoid hypoglycemia.

Method: Six T1D subjects (age 13-24y, duration $7 \pm 6 y$, A1c $7.1 \pm 1.3 \%$ ) underwent two 12-hour periods of CL control using the Medtronic Overnight CL system: with CL alone vs. $\mathrm{CL}+$ proactive snacking. Meals were identical and accompanied by pre-meal boluses. Blood glucose (BG) and insulin were measured every 30 minutes. At 3p.m., exercise commenced with a standardized protocol of four 15-minute periods of brisk treadmill walking to $65-70 \% \mathrm{HR}_{\max }$. Gatorade was provided on snacking visits prior to exercise and mid-way through the 60 -minute exercise period $(\mathrm{BG}>150 \mathrm{mg} / \mathrm{dL}=15$ grams and $<150 \mathrm{mg} / \mathrm{dL}=30$ grams).

Results: Hypoglycemia (BG $<60 \mathrm{mg} / \mathrm{dL}$ ) occurred in $50 \%$ of non-snacking visits, two during exercise and one 40-minutes post-exercise; whereas, no hypoglycemia occurred during snacking visits. The figure shows glycemic excursions during exercise with progressive drop in BG noted on non-snacking days $(\mathrm{p}=0.003)$.

Conclusion: These preliminary data support the use of a simple snacking strategy during exercise to avoid hypoglycemia while on CL insulin delivery. This may be a feasible alternative to activity monitors and dual hormone delivery as early iterations of CL systems become commercially available.

\section{1}

\section{RATE OF CHANGE ACCURACY ASSESSMENT AND LAG TIME CHARACTERIZATION OF AN IMPLANTABLE CONTINUOUS GLUCOSE MONITORING (CGM) SYSTEM IN THE PRECISE STUDY}

\section{E. Zijlstra ${ }^{1}$, A. DeHennis ${ }^{2}$, X. Chen ${ }^{2}$, M. Link ${ }^{3}$}

${ }^{1}$ Profil, Medical Technology, Neuss, Germany

${ }^{2}$ Senseonics Incorporated, Engineering, Germantown, USA

${ }^{3}$ University of Ulm, Institut für Diabetes Technologie, Ulm, Germany

Background and Aims: At times of rapid glucose fluctuations real-time subcutaneous glucose sensing performance is limited by a system's measurement delay, typically referred to as

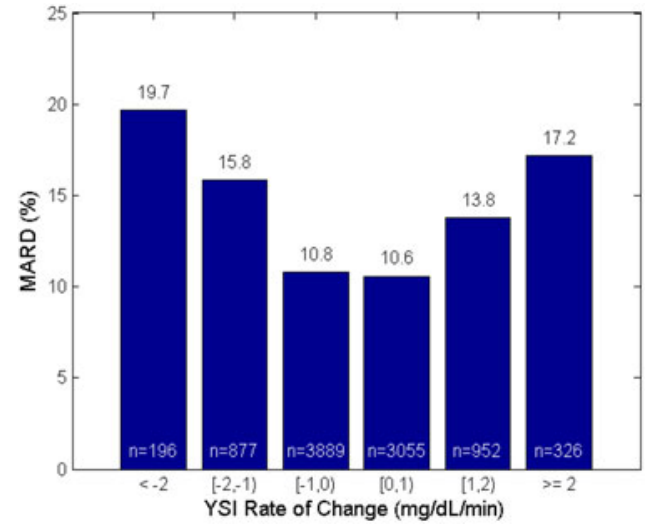

lag time. Total delay consists of an approximate 5- to 10-minute physiological delay due to glucose exchange between blood and interstitial fluid and a sensing delay due to the sensing mechanism. This analysis investigated the dependence of lag time and accuracy on glucose rate of change (ROC) for a 90-day implantable CGM system.

Method: Sensor glucose and paired YSI data from 44 subjects with T1DM participating in a prospective, single-arm, multicenter clinical study (PRECISE) were analyzed. Glucose fluctuations were achieved by regular food consumption and insulin administrations. For each sensor-YSI pair, the YSI ROC and mean absolute relative difference (MARD) were calculated. Lag time was also calculated over falling, steady state, and rising glucose using the methods of (Kovatchev et al 2009).

Results: Figure 1 shows the MARD impact across the full ROC characterization for the study. Lag time for different YSI rates of change, glucose decreasing more than $1 \mathrm{mg} / \mathrm{dL} / \mathrm{min}$, glucose stable within $1 \mathrm{mg} / \mathrm{dL} / \mathrm{min}$, and glucose increasing more than $1 \mathrm{mg} / \mathrm{dL} /$ min was $12.4 \mathrm{~min}, 10.1 \mathrm{~min}$, and $8.2 \mathrm{~min}$, respectively.

Conclusion: The PRECISE study characterized the implantable CGM system over a range of varying glucose concentrations and showed an impact on overall accuracy and lag time performance consistent with published theory of physiological glucose diffusion.

\section{2}

INPATIENT CLINICAL TRIAL OF A FULLY CLOSED-LOOP ARTIFICIAL PANCREAS USING ONLY CGM AND ACCELEROMETER DATA FOR INSULIN DOSING

F. Cameron ${ }^{1}$, T.T. Ly ${ }^{2}$, G.P. Forlenza ${ }^{3}$, S.D. Patek ${ }^{4}$, N. Baysal ${ }^{1}$, L.H. Messer ${ }^{3}$, P. Clinton ${ }^{2}$, D.M. Maahs ${ }^{3}$, B.A. Buckingham ${ }^{2}$, $\underline{B . W . B e q u e t t e}^{1}$

${ }^{1}$ Rensselaer Polytechnic Institute, Chemical and Biological Engineering, Troy, USA

${ }^{2}$ Stanford University, Pediatric Endocrinology, Stanford, USA

${ }^{3}$ University of Colorado at Denver, Pediatric Endocrinology, Aurora, USA

${ }^{4}$ University of Virginia, Systems and Information Engineering, Charlottesville, USA

Background and Aims: A risk-minimizing probabilisticbased closed-loop artificial pancreas (AP) was tested at two clinical sites on 10 subjects for 300 total hours. The controller received signals from an activity monitor and CGM. It did not receive any meal, activity, or wake announcements. The activity 


\begin{tabular}{|l|l|l|l|l|}
\hline \multirow{2}{*}{} & \multicolumn{4}{|c|}{ Time in Reference Glucose Range (m/dl) } \\
\cline { 2 - 5 } & $\%<50$ & $\% 50-70$ & $\% 70-180$ & $\%>250$ \\
\hline Mean & .03 & 1.8 & 78 & 3.8 \\
\hline Std. Dev. & .10 & 2.4 & 9.9 & 4.0 \\
\hline
\end{tabular}

sensor enabled switching between sleeping and waking control modes and made the controller less aggressive during exercise. The controller used only the subject's basal rates and total daily dose as tuning parameters.

Method: The AP was implemented on the UVA DiAs system and used a Roche Spirit Combo Insulin Pump with a Dexcom G4 Continuous Glucose Monitor and a Zephyr BioHarness 3.0 accelerometer. Therapy began at 9 AM after fasting and ended at roughly 5PM the next day. Subjects had several periods of mild exercise, engaging in activities such as walking and ultimate Frisbee. Subjects were encouraged to eat any type and size of meal that they wished.

Results: On a 24-hour basis, subjects had mean reference blood glucose/CGM values of $141 / 149 \mathrm{mg} / \mathrm{dl}$, with $80 / 72 \%$ of time spent between 70 and $180 \mathrm{mg} / \mathrm{dl}$. Meter glucose levels $<70 \mathrm{mg} / \mathrm{dl}$ prompted 1.5 hypoglycemia interventions per day. Four interventions were due to inaccurate accelerometer data. One subject accounted for 5 interventions. Subjects ate an average of $292 \pm 119 \mathrm{~g} \mathrm{CHO}$ each per study day.

Conclusion: The MMPPC was tested in-clinic against repeated unannounced meals of variable size and with mild exercise. It maintained good control and needs to be tested in an outpatient setting with more varied activity and sleeping patterns.

063

\section{OUTPATIENT CLOSED-LOOP CONTROL WITH UNANNOUNCED MODERATE EXERCISE IN ADOLESCENTS USING ZONE MODEL PREDICTIVE CONTROL}

L.M. Huyett ${ }^{1,2}$, T.T. Ly ${ }^{3}$, S. Reuschel-DiVirgilio ${ }^{3}$, S.M. Clay ${ }^{4}$,

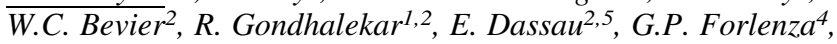
F.J. Doyle III ${ }^{2,5}$, J.E. Pinsker ${ }^{2}$, D.M. Maahs ${ }^{4}$,

B.A. Buckingham ${ }^{3}$

${ }^{1}$ University of California Santa Barbara, Department of Chemical Engineering, Santa Barbara- CA, USA

${ }^{2}$ William Sansum Diabetes Center, Research Division, Santa Barbara- CA, USA

${ }^{3}$ Stanford University, Department of Pediatrics- Division of Pediatric Endocrinology and Diabetes, Stanford-CA, USA

${ }^{4}$ University of Colorado Anschutz Medical Campus, Barbara Davis Center for Childhood Diabetes, Aurora- CO, USA

${ }^{5}$ Harvard University, John A. Paulson School of Engineering \& Applied Sciences, Cambridge-MA, USA

Background and Aims: We present the first evaluation of an artificial pancreas (AP) using the UCSB Zone Model Predictive Control and Health Monitoring System (ZMPC + HMS) algorithms in adolescents. We sought to determine the feasibility of the system in the outpatient environment with unannounced moderate exercise and announced meals.

Method: Ten adolescents (11-17 years, 5M/5F) completed $72 \mathrm{~h}$ of closed-loop control (CLC) in a hotel setting. Data on $95 \pm 14 \mathrm{~h}$ of sensor-augmented pump therapy (SAP) prior to CLC are included as a comparison. The AP system consisted of the ZMPC + HMS algorithms on the Diabetes Assistant platform (UVA), Dexcom G4P Share sensor, and Roche Accu-Chek Spirit
Table 1: Glycemic control during CLC compared with SAP. Data are shown as mean \pm standard deviation for normally distributed data and median (interquartile range) for non-normally distributed data.

\begin{tabular}{|c|c|c|c|c|c|c|}
\hline & \multicolumn{3}{|c|}{ DAYAND NIGHT } & \multicolumn{3}{c|}{ NIGHT (00:00-07:00) } \\
\hline & SAP & CLC & p-value & SAP & CLC & p-value \\
\cline { 2 - 7 } Mean CGM & $173 \pm 31$ & $151 \pm 19$ & $0.042^{*}$ & $158 \pm 45$ & $150 \pm 27$ & 0.62 \\
$\% 70-180$ & $57 \pm 17$ & $71 \pm 10$ & $0.012^{*}$ & $66 \pm 25$ & $74 \pm 20$ & 0.45 \\
$\% 80-140$ & $30(21,42)$ & $47(39,53)$ & $0.002^{*}$ & $34 \pm 18$ & $49 \pm 24$ & 0.12 \\
$\%>250$ & $17 \pm 14$ & $8.0 \pm 6.9$ & 0.088 & $11 \pm 17$ & $5.3 \pm 6.2$ & 0.37 \\
$\%<70$ & $4.2 \pm 3.1$ & $2.5 \pm 1.8$ & 0.13 & $0.96(0.0,13)$ & $0.0(0.0,0.61)$ & 0.078 \\
\hline
\end{tabular}

*Indicates a p-value $<0.05$

Combo pump. Subjects engaged in $\geq 2 \mathrm{~h}$ of mild to moderate intensity exercise daily including soccer, tennis, frisbee, and bicycling. Meal size estimation and announcement were performed by subjects.

Results: Overall, subjects spent $71 \pm 10 \%$ of time with CGM $70-180 \mathrm{mg} / \mathrm{dL}$ compared to $57 \pm 17 \%$ during SAP $(\mathrm{p}=0.012)$. The mean glucose during the study was $151 \pm 19 \mathrm{mg} / \mathrm{dL}$. Nocturnal control was safe, with $0.52 \pm 1.1 \%$ of time spent with CGM $<70 \mathrm{mg} / \mathrm{dL}$ and $74 \pm 20 \%$ of time $70-180 \mathrm{mg} / \mathrm{dL}$ (00:0007:00). Despite large meals estimated up to $120 \mathrm{~g}$ of $\mathrm{CHO}$, only $8.0 \pm 6.9 \%$ of time was spent with CGM $>250 \mathrm{mg} / \mathrm{dL}$. The system remained connected in closed-loop for $97 \pm 2 \%$ of the total study time. No adverse events or severe hypoglycemia occurred.

Conclusion: The ZMPC + HMS algorithms provided safe glucose control in the adolescent outpatient environment, limiting both hyperglycemia and hypoglycemia in the face of unannounced exercise. The control achieved by the AP resulted in significantly more time spent in the desired glycemic range than SAP.

\section{4}

\section{IN-CLINIC EVALUATION OF A PREDICTIVE LOW GLUCOSE MANAGEMENT SYSTEM (PLGM) FOR HYPOGLYCEMIA PREVENTION}

$\underline{T . B a i l e y}^{1}$, B. Buckingham ${ }^{2}$, M. Christiansen ${ }^{3}$, S. Garg ${ }^{4}$, S. Weinzimer ${ }^{5}$, B. Bode ${ }^{6}$, S. Anderson ${ }^{7}$, R. Brazg ${ }^{8}, M$. Mao $^{9}$

${ }^{1}$ AMCR Institute- Inc., Diabetes, Escondido, USA

${ }^{2}$ Stanford University, Pediatrics, Stanford, USA

${ }^{3}$ Diablo Clinical Research, Diabetes, Walnut Creek, USA

${ }^{4}$ University of Colorado- Denver, Barbara Davis Center

for Childhood Diabetes, Aurora, USA

${ }^{5}$ Yale University, Pediatrics, New Haven, USA

${ }^{6}$ Atlanta Diabetes Associates, Diabetes, Atlanta, USA

${ }^{7}$ University of Virginia, Endocrinology and Metabolism, Charlottesville, USA

${ }^{8}$ Rainier Clinical Research Center, Diabetes, Renton, USA

${ }^{9}$ Medtronic-Inc., Diabetes, Northridge, USA

Background and Aims: Prediction based on continuous glucose monitoring (CGM) data is the basis for automatic suspension and resumption of insulin delivery by a PLGM feature, which is part of the MiniMed 640G insulin pump system. This study evaluated the safety and performance of the system.

Method: Standardized increases in basal insulin delivery rates were used in-clinic to induce hypoglycemia in subjects with type 1 diabetes. The PLGM feature was set with the suspend before low limit at $65 \mathrm{mg} / \mathrm{dL}$.YSI plasma glucose values were 
Table. Mean (SD) YSI values before and after automatic pump suspensions. Each point represents 68 interpolated YSI values.

\begin{tabular}{|c|c|}
\hline Time Point, min & YSI Mean (SD), min \\
\hline-60 & $133.6(27.2)$ \\
\hline-30 & $119.8(23.6)$ \\
\hline PLGM activated & $101.2(18.5)$ \\
\hline 30 & $88.3(18.8)$ \\
\hline 60 & $83.2(19.8)$ \\
\hline 90 & $87.8(25.4)$ \\
\hline 120 & $101.6(34.6)$ \\
\hline
\end{tabular}

used to establish hypoglycemia (defined as $\geq 2$ YSI values $\leq 65 \mathrm{mg} / \mathrm{dL}$ ).

Results: Eighty subjects age 14-74 were screened. Of the 69 successfully-completed experiments, the PLGM feature was activated in 68 cases and of these, the hypoglycemic criterion was not met in 41 cases, representing a $60.2 \%$ prevention rate; hypoglycemia was not avoided in the remaining 27 cases. The mean $( \pm$ SD) YSI glucose value at the start of the 68 pump suspensions was $101.2 \pm 18.5 \mathrm{mg} / \mathrm{dL}$ and the mean $( \pm \mathrm{SD}) \mathrm{du}-$ ration of the pump suspension events was $104.9 \pm 26.7 \mathrm{~min}$. (One experiment was successfully completed without PLGM feature activation.) Mean YSI values before and up to 120 minutes after the start of the pump suspension events are shown in the Table. There were no severe adverse events such as seizures, loss of consciousness, or DKA.

Conclusion: The PLGM feature in the MiniMed 640G system prevented $60.2 \%$ of predicted hypoglycemic events without significant rebound hyperglycemia. PLGM with auto-resumption represents a further advance in the automation of insulin delivery.

\section{5}

\section{PERFORMANCE AND SAFETY OF AN INTEGRATED BIHORMONAL ARTIFICIAL PANCREAS FOR AUTOMATED GLUCOSE CONTROL AT HOME}

$\underline{\text { H. Blauw }}^{1,2}$, A.C. van Bon ${ }^{3}$, R. Koops ${ }^{2}$, J.H. DeVries ${ }^{1}$

${ }^{1}$ Academic Medical Center at the University of Amsterdam, Endocrinology, Amsterdam, Netherlands

${ }^{2}$ Inreda Diabetic BV, R\&D, Goor, Netherlands

${ }^{3}$ Rijnstate Hospital, Internal Medicine, Arnhem, Netherlands

Background and Aims: To overcome the limitations of bihormonal artificial pancreas systems that consist of separate devices we integrated all components in one wearable device (Figure). The aim of this pilot study was to compare the performance and safety of our integrated artificial pancreas to standard insulin pump therapy.

Method: Ten adult patients with type 1 diabetes completed this randomized cross-over study. Treatment with the artificial pancreas started with one day and night in the clinical research center, followed by three days at home. The control period consisted of four days of insulin pump therapy at home with blinded continuous glucose monitoring for data collection. Days two through four were predefined as the analysis period.

Results: The median [IQR] time spent in euglycemia was increased during use of the artificial pancreas (84.7 [82.2-87.8] $\%$ vs. 68.5 [57.9-83.6] $\%$ for the control, $\mathrm{p}=0.007)$. The glucose level was comparable for the two treatments (7.3 [7.0-7.6] $\mathrm{mmol} / \mathrm{l}$ for the artificial pancreas vs. 7.7 [7.0-9.0] $\mathrm{mmol} / \mathrm{l}$ for the control, $\mathrm{p}=0.123$ ). Time in hypoglycemia was $1.3[0.2-3.2] \%$ for the artificial pancreas and $2.4[0.4-10.3] \%$ for the control

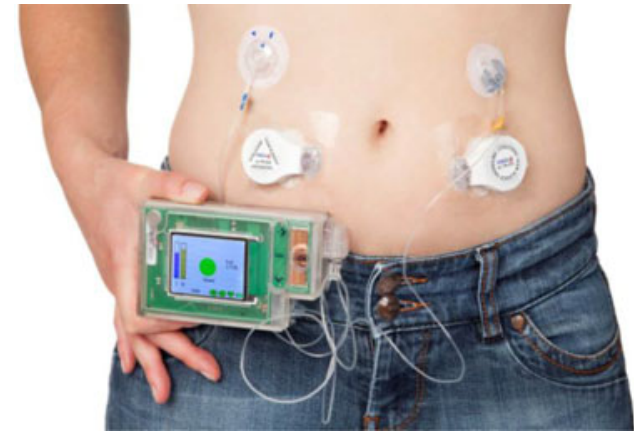

treatment $(\mathrm{p}=0.139)$. Separate analysis of daytime and nighttime showed that the improvements were mainly achieved during the night.

Conclusion: The results of this study suggest that our integrated artificial pancreas provides better glucose control than insulin pump therapy in patients with type 1 diabetes at home and that the treatment is safe.

066

OUTPATIENT 60-HOUR DAY-AND-NIGHT GLUCOSE CONTROL WITH DUAL-HORMONE ARTIFICIAL PANCREAS, SINGLE-HORMONE ARTIFICIAL PANCREAS, OR SENSOR-AUGMENTED PUMP THERAPY IN TYPE 1 DIABETES: RANDOMISED CONTROLLED TRIAL

A. Haidar ${ }^{1}$, V. Messier ${ }^{2}$, L. Legault ${ }^{3}$, M. Ladouceur ${ }^{4}$, R. Rabasa-Lhoret ${ }^{2}$

${ }^{1}$ Montreal Institute of Clinical Research, Montreal, Canada

${ }^{2}$ Montreal Institute of Clinical Research, Diabetes, Montreal, Canada

${ }^{3}$ McGill University, Pediatrics, Montreal, Canada

${ }^{4}$ The Research Center of the Université de Montréal Hospital Center, The Research Center of the Université de Montréal Hospital Center, Montreal, Canada

Background and Aims: To assess whether the dual-hormone (insulin and glucagon) artificial pancreas reduces hypoglycemia compared to the single-hormone (insulin alone) artificial pancreas in outpatient settings during the day and night

Method: In a randomized, three-way, crossover trial, we compared the dual-hormone artificial pancreas, the single-hormone artificial pancreas, and sensor-augmented pump (conventional) therapy in 23 adults with type 1 diabetes. Each intervention was applied from 08h00 Day 1 to 20:00 Day 3 (60 hours) in outpatient free-living conditions. The primary outcome was time spent with sensor glucose levels below $4.0 \mathrm{mmol} / \mathrm{L}$. A P value of less than 0.017 was regarded as significant. This trial is registered, NCT01966393.

Results: The dual-hormone system reduced time spent below $4.0 \mathrm{mmol} / \mathrm{L}, 3.5 \mathrm{mmol} / \mathrm{L}$, and $3.3 \mathrm{mmol} / \mathrm{L}$ compared to the single-hormone system by $-2.3 \%(-3.7-0.5 ; \mathrm{P}=0.072),-1.3$ $(-2.5-0.2 ; \mathrm{P}=0.017)$, and $-0.7(-2.0-0.2 ; \mathrm{P}=0.031)$, respectively. Both systems significantly $(\mathrm{P}<0.017)$ reduced hypoglycemia below $4.0 \mathrm{mmol} / \mathrm{L}, 3.5 \mathrm{mmol} / \mathrm{L}$, and $3.3 \mathrm{mmol} / \mathrm{L}$ compared to conventional therapy but the reductions were higher with the dual-hormone system than with the single-hormone system (medians $-4.0 \%$ vs $-3.4 \%$ for $4.0 \mathrm{mmol} / \mathrm{L} ;-2.7 \%$ vs 
$-2.2 \%$ for $3.5 \mathrm{mmol} / \mathrm{L}$; and $-2 \cdot 2 \% v s-1.2 \%$ for $3.3 \mathrm{mmol} / \mathrm{L})$. There were 34 hypoglycemic events $(<3.0 \mathrm{mmol} / \mathrm{L}$ for 20 minutes) with conventional therapy, 14 with the single-hormone system, and 6 with the dual-hormone system. These differences in hypoglycemia were observed while mean glucose level was similar and low in all interventions $(\mathrm{P}=\mathrm{NS})$.

Conclusion: The dual-hormone artificial pancreas had the lowest risk of hypoglycemia. Longer and larger studies are needed.

067

\section{ACCURACY AND LONGEVITY OF AN IMPLANTABLE CONTINUOUS GLUCOSE SENSOR IN THE PRECISE STUDY: A PROSPECTIVE MULTI-CENTER PIVOTAL TRIAL}

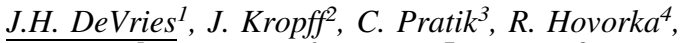

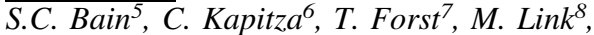
X. Chen $^{9}$, A. DeHennis ${ }^{9}$

${ }^{1}$ Academic Medical Center - University of Amsterdam, Department of Endocrinology, Amsterdam, Netherlands ${ }^{2}$ Academic Medical Center- University of Amsterdam, Department of Endocrinology, Amsterdam, Netherlands ${ }^{3}$ King's College London., London, United Kingdom ${ }^{4}$ Cambridge University Hospitals, NHS Foundation Trust, Cambridge, United Kingdom

${ }^{5}$ Swansea University, Joint Clinical Research Facility, Swansea, United Kingdom

${ }^{6}$ Profil., Neuss, Germany

${ }^{7}$ Profil., Mainz, Germany

${ }^{8}$ University of Ulm, Institut für Diabetes Technologie, Ulm, Germany

${ }^{9}$ Senseonics, Research, Germantown, USA

Background and Aims: The accuracy and longevity of a new type long term implantable continuous glucose monitoring system (CGM) was investigated over 6 months in a pivotal study. Three months data are presented here on the full study enrollment.

Method: The CGM (Eversense ${ }^{\circledR}$, Senseonics Inc.) is composed of an implantable, fluorescence-based glucose sensor and a wearable transmitter that wirelessly communicates with a smartphone-based medical app to display glucose results. In this prospective, single-arm investigation we enrolled 71 adult subjects with T1DM at 7 clinical sites. Here we report the performance of the CGM through 90 days of study. Patients used the CGM system at home and in-clinic. The CGM system was tested during five in-clinic visits (8h-24h). During these visits venous reference glucose measurements were taken (YSI2300 Stat plus) for CGM comparison.

Results: Mean absolute relative difference (MARD) for reference glucose $>75 \mathrm{mg} / \mathrm{dL}$ was $11.0 \%(\mathrm{n}=14475$, SD $10.2 \%$, $95 \%$ CI $10.9 \%, 11.2 \%)$. Mean absolute difference (MAD) for reference glucose $\leq 75 \mathrm{mg} / \mathrm{dL}$ was $14.2 \mathrm{mg} / \mathrm{dl}(\mathrm{n}=871$, SD16.2mg/ $\mathrm{dl}, 95 \% \mathrm{CI} 13.1 \mathrm{mg} / \mathrm{dl}, 15.3 \mathrm{mg} / \mathrm{dl}$ ). MARD over the full glycemic range $(40-400 \mathrm{mg} / \mathrm{dL})$ was $11.7 \%(\mathrm{n}=15346, \mathrm{SD} 11.9 \%, 95 \%$ CI $11.5 \%, 11.8 \%)$. Sensor stability over time was assessed by calculating MARD over the full glycemic range per consecutive thirty day periods and was found to be statistically equivalent at $11.6 \%$ (SD 12.3\%), 11.8\% (SD 11.5\%) and 11.9\% (SD 10.0\%) [p $=0.5017$, one-way ANOVA]. A Kaplan-Meier analysis for survivability found $82.4 \%$ functioning through 90 days.
Conclusion: The Senseonics implantable CGM system was accurate and stable over a three months period with a MARD of $11.0 \%$ for reference glucose $>75 \mathrm{mg} / \mathrm{dL}$.

\section{8}

\section{RISK ASSESSMENT OF USING NEW CONTINUOUS GLUCOSE MONITORING (CGM) SYSTEM FOR TREATMENT DECISIONS}

K. Nakamura ${ }^{1}$, E. Casal $^{2}$, D. Price ${ }^{3}$

${ }^{I}$ Dexcom Inc., San Diego, USA

${ }^{2}$ Dexcom Inc., Clinical Affair, San Diego, USA

${ }^{3}$ Dexcom Inc., Medical Affair, San Deigo, USA

Background and Aims: The Dexcom G5 Mobile CGM (G5) recently received the $\mathrm{CE}$ mark in the European Union to replace fingerstick blood glucose testing for diabetes treatment decisions in patients with Diabetes 2 years and older. Similar CGM is widely used in Artificial Pancreas research, we assessed CGMbased treatment decisions.

Method: The assessment included youth (2-17 YO) and adults (18 YO and above). 130 subjects (51A, 79Y) enrolled at $8 \mathrm{US}$ centers. 52\% Male; $57 \%$ used CSII. To assess risk of replacing fingersticks, we evaluated the incidences of readings posing risk of excessive insulin dose due to CGM reading dangerously higher than YSI: $20 \%-30 \%$ or $>30 \%$ higher when CGM $\geq 100 \mathrm{mg} / \mathrm{dL}$ and $20-30 \mathrm{mg} / \mathrm{dL}$ or $>30 \mathrm{mg} / \mathrm{dL}$ when CGM $<100 \mathrm{mg} / \mathrm{dL}$. CGM readings lower than YSI or $<20 \%$ higher than YSI would pose minimal risk of excessive insulin dose.

Results: Baseline A1C was higher in Youth $[8.5 \pm 1.5 \%$ (mean \pm s.d.) $]$ vs. Adults $(7.8 \pm 1.1 \%, \mathrm{p}=0.003)$. Patient characteristics and CGM performance were summarized in Table 1. When CGM reads $\geq 100 \mathrm{mg} / \mathrm{dL}$, overcorrecting risks were $2.3 \%$ for Youth, 3.6\% for Adults when CGM reads 20-30\% higher than YSIs; risks were $1.8 \%$ for Youth, $2.5 \%$ for Adults when CGM reads $>30 \%$ higher than YSI. When CGM reads $<100 \mathrm{mg} / \mathrm{dL}$, risk was less than $2 \%$ for all cases.

Conclusion: CGM performance was similar between age groups. Youths showed a noticeably higher average and more variations of CGM glucose readings during 7-day use. The risks of nonadjunctive use of CGM in overcorrecting were as small as $2-4 \%$.

Table 1:Accuracy and Risk Assessment between Pediatrics and Adult Users of Dexcom GS CGM

\begin{tabular}{|c|c|c|c|c|c|}
\hline & \multicolumn{2}{|c|}{ Pediatrics } & \multicolumn{2}{|l|}{ Adults } & P-values $n$ \\
\hline N Subjects & \multicolumn{2}{|l|}{79} & \multicolumn{2}{|l|}{51} & \\
\hline Characteristics & mean & sd & mean & sd & \\
\hline Age (yrs) & 12.2 & 4.6 & 46.7 & 15.8 & NA \\
\hline Gender-Male* & 41 & $52 \%$ & 27 & $53 \%$ & 0.9075 \\
\hline$B M !\left(\mathrm{kg} / \mathrm{m}^{\wedge} 2\right)^{*}-$ & 0.71 & 0.21 & 27.4 & 4.6 & -0.0001 \\
\hline Alc ( ( $)$ & 8.5 & 1.5 & 7.8 & 1.1 & 0.003 \\
\hline Diabetes Duration (years) & 5.6 & 4.2 & 24.8 & 14.5 & 0.0001 \\
\hline Insulin Delivery Method-Multiple Daily. Injections" & 32 & $41 \%$ & 24 & $47 \%$ & 0.4613 \\
\hline Average Daily BGs (times) & 6.7 & 2.3 & 5.5 & 2.0 & 0.006 \\
\hline \multicolumn{6}{|l|}{ CGM-YSI Performance } \\
\hline N(matched Pairs) & \multicolumn{2}{|c|}{2262} & \multicolumn{2}{|l|}{2263} & $\mathrm{NA}$ \\
\hline MARD & $10 \%$ & $7 \%$ & $98 \quad 1$ & $4 \%$ & 0.3261 \\
\hline$\$ 20 / 20$ & $90 \%$ & $18 \%$ & $93 \%$ & $14 \%$ & 0.2136 \\
\hline \multicolumn{6}{|l|}{ CGM-SMBG Performance } \\
\hline$N($ matched Pairs) & \multicolumn{2}{|c|}{4264} & \multicolumn{2}{|l|}{2992} & NA \\
\hline MARD & $12 \%$ & $4 \%$ & $11 \%$ & $3 \%$ & 0.1434 \\
\hline$\$ 20 / 20$ & $84 \%$ & $11 \%$ & $87 \%$ & $8 \%$ & 0.1625 \\
\hline \multicolumn{6}{|l|}{ CGM Glucose in mmol/h } \\
\hline Average at Night (10PM to 6AM) & 10.0 & 2.1 & 8.9 & 1.7 & 0.0031 \\
\hline Average in the Morning (GAM to 11AM) & 9.8 & 2.1 & 8.5 & 1.6 & 0.0003 \\
\hline Average in the Afternoon (111AM to APM) & 10.5 & 20 & 9.3 & 1.6 & 0.0006 \\
\hline Average in the Evening ( 4 PM to 10PM) & 10.3 & 24 & 9.0 & 1.6 & -0.0001 \\
\hline Overcorrecting Risks due to High CGM vs.YSI (\%) & within 20-30\% & $>30 \%$ & within $20-30 x$ & $>30 \%$ & \\
\hline For CGM $2100 \mathrm{mg} / \mathrm{dL} \quad$ & $2.3 \%$ & $1.8 \%$ & $3.6 \%$ & $2.5 \%$ & NA \\
\hline For CGM< $100 \mathrm{mg} / \mathrm{dL}$ & $1.1 \mathrm{~s}$ & $0.0 \%$ & $1.6 \mathrm{~K}$ & $0.8 \%$ & NA \\
\hline
\end{tabular}
"Wikcoxon test p-values between pediatrics and adult subjects. 
069

\section{CONTINUOUS GLUCOSE MONITORING IN T1D PATIENTS USING INJECTIONS OF INSULIN: A REPORT FROM THE T1D EXCHANGE CLINIC REGISTRY}

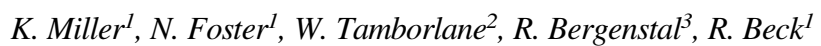

${ }^{1}$ Jaeb Center for Health Research, T1D Exchange Clinic

Network, Tampa, USA

${ }^{2}$ Yale University School of Medicine, Pediatric Endocrinology, New Haven, USA

${ }^{3}$ International Diabetes Center Park Nicollet, Endocrinology, Minneapolis, USA

Background and Aims: To describe characteristics of individuals with type 1 diabetes (T1D) using injections for insulin delivery and continuous glucose monitoring (CGM) and to compare glycemic control across insulin delivery method and CGM use.

Method: The analysis cohort included 16,306 T1D Exchange registry participants (T1D duration $>1$ year) across 57 US clinics who had data available between 6/1/2014 and 10/1/2015. Most recent $\mathrm{HbA} 1 \mathrm{c}$ was compared across 4 groups (adjusting for age and T1D duration): (1) Injections only ( $=5,745)$; (2) Pump only $(\mathrm{N}=8,131)$; (3) Injections + CGM $(\mathrm{N}=365)$; and (4) Pump+ CGM $(\mathrm{N}=2,065)$.

Results: Among the 2,430 CGM users (15\%), 365 (15\%) were using insulin injections. A Dexcom CGM was being used by $97 \%$ of MDI users and $58 \%$ of pump users. Mean SMBG among injection + CGM users was 4.1 times per day compared with 5.4 times per day in Pump + CGM users $(\mathrm{P}<0.001)$. Adjusted mean HbA1c in injection + CGM users was similar to Pump + CGM users $(7.8 \%$ vs. $7.7 \% . \mathrm{P}=0.43$ ), and lower than mean $\mathrm{HbA1c}$ in injection only users $(8.6 \%, \mathrm{P}<0.001)$ and pump only users $(8.2 \%, \mathrm{P}<0.001)$.

Conclusion: In the T1D Exchange registry, injection users of CGM have HbA1c levels similar to pump users of CGM and HbA1c levels with CGM use are substantially better than HbA1c levels in nonusers for both injections and pump. These data support the need for investigations of the obstacles to more wide spread use of CGM in injection users.

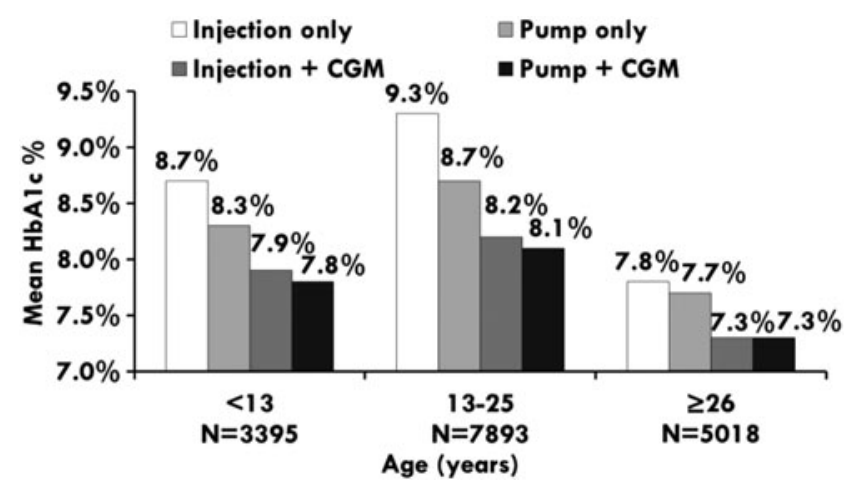

070

REAL-TIME CONTINUOUS GLUCOSE MONITORING DURING PREGNANCY IN WOMEN WITH TYPE 1 DIABETES: IS RTCGM RELATED TO BETTER GLYCAEMIC CONTROL AND KEY OBSTETRIC OUTCOMES?

\section{P. Geelhoed-Duijvestijn ${ }^{1}$}

${ }^{1}$ Medisch Centrum Haaglanden, Interal Medicine, Den Haag, Netherlands
Background and Aims: Real-time continuous glucose monitoring (RTCGM) has been shown to improve glycaemic control. Limited data are available on the effects of RTCGM during pregnancy. The current study assesses glycaemic control and obstetric outcomes in women with type 1 diabetes, comparing RTCGM with usual self measurement of blood glucose (SMBG).

Method: Patients were recruited from two hospitals. All patients were eligible. Glycaemic control was assessed by preconceptional HbA1c and HbAlc during pregnancy. Obstetric outcomes reported are premature delivery ( $\mathrm{PD},<37$ weeks), and macrosomia (Large for Gestational Age (LGA; birthweight $\geq 90$ th percentile; Very Large for Gestational Age (VLGA; birth weight $\geq 97.7^{\text {th }}$ percentile).

Results: 67 women were included; 53 (79\%) used RTCGM PD occurred in $22 \%$ of the pregnancies, $19.6 \%$ with RTCGM, $31 \%$ on SMBG $(\mathrm{p}=0.5)$. LGA occurred in $58 \%$ of pregnancies, VLGA in $31 \%$. LGA with RTCGM 61\%, with SMBG $46 \%$ $(\mathrm{p}=0.4)$. VLGA with RTCGM 37.5\%, SMG 50\%, $\mathrm{p}=0.9)$. Mean HbA1c was significantly lower before pregnancy $(52.1$ \pm 6.1 vs $67.8 \pm 18.4 \mathrm{mmol} / \mathrm{mol}, \mathrm{p}<0.001)$ and during the first trimester (6 weeks: $47.9 \pm 5.4$ vs $58.9 \pm 18.1 \mathrm{mmol} / \mathrm{mol}$, $\mathrm{p}=0.002)$ and 12 weeks $(43.9 \pm 7.0 \mathrm{mmol} / \mathrm{mol}$ vs $53.6 \pm 12.8$ $\mathrm{mmol} / \mathrm{mol} . \mathrm{p}<0.05)$, but not later in pregnancy.

Conclusion: RTCGM was associated with better early HbA1c. Premature delivery occurred less frequently with RTCGM, macrosomia more frequent. Future analysis with this expanding group will assess whether these differences persist; at this moment, better early control is not readily associated with less macrosomia.

\section{1}

\section{STRIVING FOR CONTROL: LESSONS FROM A SUCCESSFUL INTERNATIONAL TYPE 1 DIABETES (T1D) YOUTH CHALLENGE IN CRETE, GREECE}

\author{
O. Kordonouri ${ }^{1}$, A. Vazeou-Gerasimidi ${ }^{2}$, M. Scharf ${ }^{3}$, \\ M. Würsig ${ }^{I}, T$. Battelino ${ }^{4}$ \\ ${ }^{1}$ Children's Hospital AUF DER BULT, Diabetes Centre \\ for Children and Adolescents, Hannover, Germany \\ ${ }^{2}$ P\&A Kyriakou Children's Hospital, A Dept. Paediatrics- \\ Diabetes Centre, Athens, Greece \\ ${ }^{3}$ Private Practice, Center for Diabetology, Paraná, Brazil \\ ${ }^{4}$ University Children's Hospital, Department of Pediatric \\ Endocrinology/Diabetology and Metabolism, Ljubljana, \\ Slovenia
}

Background and Aims: To demonstrate whether young people with T1D using modern insulin treatment and CGM could successfully participate in extreme sport activity while maintaining good glycaemic control.

Method: The challenge took place in Crete/Greece over 4 days combining a long distance trek of different levels of severity with final destination the summit of the White Mountains at $2,080 \mathrm{~m} .11$ patients $(5$ female, age $18.2 \pm 1.3$ years, T1D duration $7.9 \pm 3.5$ years, HbA1c $7.3 \pm 0.7 \%$; mean \pm SD) from 11 SWEET centres in Belgium, Brazil, Canada, Germany, Greece, France, India, Italy, Portugal, Slovenia and Sweden participated to the challenge. Five patients were on CSII, six on MDI. All patients were well trained (pulse in resting condition $68.5 \pm 13.4$ beats/minute). Patients were wearing CGM (Medtronic) or a 
DexCom G4 system. The glycaemic targets during trekking were defined as $80-180 \mathrm{mg} / \mathrm{dl}$.

Results: In total, the group walked $54.5 \mathrm{~km}$ under varying climate conditions (temperature $14-35^{\circ} \mathrm{C}$ ). During the challenge, insulin requirements decreased significantly: total daily insulin by $31.1 \pm 16.7 \%$ ( $\mathrm{p}<.001)$, basal by $30.8 \pm 14.9 \%$ $(\mathrm{p}<.001)$, and prandial by $32.5 \pm 28.0 \%(\mathrm{p}=.023)$, with no difference between patients with CSII or MDI. No episode of severe hypoglycaemia or DKA occurred. Mean glucose levels were $170.7 \pm 60.1 \mathrm{mg} / \mathrm{dl}$ with $61.5 \pm 18.7 \%$ of CGM values in the target range, $5.4 \pm 5.4 \%$ under $80 \mathrm{mg} / \mathrm{dl}$ and $32.8 \pm 16.6 \%$ above $180 \mathrm{mg} / \mathrm{dl}$.

Conclusion: The results of this SWEET Initiative activity demonstrated that the use of sensor augmented treatment can help T1D patients to achieve extraordinary performance even under very challenging conditions while maintaining good glycaemic control and without diabetes-related acute complications.

072

\section{SPIDIMAN: CLINICAL TRIAL OF A SINGLE-PORT GLUCOSE SENSOR ON A COMMERCIAL INSULIN INFUSION SET}

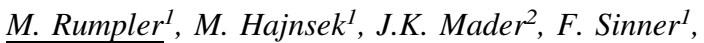
T.R. Pieber ${ }^{2}$

${ }^{1}$ Joanneum Research Forschungsgesellschaft mbH, HEALTH Institute for Biomedicine and Health Sciences,

Graz, Austria

${ }^{2}$ Medical University of Graz, Division of Endocrinology and Metabolism, Graz, Austria

Background and Aims: Continuous glucose monitoring has proven benefits for adult and pediatric patients with type 1 diabetes (T1D). In order to design a small and lightweight system for T1D patients we are combining a continuous glucose monitoring system (CGMS) with a commercial insulin infusion set to create a new single-port system.

Method: Our single-port system consists of two sensors which are directly coated onto the cannula of the insulin infusion set. Both sensors are placed subcutaneously and are interrogated in a transcutaneous way by an optical read-out unit. The first sensor is an enzymatic glucose sensor, the second sensor is an oxygen sensor to detect tissue oxygen variations.

Twelve T1D patients ( 5 females, 7 males, age $33.0 \pm 10.4$, diabetes duration $15.7 \pm 11.8 \mathrm{yrs}$ ) attended a clinical trial in a clinical research center for 12 hours. Two single-port systems were placed 24 hours prior to the trial. Patients' blood glucose dynamics was induced with high glycemic index meals followed by delayed, overdosed insulin boluses.

Results: Sensor glucose values were calculated with a retrospective calibration using a linear regression. Considering variations caused by in-house sensor production, preliminary data revealed a linear correlation of sensor and blood glucose values $\left(\mathrm{R}^{2} \leq 0.91\right)$ in the range from 50 to $200 \mathrm{mg} / \mathrm{dl}$.

Conclusion: Our single-port system successfully combines continuous glucose monitoring and insulin infusion into one device and has therefore great potential to become the central element of an artificial pancreas. Calibration procedure and standardized production are currently being optimized.

Acknowledgement: Supported by EU FP7 (\#305343SPIDIMAN).
073

\section{GLYCOMETRICS IN 70,167 PATIENTS WITH TYPE 2 DIABETES DERIVED FROM RETROSPECTIVE CONTINUOUS GLUCOSE MONITORING (CGM)}

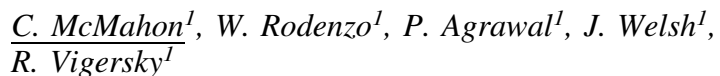

${ }^{1}$ Medtronic, Inc., Diabetes, Northridge, USA

Background and Aims: The benefit of retrospective CGM in patients with Type 1 diabetes is generally accepted, but there is limited evidence on its utility in Type 2 diabetes (T2D). Therefore, we performed glycometric analysis on over 80,000 retrospective CGM (iPro $2^{\circledR}$ ) procedures residing in our CareLink ${ }^{\circledR}$ (Medtronic MiniMed, Inc., Northridge, CA) database to understand glycemic control in a diversely treated population of patients with T2D outside of the United States.

Method: Glycometrics included mean, intra- and betweenday variability (SD, Time-in-Range, MAGE, MODD), and frequency of hypo- and hyperglycemia. Results were grouped by therapy (diet/exercise, oral agent only, insulin) at the time of the procedure. The data in all insulin-taking patients were grouped (1-2 shots/day; $\geq 3$ shots/day, or insulin pump).

Results: Data from 70,167 patients, 81,796 sensors and 353,520 patient-days from 01/01/2012 - 09/24/2015 were analyzed. The mean \pm SD glucose was $7.6 \pm 2.2 \mathrm{mmol} / \mathrm{L}$ for those on diet/exercise, $8.6 \pm 2.5 \mathrm{mmol} / \mathrm{L}$ for those on orals, and $9.4 \pm 2.6 \mathrm{mmol} / \mathrm{L}$ for those on insulin. Variability metrics were: $\mathrm{SD}=1.7,2.1$ and $2.8 \mathrm{mmol} / \mathrm{L}$, respectively; Time-in-Range $=$ $19.6 \pm 5.6,17.3 \pm 6.6$, and $14.4 \pm 6.3$ hours/day; MAGE $=3.9$ $\pm 2.1,4.6 \pm 2.1$, and $5.9 \pm 2.4 \mathrm{mmol} / \mathrm{L}$, respectively; and the MODD $=-0.22 \pm 0.76, \quad-0.17 \pm 0.93$, and $-0.19 \pm 1.18$ $\mathrm{mmol} / \mathrm{L}$, respectively. The Table shows the incidence rate of cases with hypo- and hyperglycemia in each treatment group.

Conclusion: The data demonstrate high rates of glycemic variability, hypoglycemia and hyperglycemia in those patients with T2D managed with diet/exercise, orals agents and insulin. This suggests that an iPro2 ${ }^{\circledR}$ procedure may be useful in providing actionable information in T2D regardless of underlying therapy.

Table. Percent of Sensors per Therapy Type with Glycemic Excursion beyond Threshold

\begin{tabular}{|c|c|c|c|c|c|c|}
\hline \multirow{2}{*}{ Therapy } & $\begin{array}{c}\% \\
\text { Sensors }\end{array}$ & \multirow{2}{*}{$\begin{array}{c}\text { Any } \\
\text { Hypoglycemia } \\
\text { Excursion } \\
\text { Event } \\
<2.8 \mathrm{mmoll} \text {. }\end{array}$} & \multirow{2}{*}{$\begin{array}{c}\begin{array}{c}\text { Any } \\
\text { Hypogycemia } \\
\text { Excursion } \\
\text { Event } \\
<3.3 \mathrm{mmol}\end{array} \\
3.3\end{array}$} & \multirow{2}{*}{$\begin{array}{c}\text { Any } \\
\text { Hypoglycemia } \\
\text { Excursion } \\
\text { Event } \\
<3.9 \text { mmoll. }\end{array}$} & \multirow{2}{*}{$\begin{array}{c}\text { Any } \\
\text { Hyperglycemia } \\
\text { Excursion Event } \\
>10.0 \mathrm{mmol} \text {. }\end{array}$} & \multirow{2}{*}{$\begin{array}{c}\text { Any } \\
\text { Hyperglycemia } \\
\text { Excursion Event } \\
>13.9 \text { mmoll. }\end{array}$} \\
\hline & $\begin{array}{c}\text { Incidence } \\
\text { rate }\end{array}$ & & & & & \\
\hline \multirow{2}{*}{ Diet } & $\%$ & $12 \%$ & $23 \%$ & $41 \%$ & $70 \%$ & $33 \%$ \\
\hline & events day & $0.45 \pm 0.40$ & $0.57 \pm 0.50$ & $0.78 \pm 0.74$ & $1.64 * 1.05$ & $1.06 \pm 0.86$ \\
\hline \multirow{2}{*}{ Oral } & $\%$ & $11 \%$ & $20 \%$ & $34 \%$ & $87 \%$ & $49 \%$ \\
\hline & eventsiday & $0.45 \div 0.37$ & $0.51 \pm 0.43$ & $0.65 \pm 0.58$ & $1.8 \pm 1.2$ & $1.14 \pm 1.0$ \\
\hline \multirow{2}{*}{ Insulin } & $\%$ & $20 \%$ & $33 \%$ & $48 \%$ & $95 \%$ & $74 \%$ \\
\hline & eventsiday & $0.47 \pm 0.39$ & $0.58 \times 0.48$ & $0.73 \pm 0.60$ & $1.93 \div 0.92$ & $1.23 \div 0.84$ \\
\hline
\end{tabular}

$\%$, percent of sensors with an event in the specified range.

An excursion event requires at least 2 samples ( 10 minutes) beyond the provided threshold

\section{4}

USE OF NOVEL FLASH GLUCOSE-SENSING TECHNOLOGY TO OPTIMISE GLUCOSE CONTROL IN INDIVIDUALS WITH TYPE 2 DIABETES ON INTENSIVE INSULIN THERAPY

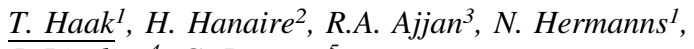
J. Riveline ${ }^{4}$, G. Rayman ${ }^{5}$ 
${ }^{1}$ Diabetes Zentrum Mergentheim, Diabetes Klinik, Bad Mergentheim, Germany

${ }^{2}$ University Hospital of Toulouse, Department of Diabetology, Toulouse, France

${ }^{3}$ University of Leeds, Leeds Institute for Cardiovascular and Metabolic Research, Leeds, United Kingdom

${ }^{4}$ Hopital Lariboisiere, Department of Endocrinology and Diabetology, Paris, France

${ }^{5}$ Ipswich Hospital NHS Trust, The Diabetes Centre, Ipswich, United Kingdom

Background and Aims: To assess the impact of a new technology on glycaemic control as an alternative to self-monitoring of capillary blood glucose levels (NCT02082184).

Method: Patients with type 2 diabetes (T2DM), on intensive insulin therapy, were recruited into a 6 month European study (26 sites). Individuals were randomised (1:2 respectively) to a control group $(\mathrm{n}=75)$, using capillary glucose-testing (FreeStyle Lite $\left.{ }^{\mathrm{TM}}\right)$, or an intervention group $(\mathrm{n}=149)$, using sensor glucose data (FreeStyle Libre ${ }^{\mathrm{TM}}$ Flash Glucose Monitoring System), for self-management and optimisation of glucose levels.

The primary outcome measure was difference in $\mathrm{HbA} 1 \mathrm{c}$ between two study groups at day 194. Pre-specified secondary outcome measures included time in hypoglycaemia and subgroup analysis by age.

Results: $\mathrm{HbA} 1 \mathrm{c}$ reduction was similar in the intervention and control group $(-3.1 \pm 0.75$ and $-3.4 \pm 1.04 \mathrm{mmol} / \mathrm{mol}$, respectively (mean $\pm \mathrm{SE}) ; \mathrm{p}=0.8259$ ). However, a difference in HbA1c was detected analysing those $<65$ years in the intervention group compared with control $(-5.7 \pm 0.96$ and $-2.2 \pm 1.31 \mathrm{mmol} / \mathrm{mol}$, respectively (mean $\pm S E) ; p=0.030$ ).

Time in hypoglycaemia, defined as glucose $<3.9 \mathrm{mmol} / \mathrm{L}$, was reduced in the intervention group compared with control at study end $(-0.47 \pm 0.134 \mathrm{hrs} /$ day (mean $\pm \mathrm{SE}) ; \mathrm{p}<0.001)$. Time in hypoglycaemia $(<3.9 \mathrm{mmol} / \mathrm{L})$ was reduced both during daytime and at night with nocturnal hypoglycaemia $(<3.9 \mathrm{mmol} / \mathrm{L}$, $11 \mathrm{pm}-6 \mathrm{am})$ cut by $1.01 \pm 0.26 \mathrm{hrs} /$ night $($ mean $\pm \mathrm{SE}) ; \mathrm{p}<0.001)$ compared with the control group.

Conclusion: Use of FreeStyle Libre by T2DM subjects on intensive insulin therapy was associated with reduction in overall hypoglycaemia during daytime and at night and significantly improved $\mathrm{HbA} 1 \mathrm{c}$ in subjects $<65$ years.

\section{5}

\section{CLINICAL ACCURACY EVALUATION OF FREESTYLE LIBRE FLASH GLUCOSE MONITORING SYSTEM WHEN USED BY CHILDREN AND YOUNG PEOPLE WITH DIABETES}

$\underline{\text { F. Campbell }}{ }^{1}$, J. Edge ${ }^{2}$, C. Acerini ${ }^{3}$, J. Hamilton-Shield $^{4}$,

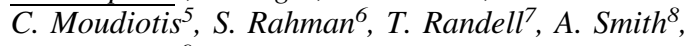
N. Trevelyan ${ }^{9}$

${ }^{1}$ Leeds Children's Hospital, Paediatrics, Leeds- West Yorkshire, United Kingdom

${ }^{2}$ Oxford Children's Hospital, Paediatrics, Oxford, United Kingdom

${ }^{3}$ Addenbrooke's Hospital, Paediatrics, Cambridge, United Kingdom

${ }^{4}$ Bristol Biomedical Research Unit in Nutrition, Paediatrics, Bristol, United Kingdom

${ }^{5}$ Royal Devon and Exeter Hosspital, Paediatrics, Exeter, United Kingdom

${ }^{6}$ Harrogate, Paediatrics, Harrogate, United Kingdom
${ }^{7}$ Nottingham Children's Hospital, Paediatrics, Nottingham, United Kingdom

${ }^{8}$ Northampton General Hospital, Paediatrics, Northampton, United Kingdom

${ }^{9}$ Southampton Children's Hospital, Paediatrics, Southampton, United Kingdom

Background and Aims: To evaluate clinical safety and accuracy of the FreeStyle Libre System when measuring interstitial glucose concentrations in the paediatric population.

Method: Paediatric subjects with type 1 diabetes $(n=87)$, aged 4-17 years from 9 sites, completed this UK study. Subjects wore a sensor (on the back of their upper arm) for up to 14 days and throughout this home-use period were asked to perform 4 capillary blood glucose (BG) tests daily using the BG (FreeStyle Optium) strip-port on the Reader, immediately followed by an interstitial glucose measurement with the Reader. Sensor data was masked to subjects until the final clinic visit where the device was unmasked for subjects to experience all functionality.

Results: A total of 5493 interstitial glucose results with paired BG results demonstrated; $83.8 \%$ of interstitial glucose results in Zone A (clinically accurate), >99\% of results in Zones A\&B (clinically acceptable) of the Consensus Error Grid and $81.2 \%$ of results within $\pm 1.1 \mathrm{mmol} / \mathrm{L}[20 \mathrm{mg} / \mathrm{dL}] / 20 \%$ of $\mathrm{BG}$ values. Clinically accurate results were obtained across all age groups: 4-7, 8-12, 13-17years. Mean absolute relative difference was $13.9 \%$. Regression analysis demonstrated high correlation to BG $(\mathrm{r}=0.95$, slope $=1.03$, intercept $=-0.23 \mathrm{mmol} / \mathrm{L}[-4.12 \mathrm{mg} /$ $\mathrm{dL}]$ ). User satisfaction questionnaires indicated high levels of acceptance for sensor wear and ease of use of the device. There were 5 unanticipated, device-related adverse events, e.g. allergic reaction, red mark, blister, abrasion.

Conclusion: Results show good agreement between the FreeStyle Libre System and capillary BG for subjects 4-17 years, indicating the device is safe and accurate within the paediatric population.

\section{6}

\section{FORMATIVE, COMPARATIVE HUMAN FACTORS STUDY OF A GLUCAGON AUTO-INJECTOR VS. RECONSTITUTED GLUCAGON IN A SIMULATED SEVERE HYPOGLYCEMIA RESCUE SITUATION}

B. Newswanger ${ }^{1}$, S. Prestrelski ${ }^{1}$, M. Cummins ${ }^{1}$, A. Andre ${ }^{2}$, K. Ching ${ }^{2}$

${ }^{1}$ Xeris Pharmaceuticals, R\&D, Austin, USA

${ }^{2}$ Interface Analysis Associates, R\&D, Saratoga, USA

Background and Aims: Hypoglycemia is highly feared by persons with diabetes and can lead to coma, seizures and automobile accidents. Currently approved glucagon products for severe hypoglycemia rescue are based on lyophilized formulations that require manual reconstitution with a vial and syringe at time of use, thus are difficult to administer and are not well accepted. Xeris is developing a room-temperature stable, soluble liquid glucagon formulation that is loaded into a prefilled, single-use two-step auto-injector intended for severe hypoglycemia rescue.

Method: A simulated-use formative human factors study was conducted with the Xeris auto-injector as compared to marketed glucagon kits (Novo, Lilly). This study was conducted with a total of 16 participants in two subgroups: 8 adult caregivers of diabetic patients and first responders experienced with glucagon 


\begin{tabular}{|l|c|c|}
\cline { 2 - 3 } \multicolumn{1}{c|}{ Comparative Performance Measures } & $\begin{array}{c}\text { Xeris } \\
\text { Auto-Injector }\end{array}$ & $\begin{array}{c}\text { Current } \\
\text { Glucagon Kit }\end{array}$ \\
\hline Successful Dose Administrations & $14 / 16(88 \%)$ & $5 / 16(31 \%)$ \\
\hline Administered With Reduced Efficacy & $0 / 16(0 \%)$ & $4 / 16(25 \%)$ \\
\hline Failed Administration & $2 / 16(13 \%)$ & $7 / 16(44 \%)$ \\
\hline Error Rate & $3.6 \%$ & $20.1 \%$ \\
\hline $\begin{array}{l}\text { Mean Total Rescue Time } \\
\text { (Start at entry of room until delivery of dose.) }\end{array}$ & $47.9 \mathrm{Sec}$. & $109.0 \mathrm{Sec}$. \\
\hline $\begin{array}{l}\text { Median Total Rescue Time } \\
\text { (Start at entry of room until delivery of dose.) }\end{array}$ & $35.0 \mathrm{Sec}$. & $96.0 \mathrm{Sec}$. \\
\hline
\end{tabular}

kits, and 8 adults naïve to the glucagon kits. Half of each participant group received training prior to the simulation.

Results: Overall, 88\% (14/16) of all participants successfully administered a rescue injection using the auto-injector compared to $31 \%(5 / 16)$ using the marketed glucagon kits $\left(\chi^{2}=10.49\right.$, $\mathrm{p}<0.05)$. The auto-injector had a statistically significant faster mean total rescue time of 47.9 seconds compared to $109.0 \mathrm{sec}-$ onds for the current kits $(\mathrm{F}(1 / 28)=12.41, \mathrm{p}<0.05)$. Interestingly, the glucagon kit failure rate was roughly similar between experienced and naïve participants.

Conclusion: Overall, $100 \%$ of participants preferred the Xeris auto-injector and reported it to be easier, safer, faster and more convenient to use. In summary, these results support continued development of Xeris' auto-injector as an alternative to current glucagon emergency kits.

077

\section{EFFECT OF VITAMIN D THERAPY ON URINARY ALBUMIN EXCRETION, RENAL FUNCTIONS AND PLASMA RENIN AMONG PATIENTS WITH DIABETIC NEPHROPATHY; A RANDOMIZED, DOUBLE-BLIND CLINICAL TRIAL}

\author{
G. Liyanage $^{1}$, S. Lekamwasam ${ }^{2}$, T. Weerarathna ${ }^{2}$, C. Liyanage $^{3}$
}

${ }^{1}$ Faculty of Medicine, Pharmacology, Galle, Sri Lanka

${ }^{2}$ Faculty of Medicine, Medicine, Galle, Sri Lanka

${ }^{3}$ Faculty of Medicine, Community Medicine, Galle, Sri Lanka

Background and Aims: Despite optimum therapy a sizable proportion of patients with proteinuria progress. Increase renin level induced by RAS (Renin Angiotensin System) blockage may contribute to this and vitamin D is found to have an inhibitory effect over RAS.

This study was conducted to examine the effects of vitamin D therapy on renal functions of patients with diabetic nephropathy (DN).

Method: A double-blind, randomized, placebo controlled study. Patients with DN (UA $>30 \mathrm{mg} / \mathrm{g}$ of creatinine) whose eGFR (estimated glomerular filtration rate) was more than $30 \mathrm{~mL} / \mathrm{min}$ were selected and their plasma renin, PTH (Parathyroid hormone), serum vitamin D, serum calcium, serum creatinine, FBS (fasting blood sugar) were done as baseline measurements. Subjects were randomized into two groups and treatment group was given vitamin D, 50000 IU $(0.25 \mathrm{ml})$ intramuscularly (IM) monthly for 6 months; control group received distilled water IM. The investigations were repeated after 6 months of therapy.

Results: Of 155 patients invited, 85 were randomly assigned to two groups. After six months, mean reduction of urinary albumin to creatinine ratio in the treatment and control group were $51.8 \mathrm{mg} / \mathrm{g}$ $(\mathrm{P}=0.06) ; 22.4 \mathrm{mg} / \mathrm{g}(\mathrm{P}<0.001)$, respectively (between group difference $P=0.001)$. Significant increase in the eGFR observed in the treatment group while eGFR remained unchanged in the control group ( $\mathrm{P}=0.006$ for the between-groups difference). Mean reduction in plasma renin in treatment group and control group were $5.85 \mathrm{pg} / \mathrm{mL}(\mathrm{P}<0.001)$ and $0.95 \mathrm{pg} / \mathrm{mL}(\mathrm{P}>0.05)$, respectively.

Conclusion: Vitamin D 50000 IU given IM for 6 months reduced urine albumin, serum creatinine, renin levels and improved eGFR.

\section{8}

\section{THE APPLICATION OF INSULIN DEGLUDEC TO ANTI-INSULIN ANITOBODY-POSITIVE PATIENTS WITH TYPE 1 DIABETES}

H. Yanai $^{1}$, H. Hamasaki ${ }^{1}$, H. Adachi $^{1}$

${ }^{1}$ Kohnodai Hospital - National Center for Global Health and Medicine, Department of Internal Medicine, Ichikawa, Japan

Background and Aims: Insulin degludec is a new basal insulin analogue that forms a depot of multi-hexamer chains after subcutaneous injection resulting in an ultra-long duration of action and stable glucose-lowering effect. Insulin analogues sometimes induce anti-insulin antibody, which induces an immunological insulin resistance and unexpected hypoglycaemia. The effect of insulin degludec on anti-insulin antibody is largely unknown.

Method: We investigated the effect of the switching the basal insulin from insulin glargine to insulin degludec on $\mathrm{HbA} 1 \mathrm{c}$, daily insulin doses and anti-insulin immunoglobulin $\mathrm{G}$ antibody (IA) levels in 10 IA-positive patients with type 1 diabetes who were treated with the intensive insulin therapy.

Results: At 3 months after the switching to insulin degludec, HbA1c tended to decrease. Daily insulin doses and the IA levels significantly decreased. At 6 months after the switching, HbAlc and daily insulin doses were still lower than those at baseline. The IA levels showed a further decrease. The reduction in IA levels at 6 months after the switching was significantly correlated with the decrease in HbA1c at 6 months after the switching.

Conclusion: The switching the basal insulin from insulin glargine to insulin degludec reduced $\mathrm{HbAlc}$, daily insulin doses, and the IA levels in IA-positive subjects with type I diabetes.

\section{9}

\section{EARLIER ONSET AND HIGHER EARLY EXPOSURE OF FASTER-ACTING INSULIN ASPART VS INSULIN ASPART IN ADULTS IS RETAINED IN CHILDREN AND ADOLESCENTS WITH T1D}

\author{
$\underline{\text { T. Danne }}^{1}$, T. Biester ${ }^{1}$, M. Fath ${ }^{1}$, T. Rikte ${ }^{2}$, O. Kordonouri ${ }^{1}$, \\ H. Haahr ${ }^{2}$ \\ 1 'Auf der Bult', Diabetes Centre for Children and Adolescents, \\ Hannover, Germany \\ ${ }^{2}$ Novo Nordisk A/S, Søborg, Denmark
}

Background and Aims: Faster-acting insulin aspart (faster aspart) is a new formulation of insulin aspart (IAsp), with faster initial absorption following subcutaneous injection. This trial assessed pharmacokinetic (PK) and pharmacodynamic (PD) profiles of faster aspart vs IAsp in children, adolescents and adults with type 1 diabetes (T1D). 
Table: PK and PD results for faster aspart vs IAsp

\begin{tabular}{|c|c|c|c|}
\hline & Treatment & tio faster aspart/lAsp & $95 \% \mathrm{CI}]$ \\
\hline & Children (n=12) & Adolescents ( $n=13)$ & Adults ( $n=15)$ \\
\hline Onset of exposure* & & & \\
\hline Onset of appearance & $0.53[0.30 ; 0.78]$ & $0.49[0.34 ; 0.65]$ & $0.45[0.32 ; 0.57]$ \\
\hline $150 \% \mathrm{C}_{\max }$ & $0.75[0.58 ; 0.94]$ & $0.79[0.67 ; 0.93]$ & $0.71[0.62 ; 0.80]$ \\
\hline Early insulin exposure & & & \\
\hline AÚCo-15 $\mathrm{min}$ & $3.24[1.62 ; 6.47]$ & $4.34[2.47 ; 7.62]$ & $5.66[3.58 ; 8.95]$ \\
\hline AUCO-30min & $1.78[1.16 ; 2.75]$ & $1.98[1.38 ; 2.85]$ & $2.47[1.85 ; 3.30]$ \\
\hline AUCo-1h & $1.26[0.95 ; 1.67]$ & $1.28[0.99 ; 1.66]$ & $1.38[1.17 ; 1.62]$ \\
\hline Glucose-lowering effect & & & \\
\hline$P G_{\text {in }}$ & $0.85[0.73 ; 0.99]$ & $0.95[0.84 ; 1.07]$ & $0.91[0.79 ; 1.03]$ \\
\hline$\triangle P G_{\text {Nal }}-1$ h & $0.68[0.49 ; 0.87]$ & $0.93[0.77 ; 1.11]$ & $0.90[0.59 ; 1.35]$ \\
\hline$\triangle P G a v a-2$ & $0.63[0.30 ; 0.92]$ & $0.95[0.67 ; 1.32]$ & $0.81[0.44 ; 1.31]$ \\
\hline
\end{tabular}

Method: Twelve children, 13 adolescents and 15 adults (mean ages: $10.4,15.1,20.2 \mathrm{y}$, respectively) received a $0.2 \mathrm{U} / \mathrm{kg}$ dose (mean: 8.3, 12.8, 15.6 U, respectively) of faster aspart or IAsp before a meal test ( $68 \%$ carbohydrates, adjusted for body weight) in a randomised, double-blind, crossover trial.

Results: Faster aspart had a significantly faster onset of appearance vs IAsp (Table). Insulin exposure at $30 \mathrm{~min}$ was higher for faster aspart vs IAsp (Table). Total exposure and maximum concentration were similar for faster aspart and IAsp for all age groups. Faster aspart had a greater glucose-lowering effect vs IAsp $\left(\Delta \mathrm{PG}_{\mathrm{av}}\right.$, significant in children). $\mathrm{PG}_{1 \mathrm{~h}}$ treatment difference (faster aspart-IAsp; estimates [95\% CI], mmol/L): children, -1.87 [ $-3.71 ;-0.04]$; adolescents, -0.64 [-2.26; 0.97]; adults, -1.10 [-2.64; 0.44]. Treatment effect did not differ significantly between age groups $\left(\mathrm{PG}_{1 \mathrm{~h}}, P=0.56 ; \Delta \mathrm{PG}_{\mathrm{av}, 0-1 \mathrm{~h}}, P=0.15 ; \Delta \mathrm{PG}_{\mathrm{av}, 0-2 \mathrm{~h}}, P=0.32\right)$.

Conclusion: Faster onset and higher early insulin exposure with faster aspart vs IAsp led to a greater early glucose-lowering effect, although this was only significant for children, who are prone to rapidly fluctuating glucose levels and unplanned food intake.

\section{0}

\section{SWITCHING TO INSULIN GLARGINE 300 U/ML (GLA-300): BENEFITS VS GLA-100 BY DURATION OF PRIOR BASAL INSULIN THERAPY}

R.C. Bonadonna ${ }^{1}$, E. Renard ${ }^{2}$, A. Cheng ${ }^{3}$, A. Fritsche , $^{4}$

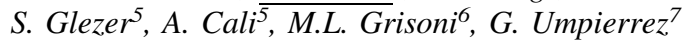

${ }^{1}$ University of Parma, Department of Clinical and Experimental Medicine, Parma, Italy

${ }^{2}$ Montpellier University Hospital, Department of Endocrinology Diabetes Nutrition and INSERM Clinical Investigation Centre 1411, Montpellier, France

${ }^{3}$ University of Toronto, St. Michael's Hospital, Toronto, Canada

${ }^{4}$ University of Tübingen, Institute for Diabetes Research and Metabolic Diseases - Helmholtz Center Munich, Tübingen,

Germany

${ }^{5}$ Sanofi, Diabetes Division, Paris, France

${ }^{6}$ AIXIAL, Biostatistics, Levallois-Perret, France

${ }^{7}$ Emory University School of Medicine, Division of Endocrinology and Metabolism, Atlanta, USA

Background and Aims: In the EDITION program, switching to Gla-300 provided comparable glycemic control with less hypoglycemia vs insulin glargine $100 \mathrm{U} / \mathrm{mL}$ (Gla-100) over 6 months of treatment in people with T2DM previously treated with basal insulin + mealtime insulin (EDITION 1) or basal insulin + oral antihyperglycemic drugs (EDITION 2). This
HbAk and hypoglycemia in T2DM: patient-level meta-analysis of EDITION 1 and 2 by duration of prior basal insulin therapN

\begin{tabular}{|c|c|c|c|c|c|}
\hline \multirow{2}{*}{$\begin{array}{l}\text { Durtion of prior basal } \\
\text { insslin therapy }\end{array}$} & \multicolumn{2}{|c|}{ Ranoomized participants, $n\left(X_{x}\right)$} & \multirow{2}{*}{ 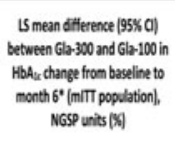 } & \multicolumn{2}{|c|}{ 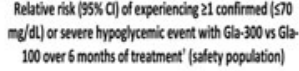 } \\
\hline & $\begin{array}{l}G l-300 \\
N=8088\end{array}$ & $\begin{array}{l}G a-100 \\
N=810\end{array}$ & & At any time (24 h) & Nocturnal (00000-05.59 h) \\
\hline Overall & - & - & $-0.03(-0.12+0.055)$ & $0.92(0.888$ to 0.97$)$ & $0.75(0.57$ to 0.84$)$ \\
\hline$x-2 y$ & 192 (23.8) & $195(24.1)$ & $0.08(-0.10100 .26)$ & $0.84(0.75$ to 0.95$)$ & 0.65 (0.49 to 0.88$)$ \\
\hline $22-5 y$ & $297(368)$ & $303(37.4)$ & $0.01(-0.14$ to 0.15$)$ & 0.56 (0.88 to 1.06) & 0.80 (0.55 to 0.99) \\
\hline $5 y$ & $319(39.5)$ & $312(38.5)$ & $-0.14(-0.2910-0.00)$ & 0.92 (0.87 to 0.58$)$ & 0.75 (0.54 to 0.87$)$ \\
\hline $\begin{array}{l}\text { Heterogenenety of } \\
\text { treatment effect accoss } \\
\text { sibgeroups, pravue }\end{array}$ & & & 0.13 & $0.19 t$ & $0.40 \div$ \\
\hline
\end{tabular}

analysis investigated the association of duration of prior basal insulin therapy with these outcomes.

Method: The effects of Gla-300 vs Gla-100 on $\mathrm{HbA}_{1 \mathrm{c}}$ and hypoglycemia were investigated in subgroups split by prior basal insulin duration: $>0-\leq 2 \mathrm{y},>2-\leq 5 \mathrm{y}$ and $>5 \mathrm{y}$, in a post hoc patient-level meta-analysis of EDITION 1 and 2.

Results: Data were available for 1618 participants; $24 \%$ of participants received basal insulin therapy for $>0-\leq 2 \mathrm{y}, 37 \%$ for $>2-\leq 5 \mathrm{y}$ and $39 \%$ for $>5 \mathrm{y}$. $\mathrm{HbA}_{1 \mathrm{c}}$ reduction remained comparable between Gla-300 and Gla-100 regardless of duration of prior basal insulin therapy (no evidence of heterogeneity of treatment effect across subgroups; $p=0.13$ ) (Table). The hypoglycemia benefit of Gla-300 in terms of lower risk of confirmed $(\leq 70 \mathrm{mg} / \mathrm{dL})$ or severe hypoglycemia at any time of day $(24 \mathrm{~h})$ and during the night (00:00-05:59 h) vs Gla-100 was consistent across the different subgroups (no evidence of heterogeneity of treatment effect across subgroups; $p>0.05$ ) (Table).

Conclusion: Switching to Gla-300 provided comparable glycemic control with hypoglycemia benefit vs Gla-100, regardless of duration of prior basal insulin therapy.

Studies sponsored by Sanofi (NCT01499082, NCT01499095)

\section{1}

\section{COMBINING AUDIO AND MOTION SENSORS FOR AUTOMATED DIETARY MONITORING}

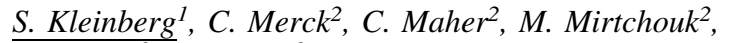
M. Zheng ${ }^{2}$, Y. Huang ${ }^{2}$

${ }^{1}$ Stevens Institute of Technology, Hoboken, USA

${ }^{2}$ Stevens Institute of Technology, Computer Science, Hoboken, USA

Background and Aims: Managing blood glucose (BG) requires understanding both dietary intake and physical activities. While physical activity can be monitored routinely through devices such as smart watches, nutrition has required human input such as through food logs or meal announcements in closed-loop BG control.

We aim to develop a fully automated dietary monitoring system that can identify meal times, amounts, and food type, based on data continuously collected from unobtrusive, bodyworn sensors. To address this, we first aim to determine the timing of eating events (such as food intake and chewing) from which other activities can be inferred. 
Method: We collected data from 6 participants (2 sessions each), wearing Google Glass (measuring head motion), a smart watch on each wrist (capturing wrist motion), and an earbud we created with an internal and external microphone (to enable cancellation of external noise). Data was collected in a laboratory environment with video annotated at the level of individual chews, food intakes, and swallows to determine ground truth. Participants ate an unrestricted set of foods and provided $\sim 6$ hours of sensor data per session.

Results: Using standard random forest methods for classification, audio sensing achieves high precision and recall for identifying meals ( $92 \%$ and $89 \%$ respectively), while motion data was required to identify intakes and episodes of drinking. Our noise cancellation procedure enables extraction of the sounds associated with eating, while removing speech sounds and preserving privacy.

Conclusion: Ultimately, such accurate automated dietary monitoring may provide input to closed-loop systems or reminders for missed insulin boluses around meals.

082

\section{A NOVEL METRIC CAPTURING THE THERAPEUTIC VALUE OF A PREDICTIVE ARTIFICIAL PANCREAS ALGORITHM}

D.A. Finan ${ }^{1}$, P.P. Vereshchetin ${ }^{1}$, T.W. McCann ${ }^{1}$, R. Venugopalan ${ }^{1}$, R.G. Naik', B.L. Levy ${ }^{1}$

${ }^{1} U S A$

The emergence of artificial pancreas (AP)-type devices in clinical practice necessitates novel tools for understanding precisely what the device does on behalf of the patient. In particular, for a predictive AP algorithm which autonomously modulates insulin delivery relative to the prescribed basal rate, it is helpful to develop a simple metric capturing, quantitatively, instances when the algorithm took significant insulinmodulating action to mitigate, if not avoid, potential hypo- and hyperglycemic excursions. The benefits of such a metric are twofold:

1) By design, the metric will capture instances when the algorithm successfully averted situations in which the patient would have been alarmed (e.g., via CGM threshold alarms), and thus had to intervene him- or herself which will help instill confidence in the system; and

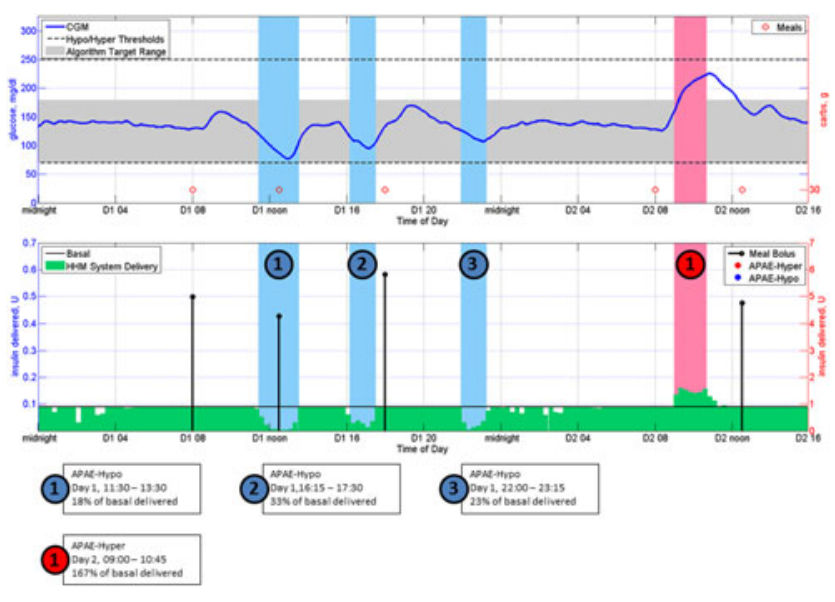

2) Proper, guided analyses of such metrics can help patients and/or HCPs fine-tune the patient's pump settings, like basal rates, to further improve glucose control.

Animas Corporation has developed and evaluated such a metric, called the Artificial Pancreas Activity Event (APAE). This metric has two analogous variations: APAE-Hypo and APAE-Hyper, which capture instances when the algorithm took significant action to mitigate, if not avoid, hypo- and hyperglycemic excursions, respectively. The metric is based both on the duration of algorithm activity and the magnitude of its insulin modulation relative to the basal rate.

Evaluation of the metric via an in-depth in silico trial using the FDA-accepted UVA/Padova Simulator demonstrated promise in enabling patients/HCPs to better understand the system's overall therapeutic value. The figure shows a representative example of the APAEs recorded for one virtual patient using Animas' Hypoglycemia-Hyperglycemia Minimizer (HHM) algorithm.

082A

\section{INSULIN DEGLUDEC (IDEG) VERSUS INSULIN GLARGINE 100 U/ML (GLA-100): OUTCOMES OF A TRIAL-LEVEL META-ANALYSIS IN PATIENTS WITH TYPE 1 DIABETES (T1D)}

\author{
Geremia Bolli $^{1}$, Beverley Balkau ${ }^{2}$, Soazig Chevalier ${ }^{3}$, \\ Jeremy Pettus 4
}

${ }^{1}$ Department of Medicine, University of Perugia School of Medicine, Perugia, Italy

${ }^{2}$ INSERM U1018, Center for Research in Epidemiology and Population Health, Paris-South University, Villejuif, France ${ }^{3}$ Sanofi, Paris, France

${ }^{4}$ Division of Endocrinology \& Metabolism, University of California San Diego, San Diego, CA, USA

Background and aims: We performed a trial-level metaanalysis of IDeg versus Gla-100 clinical studies in T1D.

Method: Efficacy and safety parameters $\left(\mathrm{HbA}_{1 \mathrm{c}}\right.$, FPG and hypoglycaemia incidence and event rates) were explored regardless of study duration and treatment regimen.

Results: There was a consistent slightly greater $\mathrm{HbA}_{1 \mathrm{c}}$ reduction with Gla-100, whereas FPG reduction was greater with IDeg (Figure). Hypoglycaemia incidence rate, defined as patients experiencing $\geq 1$ confirmed ( $<56 \mathrm{mg} / \mathrm{dL}$ ) hypoglycaemic event, was comparable for IDeg and Gla-100 during the night, at any time (24 hours) and for severe events (Figure). No significant heterogeneity of treatment effect across studies was observed for $\mathrm{HbA}_{1 \mathrm{c}}$, FPG, and hypoglycaemia incidence rate; $\mathrm{p}>0.05$. Significant heterogeneity was observed for hypoglycaemia event rate during the night and at any time ( 24 hours); $<<0.05$. A similar severe hypoglycaemia event rate was observed with IDeg versus Gla-100 (rate ratio: fixed model 0.93; random model 0.92 and fixed model 1.22; random model 1.22, respectively), with no evidence of heterogeneity of treatment effect across studies.

Conclusion: This trial-level meta-analysis of IDeg versus Gla-100 showed a slightly better $\mathrm{HbA}_{1 \mathrm{c}}$ reduction with Gla-100 and a slightly better FPG reduction with IDeg, with no difference in nocturnal, any time ( 24 hours), or severe hypoglycaemia incidence rates between treatment groups.

Financial support: Trial-meta-analysis statistical and editorial support provided by Sanofi.

Author disclosures: Geremia Bolli: has received honoraria for consulting and lecturing for Sanofi, Eli Lilly and Menarini 

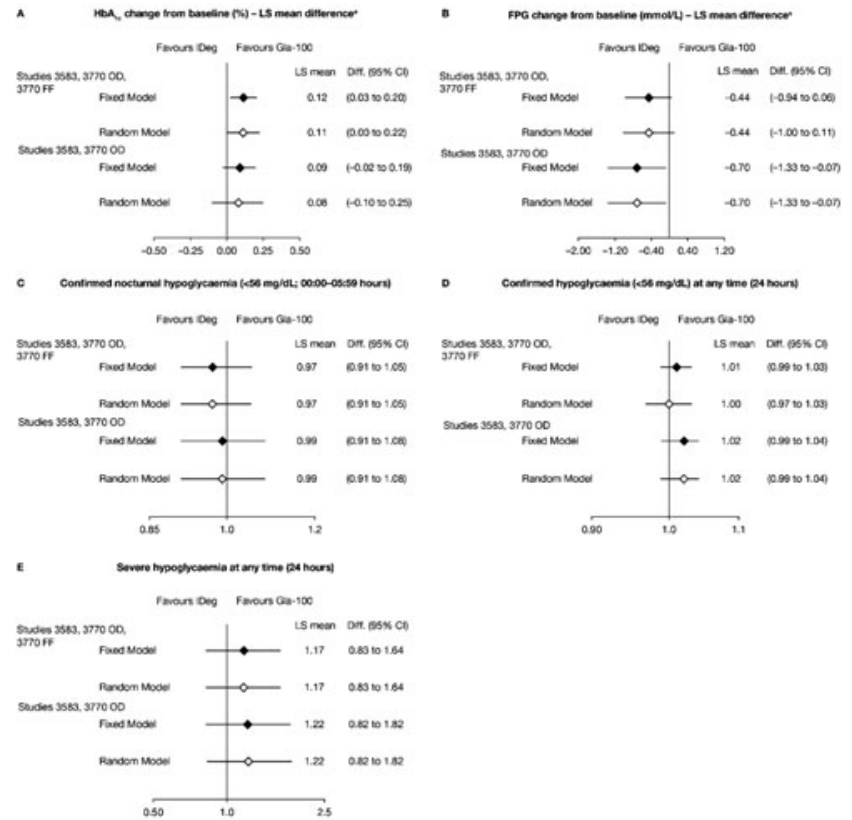

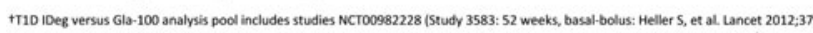
1489-1497), NCT01079234 (Study 3770: 26 weeks, basal-bolus: Mathieu C, et al. J Clin Endocrinol Metab 2013;98: 1154-1162), "Based on

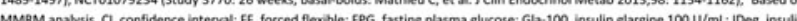

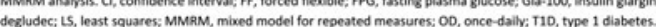

Beverley Balkau: has served on advisory boards for AstraZeneca, BMS, Novo Nordisk, and Sanofi.

Soazig Chevalier: is an employee of Sanofi.

Jeremy Pettus: has served on advisory boards for Sanofi, Tandem, and Novo Nordisk.

\section{3}

\section{SHORT-TERM COUNSELLING DURING ATHLETIC SPORTS CAMP LEADS TO LONG-TERM BENEFITS IN INDIVIDUALS WITH TYPE 1 DIABETES}

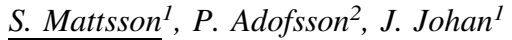

${ }^{1}$ Faculty of Health Sciences and Medicine, Örebro University Hospital, Örebro, Sweden

${ }^{2}$ Institute of Clinical Sciences- Sahlgrenska Academy

at University of Gothenburg, Department of Pediatrics,

Gothenburg, Sweden

Background and Aims: Physical exercise (PE) is associated with an increased incidence of both hypo-/hyperglycemia especially during and after PE in people with type 1 diabetes (T1D). The aim of this study was to investigate if participation in a sport camp, was associated with improved glycemic control measured as a reduction in $\mathrm{HbA} 1 \mathrm{c}$.

Method: Individuals with T1D were invited to attend at a three-day long sports camp including exercise twice daily, and an offer about medical and dietary advice. Participants used continuous glucose monitoring (CGM), and received advice on how to adjust their carbohydrate intake and insulin doses before, during and after workouts. HbA1c was assessed at baseline and after 3 months.

Results: Seven sports camps were conducted with 95 participants (Male/Female, 44/48, mean age $40.5 \pm 10.0$ yrs, mean duration of diabetes $18.7 \pm 12.3$ yrs and mean BMI $23.6 \pm 3.5 \mathrm{~kg} /$ $\mathrm{m}^{2}$ ) of which $46 / 54 \%$ were on CSII/MDI. HbA1c was significantly reduced from $59.2 \pm 9.5 \mathrm{mmol} / \mathrm{mol}$ to $56.5 \pm 8.3 \mathrm{mmol} /$ mol, (95\% CI, $1.9-4.2 \mathrm{mmol} / \mathrm{mol}), \mathrm{p}<0.001$. Participants with $\mathrm{HbAlc}<58 \mathrm{mmol} / \mathrm{mol}(\mathrm{n}=35)$, at the start of the camp, did not show any improvement in HbAlc (baseline; $49.5 \pm 5.9$, after 3 months; $49.9 \pm 7.1 \mathrm{mmol} / \mathrm{mol}$ ), participants with $\mathrm{HbA} 1 \mathrm{c}$ between $58-70 \mathrm{mmol} / \mathrm{mol}(\mathrm{n}=45)$ and $\mathrm{HbAlc}>70 \mathrm{mmol} / \mathrm{mol}(\mathrm{n}=12)$ lowered their HbA1c significantly more with $4.0 \pm 4.8$ (95\% CI, 2.6-5.5), respectively $7.0 \pm 3.8$ (95\% CI, 4.6-9.4) $\mathrm{mmol} / \mathrm{mol}$.

Conclusion: Glucose control, improved HbA1c significantly 3 months after participation in a three-day sport camp. Participants with higher $\mathrm{HbA} 1 \mathrm{c}$ or higher $\mathrm{HbA} 1 \mathrm{c}$ combined with CSII showed a more prominent $\mathrm{HbA} 1 \mathrm{c}$ reduction.

\section{4}

\section{UTILIZING SOCIAL MEDIA FOR RESEARCH AND DEVELOPMENT OF DIABETES SELF-MANAGEMENT TOOLS}

\author{
M. Bradway ${ }^{1}$, E. Arntzen ${ }^{1}$, E. Arsand ${ }^{1,2}$ \\ ${ }^{1}$ University Hospital of North Norway, Norwegian Centre \\ for Integrated Care and Telemedicine, Troms $\phi$, Norway \\ ${ }^{2}$ University of Troms $\phi$, Department of Clinical Medicine, \\ Troms $\phi$, Norway
}

Background and Aims: Individuals with diabetes are more often looking past healthcare services toward social media, such as online support communities, and mobile apps to guide their own daily diabetes self-management. This makes it more challenging for medical systems to relate to these individuals. This study aimed to determine if and how clinical research teams could use social media during development and testing of diabetes-related mHealth (mDiabetes) tools to better understand patients and their needs.

Method: Our Diabetes Research Team has used online and social media channels to engage end-users during development of the Diabetes Diary app and its systems. Analysis compares the frequency and content of social media dissemination to the number and type of followers' responses.

Results: Activities and responses between 17/4/2013-24/7/ 2015 include: the app's release on Google Play ( $n=922$ "current installs"), posts on Facebook ( $\mathrm{n}=107$ with $\mathrm{n}=1076$ "likes", $\mathrm{n}=82$ comments, $\mathrm{n}=191$ "shares", $\mathrm{n}=730$ "avg. reach", i.e. accessibility) and Twitter ( $\mathrm{n}=83$ with $\mathrm{n}=81$ "retweets" and "favourites", $\mathrm{n}=75$ followers), YouTube videos ( $\mathrm{n}=20$ with $\mathrm{n}=1037$ views), and a webpage. We also analysed $\mathrm{n}=95$ emails from users $(\mathrm{n}=50$ questions, $\mathrm{n}=27$ suggestions, $\mathrm{n}=18$ positive feedback, as main themes). Facebook-user engagement increased significantly during "contests" whereby followers were asked to provide feedback about the Pebble (smartwatch) version of the app, see Figure 1.

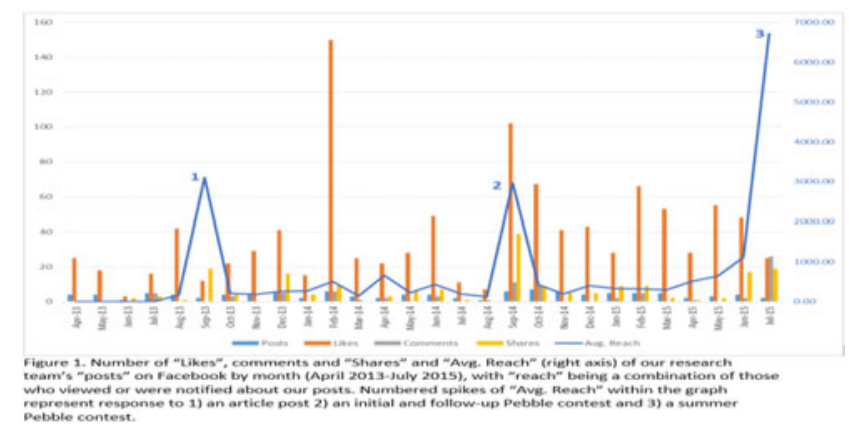


Conclusion: This study demonstrates that social media can be used to quickly and effectively communicate with endusers during mHealth-tool research and development. Further analysis and results will be presented at ATTD2016 regarding potential uses and impacts of user-engagement through these channels within mDiabetes research and development.

085

\section{PERCEIVED UTILITY OF A REMOTE MONITORING SYSTEM FOR PEDIATRIC SUBJECTS AFFECTED BY TYPE 1 DIABETES IN PEDARPAN (PEDIATRICS ARTIFICIAL PANCREAS) SUMMER CAMP}

E. Losiouk ${ }^{1}$, G. Lanzola ${ }^{1}$, R. Bonfanti ${ }^{2}$, D. Iafusco ${ }^{3}$, $\overline{\text { I. Rabbone }}^{4}$, A. Sabbion ${ }^{5}$, R. Schiaffini ${ }^{6}$, A. Galderisi ${ }^{7}$, M. Marigliano ${ }^{5}$, N. Rapini ${ }^{8}$, A. Rigamonti ${ }^{9}$, D. Tinti ${ }^{4}$, V. Vallone ${ }^{10}$, A. Zanfardino ${ }^{3}$, F. Boscari ${ }^{10}$, S. Galasso $^{10}$, A. Troncone $^{11}$, S. Del Favero ${ }^{12}$, R. Visentin $^{12}, R$. Calore $^{12}$, M.Y. Leal ${ }^{13}$, F. Di Palma ${ }^{14}$, M. Messori ${ }^{14}$, D. Chernavvsky ${ }^{15}$, L. Magni $^{14}$, D. Bruttomesso, S. Quaglini ${ }^{1}$, C. Cobelli ${ }^{17}$

${ }^{1}$ University of Pavia, Department of Electrical- Computer and Biomedical Engineering, Pavia, Italy

${ }^{2}$ Hospital San Raffaele, Pediatric Department and Diabetes Research Institute, Milan, Italy

${ }^{3}$ Second University of Naples, Department of Pediatrics, Naples, Italy

${ }^{4}$ University of Turin, Department of Pediatrics, Turin, Italy

${ }^{5}$ University of Verona, Department of Life \& Reproduction Sciences, Verona, Italy

${ }^{6}$ Bambino Gesu-Children's Hospital, Unit of Endocrinology and Diabetes, Rome, Italy

${ }^{7}$ University of Padua, Department of Woman's and Child's

Health, Padova, Italy

${ }^{8}$ Policlinico di TorVergata- University of Rome,

Pediatric Diabetology Unit, Rome, Italy

${ }^{9}$ Hospital San Raffaele, Department and Diabetes Research Institute, Milan, Italy

${ }^{10}$ University of Padova, Department of Internal Medicine-DIM, Padova, Italy

${ }^{11}$ Second University of Naples, Department of Psychology, Caserta, Italy

${ }^{12}$ University of Padova, Department of Information

Engineering, Padova, Italy

${ }^{13}$ Universidade Tecnológica Federal do Paraná, Curitiba,

Brasil

${ }^{14}$ Department of Civil Engineering and Architecture, University of Pavia, Pavia, Italy

${ }^{15}$ Center for Diabetes Technology, University of Virginia, USA

${ }^{16}$ Unit of Metabolic Diseases, Department of Internal MedicineDIM, University of Padova, Padova, Italy

${ }^{17}$ Department of Information Engineering, University of

Padova, Padova, Italy

Background and Aims: Remote monitoring of outpatients traditionally deals with transferring data to hospitals to support early detection of complications. While this mostly involves physicians, here we describe a different use-case involving diabetic children's parents as primary stakeholders of a monitoring system by analyzing its perceived usefulness.

Method: A remote monitoring service was set up within a randomized controlled trial involving 32 children during a 7-day summer camp. Parents, some located at the camp and some at home, were instructed about using the system and allowed to monitor their children in real-time during the trial. Ad-hoc developed questionnaires were administered before and after the trial, to provide both a baseline assessment of the issues that those families face and the perceived benefit of the system, once it would be used in their daily life.

Results: At the baseline, $53 \%$ of the answers expressed a negative feeling of "living with diabetes". When we asked if and how the remote monitoring could affect those aspects, the negative answers decreased to $19 \%$ (p-value $\ll 0.05$ ). When asked (0-100 scale) how much their quality of life was affected by diabetes and how much the system could improve it, the average value increased from 64.13 to $78.42(\mathrm{p}$-value $=0.03$ ). The positive parents' attitude toward the system was also proved by the fact that they would be willing to pay an average of 356.77 ([0-3000]) Euro/Year for that service.

Conclusion: The system seems to be appreciated since it reduces concern and improves calmness of the children's parents.

\section{6}

\section{CLINICAL SAFETY AND FEASIBILITY OF THE ADVANCED BOLUS CALCULATOR FOR TYPE 1 DIABETES BASED ON CASE-BASED REASONING: A 6-WEEK NON-RANDOMISED SINGLE-ARM PILOT STUDY}

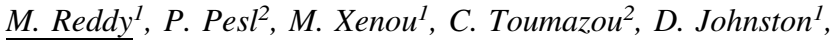
P. Georgiou ${ }^{2}$, P. Herrero ${ }^{2}$, N. Oliver $^{1}$

${ }^{1}$ Imperial College London, Division of diabetes- endocrinology and metabolism, London, United Kingdom

${ }^{2}$ Imperial College London, Centre for Bio-Inspired TechnologyDepartment of Electrical and Electronic Engineering- Institute of Biomedical Engineering, London, United Kingdom

Background and Aims: To assess safety and feasibility of the Advanced Bolus Calculator for Diabetes (ABC4D) in a freeliving environment over 6 weeks. The ABC4D is a decision support system based on case-based reasoning (CBR). It is implemented in a smartphone application and utilises continuous glucose monitoring data aiming for personalised and adaptive insulin bolus advice.

Method: Prospective non-randomised single-arm pilot study. Participants used the ABC4D smartphone application for six weeks in their home environment, attending the clinical research facility weekly for data upload, revision and adaptation of the CBR casebase. The primary outcome was post-prandial hypoglycaemia.

Results: Ten adults with T1DM on MDI of insulin (mean (SD) age 47 (17), diabetes duration 25(16), and HbA1C 68(16) mmol/ mol $(8.4(1.5) \%)$ participated. A total of 182 and 150 meal scenarios, in week 1 and week 6 respectively, were included in the analysis of post-prandial outcomes. The post-prandial and overall glycaemic outcomes comparing the first week with the final week of the study are outlined in the table below. The mean (SD) number

\begin{tabular}{|c|c|c|c|}
\hline Glycaemic outcomes & Week 1 & Week 6 & p \\
\hline Episodes of hypoglycaemia within 6 hours post-meal & $4.5(2.0-8.2)$ & $2(0.5-6.5)$ & 0.1 \\
\hline Post-prandial glucose at $120 \mathrm{~min}(\mathrm{mmol} / \mathrm{l})$ & $9.3(7.0-10.6)$ & $8.6(7.6-10.9)$ & 0.5 \\
\hline$\%$ time in hypoglycaemia $(<3.5 \mathrm{mmol} / \mathrm{)})$ & $3.0(0.4-7.1)$ & $1.7(0.3-7.2)$ & 0.7 \\
\hline$\%$ time in hypoglycaemia $<3.9 \mathrm{mmol} /$ & $5.0(0.7-9.2)$ & $3.6(0.6-9.8)$ & 0.7 \\
\hline$\%$ time in target $(3.9-10.0 \mathrm{mmol} / \mathrm{l})$ & $55.0(50.1-56.7)$ & $60.9(46.5-72.2)$ & 0.9 \\
\hline$\%$ time in hyperglycaemia $(>10 \mathrm{mmol} / \mathrm{h})$ & $40.5(26.3-47.3)$ & $36.9(18.0-43.1)$ & 0.5 \\
\hline
\end{tabular}


of post-prandial rescue carbohydrate required for hypoglycaemia was $1.8(1.7)$ versus $0.7(0.9)(\mathrm{p}=0.06)$ in week 1 and week 6 respectively. No episodes of severe hypoglycaemia occurred.

Conclusion: The ABC4D is safe for use as a decision support tool for insulin bolus dosing. A trend suggesting a reduction in post-prandial hypoglycaemia was observed in the final week compared to week 1. A 6-month powered randomised controlled trial comparing the ABC4D with a standard bolus calculator with real-time CGM is currently underway.

\section{7}

\section{USE OF THE ACCU-CHEK CONNECT SYSTEM IMPROVED FEELINGS OF CONFIDENCE, SAFETY AND REDUCED HYPOGLYCEMIA FEAR AMONG T1D CHILDREN/ADOLESCENTS AND THEIR PARENTS/ CAREGIVERS}

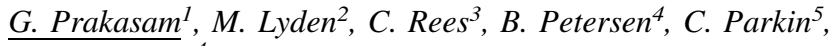
M. Schweitzer ${ }^{4}$

${ }^{1}$ Sutter Medical Center and Center of Excellence in Diabetes and Endocrinology, USA

${ }^{2}$ BioStat International- Inc., Statistics Department, Tampa, USA

${ }^{3}$ Roche Diabetes Care- Inc., Medical Affairs Department, Indianapolis, USA

${ }^{4}$ Roche Diabetes Care GmbH, Medical Affairs, Mannheim, Germany

${ }^{5}$ CGParkin Communications- Inc., Research Department, Boulder City, USA

Background and Aims: Immediate access to diabetes-related data and the ability to review these data retrospectively may improve clinical outcomes, increase feelings of confidence/safety in diabetes management and reduce fear of hypoglycemia among T1D children/adolescents and their parents/caregivers. The ACCU-CHEK ${ }^{\circledR}$ Connect system (system) consists of a blood glucose meter, which connects wirelessly, via low-energy Bluetooth, to the user's diabetes management app on their mobile device, providing parents/caregivers with real-time, remote access to their child's diabetes-related data. We assessed user perceptions of the system, regarding confidence, safety, hypoglycemia fear and preference to current diabetes management method among children/adolescents with T1D and their parents/caregivers.

Method: In this 7-day, observational, pilot study, we surveyed T1D children/adolescents and parents/caregivers who attended the Friends for Life conference, held July 6-12, 2015, in Or-

Figure. Percentage of respondents who reported feeling more confident, safer and less hypoglycemia fear.

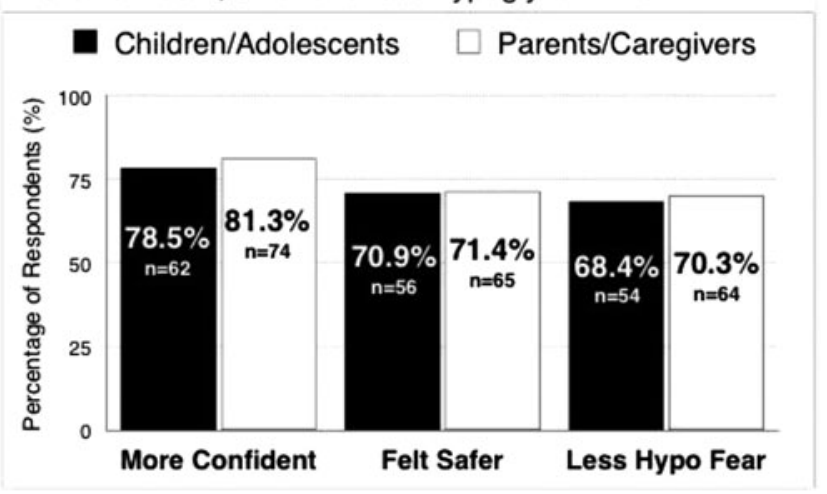

lando, Florida. Eligibility criteria for inclusion in the analysis were completion of baseline questionnaire, $\geq 3$ days of participation and completion of $\geq 50 \%$ of the questions in the parent/ caregiver post questionnaire.

Results: Ninety-one parents/caregivers (of children/adolescents $<8$ to $<18$ years) and 79 children/adolescents $(<8$ to $<18$ years) were included in the analysis. Most respondents reported that they felt more confident, safer and worried less about hypoglycemia when using the system regardless of treatment. (Figure) The majority of respondents reported they preferred using the system over their previous method of diabetes management (children/adolescents, 64.4\%; parents/caregivers, 73.3\%).

Conclusion: Use of the ACCU-CHEK ${ }^{\circledR}$ Connect system was preferred over previous methods and may help T1D children/ adolescents and their parents/caregivers reduce their fears and concerns relevant to diabetes management.

088

\section{T1D CHILDREN/ADOLESCENTS AND THEIR PARENTS/CAREGIVERS CAN EASILY LEARN AND USE THE ACCU-CHEK® CONNECT SYSTEM}

C. Rees ${ }^{1}$, G. Prakasam ${ }^{2}$, M. Lyden ${ }^{3}$, B. Petersen ${ }^{4}$, P. Fleming ${ }^{5}$, C. Parkin ${ }^{6}$, M. Schweitzer ${ }^{4}$

${ }^{1}$ Roche Diabetes Care-Inc., Medical Affairs Department, Indianapolis, USA

${ }^{2}$ Sutter Health, Medical Department, Sacramento, USA

${ }^{3}$ BioStat International- Inc., Statistics Department, Tampa, USA

${ }^{4}$ Roche Diabetes Care GmbH, Medical Affairs, Mannheim,

Germany

${ }^{5}$ Roche Diabetes Care- Inc., Clinical Department, Indianapolis, USA

${ }^{6}$ CGParkin Communications - Inc., Research Department, Boulder City, USA

Background and Aims: New diabetes management tools have the potential to improve clinical and psychosocial outcomes. However, achieving these benefits is dependent upon users' ability to use the technology and perceived value of these tools. The ACCU-CHEK ${ }^{\circledR}$ Connect system (system) consists of a blood glucose meter, which connects wirelessly, via low-energy Bluetooth, to the user's diabetes management app on their mobile device, providing parents/caregivers of T1D children/adolescents with real-time, remote access to their child's diabetes-related data. Children/adolescents can review their current and retrospective data. We assessed perceptions of the ease of learning/using the system and its usefulness in diabetes management among T1D children/adolescents and their parents/caregivers.

Method: In this 7-day, observational, pilot study, we surveyed children/adolescents with T1D and their parents/caregivers who attended the Friends for Life conference, held July 6-12, 2015, Orlando, Florida. All respondents participated in a 15-minute training session prior to system use and were asked to assess the effectiveness of their training and their satisfaction with the system in managing their child's diabetes.

Results: Ninety-one parents/caregivers (of children/adolescents $<8$ to $<18$ years) and 79 children/adolescents $(8$ to $<18$ years) were included in the analysis. The majority of respondents reported that they were satisfied with the ease of learning and ease of using the system following training. (Figure) Most were satisfied with how well the system helped them with diabetes management (children/adolescents, 93.7\%; parents/caregivers, $90.1 \%)$. 
Figure. Percentage of children/adolescents and parents/caregivers who reported satisfaction with ease of learning/using the system.

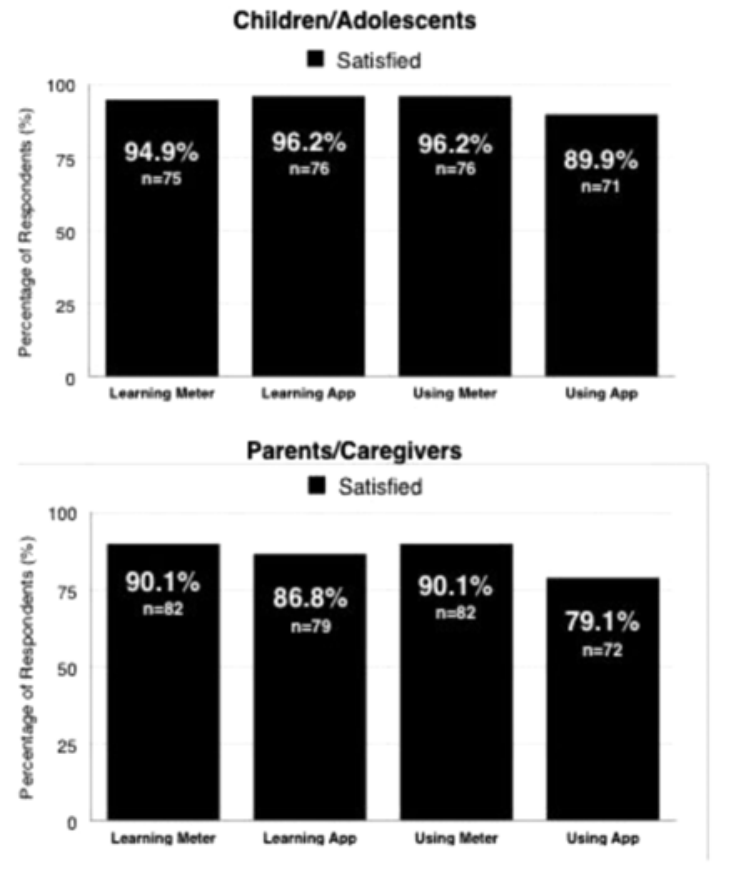

Conclusion: Our results suggest that the ACCU-CHEK ${ }^{\circledR}$ Connect system is easy to learn/use and helpful for T1D children/ adolescents and their parents/caregivers in managing their diabetes.

089

\section{SPECTRUM A MANUFACTURER INDEPENDENT EDUCATION PROGRAM FOR CONTINUOUS GLUCOSE MONITORING (CGM) FOR ALL AGE GROUPS}

M. Holder ${ }^{1}$, M. Holder et al. for the Adult and Pediatric Spectrum Group of the Working Group for DiabetesTechnology and the Working Group of Pediatric Diabetology (AGPD) in Germany ${ }^{A D G T}$

\section{${ }^{1}$ Olgahospital - Klinikum Stuttgart, Pediatric Endocrinology and Diabetology, Stuttgart, Germany}

Background and Aims: CGM is used by an increasing number of children, adolescents and adults with type-1 diabetes in Germany, however the total number is still small. Limited uptake of CGM includes economic and behavioural barriers, but also the lack of a manufacturer independent structured education program for all age groups.

Method: Based of intensive experiences in education for decades we therefore developed such a program called SPECTRUM ("Structured patient education and treatment program for self-reliant continuous glucose monitoring"). It combines technical understanding with appropriate therapeutic test interpretations. It is available in 3 versions: one for adults and two adapted for pediatric patients (parents with their children and adolescents).

Results: In several modules (each is intended to last $90 \mathrm{~min}$ with a detailed curriculum) all aspects of CGM use will be discussed interactively with the users. Module 0 (introduction) informs the patients about positive and possible negative experi- ences in long-term CGM use to provide them a realistic view of the benefits of this technology beforehand. The main modules 1 to 6 cover basic knowledge about CGM, alarm-settings, glucose trend arrows, CGM usage in everyday life and CGM software. The patients get trained how to assess and download CGM data, improve CGM use and implement it in their daily life.

Conclusion: SPECTRUM provides patients and their diabetes-teams with the opportunity to optimize CGM use in an independent and effective way. Important conditions of this new education program are independency of manufacturers and product-neutrality enabling certification after formal evaluation within the framework of large clinical trial.

090

\section{TECHNOLOGY-ENABLED SELF-MANAGEMENT SUPPORT INTERVENTION FOR PEOPLE WITH TYPE 2 DIABETES}

\author{
N. Kaufman $^{1}$ \\ ${ }^{1}$ Canary Health, Medical, Los Angeles, USA
}

Background and Aims: Individuals with type 2 diabetes improve their health if they increase self-efficacy and better managechallenges associated with living with diabetes. Technology has the capacity to bring to scale programs proven effective in-person by lowering the financial and logistical costs associated with the program and by removing barriers to participation. This study presents the results from a real-world deployment of the research-proven-effective digital translation of an in-person self-management intervention (Chronic Disease Self-management program (CDSMP)) called Better Choices Better Health ${ }^{\mathrm{TM}}$. This six week online program was developed by Stanford University based on more than 3 decades of research. Individuals participate in a peer-led intervention consisting of weekly lessons, group discussion boards and personal action planning. Results include improved self-efficacy, enhanced medication adherence, improved diabetes control and lowered depression severity.

Method: U.S. federal employees with type 2 diabetes were recruited using a multi-modal outreach and engagement approach.

Results: Of the 12,247 individuals in the outreach group with claims for diabetes, $1.1 \%$ enrolled. Women were more likely to enroll than men. Enrollees averaged 1.83 additional chronic conditions. $59 \%$ had 2 or more other additional conditions (hypertension $82.7 \%$; hyperlipidemia $87.9 \%$ ) and $41 \%$ were nonadherent with AIC testing. Participants $(\mathrm{N}=108)$ were placed into groups of 25 individuals each of whom had at least one chronic conditions. On average, participants engaged in 4.3/6 sessions (females 4.5 and males 4.0). Participants were highly satisfied with the workshops (4.4/5). The average perceived improvement in managing health was $4.3 / 5$.

Conclusion: Online CDSMP engages people with type 2 diabetes and improves their self-efficacy.

\section{1}

\section{A NOVEL APPROACH TO DEFINE BETA CELL LOSS IN TYPE 1 DIABETES: USE OF THE SITAR MODEL}

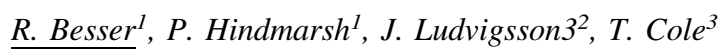




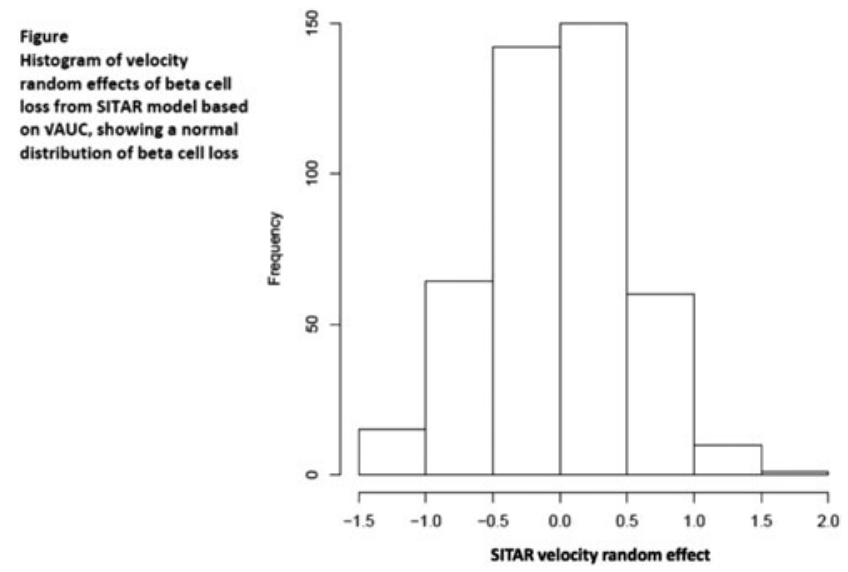

${ }^{1}$ Institute of Child Health- University College London, Genetics and Epigenetics in Health and Disease, London, United Kingdom

${ }^{2}$ Faculty of Health Sciences- Linköping Universit, Department of Clin Exp Medicine, Linköping, Sweden

${ }^{3}$ Institute of Child Health- University College London, Population- Policy and Practice Programme, London, United Kingdom

Background and Aims: C-peptide (CP) loss in Type 1 diabetes (T1D) is highly variable between individuals, and drivers affecting it are poorly understood. Aims: To use a mixed effects growth model, SITAR (SuperImposition by Translation And Rotation), to (1)identify a suitable scale to measure area under the curve $\mathrm{CP}$ (AUC), and (2)provide a definition of CP loss.

Method: CP was sampled during 1,334 150min mixed meal tolerance tests, in $421 \mathrm{~T} 1 \mathrm{D}$ patients $<18 \mathrm{yr}$, to calculate AUC, at 3,9,18,30,48 and 72mnths after diagnosis. CP loss was assessed using SITAR, which treats the individuals' serial AUC values plotted against time as 'growth curves'. Adjustments shift the mean curve up/down, and stretch/shrink the age scale, to adjust for rate of AUC fall and match individual curves. AUC and rate of CP loss were analysed on the original, log and square root scales, and further adjusted for age and gender.

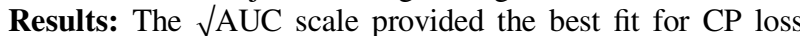
(Bayesian Criterion Information).The rate of CP loss was normally distributed. No correlation was seen between the mean and rate of fall of $\sqrt{\text { AUC. }}$ /AUC increased with age $(\mathrm{p}<0.0001)$ and was higher in females $(p=0.0004)$. The rate of $\sqrt{\text { AUC fall was similar }}$ in males and females, but fell with increasing age $(\mathrm{p}<0.0001)$.

Conclusion: SITAR provides a novel tool to assess CP loss in T1D. A definition of rapid or slow CP loss could be based on cutoffs derived from the distribution for these data, to allow stratification of patients into therapies based on their projected CP loss after diagnosis.

\section{2}

DIAGNOSIS OF CARDIOVASCULAR AUTONOMIC NEUROPATHY IN TYPE 2 DIABETIC PATIENTS WITH ST-SEGMENT ELEVATION MYOCARDIAL INFARCTION

\author{
L. Strongin $^{1}$, A. Bushueva ${ }^{1}$, I. Pochinka ${ }^{1}$, S. Botova ${ }^{1}$ \\ ${ }^{1}$ Nizhny Novgorod State Medical Academy, Endocrinology and \\ Internal Medicine, Nizhny Novgorod, Russia
}

Background and Aims: To develop criterion for the diagnosis of cardiovascular neuropathy (CAN) in type 2 diabetic (T2DM) in the subacute stage of ST-segment elevation myocardial infarction (STEMI).

Method: The study involved 77 subjects. Including 54 patients with STEMI without previous coronary history, undergoing reperfusion therapy, of which 27 were T2DM patients (group 1), 27 subjects hadn't T2DM (group 2). Group 3 consisted of 23 T2DM patients without cardiovascular diseases. Ewing's cardiovascular reflex tests (ECRT) were performed. The heart rate variability (HRV) was analyzed by time-domain, frequencydomain and non-linear methods (scattergram).

Results: ECRT and HRV showed signs of autonomic dysfunction in the both STEMI groups regardless of the presence of T2DM. The scattergram revealed significantly lower data in patients with T2DM. In 1 group a cluster analysis was performed, as variables the maximal troponin I and area of scattergram (S) were used. Two clusters were identified, in which there were no differences of troponin level and $\mathrm{S}$ levels differed significantly (in the 1 st cluster median of $S=358$, in the 2 nd $-746 \mathrm{~ms} 2$, $\mathrm{p}<0.001$ ). (Fig 1) In group 3 the ROC curve analysis was performed, ECRT and $\mathrm{S}$ were used as variables. Area under the ROC curve was 0.87 , for criterion $S \leq 571 \mathrm{~ms} 2$, sensitivity was $66 \%$, specificity $-100 \%$.

Conclusion: the study of HRV by scattergram analysis is preferable for the diagnosis of CAN in T2DM patients with STEMI, the criterion for diabetic CAN is $\mathrm{S} \leq 571 \mathrm{~ms} 2$.

\section{3}

\section{CLINICAL PERFORMANCE OF TWO INSULIN INFUSION PROTOCOLS IN THE MEDICAL INTENSIVE CARE UNIT: A COMPARISON BY MEANS OF CONTINUOUS GLUCOSE MONITORING}

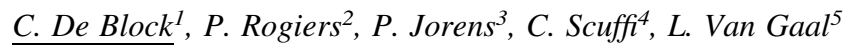

${ }^{1}$ Antwerp University Hospital, Endocrinology-DiabetologyMetabolism, Edegem, Belgium

${ }^{2}$ General Hospital Middelheim, Intensive Care Unit, Antwerp, Belgium

${ }^{3}$ Antwerp University Hospital, Intensive Care Unit, Edegem, Belgium

${ }^{4}$ A. Menarini Diagnostics, Diagnostics, Florence, Italy

${ }^{5}$ Antwerp University Hospital, Endocrinology-Diabetology-

Metabolism, Edegem, Belgium

Background and Aims: Achieving good glycemic control in the intensive care unit (ICU) requires a safe and efficient IV insulin infusion protocol (IIP) and frequent glucose measurements. We compared the clinical performance of two IIPs (Leuven versus modified Yale protocol) in patients admitted to a medical ICU, by using continuous glucose monitoring (CGM).

Method: This is a pooled data analysis of two published prospective randomized controlled trials. CGM (GlucoDay, A.Menarini Diagnostics) was performed in $55 \mathrm{MICU}$ patients (non-diabetic/ diabetic: 35/20, age $64 \pm 12 \mathrm{y}$, APACHE-II score $28 \pm 7$ ). Outcome measures were percentage of time in normoglycemia $(80-110 \mathrm{mg} /$ dl) and in hypoglycemia $(<60 \mathrm{mg} / \mathrm{dl})$, and glycemic variability (standard deviation, mean amplitude of glucose excursions).

Results: Thirty-three subjects were treated by the Yale protocol and 22 by the Leuven protocol. Mean glycemia was lower $(120 \pm 16 \mathrm{vs} 139 \pm 48 \mathrm{mg} / \mathrm{dl}, \mathrm{p}=0.034)$, percentage of time in normoglycemia was higher ( $37 \pm 15$ vs $26 \pm 11 \%, \mathrm{p}=0.001)$, and percentage of time in hypoglycemia was lower ( $2 \pm 3$ vs $5 \pm 5 \%, \mathrm{p}=0.003)$ using the Yale protocol. Glycemic variability was also smaller with the use of the Yale protocol (SD: 
$31 \pm 13$ vs $65 \pm 68 \mathrm{mg} / \mathrm{dl}, \mathrm{p}=0.008$; MAGE: $58 \pm 24$ vs $85 \pm 57 \mathrm{mg} / \mathrm{dl}, \mathrm{p}=0.018)$. Logistic regression analysis with strict glucose control (defined as a mean glycemia $\leq 110 \mathrm{mg} / \mathrm{dl}$ ) as dependent variable could not identify age, BMI, APACHE-II score, diabetes status, or type of IIP as independent parameters.

Conclusion: The Yale protocol shows better average glycemia, more time spent in normoglycemia, less time spent in hypoglycemia and less glycemic variability as compared to the Leuven protocol, but was not independently associated with strict glycemic control.

094

\section{REDUCTION IN RECURRENT INPATIENT HYPOGLYCAEMIA BY INNOVATIVE USE OF POINT OF CARE CAPILLARY GLUCOSE MONITORING}

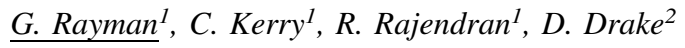

${ }^{1}$ Ipswich Hospital NHS Trust, Diabetes \& Endocrine Centre, Suffolk, United Kingdom

${ }^{2}$ Abbott Diabetes Care, Executive Installation and Support Specialist, Ipswich, United Kingdom

Background and Aims: Hypoglycaemia in inpatients with diabetes is distressing, frequent and associated with a 2-3 fold increase in mortality. The UK National Diabetes Inpatient Audit demonstrated that $\sim 10 \%$ of diabetes inpatients have a severe hypoglycaemic episode and $2 \%$ require rescue with intravenous glucose. To date, remote monitoring of Point-Of-Care (POC) capillary blood glucose $(\mathrm{CBG})$ is traditionally used by central laboratories to quality control ward based meters and individual users.

Aims: To determine whether POC CBG downloads could be adapted for use by clinical teams to identify first hypoglycaemic events to prevent recurrence.

Method: Together with Abbott Diabetes Care UK we developed an alert system based on CBG data extracted from the Abbott Precision Xceed Pro ${ }^{\mathrm{TM}}$ Web POC meters (Abbott Diabetes Care Inc., Alameda, CA, USA) linked to patients' unique identifiers, ward location, date and time. This enabled the inpatient team to quickly identify and target patients with hypoglycaemic events and so institute preventive measures. We assessed the impact of this innovation by comparing first and subsequent $\mathrm{CBG} \leq 3 \mathrm{mmol} /$ 1 in 2012 (before the alert system), 2013 and 2014.

Results: Admission rates did not significantly change but there was a small decline in first hypoglycaemic events (CBG $\leq 3 \mathrm{mmol} / \mathrm{l}) ;(676,632$ and 575 for years 2012, 2013, 2014 respectively). Recurrent hypoglycaemia significantly decreased ( $\mathrm{p}<0.001)$ from $232 / \mathrm{yr}$ to $88 / \mathrm{yr}(2012 \mathrm{v} 2013-62 \%$ reduction $)$ and 91/yr (2012v2014- 61\% reduction).

Conclusion: This study demonstrates that adapting remote monitoring of POC CBG systems for clinical use can be highly effective in preventing recurrent hypoglycaemia in inpatients with diabetes.

095

\section{HIGH GLUCOSE VARIABILITY IN HOSPITALIZED TYPE 1 DIABETES PATIENTS AT A SINGLE TERTIARY CARE US HOSPITAL: OBSERVATIONAL STUDY AND SYSTEMATIC REVIEW OF PUBLISHED LITERATURE}

V. Dadlani ${ }^{1}$, S. Tamhane ${ }^{1}$, A. Sun ${ }^{1}$, A. Sharma ${ }^{1}$, D. Delivanis ${ }^{1}$,

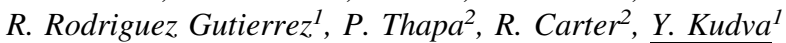

\begin{tabular}{|c|c|c|c|c|c|c|c|c|}
\hline $\begin{array}{l}\text { Patient admission } \\
\text { in unit }\end{array}$ & $\begin{array}{l}\text { No. of } \\
\text { Patients }\end{array}$ & BG_Mean & HBGI & LBGI & ADRR & \multicolumn{3}{|c|}{ Risk of ADRR (\% of subjects) } \\
\hline & & & & & & $\begin{array}{l}\text { Low } \\
(<20 \%)\end{array}$ & $\begin{array}{l}\text { Moderate } \\
(20-40 \%)\end{array}$ & $\begin{array}{l}\text { High } \\
(>40 \%)\end{array}$ \\
\hline Total & 787 & 183.3 & 12.5 & 1.5 & 34.9 & 16.1 & 48.4 & 35.5 \\
\hline Medical & 455 & 194.5 & 14.8 & 1.7 & 39.5 & 9.5 & 43.3 & 47.2 \\
\hline Metabolic & 132 & 214.6 & 18.4 & 1.2 & 48.7 & 0 & 28.8 & 71.2 \\
\hline Hyperglycemia & 121 & 217.5 & 18.8 & 0.8 & 48 & 0 & 29.8 & 70.2 \\
\hline Hypoglycemia & 11 & 186 & 14.7 & 3.8 & 55.2 & 0 & 18.2 & 81.8 \\
\hline Non Metabolic & 323 & 186.7 & 13.4 & 1.9 & 35.9 & 13.3 & 48.9 & 37.8 \\
\hline Surgical & 328 & 167.8 & 9.5 & 5.9 & 28.8 & 25.3 & 55.5 & 19.2 \\
\hline Elective & 262 & 168.3 & 9.6 & 1.4 & 28 & 27.4 & 54.6 & 18 \\
\hline
\end{tabular}

${ }^{1}$ Mayo Clinic, Endocrinology, Rochester, USA

${ }^{2}$ Mayo Clinic, Department of Health Sciences Research, Rochester, USA

Background and Aims: Glycemic variability (GV) in type 1diabetes (T1D) is challenging but has been studied rigorously only in the ambulatory setting. Limited data are available for GV in patients with T1D during hospitalization.

Method: We identified patients with T1D hospitalized during the years 2006 to 2010 and analyzed in-hospital glucose measurements to assess GV. We studied 787 patients hospitalized in different units and performed subgroup analysis. To place our data in context, we conducted a systematic review using Cochrane collaboration methodology, of GV in hospitalized T1D. Eligible studies included a cohort of T1D patients admitted to hospital for different reasons, regardless of study design, sample size and language from 1970 to 2015 . We excluded all non-original reports without original data. Search included electronic databases.

Results: GV in T1D patients was high, mean average daily risk range (ADRR) 34.9. The mean (range) ADRR for medical admissions was 39.5 (1-118) with only $9.5 \%$ being classified as low risk (ADRR <20). For the systematic review, initial search yielded 2,336 studies for screening of which 9 studies were chosen for final data extraction. Since none of them had data specific for type $1 \mathrm{DM}$, authors of included studies were contacted. Data specific for T1D are not available from any study.

Conclusion: Inpatient GV is high in T1D patients who were admitted at our tertiary care center. Review of the literature shows paucity in the data regarding inpatient glycemic variability. A prospective study to address this issue is urgently needed.

\section{6}

\section{THE EFFECT OF GLUCOSE VARIABILITY ON MICROVASCULAR COMPLICATIONS IN TYPE 1 DIABETES}

L. Cardoso ${ }^{1}$, C. Baptista ${ }^{1}$, D. Rodrigues ${ }^{1}$, L. Barros ${ }^{1}$,

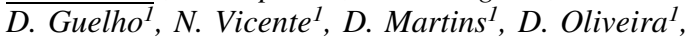

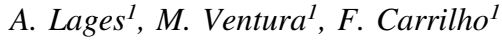

${ }^{1}$ Centro Hospitalar e Universitário de Coimbra, Department of Endocrinology, Diabetes and Metabolism, Coimbra, Portugal

Background and Aims: Evidence implicates oxidative stress as mediator of diabetic complications. Furthermore, glucose variability $(\mathrm{GV})$ is associated with oxidative stress and inflammation; however, association between GV and diabetes complications remains to be established. Our aim was to assess GV in patients with and without microvascular complications (MVC).

Method: We analysed 2,454 and 6,593 hours of continuous glucose monitoring of patients with type 1 diabetes (T1D) and labile glucose control with or without MVC, respectively. Patients were matched for A1C. All data points were collected on iPro $2^{\mathrm{TM}}$. 
Results: Most patients were females $(\mathrm{n}=36,59 \%)$ and A1C was similar on both groups $(7.7 \pm 0.9$ vs $8.0 \pm 1.2 \%, \mathrm{p}=0.372)$. Patients with MVC were older $(36.9 \pm 4.8$ vs $27.1 \pm 9.0$ years, $\mathrm{p}<0.001)$ and had longer duration of T1D $(22.7 \pm 6.1 \mathrm{vs}$ $15.4 \pm 9.8$ years, $\mathrm{p}=0.011$ ) than patients without MVC. GV measured by standard deviation $(3.5 \pm 0.8$ vs $3.5 \pm 1.1$, $\mathrm{p}=0.937)$, mean amplitude of glycemic excursions $(6.5 \pm 1.2 \mathrm{vs}$ $6.8 \pm 2.5, p=0.716)$, mean of daily differences $(3.8 \pm 0.9 v s$ $3.9 \pm 1.3, \mathrm{p}=0.796$ ) was similar between the patients with or without MVC, respectively. Interestingly, patients with neuropathy had higher glucose variability measured by lability index $(15.1 \pm 7.6$ vs $6.1 \pm 3.6, \mathrm{p}=0.02)$ and mean absolute glucose (3.4 $\pm 0.9 v s 2.3 \pm 0.7, \mathrm{p}=0.033$ ) but they were significantly older (39.0 vs 29.3 years), had longer disease duration (27.0 vs 16.9 years) and poorer glycaemic control (A1C, 9.1 vs $7.9 \%$ ) than patients without neuropathy.

Conclusion: Glucose variability was not consistently associated with higher burden of microvascular complications. Though, prospectively designed studies targeting glucose variability are needed to definitively clarify the importance of GV.

097

\section{THE EFFECT OF PROTEIN PLUS CARBOHYDRATE WITH NO FAT ON POSTPRANDIAL GLYCAEMIA IN INDIVIDUALS WITH TYPE 1 DIABETES}

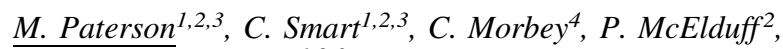
P. Lopez ${ }^{1,2,3}$, B. King ${ }^{1,2,3}$

${ }^{1}$ John Hunter Children's Hospital, Newcastle, Australia

${ }^{2}$ The University of Newcastle, Newcastle, Australia

${ }^{3}$ Mothers and Babies Research Centre, Hunter Medical Research Institute, Newcastle, Australia

${ }^{4}$ John Hunter Hospital, Newcastle, Australia

Background and Aims: Dietary protein increases postprandial glucose and insulin requirements in people with T1DM. Previously we demonstrated that $40 \mathrm{~g}$ protein in a mixed macronutrient meal and $\geq 75 \mathrm{~g}$ pure protein resulted in significant postprandial glycaemic excursions between $3-5$ hours. The aim of this study is to determine the glycaemic impact of protein consumed with carbohydrate and no fat in individuals using intensive insulin therapy (IIT).

Method: Twenty-five subjects (15 male) with T1DM, aged 7-40 years, using IIT, (HbAlc $<8.1 \% / 65 \mathrm{mmol} / \mathrm{mol})$ con-

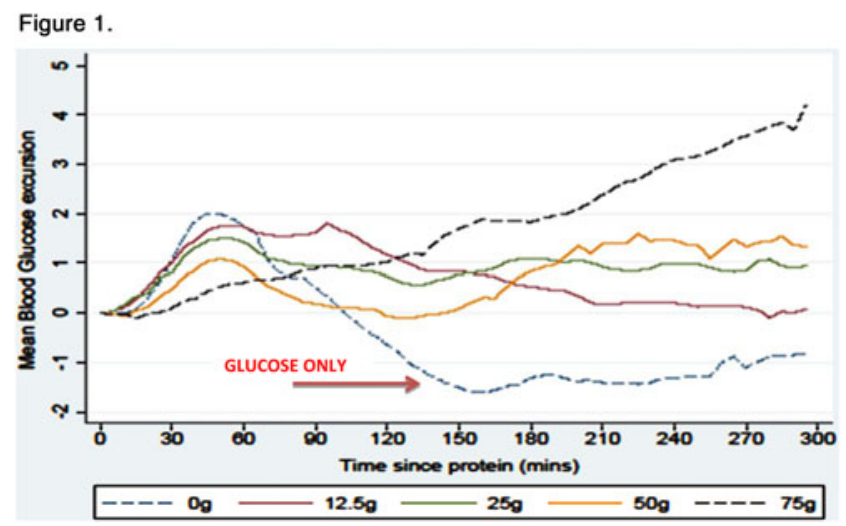

sumed 5 drinks contaning $30 \mathrm{~g}$ of glucose with added protein (whey isolate $0 \mathrm{~g} / \mathrm{kg}$ carbohydrate, $0 \mathrm{~g} / \mathrm{kg}$ lipid) of $0,12.5,25,50$ and $75 \mathrm{~g}$. Insulin was given for the glucose. Postprandial glycaemia was assessed by 5 hours of DEXCOM continuous glucose monitoring.

Results: Figure 1 shows postprandial glycaemic excursions following all test meals. Adding protein to $30 \mathrm{~g}$ glucose initially reduced glycaemic excursions from 30-60min. Compared with glucose-only, mean glycaemic excursions were greater for all protein meals from $120-180 \mathrm{~min}(\mathrm{p}<0.05)$. From $180-300 \mathrm{~min}$ the excursion from $75 \mathrm{~g}$ protein remained significantly greater than glucose-only $(\mathrm{p}=0.001)$.

A significant dose-dependent response was seen with increasing grams of protein from $180-300 \mathrm{~min}(\mathrm{p}<0.001)$

Conclusion: Increasing protein in a $\mathrm{CHO}$ no fat meal increases glucose excursions in a dose-dependent manner. Protein is thought to affect postprandial glucose via conversion of amino acids to glucose and stimulation of glucagon secretion. Consistent with these mechanisms, the blood glucose rise was delayed and slower than the rise seen with glucose alone. This study has significant clinical implications for insulin administration for protein.

098

\section{OCCURRENCE OF HYPOGLYCEMIA IN TYPE 2 DIABETES PATIENTS IN A PERSPECTIVE OF A NEW THERAPEUTIC PARADIGM}

$\underline{\text { S. Levit }}{ }^{1}$, S. Giveon ${ }^{2}$, G. Ginossar ${ }^{3}$, J. Wainstein ${ }^{4}, Y$. Toledano 5

${ }^{1}$ Assuta Medical Center - Maccabi Health Fund, Institute of Endocrinology and Diabetes, Tel-Aviv, Israel

${ }^{2}$ Sharon-Shomron District Clalit Health Services, Department of Family Practice Sackler School of Medicine Tel-Aviv University, Tel-Aviv, Israel

${ }^{3}$ Urgent Care Consultant The Royal London Hospital, A\&E and Urgent Care Centre, London, United Kingdom

${ }^{4}$ Edith Wolfson Medical Center-Holon-Israel-Sackler School of Medicine Tel-Aviv University, Institute of Diabetes, Holon, Israel

${ }^{5}$ Maccabi Health Fund, Head of Diabetes Project, Tel Aviv, Israel

Background and Aims: Hypoglycemia is an iatrogenic complication with potentially detrimental outcomes. Presented here is an innovative therapeutic algorithm, which results from a novel Gravicentric concept, based on several fundamental points: T2DM as a disorder of energy balance; obesity as the main foe; rapid reversibility of the disease; a new vision regarding the roles different pharmacological classes play in energy homeostasis.

Objectives

Primary: To evaluate a Hypoglycemia frequency and severity during a long-term implementation of our new therapeutic paradigm

Secondary: To analyze other (biochemical and anthropometric) parameters

Method: In 54 uncontrolled T2DM patients with mean disease duration of 17.5 years and mean extent of insulin therapy of 4.5 years, hypoglycemic (energy sparing) medications were substituted by GLP-1 agonists according to our Gravicentric algorithm. 
Clinical, biochemical and anthropometrical parameters during intervention were analyzed. Our Compliance and Hypoglycemia assessments were also applied.

Results: BMI dropped down from $35.1 \pm 4.8 \mathrm{~kg} / \mathrm{cm}^{2}$ before to $32.8 \pm 5.0 \mathrm{~kg} / \mathrm{cm}^{2}$ on GLP-1 analogues, $\mathrm{p}<0.01$. HBA1c decreased from $9.28 \pm 1.43$ to $8.54 \pm 1.4 \%$ on GLP-1 analogues, $\mathrm{p}<0.01$. Total Daily Dose of Insulin (TDI) reduced: $80.6 \pm 42.7 \mathrm{U} /$ day before starting GLP-1 vs. $41.0 \pm 30.7 \mathrm{U} /$ day on GLP-1, p $<0.01$. No severe hypoglycemia was observed during 27.8 months of follow-up. The non-severe hypoglycemia occurrence was negligible: approximately one episode per two months.

Conclusion: Our therapeutic scheme appeared to be very effective in terms of metabolic control with virtually no effect on hypoglycemia frequency.

These findings are proof- of- concept of our Gravicentric theory.

099

IMPROVED POSTPRANDIAL GLYCAEMIC CONTROL WITH FASTER-ACTING INSULIN ASPART VS INSULIN ASPART IN SUBJECTS WITH TYPE 1 DIABETES USING CSII IN PUMPS

B.W.Bode ${ }^{1}$, L. Hyveled ${ }^{2}$, S.C. Tamer $^{2}$, M. Demissie ${ }^{2}$

${ }^{1}$ Atlanta Diabetes Associates, Atlanta, USA

${ }^{2}$ Novo Nordisk A/S, Sфborg, Denmark

Background and Aims: Faster-acting insulin aspart (faster aspart) is insulin aspart (IAsp) set in a new formulation. This double-blind, randomised, crossover, active-controlled trial compared 14 days of continuous subcutaneous insulin infusion (CSII) of two formulations of faster aspart with CSII of IAsp in 43 adult subjects with type 1 diabetes. Only data for the faster aspart formulation undergoing further development are presented.

Method: The primary endpoint was the mean change in postprandial glucose response $2 \mathrm{~h}$ after a standardised meal test (individualised insulin dosing by bolus calculator), as evaluated by mean change in plasma glucose $\left(\Delta \mathrm{PG}_{\mathrm{av}, 0-2 \mathrm{~h}}\right)$. Subjects had blinded continuous glucose monitoring (CGM) during the trial.

Results: Faster aspart provided a significantly lower $\Delta \mathrm{PG}_{\mathrm{av}, 0-2 \mathrm{~h}}$, compared with IAsp (3.03 vs $4.02 \mathrm{mmol} / \mathrm{L}$; mean difference [ $95 \%$

Table: Glucose-lowering effect of faster aspart vs IAsp.

\begin{tabular}{|c|c|c|c|}
\hline & $\begin{array}{c}\text { Faster aspart } \\
\text { LSMean } \\
(\mathrm{mmol} / \mathrm{L}) \\
\mathrm{N}=43\end{array}$ & $\begin{array}{c}\text { IAsp } \\
\text { LSMean } \\
\left(\underset{\mathrm{mmol} / \mathrm{L})}{\mathrm{N}=42^{*}}\right.\end{array}$ & $\begin{array}{c}\text { Mean treatment } \\
\text { difference }(\mathrm{mmol} / \mathrm{L}) \\
{[95 \% \mathrm{Cl}]}\end{array}$ \\
\hline $\begin{array}{l}\Delta \mathrm{PG}_{\mathrm{av}} \\
0-2 \mathrm{~h}^{\dagger} \\
0-1 \mathrm{~h}^{2} \\
0-4 \mathrm{~h}^{+}\end{array}$ & $\begin{array}{l}3.03^{\Uparrow} \\
1.89^{\Uparrow} \\
3.59^{\Uparrow}\end{array}$ & $\begin{array}{l}4.02^{9} \\
2.39^{9} \\
4.32^{9}\end{array}$ & $\begin{array}{l}-0.99[-1.95 ;-0.03]^{*} \\
-0.50[-1.07 ; 0.07] \\
-0.73[-2.00 ; 0.54]\end{array}$ \\
\hline $\begin{array}{l}\text { IG increment } \\
\text { All meals, } 60 \mathrm{~min} \text { after } \\
\text { All meals, } 120 \mathrm{~min} \text { after } \\
\text { Breakfast, } 60 \mathrm{~min} \text { after } \\
\text { Breakfast, } 120 \mathrm{~min} \text { after }\end{array}$ & $\begin{array}{l}0.63 \\
0.50 \\
1.12 \\
0.67\end{array}$ & $\begin{array}{l}1.29 \\
1.07 \\
2.04 \\
1.79\end{array}$ & $\begin{array}{l}-0.66[-0.95 ;-0.37]^{* * *} \\
-0.58[-0.97 ;-0.19]^{* *} \\
-0.92[-1.45 ;-0.38]^{* * *} \\
-1.13[-2.03 ;-0.23]^{*}\end{array}$ \\
\hline
\end{tabular}

CI]: $-0.99[-1.95 ;-0.03]$; Table). The greater glucose-lowering effect demonstrated at meals with faster aspart vs IAsp was supported by interstitial glucose (IG) profiles, with the largest differences at breakfast (Table). The duration of low IG $(\leq 3.9 \mathrm{mmol} / \mathrm{L}$ per $24 \mathrm{~h}$ ) was significantly shorter for faster aspart $(2.03 \mathrm{~h})$ than for IAsp $(2.45 \mathrm{~h} ;-0.42[-0.72 ;-0.11]$. No new safety findings were observed with faster aspart, compared with IAsp.

Conclusion: In summary, faster aspart had a significantly greater glucose-lowering effect than the conventional IAsp formulation after a standardised meal, with findings supported by CGM for all meals, and less time spent with low glucose levels, as measured by IG.

\section{0}

\section{BLOOD GLUCOSE SENSING THROUGH SKIN BY NON-INVASIVE FINGER TOUCH METER}

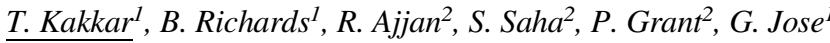 \\ ${ }^{1}$ Institute for Materials Research, School of Chemical \\ and Process Engineering-University of Leeds, Leeds, \\ United Kingdom \\ ${ }^{2}$ Leeds Institute of Cardiovascular and Metabolic Medicine, \\ Faculty of Medicine- University of Leeds, Leeds, \\ United Kingdom
}

Background and Aims: Newest progresses in self-monitoring of blood glucose (SMBG) has resulted in remarkable shift towards personalized care in glucose monitoring for patients with diabetes. Key requirement for SMBG meters is either minimally invasive or non-invasive device. We have developed a state-of-the-art non-invasive finger touch meter (GlucoSense) which senses capillary blood glucose by novel photonic glass sensor. The photonic glass offers high signal to noise ratio thus yielding a better result.

Method: When excited with pulsed laser diode it emits intrinsic fluorescence in the near infrared region (NIR) with higher absorption coefficient for glucose than other molecules present in blood such as lactates, urea. The altering glucose concentration in the blood shifts the fluorescence decay lifetime of photonic glass. Each measurement was taken by placing fingertip on the top of photonic glass sensor(shown in figure 1) and decay lifetime signal was collected with photodetector. A "proof of concept" pilot clinical study was carried out involving 12 candidates with type- 1 diabetes, attending 2 sessions of 8 hours duration.

Results: The glucose value measured from GlucoSense was compared with corresponding blood glucose values obtained from traditional SMBG meter as well as continuous glucose monitor (CGM) using Clarke error grid (CEG) analysis. CEG analysis of GlucoSense with SMBG and CGM shows clinical acceptability of 96.5 and $92.3 \%$ respectively.

Conclusion: The clinical results suggest a promising optical technique for non-invasive glucose measurements. Next stage of development is currently in progress to develop a miniaturized and more stable platform of GlucoSense.
$100 \mathrm{~A}$

(See page A-140 for this postpublication oral presentation.) 


\section{ATTD 2016 E-Poster Presentations}

101

\section{EFFICACY AND SAFETY OF INSULIN PUMP THERAPY IN TYPE 2 DIABETES: OPT2MISE STUDY - FULL STUDY REPORT}

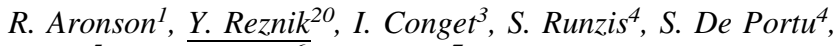
S. Lee ${ }^{5}$, J. Castaneda ${ }^{6}$, O. Cohen ${ }^{7}$

${ }^{1}$ LMC Diabetes and Endocrinology, LMC Diabetes and Endocrinology, Toronto, Canada

${ }^{2}$ University of Caen Cote de Nacre Hospital, Department of Endocrinology, Caen, France

${ }^{3}$ University Hospital Clinic, Diabetes Unit, Endocrinology and Nutrition Department, Barcelona, Spain

${ }^{4}$ Medtronic International Trading Sàrl, Diabetes, Tolochenaz, Switzerland

${ }^{5}$ Medtronic Diabetes, Medtronic Diabetes, Northridge, USA

${ }^{6}$ Medtronic Bakken Reserach Center, Medtronic Bakken

Reserach Center, Maastricht, Netherlands

${ }^{7}$ Chaim Sheba Medical Center, Institute of Endocrinology,

Tel Hashomer, Israel

Background and Aims: The OpT2mise study was a multicenter, randomized, controlled trial comparing efficacy and safety of CSII vs MDI in insulin-using patients with T2D.

Method: Subjects using MDI with persistent hyperglycemia $(\mathrm{A} 1 \mathrm{C}>8 \%$ ) after a 9-week optimization period were randomly assigned to CSII or to continue MDI in a study phase (SP) of 6 Months. In a 6-months continuation phase (CP), CSII patients continued while MDI subjects crossed to CSII.

Results: Of 331 randomized (46\% women, age $56+9.6 \mathrm{yr}$, BMI $33.4+7.3 \mathrm{~kg} / \mathrm{m} 2$, diabetes duration $15.1+8.0 \mathrm{yr}$, A1C $9.0+0.8 \%), 291$ completed the study.

At 6 months, CSII subjects had achieved significantly greater A1C reduction $(-1.1+1.2 \%$ vs $-0.4+1.1 \%, \mathrm{p}<0.001)-$ maintained at 12 months. MDI subjects crossing to CSII showed $-0.8 \%$ $\mathrm{A} 1 \mathrm{C}$ reduction, with final A1C identical in both arms. SP response rate $(\mathrm{A} 1 \mathrm{C}<8.0 \%$ ) was higher in the CSII arm (CSII 55\% vs. MDI $28 \%$ ) but by end of the $\mathrm{CP}$, both groups had achieved similar response rates of $57 \%$.

CSII total daily dose (TDD) was $520.4 \%$ lower in the SP; in the CP, MDI subjects crossing to CSII showed a $19.0 \%$ TDD reduction; final TDD was equivalent in both groups. There was no difference between groups in weight gain nor ketoacidosis. In each group, 1 patient experienced severe hypoglycemia.

Conclusion: The OpT2mise study demonstrates that CSII provides a significant advantage in glycemic control over MDI with a safe and consistent effect in long-term treatment.
102

\section{HEALTH-ECONOMIC EVALUATION OF SENSOR- AUGMENTED PUMP (SAP) VERSUS INSULIN PUMP THERAPY ALONE (CSII), IN TYPE 1 DIABETES PATIENTS, IN ITALY}

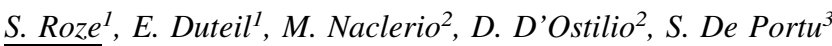 \\ ${ }^{1}$ HEVA HEOR, Health Economics, Lyon, France \\ ${ }^{2}$ Medtronic, Health Economics \& Reimbursement, \\ Milano, Italy \\ ${ }^{3}$ Medtronic, Health Economics, Health Economics, \\ Switzerland
}

Background and Aims: The objective of the study was to estimate the cost-effectiveness and to project the clinical benefits of sensor augmented pump (SAP) versus continuous subcutaneous insulin infusion therapy alone (CSII) in type 1 diabetes patients (T1D) in the Italian setting.

Method: The analysis was conducted on two types of population: a hyper-population based on Pickup's meta-analysis and a hypo-unaware population based on the Ly's study. The Core Diabetes Model was used to project the incidence of diabetes-related complications. Both cohort characteristics are presented in table 1. In Pickup's meta-analysis, $80.5 \%$ sensor usage in a population with baseline $\mathrm{HbA} 1 \mathrm{c}$ of $8.1 \%$ led to a reduction of $-0.56 \%$ versus $-0.13 \% \mathrm{HbA} 1 \mathrm{c}$, for SAP and CSII respectively.

Results: The incremental cost-effectiveness ratio (ICER), based on a societal perspective, was 44,982 € per Quality Adjusted Life Year gained (QALY) and 33,692 €/QALY, for the hyper- and hypo-unaware cohort respectively. In both populations, SAP usage delayed the mean time alive and free from complications. The acceptability curve showed that the likelihood to be cost-effective at a willingness to pay of 60,000 $€ /$ QALY was $93.1 \%$ and $99.9 \%$ for the hyper and the hypounaware population respectively.

Conclusion: These results indicate that according to commonly accepted willingness to pay threshold SAP can be considered a cost-effective therapeutic option compared to CSII alone in the Italian setting. Extensive sensitivity analysis on key drivers confirmed the robustness of results.

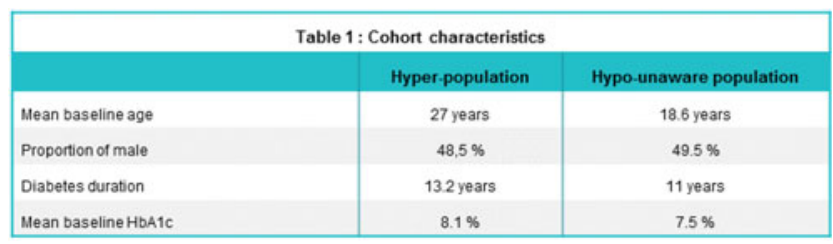


103

CONSENSUS TO DEFINE ALERT VALUES OF A PATTERN DETECTION WEB BASED MODULE

M.J.Comellas ${ }^{1}$, E. Albiñana ${ }^{2}$, R. Corcoy ${ }^{3}$, D. Fernández-García ${ }^{4}$, J. García-Alemán ${ }^{4}$, B. García-Cuartero ${ }^{5}$, C. González ${ }^{3}$, M.T. Rivero ${ }^{6}, J$. Weissmann ${ }^{7}$

${ }^{1}$ Roche Diagnostics S.L., Medical Affairs, Sant Cugat del Vallès, Spain

${ }^{2}$ Vithas Hospital Internacional Medimar, Children's Endocrinology Unit, Alicante, Spain

${ }^{3}$ Hospital Santa Creu i Sant Pau, Endocrinology, Barcelona, Spain

${ }^{4}$ Hospital Universitario Virgen de la Victoria, Endocrinology, Málaga, Spain

${ }^{5}$ Hospital Universitario Ramón y Cajal, Pediatrics, Madrid, Spain

${ }^{6}$ Complejo Hospitalario de Orense, Endocrinology, Orense, Spain

${ }^{7}$ Roche Diagnostics Deutschland GmbH, Medical Affairs, Mannheim, Germany

Background and Aims: Emminens eConecta ${ }^{\circledR}$ is a modular and customizable web-based platform that enables personalized management of Diabetes Mellitus (DM). The new eDetecta module including algorithms and alerts has been developed to facilitate pattern detection and analysis of glycemic, insulin and carbohydrate data.

To reach consensus of the default values and ranges of the eDetecta alerts and define specific profiles of alerts depending on patient age, treatment and situation.

Method: An advisory board of 7 Spanish experienced diabetologists and pediatricians worked in groups of 2-3 to define the default values according to different patient profiles (adults in MDI and CSII, hypoglycemia unawareness, pregnant women and children). Every expert filled in a template indicating agreement/ disagreement with a set of proposed default values and ranges for each alert parameter. Responses were compiled and a consensus meeting was held to discuss discrepancies and arguments behind alternative values.

Results: General settings and the 22 alerts parameters available in eDetecta have been agreed upon. $50 \%$ of these alerts have been adjusted, being the main criterion that they should apply to most of the patients and should signal situations that need attention. Following the consensus, recommended general and alerts settings were defined for different patient profiles (see table).

Conclusion: Default values and ranges currently included in the emminens eDetecta module have been agreed by a group of experts with broad clinical experience in DM. They have also defined recommendations on alert configuration to allow a personalized pattern analysis depending on different patient profiles.

\begin{tabular}{|r|c|c|c|c|c|c|}
\hline GENERAL SETाINGS & $\begin{array}{c}\text { MOI/CSII } \\
\text { adults }\end{array}$ & $\begin{array}{c}\text { Hypoglycemia } \\
\text { unawareness }\end{array}$ & $\begin{array}{c}\text { MDI/CSII } \\
\text { pregnancy }\end{array}$ & $\begin{array}{c}\text { MDI/CSII } \\
\text { pediatrics < } \\
\text { 6years }\end{array}$ & $\begin{array}{c}\text { MDI/CSII } \\
\text { pediatrics 6- } \\
18 \text { years }\end{array}$ & Range \\
\hline Calculation period (weeks) & 4 & 4 & 2 & 4 & 4 & $2-12$ \\
\hline Mininum number of tests to perform analysis of: & \multicolumn{5}{|l|}{} \\
\hline Glycemic Variability & 84 & 84 & 84 & 112 & 112 & $60-270$ \\
\hline In General & 84 & 84 & 84 & 72 & 72 & $30-120$ \\
\hline Preprandial block & 24 & 24 & 24 & 24 & 24 & $10-40$ \\
\hline Postprandial block & 10 & 10 & 10 & 12 & 12 & $5-40$ \\
\hline Bedtime block & 5 & 5 & 5 & 10 & 10 & $5-30$ \\
\hline Glycemic Limits (mg/dl) & & & & & & \\
\hline Hypo limit & 70 & 80 & 60 & 70 & 70 & $50-90$ \\
\hline Hyper limit & 160 & 160 & 140 & 180 & 160 & $100-250$ \\
\hline Hyper limit Preprandial & 150 & 150 & 110 & 160 & 160 & $100-250$ \\
\hline Hyper limit Postprandial & 180 & 180 & 140 & 180 & 180 & $120-250$ \\
\hline Overcorrection Hyper limit & 200 & 200 & 140 & 200 & 200 & $120-250$ \\
\hline
\end{tabular}

104

\section{REDUCTION IN GLYCEMIC VARIABILITY \\ IS CORRELATED WITH REDUCTIONS \\ IN BOTH HYPOGLYCEMIA AND HYPERGLYCEMIA RISK IN TYPE 1 AND TYPE 2 SUBJECTS}

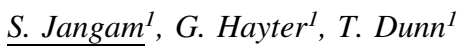

${ }^{1}$ Abbott Diabetes Care, Research and Development, Alameda, USA

Background and Aims: Therapy changes intended to minimize a patient's hypoglycemia or hyperglycemia risk often result in the reduction of one risk at the expense of another.

Method: To understand the importance of glucose variability (median $-10^{\text {th }}$ percentile) in the simultaneous reduction of hypoglycemia and hyperglycemia risk, we introduce a Glycemic Risk plot: Estimated A1c \% (eA1c) vs Minutes Below $70 \mathrm{mg} / \mathrm{dL}$ (MB70) with constant variability contours for comparing preand post-intervention risks.

Results: The plot illustrates that subjects that do not reduce variability can only decrease one of the two risks (hypoglycemia or hyperglycemia), but not both. The only way to reduce both risks is to address variability. These results were confirmed by data collected in randomized controlled trial consisting of Type 1 and Type 2 diabetes subjects on multiple daily insulin injections. For Type 1 subjects, a total of 28 subjects out of 35 (80\%) showed reduction in at least one of the risks (hypo and/or hyper) during the 100-day course of the study. Eight subjects (23\%) showed reduction in both. Similar data was observed for Type 2 subjects, where a total of 36 subjects out of $43(84 \%)$ showed reduction in at least one risk and 8 subjects $(19 \%)$ showed reduction in both.

Conclusion: All of the subjects in the Type 2 group and seven out of eight in the Type 1 group, who showed reduction in both hypoglycemia and hyperglycemia risk, showed a reduction in variability demonstrating a strong correlation between decreasing both hypo- and hyper-risk and reducing variability.

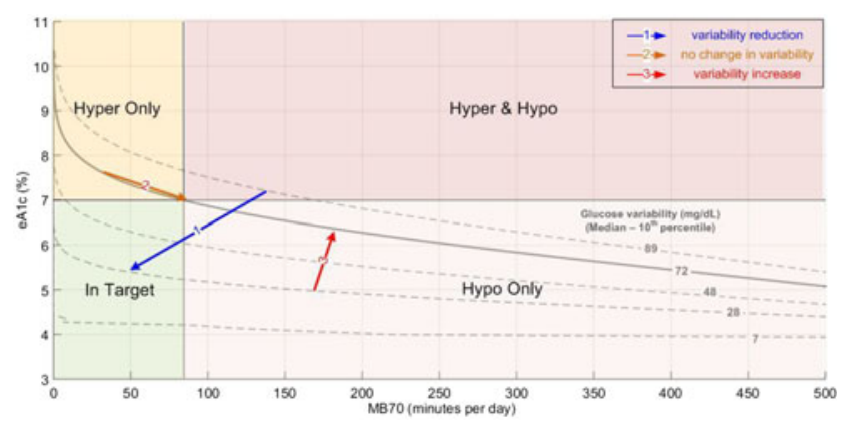

105

\section{DECREASED EFFICIENCY OF DEEP SLEEP IN CHILDREN AND ADOLESCENTS WITH NON-OPTIMALLY CONTROLLED TYPE 1 DIABETES}

\author{
M. Čiljaková ${ }^{l}$, J. Vojtkovál, P. Ďurdík ${ }^{1}$, A. Šjujanskál, \\ M. Michalovičovál, K. Pozorčiaková ${ }^{1}$, J. Lettrichovál, \\ P. Bánovčin ${ }^{1}$ \\ ${ }^{1}$ Comenius University in Bratislava- Jessenius Faculty \\ of Medicine and University Hospital Martin, \\ Department of Pediatrics, Martin, Slovakia
}


Background and Aims: Sleep restriction and changes in sleep architecture can be associated with impaired control of diabetes mellitus, predominantly in adults. Limited data are in pediatric population. The aim of the study was to determine sleep architecture and its relation to short-term or long-term metabolic compensation in children and adolescents with type 1 diabetes (T1D).

Method: 30 children with T1D (15 boys, average age 15.22 years, mean diabetes duration 9.25 years) and 10 healthy controls ( 5 boys, average age 14.40 years) were enrolled. Each subject underwent standard night polysomnographic examination and children with T1D also continuous glucose monitoring (during night polysomnography, day before and day after). Sensor glucose $<3.9 \mathrm{mmol} / \mathrm{L}$ was always compared with blood glucose, children with confirmed hypoglycemia were excluded. Parameters of short-term (average sensor glucose, glucose variability, bedtime glucose) and long-term metabolic compensation (HbA1c) of T1D were measured.

Results: Children with T1D had significantly reduced percentage of time in deep sleep (non-REM3 stage, $25.71 \%$ vs. $36.02 \%, \mathrm{p}<0.05)$ and decreased efficiency of deep sleep $(41.42 \%$ vs. $54.46 \%, \mathrm{p}<0.001)$ compared to healthy controls. Efficiency of deep sleep negatively correlated with parameters of metabolic control of T1D - with HbA1c $(r=-0.71)$, average sensor glucose $(r=-0.51)$, glucose variability $(r=-0.46)$ and bedtime glucose $(r=-0.41)$.

Conclusion: Subjects with T1D showed significantly reduced percentage of deep sleep time and decreased efficiency of deep sleep that strongly correlated with non-optimal long-term metabolic control. Further studies are needed to determine, whether changes of sleep architecture can play a role in metabolic control in children with T1D.

Supported by grant VEGA1/0262/14

\section{6}

GRADUATED TUNING FORKS PROVIDE MORE ACCURATE INDICATION THAN MONOFILAMENTS OF THE ONSET AT TWO YEARS OF FOOT ULCERS IN DIABETIC PATIENTS

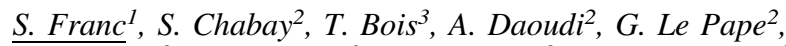

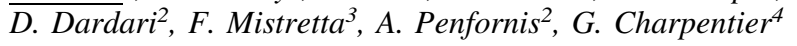

${ }^{1}$ Paris, France

${ }^{2}$ CHSF, Essonnes, Corbeil-Essonnes, France

${ }^{3}$ RCTS, Rhône, Lyon, France

${ }^{4}$ CERITD, Essonnes, Evry, France

Background and Aims: To compare graduated tuning forks vs. monofilaments in the prediction of foot ulcers in T1D patients at two years

Method: From our database of patients monitored between 1987 and 2014, an analytical population was selected of 2,485 patients as follows:

- ulcers: follow-up visit in the two years preceding onset of first ulcer,

- no ulcers: less than two years between last two follow-up visits.

Ulcer onset was predicted via monofilament and GTF (0-8) tests, with predictive accuracy being analysed using the AUC of ROC curves. Screening of predictive factors for ulcer onset was then performed using multivariate logistic regression.
Results: Patients were aged $50 \pm 15$ years, with $57.2 \%$ male and $26 \%$ T1D. $119(5 \%)$ developed ulcers after 2 years. GTF was $3.1 \pm 2.5$ in patients subsequently developing ulcers $v s$. $5.1 \pm 2.2$ in patients not developing ulcers $(p<0.0001)$. There were $40.2 \%$ negative monofilament tests in patients developing ulcers vs. $13.7 \%$ in patients without ulcers $(\mathrm{p}<0.0001)$. The AUC for the ROC curve was 0.63 for monofilament vs. 0.72 for GTF, irrespective of vibratory sensitivity $(\mathrm{p}=0.009)$. Multivariate analysis also showed $\mathrm{HbA1c}$, microalbuminuria, presence of 4 pulses and quantitative GTF values to be associated with ulcer onset. Use of a polynomial equation incorporating these parameters together with sex and diabetes duration allowed calculation of the probability of ulcer onset at two years.

Conclusion: GTF is thus more reliable than monofilament in predicting onset of foot ulcer within 2 years; used in association with other clinical and laboratory parameters, it enables the probability of onset to be calculated.

107

\section{PROPORTION OF CAPILLARY BLOOD GLUCOSE READINGS REQUIRED IN THE TARGET RANGE TO ACHIEVE TARGET HBA1C: SHIFT OF FOCUS FROM TARGET RANGE TO PROPORTION IN RANGE}

\author{
S. Sivasubramaniyam $^{1,2}$, P. Choudhary ${ }^{1}$ \\ ${ }^{1}$ King's College London, Diabete Research Group, \\ London, United Kingdom \\ ${ }^{2}$ London Northwest Healthcare Trust, Endocrinology, \\ London, United Kingdom
}

Background and Aims: Patients with type 1 diabetes are given pre- and post-meal capillary blood glucose (CBG) targets to achieve optimal glycaemic control. We evaluated the proportion of daily CBG readings between $4-10 \mathrm{mmol} / 1$ in patients achieving an acceptable HbA1c of $<7.5 \%$.

Method: CBG data were obtained for 201 adult patients treated with continuous subcutaneous insulin infusion (CSII) at a single hospital clinic using routine CSII/meter downloads for which, a concurrent HbA1c was available. Exclusion criteria were CSII $<6$ months, $<3$ CBG/day, pregnancy, haemoglobinopathy and continuous sensor use. Patients were categorised into 3 groups based on HbAlc, $[<7.5 \% / 58 \mathrm{mmol} / \mathrm{mol} \quad(\mathrm{n}=58)$; 7.5 $8.9 \% / 58-74 \mathrm{mmol} / \mathrm{mol} \quad(\mathrm{n}=107)$ and $\geq 9.0 \% / 75 \mathrm{mmol} / \mathrm{mol}$ $(\mathrm{n}=36)]$.

Results: Patients had a mean age of $43 \pm 13$ years and mean HBA1c of $8.0 \pm 1.1 \%$. $47 \%$ started CSII for poor control, $25 \%$ due to hypoglycemia and the rest during pregnancy. Downloads contained a mean of $22 \pm 6.8$ days of data per patient. Daily CBG frequency was similar between the three groups [5.6 \pm 2.0 vs. $5.6 \pm 1.9$ vs. $5.4 \pm 1.2 \mathrm{CBG} /$ day; $\mathrm{p}=0.877]$. Proportion of CBG readings between $4-10 \mathrm{mmol} / \mathrm{l}$ was $57.3 \pm 25.4$ vs. $50.6 \pm 11.1$ vs. $39.9 \pm 16.5 \% ; \mathrm{p}<0.0001 ;<4 \mathrm{mmol}$ was 13.8 vs 8.8 vs $4.4 \%$; $<<0.0001$ and $>10 \mathrm{mmol} / 1$ was $28.9 \pm 16.5$ vs. $40.6 \pm 12.1$ vs. $55.6 \pm 17.9 \% ; \mathrm{p}<0.0001$ in the three groups respectively.

Conclusion: The observation that up to $30 \%$ of CBG readings being out of target range remains compatible with achieving target $\mathrm{HbA1c}$ may be useful in diminishing anxiety around home 
CBG testing and allows a novel target for people with diabetes, in which only 3 of 5 daily tests must be in target.

108

\section{CHANGING BASAL INSULIN TO DEGLUDEC IN PATIENTS WITH INSULIN-TREATED DIABETES: THE EXPERIENCE OF AN ADULT DIABETES CLINIC IN AN URBAN HOSPITAL}

A. Caretto $^{1}$, C. Molinari ${ }^{1}$, A.M. Bolla ${ }^{2}$, A. Laurenzi ${ }^{2}$,

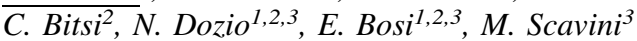

${ }^{1}$ Università Vita-Salute San Raffaele, Department of Internal Medicine, Milan, Italy

${ }^{2}$ Scientific Institute San Raffaele, Department of Internal Medicine, Milan, Italy

${ }^{3}$ Scientific Institute San Raffaele, Diabetes Research Institute, Milan, Italy

Background and Aims: Insulin preparations with pharmacokinetics matching physiological insulin profiles are developed to improve glycaemic control and rates of hypoglycaemia. Aim of this work was to describe the effect of changing basal insulin to degludec in patients with insulintreated diabetes in the real-life setting of a large urban hospital.

Method: We identified patients with insulin-treated diabetes prescribed degludec querying the MyStar Connect software of our diabetes clinic and collected demographics and general characteristics of patients and indexes of glycaemic control before and after changing to degludec.

Results: From 21/01/2015 to 21/09/2015 $\mathrm{n}=133$ patients were changed to degludec; $n=57(43 \%)$ had at least one follow-up HbA1c measurement and were included in our analysis. Median age was 40 years (IQR 21-48.5), 58\% were females, $84 \%$ had type 1 diabetes, diabetes duration was 16 years (IQR 11-24), $89 \%$ were on glargine and $11 \%$ on detemir. Reasons for changing were frequent hypoglycaemia, predinner hyperglycaemia and poor glucose control. The first follow-up visit after changing to degludec occurred after 89 days (IQR 63-112). Median HbA1c change was $-5 \mathrm{mmol} / \mathrm{mol}$ (IQR -9-1).

Data are median and interquartile range (IQR). *In a subset of $n=15$ patients, glucometers were downloaded during outpatient visits and SMBG values in the prior 3 months were analyzed.

Conclusion: A modest although significant improvement in HbA1c without any increase in hypoglycaemia was observed within 4 months in patients prescribed degludec as basal insulin. The sustainability of this improvement will need to be documented over time.

\begin{tabular}{|c|c|c|c|}
\hline & $\begin{array}{l}\text { Prior to changing to } \\
\text { degludec }\end{array}$ & $\begin{array}{l}\text { After changing to } \\
\text { degludec }\end{array}$ & $p$ \\
\hline $\mathrm{HbA1c}(\mathrm{mmol} / \mathrm{mol})$ & $64(57-77)$ & $61(54.68)$ & 0.0001 \\
\hline Basal insulin dose (U/day) & $22(14 \cdot 28)$ & $20(14-26)$ & 0.0239 \\
\hline$S M B G<70 \mathrm{mg} / \mathrm{d}^{*}$ & $19(10-27)$ & $18(7.35)$ & 0.589 \\
\hline $\begin{array}{l}\text { SMBG }<70 \mathrm{mg} / \mathrm{d} \text { l during } \\
\text { night }^{+}\end{array}$ & $1(1.5)$ & $1(0.6)$ & 0.424 \\
\hline SMBG $<50 \mathrm{mg} / \mathrm{d}^{*}$ & $1(0.5)$ & $1(0-4)$ & 0.386 \\
\hline
\end{tabular}

109

\section{HIGHER EARLY EXPOSURE AND GREATER \\ EARLY GLUCOSE-LOWERING EFFECT WITH FASTER-ACTING INSULIN ASPART VS INSULIN ASPART IN ELDERLY AND YOUNGER ADULTS WITH TYPE 1 DIABETES (T1D)}

U. Hövelmann ${ }^{1}$, E. Zijlstra ${ }^{1}$, K. Stender-Petersen ${ }^{2}$, J.B. Jacobsen ${ }^{2}$, T. Heise ${ }^{1}$, H. Haahr

${ }^{1}$ Profil, Neuss, Germany

${ }^{2}$ Novo Nordisk A/S, Sфborg, Denmark

Background and Aims: Faster-acting insulin aspart (faster aspart) is a new formulation of insulin aspart (IAsp), with faster initial absorption following subcutaneous injection. This trial compared pharmacokinetic (PK) and pharmacodynamic (PD) properties of faster aspart vs IAsp in elderly and younger adults with T1D.

Method: Subjects $(n=37,18-35 y ; n=30, \geq 65$ y) received a single dose $(0.2 \mathrm{U} / \mathrm{kg})$ of faster aspart or IAsp in an automated euglycaemic glucose clamp setting (blood glucose target 5.5 $\mathrm{mmol} / \mathrm{L}$ ), in a double-blind, two-period, cross-over trial.

Results: Onset of appearance with faster aspart vs IAsp occurred 55\% (-2.9 $\mathrm{min}$ [95\% CI: $-3.8,-1.9])$ and $47 \%(-2.8 \mathrm{~min}$ $[-4.2,-1.4])$ earlier in elderly and younger adults, respectively, with a greater early insulin exposure during the first $1 \mathrm{~h}$; total exposure was similar (Table). Earlier onset of action with faster aspart occurred in both groups (elderly, $-10.2 \min [-15.3$, $-5.1]$; younger adults, $-8.7 \mathrm{~min}[-15.1,-2.3])$ and was supported by a shorter $\mathrm{t} 50 \% \mathrm{GIR}_{\max }$ (elderly, $-5.6 \min [-9.0,-2.2]$; younger adults, $-10.3 \min [-15.4,-5.1])$.

Faster aspart demonstrated a greater early glucose-lowering effect within $2 \mathrm{~h}$ post-dose than IAsp (Table); the greatest (2-fold) difference was within the first $30 \mathrm{~min}$ (LSmean, $\mathrm{mg} / \mathrm{kg}$ : elderly

Table: PK and PD results for faster aspart vs IAsp

\begin{tabular}{|c|c|c|}
\hline & \multicolumn{2}{|c|}{ Treatment ratio faster aspart/Asp $[95 \% \mathrm{Cl}]$} \\
\hline & Elderly & Younger adults \\
\hline \multicolumn{3}{|l|}{ PK (insulin exposure) ${ }^{*}$} \\
\hline $\begin{array}{l}\text { Onset } \\
\text { Onset of appearance } \\
\text { t50\% } \mathrm{C}_{\max }\end{array}$ & $\begin{array}{l}0.45[0.30 ; 0.60] \\
0.65[0.56 ; 0.74]\end{array}$ & $\begin{array}{l}0.53[0.34 ; 0.74] \\
0.71[0.62 ; 0.81]\end{array}$ \\
\hline $\begin{array}{l}\text { Early } \\
\text { AUC } C_{0-15 \min } \\
\text { AUC }_{0-30 \mathrm{~min}} \\
\text { AUC }_{0-1 \mathrm{~h}} \\
\text { AUC }_{0-2 \mathrm{~h}}\end{array}$ & $\begin{array}{l}3.17[2.43 ; 4.13] \\
1.86[1.53 ; 2.27] \\
1.33[1.17 ; 1.52] \\
1.15[1.04 ; 1.27]\end{array}$ & $\begin{array}{l}2.51[1.78 ; 3.54] \\
1.67[1.35 ; 2.07] \\
1.26[1.07 ; 1.48] \\
1.11[0.95 ; 1.29]\end{array}$ \\
\hline $\begin{array}{l}\text { Total } \\
\text { AUC }_{0-12 \mathrm{~h}} \\
\mathrm{C}_{\max }\end{array}$ & $\begin{array}{l}1.05[0.98 ; 1.14] \\
1.11[1.00 ; 1.23]\end{array}$ & $\begin{array}{l}1.07[0.92 ; 1.25] \\
1.04[0.91 ; 1.18]\end{array}$ \\
\hline \multicolumn{3}{|c|}{ PD (glucose-lowering effect) ${ }^{\dagger}$} \\
\hline $\begin{array}{l}\text { Onset } \\
\text { Onset of action } \\
\text { t50\%GIR }\end{array}$ & $\begin{array}{l}0.65[0.51 ; 0.81] \\
0.85[0.77 ; 0.94]\end{array}$ & $\begin{array}{l}0.67[0.49 ; 0.89] \\
0.75[0.65 ; 0.86]\end{array}$ \\
\hline $\begin{array}{l}\text { Early } \\
\text { AUC }_{\text {GIR.0-30min }} \\
\text { AUC }_{\text {GIR.0-1h }} \\
\text { AUC }_{\text {GIR.0-2h }}\end{array}$ & $\begin{array}{l}2.09[1.37 ; 4.66] \\
1.42[1.14 ; 1.75] \\
1.20[1.00 ; 1.43]\end{array}$ & $\begin{array}{l}2.09[1.31 ; 4.30] \\
1.55[1.16 ; 2.07] \\
1.19[0.97 ; 1.46]\end{array}$ \\
\hline $\begin{array}{l}\text { Total } \\
\text { AUC }_{\text {GIR.0-12h }} \\
\text { GIR }_{\max }\end{array}$ & $\begin{array}{l}1.12[0.94 ; 1.35] \\
1.13[0.96 ; 1.33]\end{array}$ & $\begin{array}{l}1.03[0.90 ; 1.17] \\
1.04[0.88 ; 1.22]\end{array}$ \\
\hline
\end{tabular}

Based on free serum insulin aspart; ${ }^{\dagger} A$ total of 44 subjects ( 22 elderly, 22 younger adults) contributed to the analysis of PD parameters; ;Primary endpoint. AUC=area under the curve; $\mathrm{C}_{\max }=$ maximum observed concentration; GIR $\max =$ maximum glucose infusion rate; onset of appearance=time from dosing until the first time serum IAsp concentration $\geq l o w e r$ limit of t50\%GIR max =time to $50 \%$ of maximum glucose infusion rate. 
44.7 vs 21.4; younger adults 42.8 vs 20.5). PK/PD effects did not differ significantly between groups. Treatments were well tolerated; no safety issues were identified.

Conclusion: Earlier onset and greater early insulin exposure with faster aspart led to a greater early glucose-lowering effect vs IAsp, in both elderly and younger adults with T1D.

\section{0}

\section{AGE OF DIABETES ONSET: GLYCEMIC CONTROL AND HYPOGLYCEMIA BENEFIT WITH NEW INSULIN GLARGINE $300 \mathrm{U} / \mathrm{ML}$ IN T2DM}

S. Twigg ${ }^{1}$, K. Khunti ${ }^{2}$, T. Forst ${ }^{3}$, A. Jane $^{4}$, A. Merino-Trigo ${ }^{5}$, P. Stella ${ }^{5}$, M.L. Grisoni ${ }^{6}$, G. Galstyan ${ }^{7}$

${ }^{1}$ University of Sydney, Sydney Medical School, Sydney, Australia

${ }^{2}$ University of Leicester, Diabetes Research Centre, Leicester, United Kingdom

${ }^{3}$ Profil, Institute for Metabolic Research, Neuss, Germany

${ }^{4}$ University Medical Center Ljubljana, Department of Endocrinology Diabetes and Metabolic Diseases, Ljubljana, Slovenia

${ }^{5}$ Sanofi, Diabetes Division, Paris, France

${ }^{6}$ AIXIAL, Biostatistics, Levallois-Perret, France

${ }^{7}$ Health Care Ministry of Russian Federation, Endocrinology Research Centre, Moscow, Russia

Background and Aims: In people with T2DM treated with basal insulin + mealtime insulin (EDITION 1), basal insulin + oral antihyperglycemic drugs (OADs, EDITION 2), or OADs and insulin-naïve (EDITION 3), switching to or starting with insulin glargine $300 \mathrm{U} / \mathrm{mL}$ (Gla-300) provided comparable glycemic control to glargine $100 \mathrm{U} / \mathrm{mL}$ (Gla-100) and less hypoglycemia, over 6 months of treatment. This post-hoc analysis investigated the association of age of diabetes onset with these outcomes.

Method: Patient-level meta-analysis of efficacy and safety outcomes by age of diabetes onset subgroup $(<40 \mathrm{y}, 40-50 \mathrm{y}$, $>50 \mathrm{y}$ ) for participants in EDITION 1, 2 and 3 over 6 months of treatment.

Results: Mean duration of diabetes was lower with later diabetes onset (onset age <40 y: $16.8 \mathrm{y}$ and $16.7 \mathrm{y} ; 40-50 \mathrm{y}: 13.2 \mathrm{y}$ and 12.9 y; $>50$ y: 9.5 y and 9.5 y for Gla-300 and Gla-100). $\mathrm{HbA}_{1 \mathrm{c}}$ reduction was comparable between treatment groups regardless of age of diabetes onset (no evidence of heterogeneity of treatment effect across subgroups, $p=0.56$ ) (Table). The benefit of Gla-300 in terms of lower risk of confirmed $(\leq 70 \mathrm{mg} / \mathrm{dL})$ or severe hypoglycemia at any time of day $(24 \mathrm{~h})$ and during the night (00:00-05:59h) vs Gla-100 was consistently seen regard-

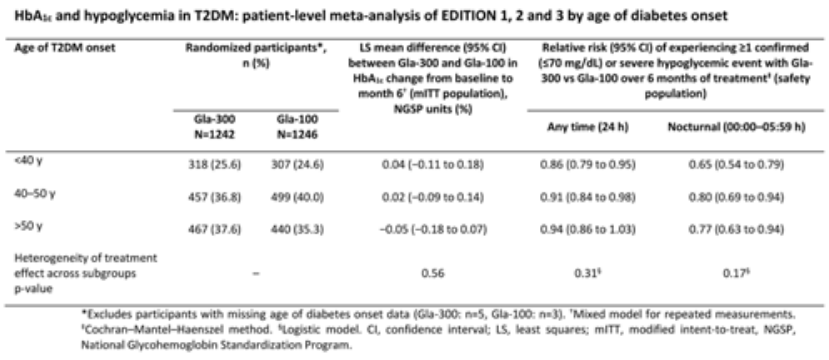

less of age of diabetes onset (no evidence of heterogeneity of treatment effect across subgroups, $\mathrm{p}>0.05$ ) (Table).

Conclusion: Starting with or switching to Gla-300 provided comparable glycemic control with less hypoglycemia vs Gla100, regardless of age of diabetes onset.

Studies sponsored by Sanofi (NCT01499082, NCT01499095, NCT01676220)

\section{1}

\section{A CLINICALLY DEFINED NOCTURNAL WINDOW FOR ANALYSIS OF HYPOGLYCEMIA: NEW INSULIN GLARGINE 300 U/ML (GLA-300) IN TYPE 2 DIABETES (T2DM)}

M.C. Riddle ${ }^{1}$, P.D. Home ${ }^{2}$, A. Avogaro ${ }^{3}$, M. Giménez Alvarez ${ }^{4}$, A. Merino-Trigo ${ }^{5}$, M.L. Grisoni ${ }^{6}$, S. Glezer ${ }^{5}$, G.B. Bolli $^{7}$

${ }^{1}$ Oregon Health \& Science University, Department of Medicine, Portland, USA

${ }^{2}$ Newcastle University, Institute of Cellular Medicine -

Diabetes, Newcastle upon Tyne, United Kingdom

${ }^{3}$ University of Padova, Department of Medicine, Padova, Italy

${ }^{4}$ Hospital Clinic of Barcelona, Diabetes Unit - Endocrinology

Department, Barcelona, Spain

${ }^{5}$ Sanofi, Diabetes Division, Paris, France

${ }^{6}$ AIXIAL, Biostatistics, Levallois-Perret, France

${ }^{7}$ University of Perugia, Medicine, Perugia, Italy

Background and Aims: Analysis of 6-month data from EDITION 1, 2 and 3 shows that in T2DM, Gla-300 causes less nocturnal (00:00-05:59h) and anytime (24h) confirmed or severe hypoglycemia than glargine $100 \mathrm{U} / \mathrm{mL}$ (Gla-100). However, this nocturnal window may not accurately reflect a real-life overnight fasting period; a post-hoc analysis evaluated a broader, overnight fasting, period.

Method: A sensitivity analysis of these data defined nocturnal exposure as 22:00h to pre-breakfast SMPG. Confirmed $(\leq 70 \mathrm{mg} / \mathrm{dL})$ or severe hypoglycemia was analyzed using both nocturnal windows in a patient-level meta-analysis of EDITION 1,2 and 3.

Results: Data were available for 2488 participants (randomized population [ $\mathrm{N}=2496]$, mean age $59 \mathrm{y}$, diabetes duration $13 \mathrm{y}$, BMI $35 \mathrm{~kg} / \mathrm{m}^{2}$ ). Median pre-breakfast SMPG time was $07: 30 \mathrm{~h}$ (IQR 07:00-08:19). More events occurred in the clinically defined window (Gla-300 3585 vs 1235; Gla-100 4730 vs 1791). The relative risk for participants with $\geq 1$ event was lower with Gla-300 vs Gla-100 with both windows (Table). Annualized event rates were also lower with Gla-300 with both windows

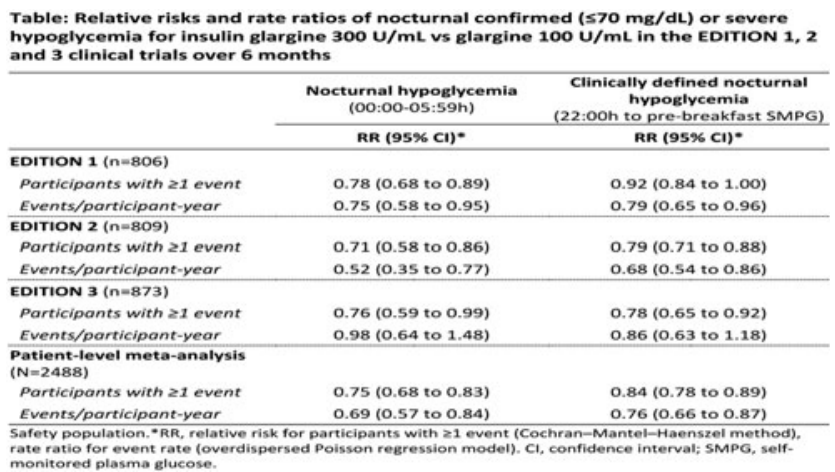


(rate ratio $31 \%$ lower 00:00-05:59h; 24\% lower in the clinically defined window). Absolute difference in numbers of events (favoring Gla-300) was greater in the clinically defined window (1145 vs 556).

Conclusion: The nocturnal hypoglycemia benefit of Gla-300 vs Gla-100 is confirmed with both windows. Greater relative reduction of percent people affected and event rates is suggested for 00:00-05:59h, but lower absolute numbers of events with Gla-300 during a clinically defined fasting period may be more clinically relevant.

Studies sponsored by Sanofi (NCT01499082, NCT01499095, NCT01676220)

112

\section{A SINGLE SUBCUTANEOUS SOMATOSTATIN RECEPTOR 2 ANTAGONIST INJECTION TO PREVENT HYPOGLYCEMIA AFTER RECURRENT HYPOGLYCEMIC EVENTS}

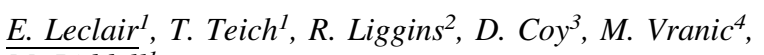
M. Riddell ${ }^{1}$

${ }^{1}$ York University, School of Kinesiology and Health Science Faculty of Health, Toronto, Canada

${ }^{2}$ The Center for Drug Research and Development, Division of Drug Delivery, Vancouver, Canada

${ }^{3}$ Tulane University Medical Center, Department of Medicine Peptide Research Labs, New Orleans, USA

${ }^{4}$ University of Toronto, Departments of Physiology and Medicine, Toronto, Canada

Background and Aims: Recurrent hypoglycemia (hypo) causes defective hormone counterregulation and increases the susceptibility to subsequent events. Pancreatic and/or circulating somatostatin levels are elevated in diabetes which inhibits counterregulation. A selective somatostatin receptor 2 antagonist (PRL-2903) improves counterregulation after four hypo events over a two day period. Here we examined the efficacy of a single subcutaneous administration of PRL-2903 in ameliorating insulin induced hypo after a four week exposure to recurrent hypo.

Method: Eight STZ-diabetic rats underwent $4 \mathrm{~h}$ of hypoglycemic challenges twice a week (48 hours apart) for 4 weeks with individualized doses of s.c. insulin based on initial blood glucose concentrations (ranging from 11-33 mM). PRL-2903 was administered $(10 \mathrm{mg} / \mathrm{kg}$ s.c.) one hour before hypo challenge on week 5.

Results: During the four weeks, hypo depth and duration became more severe and was always more severe on second challenge (BG nadir: $2.0 \pm 0.5)$ than on the first $(2.5 \pm 1.1)$

\begin{tabular}{|c|c|c|c|c|c|c|c|c|}
\hline $\begin{array}{l}\text { Time } \\
\text { Point }\end{array}$ & N & NExcluded ${ }^{t}$ & $\begin{array}{c}\mathrm{N} \\
\text { Adjusted }\end{array}$ & Nadir $<1.9 \mathrm{mM}$ & $\begin{array}{l}\text { Rescued } \\
\text { from Hypo }\end{array}$ & $\begin{array}{l}\text { Sustained } \\
\text { Hypo" }\end{array}$ & $\begin{array}{l}\text { Recovered } \\
\text { from Hypo }\end{array}$ & No Hypo \\
\hline W:4-1 & 8 & 0 & 8 & $7(88 \%)$ & $1(12 \%)$ & $7(888)$ & 0 & 0 \\
\hline Wk42 & 4 & 0 & 4 & $4(100 \%)$ & $2(50 \%)$ & $2(50 \%)$ & 0 & 0 \\
\hline Wh5-1 & 8 & 1 & 7 & $1(14 \%)$ & $1(14 \%)$ & $1(14 \%)$ & $4(57 \%)$ & $2(29 \%)$ \\
\hline Wh5.2 & 8 & 2 & 6 & $6(100 \%)$ & $5(838)$ & $3(50 \%)$ & 0 & 0 \\
\hline
\end{tabular}

$(\mathrm{p}<0.05)$ each week. PRL-2903 treatment on week 5 reduced both the depth and duration of hypoglycemia compared to week 4 (see table). Time to reach $3.5 \mathrm{mM}$ after insulin dosing was longer with PRL-2903 treatment (week 5) vs. placebo treatment (week 4). However, on the second hypo, PRL-2903 treatment was less effective, perhaps because of limited liver glycogen reserves in STZ-diabetic rats.

Conclusion: A single subcutaneous injection of PRL-2903 was efficacious in preventing insulin-induced hypo after 4 weeks of recurrent hypo events. Further work to improve and maintain liver glycogen stores may help to maintain this efficacy.

\section{3}

THE SOCIAL ACCEPTANCE OF FUTURE ARTIFICIAL PANCREAS TECHNOLOGY: PARENTS' PERCEPTIONS OF PEDARPAN (PEDIATRICS ARTIFICIAL PANCREAS)

${\text { A. } \text { Troncone }^{1}, \text { R. Bonfanti }}^{2}$, D. Iafusco ${ }^{3}$, I. Rabbone , $^{4}$, A. Sabbion $^{5}$, R. Schiaffini ${ }^{6}$, A. Galderisi ${ }^{7}$, M. Marigliano ${ }^{5}$, N. Rapini ${ }^{8}$, A. Rigamonti ${ }^{2}$, D. Tinti ${ }^{4}$, V. Vallone,

A. Zanfardino ${ }^{3}$, F. Boscari ${ }^{9}$, S. Del Favero ${ }^{10}$, S. Galasso ${ }^{9}$,

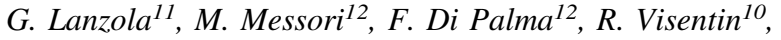
R. Calore $^{13}$, Y. Leal ${ }^{14}$, L. Magni ${ }^{15}$, E. Losiouk ${ }^{16}$,

D. Chernavvsky ${ }^{17}$, S. Quaglini ${ }^{18}$, C. Cobelli $^{19}$,

D. Bruttomesso ${ }^{20}$

${ }^{1}$ Second University of Naples, Department of Psychology, Caserta, Italy

${ }^{2}$ Pediatric Department and Diabetes Research Institute-

Scientific Institute- Hospital San Raffaele-Milan-Italy,

Department of Pediatrics, Milan, Italy

${ }^{3}$ Second University of Naples, Department of Pediatrics, Naples, Italy

${ }^{4}$ University of Turin, Department of Pediatrics, Turin, Italy

${ }^{5}$ Regional Center for Pediatric Diabetes-Clinical Nutrition \&

Obesity- University of Verona, Department of Life \&

Reproduction Sciences, Verona, Italy

${ }^{6}$ Unit of Endocrinology and Diabetes- Bambino Gesu-

Children's Hospital, Department of Pediatrics, Rome, Italy

${ }^{7}$ University of Padua, Department of Woman's and Child's

Health, Padua, Italy

${ }^{8}$ Pediatric Diabetology Unit- Policlinico di TorVergata-

University of Rome TorVergata, Department of Pediatrics,

Rome, Italy

${ }^{9}$ Unit of Metabolic Diseases- University of Padua, Department

of Internal Medicine-DIM, Padua, Italy

${ }^{10}$ University of Padua, Department of Information Engineering, Padua, Italy

${ }^{11}$ University of Pavia, Department of Electrical- Computer and Biomedical Engineering, Pavia, Italy

${ }^{12}$ University of Pavia, Department of Civil Engineering and Architecture, Pavia, Italy

${ }^{13}$ Department of Information Engineering, University of Padova, Padova, Italy

${ }^{14}$ Universidade Tecnológica Federal do Paraná, Curitiba, Brasil

${ }^{15}$ Department of Civil Engineering and Architecture, University of Pavia, Pavia, Italy

${ }^{16}$ Department of Industrial and Information Engineering,

University of Pavia, Pavia, Italy

${ }^{17}$ Center for Diabetes Technology, University of Virginia, Charlottesville, USA 
${ }^{18}$ Department of Electrical, Computer and Biomedical Engineering, University of Pavia, Pavia, Italy

${ }^{19}$ Department of Information Engineering, University of Padova, Padova, Italy

${ }^{20}$ Unit of Metabolic Diseases, Department of Internal MedicineDIM, University of Padova, Padova, Italy

Background and Aims: To explore the experiences of parents of 5-8-year-old children with type 1 diabetes participating in a clinical trial regarding artificial pancreas (AP), semistructured interviews, based on the Technology Acceptance Model, were conducted after 3 days of children's treatment.

Method: Questions focused on evaluating parents' perceived usefulness of, perceived ease of use of, trust in, and intention to use the new system. Interviews were conducted by a psychologist, and the answers, both audio-recorded and transcribed verbatim, were assessed using qualitative research methods.

Results: Altogether, 27 (22 mothers) of 30 parents were interviewed (Table 1), and their overall attitude toward AP was positive $(96 \%)$. Perceived advantages included stable glucose regulation $(52 \%)$, better quality of life for children (22\%), relief of parents' daily concerns (15\%), and reduced need for continual parental monitoring of nocturnal blood glucose $(11 \%)$, while perceived disadvantages included having to constantly wear a bulky, heavy device (37\%) and the risk of technical error (33\%). Participants were mostly confident in the positive impact of AP on diabetes control (96\%) and in children's, especially older ones', capability to use the system $(55 \%)$. The reactions of teachers and friends were reported to be generally positive, though some parents $(28 \%)$ expected an initially fearful reaction from teachers. Nearly all participants expressed trust in AP and in the quality of glucose control $(96 \%)$, as well as the intention to use the new system when available (100\%).

Conclusion: Results indicate that, thanks to the psychological and physical benefits of AP, parents expressed a strong likelihood of future acceptance.

\section{4}

\section{SINGLE- AND DUAL-HORMONE ARTIFICIAL PANCREAS FOR OVERNIGHT GLUCOSE CONTROL IN TYPE 1 DIABETES}

A. Haidar ${ }^{1}$, R.L. Rémi ${ }^{1}$, L. Legault ${ }^{2}$, E. Lovblom ${ }^{3}$, R. Rakheja ${ }^{1}$, V. Messierl , É. D'Aoust ${ }^{4}$, C.M. Falappa ${ }^{3}$, T. Justice ${ }^{3}$, A. Orszag $^{3}$, H. Tschirhart ${ }^{3}$, M. Dallaire ${ }^{1}$, M. Ladouceur $^{5}$, B.A. Perkins ${ }^{6}$

${ }^{1}$ IRCM Institut de recherches Cliniques de Montréal, Metabolic Diseases, Montréal, Canada

${ }^{2}$ McGill University Health Centre, Montreal Children's Hospital, Montréal, Canada

${ }^{3}$ Mount Sinai Hospital, Lunenfeld-Tanenbaum Research Institute, Toronto, Canada

${ }^{4}$ Université de Montréal, Hôpital Maisonneuve-Rosemont, Montréal, Canada

${ }^{5}$ Université de Montréal, Centre de Recherche, Montréal, Canada

${ }^{6}$ University of Toronto, Lunenfeld-Tanenbaum Research Institute- Mount Sinai Hospital, Toronto, Canada

Background and Aims: The added benefit of glucagon in artificial pancreas systems for overnight glucose control in type 1 diabetes has not been fully explored. We aimed to compare the efficacy of dual-hormone (insulin and glucagon) artificial pan- creas, single-hormone (insulin alone) artificial pancreas, and conventional insulin pump therapy.

Method: In a three-centre, randomized, three-arm, open-label crossover trial, we compared the three interventions in 28 participants (21 adults, 7 adolescents) with type 1 diabetes in home settings. Each intervention was activated from 21:00-07:00h over a night following exercise and a second night following a high carbohydrate/high fat meal to mimic real-life glycemic excursions. The primary outcome was the proportion of time-intarget $(4-8 \mathrm{mmol} / \mathrm{l})$ by continuous glucose monitoring from 23:00-07:00h. Analysis was by intention to treat.

Results: The median(IQR) percentage of time-in-target glucose range was $47(36,71) \%$ for conventional therapy, higher on both single-hormone $[76(65,91) \%, \mathrm{p}<0.001]$ and dual-hormone artificial pancreas $[81(68,93) \%, \mathrm{p}<0.001]$. The median(IQR) time spent below $4 \mathrm{mmol} / \mathrm{L}$ was $14(4,28) \%$ for conventional therapy, lower on both single-hormone $[5(0,13) \%, \mathrm{p}=0.004]$ and dual-hormone artificial pancreas [1 $(0,8) \%, \mathrm{p}<0.001]$. There were 14 hypoglycemic events on conventional therapy compared to 6 incidences on single-hormone artificial pancreas $(\mathrm{p}=0.059)$ and 3 incidences on dual-hormone artificial pancreas $(\mathrm{p}=0.017)$. None of these outcomes differed significantly between singleand dual-hormone configurations.

Conclusion: Single- and dual-hormone artificial pancreas systems both provided better glucose control than conventional therapy. Though the dual-hormone configuration did not increase overnight time-in-target glucose levels, an effect on lowering hypoglycemia risk cannot be ruled out.

\section{5}

\section{IN-HOME OVERNIGHT PREDICTIVE LOW GLUCOSE SUSPEND (PLGS) EXPERIENCE: DIFFERENCES ACROSS AGE GROUPS}

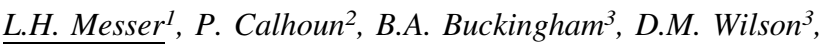
I. Hramiak ${ }^{4}$, T.T. Ly ${ }^{3}$, M. Driscoll ${ }^{4}$, D.M. Maahs ${ }^{1}$

${ }^{1}$ University of Colorado Anschutz, Barbara Davis Center for Diabetes, Aurora- CO, USA

${ }^{2}$ Jaeb Center for Health Research, Jaeb Center for Health Research, Tampa- FL, USA

${ }^{3}$ Stanford University, Pediatrics- Division of Endocrinology and Diabetes, Palo Alto- CA, USA

${ }^{4}$ St. Joseph's Health Care- London, Division of Endocrinology and Metabolism, London- Ontario, Canada

Background and Aims: Overnight predictive low glucose suspend (PLGS) reduces hypoglycemia across all ages; however, there are no reports on behavior or experience differences across age groups.

Method: As run in for a subsequent randomized clinical trial (RCT), 127 subjects (50\% male) ages $4-45$ yo utilized the experimental PLGS system nightly for 5-10 nights (PLGS Active phase). During this PLGS Active phase, we analyzed number of blood glucose (BG) checks and boluses given per age group.

During the subsequent 42 night RCT phase, we analyzed sensor use, skin reactions, errors, and why the experimental system was not used.

Results: In 821 nights of Active PLGS, subjects ages 4-6 yo (and their parents) tested BG levels $75 \%$ of nights compared with $65 \%$ of nights (7-10 yo), $53 \%$ of nights (11-14 yo), $33 \%$ of nights $(15-25$ yo) and $28 \%$ of nights $(26-45$ yo) respectively $(\mathrm{P}<0.001)$. Likewise, youngest subjects (and parents) administered insulin boluses $56 \%$ of nights during Active PLGS use compared to 


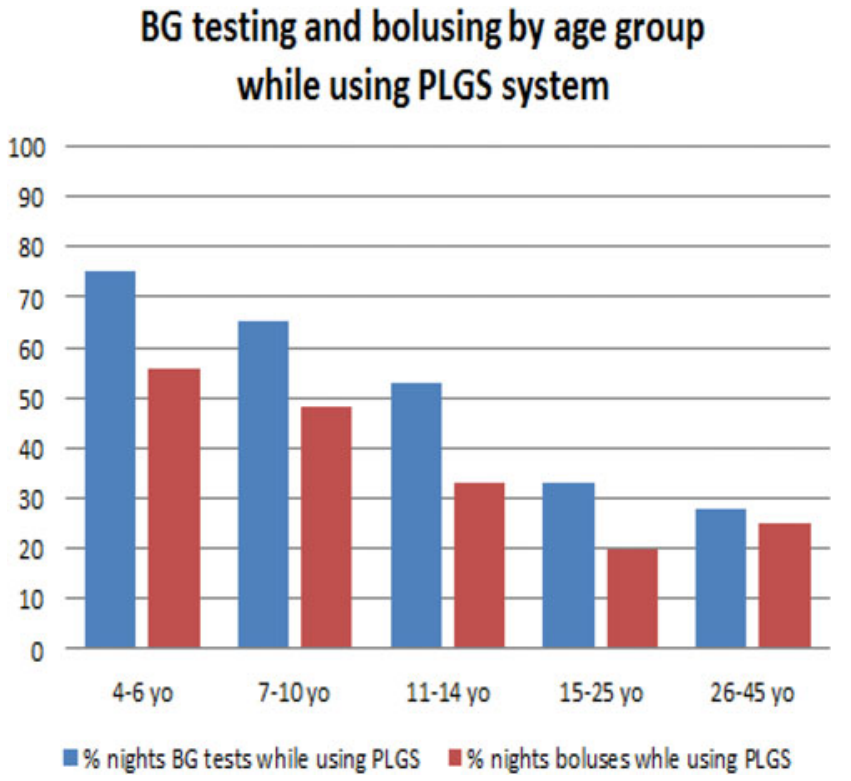

$48 \%, 33 \%, 20 \%$, and $25 \%$ respectively $(\mathrm{P}<0.001)$. This was unrelated to any study requirements.

During the RCT, subjects 4-6 yo experienced more frequent and severe skin reactions $(\mathrm{P}=0.02)$, while adult subjects $(26-45$ yo) wore individual sensors a median of 26 hours longer than the youngest subjects $(\mathrm{P}<0.001)$. Technical problems with the sensor (errors, miscalibrations, etc.), traveling, and BG levels $>270$ at bedtime (study requirement) were primary contributors to non-system use.

Conclusion: Understanding the different use patterns and challenges across age groups is needed to direct patient education to optimize use of PLGS and future artificial pancreas systems.

116

\section{HIERARCHICAL MODEL OF GLUCOSE-INSULIN REGULATION OVER 12-WEEK HOME USE OF CLOSED-LOOP INSULIN DELIVERY}

\section{Y. Ruan ${ }^{1}$, M. Wilinska ${ }^{1}$, H. Thabit ${ }^{1}$, R. Hovorka ${ }^{1}$}

${ }^{1}$ University of Cambridge Metabolic Research Laboratories, Institute of Metabolic Science, Cambridge, United Kingdom

Background and Aims: Parameters of physiological models of glucose-insulin regulation in type 1 diabetes have been estimated using data collected over a relatively short period of time and lack the description of day-to-day variability. We developed a new hierarchical model to relate subcutaneous insulin delivery and carbohydrate intake to continuous glucose monitoring over 12 weeks.

Method: Sensor glucose data (sampled every $10 \mathrm{~min}$ ), insulin aspart delivery and meal intake were analysed from 6 adults with type 1 diabetes (male/female 4/2, age $40.8 \pm 2.6$ years, BMI $25.3 \pm 3.6 \mathrm{~kg} / \mathrm{m}^{2}, \mathrm{HbA} 1 \mathrm{C} 8.2 \pm 1.0 \%$ ) who underwent a 12 -week home study of closed-loop insulin delivery. A compartment model comprised five linear differential equations with a closedform solution. Model parameters were estimated using the Markov chain Monte Carlo approach within a hierarchical Bayesian model framework.

Results: Physiologically plausible a posteriori distributions of model parameters including insulin sensitivity, time-to-peak
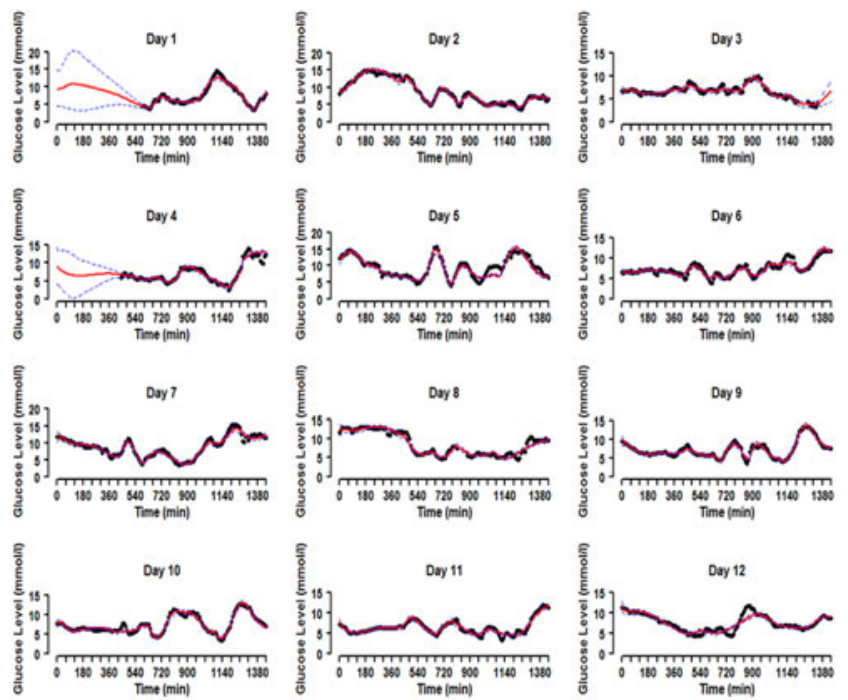

Figure 1. Model fit of CGM data over 12 days in a sample subject. Black points are CGM measurements. Red solid lines are model estimations and blue dashed lines are $95 \%$ credible intervals.

insulin action, time-to-peak gut absorption, and carbohydrate bioavailability, and good model fit were observed. Day-to-day variability of model parameters was estimated in the range 38 to $79 \%$ for insulin sensitivity and 35 to $48 \%$ time-to-peak insulin action.

Conclusion: A linear Bayesian hierarchical approach is feasible to model 12-week glucose-insulin relationship using conventionally measured data.

117

THE IMPACT OF PSYCHOLOGICAL STRESS

ON GLUCOSE LEVELS: A PRELIMINARY STUDY UTILIZING CONTINUOUS GLUCOSE MONITORING (CGM)

L. Gonder-Frederick ${ }^{1}$, J.Grabman $^{1}$, J. Shepard ${ }^{1}$, B. Kovatchev ${ }^{1}$

${ }^{1}$ University of Virginia, Psychiatry and NB Sciences, Charlottesville, USA

Background and Aims: Inconclusive data exist regarding the impact of daily psychological stress on BG fluctuations in individuals with type 1 diabetes (T1D). As glucose control system technology advances, more research is warranted to determine if stress-related BG changes should be considered in the algorithms that drive these technologies. This study examined relationships between self-reported routine daily stressors and BG profile measures generated from CGM readings.

Method: A total of 33 individuals with T1D participated in the study, which required them to wear a CGM device and record daily ratings of psychological stress, carbohydrates, and insulin boluses over a 1-week period.

Results: Within-subjects ANCOVAs, controlled for daily insulin and/or carbohydrates, found significant correlations between daily stress and indices of BG variability $(r=.172$ to .185 , $p=.011$ to .018$)$, increased \% time in hypoglycemia $(r=.153$, $p=.036)$ and decreased carbohydrate consumption $(r=-.157$, $p=.031)$. Models accounted for more variance in individuals reporting the most lability in daily stress. There was no 
relationship between stress and mean daily glucose or low/high glucose risk indices.

Conclusion: These preliminary findings suggest that daily stressors can be associated with increased glucose instability and hypoglycemia, as well as decreased carbohydrate consumption. Furthermore, results support the hypothesis that some patients are more susceptible to the effects of stress. Additional, more rigorous, studies utilizing CGM are needed to better understand the clinical impact of stress on BG and if it is a behavioral factor that should be considered in more individualized glucose control systems.

\section{8}

\section{THE BURDEN OF STRUCTURED SMBG ON DIABETES-SPECIFIC QUALITY OF LIFE AND LOCUS OF CONTROL IN NON INSULIN-TREATED TYPE 2 DIABETIC SUBJECTS: THE PRISMA STUDY}

G.T. Russo ${ }^{1}$, M. Scavini ${ }^{2}$, E. Bonizzoni ${ }^{3}$, E. Bosi ${ }^{2}$, A. Ceriello ${ }^{4}$, F. Giorgino ${ }^{5}$, A. Tiengo $^{6}$, E. Acmet $^{7}$, D. Cucinotta $^{1}$

${ }^{1}$ Policlinico Universitario Gaetano Martino, Department of Clinical and Experimental Medicine, Messina, Italy

${ }^{2}$ San Raffaele Hospital \& Scientific Institute - San Raffaele Vita-Salute University, Diabetes Research Institute, Milan, Italy ${ }^{3}$ University of Milan School of Medicine, Department of Occupational Health Clinica del Lavoro L. Devoto-Section of Medical Statistics and Biometry G.A. Maccacaro, Milan, Italy ${ }^{4}$ Institut d'Investigacions Biomèdiques August Pi Sunyer IDIBAPS, Centro de Investigacion Biomedica en Red de Diabetes y Enfermedades Metabolicas Asociadis CIBERDEM, Barcelona, Spain

${ }^{5}$ University of Bari School of Medicine, Department of Emergency and Organ Transplantation-Section of Internal Medicine-Endocrinology-Andrology and Metabolic Diseases, Bari, Italy

${ }^{6}$ University of Padova, Department of Clinical and

Experimental Medicine- Division of Metabolic Diseases, Padova, Italy

${ }^{7}$ Roche Diagnostics S.p.A., Medical Affairs, Monza, Italy

Background and Aims: SMBG has been associated with poor quality of life (QoL), suggesting that the positive effects on metabolic control could be counterbalanced by psychological distress, thus casting doubts on its use in non-insulin treated Type 2 diabetes (T2DM).

Method: In this analysis of the Prospective, Randomized Trial on Intensive SMBG Management Added Value in Non-insulinTreated T2DM Patients (PRISMA) Study, we evaluated the impact of 12-months structured SMBG on individual domains of the diabetes-specific quality of life (DSQoL) and locus of control (LOC) questionnaires, including the role of selected confounders.

Results: After 12 months intervention the DSQoL Scores (Satisfaction, Impact and Worry domains) improved compared to baseline, both in the structured SMBG (ISM=501) and active control $(\mathrm{AC}=523)$ group, without significant between-groups differences. Scores for the Internal, Chance and Powerful Others domains (LOC) improved compared to baseline, with significant between-group changes in the Chance domain $(\mathrm{P}=0.03)$. Improvements in DSQoL domain scores were associated with more SMBG measurements $(\mathrm{P}=0.007)$, older age $(\mathrm{P}=0.013)$ and male sex $(\mathrm{P}=0.01)$ for Satisfaction and with male sex $(\mathrm{P}<0.0001)$ for Worry. Improvements in LOC domain scores were associated with longer diabetes duration $(\mathrm{P}=0.0084)$, and younger age
$(\mathrm{P}<0.0001)$ for Chance, and total number of SMBG measurements $(\mathrm{P}=0.0036)$ for Internal, with intervention group close to being significant $(\mathrm{P}=0.06)$.

Conclusion: In non insulin-treated T2DM structured SMBG is not associated with deterioration of quality of life and locus of control. Improvements of domain scores occurred in both groups, and are strongly predicted by demographics and diabetes-related variables.

119

\section{INTEGRATING AN AUTOMATED DIABETES MANAGEMENT SYSTEM INTO THE FAMILY MANAGEMENT OF CHILDREN WITH TYPE 1 DIABETES: RESULTS FROM A 12-MONTH RANDOMIZED CONTROLLED TECHNOLOGY TRIAL}

K.L. McMahon ${ }^{1}$, T. Toscos ${ }^{2}$,

S.W. Ponder MD FAAP CDE

${ }^{1}$ Diabetech- LP, Research \& Development, Sausalito, USA

${ }^{2}$ Indiana University - Purdue University Fort Wayne, Informatics, Fort Wayne, USA

${ }^{3}$ Baylor Scott \& White, Pediatric Endocrinology, Temple, USA

Background and Aims: To evaluate how the use of a pervasive blood glucose monitoring (BGM) technology relates to glycemic control, reported self-care behaviors, and emotional response in children with type 1 diabetes (T1D) and their parents. (Diabetes Care, March 2012 vol. 35 no. 3 498-502)

Method: Forty-eight children aged less than 12 years (mean 8.8 years) with T1D were randomly assigned either the control group (conventional care without technology) or an experimental group (conventional care with technology), and followed for 12 months. Families in the experimental group were given the Automated Diabetes Management System (ADMS), which automatically collects blood glucose (BG) values and sends to parent(s) a 21-day BG trending report via e-mail. Measures of glycemic control (HbA1c) were collected at baseline and at quarterly diabetes clinic visits; BGM effect and diabetes selfcare behavior measures were obtained at the baseline, 6-month, and 12-month visits.

Results: Children in the experimental group had significantly $(\mathrm{P}=0.01)$ lower HbA1c at 12 months $(7.4460 .94,20.35$ from baseline) than controls (8.31 $61.24,+0.15$ from baseline). Improvement in $\mathrm{HbA} 1 \mathrm{c}$ was more profound in families using the ADMS more frequently. In addition, in these families, parents showed a significant improvement in BGM effect $(\mathrm{P}=0.03)$ and children became more meticulous in diabetes self-care $(\mathrm{P}=0.04)$. Children in both experimental and control groups experienced no change in their emotional response to BGM.

Conclusion: Using the ADMS 1-3 times/week may help children with T1D improve glycemic control, gain diabetes selfmanagement skills, and improve the emotional response of parents.

\section{0}

\section{ASSESSING THE ACCURACY OF CONTINUOUS GLUCOSE MONITORING (CGM) CALIBRATED WITH CAPILLARY VALUES USING CAPILLARY OR VENOUS GLUCOSE LEVELS AS A REFERENCE}

J. Kropff ${ }^{1}$, M. Andelin ${ }^{2}$, V. Matuleviciene ${ }^{3}$, J.I. Joseph ${ }^{4}$, S. Attvall ${ }^{3}$, E. Theodorsson ${ }^{5}$, I.B. Hirsch ${ }^{6}$, H. Imberg ${ }^{7}$, S. Dahlqvist ${ }^{2}$, D. Klonoff ${ }^{8}$, B. Haraldsson ${ }^{3}$, J.H. DeVries ${ }^{1}$, M. Lind $^{3}$ 
${ }^{1}$ Academic Medical Center - University of Amsterdam, Department of Endocrinology, Amsterdam, Netherlands ${ }^{2} \mathrm{NU}$ Hospital Group, Department of Medicine, Uddevalla, Sweden

${ }^{3}$ University of Gothenburg, Institute of Medicine, Gothenburg, Sweden

${ }^{4}$ Sidney Kimmel Medical College of Thomas Jefferson University, Department of Anaesthesiology, Philadelphia, Pennsylvania, USA

${ }^{5}$ Faculty of Health Sciences, Department of Clinical and Experimental Medicine, Linköping University, Sweden

${ }^{6}$ University of Washington, Seattle, Washington, USA

${ }^{7}$ Statistiska Konsultgruppen, Gothenburg, Sweden

${ }^{8}$ Mills-Peninsula Health Services, Diabetes Research Institute, San Mateo, California, USA

Background and Aims: Using standard venous reference for evaluation of continuous glucose monitoring (CGM) systems could negatively affect measured CGM accuracy since CGM are generally calibrated with capillary glucose and venous and capillary glucose concentration differ. We therefore aimed to to quantify the effect of using capillary versus venous glucose reference samples on estimated accuracy in capillary calibrated CGM.

Method: We evaluated 41 individuals with T1DM using the DexcomG4 CGM over 6 days. Patients calibrated their CGM devices with capillary glucose by means of the HemoCue system. On two occasions, capillary and venous samples were simultaneously measured per HemoCue. The Mean Absolute Relative Difference (MARD) was calculated using capillary and venous reference samples.

Results: Venous glucose values were $0.83 \mathrm{mmol} / \mathrm{L}(15.0 \mathrm{mg} /$ dl) lower than capillary values over all glycemic ranges, $\mathrm{p}<0.0001$. Below $4 \mathrm{mmol} / \mathrm{l}(72 \mathrm{mg} / \mathrm{dl})$, the difference was 1.25 $\mathrm{mmol} / \mathrm{l}(22.5 \mathrm{mg} / \mathrm{dl}), \mathrm{p}=0.0001$, at $4-10 \mathrm{mmol} / \mathrm{l}(72-180 \mathrm{mg} / \mathrm{dl})$ $0.67 \mathrm{mmol} / \mathrm{l}(12.0 \mathrm{mg} / \mathrm{dl}), \mathrm{p}<0.0001$ and above $10 \mathrm{mmol} / \mathrm{l}$ $(180 \mathrm{mg} / \mathrm{dl}) 0.95 \mathrm{mmol} / \mathrm{l}(17.1 \mathrm{mg} / \mathrm{dl}), \mathrm{p}<0.0001$. MARD was $11.7 \%$ using capillary values as reference compared to $13.7 \%$

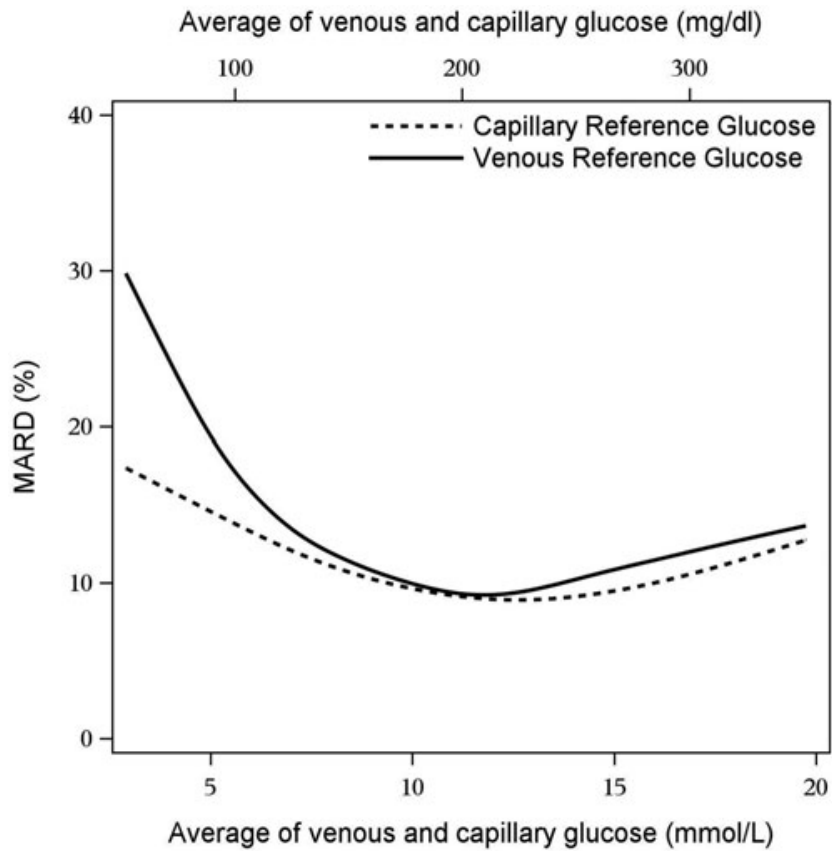

using venous samples, $\mathrm{p}=0.037$. Below $4 \mathrm{mmol} / \mathrm{l}(72 \mathrm{mg} / \mathrm{dl})$ MARD was $16.6 \%$ and $31.8 \%, \mathrm{p}=0.048$, at $4-10 \mathrm{mmol} / \mathrm{l}(72-$ $180 \mathrm{mg} / \mathrm{dl}) 12.1 \%$ and $12.6 \%, \mathrm{p}=0.32$, above $10 \mathrm{mmol} / \mathrm{l}$ $(180 \mathrm{mg} / \mathrm{dl}) 8.7 \%$ and $9.2 \%, \mathrm{p}=0.82$.

Conclusion: Using capillary glucose concentrations as reference to evaluate the accuracy of capillary calibrated CGM is associated with a lower MARD than using venous samples as reference. Capillary glucose concentrations were significantly higher than venous in all glycemic ranges.

121

\section{DIFFERENCES IN CONTINUOUS GLUCOSE MONITORING PATTERNS BETWEEN MULTIPLE DAILY INJECTION AND CONTINUOUS SUBCUTANEOUS INSULIN INFUSION THERAPY IN REAL LIFE USE OF PATIENTS WITH DIABETES}

K. Nakamura $^{1}$, C. Graham $^{2}$, A. Balo ${ }^{3}$

${ }^{1}$ Dexcom Inc., San Diego, USA

${ }^{2}$ Dexcom Inc., Global Access, San Diego, USA

${ }^{3}$ Dexcom Inc., Regulatory, San Diego, USA

Background and Aims: It is commonly believed that optimal diabetes management can best be achieved when intensive insulin treated (IIT) patients use CGM. We compared the CGM glucose patterns in patients with diabetes on MDI versus CSII therapy in real life using the Dexcom SHARE ${ }^{\mathrm{TM}}$ system.

Method: Over 67-million records of CGM glucose data over a 6-month time frame were used to identify trends in glucose control and variability between MDI and CSII users as well as users' demographics. A total of 1587 users' estimated A1C (eA1C) were evaluated over the entire measurement range $40-400 \mathrm{mg} / \mathrm{dL}$ for both groups.

Results: The eA1C from mean CGM glucose values were similar: $7.36 \%$ for MDI $(\mathrm{N}=648)$ and $7.44 \%$ for CSII $(\mathrm{N}=939)$. Both groups spend similar time within Euglycemia (70-180 mg/ $\mathrm{dL}$ ) and Hyperglycemia (glucose $\geq 240 \mathrm{mg} / \mathrm{dL}$ ): $\sim 14 \mathrm{hrs} /$ day and $\sim 3$ hrs/day, respectively. The standard deviation of glucose was slightly lower for MDI (60 mg/dL), compared with CSII (63 mg/ $\mathrm{dL}), \mathrm{p}=0.0148$. On average, users on MDI spend less time in Low glucose $<70 \mathrm{mg} / \mathrm{dL}$ ( $0.51 \mathrm{hrs} /$ day) compared with CSII (0.66 hrs/day), $\mathrm{p}=0.0002$. CGM glucose control and variability improved as users' ages increased, and the differences between MDI and CSII therapy were minimal across age groups (Figure 1).

Conclusion: The CGM glucose control were similar between MDI and CSII therapy in real life use, although there are small differences in glucose variation, and time spend in Low. Patients

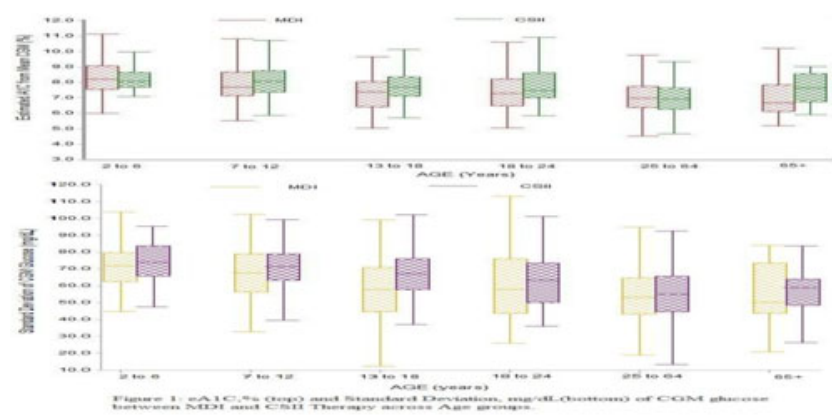


on CGM showed good glycemic control with both therapies. Physiological differences (e.g., age) result in apparent glycemic differences.

122

ACCURACY AND USER PERFORMANCE

EVALUATION OF A NEW, WIRELESS-ENABLED, BLOOD GLUCOSE METER IN DEVELOPMENT THAT LINKS TO A SMART MOBILE DEVICE VIA BLUETOOTH CONNECTIVITY

T. Bailey ${ }^{1}$, J. Wallace ${ }^{2}$, S. Pardo ${ }^{2}$, M.E. Warchal-Windham ${ }^{2}$,

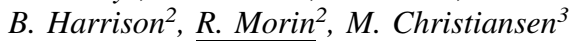

${ }^{1}$ AMCR Institute Inc., Escondido CA, USA

${ }^{2}$ Bayer HealthCare LLC, Diabetes Care, Whippany NJ, USA

${ }^{3}$ Diablo Clinical Research, Walnut Creek CA, USA

Background and Aims: To evaluate the accuracy of a new blood glucose monitoring system (BGMS) in development for use with CONTOUR Plus test strips, in the laboratory and clinical settings. The BGMS features an easy-to-use wirelessenabled blood glucose meter that links to a smart mobile device via Bluetooth connectivity.

Method: In the laboratory study, fingertip blood samples from 100 subjects were tested in duplicate using each of 3 test strip lots. BGMS results were compared with YSI reference results and assessed per ISO 15197:2013 accuracy criteria ( $\geq 95 \%$ of results within $\pm 15 \mathrm{mg} / \mathrm{dL}[ \pm 0.8 \mathrm{mmol} / \mathrm{L}]$ or $\pm 15 \%$ of reference for glucose $<100 \mathrm{mg} / \mathrm{dL}[<5.6 \mathrm{mmol} / \mathrm{L}]$ or $\geq 100 \mathrm{mg} / \mathrm{dL}[\geq 5.6$ $\mathrm{mmol} / \mathrm{L}]$, respectively). In the clinical study, 134 subjects with diabetes, who had never used this BGMS previously, enrolled at 2 clinical sites. The primary objective was to evaluate BGMS accuracy using subject fingertip self-tests, based on ISO 15197:2013 accuracy criteria (see above).

Results: In the laboratory study (glucose range, $37-526 \mathrm{mg}$ / dL [2.1-29.2 mmol/L]), 99.0\% (594/600) of BGMS results met the accuracy criteria; additionally, $96.3 \%$ (578/600) of results were within $\pm 10 \mathrm{mg} / \mathrm{dL}( \pm 0.6 \mathrm{mmol} / \mathrm{L})$ or $\pm 10 \%$ of the YSI reference result. In the clinical study (glucose range, 44.3$474.5 \mathrm{mg} / \mathrm{dL}$ [2.5-26.3 mmol/L]), 99.2\% (133/134) of subject fingertip self-test results met the accuracy criteria; moreover, $95.5 \%(128 / 134)$ of results were within $\pm 10 \mathrm{mg} / \mathrm{dL}( \pm 0.6 \mathrm{mmol} / \mathrm{L})$ or $\pm 10 \%$ of the YSI reference result.

Conclusion: The BGMS exceeded ISO 15197:2013 accuracy criteria in the laboratory (Section 6.3) and in a clinical setting when used by untrained subjects (Section 8 ).

\section{3}

\section{PERFORMANCE OF A FOURTH-GENERATION GLUCOSE SENSOR WITH TWO DIFFERENT REAL-TIME DISPLAY DEVICES IN ADULTS}

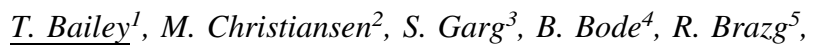
J. Shin' ${ }^{6}$ F. Kaufman

${ }^{1}$ AMCR Institute- Inc., Diabetes, Escondido, USA

${ }^{2}$ Diablo Clinical Research, Diabetes, Walnut Creek, USA

${ }^{3}$ University of Colorado Denver, Barbara Davis Center

for Childhood Diabetes, Aurora, USA

${ }^{4}$ Atlanta Diabetes Associates, Diabetes, Atlanta, USA

${ }^{5}$ Rainier Clinical Research Center, Diabetes, Renton, USA

${ }^{6}$ Medtronic-Inc., Diabetes, Northridge, USA
Table. Fourth-generation Glucose Sensor Accuracy and Precision

\begin{tabular}{|c|c|c|c|}
\hline YSI Glucose Ranges & $\leq 75 \mathrm{mg} / \mathrm{dL}^{*}$ & $75.180 \mathrm{mg} / \mathrm{dL}$ & $>180 \mathrm{mg} / \mathrm{dL}$ \\
\hline \multicolumn{4}{|c|}{ Standalone CGM vs. YSI (accuracy) } \\
\hline Mean $A R D \pm S D, \%$ & $9.51 \pm 9.54$ & $9.74 \pm 9.41$ & $9.47 \pm 7.92$ \\
\hline Median ARD, \% & 7.30 & 6.89 & 7.61 \\
\hline Bias, $m g / d L$ & -2.06 & -5.99 & -17.39 \\
\hline \multicolumn{4}{|l|}{640 G vs. YSI (accuracy) } \\
\hline Mean $A R D \pm S D, \%$ & $8.95 \pm 7.33$ & $10.10 \pm 9.51$ & $9.95 \pm 8.50$ \\
\hline Median ARD, \% & 7.25 & 7.25 & 7.96 \\
\hline Bias, $m g / d L$ & -1.48 & -5.33 & -17.74 \\
\hline \multicolumn{4}{|c|}{ Standalone CGM vs. $640 \mathrm{G}$ (precision, $640 \mathrm{G}$ used as reference) } \\
\hline Mean $A R D \pm S D, \%$ & $9.85 \pm 11.68$ & $8.30 \pm 9.25$ & $7.20 \pm 7.54$ \\
\hline Median ARD, \% & 6 & 5.61 & 4.95 \\
\hline Bias, $m g / d L$ & 5.39 & 0.66 & -2.87 \\
\hline
\end{tabular}

- for values $575 \mathrm{mg} / \mathrm{dL}$, values are expressed as absolute differences in mg/dL

Background and Aims: A fourth-generation subcutaneous glucose sensor was evaluated for 7 days of continuous wear in adults to establish its performance characteristics.

Method: After a 7-day run-in phase, each subject wore fourthgeneration sensors attached to transmitters specific for data processing and display on either a standalone CGM connected to a smartphone or the MiniMed 640G insulin pump. Subjects underwent 12-hour in-clinic visits on days 1, 3, and 7 for frequent sample testing of plasma glucose with a reference instrument (YSI). All visits included hypoglycemic and hyperglycemic challenges. Sensor glucose (SG) values were compared with YSI glucose values for accuracy and precision analyses.

Results: Eighty-nine subjects were enrolled with 82 completing the study. A total of 11,619 paired YSI-SG paired points were available from the standalone CGM and 12,090 from the 640G pumps. Clarke Error Grid analysis showed $99.1 \%$ of the paired points in the clinically acceptable A + B zones for each dataset. Accuracy (sensor vs. reference value) and precision (640G sensor vs. standalone CGM sensor) statistics are provided in the Table for reference glucose concentrations in the hypoglycemic $(\leq 75 \mathrm{mg} / \mathrm{dL})$, normoglycemic $(75-180 \mathrm{mg} / \mathrm{dL})$, and hyperglycemic $(\geq 180 \mathrm{mg} / \mathrm{dL})$ ranges. The overall MARD was $10.35 \pm 10.39 \%$ for the standalone CGM and $10.55 \pm 9.62 \%$ for the $640 \mathrm{G}$ system; overall precision was $8.39 \pm 9.93 \%$.

Conclusion: The fourth-generation glucose sensor showed excellent accuracy and precision in hypoglycemic, normoglycemic, and hyperglycemic ranges with either the standalone CGM or $640 \mathrm{G}$ devices. Results of this fourth-generation sensor support its use in closed-loop systems.

124

ANALYSIS OF 30-DAY READMISSIONS AMONG PRIVATELY INSURED TYPE 1 \& TYPE 2 DIABETES PATIENTS IN THE U.S

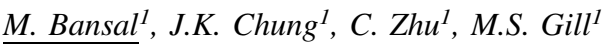

${ }^{1}$ Medtronic, Diabetes, Northridge, USA

Background and Aims: Diabetes is a chronic disease with major complications leading to high inpatient utilization and significant medical expenditure. The use of appropriate technology to track and coach the patients in this progressive disease becomes imperative to control costs and enhance the quality of hospitals. This analysis presents the most burdensome (high costs and admissions) diagnosis among the diabetes population that are hospitalized and readmitted within 30 days. 
Method: We conducted a retrospective observational analysis using a national employer-based insurance claims database (2009-2013). We included patients with diagnosis of Type 1 or Type 2 diabetes, aged 18-64 years and continuous enrollment in an insurance plan for 2 years in the analysis. The patients were either on insulin therapy, non-insulin injectable or oral antidiabetic drugs. The Diagnosis Related Groups (DRGs) were used to classify the reasons for admissions for the first four hospitalizations within 30 days of discharge. Top 20 DRGs with highest total costs inflated to 2014 dollars were evaluated.

Results: Approximately, 30-40\% of the total Type 1 and Type 2 patients were readmitted where majority of the readmissions occurred within 30 days of discharge. Diabetes related events including hypoglycemia, ketoacidosis or other complications such as sepsis/infections, amputations and pneumonia were primary attributable reasons for high costs and admissions among type 1 diabetes patients. Cardiovascular complications and sepsis/infections were primary reasons for readmissions for Type 2 diabetes patients.

Conclusion: Education on glycemic control, protocols on avoiding infections post-discharge and advanced remote monitoring with appropriated coaching can help diabetes patients avoid readmissions to the hospitals.

125

\section{RECURRENCE ANALYSIS OF HEART RATE VARIABILITY AS A PROMISING METHOD FOR CARDIAC AUTONOMIC NEUROPATHY PREDICTION IN PATIENTS WITH DIABETES MELLITUS}

$\underline{\text { S. Mala }}{ }^{1}$, L. Riedlbauchova ${ }^{2}$,J. Schlenker ${ }^{3}$, M. Jirina ${ }^{4}$, V. Potockova ${ }^{5}$, J. Kulhankova ${ }^{6}$, K. Stechova ${ }^{1}$, M. Kvapil ${ }^{1}$

${ }^{1}$ 2nd School of Medicine - Charles University and University Hospital Motol, Department of Internal Medicine, Prague, Czech Republic

${ }^{2}$ 2nd School of Medicine - Charles University and University Hospital Motol, Department of Cardiology, Prague, Czech Republic

${ }^{3}$ Czech Technical University, Faculty of Biomedical

Engineering, Prague, Czech Republic

${ }^{4}$ Czech Technical University, Faculty of Information

Technology, Prague, Czech Republic

${ }^{5}$ 2nd School of Medicine - Charles University and University Hospital Motol, Department of Neurology, Prague, Czech Republic

${ }^{6}$ 2nd School of Medicine - Charles University and University Hospital Motol, Department of Primary Care, Prague, Czech Republic

Background and Aims: Cardiovascular autonomic neuropathy $(\mathrm{CAN})$ represents a frequent and serious complication of diabetes mellitus and is associated with increased morbidity and mortality. However an early detection of CAN is still difficult. Nowadays CAN presence is evaluated by heart rate variability (HRV) analysis but recently the importance of non-linear methods of HRV analysis started to be discussed as well. The aim of our study was to assess whether recurrence quantification analysis (RQA) of HRV (one of the non-linear methods) is suitable for CAN prediction.

Method: 45 healthy controls and 36 diabetic patients with confirmed CAN were analysed. Groups were sex and age-matched. Consecutive sequence of R-R intervals derived from continual ECG that was recorded during deep breathing test was subjected

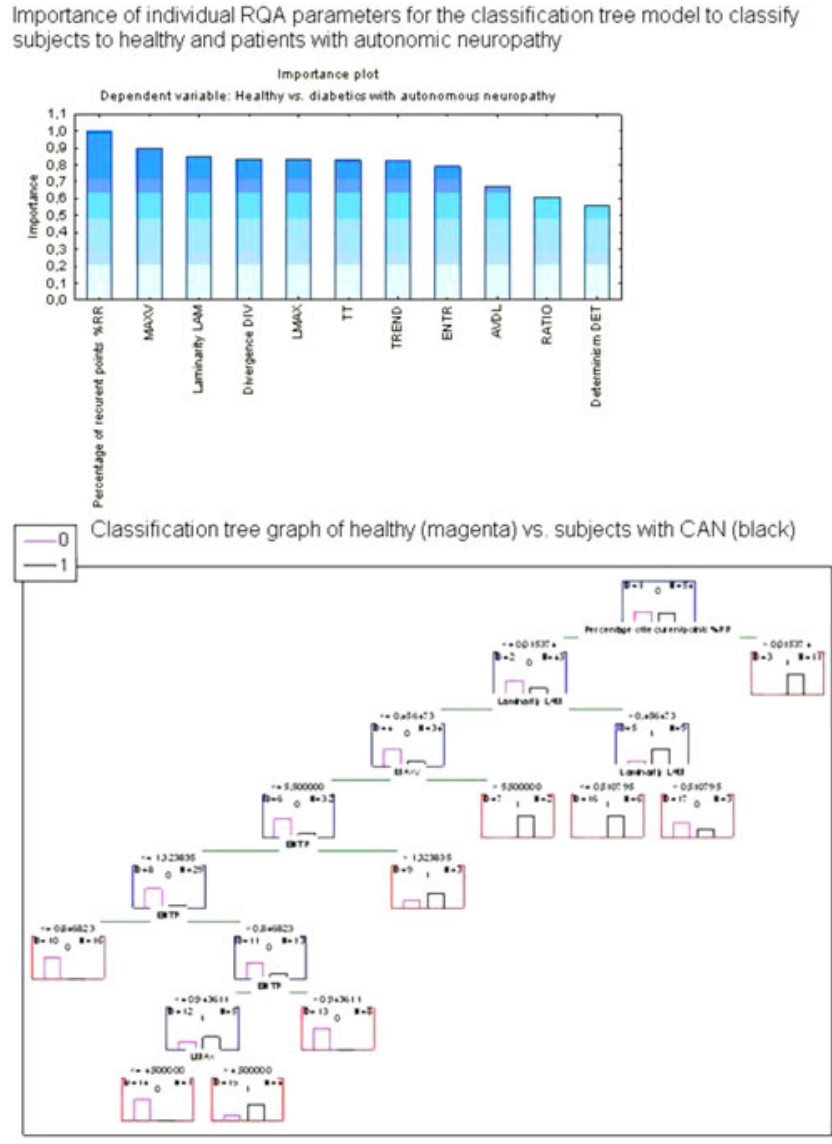

The graph holds for $1.5 \%$ RR setting of ROA in de ep bre athing test. The minimal number for a node split is 5 samples.

The accuracy on the testing set is $80 \%$, sensitivity $76,9 \%$, spedificity $82,4 \%$.

to RQA. In the presence of CAN HRV is reduced, i.e. recurrence is frequent. Its graphical representation is a recurrence plot described by various RQA parameters. The predictive value of each RQA parameter was determined by calculating the $\chi 2$ statistics between individual parameters and predicted values of the health state. The predictive values of RQA parameters was further tested by a data-mining method. Five most predictive parameters were used for classification tree construction and accuracy calculation.

Results: The accuracy of CAN testing by RQA of HRV was $80 \%$ with sensitivity $77 \%$ and specificity $82 \%$.

Conclusion: RQA of HRV seems to be a suitable method for CAN prediction.

\section{6}

DIABETIC FOOT OSTEOMYELITIS SELECTED ANTIBIOTHERAPY IN PATIENTS WITH GASTROINTESTINAL BY PASS / PROLONGED IV SHOULD BE PREFERRED TO ENTERAL

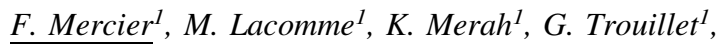
K. Mohammedi $^{2}$, L. Potier ${ }^{2}$, V. Labbe Gentils ${ }^{3}$, R. Bouzriba ${ }^{1}$

${ }^{1}$ Hôpital Européen de Paris GVM Care Research,

Seine St Denis, Aubervilliers, France 
${ }^{2}$ AP HP Hôpital Bichat - Claude Bernard Service

d'Endocrinologie Diabète Paris France - INSERM U872

Paris, France

${ }^{3}$ Hôpital Jean Verdier, Seine St Denis, Bondy, France

Background and Aims: Failure of an oral selected antibiotherapy after radio bone biopsy (RGBB) was observed in diabetic foot osteomyelitis (DFO) patients with gastro intestinal bypass (GIBP) whereas success was achieved in others diabetics patients without GIBP

We gained success in these patients with GIBP with prolonged selected intra venous (IV) rather than enteral antibiotherapy

Method: DFO in our multicenters network, had selected antibiotherapy after RGBB and wound debridement

Patients $(n=75)$, aged 44 to 92 , were included from october 2013 to april 2015

PEDIS 3 to 4 - IDSA moderate to severe - UT 3C 3D

All had a deficient bone structure on clinical (probe bone test) or imaging basis (Xr, CTscan, MRI).

Loco regional anesthesia $(n=43)$ or none $(n=32)$ were proposed.

Patients had GIPB $(n=3)$ being still diabetic.

We had no complication and $100 \%$ success in bacterial retrieval.

Gram $+68 \%$ gram $-28 \%$ anaerobie $4 \%$.

IV broad spectrum antiobiotherapy was given in first intention.

Selected enteral antibiotherapy after bacterial identification were prescribed afterwards.

Follow up was done at one, three and six months.

Results: Enteral relay was ineffective when patients $(n=3)$ had GIPB. We observed they went back to PEDIS 4 or IDSA severe in less than 3 days.

Soft tissu infection and biological degradation were patent.

As the same antibiotherapy was effective with IV, it was prolonged 4 to 6 weeks.

Conclusion: DFO best treatment is a prolonged oral antibiotherapy after radio guided bone biopsies (RGBB).

Long term IV antibiotherapy should be discussed for patient with GIBP.

\section{7}

\section{IMPAIRED RED BLOOD CELL DEFORMABILITY IS ASSOCIATED WITH DIABETIC COMPLICATIONS IN PATIENTS WITH TYPE 2 DIABETES}

\author{
J.S. Moon $^{1}$, K. Yu Kyoung ${ }^{2}$, W. Kyu Chang ${ }^{1}$ \\ ${ }^{1}$ Yeungnam University School of Medicine, Department \\ of Internal Medicine, Daegu, Republic of Korea \\ ${ }^{2}$ Yeungnam University School of Medicine, Department \\ of Labortory Medicine, Daegu, Republic of Korea
}

Background and Aims: Red blood cell (RBC) deformability is an ability of RBC to change shape under stress. RBC deformability has been known to be decreased in atherosclerosis and diabetes. But, little is known about the association between impaired RBC deformability and type 2 diabetes (T2D). We attempted to clarify whether RBC deformability is related with diabetic complications.

Method: This was a cross-sectional study, and 452 T2D patients who visited in university hospital were enrolled. Patients with end-stage renal disease and who are taking a pentoxifylline and ginkgo biloba were excluded. RBC deformability was measured by using a Rheoscan-D ${ }^{\circledR}$ (Rheo-Meditech, Seoul, Korea), and expressed as elongation index at $3 \mathrm{~Pa}$ (EI@3P, \%). We divided the EI@3P into tertile (T1, T2, and T3 from lowest to highest EI@3P).

Results: 441 patients (mean age $60.30 \pm 11.98$ years, $M=237$ ) were finally included. EI@3P showed inversely related with the HbA1c, fasting glucose, and positively with HOMA-B. EI@3P was lower in patients with microvascular complications than those without complications $(30.86 \%$ vs. $31.09 \%, p<0.05)$, and especially in group with retinopathy $(30.52 \%$ vs. $31.16, p<0.05)$. After adjustment for age, sex, history of hypertension and smoking, and lipid profiles, EI@3P remained significantly associated with the prevalence of diabetic retinopathy (Odd ratio for T1 compared with T3, 2.36; 95\% confidence interval, 1.134.93; $\mathrm{p}=0.023$ ).

Conclusion: In patients with $\mathrm{T} 2 \mathrm{D}$, there are significant relationship between EI@3P and glycemic control, HOMA-B and retinopathy. These results suggest that impaired RBC deformability is significantly related with diabetic microangiopathy, especially diabetic retinopathy.

128

\section{NEW TECHNOLOGY FOR THE TREATMENT OF RETROGRADE EJACULATION IN PATIENTS WITH DIABETES MELLITUS TYPE 1}

G. Galstyan ${ }^{1}$, D. Kurbatov ${ }^{2}$, Y. Shwarts ${ }^{3}$, A. Lepetukhin ${ }^{3}$, R. Rozhivanov ${ }^{3}$

${ }^{1}$ Endocrinology Research Center, Diabetic Foot Clinic, Moscow, Russia

${ }^{2}$ Endocrinology Research Center, Andrology and Urology, Moscow, Russia

${ }^{3}$ Endocrinology Research Center, Andrology and Urology, Moscow, Russia

Background and Aims: Retrograde ejaculation (RE) in patients with Diabetes Mellitus Type 1 (DMT1) is a complication of an autonomic neuropathy. Research of the influence of average daily trips blood glucose in the manifestation and progression of RE and to assess the effectiveness of new endoscopic method of RE correction.

Method: 30 patients, age of $32 \pm 5.7$ years with total RE included. HbA1c before operation $7.4 \pm 1.3 \%$. Analyzed the "diabetes diary" of the last 3 years before the manifestation of RE. All results were compared with control group without RE. During the operation biocompatible material was injected under mucous layer of a posterior urethra. The spermogram was examined in 1 week after the operation.

Results: Patients with RE had larger average daily blood glucose excursions: $7.1-11.1-12.8 \mathrm{mmol} / \mathrm{l}$ and it is significantly different from control group $(\mathrm{p}<0.001)$. Restoration of antegrade emission of ejaculate achieved at 22 patients. The effect of operation maintained during $7 \pm 5$ months. The spouses of the 4 men had normal pregnancies after surgery. Born were 3 children. $50 \%$ of 22 patients with effective operation have their semen cryopreserved.

Conclusion: The amplitude of the fluctuations in blood glucose is a risk factor for RE. The applying of new method provides highly effective restoration of a physiological passage of the ejaculate. 
129

\section{ONLINE GLUCOSE SENSOR ERROR DETECTION AND RECONCILIATION BY FUNCTIONAL REDUNDANCY}

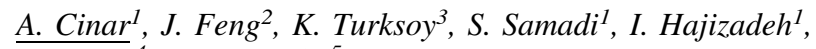
L. Quinn $^{4}$, E. Littlejohn

${ }^{1}$ Illinois Institute of Technology, Chemical and Biological Engineering and Biomedical Engineering, Chicago, USA

${ }^{2}$ Illinois Institute of Technology, Chemical and Biological Engineering, Chicago, USA

${ }^{3}$ Illinois Institute of Technology, Biomedical Engineering, Chicago, USA

${ }^{4}$ University of Illinois at Chicago, College of Nursing, Chicago, USA

${ }^{5}$ University of Chicago, Biological Sciences Division, Chicago, USA

Background and Aims: CGM readings provide critical information to artificial pancreas (AP) control systems that rely on real time glucose concentration information to calculate insulin infusion rates. CGM readings can be erroneous or missing due to several factors and can cause the computation of incorrect insulin infusion rates to cause hypoglycemia or hyperglycemia. Sensor readings can show jumps and drifts, may have outliers, missing values and increased sensor noise. Algorithms are needed to detect and modify these signals, and replace the erroneous values with best estimates for use in AP systems.

Method: An online sensor error detection and functional redundancy system is developed to detect CGM errors, and replace erroneous values with model-based estimates. The system relies on an outlier-robust Kalman filter (ORKF) and a locally-weighted partial least squares (LW-PLS) regression model to leverage the advantages of measurement error elimination with ORKF and data-driven prediction with LW-PLS. Over 2000 CGM sensor errors were added to original CGM signals from 10 clinical experiments to test the proposed system and the corrupted signals were analyzed for error detection and reconciliation of glucose measurements.

Results: Results indicate that the system can successfully detect most of the erroneous signals and substitute them with reasonable estimated values computed by functional redundancy system. Correct error detection ranged from $70.9 \%$ to $97.4 \%$ for various types of sensor errors that lasted for 2-4 consecutive sensor readings. Successful reconciliation of glucose concentrations ranged from $81.9 \%$ to $98 \%$ for the same errors.

Conclusion: The methods developed reduce the impact of sensor errors on AP operation significantly.

130

\section{A LOW-POWER BIO-INSPIRED ARTIFICIAL PANCREAS}

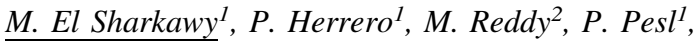
P. Georgiou ${ }^{1}$, D. Johnston ${ }^{2}$, N. Oliver ${ }^{2}$, C. Toumazou ${ }^{1}$, S.B. Seechurn ${ }^{2}$, N. Jugnee ${ }^{2}$, D.V. Pavitt ${ }^{2}$

${ }^{1}$ Imperial College London, EEE, London, United Kingdom ${ }^{2}$ Imperial College London, Department of Medicine, London, United Kingdom

Background and Aims: Existing closed-loop insulin delivery systems based on tablet/smartphone devices commonly suffer from limited usability due to the large power consumption levels associated with the devices. In this work, we present a handheld low-power system that runs an innovative bio-inspired glucose controller to form an artificial pancreas with the goal of maintaining tight glycaemic control in people with type 1 diabetes for prolonged periods of time.

Method: The Bio-inspired Artificial Pancreas (BiAP) handheld device has been designed to contain all the necessary electronics to communicate to either a Medtronic Enlite or a Dexcom G4 continuous glucose sensor and has been optimised to run the bio-inspired controller with low-power consumption. The calculated insulin dose can be transmitted to a Cellnovo insulin pump via an integrated system-on-chip radio . A user interface consisting of 3 buttons and an OLED screen allows the user to announce meals and exercise, as well as to calibrate the glucose sensor. The control unit incorporates a buzzer that is used to alert the user of any unexpected event. Data logging is performed on a micro-SD card. A 3D-printed enclosure $(9 \times 5 \times 2.4 \mathrm{~cm})$ has been designed for protective purposes and facilitating usability. In addition to the utilisation of low power electronics, power consumption was optimised via software.

Results: The system has been clinically validated by conducting more than 60 trials on people with Type- 1 diabetes. The BiAP handheld currently lasts for 3 days on a single battery charge.

Conclusion: A custom-made low-power Bio-inspired Artificial Pancreas is a suitable platform for long-duration ambulatory clinical trials.

131

\section{DO TYPE 1 DIABETIC PATIENTS REALLY} WANT AN ARTIFICIAL PANCREAS ?

\author{
S. Franc ${ }^{1}$, I. Xhaard ${ }^{2}$, L. Orlando ${ }^{2}$, M. El Makni ${ }^{2}$, \\ $\overline{\text { M.H. Petit }}{ }^{2}$, C. Randazzo ${ }^{2}$, G. Charpentier ${ }^{2}$ \\ ${ }^{1}$ Centre Hospitalier Sud-Francilien, Essonnes, \\ Corbeil-Essonnes, France \\ ${ }^{2}$ CERITD, Essonnes, Evry, France
}

Background and Aims: The artificial pancreas (AP) could provide a solution to the constraints currently imposed upon patients presenting type1-diabetes(T1D). But how does the reality of APs differ from what patients themselves imagine?

The aim was to determine how many patients would wish to have an AP after receiving detailed system information.

Method: 101-T1D-patients completed 2 identical questionnaires both before and after a session on this subject. The session involved a comprehensive presentation of AP.

Results: $42 \%$ patients imagined that PA involves grafting of an artificial organ, $27 \%$ thought a device carried on the body like a pump, $17 \%$ imagined several devices carried on the body and $18 \%$ thought it referred to a smartphone application. Following the information session, these proportions were $27 \%, 42 \%$ and $68 \%$ respectively.

Most of the patients expressed a desire to have an AP. following this information, their desire was greater, and with the number of patients feeling it was extremely likely that they would have an AP in place of their insulin-pump rising from $24 \%$ to $41 \%$.

Logistical-regression-analysis suggests that the desire to have an AP is determined by 2 factors: the recency of onset of T1D $(O R=$ $0.94 /$ year of duration; $\mathrm{p}=0.014$ ) and ongoing treatment with pump therapy vs. multiple daily injections $(\mathrm{OR}=0.26 ; \mathrm{p}=0.058)$. Dissatisfaction with current therapy, the hope of improved $\mathrm{HbA} 1 \mathrm{c}-\mathrm{levels}$ or decreased-risk-of-hypoglycaemia or associated complications, the hope of improved freedom or comfort, and the length of time needed to develop the device did not appear to be significantly correlated with hopes about AP. 
Conclusion: 101-T1D-patients attending an information session on AP, $67 \%$ expressed a wish to use such a device, while $6 \%$ rejected the idea.

\section{2}

THE EFFICACY OF SINGLE- AND DUAL-HORMONE ARTIFICIAL PANCREAS SYSTEMS AT REGULATING GLUCOSE LEVELS DURING CONTINUOUS AND INTERVAL EXERCISE IN TYPE 1 DIABETES: RANDOMISED FOUR-WAY TRIAL

N. Taleb ${ }^{1}$, A. Haidar ${ }^{2}$, C. Suppere ${ }^{1}$, A. Emami ${ }^{3}$, V. Messier ${ }^{1}$, L. legault $^{4}$, M. Ladouceur ${ }^{5}$, J.L. Chiasson ${ }^{5}$, R.L. Remi ${ }^{1}$

${ }^{1}$ Montreal Institute of Clinical Research, Diabetes, Montreal, Canada

${ }^{2}$ Montreal Institute of Clinical Research, Montreal, Canada

${ }^{3}$ McGill University, Computer Science, Montreal, Canada

${ }^{4}$ McGill University, Pediatrics, Montreal, Canada

${ }^{5}$ The Research Center of the Université de Montréal Hospital Center, The Research Center of the Université de Montréal Hospital Center, Montreal, Canada

Background and Aims: To assess whether the dual-hormone (insulin and glucagon) artificial pancreas reduces hypoglycemia compared to the single-hormone (insulin alone) artificial pancreas during two types of exercises.

Method: We conducted a randomized, crossover study comparing the single-hormone and the dual-hormone systems in 17 adults with type 1 diabetes (age of $37.2 \pm 13.6$ years, HbA1c $8.0 \pm 1.0 \%$ ) during two types of exercises: moderate-intensity continuous exercise $\left(60 \% \mathrm{VO}_{2 \text { peak }}\right.$ for $\left.60 \mathrm{~min}\right)$ and high-intensity interval exercise (2-min alternating intervals at $85 \%$ and $50 \%$ $\mathrm{VO}_{2 \text { peak }}$ for $40 \mathrm{~min}$, with two 10 -min intervals at $45 \% \mathrm{VO}_{2 \text { peak }}$ at the start and the end of the sessions), both exercises matched for overall energy expenditure. The artificial pancreas systems were applied from 15:30 until 19:30, exercise started at 18:00 and announced 20 minutes earlier to the systems.

Results: During the single-hormone visits compared to the dual-hormone visits: exercise-induced hypoglycemia (plasma glucose $\leq 3.3 \mathrm{mmol} / 1$ with symptoms or $<3$ regardless of symptoms) was observed in $31.25 \%(10 / 32)$ vs. $9 \%$ (3/33) of interventions $(\mathrm{p}=0.02)$; the percentage of time with glucose levels less than $4.0 \mathrm{mmol} / \mathrm{l}$ was $11(0-46.7) \%$ vs. $0(0,0) \%(\mathrm{p}=0.0001)$; time with glucose levels between 4.0 and $10.0 \mathrm{mmol} / \mathrm{l}$ was $71.4(53.2-100) \%$ vs. $100(100-100) \%(\mathrm{p}=0.003)$. There was no statistically significant difference in insulin delivery or plasma insulin concentrations. Higher doses of glucagon were needed during the continuous exercise $14.4(9.5-16.9) \mathrm{mg}$ vs. the interval exercise sessions 8.5(4.6-16.9) $\mathrm{mg}(\mathrm{p}=0.03)$.

Conclusion: The dual-hormone artificial pancreas is offering a tighter control and a better potential to prevent hypoglycemia during two types of exercise in adults with type 1 diabetes.

\section{3}

DIABETIC TREATMENT USING ACTIVIN A- AND BMP4-GRAFTED POLYVINYL ALCOHOL-ALGINATEGELATIN HYDROGEL TO DIFFERENTIATE INDUCED PLURIPOTENT STEM CELLS TOWARD PANCREATIC CELLS

\footnotetext{
Y.C. Kuo ${ }^{1}$, I.H. Lee ${ }^{1}$

${ }^{1}$ National Chung Cheng University, Department of Chemical Engineering, Chiayi, Taiwan
}

Background and Aims: This study examines the capacity of activin A- and BMP4-grafted polyvinyl alcohol-alginate-gelatin (PVA-Alg-Gel) hydrogel to differentiate induced pluripotent stem cells (iPSCs) toward pancreatic cells.

Method: Activin A and BMP4 are conjugated in PVA-AlgGel hydrogel and iPSCs are seeded in activin A- and BMP4grafted PVA-Alg-Gel hydrogel for producing endodermic and pancreatic cells.

Results: An increase in the weight percentage of PVA and Alg enhanced the porosity and swelling ratio of PVA-Alg-Gel hydrogel, respectively. In addition, an increase in the concentration of activin A and BMP4 in PVA-Alg-Gel hydrogel slightly reduced the viability of iPSCs. A higher concentration of activin A yielded a lower quantity of SSEA-1 and a higher quantity of SOX-17 expressed by differentiating iPSCs. This demonstrates that the activin A-grafted PVA-Alg-Gel hydrogel can activate the endodermic differentiation. Moreover, an increase in the concentration of BMP4 in PVA-Alg-Gel hydrogel increased the quantity of PDX-1 expressed by differentiating iPSCs. This suggests an induction of differentiation toward pancreatic cells.

Conclusion: Activin A- and BMP4-grafted PVA-Alg-Gel hydrogel are effective in differentiate iPSCs toward pancreatic cells and can be used for diabetic preclinical trials.

\section{4}

\section{WHAT INFLUENCES A PHYSICIAN'S INTENTION TO PRESCRIBE AN ARTIFICIAL PANCREAS TO INDIVIDUALS WITH TYPE 1 DIABETES?}

\author{
$\underline{\text { T. Oukes }}^{1}$, C. Uncu ${ }^{1}$, A. von Raesfeld \\ ${ }^{1}$ University of Twente, NIKOS, Enschede, Netherlands
}

Background and Aims: Recently, researchers have started to study the views of individuals with type 1 diabetes on artificial pancreas (AP) technology. However, few studies have attempted to investigate the beliefs and attitudes of physicians. Therefore, we aimed to study how individual characteristics, product characteristics and social influences impact a physician's intention to prescribe an AP.

Method: 105 endocrinologists from Germany $(n=20)$, Austria $(n=13)$ and the Netherlands $(n=72)$ filled out an online questionnaire after a personal e-mail invitation. The questions were adapted from existing 7-point Likert scales (1-7) used in Diffusion Theory, the Technology Acceptance Model and the Technology Readiness Index. Multiple hierarchical regression was used to analyse the data.

Results: The mean endocrinologists' intention to prescribe the AP was 5.45 (Table 1). This was positively influenced by an optimistic and innovative attitude towards new technologies. A pessimistic attitude did not decrease the intention to prescribe an AP. Additionally, the intention to prescribe was higher when endocrinologists perceived that an AP would be easy to use and compatible with their way of working. Device usefulness had no impact. Furthermore, the intention to prescribe was higher when people in the social environment of endocrinologists, especially their patients, thought that they should prescribe it.

Conclusion: Endocrinologists from three European countries appear to have the intention to prescribe an AP once it becomes available. However, this intention is influenced by their attitude towards new technologies, easy of use and compatibility of the $\mathrm{AP}$, and their social environment. 


\begin{tabular}{|c|c|c|c|c|c|}
\hline & $\mathrm{N}$ & Minimum & Maximum & Mean & Std. Deviation \\
\hline Optimism & 105 & 2.13 & 6.75 & 4.61 & 0.87 \\
\hline Innovativeness & 105 & 1.50 & 6.83 & 4.76 & 0.98 \\
\hline Pessimism & 105 & 1.43 & 5.14 & 3.24 & 0.79 \\
\hline Usefulness & 105 & 1.00 & 7.00 & 4.30 & 1.15 \\
\hline Compatibility & 105 & 1.00 & 7.00 & 4.94 & 1.05 \\
\hline Complexity & 105 & 1.00 & 6.00 & 3.29 & 1.01 \\
\hline Social influence (general) & 105 & 1.00 & 7.00 & 4.01 & 1.35 \\
\hline Social influence of colleagues & 101 & 1.00 & 7.00 & 3.69 & 1.28 \\
\hline Social influence of superiors & 97 & 1.00 & 6.00 & 3.21 & 1.37 \\
\hline Social influence of subordinates & 99 & 1.00 & 6.50 & 3.57 & 1.48 \\
\hline Social influence of patients & 72 & 1.00 & 7.00 & 5.19 & 1.17 \\
\hline Intention to prescribe & 105 & 1.00 & 7.00 & 5.45 & 1.15 \\
\hline
\end{tabular}

135

\section{AUTOMATIC DETECTION OF EXERCISE IN PEOPLE WITH TYPE 1 DIABETES USING AN UNSCENTED KALMAN FILTER}

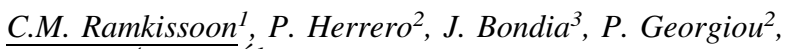
N. Oliver ${ }^{4}$, J. Vehi 1

${ }^{1}$ Institut d'Informàtica i Aplicacions, Universitat de Girona, Girona, Spain

${ }^{2}$ Centre for Bio-Inspired Technology-Institute of Biomedical Engineering, Imperial College London, London, United Kingdom

${ }^{3}$ Instituto Universitario de Automatica e Informática Industrial, Universitat Politènica de València, València, Spain

${ }^{4}$ Division of Diabetes- Endocrinology and Metabolic Medicine, Imperial College London, London, United Kingdom

Background and Aims: Physical activity in type 1 diabetes mellitus has been found to have varying degrees of effect on glycaemic control depending on the type, intensity and duration of the exercise. Such effect is associated with an imbalance between hepatic glucose production and glucose disposal into the muscle, increased insulin sensitivity and impaired counter-regulatory hormonal response. In the context of an artificial pancreas, automatic detection of exercise has the potential to significantly improve glycaemic control.

This work aims to develop a new methodology for automatically detecting exercise that only requires data from a continuous glucose monitor (CGM) and the insulin delivered to the subject.

Method: The glucose-insulin Minimal Model was extended, by adding an insulin absorption model and an auxiliary parameter used to describe disturbances. Increases in this parameter may indicate meal ingestions whereas decreases may indicate exercise. The disturbance parameter was estimated using an Unscented Kaman Filter. Two thresholds were introduced to detect exercise: a first threshold to indicate the possibility of an abnormal event; and a second threshold, based on an area-underthe-curve, to indicate exercise. The method was tested on data from 7 closed-loop trials including a period of moderate intensity structured exercise.

Results: Overall, the results obtained were satisfactory with an average detection time of 22 minutes, accuracy of $96 \%$, sensitivity of $100 \%$ and a specificity of $96 \%$.

Conclusion: The presented technique has the potential to be a viable approach to detect physical exercise in the context of an artificial pancreas. Improvements and further testing are necessary to confirm such hypothesis.

\section{6}

\section{A RUN-TO-RUN ALGORITHM FOR INSULIN TO CARBOHYDRATE RATIO ADAPTATION IN SENSOR-AUGMENTED PUMP THERAPY OF TYPE 1 DIABETES}

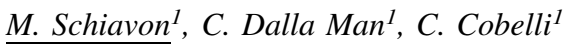 \\ ${ }^{1}$ University of Padova, Department of Information Engineering, \\ Padova, Italy
}

Background and Aims: The insulin-to-carbohydrate ratio (CR) is a parameter used in type 1 diabetes (T1D) therapy to optimally calculate the pre-meal insulin bolus and it is tuned by physician based on patient diary. However, CR is known to vary over time, within and between days.

The aim of this work is to use CGM and subcutaneous insulin delivery (CSII) to adapt patient CR to follow physiological and/ or behavioral changes.

Method: To do that, we propose a run-to-run algorithm for CR adaptation based on the calculation of an index for insulin sensitivity, from sensor and pump data (Schiavon et al., 2014), the area under CGM and CSII curves and the position of the subject in the Control Variability Grid (CVGA) (Magni et al., 2008). The method was tested in silico using the UVA/Padova T1DM simulator (Dalla Man et al., 2014), by simulating a 40day scenario, with randomness in meal timing and amount, starting from three conditions: nominal patient-specific CR, $\mathrm{CR}$ reduced and increased by $20 \%$. In addition, the effect of systematic mal-adapted basal insulin infusion rate was considered.

Results: In all simulations, CR was updated every 3 days and different gains were tested. Based on simulations, a safe and effective gain was selected, which improves the glycemic control in a significant percentage of virtual subjects.

Conclusion: The run-to-run algorithm for $\mathrm{CR}$ adaptation shows in silico an improvement of T1D therapy which needs to be confirmed clinically. In addition, it will be worth exploring the potential of the method for improving post-prandial glycemic control in closed-loop.

\section{7}

\section{APPLICATIONS OF AN ARTIFICIAL INTELLEGENT SYSTEM ON THE ASSESSMENT OF EFFECTIVENESS OF DIABETES CONTROL FOR ICU PATIENTS}

\section{J.C. Chen ${ }^{1}$}

${ }^{1}$ National YunLin University of Science and Technology, Information Management, Douliu, Taiwan

Background and Aims: Appropriate blood sugar control might greatly reduce the mortality and morbidity of surgical patients in ICU. However, for somewhat unknown reasons, the control was less effective than expected. The aim of this study was to investigate the effectiveness of glucose control for those critically ill patients after insulin injection.

Method: Data on 231 patients (2492 records) were collected covering four years in an ICU. For each patient, insulin dosage was based on health condition as well as his (her) glycemic curve and the therapeutic effect of insulin dosage. An artificial 
intelligent system was used to differentiate the results of applying insulin dosage on each patient.

Results: The study result shows that poor glycemic control with their glycemic curves outside of the average range led ICU patients to suffer sepsis, respiratory failure, pneumonia, and blood glucose control becoming more difficult along with a wider range of costs. It could predict the outcome quite accurately after injection of a certain dose of insulin.

Conclusion: The proposed system may help doctors to effectively assess their patients in determining insulin dose for better glucose control in an ICU setting.

\section{8}

\section{NEONATAL HYPOGLYCEMIA CONTINUOUS GLUCOSE MONITORING: A RANDOMIZED CONTROLLED TRIAL IN PRETERM INFANTS}

A. Galderisi $^{1}$, A. Facchinetti ${ }^{3}$, G.M. Steil ${ }^{2}$, P. Ortiz-Rubio ${ }^{2}$, ${\text { C. } \text { Cobelli }^{3} \text {, D. Trevisanuto }}^{1}$

${ }^{1}$ University-Hospital of Padua, Department of Woman's and Child's Health - Neonatal Intensive Care Unit, Padova, Italy

${ }^{2}$ Harvard Medical School, Boston Children's Hospital, Boston, USA

${ }^{3}$ University of Padova, Department of Information Engineering, Padova, Italy

Background and Aims: Neonatal hypoglycemia is associated with brain injury and impaired neurodevelopmental outcomes in very low birth weight infants (VLBWI). Glycemic monitoring is usually performed by capillary or central line sampling but does not identify up to $81 \%$ of hypoglycemic episodes in preterm newborns. We aim to assess if a continuous glucose monitor (CGM) can be used without undo pain, and whether it reduces the incidence of hypoglycemia.

Method: Newborns $\leq 32$ weeks gestational age and/or of birthweight $\leq 1500 \mathrm{~g}$ were randomized to receive glucose control (target glucose $72-144 \mathrm{mg} / \mathrm{dl}$ ) during the first 7 days of life using a Dexcom-G4-Platinum-CGM vs standard care practice. CGMmonitored patients had glucose infusion rates (GIR) adjusted every 3 hours using a glucose-control algorithm that incorporated CGM values, as well as CGM hypoglycemic alarms. Patients receiving standard care wore blinded CGMS without alarms, and GIR was modified according to 2-3 daily capillary glucose levels. Pain at insertion was evaluated with the validated Premature Infant Pain Profile (PIPP) scale.

Results: We report preliminary results on 7 patients. Mean \pm SD gestational age was $28 \pm 1$ wks and birthweight was $1140 \pm 144 \mathrm{~g}$. Mean Absolute Relative Difference CGM v. HeelPrick was 16.9\%. Sensor insertion was not more painful than HeelPrick (PIPP median (range), $4(3-15)$ v. $6(3-18)$ respectively). No complications were observed.

Conclusion: CGM with DexcomG4Platinum is feasible and safe in VLBWI. The results of this ongoing RCT will clarify the role of CGM in glycemic management of VLBWI.

Acknowledgments: Dexcom Inc, California, USA provided the materials of the study.

\section{9}

DIABETIC RETINOPATHY IN DIABETES MELLITUS TYPE 1 PATIENTS AND DAILY BLOOD GLUCOSE EXCURSIONS: 1994-2014 FOLLOW-UP STUDY

L. Bolotskaya $^{1}$, E. Bessmertnaya ${ }^{2}$, A. Vitebskaya ${ }^{3}$
${ }^{1}$ Endocrinology Research Center, Epidemiology, Moscow, Russia ${ }^{2}$ Endocrinology Research Center, Ophthalmology, Moscow, Russia ${ }^{3}$ I.M. Sechenov First Moscow State Medical University, Pediatric Endocrinology, Moscow, Russia

Background and Aims: Observational program included 124 patients with diabetes mellitus type 1 (DM1): 77 girls $(62.1 \%)$, 47 boys $(37.9 \%)$. Age of DM1 manifestation was $12.9 \pm 6.1$ years.

Determination of the correlation between average daily blood glucose excursion and manifestation/progression of DR during 20 year period.

Method: Patients consulted endocrinologist with their blood sugar diaries once every 3 months and ophthalmologist twice a year. In 2010-2014 years Continuous Glucose Monitoring System (CGMS) was used twice a year to define the results of every day measurements using glucometer. Correlation between average daily blood glucose excursion and DR diagnosis were studied.

Results: In 20 years 63 patients $(50.8 \%)$ had no diabetic retinopathy (DR), 43 patients $(34.7 \%)$ developed non-proliferative DR, 18 patients $(14.5 \%)$ had proliferative DR and underwent laser coagulation. Age at DR diagnosis was $8.1 \pm 2.3$ years, duration of disease $-3.2 \pm 1.8$ years, glycosylated hemoglobin (HbA1c) level $8.8 \pm 0.5 \%$. Patients without DR and with non-proliferative DR had low average daily blood glucose excursions (Min-Medium-Max): $1.4-1.7-2.4 \mathrm{mmol} / \mathrm{l}$ and $1.9-2.5-2.8 \mathrm{mmol} / \mathrm{l}$, and were not significantly different ( $\mathrm{p}$-Wilk $>0.05$ ). Patients with proliferative DR had significantly larger average daily blood glucose excursions: $6.2-10.5-12.2 \mathrm{mmol} / \mathrm{l}$, p-Wilk $<0.001$.

Conclusion: Large average daily blood glucose excursion in patients with DM1 leads to development and progression of diabetic retinopathy to proliferative stage.

\section{0}

\section{A BIHORMONAL GLUCOSE CONTROLLER BASED ON THE PARACRINE INTERACTION BETWEEN BETA CELL AND ALPHA CELL}

P. Herrero $^{1}$, J. Bondia ${ }^{2}$, G. Amparo ${ }^{3}$, N. Oliver $^{4}$, C. Toumazou $^{4}$, P. Georgiou ${ }^{1}$

${ }^{1}$ Imperial College London, Electrical and Electronic Engineering, London, United Kingdom

${ }^{2}$ Universidad Politécnica de Valencia, Ingeniería de Sistemas y Automática, Valencia, Spain

${ }^{3}$ Universidad Politécnica de Mandrid, Tecnología fotónoca y bioengineniería, Madrid, Spain

${ }^{4}$ Imperial College London, Endocrinology and Metabolic Medicine, London, United Kingdom

Background and Aims: To date, most bihormonal closedloop systems for glucagon and insulin delivery have been based on two independent controllers (e.g. MPC+PID or PID+PID). However, there is a well-established paracrine interaction between the secretion of insulin and glucagon. In this work, we present a novel biologically inspired glucose control strategy that accounts for such coordination.

Method: A model of the beta-cell insulin secretion and a model of the alpha-cell glucagon secretion were selected for this purpose. To account for the autoinhibition of the two hormones, two feedback loops were added to the original models. Subcutaneous absorption models were employed to estimate plasma concentration levels of both hormones. Additional terms modelling the potentiation of insulin secretion by plasma glucagon levels and the suppression of glucagon secretion by plasma insulin levels were included. 
The UVa-Padova T1DM Simulator v3.2 was used to validate the proposed technique. The 10 adult virtual subjects over a 1week scenario were used for testing purposes. The proposed coordinated strategy was evaluated against an uncoordinated version of the same controller. Results are expressed as mean \pm SD.

Results: While maintaining the percentage time in the target range $70-180 \mathrm{mg} / \mathrm{dL}$ (coordinated vs. uncoordinated) $(82.3 \pm 2.6$ vs. $78.1 \pm 10.9, p=0.2$ ), the coordinated strategy managed to significantly reduce the percentage time in hypoglycemia $(0.36 \pm 1.12$ vs. $5.1 \pm 4.1, \mathrm{p}<0.01)$ by delivering significantly less insulin (U/day) $(38.8 \pm 6.7$ vs. $53.1 \pm 15.3, \mathrm{p}<0.01)$ and glucagon (mg/day) $(0.46 \pm 0.37$ vs. $1.34 \pm 0.4, \mathrm{p}<0.01)$.

Conclusion: A bihomornal glucose controller based on the beta-cell and alpha-cell interacting physiology has the potential to improve glycaemic control in a type 1 diabetes adult population.

141

\section{PERFORMANCE OF A HYBRID CLOSED-LOOP SYSTEM IN TYPE 1 DIABETES PATIENTS WITH MEAN A1C $<7 \%$}

J. Ilany ${ }^{1}$, B. Grosman ${ }^{2}$, N. Konvalina ${ }^{1}$, C. Mylonas ${ }^{1}$, A. Roy ${ }^{2}$, N. Kurtz ${ }^{2}$, D. Wu ${ }^{2}$, N. Parikh ${ }^{2}$, G. Voskanyan ${ }^{2}$, R. Gottlieb ${ }^{2}$, F. Kaufman ${ }^{2}$, O. Cohen ${ }^{2}$

${ }^{1}$ Sheba medical center, Institute of Endocrinology, Ramat Gan, Israel ${ }^{2}$ Medtronic MiniMed, 18000 Devonshire Street, Northridge. CA 91325, USA

Background and Aims: Hybrid closed-loop (HCL) systems require continuous glucose monitoring (CGM) data and userprovided estimates of upcoming carbohydrate intake to make appropriate adjustments to insulin delivery. A novel HCL system was tested in a protected home setting.

Method: The HCL system includced the Medtronic MiniMed 670G pump, new-generation sensors and transmitters, and an algorithm that addresses the pharmacodynamics of subcutaneous insulin as well as CGM limitations. It was tested in a pre-pivotal trial that enrolled 8 subjects with type 1 diabetes and experience with sensor-augmented pump therapy. Subjects mean \pm SD age was $35.1 \pm 11.4$ years; baseline A1C was $6.4 \pm 0.7 \%$ (range 5.5-7.6\%). The study included an 8-day run-in period without automatic insulin adjustments followed by an 8-day period of HCL use. Sensor glucose (SG) values from the run-in and HCL phases were compared.

Results: The percentage of time spent in euglycemia (70$180 \mathrm{mg} / \mathrm{dL}$ ) was $72 \%$ during the run-in and $81 \%$ during the HCL phase $(\mathrm{p}=0.29)$. The percentage of time spent in hypoglycemia (SG $<70 \mathrm{mg} / \mathrm{dL}$ ) was $8 \%$ during the run-in and $1 \%$ during the HCL phase $(p=0.78)$. There were no adverse events.

Conclusion: In this small pre-pivotal trial, use of the HCL system in a group of type 1 subjects with low to slightly above target $\mathrm{A} 1 \mathrm{C}$ values was associated with increased time in the target range and decreased time in the hypoglycemic range. Larger and longer-term studies of this HCL system in different outpatient settings are warranted.

142

HOW CAN WE FIND REGULARITIES IN DIURNAL BS/TIME CURVE DESPITE CHAOTIC LIFESTYLE AND DIURNAL MEAL INTAKE AND ACTIVITY?

\section{K. Khodabakhshi Pirkalani ${ }^{1}$}

${ }^{1}$ Mehr Medical Group, Internal Medicine/Gynecology, Tehran, Iran
Background and Aims: The BS/time curve looks very chaotic in both normal and diabetic patients due to untimely physical activity, meal intake and quality and quantity of them in addition to different mental status and rest and sexual activity. We wondered whether the slopes of this curve might have some regularity.

Method: Twenty four patients with type II DM in addition to ten normal controls were evaluated by BS control every 15 minutes for $16 \mathrm{~h} / 24 \mathrm{~h}$ for 3 consecutive days. More frequent evaluations were omitted due to absent equipments. In all, the exact BS/time curves were plotted via mathematical extrapolation and best fit curve methods. First and second derivative of the primary curves were plotted with the MATLAB software.

Results: Although the primary curve and its first derivatives were completely chaotic in regard to slopes and amplitudes, the second derivative showed a curious regularity in that it crossed the $\mathrm{X}$ axis at fixed intervals meaning that at these points the innate $\mathrm{BS}$ reducing properties wane irrespective of the $\mathrm{BS}$ values both in normal and diabetic patients.

Conclusion: Appearance of these regularities gives clinicians the opportunity to both exploit innate BS reducing activity and prevent superimposition of external and internal potentials giving rise to hypoglycemic attacks. The revolutionary breakthrough in this regard will symbolize itself into this phrase:

In contrast to conventional practice where insulin is delivered whenever it is needed (high BS), we deliver insulin whenever it is most effective (when the first derivative changes its slop direction) so that very low doses can accomplish huge jobs.

\section{3}

\section{IS BS MONITORING REALLY NEEDED IN TYPE II DM UNDER AUDITORY INSULIN THERAPY?}

\author{
K. Khodabakhshi Pirkalani ${ }^{1}$ \\ ${ }^{1}$ Mehr Medical Group, Internal Medicine/Gynecology, Tehran,
} Iran

Background and Aims: Although strict BS monitoring is stressed by physicians many practical restrains remain. We hereby suggest that this point is neither mandatory nor of clinical significance. Since the advent of auditory insulin instillation treatment of type II DM became extremely easier with higher patients' convenience.

Method: Twenty eight patients with type II DM of over 10 years from diagnosis were treated with Metformin 2-3 gram / $\mathrm{d}+100 \mathrm{mg}$ Sitagliptine in addition to 8-15 units of auditory insulin 30 minutes before meal. Half were advised to monitor their BS 12 times a week (6 fasting and $63 \mathrm{hpp}$ ) while the other half were let free. After three months all were subjected to a series of evaluations.

Results: The mean HbA1C reductions in both groups were comparable at $1.8 \%$. The number of real and subjective hypoglycemic attacks were 3 (never severe) and seven/month and comparable. Voluntary change of the auditory insulin doses was never seen in the fixed dose group and only at a maximum of 4 units in the monitoring group. Besides, this was not used as a routine practice by them (with a mean of 8 times/months).

Conclusion: Omitting BS monitoring in type II DM patients who are under auditory insulin instillation is feasible and without major acute risk. Combination of auditory insulin instillation with Metformin and Sitagliptine gives satisfactory short course BS control without major hypoglycemic attacks and acceptable $\mathrm{HbA} 1 \mathrm{C}$ reductions. Longer follow ups are underway but it seems that no difference with these findings will possibly emerge. 


\section{4}

\section{AUTOMATED INSULIN DELIVERY “IN THE WILD"}

R. Kircher ${ }^{1}$, D. Matheson ${ }^{2}$, R. Mauseth ${ }^{3}$

${ }^{1}$ Mason Clinic, Seattle, USA

${ }^{2}$ Dose Safety. Inc., Operations, Seatte, USA

${ }^{3}$ Veterans Administration Medical Center, Seattle, USA

Background and Aims: The objective of this research is to improve the safety of automated insulin delivery when encountering everyday system incidents including: sensor changes/ calibrations, system startup/shutdown, Continuous Glucose Monitor (CGM) and insulin pump communication errors, infusion set changes, insulin refills and battery changes.

Method: Our new autodosing mode control (ADMC) module allows closed-loop insulin dosing only when interfacing subsystems are operating normally. The ADMC suspends insulin dosing and reverts to the user's preprogrammed basal when it detects something is wrong. Automated insulin delivery resumes when problems are resolved.

Forty (40) system incidents, resulting in missed automated insulin doses, occurred in 17 Daily Living clinical studies in the CRC. In each case, because the ADMC was not yet implemented, the on site engineer had to intervene and manually restore closedloop dosing.

Results: We analyzed the 40 system incidents given the ADMC module logic and concluded that 38 (95\%) of the incidents would have been detected and the closed loop dosing temporarily suspended by the ADMC. The remaining 2 incidents were caused by operator error and not detectable by the ADMC.

Conclusion: The retrospective analysis of system incidents in prior clinical studies showed that the ADMC module would have detected $95 \%$ of the system faults and temporarily suspended fuzzy logic dosing module (FLDM) dosing. The closed-loop FLDM does not depend on prior insulin administration and gracefully tolerates periods when dosing is suspended by the ADMC. The FLDM, in conjunction with the ADMC, will safely administer automated insulin delivery "in the wild".

\section{5}

\section{A COMPARTMENTAL MODEL OF THE EFFECT OF ENDOGENOUS ADRENALINE ON INSULIN-GLUCOSE DYNAMICS}

\section{Lewis $^{1}$, N. Ramroop Singh ${ }^{2}$, B. Aufderheide ${ }^{3}$}

${ }^{1}$ Port of Spain, Trinidad and Tobago

${ }^{2}$ The University of Trinidad and Tobago, Biomedical Engineering, O'Meara, Trinidad and Tobago

${ }^{3}$ The University of Trinidad and Tobago, Process Engineering, Pt. Lisas, Trinidad and Tobago

Background and Aims: Previous studies have shown that ingesting caffeine adversely affects insulin-glucose dynamics in humans. Four main pathways have identified the mechanism through which this occurs: 1) increasing non-esterified fatty acid (NEFA) content in plasma; 2) inhibiting phosphodiesterase (PDE) activity; 3) inhibiting cyclic adenosine monophosphate (cAMP) activity; and 4) increasing adrenaline production.

It has been shown that an exogenous bolus of adrenaline causes increased plasma glucose levels mainly due to gluconeogenesis, a compensatory increase in insulin secretion, and a counterintuitive reduction in glucose uptake by body cells.
Method: A study, using male Sprague Dawley rats as an acceptable human physiology model, was conducted to determine the levels of adrenaline, insulin, and glucose in the blood for caffeine doses of $0,1,2$, and 4 "cups". This data was used to fit the mass transfer parameters of a multi-compartmental pharmacokinetic/pharmacodynamics model.

Results: The model describes the levels of endogenous adrenaline produced due to the caffeine stimulus, the consequent rise in blood glucose levels, and the time taken by the insulin to return plasma glucose levels to within its acceptable range.

Conclusion: This model can be used as the baseline to refit parameters for data derived from male human study to predict plasma glucose spikes. It may also be used to compare changes in response to caffeine stimulus between males and females.

146

\section{GLUCAGON PEN MAKES BIHORMONAL DELIVERY MORE PATIENT FRIENDLY AND ALLOWS BETTER BG CONTROL IN SPITE OF IMPRECISE MEAL ESTIMATIONS}

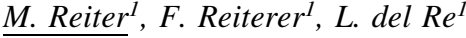

${ }^{1}$ Johannes Kepler University of Linz, Institute for Design and Control of Mechatronical Systems, Linz, Austria

Background and Aims: We propose a novel method to realize an artificial pancreas with an insulin pump and a glucagon pen. We show that the benefits of a second hormone can also be reached with pen injections, which makes a second pump for glucagon unnecessary.
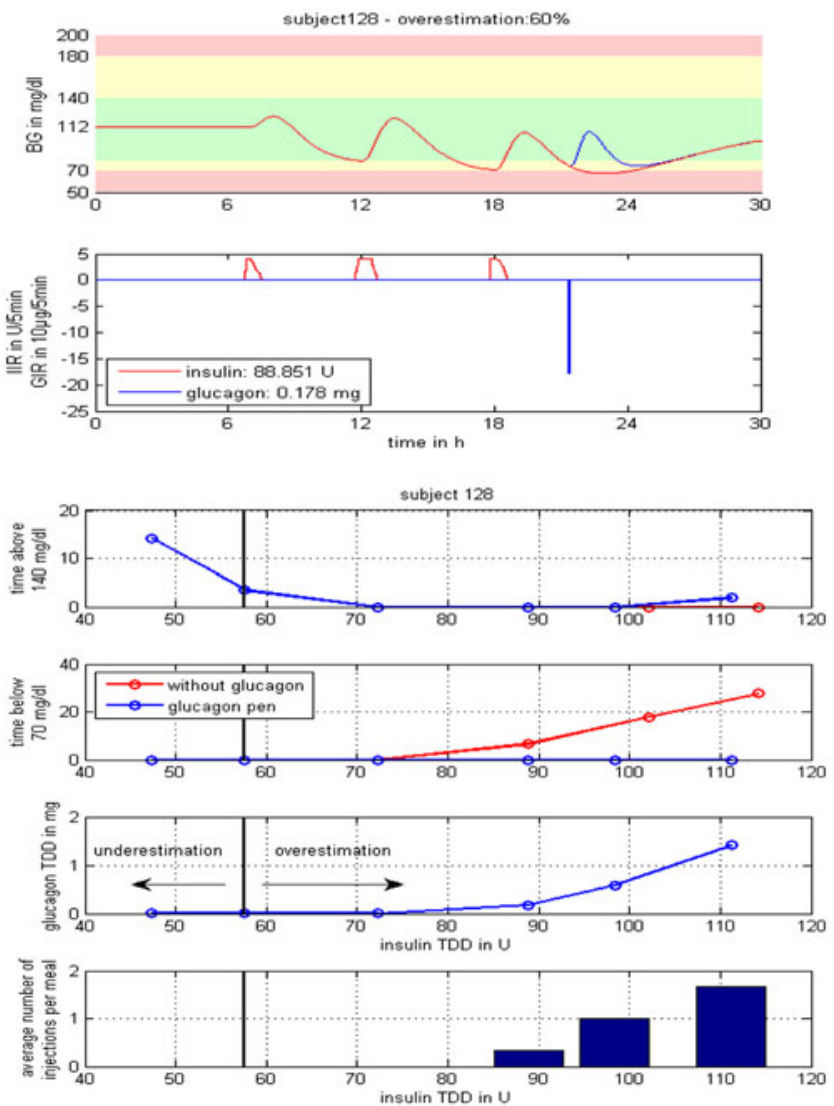
Method: An existing simulation model for the human glucoregulatory system was used to develop a predictive control that administers insulin doses and, in case of impending hypoglycemia, calculates an optimal amount of glucagon for pen injection. We considered errors in the carbohydrate (CHO) estimations for the meal announcements and tested the controller in-silico using the simulation model and data of two patients.

Results: The risk of hypoglycemia associated with a $\mathrm{CHO}$ meal overestimation can be removed by glucagon pen injection. The injection is necessary only if the overestimation is sufficiently large (e.g. $>60 \%$ for patient 128 ). The amount of infused insulin and the time spent in hyperglycemia is not affected by these glucagon injections, but a higher or earlier insulin delivery is possible increasing the time in the euglycemic range.

Conclusion: The proposed method shows that a bihormonal controller can also be realized with a glucagon pen that is used only for the case of impending hypoglycemia. It can be used more easily than a two pump approach and could encourage diabetics to tune their BG control more aggressively because in the seldom case of extreme $\mathrm{CHO}$ overestimation they can rely on the safety feature of the controller.

147

\section{EFFECT OF A MORE AGGRESSIVE PRE-MEAL BOLUS ON MEDTRONIC'S HYBRID CLOSED-LOOP SYSTEM}

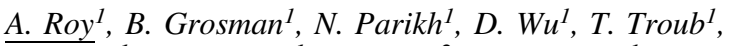

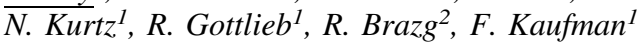

${ }^{1}$ Medtronic, Inc., R\&D, Northridge, USA

${ }^{2}$ Rainer Clinical Research Center, Clinical Research, Renton, USA

Background and Aims: To arrive at appropriate insulin delivery decisions, hybrid closed-loop (HCL) systems require input from continuous glucose monitoring (CGM) sensors and from users who provide the system with estimates of upcoming carbohydrate intake (meal announcements). We investigated whether a more aggressive carbohydrate-to-insulin (CHO:I) ratio

\begin{tabular}{|c|c|c|c|}
\hline \multicolumn{4}{|c|}{ Table 1. Control Types, CHO:I ratios, and Glycemic Outcomes } \\
\hline Experiment days & $1-3$ & $4-7$ & $8-11$ \\
\hline Open-loop or HCL & Open-loop & $\mathrm{HCl}$ & $\mathrm{HCL}$ \\
\hline CHO:I Ratio Category & Patients' Pre-study & Patients' Pre-study & More Aggressive \\
\hline CHO:I Ratio (g/U) & $14.2 \pm 5.1$ & $14.2 \pm 5.1$ & $10.0 \pm 4.2$ \\
\hline \multicolumn{4}{|c|}{ Mean \pm SD Meal-Related SG Values (mg/dL) } \\
\hline Start of meal & $147 \pm 53$ & $148 \pm 41$ & $142 \pm 35$ \\
\hline Postprandial peak & $214 \pm 44$ & $220 \pm 51$ & $194 \pm 41$ \\
\hline 4-h postprandial & $148 \pm 48$ & $152 \pm 56$ & $142 \pm 46$ \\
\hline \multicolumn{4}{|c|}{ Mean $\pm S D$ Overall Percentages of Time Spent in Various $S G$ Ranges } \\
\hline$<70 \mathrm{mg} / \mathrm{dl}$ & $1.8 \pm 1.8$ & $0.5 \pm 0.5$ & $0.8 \pm 0.5$ \\
\hline 70 to $180 \mathrm{mg} / \mathrm{dl}$ & $66.1 \pm 17.5$ & $73.1 \pm 12.4$ & $79.6 \pm 6.6$ \\
\hline$>180 \mathrm{mg} / \mathrm{dl}$ & $32.2 \pm 17.2$ & $26.5 \pm 12.3$ & $19.5 \pm 6.5$ \\
\hline $\begin{array}{l}\text { CHO:1, carbohydrate to insu } \\
\text { SD, standard deviation; SG, }\end{array}$ & $\begin{array}{l}\text { Li, hybrid closed-10op; } \\
\text { glucose }\end{array}$ & & \\
\hline
\end{tabular}

(i.e., more insulin for a given carbohydrate estimate) would safely lower postprandial glycemia in a supervised outpatient setting.

Method: Ten insulin pump users with established CHO:I ratios were studied for 12 days with no activity or dietary restrictions. The 12-day hotel stay was divided into 3 treatment periods: Open-loop control with patients' pre-study CHO:I ratios on days $1-3$, HCL with patients' pre-study CHO:I ratios on days 4-7, and HCL with more aggressive CHO:I ratios that were decreased by an average (range) of $30 \%(18 \%-50 \%)$ on days $8-11$.

Results: Sensor glucose (SG) values from each of the 3 treatment periods were analyzed (Table 1). Compared to openloop control, periods of HCL control were characterized by fewer $\mathrm{SG}$ values $<70 \mathrm{mg} / \mathrm{dL}$. The more aggressive CHO:I ratio was associated with a significant reduction in postprandial peak $\mathrm{SG}$ values $(\mathrm{p}<0.02)$. Transitioning from open-loop to HCL control with patients' pre-study CHO:I ratios was associated with an increase in the percentage of time with SG values in the 70$180 \mathrm{mg} / \mathrm{dL}$ range, as was transitioning from HCL with patients' pre-study CHO:I ratios to HCL with the more aggressive CHO:I ratios $(\mathrm{p}<0.03)$.

Conclusion: The HCL system provided good glycemic control in this supervised outpatient setting. More aggressive premeal bolus dosing attenuated postprandial glycemic excursions without causing additional hypoglycemia.

\section{8}

\section{DOES THE ADDITION OF GLUCAGON TO A CLOSED LOOP SYSTEM IMPACT ON POST EXERCISE GLYCAEMIA?}

S. Seechurn ${ }^{1}$, M. Reddy ${ }^{2}$, N. Jugnee ${ }^{3}$, M. El Sharkawy ${ }^{4}$,

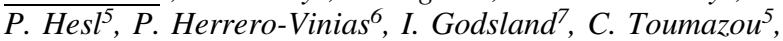
G. Pantelis ${ }^{5}$, N. Oliver ${ }^{7}$

${ }^{1}$ Imperial College, Department of Diabetes, Endocrinology \& Metabolism, London, United Kingdom

${ }^{2}$ Imperial College, Department of Diabetes, Endocrinology \& Metabolism, London, United Kingdom

${ }^{3}$ Imperial College, Dept of Diabetes, Endocrinology \&

Metabolism, London, United Kingdom

${ }^{4}$ Imperial College, Centre for Bioinspired Technology, London, United Kingdom

${ }^{5}$ Imperial College, Centre for Bioinspired Technology, London, United Kingdom

${ }^{6}$ Imperial College, Centre for Bioinspired Technology, Imperial College, London, United Kingdom

${ }^{7}$ Imperial College, Dept of Diabetes, Endocrinology and Metabolism, London, United Kingdom

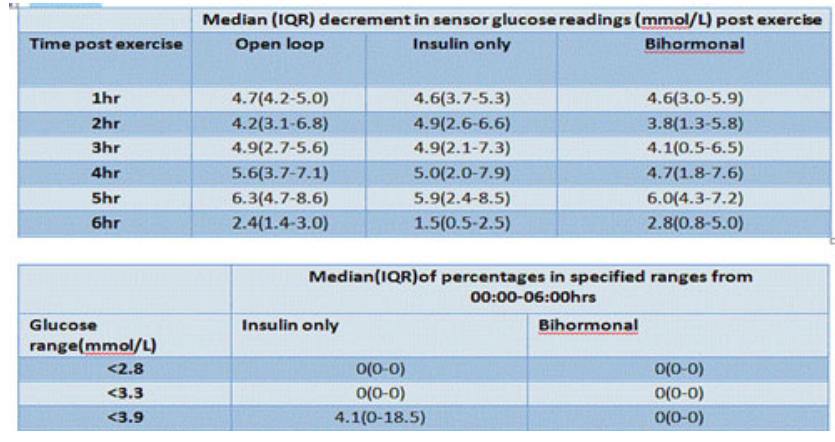


Background and Aims: Exercise is a risk factor for immediate and late hypoglycaemia in type 1 diabetes. Closed loop insulin delivery systems have the potential to optimise glucose control but face challenges in managing day to day variability from food, exercise, stress and other external factors. The addition of glucagon to a closed loop system may enable aggressive management and reduce the risk of hypoglycaemia around exercise.

Method: 6 participants with insulin pump treated type 1 diabetes participated in an overnight 25 hour study on 3 occasions using open-loop (standard pump therapy), insulin only closedloop and the bihormonal closed-loop in random order. Each participant underwent a moderate intensity exercise on a Cosmed static bike 2 hours after lunch. A Dexcom G4 sensor was used with a bio-inspired control algorithm and Roche Accu-Chek Combo pumps.

Results: The decrement in sensor glucose peaks 5 hours post exercise and was at least $5.5 \mathrm{mmol} / \mathrm{L}$. There was no significant difference in the decrement over 6 hours post exercise, and the number of events $<3.9 \mathrm{mmol} / \mathrm{L}$ between insulin only and bihormonal groups.

Conclusion: The addition of glucagon to a closed loop insulin delivery system does not impact on the post-exercise glucose decrement

\section{9}

\section{DOES THE ADDITION OF GLUCAGON TO THE CLOSED LOOP INSULIN PUMP ADD ANY BENEFIT?}

S. Seechurn ${ }^{1}$, M. Reddy ${ }^{2}$, N. Jugnee ${ }^{1}$, P. Herrero ${ }^{3}$, M. El Sharkawy ${ }^{4}$ P. Pesl ${ }^{5}$, I. Godsland 6 , C. Toumazo, G. Pantelis ${ }^{4}, N$. Oliver ${ }^{7}$

${ }^{1}$ Imperial College, Department of Diabetes, Endocrinology \& Metabolism, London, United Kingdom

${ }^{2}$ Imperial College, Department of Diabetes, Endocrinology \& Metabolism, London, United Kingdom

${ }^{3}$ Imperial College, Centre for Bioinspired Technology, London, United Kingdom

${ }^{4}$ Imperial College, Centre for Bioinspired Technology, London, United Kingdom

${ }^{5}$ Imperial, Centre for Bioinspired Technology, London, United Kingdom

${ }^{6}$ Imperial College, Department of Diabetes, Endocrinology \& Metabolism, London, United Kingdom

${ }^{7}$ Imperial College, Diabetes, Endocrinology \& Metabolism, London, United Kingdom

Background and Aims: The ultimate aim of closed loop insulin delivery is to achieve euglycaemia without risk of hypoglycaemia. The addition of glucagon to insulin in a closed loop system -a bihormonal system - may enable this. Our study compares a bihormonal system with an insulin only closed loop and standard insulin pump therapy.

Method: 6 participants with insulin pump treated type 1 diabetes participated in an overnight $25 \mathrm{hr}$ study on 3 occasions using standard pump therapy, insulin only closed loop and the bihormonal closed loop in random order. Participants had a $50 \mathrm{~g}$ carbohydrate lunch, 80g carbohydrate dinner and 40g carbohydrate breakfast. Meals were announced to the controller. Each participant underwent a moderate intensity static bike exercise 2 hours after lunch. A Dexcom G4 sensor was used with a bioinspired control algorithm and two Roche Accu-Chek Combo pumps.

\section{Results:}

\begin{tabular}{|l|l|l||l|}
\hline \multicolumn{2}{|l|}{} & Median (IQR) of percentage of sensor readings in specified range \\
\hline Glucose(mmol/L) & Opened Loop & Insulin Only & Bihormonal \\
\hline$<2.8$ & O(0-0) & $0(0-0.2)$ & $0(0-0)$ \\
\hline$<3.3$ & $0(0-1.0)$ & $0(0-0.2)$ & $0(0-0.2)$ \\
\hline$<3.9$ & $4.7(1.8-12.0)$ & $1.3(0.4-7.2)$ & $2(0.4-3.0)$ \\
\hline $3.9-7.8$ & $48.9(40.7-55.5)$ & $54.1(51.8-56.9)$ & $57.5(51.6-64.4)$ \\
\hline$>7.8$ & $45.1(44.2-46.2)$ & $43.6(39.8-46.5)$ & $36.3(29.9-47.8)$ \\
\hline $3.9-10$ & $64.5(58.4-79.0)$ & $73.3(70.8-82.6)$ & $77.2(70.7-87)$ \\
\hline$>10$ & $23.7(18.5-29.2)$ & $22(15.5-26.8)$ & $16.1(12.0-23.9)$ \\
\hline$>15$ & $0(0-3.6)$ & $0(0-4.5)$ & $0(0-1.7)$ \\
\hline
\end{tabular}

Conclusion: A trend towards reduction in hypoglycaemia $(<3.9 \mathrm{mmol} / \mathrm{l})$ and increased time in target $(3.9-10 \mathrm{mmol} / \mathrm{L})$ was observed with our bihormonal and insulin only closedloop system when compared to open-loop. However, there were no significant differences between insulin only and the bihormonal arms in the number of events in any of the specified ranges but our study was limited by the small population size.

150

\section{ESTIMATES OF DAY-TO-DAY INTRA-PERSONAL VARIABILITY IN OVERNIGHT BASAL INSULIN REQUIREMENT IN CSII THERAPY}

\author{
F.STAHL ${ }^{1}$, R. Johansson ${ }^{1}$, M. Landin-Olsson ${ }^{2}$
}

${ }^{1}$ Lund University, Department of Automatic Control, Lund, Sweden

${ }^{2}$ Lund University, Department of Endocrinology, Lund, Sweden

Background and Aims: Day-to-day variability in insulin basal requirement (IR) is one possible explanation for reoccurring episodes of nocturnal hypoglycemia $(\mathrm{NH})$. This was investigated in CSII patients monitored by CGM, aiming at a correlation analysis between $\mathrm{NH}$ and estimated IR.

Method: 29 patients on CSII therapy, enrolled at Lund University Hospital, Sweden, were monitored at home with the Medtronic Sof (6 patients), Enlite (8 patients) and the Dexcom G4 (15 patients) sensors for more than 36 days. CGM glucose traces for the overnight periods (from midnight until breakfast), together with the corresponding insulin dosage data, were extracted from the data material for each patient. Data that contained reports of meals were discarded.

A nonparametric modeling approach using a combined pharmacokinetic/pharmacodynamics model was used to estimate the insulin action of the rapid-acting insulin and the IR for each patient. The IR estimate was allowed to change in-between days.

Results: The estimated models could reproduce the overnight glucose curves with good accuracy in a leave-one-out crossvalidation test (median RMSE $11.6 \mathrm{mg} / \mathrm{dl}$ ). The corresponding IR estimates displayed a large day-to-day variability with a mean $\mathrm{CV}$ of $26 \%$, and was significantly lower than the average for dates where $\mathrm{NH}$ occurred $(\mathrm{p}<0.001)$.

Conclusion: The results indicate that day-to-day variability in overnight IR can be significant and a possible source to NH due to the associated mismatch between the basal program and the true insulin need. Further research is needed to investigate the underlying reasons and mechanisms to the variability as well as the possibilities to reduce or foresee these changes. 
151

\section{SENSOR OPERATION DURATION DOES NOT AFFECT OVERNIGHT CLOSED-LOOP PERFORMANCE: A RANDOMISED CLINICAL TRIAL}

M. Tauschmann ${ }^{1}$, J.A. Allen ${ }^{1}$, M.E. Wilinska ${ }^{1}$, H. Thabit ${ }^{1}$, C.L. Acerini ${ }^{2}$, D.B. Dunger ${ }^{1}$

${ }^{1}$ University of Cambridge Metabolic Research Laboratories, Institute of Metabolic Science, Cambridge, United Kingdom

${ }^{2}$ University of Cambridge, Paediatrics, Cambridge, United Kingdom

Background and Aims: Closed-loop (CL) systems modulate insulin delivery based on continuous glucose monitor (CGM) sensor values. CGM accuracy varies with sensor life, being least accurate on day 1 of sensor operation. We evaluated the effect of sensor life on CL performance comparing CL efficacy on day 1 of sensor insertion to day 3-4.

Method: In an open-label, two-period cross-over study, 12 adolescents on insulin pump (age 16.7 \pm 1.9 years; HbA1c $66 \pm 10 \mathrm{mmol} / \mathrm{mol}$; duration of diabetes $8.7 \pm 3$.6years; mean \pm SD) attended a clinical research facility on two overnight occasions. In random order, participants received CL on day 1 or on day 3-4 after sensor insertion. During both periods, glucose was controlled by a model predictive algorithm informed by sensor values. Plasma glucose was measured every 30-60min.

Results: During the overnight period (22h30 to $7 \mathrm{~h} 30$ ), mean plasma glucose was $7.9 \pm 1.6 \mathrm{mmol} / \mathrm{l}$ on day 1 of sensor insertion vs $7.8 \pm 1.8 \mathrm{mmol} / 1$ on day $3-4$ of sensor insertion $(\mathrm{p}=0.30)$. Proportion of time spent in the target glucose range (3.9$8.0 \mathrm{mmol} / \mathrm{l})$ was $58 \pm 32 \%$ vs $56 \pm 36 \%(\mathrm{p}=0.34)$. Percentage time above $8.0 \mathrm{mmol} / \mathrm{l}$ was $39 \pm 33 \%$ vs $43 \pm 36 \%(\mathrm{p}=0.53)$, and time spent below $3.9 \mathrm{mmol} / 1$ was $0.0 \%$ [ 0.0 to 3.2 ] vs $0.0 \%$ [0.0 to 1.8 ] (median [IQR]; $\mathrm{p}=0.78$ ). CGM measurements tended to be less accurate on day 1 compared to day 3-4 (mean absolute relative difference between paired CGM and plasma glucose: $16.5 \%$ [12.4 to 26.9] vs $13.0 \%$ [7.4 to 19.3 ], $\mathrm{p}=0.09$ ).

Conclusion: Overnight closed-loop glucose control informed by CGM sensor values on day 1 or day 3-4 after sensor insertion is comparable. Sensor life does not appear to affect closed-loop performance.

\section{2}

GLYCEMIC PROFILES DURING USE OF INSULIN PUMPS WITH AUTOMATED INSULIN MANAGEMENT FEATURES: SUSPEND BEFORE LOW/AUTO-RESUME COMPARED TO SUSPEND ON LOW

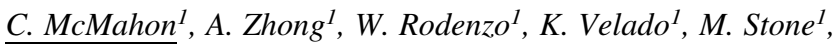
P. Agrawal ${ }^{1}$, B. Kannard ${ }^{1}$, R. Shah ${ }^{1}$

${ }^{1}$ Medtronic - Inc., Diabetes, Northridge, USA

Table. Average Glycemic Parameters with Different Insulin Delivery Strategies. MiniMed 640G: Suspend before Low and Auto-Resume; MiniMed Veo: Low Glucose Suspend

\begin{tabular}{|c|c|c|c|c|}
\hline Parameter: Mean & $\begin{array}{c}\text { MiniMed } \\
640 G\end{array}$ & $\begin{array}{c}\text { MiniMed } \\
\text { Veo }\end{array}$ & $\begin{array}{c}\Delta, \text { Intra-User } \\
\text { Difference }\end{array}$ & $\mathbf{p}$ \\
\hline Hypoglycemia, min/day & 43 & 68 & -25 & $<0.001$ \\
\hline Hyperglycemia, min/day & 46 & 50 & -4 & 0.01 \\
\hline Hypoglycemia AUC, mg/dL $\times$ day & 0.32 & 0.44 & -0.12 & $<0.001$ \\
\hline Hyperglycemia AUC, mg/dL $\times$ day & 2.06 & 2.47 & -0.45 & $<0.001$ \\
\hline Hypoglycemic excursions per day & 0.87 & 1.13 & -0.26 & $<0.001$ \\
\hline Hyperglycemicexcursions per day & 0.48 & 0.54 & -0.06 & $<0.001$ \\
\hline
\end{tabular}

SG, sensor glucose: AUC, area under the glucose concentration-time curve.

Hypoglycemia defined as SG $\leq 3.9 \mathrm{mmol}$ ( $(570 \mathrm{mg} / \mathrm{dL}$ ): hyperglycemia defined as SG $\geq 16.7 \mathrm{mmolh}$ ( $\geq 300 \mathrm{mg} / \mathrm{dL}$ ). Hypoglycemic and hyperglycemic excursions defined as $\geq 2$ consecutive hypoglycemic or hyperglycemic SG values.
Background and Aims: The MiniMed 640G system includes Suspend before Low, which suspends insulin delivery in advance of predicted hypoglycemia, and Auto-resume, which automatically restarts basal insulin upon recovery. Its Suspend on Low feature is similar to the Low Glucose Suspend (LGS) feature of the Veo pump system. We compared the real-world outcomes of users in Europe and Australia who switched to MiniMed 640G from the MiniMed Veo system.

Method: We identified 851 people who had uploaded $\geq 7$ days of data from each of the systems to the CareLink personal database before 9/29/2015. Hypoglycemia and hyperglycemia were defined as sensor glucose (SG) values $\leq 3.9$ and $\geq 16.7$ $\mathrm{mmol} / \mathrm{L}$ ( $\leq 70$ and $\geq 300 \mathrm{mg} / \mathrm{dL}$ ), respectively. We compared hypoglycemia and hyperglycemia rates while on the different pump systems using paired t-tests.

Results: Glycemic outcomes are compared in the Table. Of the 851 users, $672(79 \%)$ experienced less hypoglycemia with the $640 \mathrm{G}$ system. While on Veo, $86 \%$ of the users enabled LGS (an average of $82 \pm 30 \%$ of the time) and $100 \%$ of the $640 \mathrm{G}$ users enabled one or more of the advanced insulin management features (an average of $88 \pm 24 \%$ of the time for Suspend before Low).

Conclusion: Users of the $640 \mathrm{G}$ system with its Suspend before Low feature who switched from the Veo system had lower rates of hypoglycemia, without increasing hyperglycemia. Automatic suspension and resumption of insulin delivery is a viable strategy for improving glycemic outcomes in insulin-requiring patients.

\section{3}

\section{REAL-WORLD ASSESSMENT OF AUTOMATIC INSULIN RESUMPTION ALGORITHM}

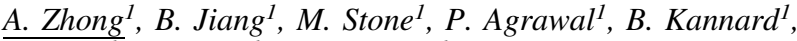

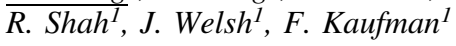 \\ ${ }^{1}$ Medtronic - Inc., Diabetes, Northridge, USA
}

Background and Aims: The MiniMed 640G system includes the SmartGuard feature that allows for the automatic suspension of insulin delivery using a predictive low glucose management (PLGM) algorithm; basal insulin delivery can be resumed manually at any time, or automatically if recovery from hypoglycemia is predicted. Glycemic outcomes from manual resumption and automatic pump resumption strategies were compared.

Method: Data from 9,584 patients who voluntarily uploaded to the CareLink Personal Database (Medtronic MiniMed, Inc., Northridge, CA) were evaluated. Manually-resumed and automatically-resumed events were compared. Recovery time was defined as the interval from pump resumption to the start of the first 20-minute interval with sensor glucose (SG) values 3.8-6.6 $\mathrm{mmol} / \mathrm{L}$. Moderate and extreme hypoglycemia were defined as

Table. Parameters Associated with Manual vs. Automatic
Resumption Strategies for Basal Insulin Delivery
\begin{tabular}{|c|c|c|c|c|}
\hline & $\begin{array}{c}\text { Manual } \\
\text { Resumption }\end{array}$ & $\begin{array}{c}\text { Automatic } \\
\text { Resumption }\end{array}$ & $\Delta, \%$ & $p$ \\
\hline Pump Suspension Events, N & 66,374 & 101,162 & 52.4 & \\
\hline Predictive SG Suspend Threshold (mg/dL), \\
mean \pm SD & $65.2 \pm 8.5$ & $65.2 \pm 8.5$ & 0.0 & 0.99 \\
\hline Duration of pump suspension (min), mean \pm SD & $32.6 \pm 28.9$ & $57.5 \pm 24.8$ & 43.3 & $<0.001$ \\
\hline Recovery time (min), mean & 42.0 & 30.4 & -27.6 & $<0.001$ \\
\hline Extreme hypoglycemia (\% of SG values) & 1.2 & 0.4 & -66.7 & $<0.001$ \\
\hline Moderate hypoglycemia (\% of SG values) & 7.2 & 3.9 & -45.8 & $<0.001$ \\
\hline Euglycemia (\% of SG values) & 73.0 & 73.0 & 0.0 & 0.54 \\
\hline
\end{tabular}


$\mathrm{SG}<3.8$ and $<2.7 \mathrm{mmol} / \mathrm{L}$, respectively; euglycemia was defined as $\mathrm{SG}$ values from $3.8-10.0 \mathrm{mmol} / \mathrm{L}$.

Results: Of the 167,536 predictive pump suspension events analyzed, $39.6 \%$ were manually resumed and $60.4 \%$ were automatically resumed. Of the manually-resumed events, $18.2 \%$ were resumed immediately after the suspension event started and $56.4 \%$ were resumed within 30 minutes. . Compared to automatically-resumed events, manually-resumed events had shorter durations, longer recovery times, and more time in extreme and moderate hypoglycemia (Table). Time in control was comparable between two methods (73.0\% for both automatic resumption and manual resumption).

Conclusion: Users of the MiniMed 640G system and its automated insulin management features may benefit from increased reliance on the algorithm governing automatic resumption of insulin delivery and decreased reliance on manual resumption.

\section{4}

\section{REAL-WORLD PERFORMANCE OF A PREDICTIVE LOW-GLUCOSE MANAGEMENT ALGORITHM IN PEDIATRIC PATIENTS}

A. Zhong ${ }^{1}$, C. McMahon ${ }^{1}$, W. Rodenzo ${ }^{1}, K$. Velado $^{1}$, M. Stone ${ }^{1}$,

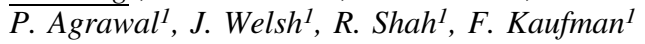

${ }^{1}$ Medtronic - Inc., Diabetes, Northridge, USA

Background and Aims: The MiniMed 640G system allows for automatic suspension of insulin delivery before hypoglycemia occurs by employing a predictive low glucose management (PLGM) algorithm. We evaluated implications of routine use of this feature by analyzing sensor glucose $(\mathrm{SG})$ values that were voluntarily uploaded to the CareLink Personal database by patients whose self-reported age was $<16$ years.

Method: Hypoglycemia and hyperglycemia were defined as SG values below or above various threshold SG values, respectively, and were compared for time intervals in which the feature was enabled versus intervals when it was not.

Results: Data were analyzed from 587 pediatric patients who uploaded into the CareLink system between 1/13/2015 and 8/26/ 2015 (26,490 patient-days), 75.5\% of time with PLGM on which resulted in 64,833 predictive suspension events (3.2 per patientday). With respect to hypoglycemia, $\mathrm{SG}$ values $\leq 2.7 \mathrm{mmol} / \mathrm{L}$ occurred $0.4 \%$ of the time when the feature was enabled vs. $0.9 \%$ of the time when it was disabled $(\mathrm{p}<0.001)$. SG values $\leq 4.4$ $\mathrm{mmol} / \mathrm{L}$ occurred $5.3 \%$ of the time when the feature was enabled vs. $8.0 \%$ of the time when it was disabled $(\mathrm{p}<0.001)$. With respect to hyperglycemia, SG values $\geq 13.3 \mathrm{mmol} / \mathrm{L}$ occurred $15.0 \%$ of the time when the feature was enabled vs. $15.5 \%$ of the time when it was disabled $(\mathrm{p}=0.7)$. SG values $\geq 16.7 \mathrm{mmol} / \mathrm{L}$ occurred $4.8 \%$ of the time when the feature was enabled vs. $5.4 \%$ of the time when it was disabled $(\mathrm{p}=0.5)$.

Conclusion: In children, routine use of the PLGM algorithm appears to lessen severe and moderate hypoglycemia with no significant increase in hyperglycemia.

\section{5}

\section{EXPERIENCE OF PARENTS AND THEIR SUPPORTIVE ROLE FOR CHILDREN WITH TYPE 1 DIABETES}

\section{G. Aparício $^{1}$, M. Macedo Silva ${ }^{1}$, E. Batoca Silva ${ }^{1}$}

${ }^{1}$ Polytechnic Institute of Viseu, Health School, Viseu, Portugal

Background and Aims: Type 1 Diabetes Mellitus is a growing chronic disease that affects increasingly more children, involving changes in their lifestyle, social relationships and economic costs. Its effects are felt not only by children/adolescents but also by their parents, who provide support by their parental role.

To identify feelings experienced by parents after the child diagnosis and reflect on the influence of care provided by health professionals in the acceptance and adaptation to diabetes.

Method: Qualitative/phenomenological study developed using semi structured interviews and content analysis in 14 parents of children/adolescents with Type 1 diabetes, which are monitored in a Central hospital in Portugal.

Results: Seven analysis categories emerged: impact of diabetes in family, feelings/emotions experienced by parents, changes in family life, diet and parenting role, experiences with siblings, fears, social support and opinion regarding health professionals support. These categories showed that they felt the implications of diabetes in personal and social life, requiring restructuring of family dynamics and also changes in all family diet. From the feelings experienced by parents, stands the fear and anxiety regarding the future of the child due to inherent complications of diabetes. We also found that parents felt that the health professional's support, particularly by nurses is very important in the acceptance and adaptation to diabetes.

Conclusion: This research can be a useful help for health professionals in clinical practice helping them to reflect and minimize the adverse emotional effects of diabetes and to promote the development of the parental role providing a better quality of life.

156

SYSTEM FOR ENABLING CLINICIANS TO RELATE TO A MOBILE HEALTH APP: PRELIMINARY RESULTS OF THE NORWEGIAN TRIAL IN THE EU FI-STAR PROJECT

\section{Bradway ${ }^{1}$, A. Grøttland ${ }^{1}$, H. Blixgård ${ }^{1}$, A. Giordanengo ${ }^{1}$, E. Arsand ${ }^{1,2}$ \\ ${ }^{1}$ University Hospital of North Norway, Norwegian Centre for Interated Care and Telemedicine, Troms $\phi$, Norway ${ }^{2}$ University of Troms $\phi$, Department of Clinical Medicine, Troms $\phi$, Norway}

Background and Aims: Since the rise of the fast-paced environment of mobile self-management technologies, such as diabetes apps, the medical world has begun to adapt. It is becoming increasingly important to involve clinicians in the development and assessment of such tools.

Method: Together with patients and clinicians, we developed a secure, comprehensive and patient-initiated data-sharing system, allowing the data (BG, insulin boluses, physical activity, and diet) gathered from a patient's own Diabetes Diary

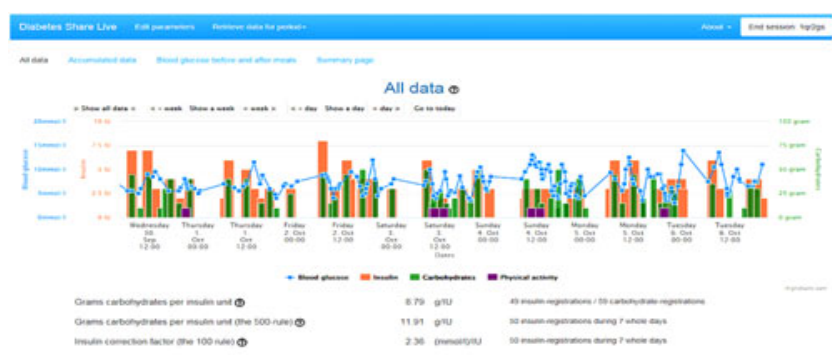
Figure 1: Print-screen of the Diabetes Share Live analysis system, enabling the clinicians to view and
analyze the patient-gathered diabetes data from their Diabetes Diary mobile app. 
smartphone app (DD) to be transferred to a web-based portal, the Diabetes Share Live (DSL). Surveys distributed during the trial assessed medical personnel's $(n=3)$ experiences during initial (T0) and follow-up (T3=3months) consultations per patient $(n=20)$ using the DD\&DSL System (Figure 1).

Results: Topics included: 1) how DD\&DSL supported consultation aims and 2) useful functionalities. Percent responses (Yes, No) were compared for cases when DD\&DSL was fully utilized, i.e. excluding "NA" $(n=15$ at T0, $n=10$ at T3). Clinicians reported that DD\&DSL increased their understanding of patients' situations (23\% increase of "Yes" responses, T0 to T3) and patients' understanding of their own situations (10\% increase of "Yes" responses, T0 to T3). Clinicians consistently reported "Cumulative-BS" as a useful functionality $(100 \%$ at $\mathrm{T} 0$ and $\mathrm{T} 3)$. Free-text responses explain that "correct factors for carbo- and insulin-sensitivity require the patient to log all data" and "looking at graphs and trends together gave more overview".

Conclusion: This study suggests that, up to 3-months, if patients provide enough data through systems such as DD\&DSL, clinicians can successfully use these tools to better relate to patients and their smartphone apps.

157

\section{DEVELOPING METHODS FOR NON-INVASIVE ASSESSMENT OF SKIN MICROCIRCULATION IN PATIENTS WITH TYPE 2 DIABETES}

\author{
D. Kulikov $^{1}$, A. Glazkov ${ }^{2}$, P. Kulikova ${ }^{1,2}$, Y. Kovaleva ${ }^{1}$, \\ A. Dreval
}

${ }^{1}$ Moscow Regional Research and Clinical Institute MONIKI, Endocrinology department, Moscow, Russia

${ }^{2}$ Moscow Regional Research and Clinical Institute MONIKI,

Laboratory of medical and physics research, Moscow, Russia

Background and Aims: Laser Doppler flowmetry (LDF) is a widely known method for the microcirculation assessment. The prospects for its use in evaluation of microcirculation in patients with diabetes are extremely broad. Unfortunately, high variability of the measurements makes this method inappropriate for clinical application.

The aim of this research is to develop methods for assessment of cutaneous blood microcirculation in patients with type 2 diabetes (DM) using LDF. The best way of doing this is reducing the variability of measurements and increasing the difference between healthy and ill individuals.

Method: We achieved this goal by applying combined functional tests (using postural-heating impacts) and calculating diagnostic indexes. 10 patients with DM and 10 healthy volunteers were included in this study. Blood microcirculation was measured on the lower limbs using LAKK-02 complex.

Results: Developed algorithms allowed us to reveal significant differences between the groups. Median perfusion during combination of impacts was $18.1[13.9 ; 22.4]$ perfusion units (PU) in control group and $10.8[9.1 ; 13.9]$ PU in diabetes group $(\mathrm{p}=0,023, \mathrm{U}$-test). Calculation of relative microcirculation index (Irel) allowed us to increase the difference between groups: 5,6 $[4,3 ; 7,5]$ in control group and $2,1[1,4 ; 3,3]$ in diabetes patients $(\mathrm{p}=0,001, \mathrm{U}$-test). This index also allowed us to classify patients into groups with $80 \%$ sensitivity and specificity.

Conclusion: Our results can be the first step on the way to the clinical use of LDF for diabetes patients.
158

VARIATION IN POINT-OF-CARE TESTING OF HBA1C
IN DIABETES CARE IN GENERAL PRACTICE

\section{T. Kristensen $^{1}$ \\ ${ }^{1}$ Institute of Public Health, Research Unit of general practice, Odense, Denmark}

Background and Aims: Point-of-care testing (POCT) for HbA1c may result in improved diabetic control, better patient outcomes and enhanced clinical efficiency with fewer patient visits and subsequent reductions in costs. In 2008, the Danish regulators agreed to create a new fee for the remuneration of POCT of HbAlc in primary care.

The aim of this study is to describe and analyze the variation in use of POCT of HbAlc among diabetes patients in Danish general practice and municipalities.

Method: We use register data from the year 2011 to define a population of 172,906 diabetes patients. The POCT fee is used to measure the amount of POCT of HbA1c among diabetes patients. Next we apply descriptive statistics to analyze variation in the prevalence of POCT versus laboratory testing at patient, clinic and municipality level. We include patient characteristics such as gender, age, socioeconomic markers, health care utilization, case mix markers and municipality classifications.

Results: Only the Capital Region of Denmark has allowed GPs to use this new incentive for POCT. There were significant variations in the use of POCT across Danish regions, municipalities, clinics and patients. The number of diabetes patients per 1000 patients was larger in POCT clinics than Non-POCT clinics.

Conclusion: It is relevant to reassess the system for POCT of $\mathrm{HbA} 1 \mathrm{c}$ in general practice across the five Danish regions and remedy variation in use of POCT of HbA1c in the Capital Region to avoid variation that is based on local medical opinion and/or supply of resources rather than patients' health care needs and preferences.

\section{9}

\section{DIABETIC AUTONOMIC CARDIAC NEUROPATHY COULD BE EARLY DETECTED BY USING TWENTY-FOUR HOURS HEART RATE VARIABILITY ANALYSIS}

\author{
I. Kurcalte $^{1}$, A. Lejnieks $^{1}$, I. Zarina-Tonne ${ }^{2}$, A. Kalinin ${ }^{1}$, \\ O. Kalejs ${ }^{2}$, R. Erts ${ }^{2}$ \\ ${ }^{1}$ Riga East Clinical University Hospital, Clinic of \\ cardiovascular diseases, Riga, Latvia \\ ${ }^{2}$ Riga Stradins University, Internal diseases, Riga, Latvia
}

Background and Aims: Introduction: Cardiac autonomic neuropathy $(\mathrm{CAN})$ is often underestimated diabetes-related long term complication. CAN has been detected at time of diagnosis in type 2 diabetes patients (T2DM). CAN can occur before DM is evident clinically. During CAN sub-clinical phase, heart rate variability (HRV) can help to detection CAN before disease is symptomatic. Cardiac autonomic reflex tests (CARTs) and shortterm HRV are recommended for CAN diagnostics.

Aim: to compare twenty-four hours HRV parameters in patients with T2DM and without and with CARTs in DM patients.

Method: In cohort of 51 T2DM patients and 49 age and gender matched controls HRV (5 Time, 4 Frequency Domain, 2 circadian indexes) computed from 24-hours ECG monitoring (Holter, HM) was compared between groups and with 2 CARTs (deep breathing, 
lying-to-standing). No patient had decompensated heart, kidney, endocrine or other disease which could affect HRV.

Results: Only 32 (62.7\%) DM patient had one or both positive CARTs (CARTpos), whereas all patients had changed one and more 24-hours HRV parameter, a sum of those was higher in T2DM patients $(4[4.5]$ vs 1 [2], $\mathrm{p}<.001)$ and CARTpos patients $(5[5]$ vs $3[2], p<.01)$. ROC curve showed fair diagnostic effectiveness of $\mathrm{HM}$ based $\mathrm{HRV}$ for $\mathrm{CAN}$ detection $(\mathrm{AUC}=.74$ $(.583 ; .899)$ with cut-off point of 4 positive tests (sensitivity $72 \%$, specificity $71 \%$, p-.002).

Conclusion: HRV should be analysed for every DM patient exposed to $\mathrm{HM}$ for cardiac autonomic function evaluation. Inclusion of 24-hours HRV in T2DM patient investigation could improve CAN detection at early subclinical stages. 24-hours HRV can be discussed for DM patients with negative CARTs.

\section{0}

\section{INTELLIGENT MONITORING SYSTEM OF PREVENTION OF THE DIABETIC FOOT}

C.R. Maia Leite ${ }^{1}$, R.A. Medeiros ${ }^{\text {I, S.R.F. Rosa }}{ }^{2}$, J. Dullius ${ }^{3}$, L.D.A. Santana ${ }^{4}$

${ }^{1}$ UERN, INFORMATIC, Mossoró, Brazil

${ }^{2}$ University of Brasilia, Faculty of Gama, Brasília, Brazil

${ }^{3}$ University of Brasilia, Coordinator of Doce Desafio Program Diabetes Education and Physical Activities, Brasília, Brazil

${ }^{4}$ UNB, University of Brasilia-Campus Ceilândia, Brasília, Brazil

Background and Aims: Neuropathies and/or angiopathies are complications of diabetes that result in changes in the lower limbs who subsequently evolve for the diabetic foot, a problem which represents one of the most devastating complications of diabetes and which can lead to, amputation and even death. Given the context, the objective of this work is to develop an Intelligent Monitoring System Diabetic Foot Prevention (SIM2PeD), allowing the personalized care from routine of each individual.

Method: The SIM2PeD (Figure 1) consists of an integrated platform with mobile device capture data of individuals entitled SIM2PeD Mobile, and a web device for monitoring the medical team called SIM2PeD Web. In SIM2PeD Mobile individuals receive alerts on precautions in accordance with its location and activity. After the capture of information is passed on to the expert system (intelligent module) that generates recommendations from answered care. The Web SIM2PeD generates graphics of tracking of device usage for the medical staff through answers.

Results: The experiments conducted in real environment led to the conclusion that the system performance satisfactory and suitable for the remote monitoring of self-care activities in patients with any disease and presents great potential for application in various treatments.

Conclusion: Experiments were carried out in order to verify the performance and dynamics of the foot care system use through mobile SIM2PeD in patients with that problem.

\section{1}

\section{SCREENING FOR AUTONOMIC CARDIOVASCULAR NEUROPATHY IN PATIENTS WITH TYPE 1 DIABETES MELLITUS}

\author{
R. Mukharyamova ${ }^{1}$, F. Valeeva $^{1}$, S. Mayanskaya \\ ${ }^{1}$ Kazan State Medical University, Therapy, Kazan, Russia
}

Background and Aims: Cardiovascular autonomic neuropathy (CAN) increases morbidity and mortality in diabetes and has great predictive power for cardiovascular events. The aim of our study was the optimizing of the diagnostics of CAN in patients with type 1 diabetes mellitus (DM).

Method: The study involved the examination of 103 randomly type 1 (insulin-dependent) DM patients (60 males) with average age $37,1 \pm 10,4$, average duration of DM 10,3 $\pm 3,4$. It employed tests of autonomic function (30:15, E/I ratio, Valsalva ratio and postural change in systolic blood pressure) and 24-h heart rate variability spectral analysis. Special autonomic tests diagnostic scales (ATDS) that calculate R-R intervals ratio during the tests on the basis of ECG data were also used. CAN was defined as the presence of at least 3 abnormalities among 7 indices.

Results: 37 (35.9\%) patients were suffering CAN in the research group. Mention of CAN in diagnosis was found only in 3 $(8,1 \%)$ patients. Among patients with CAN the disease duration was more than 5 years in 32 (86\%). 25 cases of CAN (67\%) were diagnosed on the basis of the autonomic tests data and by using ATDS. Among the tests of autonomic function, E/I ratio showed the highest sensitivity $(92,3 \%)$ and specificity $(86 \%)$.

Conclusion: The results indicate that the prevalence of CAN in DM is high with low level of diagnostics. Autonomic tests can be used as a screening in patients with DM duration over 5 years. The use of ATDS can accelerate and optimize the diagnostic process of CAN in patients with type 1 diabetes.

\section{2}

\section{PREVENTION OF METABOLIC SYNDROME AND COMPONENTS IN SUBJECTS WITH IMPAIRED FASTING GLUCOSE BY TELEPHONE-DELIVERED LIFESTYLE INTERVENTION USING SELF-HELP DEVICES}

N. Sakane $^{1}$, K. Kotani $^{1,2}$, K. Takahashi ${ }^{1,3}$, Y. Sano $^{1,4}$, K. Tsuzaki $^{1}$,

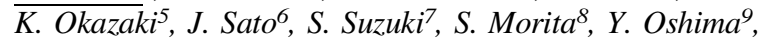
K. Izumi ${ }^{10}$, M. Kato ${ }^{11}$, N. Ishizuka ${ }^{12}$, M. Noda ${ }^{11}$, H. Kuzuya ${ }^{1,13}$

${ }^{1}$ Institute of Clinical Research Center- NHO Kyoto Medical Center, Department of Preventive Medicine, Kyoto, Japan ${ }^{2}$ Jichi Medical University, Division of Community and Family Medicine-, Shimotsuke, Japan

${ }^{3}$ Hyogo Health Service Association, Kobe, Japan

${ }^{4}$ Kanagawa University of Human Services Faculty of Health \& Social Services School of Nutrition \& Dietetics, Yokosuka, Japan ${ }^{5}$ Nagoya University Graduate School of Medicine, Department of Development for Community-oriented Healthcare System, Nagoya, Japan

${ }^{6}$ Nagoya University Graduate School of Medicine, Department of General Medicine/Family and Community Medicine,

Nagoya, Japan

${ }^{7}$ Nagoya City University Graduate School of Medical Sciences, Department of Public Health, Nagoya, Japan

${ }^{8}$ Kyoto University Graduate School of Medicine, Department of Biomedical Statistics and Bioinformatics, Kyoto, Japan

${ }^{9}$ University of Marketing and Distribution Sciences, Kobe, Japan

${ }^{10}$ National Center for Global Health and Medicine, Department of Diabetes and Metabolic Medicine, Tokyo, Japan

${ }^{11}$ Diabetes Research Center- National Center for Global Health and Medicine, Department of Diabetes Research, Tokyo, Japan ${ }_{12}^{12}$ ancer Institute hospital, Clinical trial department, Tokyo, Japan

${ }^{13}$ Takeda Hospital, Kyoto, Japan

Background and Aims: To determine the effects of one-year telephone-delivered lifestyle intervention on the development of 
metabolic syndrome (MetS) in subjects with impaired fasting glucose (IFG).

Method: The prospective, cluster-randomized, controlled trial, the Japan Diabetes Outcome Intervention Trial-1 (J-DOIT1), included 43 groups (clusters) from health care divisions and 1,597 subjects aged 20-65 years with IFG and without Mets were randomized to a telephone-delivered intervention arm $(n=753)$ or a self-directed control arm with self-help devices $(n=844)$. After setting goals, a weight scale and pedometer with a storage function were provided. They could send accumulated data to the lifestyle support center via a transmitter. The health care provider set the personal action plan and monitored progress toward each subject's goals regularly. The primary outcome was the development of MetS. The secondary outcomes were the improvement of metabolic components.

Results: During the median follow-up period of 4.9 years, $5.8 \%$ of the intervention arm and $8.1 \%$ of the control arm developed MetS. Overall, the hazard ratio (HR) for MetS development was $0.75(95 \%$ confidence interval $(\mathrm{CI}), 0.52-1.09 ; \mathrm{P}=0.14)$ in the intervention group. The HR in subjects with a body mass index (BMI) $\geq 23$ was significantly reduced to 0.63 (95\% CI, 0.41-0.95; $\mathrm{P}=0.03$ ), but not in subjects with $\mathrm{BMI}<23$. The intervention reduced the proportion of higher serum triglyceride levels.

Conclusion: The telephone-delivered lifestyle intervention using self-help devices effectively prevented the development of MetS in overweight and obese subjects and reduced serum triglyceride levels.

\section{3}

\section{AMPK PHOSPHORYLATION OF FXR AT THREONINE} CONTROLS HEPATIC GLUCOSE PRODUCTION

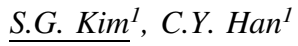 \\ ${ }^{1}$ Seoul National University, College of Pharmacy, Seoul, \\ Republic of Korea
}

Background and Aims: Farnesoid X receptor (FXR) controls the expression of hepatic genes involved in energy metabolism. Nevertheless, the regulatory signal of FXR in glucose-lowering effect and the underlying mechanisms are largely unknown. Given the functional link between SIRT1 and AMPK, this study investigated the effect of AMPK on the phosphorylation of FXR and examined its role for SIRT1-mediated hepatic glucose metabolism.

Method: Immunoblotting, reporter gene, real-time PCR and functional assays were done to evaluate FXR activation and glucose metabolism.

Results: The overexpression of SIRT1 increased FXR response element-dependent transactivation, whereas a deficiency of SIRT1 had the opposite effect. SIRT1-mediated FXR transactivation was significantly blocked by the inhibition of AMPK. The impact of SIRT1 and AMPK on the regulation of FXR function was strengthened by the pharmacological effect of ajoene. AMPK directly phosphorylated FXR at Thr 156 residue as shown by in vitro kinase, oligopeptide competition and point-mutation analyses. The overexpression of FXR-T156A mutant abolished AMPK-mediated FXR phosphorylation and the binding of FXR to co-activator. The inhibition of glucose-6-phosphatase expression or hepatocyte glucose production by CA-AMPK or ajoene was abrogated by either FXR knockdown or FXR-T156A mutant overexpression, supporting that AMPK phosphorylation of FXR contributes to the control of glucose production in hepatocytes. Finally, ajoene treatment lessened hyperglycemia and sensitized insulin signaling in high fat diet-induced obese mice.
Conclusion: AMPK phosphorylates FXR at Thr156 downstream of SIRT1, which leads to the inhibition of hepatic glucose production.

\section{4}

\section{ORTHOSTATIC HYPOTENSION IN DIABETIC PATIENTS: 10-YEAR FOLLOW-UP STUDY}

L. Gaspar ${ }^{1}$, P. Kruzliak ${ }^{2}$, A. Komornikova ${ }^{1}$, Z. Celecova ${ }^{1}$,

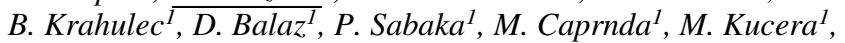
L. Rodrigo ${ }^{3}$, M. Soucek ${ }^{2}$, Y. Uehara $^{4}$, A. Dukat ${ }^{1}$

${ }^{1}$ Comenius University and University Hospital, 2 nd Department of Internal Medicine, Bratislava, Slovakia

${ }^{2}$ St. Anne's University Hospital, 2nd Department of Internal Medicine, Brno, Czech Republic

${ }^{3}$ Central University Hospital of Asturias HUCA, Department of Gastroenterology, Oviedo, Spain

${ }^{4}$ Faculty of Home Economics- Kyoritsu Women's University, Division of Clinical Nutrition, Tokyo, Japan

Background and Aims: Cardiovascular autonomic neuropathy in diabetics is a common but often underestimated and underdiagnosed complication of diabetes mellitus. One of the most clinical apparent forms of cardiovascular autonomic neuropathy is orthostatic hypotension.

To retrospectively assess the association of the orthostatic hypotension $(\mathrm{OH})$ with macrovascular and microvascular complications of diabetes mellitus and to determine its effect on mortality.

Method: We retrospectively analysed 187 patients with diabetes mellitus (60 patients with diabetes type 1 and 127 patients with diabetes type 2). Patients were divided into groups according to presence or absence of $\mathrm{OH}$ and type of diabetes. Association of $\mathrm{OH}$ with macrovascular and microvascular complications was evaluated and the effect of $\mathrm{OH}$ on 10-year allcause mortality was also assessed.

Results: $\mathrm{OH}$ was present in $31.7 \%$ of patients with diabetes type 1 (DM1) and in $32.3 \%$ of patients with diabetes type 2 (DM2). $\mathrm{OH}$ was positively associated with the prevalence of myocardial infarction in DM1 $(\mathrm{OR}=10.67)$ and with prevalence of stroke in DM2 $(\mathrm{OR}=3.33)$. There was also a strong association of $\mathrm{OH}$ and the prevalence of peripheral artery disease in both DM1 $(\mathrm{OR}=14.18)$ and DM2 $(\mathrm{OR}=3.26)$. Patients with both types of diabetes and $\mathrm{OH}$ had significantly higher prevalence of nephropathy (DM1 OR=8.68, DM2 OR=3.24), retinopathy (DM1 OR=8.09, DM2 OR=4.08) and peripheral neuropathy (DM1 OR=17.14, DM2 OR=7.51). Overall 10 year mortality rate was higher in diabetic patients with $\mathrm{OH}$.

Conclusion: Presence of $\mathrm{OH}$ in diabetics is associated with higher prevalence of macrovascular and microvascular complications of diabetes mellitus and also with higher 10-year mortality.

\section{5}

\section{AN INTEGRATED APPROACH TO THE TREATMENT OF OBESITY IN ADOLESCENTS LIVING IN THE SAMARA REGION}

E. Mikhailova $^{1}$, T. Kaganova ${ }^{2}$, G. Sabirova ${ }^{1}$, $\overline{\text { A. Kuluashova }}^{1}$, O. Pyrenkova ${ }^{1}$

${ }^{1}$ Samara Children's Hospital 1, Endocrinology, Samara, Russia ${ }^{2}$ Samara State medical university, Pediatric, Samara, Russia 
Background and Aims: According to statistics, over the last decade the incidence of overweight in children and adolescents of the Samara region has grown from 15 to $21 \%$, leading to an increase in the incidence of type 2 diabetes from 0.2 to $1 \%$ of the number of newly diagnosed cases.

Objective: To evaluate the effectiveness of an integrated approach to the treatment of obesity in the Samara region.

Method: To evaluate the efficacy of the treatment of obesity in 30 adolescents involving a nutritionist, a child psychologist, endocrinologist for 6 months. The control group of 25 patients was observed only by endocrinologist. The study undertook teenagers at high risk of developing metabolic syndrome. Assessed performance indicators BMI, the ratio of fat and muscle tissue measured by bioimpedance measuring "MEDASS ABC-01," satisfaction with the quality of life assessment using a questionnaire Peds QL. Consultation endocrinologist, nutritionist monthly, psychologist advised patients twice. All patients were trained in the school of obesity.

Results: In the study group the reduction of BMI was $2,3 \pm 1,0$; in the control group - $1,0 \pm 0,8, \mathrm{p}<0,05$; The decrease in BMI was noted by adipose tissue. In assessing the results of questionnaires in the intervention group became significantly higher self-esteem, the ability to integrate into society.

Conclusion: An integrated approach to obesity therapy is recommended to enhance its effectiveness and reduce the risk of type 2 diabetes.

\section{6}

\section{MORIN RESTORES AN IMPAIRED FAT AND GLUCOSE METABOLISM IN HIGH-FAT DIET-INDUCED OBESE MICE}

\section{J. Naowaboot $^{1}$, S. Wannasiri ${ }^{1}$}

${ }^{1}$ Thammasat University, Preclinical Science, Pathumthani, Thailand

Background and Aims: Morin, a natural bioflavonoid and found in the Moraceae family, has several properties such as antioxidant, anticarcinogenic and anti-inflammatory activities. The present study was designed to evaluate the effect of morin on impaired fat and glucose metabolism in mice fed with a high-fat diet.

Method: Five-week-old ICR mice were fed with normal or high-fat ( $60 \mathrm{kcal} \%$ fat) diet for 12 weeks. After first 6 weeks of induction, mice were divided into 4 groups of 8 mice each: normal control mice, obese control mice, and obese mice treated with morin (50 and $100 \mathrm{mg} / \mathrm{kg} / \mathrm{day})$.

After 6 weeks of treatments, the fasting blood glucose, serum insulin, serum leptin, intraperitoneal glucose tolerance test (IPGTT), serum lipid profile, hepatic glycogen content, and hepatic lipogenic genes and gluconeogenic enzyme protein expression were determined.

Results: After 6 weeks of treatment, morin significantly reduced the elevated blood glucose and serum leptin levels, and lowered insulin resistance. Moreover, the serum lipid profiles were reduced. Interestingly, morin reduced the expression of hepatic lipogenic genes; sterol regulatory element binding protein 1c (SREBP1c), fatty acid synthase (FAS), and acetyl-CoA carboxylase (ACC). The effect of morin on regulating glucose homeostasis was also investigated, we found that morin increased the hepatic glycogen contents and suppressed the protein expression of gluconeogenic enzyme; phosphoenolpyruvate carboxylase (PEPCK).

Conclusion: Our findings demonstrate that morin improves fat and glucose homeostasis in HFD-induced obesity by suppressing the lipogenesis and gluconeogenesis activities in liver tissue.

167

\section{DIABESITY: METHODS OF CONTROL FOR PREVENTION OF DIABETES IN OBESE PEOPLE}

\section{I.C. Okonkwo $^{1}$}

${ }^{1}$ Pirogov National Medical University, Internal Medicine, Vinnitysa, Ukraine

Background and Aims: About 10 obese patients were examined in Ukraine, 10 in the United States and 10 in Africa from 2013-2015. Patients were also examined for diabetes using different diagnostic methods such as random blood sugar test, Fasting blood sugar test, oral glucose tolerance test and glycated hemoglobin test. Family history and other anamnesis was collected including their feeding habits. During research we found out that the socio-economic relationship especially between the high and middle class contribute hugely to increase in obesity.

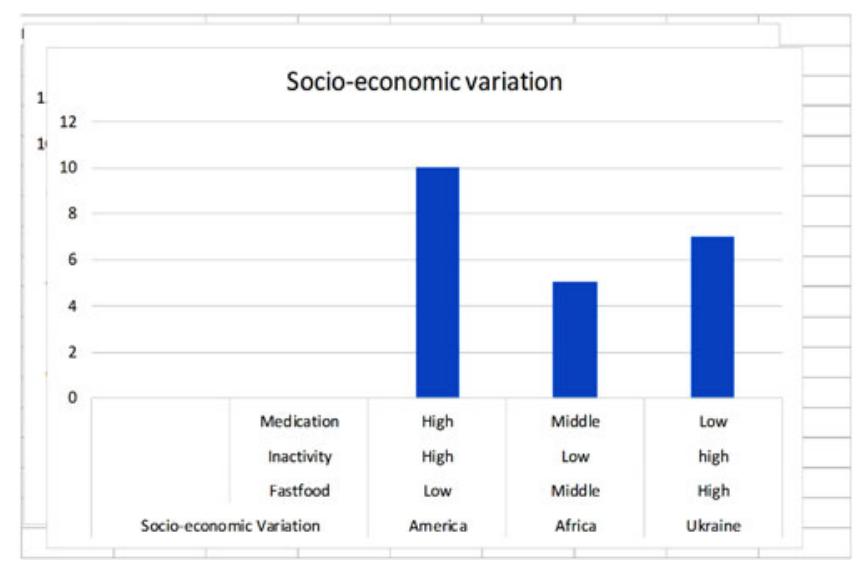

Results: Lifestyle - Particularly diet and how frequently we eat are given emphasis in the developed world today. Physical Activity- As noted in the case study, this plays an important role in obesity and diabetes. Socio-economic variation- The relationship between the high, middle, and low class should be designed in a way that benefits all in the society. Medications: Hormonal and other medications should be recommended and indicated.

Conclusion: Control of obesity in a diabetic or nondiabetic patient can be controlled if socio-economic problems are addressed with the right educational facts and theories. Everyone has a role to play from the government to the high, middle, and low class. For patients with type 2 diabetes maintaining a regular medical checkup and following the advice of their physicians are recommended. 
168

\section{CYTOKINE PROFILE IN PATIENTS WITH TYPE 2} DIABETES MELLITUS

K. Sharafetdinov $^{1,2}$, O. Plotnikova $^{1}$, I. Semenchenko ${ }^{1}$, $\overline{V .}$ Pilipenko $^{1}, T$. Sentsova ${ }^{3}$

${ }^{1}$ Institute of Nutrition of Russian Academy of Sciences, Department of Metabolic Diseases, Moscow, Russia ${ }^{2}$ Russian Medical Academy of Post-Graduate Education, Chair of Dietology and Nutriciology, Moscow, Russia ${ }^{3}$ Institute of Nutrition of Russian Academy of Sciences, Department of Clinical Biochemistry- Immunology and Allergology, Moscow, Russia

Background and Aims: To investigate cytokine profile in patients with type 2 diabetes mellitus (DM) and varying degrees of obesity.

Method: 91 patients with type 2 DM were examined. All patients were receiving standard antihyperglycemic therapy; mean age was $46.1 \pm 0.82$ years, mean duration of disease was $7.2 \pm 1.43$. They were divided into the following groups according to their degree of obesity: grade I obesity $(n=30)$, grade II obesity $(n=31)$, grade III obesity $(n=28)$ and 18 healthy volunteers. The patients were underwent biochemical analysis, determination of the cytokine profile and estimation of TNF- $\alpha$, IL-1, IL-4, TGF- $\beta 1$ by enzyme-linked immunosorbent assay.

Results: It is shown that excess in body weight is accompanied by increased of proinflammatory cytokines (TNF- $\alpha$, IL-1, TGF- $\beta 1$ ) due to lower the reduction of inflammatory cytokines (IL-4) in patients with type 2 DM and obesity.

Conclusion: The data obtained indicates that cytokine profile play a critical part in pathogenesis of type $2 \mathrm{DM}$ in association with obesity.

169

\section{CALIBRATIONS OF DEXCOM G4 PLATINUM REDUCED TO ONE PER DAY BY A TIME-VARYING DAY-SPECIFIC BAYESIAN PRIOR}

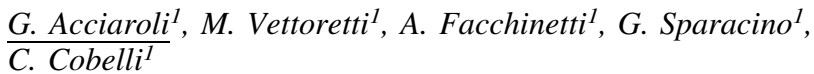

${ }^{1}$ University of Padova, Information Engineering, Padova, Italy

Background and Aims: In CGM sensors, the current signal generated by glucose-oxidase is transformed to glucose concentration by a calibration function whose parameters are periodically updated matching SMBG references, suitably collected by the patient, usually twice a day. The aim is to reduce such a frequency to once a day, by refining a recent calibration algorithm, while preserving accuracy.

Method: The database consists of 57 CGM signals collected by the Dexcom G4® Platinum for 7 days. The Bayesian calibration algorithm (Vettoretti et al., IEEE Trans Biomed Eng, 2015) was further developed by adding a prior specific for the first 12h (Acciaroli et al., Proc IEEE EMBS 2015). Available BG references on days 1, 4 and 7 were used to assess the CGM accuracy achievable with Bayesian calibrations every $12 \mathrm{~h}$ and $24 \mathrm{~h}$ and with the original manufacturer calibration (every $12 \mathrm{~h}$ ) by calculating metrics such as Mean Absolute Relative Difference (MARD), Percentage of Accu- rate Glucose Estimates (PAGE) and percentage of data lying in the A-zone of the Clarke Error Grid (CEGA-A).

Results: Considering Bayesian calibration, a non statistically significant worsening of accuracy is noted passing from $12 \mathrm{~h}$ (11.67\% MARD) to $24 \mathrm{~h}$ (11.94\% MARD) inter-calibration interval. Notably, CGM accuracy with $24 \mathrm{~h}$ inter-calibration interval is statistically better ( $p$-value of 0.0342) than that obtained with the original manufacturer calibration $(13.05 \%$ MARD).

Conclusion: For the Dexcom G4® Platinum sensor, the use of a time-varying calibration function and suitable Bayesian priors allows to halve the frequency of calibrations from 2 to 1 per day.

\section{0}

IMPACT OF SHORT-TERM PROFESSIONAL CONTINUOUS GLUCOSE MONITORING ON GLYCEMIC CONTROL AND HYPOGLYCEMIA AMONG PATIENTS WITH TYPE 1 DIABETES

\section{A. Al Hayek ${ }^{1}$, F. Al Sabaan ${ }^{2}$, M. Al Dawish ${ }^{2}$}

${ }^{1}$ Prince Sultan Military Medical City, Diabetes Treatment Center- Department of Endocrinoloy and Diabetes, Riyadh, Kingdom of Saudi Arabia

${ }^{2}$ PSMMC, Endocrinology, Riyadh, Kingdom of Saudi Arabia

Background and Aims: The aim of this study was to find out the evolving role of professional continuous glucose monitoring (PCGM) on hemoglobin A1c (HbA1c) and the frequency of hypoglycemia.

Method: This was a 3-month, prospective study conducted among patients (aged 15-24 years) with type 1 diabetes mellitus who attended a diabetes clinic [Diabetes Treatment Center, Prince Sultan Military Medical City (PSMMC), Saudi Arabia] due to recurrent unexplained hypoglycemia

\begin{tabular}{|c|c|c|c|c|c|c|}
\hline MERRIC & DAY & $\begin{array}{l}\text { Fatory allibation } \\
\text { (12h frequency) }\end{array}$ & $\begin{array}{l}\text { Baysiancalthation } \\
\text { (12hfrequenoy) }\end{array}$ & $\begin{array}{l}\text { Byejian collodion } \\
\text { (24htriequency) }\end{array}$ & $\begin{array}{l}\text { pralue (fratory } \\
\text { vi. Bajesian 12h) }\end{array}$ & $\begin{array}{l}\text { pralue(fatory } \\
\text { va Bajesian 24h) }\end{array}$ \\
\hline MARD & $\begin{array}{l}1 \\
4 \\
7 \\
1,47\end{array}$ & $\begin{array}{l}15.98 \\
9.07 \\
8.81 \\
13.05\end{array}$ & $\begin{array}{l}12.65 \\
9.06 \\
8.77 \\
11.67\end{array}$ & $\begin{array}{l}13.06 \\
9.24 \\
9.69 \\
11.94\end{array}$ & $\begin{array}{l}0.0063 \\
0.1832 \\
0.6421 \\
0.0248\end{array}$ & $\begin{array}{l}0.0243 \\
0.4154 \\
0.9651 \\
0.0342\end{array}$ \\
\hline PAGE & $\begin{array}{l}1 \\
4 \\
7 \\
1,4,7\end{array}$ & $\begin{array}{l}76.92 \\
93.33 \\
93.75 \\
78.01\end{array}$ & $\begin{array}{l}85.11 \\
95.83 \\
95.83 \\
88.65\end{array}$ & $\begin{array}{l}80.95 \\
95.83 \\
94.00 \\
87.50\end{array}$ & $\begin{array}{l}0.0026 \\
0.0023 \\
0.4684 \\
0.0097\end{array}$ & $\begin{array}{l}0.0057 \\
0.0186 \\
0.7427 \\
0.0099\end{array}$ \\
\hline CEGAA & $\begin{array}{l}1 \\
4 \\
7 \\
1,4,7\end{array}$ & $\begin{array}{l}81.25 \\
91.67 \\
93.62 \\
77.78\end{array}$ & $\begin{array}{l}89.13 \\
93.62 \\
91.49 \\
87.22\end{array}$ & $\begin{array}{l}85.42 \\
95.45 \\
91.84 \\
85.71\end{array}$ & $\begin{array}{l}0.0181 \\
0.1485 \\
0.9439 \\
0.0792\end{array}$ & $\begin{array}{l}0.0303 \\
0.0814 \\
0.8412 \\
0.0757\end{array}$ \\
\hline
\end{tabular}


unawareness episodes between July 2014 and December 2014. The respondents were purposively and conveniently selected and they were asked to wear the PCGM device (iPro ${ }^{\circledR} 2$; Medtronic MiniMed, Inc., Northridge) for 5 days. The PCGM results were collected by the diabetic educator and reviewed by the treating physician on the same day as removal of the device. Clinical and demographic data were also collected.

Results: Overall, 56 patients were included in the study. The mean $( \pm \mathrm{SD})$ age of the study cohort was $18.1 \pm 1.82$ years and $27(48.2 \%)$ patients were male. Compared with baseline, nonsignificant but positive differences were observed in HbAlc levels in both male and female patients and in those who were older (aged 20-24 years). Similar results were observed in the frequency of hypoglycemia and a significant change was observed for female patients $(\mathrm{P}<0.05)$. Compared with baseline, a significant positive difference was observed in patients' overall frequency of hypoglycemia by the end of the study $(\mathrm{P}<0.001)$.

Conclusion: PCGM is a valuable tool for detecting episodes of hypoglycemia and may help to decrease HbAlc levels and reduce the frequency of hypoglycemia.

171

\section{PATIENT FEEDBACK WITH THE FIRST 90-DAY IMPLANTABLE GLUCOSE SENSOR IN THE PRECISE STUDY}

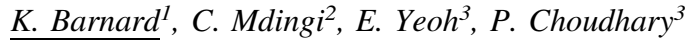 \\ ${ }^{1}$ Bournemouth University, Faculty of Health \& Social Sciences, \\ Bournemouth, United Kingdom \\ ${ }^{2}$ Senseonics Ltd, Clinical Sciences Department, Germantown, \\ USA \\ ${ }^{3}$ Kings College London, Diabetes Centre, London, \\ United Kingdom
}

Background and Aims: A new implantable CGM system consisting of a glucose sensor, body-worn smart transmitter and smartphone app was assessed. This analysis reports participant feedback on use of the system continuously for 90 days during the multisite PRECISE study.

Method: A study-specific quantitative and qualitative psychosocial and attitudinal questionnaire was administered to participants with $\mathrm{T} 1$ and $\mathrm{T} 2$ diabetes. The questionnaire included questions on participants' impression of the features and usability of the CGM system.

Results: Fifty participants across the UK $(n=10)$ and Germany $(n=40)$ completed the questionnaire. Of these, 45 had T1D, 5 had T2D and were equally split between MDI and insulin pump therapy regimen ( $n=25$ each). Forty-four percent $(44 \%$, $n=22$ ) were experienced (current or previous) CGM users and $56 \%(n=28)$ were inexperienced (first time) CGM users. Overall, the system was rated highly on ease of use, convenience and comfort with $92 \%$ indicated that they did not experience pain or discomfort when using the sensor. $84 \%$ would choose to be inserted again with $93 \%$ of CGM naïve participants (86\% experienced) reporting minimized burden of diabetes. Experienced vs inexperienced CGMs users had similar ratings with noted dissimilarity in transmitter comfort ( $82 \%$ vs $71 \%)$, alarm warned of highs/lows ( $81 \%$ vs $96 \%$ ), and would use everyday for diabetes management (93\% vs $77 \%)$.
Conclusion: The CGM sensor was acceptable to participants and using the system was associated with minimized burden of diabetes. Psychosocial functioning and factors important to quality of life were positively associated with the device for users.

\section{2}

\section{PSYCHOSOCIAL BENEFITS ASSOCIATED WITH IMPLANTABLE LONG-TERM CGM SENSOR}

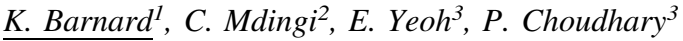

${ }^{1}$ Bournemouth University, Faculty of Health \& Social Sciences, Bournemouth, United Kingdom

${ }^{2}$ Senseonics, Clinical Sciences Department, Germantown, USA

${ }^{3}$ Kings College London, Diabetes Centre, London, United Kingdom

Background and Aims: The aim of the current study was to explore the psychosocial outcomes associated with wearing an implantable CGM.

Method: Participants involved in the PRECISE study were asked to complete questionnaires to assess factors important to diabetes-related quality of life (QOL) after the use of the CGM system for approximately 90 days.

Results: Fifty-one participants across UK $(n=10)$ and Germany $(n=41)$ completed the questionnaires. Of these, $90 \%$ had $\mathrm{T} 1 \mathrm{D}, 51 \%$ followed an insulin pump therapy regimen, and $45 \%$ of the participants were previous CGM users.

CGM Impact Scale results show $86 \%(n=44)$ of participants reported feeling better (14\% neutral) about their diabetes control with $90 \%$ CGM naïve participants and $81 \%$ experienced CGM users reporting increased confidence about their diabetes. As well as $73 \%(n=37)$ feel more safe $(27 \%$ neutral $)$ while sleeping and $78 \%(n=39)$ are confident $(22 \%$ neutral $)$ about avoiding serious hypoglycemia. Previous CGM users showed the same results as first time CGM users $(p=0.45)$. Responses correspond with an average improvement in HbA1c from 7.51 to $7.05(\mathrm{p}<.0001)$ over the 90 days use of the CGM. Eighty-four percent $(84 \%)$ would choose to be inserted again.

Diabetes related distress data was comparable for all participants with no deterioration associated with implantable CGM use. $93 \%$ of first time users and $77 \%$ experienced CGM users would like to continue using the system to help manage their diabetes more effectively.

Conclusion: Participants reported positive experiences of using a novel implantable CGM over a 90 day period. Benefits included increased self-efficacy and reduced diabetes related burden whilst using the system.

\section{3}

\section{PERFORMANCE EVALUATION OF THREE BLOOD GLUCOSE METERS SYSTEMS}

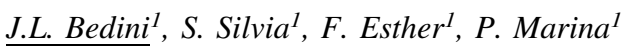 \\ ${ }^{1}$ Hospital Clínic, Laboratorio Core, Barcelona, Spain
}

Background and Aims: To evaluate the performance of three different blood glucose monitoring systems (BGMS), Contour ${ }^{\circledR}$ XT (Bayer Healthcare), OKmeter Cameo ${ }^{\circledR}$ (OK Biotech) ${ }^{\circledR}$ and TRUEresult ${ }^{\circledR}$ (Nipro Diagnostics) by measuring imprecision 
and accuracy in comparison with the hexokinase (HK) local method to determine if they fulfilled the following accuracy criteria:

ISO 15197:2013. $\geq 95 \%$ of results must be within either $\pm 15 \mathrm{mg} / \mathrm{dL}$ of the analyzer result, for glucose $<100 \mathrm{mg} / \mathrm{dL}$, or $\pm 15 \%$ for glucose $\geq 100 \mathrm{mg} / \mathrm{dL}$.

\section{FDA Draft SMBG/OTC 2014 Guidance}

a) $\geq 95 \%$ of the results must be within $\pm 15 \%$ of the analyzer results (entire range).

b) $\geq 99 \%$ of the results must be within $\pm 20 \%$ (entire range).

Clinical Laboratory Standards Institute (CLSI)

a) $\geq 95 \%$ of the results must be within either $\pm 12 \mathrm{mg} / \mathrm{dL}$ of the analyzer result, for samples $<100 \mathrm{mg} / \mathrm{dL}$, or $\pm 12.5 \%$ for samples $\geq 100 \mathrm{mg} / \mathrm{dL}$.

b) $\leq 2 \%$ of results present differences $>20 \%$ when glucose is $\geq 75 \mathrm{mg} / \mathrm{dL}$ or $>15 \mathrm{mg} / \mathrm{dL}$ when glucose is $<75 \mathrm{mg} / \mathrm{dL}$.

Method: Imprecision was evaluated by measuring two glucose concentration levels 20 times. Accuracy was measured by comparing results with those obtained with a Dimension EXL (DEXL) analyzer (Siemens Healthcare) that uses HK for glucose measurement. One hundred samples (lithium heparin) were analyzed in all the meters and the DEXL. Time from the first meter result to the time blood was centrifuged before being analyzed in the DEXL analyzer was always less than 15 minutes.

\section{Results:}

\begin{tabular}{|c|c|c|c|c|c|c|c|}
\hline & \multicolumn{3}{|l|}{ Ingrevision CV(y) } & & & \\
\hline & & Lowlevel $(70 \mathrm{mg} / \mathrm{d}$ (b) & \multicolumn{2}{|c|}{ Highters:(120 mg/oll) } & & & \\
\hline \multicolumn{2}{|c|}{ ContourXT } & 18 & \multicolumn{2}{|c|}{2,1} & & & \\
\hline \multicolumn{2}{|c|}{ ORMeter Cameo } & 5,9 & \multicolumn{2}{|c|}{4,2} & & & \\
\hline \multicolumn{2}{|c|}{ TRUEresult } & 5,6 & \multicolumn{2}{|c|}{4,1} & & & \\
\hline Criteria & Definicion & & & $\begin{array}{l}\text { Ninimum \% } \\
\text { Accepitable }\end{array}$ & $\begin{array}{l}\text { \%obtinined } \\
\text { Contour XT }\end{array}$ & $\begin{array}{l}\text { Sottained } \\
\text { OKmeter }\end{array}$ & $\begin{array}{l}\text { Yobtained } \\
\text { TRUEresult }\end{array}$ \\
\hline 150 & $\pm 15 \mathrm{mg} / \mathrm{dl} / \mathrm{l}$ & $100 \mathrm{~m} / \mathrm{d} / \mathrm{L} / \mathrm{or}+15 \% / \mathrm{lbb}$ & ( $\mathrm{me} / \mathrm{dll})$ & 95,0 & 99 & 75 & 81 \\
\hline ASt- & $\pm 12 \mathrm{me} / \mathrm{dl} / \mathrm{l}$ & $400 \mathrm{me} / \mathrm{dl} / \mathrm{or}+12.5 \%$ (1) & $100 \mathrm{~m} / \mathrm{dll}$ ) & 95,0 & 99 & 67 & 74 \\
\hline aSt-b & $>15 \mathrm{me} / \mathrm{dl} / \mathrm{k}$ & $75 \mathrm{me} / \mathrm{dl|} \mid \mathrm{or}>20 \mathrm{~S}$ (lab? & $\mathrm{me} / \mathrm{dl}$ ) & $\Omega$ & 0 & 14 & 11 \\
\hline FDA-d & s15\%/entire & & & 95,0 & 99 & 69 & 72 \\
\hline FDA-b & so\%/entire & & & 99,0 & 99 & 82 & 84 \\
\hline
\end{tabular}

Conclusion: Only Contour ${ }^{\circledR}$ XT fulfills all the accuracy criteria. It is necessary to evaluate BGMS under routine conditions to assess their quality.

\section{4}

AN AVERAGE DAILY NUMBER OF STEPS NEGATIVELY CORRELATES WITH AN AVERAGE GLYCEMIC VALUE IN TYPE I DIABETIC PATIENTS: COMPARISON BETWEEN CGM AND PEDOMETER RECORDS

J.Broz ${ }^{1}$, A. Holubova ${ }^{2}$, J. Muzik ${ }^{2}$, M. Oulicka ${ }^{2}$, M. Muzny ${ }^{2}$,

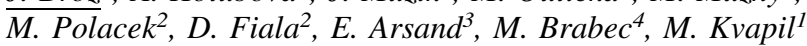

${ }^{1}$ Second Medical Faculty- Charles University, Department of Internal Medicine, Prague, Czech Republic

${ }^{2}$ Czech Technical University in Prague, Faculty of Biomedical Engineering, Prague, Czech Republic

${ }^{3}$ UiT - The Arctic University of Norway, Department of Clinical Medicine, Tromso, Norway

${ }^{4}$ Academy of Science the Czech Republic, Institute of Computer Science, Prague, Czech Republic

Background and Aims: Physical activity has great impact on blood glucose levels in patients with diabetes mellitus. The objective of the study was to determine the daily steps taken and to analyze their effect on glycemic values of Type I diabetic patients.

Method: 34 patients with type 1 diabetes have been included into the study (20 women and 14 men). Data from 31 of these patients have been evaluated. Each patient was equipped with an electronic pedometer Flex (Fitbit, Inc.) and a continuous glucose monitoring system Guardian REAL-Time (Medtronic Inc.). Multiple regression analysis and GAM (Generalized additive model) were used for the statistical model-based data processing.

Results: The system was used by patients for approx. $5.82 \pm 0.89$ days on average. In total, 120 days of usage were evaluated. The average number of steps per patient was $10,285 \pm 3172 / 24$ hours, the lowest average daily steps among the patients was 4241 and the largest number was 15,790. We proved a negative correlation between the average number of steps per day and the average daily blood glucose value (GAM). The relationship between the intraday numbers of steps and the blood glucose levels was not found.

Conclusion: The performed simulations show a correlation between the average number of steps per day and the average daily blood glucose values. This finding is in concordance with general knowledge about relationship between glycemia and physical activity but also suggests that pedometer could identify days (those with less physical activity) with increased needs of insulin.

175

\section{MARD RANGE OF GUESSING BLOOD GLUCOSE VALUES IN PEOPLE WITH T1DM}

\author{
E. Budiman ${ }^{1}$ \\ ${ }^{1}$ Abbott Diabetes Care, Alameda, USA
}

Background and Aims: Many people with type 1 diabetes mellitus (T1DM) believe they can accurately estimate their glucose value. However, studies show that $<40 \%$ guesses fall within the Clarke Zone A. A recent study of blood glucose monitoring (BGM) systems correlate $6.5 \%$ as the lower estimate of mean absolute relative deviation (MARD) whose $95 \%$ of the points fall within ISO15197:2013 boundaries $( \pm 15 \mathrm{mg} / \mathrm{dL}$ below $100 \mathrm{mg} / \mathrm{dL} ; \pm 15 \%$ otherwise $)$. A recollection-based model is postulated to estimate the MARD range of guessing BG.

Method: The model takes recent SMBG measurements around similar time-of-day from $\mathrm{N}$ past days as the input, and takes the $X^{\text {th }}$ percentile of these values as the blood glucose (BG) estimate. $\mathrm{N}$ values from 1 to 9 , and $\mathrm{X}$ values at $5 \%$ increments from $0 \%$ to $100 \%$ are examined. $6482 \mathrm{BG}$ values from $60 \mathrm{~T} 1 \mathrm{DM}$ patients in a previously published study are used to develop the 


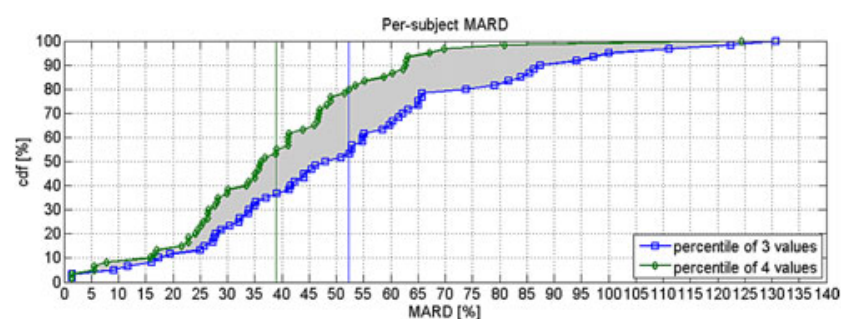

model. Aggregate Clarke Zones from self-estimation studies by T1DM patients are used to evaluate the model.

Results: Setting $X=45$ percentile and $N$ at either 3 or 4 past values provided the best Clarke Zone pattern agreement to self-estimation study results. These models predict an average $( \pm$ standard deviation) per-subject MARD of guessing in people with T1DM to be between $39 \%( \pm 21 \%)$ and $52 \%( \pm 28 \%)$. The averages are represented by vertical lines.

Conclusion: On average, guessing may result in 6 to 8 times worse MARD in patients with T1DM relative to using BGM systems whose $95 \%$ of the points are within the ISO15197:2013 boundaries $( \pm 15 \mathrm{mg} / \mathrm{dL}$ below $100 \mathrm{mg} / \mathrm{dL} ; \pm 15 \%$ otherwise).

176

\section{CONTINUOUS NON-INVASIVE GLUCOSE MONITORING BY SENSOR FUSION OF NEAR INFRARED LIGHT AND BIOIMPEDANCE MEASUREMENTS: RESULTS OF A PROOF OF CONCEPT STUDY}

S.M. Carlsen $^{1}$, S.C. Christiansen ${ }^{1}$, A.L. Fougner ${ }^{2}$, T. Karstang ${ }^{3}$, O.M. Staal ${ }^{3}$

${ }^{1}$ Trondheim University Hospital, Dept of Endocrinology, Trondheim, Norway

${ }^{2}$ Norwegian University of Science and Technology, Department of Engineering Cybernetics, Trondheim, Norway

${ }^{3}$ Prediktor Medical AS, Gamle Fredrikstad, Fredrikstad, Norway

Background and Aims: The GlucoPred (size of a large watch) is a new device that placed on the skin continuously measures glucose levels by combining data (sensor fusion) from near infrared (NIR) spectroscopy and bioimpedance measurements.

Method: Nine patients with diabetes type 1 wore 6 GlucoPreds symmetrically on the extremities, and a total of 12 placements were investigated. Five glucose intervals ranging from hypoglycemia $(\leq 2.8 \mathrm{mmol} / \mathrm{L})$ to hyperglycemia $(\geq 14.0$ $\mathrm{mmol} / \mathrm{L})$ were investigated. Intravenous glucose levels were measured every 5 minute throughout the procedure. Cross validation was used: Data from each sensor was randomly split in four sections, and three sections were used to calibrate the sensor against measured intravenous glucose levels which then were used by the GlucoPred to estimate the glucose levels in the fourth section. For sensor fusion, data from one sensor was used for calibration, and then performance was analyzed on another sensor (position).

Results: Analysing data from each sensor individually, using 4 cross-validation segments, shows that $91.8 \%$ of the bio-impedance and $95.5 \%$ of the NIR glucose estimates fell within zone A or B of the Consensus Error Grid. For sensor fusion, trained/ calibrated with data from one sensor and tested on another sensor, $87.5 \%$ of glucose estimates fell in zone A and B. The results also indicate that the best sensor position is at the anterior/posterior upper arm.

Conclusion: The performance of GlucoPred in this short term study is comparable to commercially available subcutaneous continuous glucose measuring devices.

177

\section{METABOLIC EVALUATION IN A STANDARD CLINICAL SETTING OF A GROUP OF PATIENTS 7 YEARS AFTER A CLINICAL TRIAL WITH SENSOR-AUGMENTED PUMP}

M. Domínguez-Löpez ${ }^{1}$, M. Ruiz de Adana-Navas ${ }^{1}$, N. Colomo ${ }^{1}$, M. Carera $^{2}$

${ }^{1}$ Hospital Regional Universitario, Endocrinología, Málaga, Spain ${ }^{2}$ Psychology department, Endocrinology, Malaga, Spain

Background and Aims: Several trials showed that SAP therapy is safe and effective to reduce hypoglycemic events and improve glycemic control with high treatment satisfaction. Nevertheless patient adherence and sensor use in trials usually decrease with time.

Our objective was to evaluate metabolic control and adherence to SAP therapy 7 years after initiation of SAP in a clinical trial.

Method: In 2008 we recluited 30 patients in treatment with insulin pump (CSII) to start SAP for 6 months. After the trial the patients had the opportunity to maintain the SAP treatment, but only 15 patients desired to go on with SAP therapy despite a high global evaluation of the device. We evaluate the 25 patients again 7 years after returning to the standard clinical follow-up.

Results: 7 years after returning to standard clinical care, only 6 of the 30 patients $(20 \%)$ were still continuosly using the sensor. The HbA1c in patients in treatment with CSII was $7.8 \%$ and in SAP patients was $7.5 \%$ with a statistically significant difference of $0.3 \%$. No statistically differences were found in quality of life between CSII group and SAP group. Patients in SAP therapy were using it mean $60 \%$ of the time. Most problems were referred with accuracy and alarms and daily life interference (noSAP group).

Conclusion: Our results show that in standard clinical setting still long-time benefits can be found in type $1 \mathrm{DM}$ patients treated with SAP after a clinical trial. Nevertheless, new strategies to improve patient adherence to SAP should be developed.

178

\section{CONTINUOUS GLUCOSE MONITORING: PATIENTS' PERCEPTION AND BARRIERS TO ADHERENCE}

A.El-Laboudi $^{1}$, D. Neal ${ }^{2}$, N. Oliver ${ }^{1}$

${ }^{1}$ Imperial College London, Diabetes- Endocrinology \& Metabolic Medicine, London, United Kingdom

${ }^{2}$ The NIHR/Wellcome Trust Imperial Clinical Research

Facility, London, United Kingdom

Background and Aims: Despite evidence for benefit from continuous glucose monitoring (CGM), it has not been widely implemented in clinical practice. The aim of this mixed-method study was to capture people with type 1 diabetes and caregivers' views of CGM, aiming to identify barriers to CGM use in adults and children.

Method: An online questionnaire was designed using ranking questions (quantitative) and open-ended questions (qualitative) 


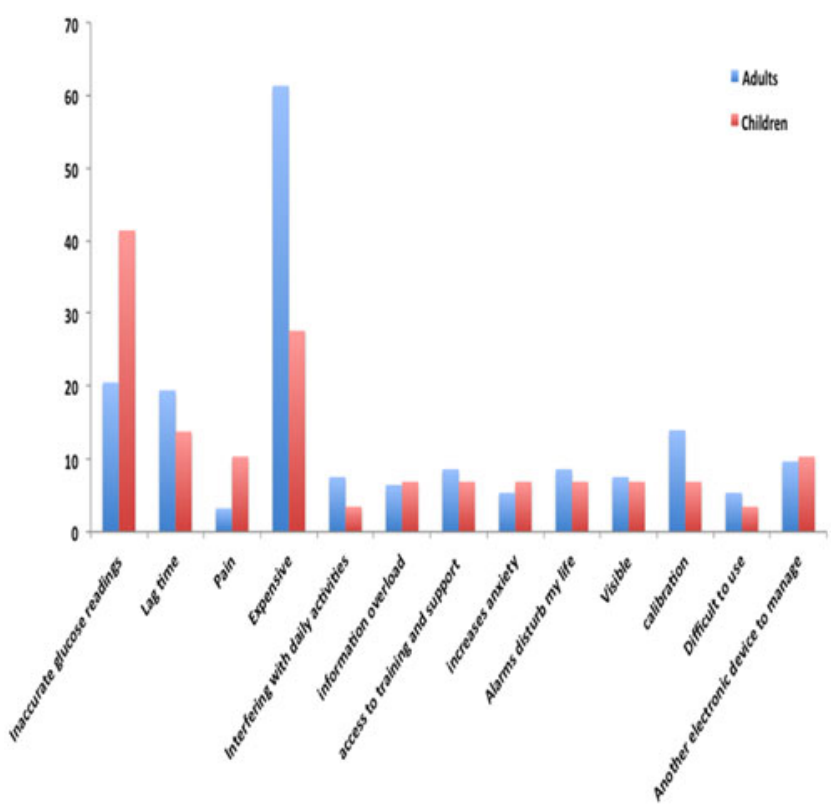

to evaluate respondents' perception of CGM. Quantitative data were analysed using Excel and SPSS 21.0. Qualitative data were analysed according to the principles of thematic analysis using Atlas.ti software.

Results: There were a total of 214 respondents (83\% adults and $17 \%$ caregivers of children). The majority were people with type 1 diabetes $(92.5 \%)$, living in the UK $(74.8 \%)$, and either current or previous CGM users $(57 \%)$.

The majority of adult patients with current or previous experience in CGM (64\%) ranked cost as the top limitation to CGM use. This was followed by lack of accuracy (21\%). This order was reversed for the children group, where CGM inaccuracy was ranked as the most important limitation (43\%) followed by cost (28\%) (Figure 1). Further analysis revealed that $33.3 \%$ of adult respondents from North America ranked cost as the top limitation compared to $67.7 \%$ in UK.

These findings were confirmed by qualitative data analysis.

Conclusion: Cost and inaccuracy represent important barriers to compliance with CGM use. However, their relative importance varies according to age of CGM-users and their geographical location.

179

\section{AMBULATORY GLUCOSE PROFILE CHARACTERIZATION OF JUVENILE DIABETES RESEARCH FOUNDATION-CONTINUOUS GLUCOSE MONITORING TRIAL DATA}

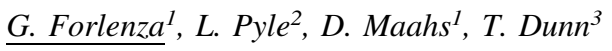

${ }^{1}$ University of Colorado Denver, Barbara Davis Center, Aurora, USA

${ }^{2}$ University of Colorado Denver, Pediatrics/Biostatistics and Informatics, Aurora, USA

${ }^{3}$ Abbott Diabetes Care, Diabetes Care, Alameda, USA

Background and Aims: The purpose of this analysis is to evaluate the Ambulatory Glucose Profile (AGP) characteristics from the JDRF-CGM trial dataset for the study-specified age (8-14, 15-24 and over 25 years) and A1c groups (above and

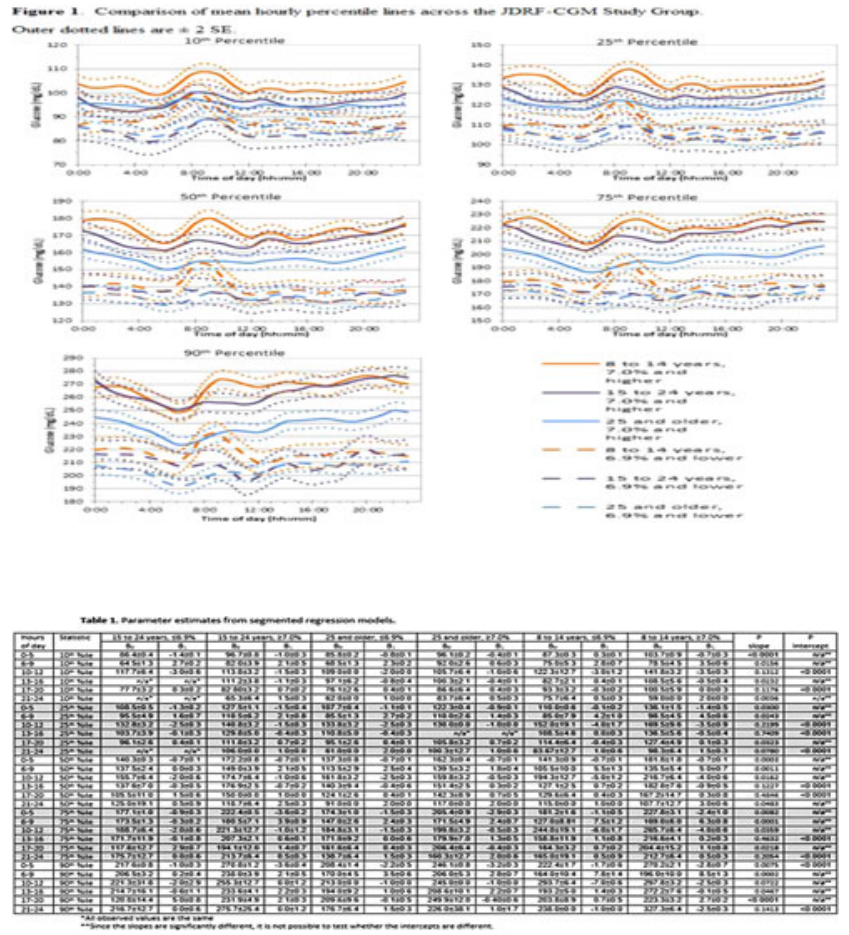

below $7.0 \%$ ) to demonstrate clinical usefulness of the AGP profile.

Method: The JDRF-CGM trial dataset was analyzed to create all available 14-day AGPs around each A1c measurement timepoint, (1101 AGPs for 393 subjects). For each hour of the day glucose value percentiles were calculated. Mean hourly percentile curves were calculated for the 6 groups. Segmented regression produced estimates of the slope and intercept for each group-time period. ANCOVA was used to first test whether the slopes of the lines were significantly different. If the slopes were not different, then the intercepts were tested.

Results: Comparing the hourly AGP lines across A1c and age groups showed significant differences between groups for all time-period segments for either rate of change in glucose or starting value of glucose. For all but the oldest well-controlled group, median glucose rose significantly over the period from 21:00 to 24:00, fell significantly from 0:00 to 5:00, and then rose from 6:00 to 9:00 for all groups.

Conclusion: This analysis supports clinical focus on the overnight period for all T1D patients with above-target A1c values, as well as particular focus on the morning meal period for T1D children $<14$ years. AGP-based analysis of the proposed segments identifies distinguishable periods of glycemic trends which may be useful clinically.

180

\section{IS FEAR ABOUT HYPO- AND HYPERGLYCEMIA RELATED TO GLUCOSE LEVELS OF PATIENTS WITH TYPE 1 DIABETES?}

\section{$\underline{\text { J. Grabman }}^{1}$, J. Shepard ${ }^{1}$, L. Gonder-Frederick ${ }^{1}$ \\ ${ }^{1}$ University of Virginia, Psychiatry and NB Sciences,} Charlottesville, USA

Background and Aims: Type 1 diabetes management requires patients to balance the daily risks of both hypo- and 
hyperglycemia. Little is known about how daily levels of concern about hypo-/hyperglycemia are associated with blood glucose (BG) fluctuations. This study explored the relationship between patients' daily self-reported hypo-/hyperglycemic fear and BG profile measures obtained from continuous glucose monitoring (CGM).

Method: Thirty-eight individuals with type 1 diabetes wore a CGM for $\geq 7$ days. In addition, participants recorded a single daily rating of hypo-/hyperglycemic fear, carbohydrate consumption, and insulin bolus units.

Results: Within-subjects ANCOVAs, controlling for daily carbohydrates and/or insulin, revealed that increased hypoglycemia fear was associated with increased carbohydrate consumption $(r=.24, p=.001)$ and there was a trend toward increased time in hypoglycemia $(r=.13, p=.079)$. Increased hyperglycemia fear corresponded with greater average BG $(r=.21, p=.009)$, time in hyperglycemia $(r=.16, p=.044)$, insulin bolus units $(r=.24, p=.002)$, hyperglycemic risk $(r=.20$, $p=.011)$ and measures of BG variability ( $r=.18$ to $.29, p=.023$ to $<.001)$, with a trend toward decreased carbohydrate consumption $(r=-.13, p=.098)$.

Conclusion: These initial findings suggest that fear of hypo-/ hyperglycemia in patients with type 1 diabetes are to some extent a reflection of actual metabolic challenges in daily diabetes management. Furthermore, some may engage in adaptive behaviors to maintain BG control. More controlled studies are needed to understand the relationship between daily diabetesrelated concerns and their clinical implications.

\section{1}

THE IMPACT ON QUALITY OF LIFE, GLUCOSE
MONITORING FREQUENCY AND SAFETY OF NOVEL
GLUCOSE-SENSING TECHNOLOGY USED BY
INDIVIDUALS WITH TYPE 2 DIABETES ON
INTENSIVE-INSULIN THERAPY

T. Haak ${ }^{1}$, H. Hanaire ${ }^{2}$, R.A. Ajjan ${ }^{3}$, N. Hermanns ${ }^{1}$, J.P. Riveline ${ }^{4}$, G. Rayman ${ }^{5}$

${ }^{1}$ Diabetes Zentrum Mergentheim, Diabetes Klinik, Bad Mergentheim, Germany

${ }^{2}$ University of Diabetology, Department of Diabetology, Toulouse, France

${ }^{3}$ University of Leeds, Leeds Institute for Cardiovascular and Metabolic Research, Leeds, United Kingdom

${ }^{4}$ Hopital Lariboisiere, Department of Endocrinolgy

and Diabetology, Paris, France

${ }^{5}$ Ipswich Hospital NHS trust, The Diabetes Centre, Ipswich, United Kingdom

Background and Aims: To assess the impact of new glucosesensing technology as an alternative to self-monitoring of blood glucose (SMBG) on testing frequency, quality of life and device safety (NCT02082184).

Method: A 6 month European study (26 sites), recruited patients with type 2 diabetes (T2DM), on intensive-insulin therapy. Randomisation (1:2 respectively) was to control group $(n=75)$ using capillary blood-glucose testing (FreeStyle Lite ${ }^{\mathrm{TM}}$ ), or intervention group $(n=149)$ using sensor glucose data (FreeStyle Libre $^{\mathrm{TM}}$ Flash Glucose Monitoring System).

Pre-specified secondary outcome measures included; SMBG frequency, quality of life measurements and safety analysis.

Results: Intervention group SMBG frequency fell from $3.8 \pm 1.4$ [median 3.7] tests/day at baseline (days 1-15) to $0.3 \pm 0.7$ [median 0.1 ] tests/day from randomisation. However in this time period, sensor scanning for glucose results was $8.3 \pm 4.3$ [median 6.9] times/day.

Diabetes-Treatment-Satisfaction Questionnaire (DTSQ) showed increased overall treatment satisfaction for intervention vs. control (13.1 \pm 0.50 (adjusted mean \pm SE) vs. $9.0 \pm 0.72$ $(\mathrm{p}<0.001))$. Diabetes Quality of Life $(\mathrm{DQoL})$ also showed increased treatment satisfaction for intervention vs. control $(-0.2 \pm 0.04$ (adjusted mean \pm SE) vs. $0.0 \pm 0.06(\mathrm{p}=0.0259)$ ).

During FreeStyle Libre wear (intervention (6 months) and control group (4 weeks, masked)) there were; 158 occurrences of skin symptoms/sensor-insertion events (e.g. mild pain, bruising, itching, erythema), normally expected with device use. There were 9 instances of device-related adverse events (e.g. infection, allergy) from 6 subjects. No device-related serious adverse events were reported.

Conclusion: Use of FreeStyle Libre by individuals with T2DM was associated with reduced SMBG frequency and increased treatment satisfaction, suggesting a high level of confidence using Sensor glucose data to support self-management.

182

\section{SIMPLIFYING CALIBRATION PRACTICE FOR HOME USE NON INVASIVE GLUCOSE MONITORING DEVICE: SHORTENING PROCEDURE DURATION}

A. Gal ${ }^{1}$, I. Harman-Boehm ${ }^{2}$, A. Drexler ${ }^{3}$, E. Naidis ${ }^{1}$, Y. Mayzel ${ }^{1}$, N. Goldstein ${ }^{1}$, K. Horman ${ }^{1}$, Y. Krasilshchikov ${ }^{1}$

${ }^{1}$ Integrity Applications Ltd., R\&D, Ashkelon, Israel

${ }^{2}$ Soroka University Medical Center, Internal Medicine and the Diabetes Unit, Be'er Sheva, Israel

${ }^{3}$ David Geffen School of Medicine, Division of EndocrinologyDiabetes and Hypertension, Los Angeles, CA, USA

Background and Aims: GlucoTrack ${ }^{\circledR}$, a non-invasive glucose monitoring device for home-use, requires bi-annual calibration by using an invasive reference device. Previous calibration procedure took about 2.5 hours and required a minimum of seven invasive measurements. In order to simplify the calibration practice, a shorter calibration scheme was developed.

Method: A new algorithm for a shorter calibration procedure was developed, based on a dataset collected from 53 type 2 subjects during clinical trials $(\sim 4,300$ paired GlucoTrack and invasive readings). The new algorithm enables calibration procedure of $<30$ minutes, with only three paired GlucoTrack and invasive measurements. The new scheme was evaluated off-line on an independent dataset of 117 type 2 subjects $(\sim 8,200$ paired readings), who performed the original calibration procedure in real-time. The new calibration parameters were set individually, based on first three original calibration points and then applied to measurement data. The original and off-line algorithms' performances were compared based on Clarke Error Grid (CEG) and Mean Absolute Relative Difference (MARD).

Results: CEG analysis shows $94.7 \%$ of the original calibration algorithm points in the clinically acceptable A $+\mathrm{B}$ zones, with MARD of $31.2 \%$, whereas $95.7 \%$ of the new, shorter calibration procedure points are within the acceptable A + B zones, with MARD of $24.2 \%$.

Conclusion: Applying the new algorithm positively affects both calibration procedure simplicity and device performances. These results support the validity of the newly developed calibration scheme for type 2 diabetes patients, while validity duration remains 6 months. Ultimately, implementing a shorter, simpler and less-painful calibration procedure is expected to improve device's usability and user satisfaction. 
183

SIMULATING THE SAFETY OF DOSING DECISIONS USING CGM: A DIRECT COMPARISON OF DEXCOM CGM SYSTEMS USING 505 SOFTWARE ALGORITHM AND BLOOD GLUCOSE MEASUREMENTS

$\underline{\text { L. Jepson }}^{1}$, N. Bhavaraju ${ }^{1}$, D. Price ${ }^{2}$

${ }^{1}$ Dexcom, R\&D, San Diego, USA

${ }^{2}$ Dexcom, Clinical, San Diego, USA

Background and Aims: Confirming previous analyses by Nandagopalan et al., a Monte Carlo simulation assessed the impact of determining a pre-meal insulin dose using continuous glucose monitoring (CGM) data and self-monitored blood glucose (SMBG) on post-meal glycemic outcomes. The simulation quantifies the collective impact of differences between SMBG and CGM on meal-time insulin dosing including: measurement accuracy, availability of rate of change (ROC) information, and the ability of CGM alerts to minimize post-meal hypoglycemia.

Method: The insulin dose is calculated from pre-meal glucose (either SMBG or CGM) and estimated carbohydrate (CHO) count, allowing CGM users an adjustment based on ROC. The pre-meal SMBG and CGM measurements and CGM alerts are simulated using data derived from clinical trials performed with CGM systems utilizing the 505 software and the Bayer Contour Next glucose meter.

Results: Insulin doses determined using SMBG and CGM resulted in similar rates of hypoglycemia and hyperglycemia if pre-meal glucose levels were stable. With CGM-based dosing, glycemic outcomes were consistent across different pre-meal glucose ROCs. With SMBG-based dosing, glycemic outcomes were highly dependent on pre-meal glucose trend, with higher rates of hypoglycemia for falling pre-meal glucose and higher rates of hyperglycemia for rising pre-meal glucose. The addition of CGM low glucose alerts greatly reduced the overall frequency of unmitigated hypoglycemia relative to SMBG.

Conclusion: Based on this simulation, use of ROC information and real-time alerts largely mitigate potential hypoglycemia risks associated with CGM-based dosing decisions and often improve glycemic outcomes. Additional studies are needed to confirm the safety of CGM-based dosing.

\section{4}

\section{COMPARING IPRO WITH LIBRE FOR SENSOR ACCURACY AND USER FRIENDLINESS}

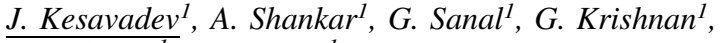 \\ S. Jothydev ${ }^{1}$ P.B. Pillai ${ }^{1}$ \\ ${ }^{1}$ Jothydev's Diabetes Research Centre, Diabetes, \\ Thiruvanathapuram, India
}

Background and Aims: Continuous glucose monitoring (CGM) systems use a tiny sensor inserted under the skin to check glucose levels in interstitial fluid. The sensor stays in place for several days to 2 weeks. CGM can be used to supplement blood glucose meter readings for more informed treatment decisions and improved glycemic control.

Method: This study examined ability of a new device, Libre Pro, to detect glucose levels compared with a currently available device, iPro2, in healthy individuals and diabetes patients. Two each of type 1 diabetes, type 2 diabetes, gestational diabetes mellitus, prediabetes and healthy individuals participated in the study. Both sensors were initiated simultaneously in the study participant. Blood glucose values were also monitored with calibrated glucose meter. Data

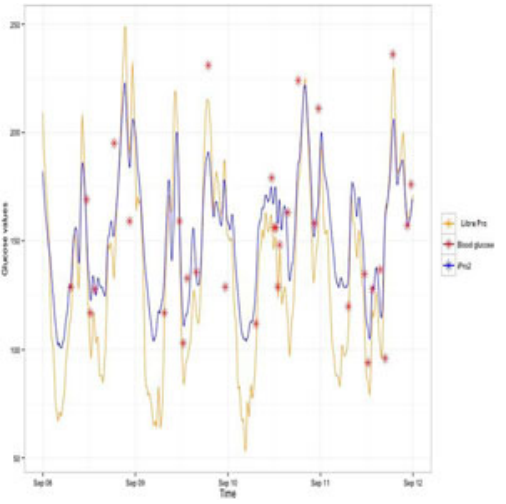

from day 2 to day 6 were analyzed. Statistical methods, including concordance analysis, were used to analyze results.

Results: The difference in the glucose values of the shown graph (Fig.1), of Libre Pro from iPro2 was $-15.8 \mathrm{mg} / \mathrm{dL}$ (SD 49.2, mean absolute percentage error $28.5 \%$ ). A concordance analysis showed that the concordance correlation coefficient 0.15 .

Conclusion: The overall glycemic pattern generated by the two devices were similar. The major advantages of Libre Pro sensor were that it precludes finger pricks for calibration, its smaller size, simplified insertion and data downloading techniques and 14 days of sensor life. Mean absolute percentage error being $28.5 \%$, randomized controlled trials in patients with rapidly fluctuating blood glucose, are warranted to better prove accuracy and reliability of this new sensor.

\section{5}

\section{CONTINUOUS GLUCOSE MONITORING IN THE DIAGNOSIS AND FOLLOW UP OF IMPAIRED GLUCOSE TOLERANCE IN OBESE CHILDREN AND ADOLESCENTS}

\section{$\underline{\text { M. Kocova }}^{1}$ \\ ${ }^{1}$ University Children's Hospital, Endocrinology \& Genetics, Skopje, FYR Macedonia}

Background and Aims: The epidemic of childhood obesity during the last few decades had an impact on the increased appearance of impaired glucose tolerance (IGT) and type 2 diabetes (DM2) in this young age group.

Continuous glucose monitoring system (CGMS) might help in defining the daily glucose profile in children with IGT detected on OGTT. It might also be a useful tool for follow up of children and adolescents with IGT.

Method: A total of 230 obese children (107 girls), at the ages of 6-18 years were analyzed. Obesity was assessed according to CDC criteria. Standard OGTT accompanied by 4 points of insulinemia was performed. HOMA index was calculated according to the standard formula. HbAlc was measured in children with peak glycemia $>7.8 \mathrm{mmol} / \mathrm{l}$. CGMS (Guardian) was applied in the same cohort for 5 days average.

Results: 28 children (mean age $11+1.8$ years) had IGT $(12.2 \%)$. BMI was $30+1.8 \mathrm{~kg} / \mathrm{m}^{2}$. Peak glycemia was $8.9+1.1 \mathrm{mmol} / \mathrm{l}$. HOMA index was $4.86+2.89$, and peak insulinemia $127.3+26.4$ $\mathrm{mIU} / \mathrm{dl}$ (range 40.7-333 mIU/dl). Only 6 children had higher HbAlc (6.6-6.8\%). CGMS profiles confirmed periods with hyper- and hypoglycemia.

Body mass index correlated well with insulinemia $(\mathrm{p}<0.001)$, but not with the peak glycemia $(\mathrm{p}=2.1)$. Peak glycemia was in 
correlation with the HOMA index $(\mathrm{p}=0.03)$. Four children $(14.3 \%)$ developed overt DM2 within five years of follow up, all at the age $>12$ years. Repeated CGMS profiles were predictive of worsened metabolic control.

Conclusion: There is significant risk of IGT and DM2 in obese children. CGMS is a useful tool in close monitoring of glycemic impairment in children with IGT.

\section{6}

\section{CALIBRATION POINT ACCURACY CHARACTERIZATION ON THE PERFORMANCE OF A LONG-TERM, IMPLANTABLE CGM: A SUBANALYSIS OF THE PRECISE STUDY}

\section{J. Kropff $^{1}$, J.H. DeVries ${ }^{1}$, R. Rastogi ${ }^{2}$, A. Dehennis ${ }^{2}$}

${ }^{1}$ Academic Medical Center - University of Amsterdam, Department of Endocrinology, Amsterdam, Netherlands

${ }^{2}$ Senseonics, Research, Germantown, USA

Background and Aims: Continuous Glucose Monitoring (CGM) systems use Self Monitored Blood Glucose (SMBG) measurements for calibration to compensate for in vivo sensorto-sensor differences and for sensitivity changes over implanted sensor life-time. An algorithm allowing weighting of previously entered SMBG's has been developed to compensate for the errors in the current SMBG entered for calibration. This study aimed to assess the impact of calibration point accuracy on the performance of the CGM system.

Method: Data from 44 subjects from a prospective, single arm, multicenter CGM pivotal study was used. Subjects used their study issued Accu Chek Aviva for SMBG calibration twice per day. CGM and SMBG accuracy expressed as mean absolute relative difference (MARD) was assessed during five in-clinic visits (8h-24h) against venous YSI reference glucose measurements (YSI2300 Stat plus). Primary outcome was CGM accuracy (MARD, CGM-YSI), binned per concomitant SMBG accuracy used to calibrate the CGM (MARD SMBG-YSI, 0-10\%, 10 $20 \%, 20-30 \%,>30 \%$ ).

Results: CGM accuracy for 44 subjects over the $40-400 \mathrm{mg} / \mathrm{dL}$ glycemic range was $12.0 \%(\mathrm{n}=10455, \mathrm{SD}=11.6 \%, 95 \% \mathrm{CI}=11.8 \%$ -

Figure 1: CGM performance binned per calibration point accuracy.

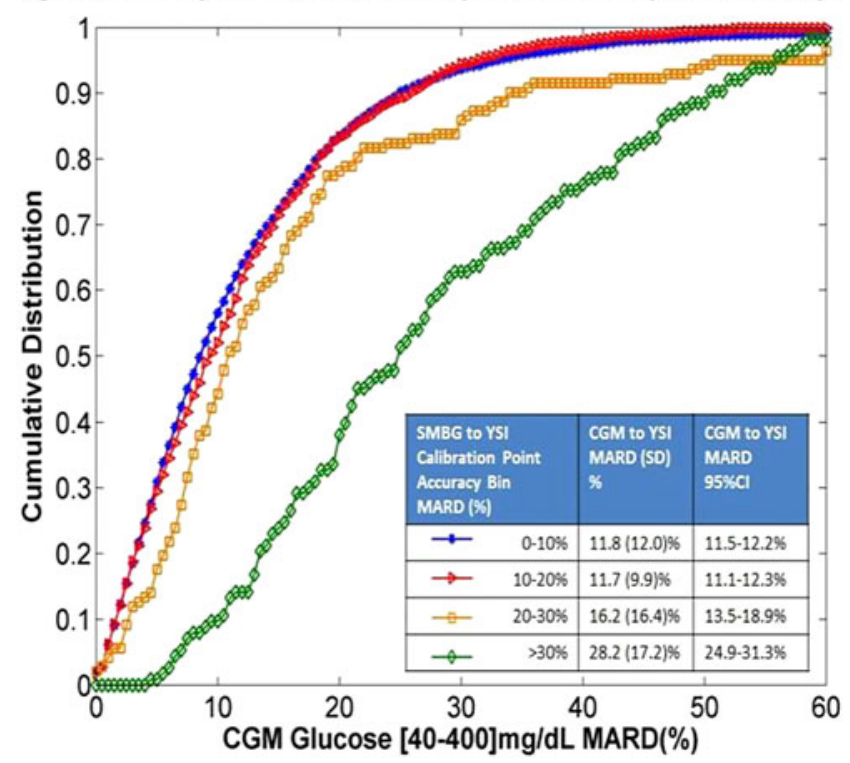

12.2\%). Overall SMBG MARD was $6.9 \%(\mathrm{n}=6268, \mathrm{SD}=6.6 \%$, $95 \% \mathrm{CI}=6.7 \%-7.1 \%$ ). CGM MARD binned per concomitant SMBG calibration accuracy summary is shown in Figure 1. Wilcox rank sum analysis showed significant differences between the accuracy results from calibration points with MARD $20 \%$ or greater $(\mathrm{p}<0.01)$. However, no impact in performance was found for CGM accuracy for calibration points below $20 \%$ $(\mathrm{p}=0.17)$.

Conclusion: Calibration point (SMBG-YSI) accuracy was found to significantly impact CGM performance. Calibration accuracy below 20\% MARD did not significantly impact the performance of the CGM.

187

\section{THE COMPARISON OF GLUCOSE READINGS OF CONTINUOUS GLUCOSE MONITORING AND BEDSIDE ARTIFICIAL PANCREAS DURING GLUCOSE CLAMP STUDY}

A. Kuroda $^{1}$, M. Matsuhisa ${ }^{1}$, S. Taniguchi ${ }^{1}$, Y. Akehi ${ }^{1}$, H. Mori ${ }^{1}$, M. Tamaki ${ }^{1}$, R. Suzuki ${ }^{I}$, Y.H. Otsuka ${ }^{1}$

${ }^{1}$ The University of Tokushima, Diabetes Therapeutics and Research Center, Tokushima, Japan

Background and Aims: Glucose values of continuous glucose monitoring (CGM) have time delay compared with plasma glucose values (PG). Artificial pancreas (STG-55, Nikkiso, Japan) (AP) measures venous blood glucose directly, but it has also time delay because of a long tubing line from vessel to the glucose sensor.

To investigate accuracy and time delay of CGM and AP in comparison with PG during hyperglycemic and euglycemic glucose clamp conditions.

Method: Two healthy volunteers and 6 patients with type 2 diabetes were recruited in this experiment. CGM (Enlite sensor, Medtronic, Northridge, CA) was attached on the day before the experiment. The calibrations of CGM sensor glucose were done just before and after the clamp study. After an overnight fasting, hyperglycemic $(200 \mathrm{mg} / \mathrm{dL})$ clamp was performed for $90 \mathrm{~min}$ utes, followed by euglycemic (100 mg/dL) clamp for 90-120 minutes using AP. AP and CGM values were compared with PG.

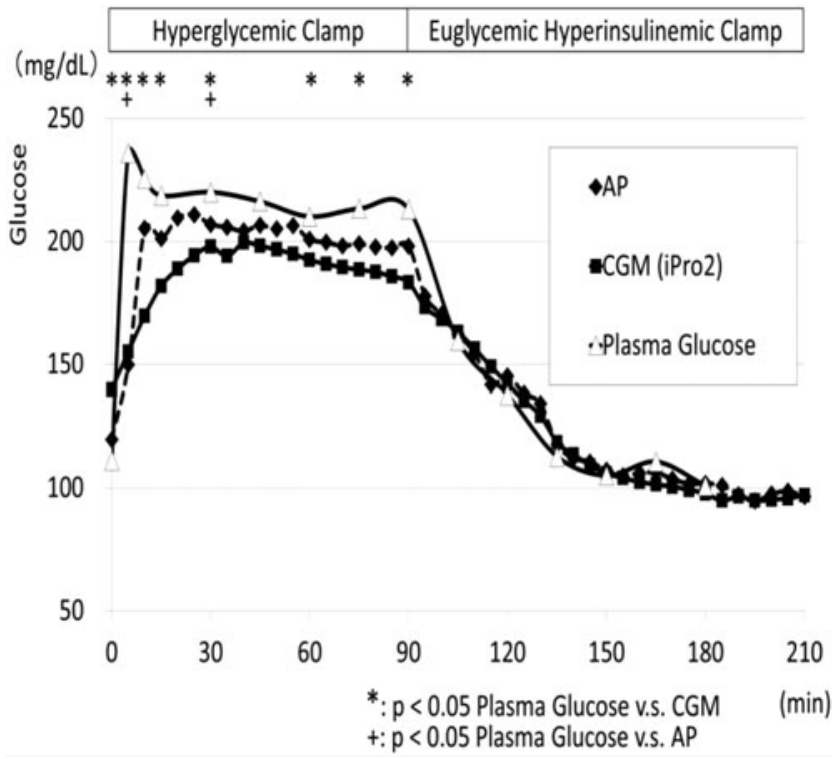


Results: AP values were significantly lower than PG at 5 and 30 minute, and delayed 5 minutes from PG during glucose rising of hyperglycemic clamp. CGM value at 0 minute was significantly higher than PG value; however, CGM values were significantly lower afterwards and were more behind than AP values during hyperglycemic clamp. On the other hands, there were no significant differences between CGM, AP and PG values during gradual decrease of PG in euglycemic clamp.

Conclusion: Both CGM and AP did not follow nonphysiological acute glucose-rising, however, matched well during physiological glucose-lowering change.

\section{8}

\section{SUPPORT VECTOR REGRESSION FOR MID-TERM NOCTURNAL GLUCOSE PREDICTION FROM CONTINUOUS GLUCOSE MONITORING AND INSULIN DELIVERY INFORMATION}

Y. Leal $^{1}$, L. Gonzalez-Abril ${ }^{2}$, R. Visentin ${ }^{1}$, S. Del Favero ${ }^{1}$, M. Vettoretti ${ }^{1}$, A. Facchinetti ${ }^{1}$, G. Sparacino ${ }^{1}$, C. Cobelli ${ }^{1}$

${ }^{1}$ University of Padova, Department of Information Engineering, Padova, Italy

${ }^{2}$ University of Seville, Department of Applied Economics I, Seville, Spain

Background and Aims: Literature glucose prediction methods normally employ 15-45 minute prediction horizons (PHs). Coping with longer PHs (hours) has been scarcely investigated, mostly because of the lack of continuous glucose monitoring (CGM) recordings with the needed minimal duration (weeks). Given the recent improvements in sensors lifetime, here we study feasibility of "mid-term" glucose prediction $(\mathrm{PH}=2 \mathrm{~h})$ using simulated data. In particular, a Support Vector Regression (SVR) prediction algorithm, fed by past CGM and insulin delivery information, is developed.

Method: The method has been tested on 100 in silico type 1 diabetic (T1DM) adults by using the UVA/Padova T1DM simulator, which incorporates a reliable open-loop manual control with realistic measurement noise and perturbations of meal and insulin injections (ref. abstract Visentin). Subjects underwent 2-weeks in sensor-augmented insulin-pump therapy, with three meals per day. Individualized SVR models with RBF kernel were trained for mid-term nocturnal glucose prediction (from 23:00 to 7:00). The first 10-days were used for tuning and training, the other 4-days for testing.

Results: For each patient, 1444 glucose estimations were obtained for testing. RMSE, MAD, MARD resulted 13.1(8.6) $\mathrm{mg} / \mathrm{dL}, 8.4(5.3) \%$, and 7.2(6.5)\%, respectively. Glucose-specific prediction metrics known gRMSE, gMAD and gMARD resulted 13.6(8.7) mg/dL, 9.2(5.7)\%, 7.3(6.9)\% respectively.

Conclusion: Simulation results suggest that the developed SVR methodology can be a viable approach to achieve sufficiently accurate mid-term glucose prediction, at least during nighttime. Next steps will include test of the SVR algorithm feasibility for noctural predictions on a wider dataset, comprising real data also, and an analysis of sensitivity/specificity of hypo-/ hyper- events mid-term forecast.

\section{9}

\section{HYPOGLYCEMIA AFTER BARIATRIC SURGERY: DETECTION RATES OF CONTINUOUS GLUCOSE MONITORING}

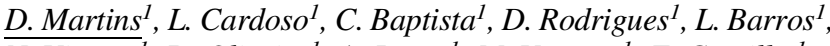

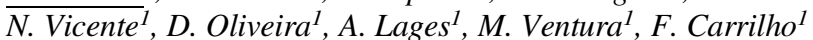

${ }^{1}$ Centro Hospitalar e Universitário de Coimbra, Serviço de Endocrinologia- Diabetes e Metabolismo, Coimbra, Portugal

Background and Aims: Hypoglycemia is a rare and underestimated complication of bariatric surgery, as Roux-en-Y gastric bypass (RYBG) or gastric sleeve. Continuous glucose monitoring (CGM) has been a valuable tool to detect hypoglycemia and characterize glycaemic variability in these patients.

Method: CGM was used in twelve patients after undergoing bariatric surgery (RYBG or sleeve) and clinical suspicion of hypoglycemia, during seven days, to assess the frequency of hypoglycemic episodes under real life conditions. CGM was done using iProTM2 CGM device (Medtronic, Northridge, CA) in all patients.

Results: A total of 21,960 glucose data points were retrieved from CGM and evaluated herein. Ten of the 12 patients $(83.3 \%)$ had previously undergone RYBG surgery and only $2(16.7 \%)$ had undergone gastric sleeve, with a mean time after surgery of $3.25 \pm 1.54$ years. All patients had at least one episode of hypoglycemia (glucose $<70 \mathrm{mg} / \mathrm{dL}$ ), furthermore, eleven patients $(91.67 \%$ ) had at least one episode of hypoglycemia below $55 \mathrm{mg} /$ dL. Total hypoglycemia exposure was 11.79 hours per patient and CGM. During the monitoring period, 157 (81.35\%) out of 193 hypolgycemic episodes occurred in the post-prandial state. Glucose variability was evaluated by MAGE $(3.20 \pm 0.94 \mathrm{mmol} / \mathrm{L})$ and CONGA (4.2 $\pm 0.60 \mathrm{mmol} / \mathrm{L})$. Area under the curve for glucose $<70 \mathrm{mg} / \mathrm{dL}$ was 1.025 and for glucose $>180 \mathrm{mg} / \mathrm{dL}$ was 1.69 .

Conclusion: Hypoglycemia after RYBG or sleeve occurred mostly in the post-prandial period. Glucose variability, particularly MAGE, was affected by glucose fluctuations. Thus, CGM is a valuable diagnostic tool and may have a role evaluating treatment response to dietary modifications, drug therapy or surgical reintervention.

\section{0}

\section{REGRESSION ANALYSIS ON IN-VITRO RATS INDUCED BLOOD GLUCOSE LEVEL DETECTION TOWARDS NON-INVASIVE DIABETES MONITORING ON HUMAN VIA NEAR INFRARED SPECTROSCOPY METHOD}

Z. Md Yusof $^{1}$, N.N. Mohd Yatim ${ }^{1}$, N.A. Mohd Aziz, ${ }^{1}$ A.R. Laili ${ }^{1}$, M.H. Hisham ${ }^{1}$, R. Sabri ${ }^{2}$, R. Muhamad Noor ${ }^{3}$, N. Nordin ${ }^{3}$, S. Abdul Rahman ${ }^{4}$, H. Ahmad

${ }^{1}$ MIMOS Berhad, Photonics R\&D, Kuala Lumpur, Malaysia ${ }^{2}$ MIMOS Berhad, ATE Digital Electronics Design, Kuala Lumpur, Malaysia

${ }^{3}$ Cyberjaya University College of Medical Sciences, Medical Sciences Division- Faculty of Medicine, Cyberjaya, Malaysia ${ }^{4}$ Cyberjaya University College of Medical Sciences, Faculty of Pharmacy, Cyberjaya, Malaysia

${ }^{5}$ Universiti Putra Malaysia, Department of Veterinary Preclinical Science-Faculty of Veterinary Medicine, Serdang, Malaysia

Background and Aims: The importance of good controlled glycaemic response and level of blood glucose in a person with diabetes will be best achieved when there is a frequent monitoring via non-invasive method of measurement. Poor control will lead to complications of many other associate diseases such as renal failure, stroke, blindness and many others. In our study, we introduced a method of blood glucose level detection using a noninvasive and non-destructive spectroscopy based optical sensor.

Method: A light spectrum in Near Infrared (NIR) region was used to shine and detect based on diffuse reflectance by the 
sample under test. The gathered spectrum of light will be analysed with a Chemometrics tools. A prediction model also developed in order to give results in $\mathrm{mmol} / \mathrm{l}$ glucose level for each measurement of sample. A complete in-vitro experiment on Sprague Dawley rats conducted towards the objective of having a non-invasive approach of blood glucose test on human. We shared our study on regression analysis for 30 male rats aged seven to eight weeks old.

Results: The rats were divided into three groups and induced with different diabetes level namely hypoglycaemia, normal glycaemia and hyperglycaemia. Variable selection was applied in order to get significant variable of sample for better prediction model. Multiple Scattering Corrector and Savitzky Golay smoothing methods used during analysis.

Conclusion: Root Mean Square Error (RMSE) and prediction Coefficient of determination $\left(\mathrm{R}^{2}\right.$ Pred) were observed to validate the model. We successfully achieved $1.32 \mathrm{mmol} / \mathrm{l} \mathrm{RMSE}$ and 0.9941 $\mathrm{R}^{2}$ Pred which gave a good promise for application on human.

\section{1}

\section{A NEW, WIRELESS-ENABLED, BLOOD GLUCOSE METER IN DEVELOPMENT THAT LINKS TO A SMART MOBILE DEVICE VIA BLUETOOTH CONNECTIVITY: ACCURACY AND USER PERFORMANCE EVALUATION}

M. Christiansen ${ }^{1}$, C. Greene ${ }^{2}$, S. Pardo , $^{2}$ M.E. Warchal-Windham ${ }^{2}$, B. Harrison ${ }^{2}$, R. Morin ${ }^{2}$, T. Bailey ${ }^{3}$

\footnotetext{
${ }^{1}$ Diablo Clinical Research, Walnut Creek CA, USA

${ }^{2}$ Bayer HealthCare LLC, Diabetes Care, Whippany NJ, USA

${ }^{3}$ AMCR Institute Inc., Escondido CA, USA
}

Background and Aims: To evaluate accuracy, in the laboratory and clinical settings, of a new blood glucose monitoring system (BGMS) in development for use with CONTOUR Next test strips. The BGMS features an easy-to-use wireless-enabled blood glucose meter that links to a smart mobile device via Bluetooth connectivity.

Method: In a laboratory study, fingertip blood samples from 100 subjects with diabetes were tested in duplicate using each of 3 test strip lots. Accuracy was assessed per ISO 15197:2013 accuracy criteria. In a 2-center clinical study, 375 subjects with $(n=332)$ or without $(n=43)$ diabetes, who had never used this BGMS previously, completed; the primary objective was to assess accuracy of the BGMS using fingertip self-test results based on ISO 15197:2013 accuracy criteria. Secondary objectives included accuracy per FDA Draft SMBG Guidance 2014 Section C (95\% of results within $\pm 15 \%$ and $99 \%$ within $\pm 20 \%$ of the laboratory method across the entire tested range).

Results: In the laboratory study, all 600 readings (glucose range, $36-643 \mathrm{mg} / \mathrm{dL}$ [2.0-35.7 mmol/L]) met ISO 15197:2013 accuracy criteria. In the clinical study, among subjects with diabetes (glucose range, $32-458 \mathrm{mg} / \mathrm{dL}$ [1.8-25.4 mmol/L]), $99.4 \%$ (327/329) of subject fingertip self-test results met ISO 15197:2013 accuracy criteria. Considering both subjects with and without diabetes, 99.5\% (370/372) of fingertip self-test results were within $\pm 15 \%$ and $99.7 \%$ (371/372) were within $\pm 20 \%$ of the laboratory method.

Conclusion: The BGMS exceeded ISO 15197:2013 accuracy criteria in the laboratory and a clinical setting and exceeded FDA Draft 2014 criteria in a clinical setting.
192

\section{CONTINUOUS GLUCOSE MONITORING SYSTEM (CGMS) IN THE MULTI-STEP APPROACH TO DIAGNOSING HYPOGLYCEMIA IN PEDIATRIC AGE}

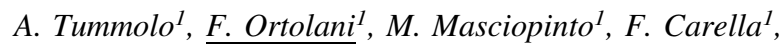
A.M. Dimauro ${ }^{1}$, M.P. Natale ${ }^{1}$, M. Vendemiale $^{2}$, D. Cornacchia ${ }^{2}$, F. Papadia ${ }^{1}$, E. Piccinno ${ }^{1}$

${ }^{1}$ Pediatric Hospital “Giovanni XXIII”, Metabolic DiseasesClinical Genetics and Diabetology, Bari, Italy

${ }^{2}$ Pediatric Hospital "Giovanni XXIII", Clinical Psychology, Bari, Italy

Background and Aims: The most common application of Continuous Glucose Monitoring Systems (CGMS) is the identification of hypoglycemic events in type 1 Diabetes Mellitus. In recent years, however, there has been a growing interest in the potential application of this technology to other disorders. In particular, the diagnostic approach to inherited metabolic disorders (IMDs) may benefit from it, allowing a real-time characterization of hypoglycemia, which may be the first sign of many IMDs.

Method: In our study, CGMS was integrated in a multistep diagnostic approach to IMDs. Results were interpreted in the light of clinical and laboratory investigations. MiniMed (Medtronic, Northridge, CA) CGMS System Gold was used in 14 pediatric patients referred to our unit for persistent hypoglycemia.

Results: Six patients $(42 \%)$ had normal glucose trend. Five patients $(35 \%)$ showed an abnormal glycemic pattern but no signs of specific IMDs. In 3 cases $(21 \%)$ an IMD was suspected on the basis of the glycemic pattern. Of the 2 out of 3 patients for whom further investigations could be performed, one case experienced long fasting hypoglycemia associated with hypoketosis and hepatomegaly (suspected defects of fatty acids betaoxidation). The latter case showed recurrent hypoglycemia with tendency to nocturnal hypoglycemia and no increase of postprandial glycemia (suspected congenital hyperinsulinism).

Conclusion: This diagnostic approach highlighted the high incidence of false positives for significative hypoglycemia, allowing a more rapid diagnosis for those cases suggestive for IMDs. Therefore CGMS may be applied to the diagnostic approach of IMDs and can represent the basis for the following diagnostic work-up.

193

\section{USE OF SENSOR-AUGMENTED INSULIN PUMP THERAPY: INCLUSION CRITERIA AND RESULTS AFTER 1 YEAR OF FOLLOW-UP}

M. Pazos-Couselo ${ }^{1}$, G.R. María ${ }^{1}$, F. Gude ${ }^{2}$, F. Casanueva ${ }^{1}$, $\overline{\text { J.M. García-Löpez }}^{1}$

${ }^{1}$ Universitary Hospital Santiago de Compostela, Endocrinology and Nutrition Service, Santiago de Compostela, Spain

${ }^{2}$ Universitary Hospital Santiago de Compostela, Clinical Epidemiology Unit, Santiago de Compostela, Spain

Background and Aims: Sensor-augmented insulin pump (SAP) therapy contributes to improve metabolic control and to reduce the hypoglycemia events. The aim of the study was to evaluate the metabolic control and hypoglycemia events after 1 year with SAP therapy in type 1 diabetes patients.

Method: Retrospective observational study. 16 patients (11 women) with at least 1 year of SAP treatment were included. 


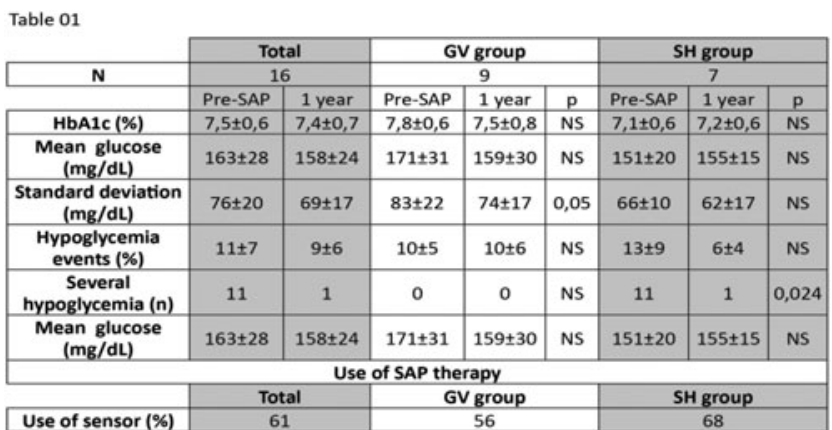

The inclusion criteria to start SAP therapy were high glycemic variability $(\mathrm{GV})$ or at least 1 episode of several hypoglycemia $(\mathrm{SH})$ in the year before the initiation of treatment with sensor in real time.

We analyzed glycemic control and hypoglycemia events at baseline and after 1 year of follow up in the whole sample and separated by inclusion criteria ( $\mathrm{GV}$ and $\mathrm{SH})$. Furthermore we evaluated the use of the sensor.

Results: 16 DM1 patients (11 women, 5 men), age between 23 and 62 (mean: 40 years old); Body mass index (BMI) $27.2 \pm 3.6 \mathrm{~kg} /$ $\mathrm{m}^{2}$; Diabetes duration $22 \pm 10$ years; Duration of continuos subcutaneos insulin infusion (CSII) treatment $32 \pm 23$ months.

Glycemic control data, hypoglycemic events and use of sensor are shown in Table 1.

The main cause of disruption of the monitoring was the unavailability of the sensors (hypoglycemia group) and patient's decision (glycemic varibility group).

Conclusion: In patients enrolled in SAP therapy by hypoglycemia there was a decrease in the number of hypoglycemic events (especially several hypoglycemia) without worsening metabolic control.

In patients with high glycemic variability SAP therapy improved the metabolic control with a significant decrease of SD.

Patients of the SH group used the sensor more than the GV group.

194

\section{DEVELOPMENT OF A NEW GLUCOSE DEHYDROGENASE MUTANT WITH DIRECT ELECTRON TRANSFER AND ENHANCED STABILITY AND SPECIFICITY}

A. Pfützner ${ }^{1}$, S. Ben Zeev ${ }^{2}$, C. Guttman ${ }^{2}$, I. Gofberg ${ }^{2}$, D. Omer ${ }^{2}$,

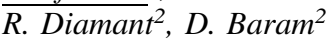

${ }^{1}$ Pfützner Science \& Health Institute, Diabetes Center and Practice, Mainz, Germany

${ }^{2}$ Smartzyme Biopharma, R\&D, Ness Ziona, Israel

Background and Aims: Accuracy of current blood glucose measurement systems using glucose dehydrogenase (GDH) have been reported to be influenced by temperature sensitivity, interfering substances (e.g. xylose), and by the sensitivity of the indirect electron transfer via mediator molecules. These conditions may alone or jointly result in a profound bias of the BG measurement result.

Method: We used our proprietary Provolution ${ }^{\mathrm{TM}}$ process to generate several variants of GDHs from a bacterial source. All variants were screened using not only biochemical assays, but also using electrochemical assays, in order to identify the most suitable candidates. Mutants were tested both in vitro and with physiological fluids. In tests which involved physiological fluid, heparinized whole blood was freshly drawn from healthy volunteers and diabetic patients and manipulated to contain different glucose concentrations and different xylose concentrations. Samples were tested at different temperature conditions with Smartzyme electrodes and the resulting signals were analyzed in order to conclude the interference percentage of the original sample.

Results: The finally selected candidates were (1) characterized by efficient direct electron transfer without need of a mediator molecule, (2) high temperature stability, and (3) no xylose interference. The signal resulting from xylose was below the signal to noise ratio.

Conclusion: By means of the Provolution ${ }^{\mathrm{TM}}$ technology, we have been able to develop a GDH mutant that showed major advantages to currently applied enzymes. Clinical studies are now warranted to demonstrate the suitability of this GDH mutant for glucose sensing devices, like self-monitoring blood glucose (SMBG) systems and continuous glucose monitors (CGMs).

\section{5}

\section{EXTRACTION OF INTERSTITIAL FLUID AND SENSING OF GLUCOSE LEVELS USING MICRONEEDLES AND MILLIMETRE WAVE SPECTROSCOPY}

\author{
P. Rangsten $^{1}$, M. Renlund ${ }^{1}$
}

${ }^{1}$ Ascilion AB, R\&D, KISTA, Sweden

Background and Aims: A minimal invasive method of sampling bodily fluids is a prerequisite for glucose monitoring. This paper presents the demonstration of extraction of interstitial fluid (ISF) utilizing a microneedle array as well as a non-electrochemical sensing method for measurement of glucose levels in bodily fluids.

Method: An extraction chip, 10x10 mm in size, incorporating more than 200 hollow microneedles with a length of a few hundred micrometers were designed and manufactured. (See Figure 1.) The chipset was manufactured in monocrystalline silicon using micro mechanical system (MEMS) technology. The

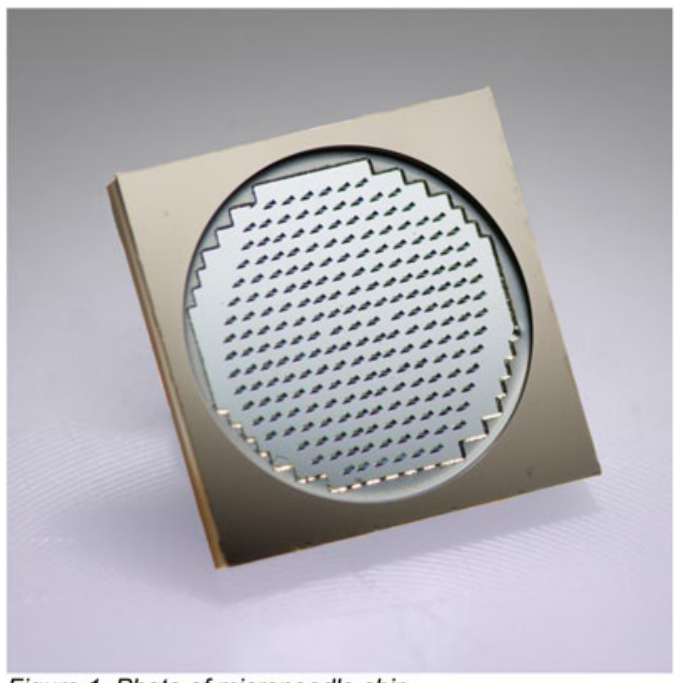

Figure 1. Photo of microneedle chip. 
length of the needles enabled extraction at the same time as they were short enough to not generate discomfort during sampling, as they didn't reach any sensory receptors.

The sensing method is based on radio frequency (RF) spectroscopy where the complex permittivity changes are measured using a resonant cavity sensor. The concept of glucose measuring using RF spectroscopy was proven both on macroscopic prototypes (cm-scale) and on a miniaturised low-cost MEMS chip.

Results: Bodily fluids were extracted using microneedle arrays. The microneedles were robust enough to puncture human skin and showed the capability of extracting and delivering ISF to a microchannel system through capillary action solely. The sensor principle was evaluated through comparison with a commercial self-monitoring blood glucose monitor from Roche (AccuChek Aviva Nano).

Conclusion: The first milestones towards realization of a minimal invasive spectroscopy-based glucose sensor system have been reached. The combination of microneedles and RF spectroscopy enables the possibility of a pain-free and accurate method for glucose measurements.

\section{6}

\section{ASSESSMENT OF HYPOGLYCEMIC EVENTS FROM A FEASIBILITY STUDY: TESTING REPEAT INSERTIONS OF LONG TERM, FULLY IMPLANTABLE CGM SYSTEM}

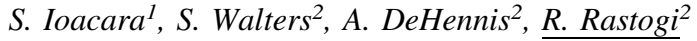

1 “Elias” Emergency University, Department of EndocrinologyDiabetes and Metabolic Diseases, Bucharest, Romania

${ }^{2}$ Senseonics Incorporated, Research, Germantown, USA

Background and Aims: Nocturnal hypoglycemia is a common concern for diabetes patients treated with insulin. Long term continuous glucose monitor (CGM) use has shown benefits in reduction of nocturnal hypoglycemia. A fully implantable CGM system has been developed that enables a single sensor to last between 3-6 months, with the implant procedure lasting approximately 5 mins for each insertion.

Method: Long term CGM data of 19 subjects with repeated insertions enrolled in a feasibility study in Bucharest, Romania was used. Subjects were implanted with 2 CGM sensors on bilateral arms and were instructed to follow their usual diabetes management regimen, for up to 6 months (sensor life). During the study, subjects recorded 5-7 SMBG per day. This analysis includes 10 subjects re-enrolled 2 times, 8 subjects re-enrolled 3 times and 1 subject re-enrolled 4 times over the duration of 2 years.

Results: Fear of and percent of hypoglycemic events, as confirmed by SMBG reference, was low during first exposure to the CGM system $8.0 \%$ ( $n=19$ subjects), and remained so at second $6.4 \%(\mathrm{n}=19$ subjects), third $6.7 \%(\mathrm{n}=9$ subjects $)$ and forth re-insertion $7.7 \%(\mathrm{n}=1$ subject). A similar finding was noticed in nocturnal hypoglycemic events, 6.9\%, 6.7\%, 6.8\% and $5.6 \%$ was also observed for successive exposure to the system.

Conclusion: Repeated exposure to prolonged use of the study CGM showed persistence of initial benefits and even a small reduction in the hypoglycemic events. System design and performance translated into a high patient acceptance and integration into routine daily life.
PROJECTION OF HEALTH-ECONOMIC BENEFITS
OF SENSOR-AUGMENTED PUMP (SAP) VERSUS INSULIN PUMP THERAPY ALONE (CSII), IN TYPE 1 DIABETES PATIENTS, IN DENMARK

S. Roze $e^{1}$, E. Duteil ${ }^{1}$, A. Delbaere ${ }^{2}$, S. De Portu ${ }^{2}$, A.S. Brandt ${ }^{3}$, M. Ridderstråle 4

${ }^{1}$ HEVA HEOR, Health Economics, Lyon, France

${ }^{2}$ Medtronic, Health Economics, Tolochenaz, Switzerland

${ }^{3}$ Medtronic Danmark, Health Economics, Kobenhavn, Denmark

${ }^{4}$ Steno Diabetes Center, Biomedical research, Gentofte, Denmark

Background and Aims: To assess the cost-effectiveness and to project the clinical benefits of Sensor-Augmented-Pump (SAP) versus Continuous Subcutaneous Insulin Infusion alone (CSII) in type 1 diabetes patients in Denmark.

Method: The Core Diabetes Model was used to project the incidence of diabetes-related complications over a lifetime horizon based on a meta-analysis comparing SAP vs. CSII comprising 892 patients. In this meta-analysis, $80.5 \%$ sensor usage in a hyperglycemic population with a baseline HbA1c of $8.1 \%$ would lead to an $\mathrm{HbA} 1 \mathrm{c}$ reduction of $0.56 \%$ for SAP and $0.13 \%$ for CSII. Data from a hypoglycemia-prone population $(n=95)$ was also simulated. The quality of life was adjusted for a reduced fear of hypoglycemic event in the SAP arm.

Results: The incremental cost-effectiveness ratio was 156'082 DKK per Quality Adjusted Life Year gained (QALY) based on societal perspective. SAP-related additional costs were partially offset by the savings due to the reduction in diabetes long-term complications and lower frequency of finger-prick tests. Thanks to the HbAlc reduction difference, SAP delayed the onset of complications for amputation, end-stage renal disease, myocardial infarction and severe vision-loss by 1.39, 1.37, 1.23 , and 1.05 years, respectively. The acceptability curve showed that the likelihood to be cost-effective at a willingness-

\begin{tabular}{|l|c|c|c|}
\hline \multicolumn{3}{|l|}{ Table 1: Time alive before the onset of complications in the hyperglycemic population [in years] } \\
\hline & SAP & CSII alone & Delta \\
\hline Proliferative Retinopathy & 27.56 & 26.08 & 1.48 \\
\hline Microalbuminuria & 14.55 & 13.16 & 1.39 \\
\hline Gross Proteinuria & 29.46 & 27.8 & 1.66 \\
\hline End-Stage Renal Disease & 32.23 & 30.86 & 1.37 \\
1st Ulcer & 26.73 & 25.07 & 1.66 \\
\hline Amputation & 30.97 & 29.58 & 1.39 \\
\hline Neuropathy & 18.65 & 16.87 & 1.78 \\
\hline Peripheral Vascular Disease & 31.33 & 30.07 & 1.26 \\
Congestive Heart Failure & 31.36 & 30.06 & 1.3 \\
\hline Angina & 30.97 & 29.73 & 1.24 \\
Myocardial Infarction & 31.44 & 30.21 & 1.23 \\
Stroke & 30.59 & 29.46 & 1.13 \\
Cataract & 28.25 & 27.28 & 0.97 \\
Macular Edema & 20.97 & 19.88 & 1.09 \\
Severe Vision Loss & 25.01 & 23.96 & 1.05 \\
\hline
\end{tabular}


to-pay of $189^{\prime} 000$ DKK/QALY was $72 \%$. The same trends applied for the hypoglycemia-prone population.

Conclusion: According to commonly accepted thresholds, SAP vs. CSII can be considered good value for money in the Danish setting both in the hyperglycemic and hypoglycemiaprone patients. Extensive sensitivity analysis on key drivers confirmed the robustness of results.

\section{8}

THE RELATION BETWEEN GLUCOSE VARIABILITY AND PAINFUL NEUROPATHY IN TYPE 2 DIABETES

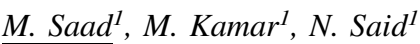

${ }^{1}$ Zagazig University- Faculty of Medicine, Internal Medicine, Zagazig, Egypt

Background and Aims: Irrespective of the magnitude of hyperglycemia, glucose variability, may confer an additional risk for the development of micro- and macrovascular diabetic complications. This study aimed to assess within-day blood glucose excursions in patients with type 2 diabetes mellitus (DM) measuring it by two different methods and correlate it with diabetic peripheral neuropathy.

Method: The study included 33 type 2 DM who were equally divided into 3 groups: group I (diabetic patients), group II (diabetic patients with neuropathy) and group III (diabetic patients with painful neuropathy). All participants were subjected to thorough clinical assessment, laboratory investigations and glucose variability calculation using the mean amplitude of glucose excursions (MAGE index) and continuous glucose monitoring (CGM) for a sample of patients.

Results: Patients with neuropathy had significantly higher fasting blood glucose and postprandial blood glucose when compared with patients without $(\mathrm{P}=<0.0001)$. Furthermore, patients with painful neuropathy had significantly higher MAGE levels when compared with those with non-painful neuropathy and the latter group had significantly higher MAGE levels when compared with diabetic patients without neuropathy (mean \pm SD $183.88 \pm 13.22$, $120.06 \pm 9.73,75.88 \pm 7.43$ respectively $\mathrm{p}<0.0005$ ). Moreover, continuous glucose monitoring revealed a distinguished pattern of glucose variability in DM patients with painful neuropathy as compared to patients with neuropathy and those without plasma glucose $>180 \mathrm{mg} / \mathrm{dl}(91.0 \%, 48 \%, 43.0 \%)$ respectively.

Conclusion: Glucose variability may be implicated in diabetic neuropathy particularly its painful form. MAGE and other glucose variability assessment measures are suggested to be incorporated in DM follow up armamentarium.

199

\section{RT-CGMS REVEALED THAT MEAL CONTENT ESTIMATION IS STILL A PROBLEM EVEN IN WELL EDUCATED DM1 PATIENTS}

$\underline{\text { K. Stechova }}^{1}$, P. Pithova ${ }^{1}$, M. Kvapil ${ }^{1}$

${ }^{1}$ Charles University - 2nd Faculty of Medicine and University Hospital Motol, Dpt.of Internal Medicine, Prague, Czech Republic

Background and Aims: The main goal of our study was by using RT-CGMS and patient logbook including meal photodocumentation to analyse if patients evaluate properly factors influencing glycaemia (meal carbohydrate content, physical activity etc.).

Method: Twenty patients treated for DM1 at least 1 year received RT-CGMS for 2 weeks. They wrote recorded detailed logbook including estimation of meal carbohydrate content and glycaemic index (GI) plus documented each meal by their smart phone camera. Patient's characteristics: F/M 9/11; median of age 24 years (18-48 years); 17 used insulin pumps, 3/20 MDI regime; median HbAlc $60 \mathrm{mmol} / \mathrm{mol}$ (48-73 mmol/mol). Data were analysed by SPSS SW v.22.

Results: Median of time in normoglycaemia was $47 \%$ (33$63 \%$ ) and no serious hypoglycaemia as well as hyperglycaemia occurred. Patients suffered from mild hypoglycaemia only which were connected in $40 \%$ to previous physical activity and in $36 \%$ to hyperglycaemia overcorrection. Glycaemia over target range occurred in $63 \%$ after meal and was strongly correlated to meal carbohydrate content underestimation $(\mathrm{p}<0,001)$ but mistakes in GI evaluation were insignificant. It is noteworthy that patient's diet was mostly unhealthy (inappropriate fat and protein content). Patients insufficiently used advanced insulin pump features (bolus calculators, different bolus types etc.) and/or online dietary advisors but they were satisfied with using smart phones as the quick and convenient meal logbook.

Conclusion: Glycaemia over the target range was strongly connected to meal carbohydrates content underestimation. Patients were reluctant to use advanced technological tools so the crucial point still remains re-education and patient's motivation.

Supported by the Czech Ministry of Health Project No.15-25710A(P08 panel).

\section{0}

\section{LONG TERM EFFICACITY OF REAL-TIME CONTINUOUS GLUCOSE MONITORING IN T1DM PATIENTS TREATED BY INSULIN PUMP : A MONOCENTRIC EXPERIENCE}

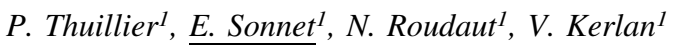 \\ ${ }^{1}$ CHRU Brest, Endocrinology-Diabetology, Brest, France
}

Background and Aims: To evaluate the efficacy of long-term real-time continuous glucose monitoring (RT-CGM) in diabetic type 1 patients (T1DM) in a retrospective cohort study.

Method: The inclusion criteria were: T1DM patients, age $\geq 18$ years old, treated by an external insulin pump, with a RT-CGM for at least three months. Patients were followed-up until March 2015. Evolution of A1c and incidence of acute T1DM complications before / after RT-CGM were studied. The change between the first and the last month of RT-CGM data (extracted with CarelinkPro ${ }^{\circledR}$ software) and glycemic variability index (LBGI, HBGI, MODD and MAGE, calculated with EasyGV® software) were analyzed.

Results: Twenty-eight T1DM patients (19 women, 9 male, mean age 40 years, mean duration of diabetes 25,7 years) had a RT-CGM between 2011 and 2015 (mean follow up of 22 months). A1c at the end of follow-up was reduced (8.24\% versus 7.54\%: $-0.7 \% ; \mathrm{p}=0.001)$. The number of admission to Emergency Department and number of days of hospitalization decreased of $65 \%(\mathrm{p}=0.03)$ and $79 \%(\mathrm{p}<0.001)$, respectively. At the end of follow-up, CGM use was lower (64.5 vs $86.4 \%$ of the time; $\mathrm{p}=0.002)$. Glycemic index were not changed.

Conclusion: Our study shows long-term benefits of the RTCGM, with a persistent decrease of A1c and decrease of the incidence of acute T1DM complications despite lower use of CGM. 


\section{1}

COMPARISON IN A PROSPECTIVE STUDY BETWEEN THE EXTERNAL INSULIN PUMP MINIMED 640G® AND VEO IN T1DM PATIENTS CARRYING A REAL TIME CONTINUOUS GLUCOSE MONITORING DEVICE

P. Thuillier ${ }^{1}$, E. Sonnet ${ }^{1}$, N. Roudaut ${ }^{1}$, V. Kerlan ${ }^{1}$

${ }^{1}$ CHRU Brest, Endocrinology-Diabetology, Brest, France

Background and Aims: To assess the effectiveness and satisfaction of patients with type 1 diabetes mellitus (T1DM) on the external insulin pump Minimed 640G ${ }^{\circledR}$.

Method: In May 2015, T1DM patients, age $\geq 18$ years old, treated by a Minimed Veo insulin pump and carrying a real time continuous glucose monitoring device (RT-CGM), were asked to change their pump to the latest generation external insulin pump : Minimed 640G®. A1c level, numbers of total and nocturnal hypoglycemia episodes per week and area under the curve (AUC $\leq 70 \mathrm{mg} / \mathrm{dl}$ ) initially and at 3 months were compared. Patient satisfaction about their device initially and at 3 months, was assessed with ITSQ (Insulin Treatment Satisfaction Questionnaire). At three months, patients had to answer to 5 additional questions comparing the two pumps (global comparison, ergonomy, glycemic variability, discretion and adaptation to their lifestyle).

Results: Eleven patients were included. At 3 months, median A1c was similar (7.6 vs. 7.6, $\mathrm{p}=0.89$ ). Number of global hypoglycemic episodes was reduced by $31 \%(\mathrm{p}=0.03)$. Number of nocturnal hypoglycemia, AUC $\leq 70 \mathrm{mg} / \mathrm{dl}$ were not changed. ITSQ questionnaire score was improved (129 versus 138), but not significantly $(\mathrm{p}=0.075)$. The confidence of the patient in his pump to avoid hypoglycemia $(\mathrm{p}=0.009)$ and patient satisfaction with his device $(\mathrm{p}=0.027)$ were higher. In direct comparison, patients describe the superiority of $640 \mathrm{G}$ on all criteria except on discretion.

Conclusion: The pump Minimed 640G ${ }^{\circledR}$ allows a significant reduction in A1c without increasing hypoglycemia.

\section{2}

\section{RESULTS OF SELF-CONTROL OF GLUCOSE LEVEL AND CONTINUOUS GLUCOSE MONITORING IN WOMEN WITH GESTATIONAL DIABETES}

\section{O. Ulyanova $^{1}$, Z. Taubaldieva ${ }^{1}$ \\ ${ }^{1}$ National Scientific Medical Research Center, Endocrinology Department, Astana, Kazakhstan}

Background and Aims: Gestational diabetes is generally characterized by medium hyperglycemia and glucose levels which are usually normalized by introducing a low-carbohydrate diet. Usually pregnant women control their own blood glucose by glycometer. The continuous glucose monitoring (CGM) is a research method that allows to get a detailed assessment of fluctuations in blood glucose throughout the day.

Method: 3 days CGM (CGMS gold, Medtronic) and selfcontrol of glucose levels (4 times per day) by glucometer (ACCU-CHEK Active, Roche) were performed in 5 women (age range from 27 to $45 \mathrm{y}$ ) with gestational diabetes with normal levels of $\mathrm{HbA} 1 \mathrm{C}(\mathrm{HbA} 1 \mathrm{C}<6 \%)$ and $\mathrm{C}$-peptide. The mean minimal and maximal glucose daily levels, measured during 3 days by CGM and glucometer were analyzed.The amount of high ( $>10,0 \mathrm{mmol} / \mathrm{l}$ ) glucose excursion was analyzed.

Results: According to CGM data, the mean minimal glucose daily level measured by CGM were less then the mean minimal glucose daily level measured by glucometer $-3,28 \mathrm{mmol} / \mathrm{l}$ and
$4,82 \mathrm{mmol} / \mathrm{l}(\mathrm{p}<0,05)$, respectively. The mean maximal glucose daily level measured by CGM consisted $9,6 \mathrm{mmol} / \mathrm{l}$ against 8,4 $\mathrm{mmol} / \mathrm{l}$ measured by glucometer $(\mathrm{p}<0,05)$. The one of the women had 1 high glucose excursion every day during 3 days.

Conclusion: Thus, the mean minimal and maximal glucose daily levels measured by CGM system and glucometer were significantly different in women with gestational diabetes. Women with gestational diabetes with blood glucose level greater than $8.5 \mathrm{mmol} / \mathrm{l}$ measured by self-control by using a glucometer are required to undergo CGM for the timely treatment of insulin.

203

NON-ADJUNCTIVE USE OF CGM IN T1D THERAPY: IN SILICO ASSESSMENT OF USING GLUCOSE TREND INFORMATION FOR INSULIN DOSING

${\text { M. } \text { Vettoretti }^{1}, \text { A. Facchinetti }}^{1}$, G. Sparacino ${ }^{1}$, C. Cobelli ${ }^{1}$

${ }^{1}$ University of Padova, Department of Information Engineering, Padova, Italy

Background and Aims: CGM sensors are expected to improve T1D management thanks to hypo/hyperglycemic alarms and glucose trend information. By using a recently developed model of T1D patient decision-making, here we test, in silico, SMBG-based vs CGM-based insulin dosing (non-adjunctive use) and, in particular, the efficacy of correcting meal doses according to the glucose trend obtained from the CGM sensor.

Method: The decision-making model includes the last version of the UVA/Padova T1D simulator into which models of SMBG \& CGM errors and insulin therapy schemes have been incorporated. For 30 virtual patients, a single breakfast was simulated. Patient insulin sensitivity was suitably modulated in order to obtain an increasing or decreasing trend at meal-time. Two therapies were compared: 1) standard SMBG-based, and 2) nonadjunctive CGM-based, with insulin bolus increased/decreased by $\mathrm{P} \%$ when an increasing/decreasing glucose trend was detected by CGM. Six values for P were tested: 0, 10, 20, 30, 40 and 50.

Results: When meal-time trend is decreasing, non-adjunctive CGM with $\mathrm{P}_{\mathrm{opt}}=30 \%$, compared to $\mathrm{SMBG}$, increased time in euglycemia by $9 \%$ and reduced time in hypoglycemia and number of hypoglycemic events by $11 \%$ and $33 \%$, respectively. When meal-time trend is increasing, non-adjunctive CGM with $\mathrm{P}_{\text {opt }}=40 \%$, compared to SMBG, reduced time in hyperglycemia by $15 \%$ without increasing time in hypoglycemia.

Conclusion: The information provided by CGM in terms of trends and alarms allows to determine a more effective insulin meal bolus by increasing time in euglycemia and reducing hypoglycemia risk, especially when the trend at meal is decreasing.

\section{4}

\section{ACCURACY OF THE CONTINUOUS GLUCOSE SENSOR USED WITH THE PREDICTIVE LOW GLUCOSE MANAGEMENT SYSTEM}

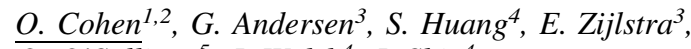
O. O'Sullivan', J. Welsh ${ }^{4}$, J. Shin ${ }^{4}$

${ }^{1}$ Chaim Sheba Medical Center, Diabetes, Ramat Gan, Israel ${ }^{2}$ Medtronic World Trade Corporation, Diabetes, Ramat Gan, Israel ${ }^{3}$ Profil, Clinical Pharmacology, Neuss, Germany

${ }^{4}$ Medtronic-Inc., Diabetes, Northridge, USA

${ }^{5}$ Medtronic International Trading Sàrl, Diabetes, Tolochenaz, Switzerland 


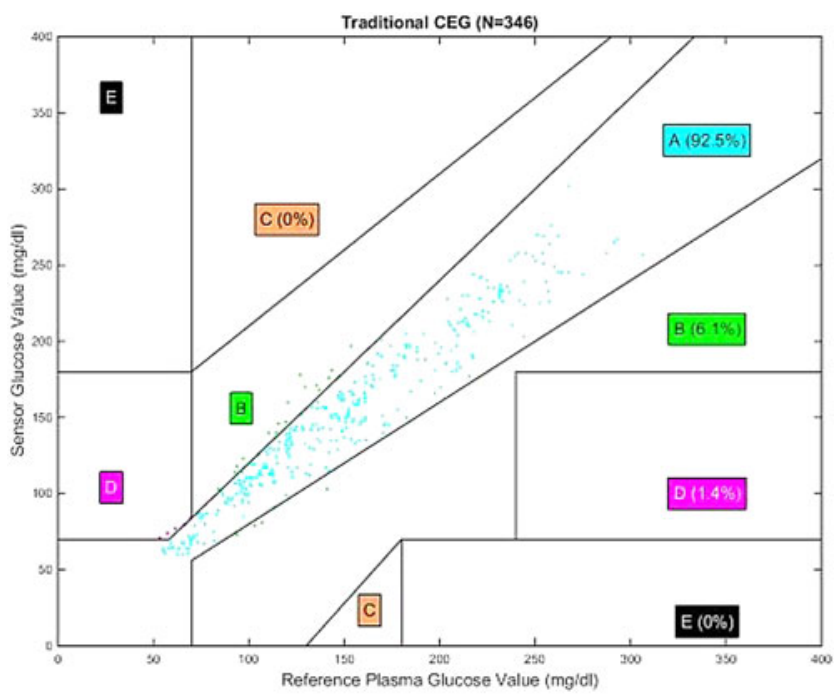

Background and Aims: The current Enlite glucose sensor, now used with the MiniMed 640G pump system (not available in the United States), benefits from design changes and an enhanced signal processing algorithm. This study evaluated performance characteristics of the sensor in patients with type 1 diabetes in a clinical research center setting.

Method: Sensors were worn for a 6-day test period by 24 adults ( 9 female) aged 21-61 years. The test period followed a 6-day runin period to familiarize subjects with the system. The $640 \mathrm{G}$ pumps were used solely for receiving and processing sensor signals and displaying sensor glucose (SG) readings. Day 3 of the test period included a clinic visit with a 4-hour interval of frequent (every $15 \mathrm{~min}$ ) reference plasma glucose measurements (YSI).

Results: A total of 346 paired YSI-SG data points were analyzed. The overall MARD $( \pm \mathrm{SD})$ was $9.1 \pm 7.1 \%$ and median absolute relative difference (ARD) 7.7\%. The overall within$20 \%$ agreement rate was $93.1 \%$ and overall mean bias was $2.4 \pm 16.0 \mathrm{mg} / \mathrm{dL}$. For the 32 pairs in which the reference value was $\leq 75 \mathrm{mg} / \mathrm{dL}$, the mean and median ARDs were $10.6 \pm 8.5 \%$ and $8.4 \%$. For reference values $\leq 75 \mathrm{mg} / \mathrm{dL}$, the bias was $4.8 \pm 7.1 \mathrm{mg} / \mathrm{dL}$. Sensors had a mean functional life of 131.3 hours (95\% CI, 116.9-145.7 hours) and a median functional life of 141.4 hours. Clarke Error Grid analysis (figure) showed 98.6\% of paired points in the clinically acceptable A + B zones.

Conclusion: Glucose values from the current Enlite sensor were in good agreement with reference values, confirming its acceptability for use with the MiniMed 640G system.

\section{5}

\section{TRUSTING DIABETES TECHNOLOGY OR PATIENTS?}

M. Yafi ${ }^{1}$, K. Collins ${ }^{1}$, C. Bowden ${ }^{1}$, C. Hughes ${ }^{1}$

${ }^{1}$ University of Texas at Houston Health Science Center, Pediatric Endocrinology, Houston, USA

Background and Aims: The advancement of diabetes technology has offered multiple methods of monitoring glucose levels. Consistent testing with glucometers and CGMS remain a cornerstone in management. It is still important, however, to evaluate the correct utilization of these testing methods in children and adolescents who may have the habit of reporting false FBSs.
Method: We present 2 cases in pediatric adolescents with diabetes that challenged the technology use of glucometers for monitoring FBSs:

- 16 yrs female T1DM

- $\mathrm{HbA} 1 \mathrm{C} 12.8 \%$

- Reported FBSs: $100-200 \mathrm{mg} / \mathrm{dl}$

The patient admitted using the control solution provided with her home blood glucose meter to do the tests, instead of using her own blood.

- 18 yrs female T1DM

- HbA1C $13.2 \%$

- Download of glucometer normal FBSs below $110 \mathrm{mg} / \mathrm{dl}$ Her boyfriend admitted "offering himself" to use his blood test on the glucometer to make her feel better about diabetes.

Results: When discrepancies arise between the use of diabetes technology at home and in professional settings, further evaluation is needed. This may include repeating the $\mathrm{HbA} 1 \mathrm{C}$ testing or evaluating the testing devices for any malfunctions. This may also require replacing the current testing device (glucometer), offering a higher level of testing such as Continuous Glucose Monitor System (CGMS) or even therapy such as closed-loop sensor with insulin pump.

Conclusion: Facing discrepancies between different testing methods, the healthcare providers should evaluate the following:

1- Technology/device: Evaluate malfunction

2- Human aspect/persons involved or witnessing testing: Perform testing under the supervision of the healthcare provider and explore the possibility of false testing.

\section{6}

EFFICACY OF BASAL-BOLUS INSULIN REGIMES IN HOSPITALIZED NON-CRITICALLY ILL PATIENTS WITH TYPE 2 DIABETES: A SYSTEMATIC REVIEW AND META-ANALYSIS

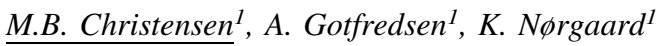

${ }^{1}$ Hvidovre University Hospital, Department of Endocrinology, Hvidovre, Denmark

Background and Aims: Hyperglycemia during hospitalization is associated with increased rates of complications and longer hospital stays. Basal-bolus insulin therapy (BBI) is recommended for inpatient diabetes management of non-critically ill patients on empirical basis. We aimed to assess the efficacy and safety of BBI therapy by summarizing all evidence from studies of BBI versus sliding scale insulin therapy (SSI) for hospitalized non-critically ill diabetic patients.

Method: An electronic search for studies comparing BBI therapy with SSI therapy was conducted. Primary outcome was mean daily blood glucose (BG) during admission. Secondary outcomes were hypoglycemia and length of hospital stay. Results

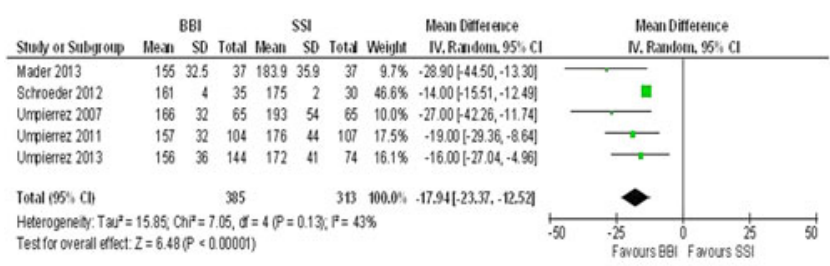

Figure 1: Meta-analysis of mean daily blood glucose with basal-bolus insulin versus sliding scale insulin (SSI) 


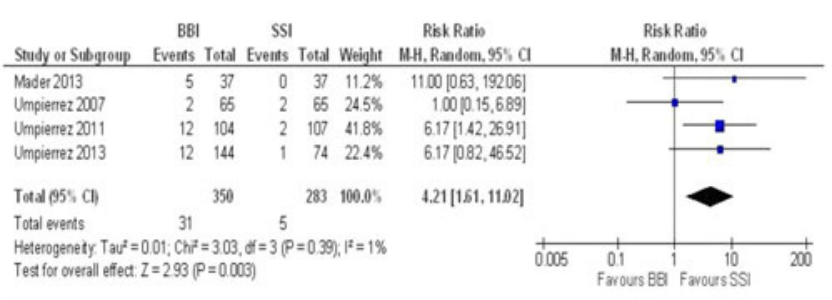

Figure 2: Riskratio of hypoglycemia (BGS60 mg/dl) with basal-bolus insulin (BBI) versus sliding scale insulin (SSI)

of included randomized controlled trials (RCT) were pooled and meta-analyzed to provide estimates of the efficacy of BBI therapy.

Results: Five RCTs and seven observational studies were included in the review. Meta-analysis of RCTs showed significantly lower mean daily blood glucose with BBI than with SSI. Mean difference in daily BG between the two regimes ranged from $14-28.9 \mathrm{mg} / \mathrm{dl}$. The incidence of hypoglycemia ranged from $2 \%$ to $29 \%$. BBI therapy was associated with increased risk of mild hypoglycemia (BG $\leq 70 \mathrm{mg} / \mathrm{dl}$, RR 5.75 ; $95 \%$ CI 2.79 11.83), (BG $\leq 60 \mathrm{mg} / \mathrm{dl}, \mathrm{RR} 4.21 ; 95 \%$ CI 1.61-11.02) compared with SSI therapy. There was no difference in risk of severe hypoglycemia (BG $\leq 40 \mathrm{mg} / \mathrm{dl}$ ) and no difference in mean length of stay.

Conclusion: Basal-bolus insulin for the inpatient diabetes management results in significant lower mean daily blood glucose than sliding scale insulin but is associated with increased risk of mild hypoglycemia.

\section{7}

\section{HYPOGLYCAEMIA PREVENTION IN CHILDREN WITH TYPE 1 DIABETES BY USING SMARTGUARD ALGORITHM IN SENSOR-AUGMENTED PUMP THERAPY}

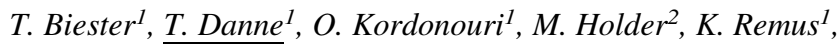
T. Wadien ${ }^{2}$, A. Thomas $^{3}$

${ }^{1}$ Kinderkrankenhaus auf der Bult, Janusz-Korczak-Allee 12, D-30173 Hannover, Germany

${ }^{2}$ Olgahospital Stuttgart, Kinderklinik, Kriegsbergstr. 62, D-70176 Stuttgart, Germany

${ }^{3}$ Medtronic GmbH Meerbusch, Germany

Sensor-augmented insulin pump (SAP) with the "MiniMed ${ }^{\circ} 640 G$ " system features the PLGM algorithm (predictive low glucose management $=$ "SmartGuard") which stops insulin pump delivery based on predicted sensor glucose levels. This may offer advanced protection against hypoglycaemia.

The present study with pediatric patients in Germany had 3 phases: phase 1 - CSII with MM640G (4 weeks), phase 2 - SAP with MM640G without SmartGuard (2 weeks), phase 3 MM640G with SmartGuard (6 weeks). Following settings for SmartGuart were applied in the study: switch off before Low: $70 \mathrm{mg} / \mathrm{dL}$, threshold for hypoglycemia: $70 \mathrm{mg} / \mathrm{dL}$. Primary outcome was the potential reduction in the frequency of hypogly-

\begin{tabular}{|l|l|l|l|}
\hline & \multicolumn{1}{|c|}{ Phase 1 } & \multicolumn{1}{c|}{ Phase 2 } & P-value \\
& Without SmartGuard & with SmartGuard & \\
\hline Excursions/day $<70 \mathrm{mg} / \mathrm{dl}$ & $0.96 \pm 0.59$ & $0.61 \pm 0.39$ & 0.050 \\
\hline Excursions/day $=40 \mathrm{mg} / \mathrm{dl}$ & $0.12 \pm 0.12$ & $0.04 \pm 0,03$ & 0.024 \\
\hline AUC/Tag $<70 \mathrm{mg} / \mathrm{dl}\left(\mathrm{mg} / \mathrm{dl}{ }^{*}\right.$ day) & $0.51 \pm 0.60$ & $0.23 \pm 0.13$ & 0.057 \\
\hline Time/day $<70 \mathrm{mg} / \mathrm{dL}(\mathrm{min})$ & $59.0 \pm 55.6$ & $21.7 \pm 18.5$ & 0.025 \\
\hline
\end{tabular}

cemic episodes and hypoglycemic intensity (AUC and time $<70 \mathrm{mg} / \mathrm{dl}$ ).

The interim analysis of 18 patients (age 9.9 \pm 3.8 years, dia-

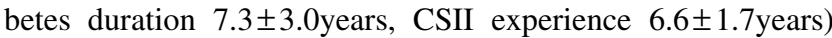
showed $2.64 \pm 0.54$ "interruptions before Low" per day and patient with SmartGuard. The total pump stoppage time was $149 \pm 36$ min per day. The pump stoppage time per event was $62.1 \mathrm{~min}$. On average, the lowest glucose value during insulin shutdown was observed after $75.1 \mathrm{~min}$. In $10 \%$ of cases the glucose values were lower than $55 \mathrm{mg} / \mathrm{dL}$. The predictive low glucose shutdown of insulin delivery occurred at a glucose level of $107.5 \mathrm{mg} / \mathrm{dL}$, and the average level at resumption was $108.0 \mathrm{mg} / \mathrm{dL}$. Comparing phase of SAP with/without SmartGuard, decreased hypoglycemia events and intensity by using SmartGuard (table).

The present study provides evidence for further reducing the risk for hypoglycemia with SmartGuard without compromising the safety of SAP therapy.

\section{8}

\section{CLINICAL AND ECONOMIC CONSEQUENCES OF TYPE 1 DIABETES IN REAL-LIFE SETTING IN SPAIN}

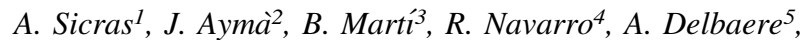
J. Debén

${ }^{1}$ Badalona Serveis Assistencials, Dirección de Planificación, Badalona- Barcelona, Spain

${ }^{2}$ Hospital Municipal de Badalona, Servicio de Endocrinología, Badalona- Barcelona, Spain

${ }^{3}$ Medtronic Ibérica S.A., Health Economics \& Outcomes Research, Madrid, Spain

${ }^{4}$ Hospital Germans Trias i Pujol, Documentación Médica, Badalona. Barcelona, Spain

${ }^{5}$ Medtronic international Sarl, Health Economics \& Outcomes Research, Tolochenaz, Switzerland

${ }^{6}$ Medtronic Ibérica S.A., Marketing, Madrid, Spain

Background and Aims: Type 1 Diabetes Mellitus (T1DM) is a chronic disease with major societal impact. The aim of this

Rate of diabetes related complications during last year of study (new cases) in the 2 groups

\begin{tabular}{lrrrr}
\hline Groups & HbA1c $\leq 7 \%$ & HbA1C $>7 \%$ & Total & \\
Number of patients, \% & $\mathrm{N}=271(45.1 \%)$ & $\begin{array}{r}\mathrm{N}=330 \\
(54.9 \%)\end{array}$ & $\begin{array}{r}\mathrm{N}=601 \\
(100 \%)\end{array}$ & $\mathrm{p}$ \\
\hline Hypoglycemia / Hyperglycemia & $36.2 \%$ & $53.6 \%$ & $45.8 \%$ & 0.002 \\
- Hypoglycemia mildmoderate & $29.5 \%$ & $47.0 \%$ & $39.1 \%$ & $<0.001$ \\
- Hypoglycemia severe & $10.3 \%$ & $25.5 \%$ & $23.3 \%$ & $<0.001$ \\
- Hyperglycemia (DKA) & $4.5 \%$ & $6.7 \%$ & $5.5 \%$ & 0.247 \\
\hline Cardiovascular events & $6.7 \%$ & $10.9 \%$ & $8.7 \%$ & 0.048 \\
- Ischemic cardiopathy & $2.2 \%$ & $3.1 \%$ & $3.0 \%$ & 0.725 \\
- Cerebrovascular disease & $2.0 \%$ & $2.7 \%$ & $2.5 \%$ & 0.575 \\
- Heart failure & $1.2 \%$ & $3.1 \%$ & $2.5 \%$ & 0.286 \\
\hline Microvascular complications & & & & \\
- Retinopathy & $11.4 \%$ & $22.0 \%$ & $16.2 \%$ & $<0.001$ \\
- Neuropathy & $3.1 \%$ & $9.6 \%$ & $6.1 \%$ & $<0.001$ \\
- Nephropathy & $1.8 \%$ & $4.9 \%$ & $2.9 \%$ & 0.012 \\
\hline
\end{tabular}


study was to assess the burden of the disease of T1DM in the Spanish setting.

Method: A retrospective cohort study was conducted in the Badalona healthcare region. T1DM patients ( $\geq 15$ years) with access to care between 2005-2014 were included in the analysis. Sociodemographic data, diabetes related complications, treatment and health care resources utilizations were collected. Associated direct and indirect costs were calculated. Data were analyzed by means of logistic regression and ANCOVA $(\mathrm{p}<0.05)$ and results are presented for subgroups of patients defined by HbA1c levels $(\leq 7 \%$ or $>7 \%$ ).

Results: A total of 601 patients were included ( $54.1 \%$ men) with an average age of 49.1 years. DMT1 prevalence was $0.58 \%$ and the incidence rate 26.2/100,000 people/year. Out of the total population $54.9 \%$ had poor glycemic control (HbA1c > 7\%) and $12 \% \mathrm{HbA} 1 \mathrm{c}$ $>9 \%$. Patients with $\mathrm{HbA1c} \leq 7 \%$ had less severe hypoglycemic events $(10.3 \%$ vs $25.5 \% ; \mathrm{p}<0.001)$, cardiovascular events $(6.7 \%$ vs $10.9 \% ; \mathrm{p}=0.048)$ and mean total costs $€ 2,542$ vs $€ 3,433(\mathrm{p}<0.001)$ compared to the ones with $\mathrm{HbAlc}>7 \%$.

Conclusion: The T1DM incidence slightly increased over time of observation. Diabetes has a significant impact on the healthcare system. Resources utilization and costs of care are high, especially for patients with poor glycemic control, severe hypoglycemic events and metabolic syndrome. Potential savings can be obtained if better glycemic control can be achieved in T1DM patients.

209

\section{ACCURACY OF CONTINUOUS GLUCOSE MONITORING IN PATIENTS AFTER MAJOR ABDOMINAL SURGERY}

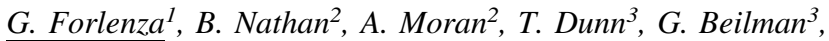 \\ T. Pruett ${ }^{3}$, B. Kovatchev ${ }^{4}$, M. Bellin ${ }^{2}$ \\ ${ }^{1}$ University of Colorado Denver, Barbara Davis Center, Aurora, USA \\ ${ }^{2}$ University of Minnesota, Pediatric Endocrinology, \\ Minneapolis, USA \\ ${ }^{3}$ University of Minnesota, Transplant Surgery, Minneapolis, USA \\ ${ }^{4}$ University of Virginia, Center for Diabetes Technology, \\ Charlottesville, USA
}

Background and Aims: Among post-surgical and critically ill patients, dysglycemia is associated with increased complications. Continuous glucose monitoring (CGM) in the inpatient population may enhance glycemic control. CGM may be compromised, however, by postsurgical complications such as edema or vascular changes. In this project we utilize Clarke Error Grid (CEG) and Surveillance Error Grid (SEG) analysis to evaluate CGM performance after Total Pancreatectomy with Islet AutoTransplantation.

Method: This sub-analysis looked at Medtronic Enlite 2 CGM glucose v. YSI serum glucose in 7 post-transplant patients $(86 \%$ female; $38.6 \pm 9.4$ years) on artificial pancreas for 72 hours at transition from IV to subcutaneous insulin. Sensor recalibration occurred for ARD $\geq 20 \% \times 2, \geq 30 \% \times 1$, or by investigator discretion based on trend.

Results: Sensor analysis showed MARD of $11.0 \pm 11.5 \%$. The sensors were recalibrated 8.3 times/day; active sensor was switched 1.4 times/day. Calibration factor was $7.692 \pm 3.786 \mathrm{mg} /$ $\mathrm{nA} * \mathrm{dL}$ (target $=1.5$ to $20 \mathrm{mg} / \mathrm{nA} * \mathrm{dL}$ ). CEG analysis showed $86.1 \%$ of pairs in Zone $\mathrm{A}$ and $99.4 \%$ of pairs in Zones $\mathrm{A}+\mathrm{B}$ (Figure 1 and Table 1). SEG analysis showed $92.22 \%$ of pairs in the "no risk" zone, $5.96 \%$ of pairs in the "slight, lower" risk zone,

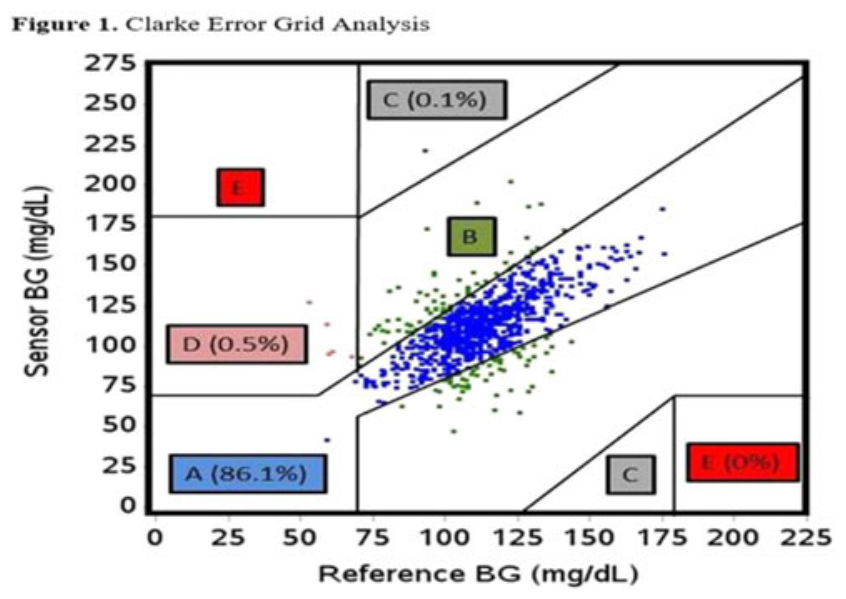

Figure 2. Surveillance Error Grid Analysis
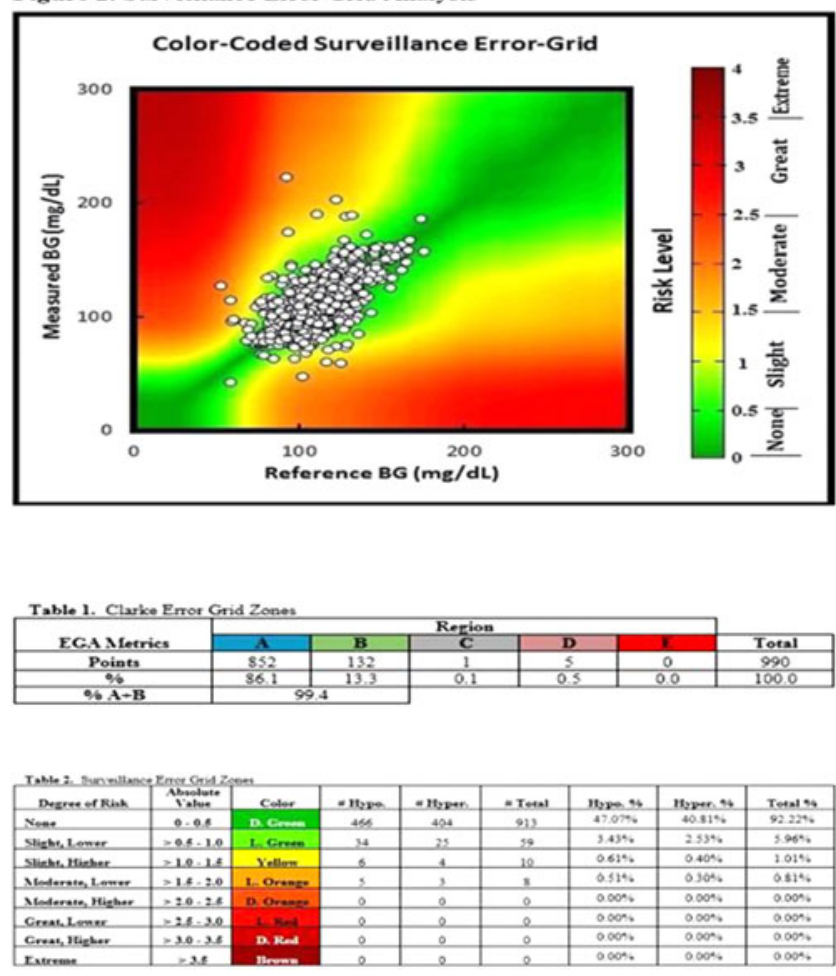

$1.01 \%$ of pairs in the "slight, higher" risk zone and only $0.81 \%$ of pairs in the "moderate, lower" risk zone (Figure 2 and Table 2).

Conclusion: Overall performance of the Medtronic Enlite 2 CGM in the post-transplant population was reasonably good with "no risk" or "slight, lower" risk by SEG analysis and high CGM-YSI agreement by CEG analysis, however frequent recalibrations were required in this population.

\section{0}

\section{FEATURES OF GASTRIC EMPTYING IN PATIENTS WITH TYPE 2 DIABETES MELLITUS DEPENDING TO THE GLYCEMIC CONTROL}

\section{Kostitska ${ }^{1}$}

${ }^{1}$ Ivano-Frankivsk National Medical University, Endocrinology, Ivano-Frankivsk, Ukraine 
Background and Aims: Gastroparesis is the most overlooked chronic complication of diabetes mellitus (DM). Several factors including hyperglycaemia, obesity, hyperinsulinaemia have been found to be associated with delayed on motor function of stomach. The aim of the study is to assess the prevalence, risk factors for diabetic gastroparesis (DG) and to investigate the noninvasive method: ${ }^{13} \mathrm{C}$-octanoic breast test $\left({ }^{13} \mathrm{C}-\mathrm{OBT}\right)$ of preclinical diagnosis for gastric emptying.

Method: We have studied 25 subjects (10 male/15 female, mean age $-48.7 \pm 6.6$ years) with type $2 \mathrm{DM}$ with the duration of $13.5 \pm 4.7$ years, mean value of $\mathrm{HbA}_{1} \mathrm{C}$ was $9.2 \pm 1.1 \%$, body mass index (BMI) $-34,6 \pm 2,8 \mathrm{~kg} / \mathrm{m}^{2}$ and 10 healthy volunteers $(5$ male/5 female, mean age was $45.4 \pm 9.6$ years, BMI$\left.18,6 \pm 1,1 \mathrm{~kg} / \mathrm{m}^{2}\right)$ as the control group. Was made synchronous monitoring of glycemia ( 0 rain - start of the test and during the test after 60, 150, 240 min) and ${ }^{13} \mathrm{C}$-OBT.

Results: ${ }^{13} \mathrm{C}$-OBT we have found out that $9(36 \%)$ patients has been diagnosed the mild degree of low gastric motility ( $\left.\mathrm{T}^{1 / 2}-89,2 \pm 3,1 \mathrm{~min}\right), 12(48 \%)$ diabetics - moderate degree $\left(\mathrm{T}^{1 / 2}\right.$ $95.4 \pm 11.2 \mathrm{~min}), 4(16 \%)$ subjects diagnosed severe degree $\left(\mathrm{T}^{1} 1 / 2-\right.$ $103.5 \pm 13.5 \mathrm{~min})$. Glycemia during ${ }^{13} \mathrm{C}$-OBT: $0 \mathrm{~min}-10,3 \pm 4,1$ $\mathrm{mmol} / \mathrm{L}, 60 \mathrm{~min}-11,3 \pm 4,3 \mathrm{mmol} / \mathrm{L}, 150 \mathrm{~min}-11,2 \pm 3,9 \mathrm{mmol} / \mathrm{L}$, $240 \mathrm{~min}-9,9 \pm 4,1 \mathrm{mmol} / \mathrm{L}$. Significant correlations were observed between results of ${ }^{13} \mathrm{C}-\mathrm{OBT}$ and BMI $(\mathrm{r}=0,84, \mathrm{p}<0,01)$ and $\mathrm{HbA}_{1} \mathrm{C}(\mathrm{r}=0,81, \mathrm{p}<0,01)$, but there were no significant associations with the glycemia during the ${ }^{13} \mathrm{C}-\mathrm{OBT}$.

Conclusion: Obesity and hyperglycemia are diagnostical markers of delays gastric emptying in patients with type $2 \mathrm{DM}$.

\section{1}

\section{DIABETES IN PREGNANCY: ARE WE ON THE RIGHT TRACK?}

\author{
G. Mlawa ${ }^{1}$, S. Moyo $^{1}$, B. Ajay ${ }^{1}$ \\ ${ }^{1}$ Croydon University Hospital, Diabetes \& Endocrine/ \\ Obstetrics \& Gynaecology, London, United Kingdom
}

Background and Aims: 2-5\% of pregnancies in the UK are complicated by diabetes. $87.5 \%$ of these are affected by gestational diabetes and $7.5 \%$ by pre-existing diabetes type 1 and $5 \%$ by type 2 . Diabetes in pregnancy is associated with increased risks to the woman and foetus. Croydon is socially deprived area with diverse ethnicity. Maternity services must ensure that diabetic women are seen antenatally in multidisciplinary setting.

The aim was to assess whether pregnant diabetic women were seen in clinic as per guidelines and to determine the outcome of all diabetic pregnancies in line with the below audit standards.

Method: Retrospective study/audit involving 257 pregnant diabetic women. The following demographic data was collected: type of diabetes, ethnicity, weight of baby at birth $<2.5 \mathrm{~kg}$ or $>4.5 \mathrm{~kg}$, admission to special baby care unit, delivery by caesarean section and type of treatment.

Standards: All previous Gestational diabetes women referred to the joint antenatal clinic $-100 \%$

All diabetic women seen in the multidisciplinary antenatal clinic $-100 \%$

Care plan documented $-100 \%$

Results: There were 2 Type 1 diabetes women, 21 Type 2 diabetes, and 234 Gestational diabetes, (87 diet controlled, 116 on metformin, and 31 on both insulin and metformin).

Of the 257 women, 99 had a caesarean section (38.5\%). 26/257 (10\%) babies required admission to the neonatal unit. $3 \%(8 / 257)$ of all diabetic women had babies who needed admission for neonatal hypoglycaemia. $88 \%$ of all babies born had a normal weight.
Conclusion: The study showed our practice is compliant with the guidelines. It also showed that being of an Asian ethnicity puts you at a higher risk of developing diabetes.

\section{2}

\section{THE IMPACT OF PARITY ON DIETARY INTAKES IN WOMEN WITH GESTATIONAL DIABETES MELLITUS}

\author{
S. Nomachi ${ }^{1}$, F. Nagumo ${ }^{1}$, Y. Emori ${ }^{2}$, H. Shimano ${ }^{3}$, \\ H. Hamada \\ ${ }^{1}$ University of Tsukuba, Graduate School of Comprehensive \\ Human Science, Tsukuba, Japan \\ ${ }^{2}$ University of Tsukuba, Maternity Nursing and Midwifery- \\ Faculty of Medicine, Tsukuba, Japan \\ ${ }^{3}$ University of Tsukuba, Department of Internal Medicine- \\ Faculty of Medicine, Tsukuba, Japan \\ ${ }^{4}$ University of Tsukuba, Department of Maternal-Fetal \\ Medicine Faculty of Medicine, Tsukuba, Japan
}

Background and Aims: Gestational diabetes mellitus (GDM) is one of the most common pregnancy complications with a prevalence of $12.4 \%$ in Japan. Nutrition therapy is an integral part of the treatment of GDM. A relationship between parity and dietary intakes in GDM has not been established. The purpose of this study was to investigate the effects on maternal parity and dietary intakes in women with GDM.

Method: A study was conducted from March to August 2015. Sixty-four Japanese women (38 primipara, 26 multipara) were enrolled in the study. Dietary habits during the last month of their gestation were assessed with a self-administered dietary history questionnaire. This study was approved by the ethics committee of the University of Tsukuba hospital review board.

Results: No significant differences were observed by the parity in age, pre-pregnancy BMI, weight, and $\mathrm{HbA} 1 \mathrm{c}$ level.The mean energy intake and the percentage of kilocalories from carbohydrate were significantly higher in the multipara women than in the primipara women $(1,460 \mathrm{kcal}$ vs. $1,351 \mathrm{kcal}, \mathrm{p}<.05$; $54.3 \%$ vs. $50.7 \%, \mathrm{p}<.05)$. In multipara, the mean dietary fiber and folic acid intakes were significantly lower than the recommended dietary allowance $(15.3 \mathrm{~g} /$ day vs. $17 \mathrm{~g} / \mathrm{day}, \mathrm{p}<.05$; $240 \mu \mathrm{g} /$ day vs. $480 \mu \mathrm{g} / \mathrm{day}, \mathrm{p}<.001)$.

Conclusion: Parity has a positive effect on dietary intakes in women with GDM. The dietary carbohydrate intake and inadequate dietary patterns may cause pregnancy complications and increase the risk of developing type 2 diabetes mellitus in pregnant women. The multipara women with GDM should be encouraged to eat a well-balanced diet, both during and after pregnancy.

\section{3}

\section{LONG TERM TREATMENT WITH IMPLANTABLE PUMP IN PATIENTS WITH TYPE 1 DIABETES: EVIDENCE THAT THE INTRAPERITONEAL ROUTE HAS NO DELETERIOUS EFFECT ON THE LIVER FUNCTION}

$\underline{\text { S. Baillot-Rudoni }}{ }^{1}$, B. Bouillet ${ }^{1}$, P. Buffier ${ }^{1}$, G. Vaillant $^{1}$, ${\text { A. } \text { Farret }^{2} \text {, P. Schaepelynck }}^{3}$, J.M. Petit ${ }^{1}$, B. Vergès ${ }^{1}$

${ }^{1}$ Hospital, Endocrinology-Diabetology and Metabolic Diseases, Dijon, France

${ }^{2}$ Hospital, Diabetology and Metabolic Diseases, Montpellier, France

${ }^{3}$ Hospital, Endocrinology, Marseille, France 
Background and Aims: In rare cases, treatment with implantable pumps in type 1 diabetes (T1D) has led to focal hepatic steatosis. Furthermore, recent studies have shown conflicting data about the possibility of steatosis in T1D. In T1D patients, we investigated whether implantable pumps were more likely than subcutaneous therapy to induce biological modifications of hepatic function.

Method: Seventeen patients receiving intraperitoneal insulin (G1) were matched with 17 patients receiving subcutaneous insulin (G2) for sex, age and duration of diabetes. We compared transaminases, lipoproteins, triglycerides and BMI before internal/external pump and at the end of the study.

Results: Initially, the two groups were comparable for BMI, transaminases and lipoproteins; there was a trend towards better metabolic control in G 1 than in $\mathrm{G} 2(\mathrm{p}=0.07)$. The mean duration of each treatment type was comparable (12.8 $\pm 5.2 / 11 \pm 7.2$ years -G1/G2-). In 2015 mean ALAT were similar in the two groups $(26.4 \pm 11.4 / 27 \pm 15.4 \mathrm{UI} / \mathrm{L}-\mathrm{G} 1 / \mathrm{G} 2-)$; there were no differences between the 2 groups for LDL, HDL cholesterol and triglycerides, or for $\mathrm{HbA} 1 \mathrm{c}$ and $\mathrm{BMI}$; in G1, HDL cholesterol level was higher at the end of the study than at inclusion $(\mathrm{p}=0.03)$.

Conclusion: Lipoproteins, triglycerides and ALAT in patients treated with an implantable pump over 12 years were no higher than those in patients treated with an external pump. However, HDL cholesterol level improved in the group of patients treated with an implantable pump, which argues against an increased metabolic risk with the intraperitoneal route in type 1 diabetic patients.

\section{4}

\section{SERIOUS GAMING IN DIABETES: COMBINING APPS AND GAMING PRINCIPLES IN A HOLISTIC DIABETES ENVIRONMENT}

E. Arsand $^{1,2}$, A. Makhlysheva ${ }^{3}$, M. Bradway ${ }^{1}$, T. Chomutare $^{1}$, $\overline{\text { S.G. Johansen }}{ }^{1,4}$, H. Blixgård ${ }^{1}$, G. Hartvigsen ${ }^{4}$

${ }^{1}$ University Hospital of North Norway, Norwegian Centre for Integrated Care and Telemedicine, Troms $\phi$, Norway

${ }^{2}$ UiT - The Arctic University of Norway, Department of Clinical Medicine, Tromso, Norway

${ }^{3}$ University Hospital of North Norway, Norwegian Centre for Integrated Care and Telemedicine, Tromso, Norway

${ }^{4}$ UiT - The Arctic University of Norway, Department

of Computer Science, Tromso, Norway

Background and Aims: There have been many attempts to design engaging games for children and adolescents with diabetes. However, it is often challenging to sustain the users' engagement in these games. The project "Play and learn with Diabetes Friends" aims to combine mobile phone-based elements such as the Diabetes Diary app (www.diabetesdagboka.no), a quiz app, mobile games, and a social network app.

Method: By allowing users to play the same games and quizsets as other users, the social network provides a positive yet competitive environment as they are motivated to achieve better scores. However, rewards are not predicated upon their blood glucose values. This approach is chosen based on the assumption that engaging in such a chronic illness is challenging enough for this age group of patients that the focus should be on rewarding their involvement in the diabetes management process, rather than on improving health factors.

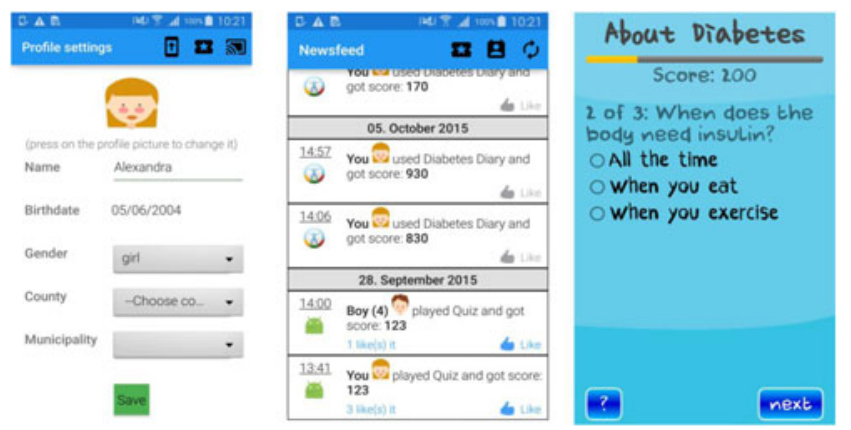

Results: With the aim of encouraging and guiding this group to better self-manage their diabetes, we developed a holistic support system consisting of a collection of tools which work together to form a motivational and educational environment (Figure 1). This system displays anonymous users' activities from the diabetesrelated apps mentioned above, calculates scores, awards badges for a user's engagement in diabetes management, and allows users to "like" one another's achievements and activities.

Conclusion: The proposed concept is currently being tested and will be available on Google Play prior to ATTD2016, where details about how it is received by patients, healthcare workers and the Norwegian Diabetes Association, will be presented.

\section{5}

RESULTS OF THE RANDOMIZED TRIAL OF LONGACTING INSULIN GLARGINE TITRATION WEB TOOL (LTHOME) VS. ENHANCED USUAL THERAPY OF GLARGINE TITRATION (INNOVATE TRIAL)

H. Bajaj ${ }^{1}, \underline{\text { K. Venn }}{ }^{1}, C . Y e^{1}$, R. Aronson ${ }^{1}$

${ }^{1}$ LMC Diabetes \& Endocrinology, Diabetes, Toronto, Canada

Background and Aims: Basal insulin titration in the realworld is often prolonged and unsuccessful, due to titration complexity and hypoglycemia. LTHome is a web tool that applies a rules engine-based algorithm providing insulin titration advice directly to the patient.

Method: INNOVATE is a randomized, investigator-initiated trial evaluating the safety and efficacy of basal insulin glargine titration by LTHome compared to Enhanced Usual Therapy (EUT - healthcare provider driven diabetes education program)

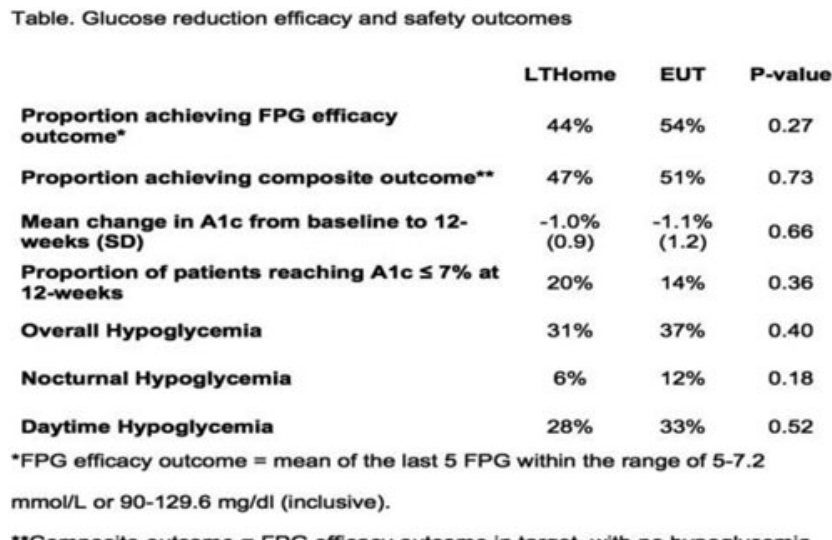

*Composite outcome = FPG efficacy outcome in target, with no hypoglycemia

LTHome $=$ web-tool. EUT $=$ Enhanced Usual Therapy 
over 12 weeks. Important inclusion criteria for patients scheduled to initiate or titrate basal insulin were: type 2 diabetes, 1875 years, computer literacy, Fasting Plasma Glucose (FPG) $>7$ $\mathrm{mmol} / \mathrm{L}$ and A $1 \mathrm{c}>7.0 \%$. INNOVATE protocol was approved by ethics board. INNOVATE trial is supported by Sanofi.

Results: One hundred thirty nine subjects, with comparable baseline characteristics, were randomized to LTHome or EUT. Results are outlined in the Table. Composite endpoint (mean of last $5 \mathrm{FPG}$ within the range of 5-7.2 $\mathrm{mmol} / \mathrm{L}$ prior to 12 weeks trial end + no documented hypoglycemia during the trial period) was achieved in $47 \%$ of LTHome users vs. $51 \%$ in EUT $(\mathrm{p}=0.73)$. A1c reduction $(-1.0 \%$ and $-1.1 \%, \mathrm{p}=0.66)$ and proportion achieving A $1 \mathrm{c}$ target $\leq 7 \%(14 \%$ and $20 \%, \mathrm{p}=0.36)$ were similar between the LTHome and EUT arms, respectively. Similar hypoglycemia incidence was observed with LTHome and EUT - with no severe hypoglycemia reported.

Conclusion: LTHome - with its rules-engine based algorithm - may offer an effective and safe real-world alternative to EUT, for basal insulin initiation and titration.

\section{6}

PATIENT SATISFACTION SCORE CHANGES IN THE RANDOMIZED TRIAL OF LONG-ACTING INSULIN GLARGINE TITRATION WEB TOOL (LTHOME) VS ENHANCED USUAL THERAPY OF GLARGINE TITRATION (INNOVATE)

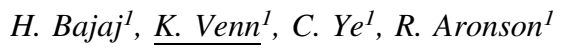

${ }^{1}$ LMC Diabetes \& Endocrinology, Diabetes, Toronto, Canada

Background and Aims: Basal insulin initiation and titration in the real-world is commonly performed under the guidance of diabetes education programs (DEP). Use of health information technology for insulin titration - as an alternative to DEP - may offer benefits in terms of greater treatment satisfaction, quality of life as well as self-efficacy parameters. LTHome is a web tool that applies a rules engine-based algorithm providing insulin titration advice directly to the patient.

Method: INNOVATE is a randomized, investigator-initiated trial, supported by Sanofi, that evaluates the safety and efficacy of basal insulin glargine titration by LTHome compared to Enhanced Usual Therapy (EUT - healthcare provider driven DEP) over 12 weeks. Important inclusion criteria for patients

\begin{tabular}{|c|c|c|c|c|}
\hline & & mean & SD & p value \\
\hline Change DTSQs & LTHome & 3.2 & $\begin{array}{l}7.1 \\
5.0\end{array}$ & 0.69 \\
\hline Change HFS & $\begin{array}{c}\text { LTHome } \\
\text { EUT }\end{array}$ & $\begin{array}{l}0.0 \\
4.1\end{array}$ & $\begin{array}{c}9.6 \\
12.5\end{array}$ & 0.04 \\
\hline Change Total DDS Score & LTHome & $\begin{array}{l}-8.8 \\
-3.1\end{array}$ & $\begin{array}{l}17.5 \\
13.5\end{array}$ & 0.04 \\
\hline Change Emotional Burden & $\begin{array}{c}\text { LTHome } \\
\text { EUT }\end{array}$ & $\begin{array}{l}-3.1 \\
-1.1\end{array}$ & $\begin{array}{l}5.9 \\
4.2\end{array}$ & 0.03 \\
\hline Change Physician Distress & $\begin{array}{c}\text { LTHome } \\
\text { EUT }\end{array}$ & $\begin{array}{l}-0.9 \\
0.4\end{array}$ & $\begin{array}{l}4.9 \\
4.2\end{array}$ & 0.10 \\
\hline Change Regimen Distress & $\begin{array}{c}\text { LTHome } \\
\text { EUT }\end{array}$ & $\begin{array}{l}-4.3 \\
-2.0\end{array}$ & $\begin{array}{l}5.6 \\
5.0\end{array}$ & 0.02 \\
\hline Change Interpersonal Distress & $\begin{array}{c}\text { LTHome } \\
\text { EUT }\end{array}$ & $\begin{array}{l}-0.5 \\
-0.3\end{array}$ & $\begin{array}{l}3.6 \\
2.7\end{array}$ & 0.61 \\
\hline Change WHOS Score & $\begin{array}{c}\text { LTHome } \\
\text { EUT }\end{array}$ & $\begin{array}{l}1.1 \\
1.3\end{array}$ & $\begin{array}{l}3.8 \\
3.9\end{array}$ & 0.83 \\
\hline Change WHO5 Percentage & $\begin{array}{c}\text { LTHome } \\
\text { EUT }\end{array}$ & $\begin{array}{l}4.5 \\
5.1\end{array}$ & $\begin{array}{l}15.3 \\
15.6\end{array}$ & 0.83 \\
\hline
\end{tabular}

DTSQ $=$ Diabetes Treatment Satisfaction Questionnaire

HFS $=$ Fear of hypoglycemia Score

DDS $=$ Diabetes Distress Score

WHO5 $=$ WHO-5 well-being index scheduled to initiate or titrate basal insulin were: type 2 diabetes, 18-75 years, computer literacy and $\mathrm{HbA} 1 \mathrm{c}>7.0 \%$.

Results: One hundred thirty nine subjects, with comparable baseline characteristics, were randomized to LTHome or EUT. At the end of trial period of 12-weeks, a significant difference in change of satisfaction scores in favor of the LTHome arm was observed compared to EUT: 1. Fear of hypoglycemia Score (HFS) change was 4.1 in EUT vs. 0.0 in LTHome, $\mathrm{p}=0.04 ; 2$. Diabetes Distress Scale (DDS) reduction was -3.1 in EUT vs. -8.8 in LTHome, $\mathrm{p}=0.04$.

Conclusion: LTHome as an alternative to EUT for basal insulin initiation and titration in the real world may offer better patient satisfaction - mainly driven by a perceived fear of hypoglycemia, emotional burden and regimen distress.

\section{7}

\section{CLINIC DOWNLOADING AND VIEWING OF GLUCOSE LOGBOOKS IS VERY POPULAR WITH YOUNG DIABETES IMPROVERS}

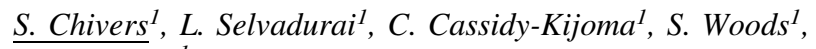 \\ E. Holloway ${ }^{1}$ \\ ${ }^{1}$ Croydon University Hospital, Paediatrics, London, \\ United Kingdom
}

Background and Aims: Croydon (UK) has a population of high ethnic diversity and social deprivation. 115 children and young people have diabetes mellitus and mean $\mathrm{HbA} 1 \mathrm{c}$ of $75.8 \mathrm{mmo} / \mathrm{mol}$. In March 2014, a multidisciplinary service commenced and use of electronic download (Diasend ${ }^{\mathrm{TM}}$ ) began for glucometers and pumps during clinic visits (in light of new UK Best Practice for diabetes mellitus).

Aim: Identify patients with improved diabetes control and which factors have contributed most.

Method: 33 patients with established type 1 diabetes were identified as improvers. They had sustained improved HbA1c ( $>10 \%$ reduction in $\mathrm{HbA} 1 \mathrm{c}$ on at least 2 values 3 months apart)
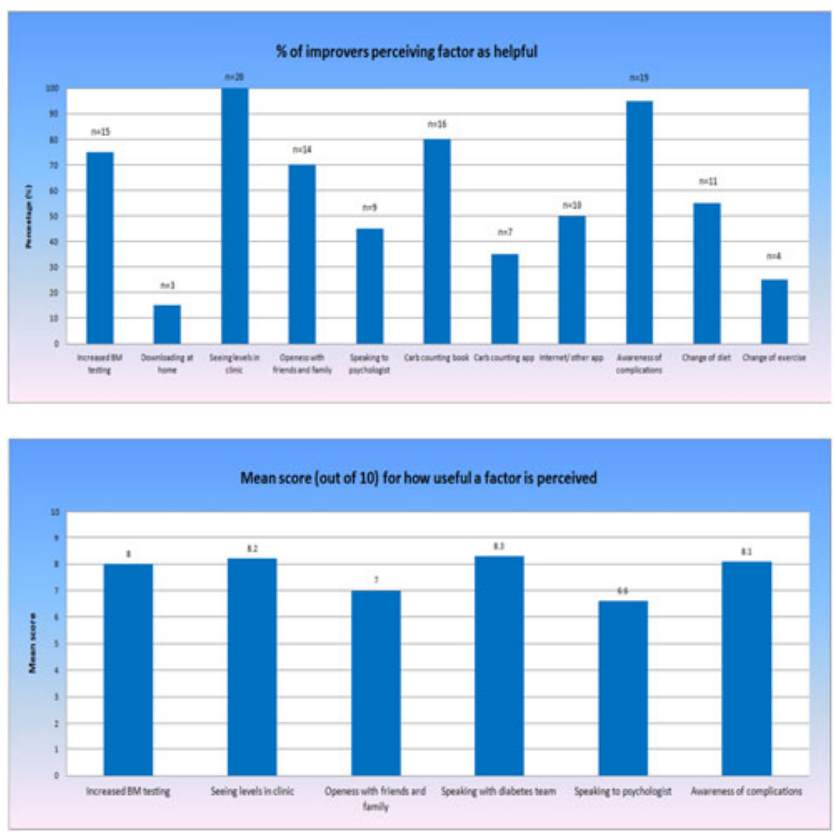
between March 2014 and September 2015. A telephone survey of 24 questions on various topics including the use of technology in clinic and at home.

Results: There were 20 respondents. 7 questions were scored by respondents (usefulness as a score out of 10, higher indicating more useful). 3 received the most positive feedback:

1. Reviewing blood glucose levels on the computer screen with a clinician to discuss during clinic visits $(\mathbf{1 0 0 \%}$ of respondents thought helped, mean score of 8.2)

2. Increased awareness of complications of diabetes $(\mathbf{9 5 \%}$ of respondents, mean score of 8.1)

3. Increased blood glucose test frequency $\mathbf{7 5 \%}$ of respondents, mean score of 8.0)

Conclusion: All patients think seeing their blood glucose levels with the team during clinic consultations has helped them improve their $\mathrm{HbA1c}$. Increased testing frequency and awareness of diabetes complications were also positive factors in helping improve control. Surprising negative results were the lack of perceived benefit of smartphone apps and downloading at home.

218

USING ONLINE FORUMS TO BETTER UNDERSTAND MOTIVATIONS BEHIND TECHNOLOGY UPTAKE AMONG TYPE 2 DIABETES PATIENTS, USING CGM AS THE USE-CASE

\section{T. Chomutare ${ }^{1}$, M. Bradway $^{1}$, E. Arsand ${ }^{1}$, G. Hartvigsen ${ }^{1}$ \\ ${ }^{1}$ University Hospital of North Norway, NST, Troms $\phi$, Norway}

Background and Aims: Many individuals with Type 2-diabetes (T2D) are seeking guidance from their peers via online diabetes-forums, outside of the clinical community. These conversations often include suggestions that are inconsistent with their physicians' recommendations, including the use of CGMs for selfmanagement. We aimed to demonstrate what understanding can be gained from these diabetes-forums regarding desires, knowledge gaps and motivations of individuals seeking alternative management options by analysing conversations surrounding technology use among T2D patients, with CGM as the use-case.

Method: Two reviewers manually analysed a set of comments $(n=130)$ within discussions $(n=10)$ between members with T2D $(n=53)$ from an American online diabetes-forum. Conversations were identified based upon presence of the term "CGM". Reviewers identified major topics discussed as well as personal characteristics, i.e. unsolicited personal details revealed by forum-members.

Results: The four most commonly reported "topics" within these discussions were: short-term use of CGMs and other tools, general self-management concepts, own experiences and selfmanagement practices and frustration with current clinical treatment. Three most commonly reported "Personal characteristics" were: years since diagnosis, self-management tools used, and lifestyle habits.

Conclusion: While individuals value their peers' guidance to fill knowledge-gaps, they still report a strong desire for the medical community to better understand what is personally effective for an individual's self-management, including the option of using CGMs for short-term observations. Profiling, i.e. a combination of personal details and individual concerns, can provide the medical community with a means to better relate to and treat their patients. Further analysis and potential uses of these insights will be presented at ATTD2016.
219

\section{ENHANCING TYPE 2 DIABETES ADVERSE EVENTS' RISK PREDICTION THROUGH DRUG PURCHASING BEHAVIORAL PATTERNS}

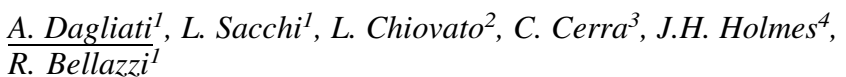

${ }^{1}$ University of Pavia, Dipartimento di Ingegneria Industriale

e dell'Informazione, Pavia, Italy

${ }^{2}$ IRCCS Fondazione Maugeri, Diabetology Endocrinology, Pavia, Italy

${ }^{3}$ ASL Pavia, Purchasing and Control, Pavia, Italy

${ }^{4}$ University of Pennsylvania, Biostatistics and Epidemiology, Philadelphia, USA

Background and Aims: Understanding which factors trigger a worsened disease control is a crucial step in Type 2 Diabetes (T2D) patient management. The Mosaic project, funded by the European Commission, has been designed to integrate heterogeneous data sources and provide decision support in chronic T2D management through patients' stratification. We exploited administrative data as population marker: leveraging on drug exposure patterns our method stratifies the population through purchasing behaviors.

Method: We analyzed 1.020 T2D patients. Daily doses defined on prescriptions (DDD) were exploited as a proxy for adherence. We used DDD to build Metformin exposure profiles for each patient from the date of diagnosis in one-month intervals. In order to define behavioral patterns in drug purchasing, we calculated the Proportion of Days Covered (PDC) as the number of months covered by prescription refills over the observation period since diagnosis. PDC of less than $90 \%$ indicated discontinuous purchases. We included PDC values in a multivariate predictive model for Nephropathy.

Results: Mean PDC was $71.8 \%$ (s.d.=25.6). In the model for the risk of Nephropathy at 3 years after the first visit we found that irregular purchasing patterns of Metformin are related to a higher risk of developing the complication (Odds Ratio $=2.8$, $\mathrm{p}-$ value $<0.01$ ). The Area Under Curve for the model, validated with a leave-one-out strategy, is 0.683 .

Conclusion: This result suggests that drug persistence and discontinuation are associated with adverse T2D outcomes, in this case nephropathy. This extensive approach emphasized the exploitation of administrative data to identify patterns able to explain clinical conditions.

\section{0}

\section{MHEALTH ENABLED REMOTE MONITORING IMPROVES DIABETES OUTCOMES}

\author{
J. Fischer ${ }^{1}$, V. Singh ${ }^{2}$ \\ ${ }^{1}$ Diabetes Glandular Disease Clinic, Endocrinology, \\ San Antonio, USA \\ ${ }^{2}$ Glooko, Data Science, Palo Alto, USA
}

Background and Aims: The concept of remote monitoring in diabetes management is increasingly discussed as a method for improving quality of patient care while lowering costs. mHealth solutions that leverage data from patients' existing diabetes devices can facilitate broader diabetes population management and remote monitoring; however, little substantive research has been conducted to understand the value of mHealth-driven clinical interactions on patient outcomes in diabetes. 


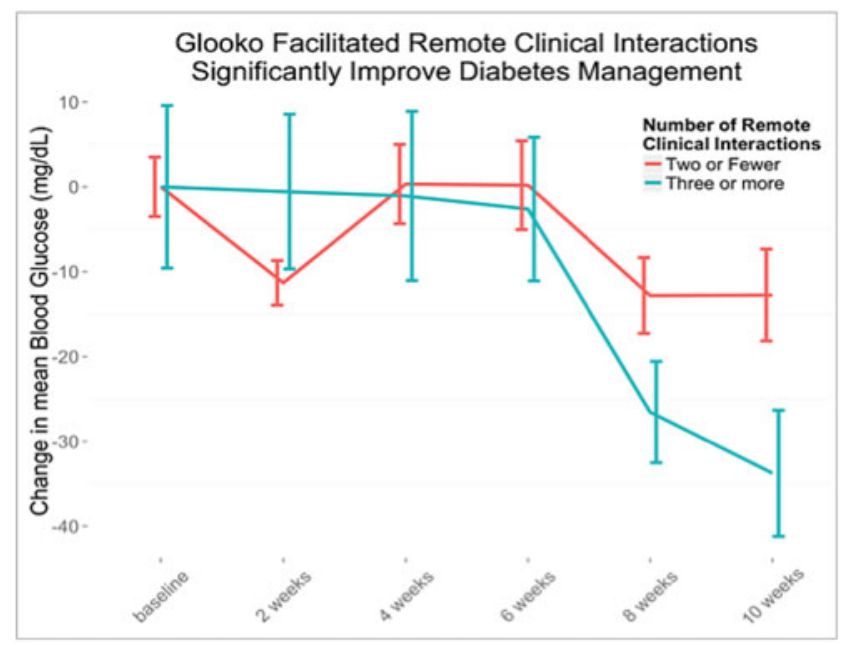

Method: 53 adult subjects with Type 2 diabetes were recruited from the Diabetes \& Glandular Disease (DGD) Clinic in San Antonio, Texas. Participants owned Glooko compatible smartphones and SMBG meters, and met these inclusion criteria: 1) an A1c greater than or equal to $8.5 \%$ and/or 2) insulin naive. Subjects consented to participate in a 2-week run-in period followed by 10 weeks of intervention. Participants in both control and intervention groups were given Glooko, trained on Glooko's mobile and web apps and the clinical staff had remote access to subject data via Glooko's Population Tracker, including subjects' most recent blood glucose readings, food, medication/ insulin, and activity data.

Results: 36 patients adhered to the 12-week study protocol and were included in the final analysis. All participants exhibited a significant mean BG decrease of $16.16 \mathrm{mg} / \mathrm{dL}(\mathrm{p}=0.026)$. Importantly, subjects who received $\geq 3$ clinical interactions exhibited a mean reduction of $33.7 \mathrm{mg} / \mathrm{dL}(\mathrm{p}=0.035)$, which was significantly more than the control group.

Conclusion: These results indicate that both Glooko facilitated self-management and patient remote monitoring by nurse practitioners led to a significant decrease in mean BG levels and glycemic variability over a 10 -week period.

221

\section{THIRTEEN DIABETES PROJECT (DIAB 13): AN INNOVATIVE INTEGRATED MANAGEMENT FOR DIABETIC PATIENT}

O.E. Disoteo ${ }^{1}$, E. Spreafico ${ }^{1}$, E. Cimino ${ }^{1}$, G. Pizzi ${ }^{1}$, A.C. Bossi ${ }^{2}$,

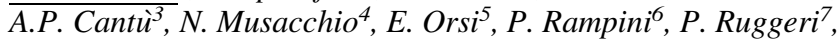
L. Sciangula ${ }^{8}$, R. Trevisan ${ }^{9}$, G. Borghi ${ }^{10}$, F. Auxilia ${ }^{3}$, G. Pozza ${ }^{11}$

${ }^{1}$ A.O. Ospedale Niguarda, SSD Diabetologia, Milano, Italy

${ }^{2}$ AO Treviglio, Diabetologia, Bergamo, Italy

${ }^{3}$ Università degli Studi, Dip. Scienze Biomediche per la Salute, Milano, Italy

${ }^{4}$ AO ICP, Poliambulatorio, Cusano Milanino MI, Italy

${ }^{5}$ IRCCS Fondazione Ospedale Maggiore Policlinico, Diabetologia e Dietologia dell'U.O. di Endocrinologia

e Diabetologia, Milano, Italy

${ }^{6}$ AO Fatebenefratelli, SSD di Malattie Endocrine-Diabetologia, Milano, Italy

${ }^{7}$ AO Cremona, Centro Diabetologico, Cremona, Italy
${ }^{8}$ AO Sant'Anna, SSD di Diabetologia ed Endocrinologia del Presidio Ospedaliero di Mariano Comense, Como, Italy ${ }^{9}$ AO Ospedali Riuniti, Malattie endocrine - Diabetologia, Bergamo, Italy

${ }^{10}$ CEFRIEL, -, Milano, Italy

${ }^{11}$ IRCCS San Raffaele, Diabetologia, Milano, Italy

Background and Aims: "Diab 13", promoted by Italian National Health Board and Lombardy Regional Government, started in 2013 with the aim to evaluate effectiveness of an integrated management of type 1 and complicated type 2 diabetic patients. The project includes two different aspects: the Ambulatory Day Service (DSA) and the digital network shared among 8 Specialistic Diabetes Centers involved in the study with the General Medical Practitioner (GMP) and the patient.

Method: Diab 13 proposes to improve care quality provided and patient, GMP, Specialist integration using medical records of Lombardy Network Social Health Information System (SISS), Electronic Health Record (ESF) and Diagnostic Therapeutic Pathways (PDTA) shared.

The "DSA box" consists in blood tests, ECG, eyes examination, carotid doppler ultrasound and, if necessary, specific evaluations and finally diabetologic visit. The other patients "not DSA" are followed according to the usual standard outpatient care.

Results: During two year the diabetologists have collected data from 17.137 patients divided in DSA (1.444 patients) and not DSA (15.693) for more than 31.099 reports in 2 years of follow up.

Our preliminary data highlights the difficulties of aligning the hospital digital systems, the modalities to collect medical records and the different clinical reality.

Conclusion: This network improves the access to clinical data by patient himself, GPM and other specialist involved in the treatment of diabetic to ameliorate the Diabetes Care. This could be also an opportunity to consolidate a wide database to know the patient's clinical condition and treatment in our region.

\section{2}

\section{SMARTPHONE APPLICATION FOR CARBOHYDRATE COUNTING IN MEALS}

K. Dovc ${ }^{1}$, T. Pangersic Litaj ${ }^{2}$, S. Litaj Pangersic ${ }^{2}$, A. Sircelj Campa ${ }^{3}$, N. Bratina ${ }^{1}$

${ }^{1}$ University Children's Hospital, Department of EndocrinologyDiabetes and Metabolism, Ljubljana, Slovenia

${ }^{2}$ FCB d.o.o, /, Ljubljana, Slovenia

${ }^{3}$ University Children's Hospital, Unit for Nutrition- Diet

Therapy and Hospital Nutrition, Ljubljana, Slovenia

Background: The right estimation of the carbohydrate consumption is an important part of good metabolic control. Mobile applications can provide children and adolescents with diabetes useful tool for better carbohydrate count control.

Main feature of this application is that it calculates carbohydrates, fats, proteins and other nutrient values from weight of consumed food. It uses well-edited database consisting of local finished meals and also local and international food products available. It also features easy selection with multiple portionsize pictures (according to children age groups) of selected food items to simplify weight input. As food is added into the application, sums of all nutrient values are calculated and displayed. 
User can also save typical complete meals as templates to speed up the input process.

Methods: Primary user group of this application are children with diabetes. Design and user experience are adapted to this user group, featuring vibrant icons and easy step-by-step instructions.

Application will be developed in three phases. First we will build a dynamic webpage with database of foods nutrients and individualised according to age portions. In the second phase we will build "web-view application" and publish it on Google Play store. In the last phase, the web page will be reproduced as "native application" with complete nutrients database and images, so it will work on mobile phones without internet connection, allowing users to use it everywhere on the go.

Results: A study will start early 2016, results will be reported in 2017.

Conclusion: A study will start early 2016, results will be reported in 2017.

223

\section{AGREEMENT BETWEEN GLYCAEMIC VARIABILITY CALCULATORS: ANOTHER LIMITATION IN MEASURING GLYCAEMIC VARIABILITY}

\section{A. El-Laboudi ${ }^{1}$, N. Oliver ${ }^{1}$}

${ }^{1}$ Imperial College London, Diabetes- Endocrinology \& Metabolic Medicine, London, United Kingdom

Background and Aims: Despite evidence supporting an independent role for glycaemic variability $(\mathrm{GV})$ in diabetes related complications, the use of GV measures in clinical practice is limited by uncertainty of the significance of these measures and the lack of a gold standard measure. Several software programmes have been developed for automated calculation of GV indices, which can facilitate the use of these measures in clinical practice. Our aim was to compare the degree of agreement between two of the GV calculators.

Method: Two software programmes (EasyGV (v8.8.2.R2) and Rodbard GV calculator) were used to calculate GV measures of 10 continuous glucose monitoring traces of subjects with type 1 diabetes. The correlation between the two calculators in mea-

\begin{tabular}{|c|c|c|c|c|c|c|}
\hline & $\begin{array}{c}\text { CONGA1 } \\
\text { (EasyGV) }\end{array}$ & $\begin{array}{c}\text { MODD } \\
\text { (EasyGV) }\end{array}$ & $\begin{array}{c}\text { SD } \\
\text { (Rodbard) }\end{array}$ & $\begin{array}{c}\text { CONGA1 } \\
\text { (Rodbard) }\end{array}$ & $\begin{array}{c}\text { MODD } \\
\text { (Rodbard) }\end{array}$ & $\begin{array}{c}\text { SD } \\
\text { (CareLink) }\end{array}$ \\
\hline $\begin{array}{c}\text { SD } \\
\text { (EasyGV) }\end{array}$ & .212 & $.855^{\prime \prime}$ & $1.000^{\prime \prime}$ & $.782^{*}$ & $.745^{\circ}$ & $1.000^{\prime \prime}$ \\
\hline $\begin{array}{c}\text { CONGA1 } \\
\text { (EasyGV) }\end{array}$ & .115 & .212 & -.164 & .067 & .212 \\
\hline $\begin{array}{c}\text { MODD } \\
\text { (EasyGV) }\end{array}$ & & $.855^{\prime \prime}$ & $.806^{\prime \prime}$ & $.758^{\circ}$ & $.855^{\prime \prime}$ \\
\hline $\begin{array}{c}\text { SD } \\
\text { (Rodbard) }\end{array}$ & & & $.782^{\prime \prime}$ & $.745^{\circ}$ & $1.000^{\prime \prime}$ \\
\hline $\begin{array}{c}\text { CONGA1 } \\
\text { (Rodbard) }\end{array}$ & & & & & .564 & $.782^{\prime \prime}$ \\
\hline $\begin{array}{c}\text { MODD } \\
\text { (Rodbard) }\end{array}$ & & & & & $.745^{\circ}$ \\
\hline
\end{tabular}

**. Correlation is significant at the 0.01 level (2-tailed).

*. Correlation is significant at the 0.05 level (2-tailed). suring standard deviation (SD), mean of daily difference (MODD) and continuous overall net glycaemic action (CON$\mathrm{GA}_{1}$ ) was evaluated using Spearman's correlation analysis. Correlation with SD reported by CareLink Professional software was also evaluated.

Results: The correlation coefficients between the two calculators in the measurement of SD, MODD and CONGA 1 were 1 , 0.75 and -0.16 , respectively (Table 1 ). The correlation coefficient between the two software programmes and CareLink Professional in measuring SD was 1.

Conclusion: Despite the perfect correlation between the two evaluated GV calculators in measuring SD, there is no agreement in measuring $\mathrm{CONGA}_{1}$ and moderate agreement in measuring MODD. This represents another limitation to the potential use of GV measures in clinical practice. There is a need for data supporting a gold standard measure and an agreement of measurement methodology.

\section{4}

A DEVICE ACQUIRING, DIGITIZING, AND SENDING THE RESULTS OF EVERY GLUCOMETER IN DIGITAL FORMAT FOR TELEMEDICINE PURPOSES

A. Gnasso ${ }^{1}$, M.F. Spadea ${ }^{2}$, S. Scaramuzzino ${ }^{1}$, $\overline{\text { G. Pileggi }}^{1}$, P. Zaffino ${ }^{1}$, C. Carallo $^{3}$

\section{1 “Magna Gracia” University, Department of Clinical and Experimental Medicine, Catanzaro, Italy ${ }^{2}$ Massachusetts General Hospital and Harvard Medical School, Department of Radiation Oncology, Massachusetts, USA \\ ${ }^{3}$ Imperial College London, Department of Chemical Engineering, London, United Kingdom}

Background and Aims: Here it is proposed a technical device aimed at home monitoring of vital, anthropometric, and biochemical values. These parameters are used in the most common chronic diseases such as diabetes mellitus, respiratory and heart failure, arterial hypertension, obesity. In these clinical settings, the data of interest are generally numeric values of blood glucose, arterial oxygen saturation, blood pressure and heart rate, body weight. These values are detected by electronic devices, generally already owned by the patient. In the great majority of the measurements, a direct intervention of the medical personnel is not needed, but only an electronic check against expected values; in fact, these clinical settings are well suited for telemedicine. The difficulty is to send the clinical data to the telemedicine center.

Method: Currently, it is in use to provide (replace) the patient's instrumentations with dedicated Wi-Fi devices, connected by telephone lines (cable or ether) with the telemedicine center. Conversely, the proposed patented system can be added to the patients' current instrumentations.

Results: The main strengths of the proposed device are: it saves the used devices; it does not alter the habits of the patient regarding their known measurement tools; given its low cost, it is applicable also to the monitoring of healthy subjects; ductility, as it can adapt itself to all tools on the market.

Conclusion: The proposed technical device should improve home care processes regarding diabetes mellitus and some other chronic diseases. 


\section{5}

\section{UTILIZING TECHNOLOGY AND ALTERNATIVE APPROACHES FOR DISPLAYING VITAL INFORMATION AND CREATING ENGAGEMENT FOR CHILDREN AND ADOLESCENTS WITH DIABETES}

G. Hartvigsen ${ }^{1}$, M. Muzny ${ }^{2}$, E. Arsand ${ }^{2}$, A. Makhlysheva ${ }^{2}$, H. Blixgård ${ }^{2}$, T. Chomutare ${ }^{2}$, M. Bradway ${ }^{2}$, S.G. Johansen ${ }^{2}$, A. Giordanengo ${ }^{2}$

${ }^{1}$ University of Troms $\phi$ - The Arctic University of Norway, Department of Computer Science, Troms $\phi$, Norway

${ }^{2}$ University Hospital of North Norway, Norwegian Centre for

Integrated Care and Telemedicine, Troms $\phi$, Norway

Background and Aims: Living with diabetes is not easy, particularly when you are young. Because diabetes self-management for a child requires a different support structure than that of an adult, we aimed to make concerned family members aware of their child's blood glucose (BG) regulation mainly by enhancing functionality of commonly used equipment, thereby enabling large circle of relatives to help their kids during challenging situations.

Method: We meet this challenge by developing and researching smart technologies and applications for children with diabetes and their relatives including tailoring the Diabetes Diary app (Diabetesdagboka, GooglePlay/AppStore) and a smartwatch version (Pebble app store).

Results: The resulting portfolio of tools includes (Figure 1):

- Colour signalization: Automatically adjusted lamp in the family's home indicates how the child is doing by changing colours and blinking. Similarly, colour can be displayed on walls, smartwatches, smartphones, pads and computers.

- Info panel: Smart panel running Nightscout application at home plays an alarm and displays updated information received from the Diabetes Diary independently of where the child is located.

- Smartwatch: Alarms and vital information from the child is available on a Pebble that communicates with the smartphone-based diary.

- Radio: Through transferring BG-values as radio signals, the FM-RDS radio announces and displays incoming BG-values.

- SMS: The diary app sends an SMS to the child's parents' phone(s) every time updates are made to the diary.

Conclusion: The described ways of utilizing technology for children's diabetes treatment support illustrates how technology can be used by their parents to support the children's diabetes management.

226

\section{ALERTS, NOTIFICATIONS, REMINDERS: TELEMEDICINE SOLUTION NOT TO BURDEN BUT TO SUPPORT PATIENTS WITH DIABETES}

A. Holubova $^{1}$, J. Muzik ${ }^{2}$, E. Årsand ${ }^{3,4}$, M. Muzny $y^{3,5}$, J. Broz', M. Vlasakova ${ }^{7}$, J. Kaspar ${ }^{1}$, K. Hana ${ }^{1}$

${ }^{1}$ Czech Technical University in Prague, Faculty of Biomedical Engineering, Kladno, Czech Republic

${ }^{2}$ Czech Technical University in Prague, Faculty of Biomedical Engineering, Prague, Czech Republic
${ }^{3}$ University Hospital of North Norway, Norwegian Centre for Integrated Care and Telemedicine, Troms $\phi$, Norway

${ }^{4}$ UiT-The Arctic University of Norway, Department of Clinical Medicine, Troms $\phi$, Norway

${ }^{5}$ First Medical Faculty of Charles University, Spin-off Application Centre, Prague, Czech Republic

${ }^{6}$ University Hospital in Motol, Internal Clinic of the 2nd Faculty of Medicine, Prague, Czech Republic

${ }^{7}$ Charles University in Prague, 1st Faculty of Medicine,

Prague, Czech Republic

Background and Aims: Having diabetes means everyday and continuous control of patients' health conditions. Although new technologies provide numerous tools that can help in self-management and patients are initially willing to use such, the adherence decreases over time. Reasons can be low motivation, time consumption, and inability of an adequate and timely reaction to change of the monitored parameters.

Regarding the approach applied in the system presented, our aim is to find the best possible combination of reminders and alerts and implement these processes as fully automatic.

Method: Building on our system that collects and stores data in a cloud-based solution, data from several devices and apps for diabetes telemonitoring, we have implemented alerts, notifications, and reminders for helping the patients to manage their activities, to motivate them, and to call their attention to situations in which they make mistakes frequently and which might not be discovered on time.

Results: Our supervision system consists of: (1) a security system that monitors blood glucose level via the Nightscout application and generates alarms for critically low and high values under defined conditions; (2) a monitoring server system that sends e-mail notifications about patients' physical activity; (3) a reminder system for patients to check post-meal glycoses via Pebble smartwatch; and (4) social websites and e-mail support to increase patients' awareness and motivation.

The system has been already tested by diabetic patients.

Conclusion: Diabetes telemedicine solution that provides alerts and reminders may improve motivation in patients' selfmanagement, and therefore, the improvements of the illness.

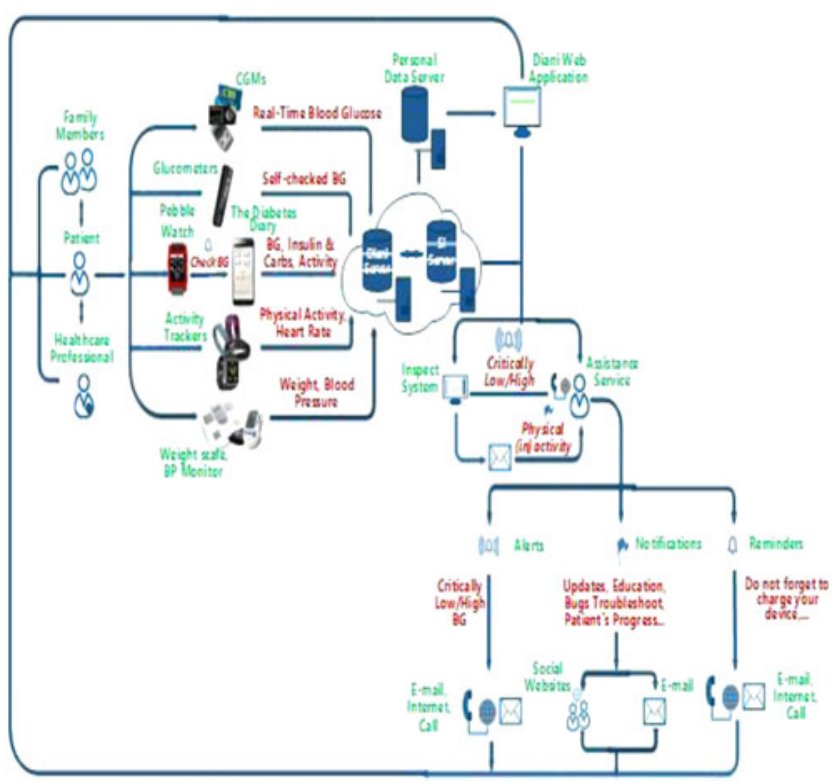


227

"MELLITUSONE.", A NEW TECHNOLOGICAL APPROACH TO RECORD, ACCESS, SHARE AND IMPROVE THE GLYCEMIC CONTROL

R.C. Jacomassi ${ }^{1}$, L.L. Galastri ${ }^{1}$, R.J. Pineda-Wieselberg ${ }^{1}$, M.T.U. Barone ${ }^{1}$

${ }^{1}$ Brazilian Juvenile Diabetes Association ADJ Diabetes Brasil, Brazilian Young Leaders Training, São Paulo, Brazil

Background and Aims: The medical sector has joined forces with the technological field in order to produce devices that facilitate health monitoring and diabetes management. In this context, for patients who have diabetes and for their health care team (HCT), collecting and reviewing daily data are extremely important. Our goal was to develop and test an affordable technological alternative for most people with diabetes who use insulin, in order to record and share data with their HCT.

Method: A Microsoft ${ }^{\circledR}$ Excel ${ }^{\circledR}$ dynamic spreadsheet, called "MellitusOne.", was created. On its first and second sheets data are inputted. On the other two sheets automated charts and graphs are presented and a friendly print layout is available. The dynamic sheet was extensively tested by the developers and then by volunteers. Instructions were made available on the YouTube and Facebook (facebook.com/mellitusone).

Results: From the 8 surveyed people, seven declared that would continue using it (main reasons: easiness to see glycemic variation on the graphs, ( 5 answers), and easiness to use, (2 answers). After more improvements, the second group tested it. All other four volunteers declared that would continue using it, and the reason raised were the same. The main suggestions we are working on were: increase from 20 to 30 days the final report, add cells for more than one fixed insulin type/dose, and other types of graphs.

Conclusion: The results show us that "MellitusOne." is useful, and may be beneficial to this population.

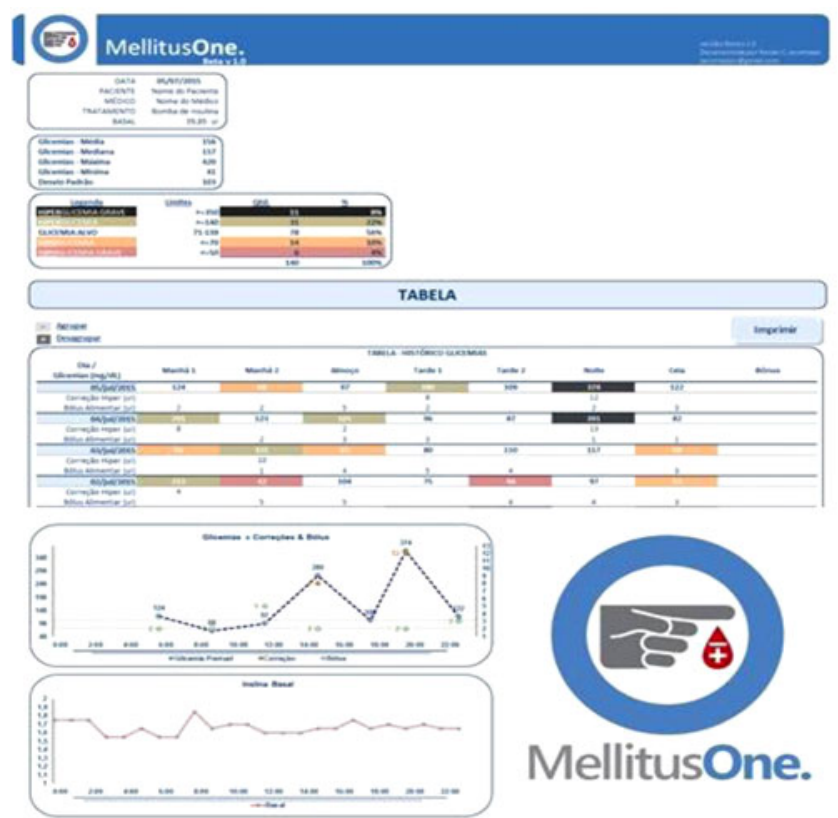

228

\section{DESIGNING MOTIVATIONAL AND EDUCATIONAL DIABETES VIDEO GAMES INVOLVING CHILDREN AS A CREATIVE RESOURCE}

S.G. Johansen $^{1,2}$, A. Makhlysheva ${ }^{2}$, E. Arsand ${ }^{2,3}$, M. Bradway ${ }^{2}$ G. Hartvigsen ${ }^{1,2}$

${ }^{1}$ The Arctic University of Norway, Department of Computer Science-UiT, Troms $\phi$, Norway

${ }^{2}$ University Hospital of North Norway, Norwegian Centre for Integrated Care and Telemedicine, Troms $\phi$, Norway

${ }^{3}$ The Arctic University of Norway, Department of Clinical Medicine-UiT, Troms $\phi$, Norway

Background and Aims: The biggest challenge with creating serious games to motivate behaviour change in children is that behaviourists are rarely game designers. When pedagogic concerns are the main catalyst for making games for children and adolescents with diabetes, the results may often be educational, but usually in some didactic style with limited interactivity. Consequently, they tend to miss the most essential engagement mechanism of traditional games-having fun. Therefore, the challenge is to both take into account the lessons the game is to impart, and how to make the game engaging.

Method: We invited children aged 8-12 with diabetes to a workshop where they were asked to draw a game about diabetes that they would enjoy playing with their friends. Then, we combined this input with game-design know-how from game developers in Troms $\varnothing$ (see Figure 1).

Results: A game for children with diabetes, combining insights gained from the workshop and game mechanics built on a template from "World of Warcraft" — one of the world's most successful games - was developed.

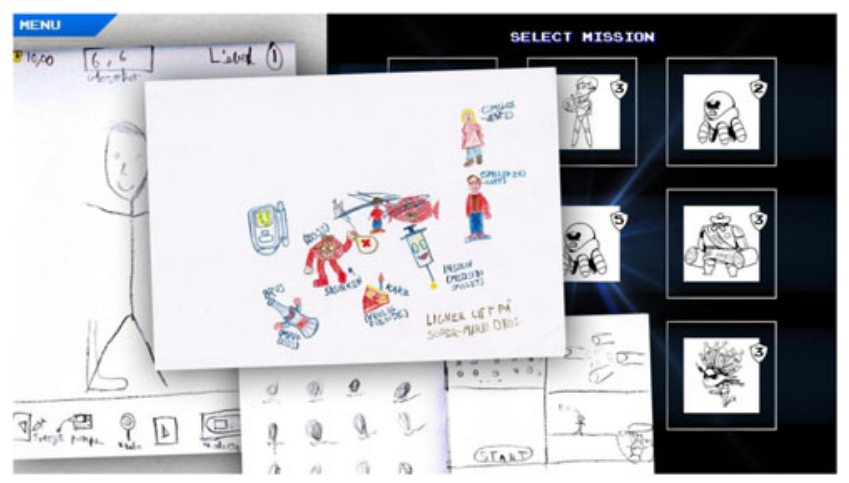

Conclusion: The goal was to create a fun game which allows children with diabetes to play and experiment with aspects of diabetes management on virtual subjects. Future work will expand children participation by allowing them to create characters and come up with stories. This should increase interest in the game, as well as tailor it to the specific age group. Concrete layouts and concepts from the design process and workshop will be discussed at ATTD 2016.

\section{9}

PERIODIC FOLLOW UP WITH TELEMANAGEMENT SYSTEM FOR 15 YEARS AND SIGNIFICANT REDUCTION IN VASCULAR COMPLICATIONS IN T2DM

\author{
J. Kesavadev ${ }^{1}$, A. Shankar ${ }^{1}$, G. Krishnan $^{1}$, S. Jothydev ${ }^{1}$
}




\author{
'Jothydev's Diabetes Research Centre, Diabetes, \\ Thiruvanathapuram, India
}

Background and Aims: We evaluated treatment outcomes of a telemedicine program run at our centre since 1999. Diabetes Tele Management System (DTMS $®$ ) makes use of customized software, a decision support system aided by multidisciplinary team, telecommunication using telephone/email/secure website to ensure multidrug compliance and cost effective attainment of treatment targets.

Method: EMR of 904 T2D who continues to be on DTMS ${ }^{\circledR}$ follow up for 15 years were analyzed to assess long-term outcomes. These patients had no major vascular complications at baseline. Regular DTMS ${ }^{\circledR}$ follow up included a minimum of one telephone/email reporting of blood sugars and a physical visit once every 3-6 months/year. Regular reminders from DTMS ${ }^{\circledR}$ team ensured adherence to the drugs and lifestyles.

Results: The mean age was $49.58 \pm 11.4$ and mean age of diagnosis was $44.82 \pm 11.3$. Patients on regular DTMS $®$ follow up had fewer complications, lesser hospitalisations and better glycemic control with an average HbA1c of $7.7 \pm 1.2 \% .11$ patients developed coronary artery disease, 6 had retinopathy requiring laser therapy and 9 progressed to stage 5 CKD. Renal, neurological and retinal complications were observed in younger age group, with an early onset of diabetes, who failed to maintain regular DTMS ${ }^{\circledR}$ follow up.

Conclusion: This retrospective study of surviving patients on 15 years of DTMS ${ }^{\circledR}$ follow up, illustrates significant reduction in micro and macrovascular complications. Diabetes being a progressive disease, a structured tele management program will effectively reduce disease complications and prove cost effective in the long term, provided patients are continuously motivated to use it.

\section{0}

\section{MODEL-BASED INTERPRETATION OF ORAL GLUCOSE TOLERANCE TEST}

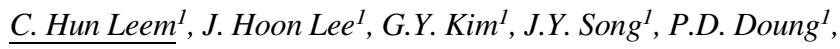 \\ ${ }^{1}$ University of Ulsan College of Medicine/Asan Medical Center, \\ Department of Physiology, Seoul, Republic of Korea
}

Background: Oral glucose tolerance tests (OGTTs) are used commonly to diagnose diabetes mellitus (DM). The changes on blood glucose and insulin by OGTTs contain information of the intestinal absorption, hepatic control of glucose and insulin, pancreatic insulin secretion and peripheral tissue glucose and insulin control.

Aim: We developed an appropriate dynamic model to reveal the above information from OGTT data.

Method: We developed an OGTT model containing five compartments for insulin dynamics and two compartments for glucose dynamics based on previous reports. Simplex and Levenberg-Marquardt algorithms were then used to fit the data obtained from 42 normal subjects ( 24 males and 20 females) and eight subjects with DM.

Results: We found clear gender differences in the intestinal glucose absorption kinetics, glucose sensitivity in the pancreas, maximal insulin production capacity and endogenous glucose production. The differences between normal and DM subjects in glucose and insulin dynamics in the pancreas, liver and peripheral tissues, such as insulin resistance, insulin secretion and the relative roles of glucose disposal in each organ, were demonstrated clearly and quantitatively in a time-dependent manner. This study revealed the quantitative dynamic interaction between glucose and insulin using OGTT data and revealed organ function during the OGTT.
Conclusions: Using this approach, we identified the dysfunctional organs for glucose and insulin regulation. Data produced using this model will allow a personalized and targeted approach for health issues related to glucose and insulin. (Supported by the grant No. NRF-2015M3A9B6028310, NRF2014M3A9D7034366 \& 2015K000247 from MSIP/COMPA)

\section{1}

\section{A RELEVANT GLUCOSE-INSULIN MODEL: VALIDATION USING CLINICAL DATA}

N. Magdelaine $^{1}$, L. Chaillous ${ }^{2}$, I. Guilhem ${ }^{3}$, J.Y. Poirier ${ }^{3}$, M. Krempf ${ }^{2}$, A.L. Fournier Guilloux ${ }^{2}$, C. Moog ${ }^{1}$, E. Le Carpentier ${ }^{4}$

${ }^{1}$ IRCCyN, Commande, Nantes, France

${ }^{2}$ CHU Nantes, Endocrinology Diabetes \& Metabolism, Nantes, France

${ }^{3}$ CHU Rennes, Endocrinology Diabetes \& Metabolism, Rennes, France

${ }^{4}$ IRCCyN, ADTSI, Nantes, France

Background and Aims: A relevant glucose-insulin model with realistic asymptotic properties has been introduced ("A long-Term Model of Glucose-Insulin Dynamics for Type-1 Diabetes", Magdelaine et al., IEEE TBME 2015). Its parameters provide the computation of the tools for functional insulin therapy like basal rate, insulin sensitivity factor and insulin to carbohydrates ratio.

The objective is to validate this model using clinical data of a larger number of patients and to prove its ability to predict the behavior of glycemia and insulinemia dynamics.

Method: Data (CGM, insulin injections, carbohydrates) from 12 type-1 diabetic subjects were collected. An unconstrained optimization algorithm estimated the model's parameters on $n$ samples. Then, the estimated model was simulated on a set of $n / 2$ samples as a cross-validation.

Results: The estimation was performed over at least 24 hours. The model-fit was very satisfactory as the mean standard deviation (SD) was $26 \mathrm{mg} / \mathrm{dl}$. The cross-validation (performed over
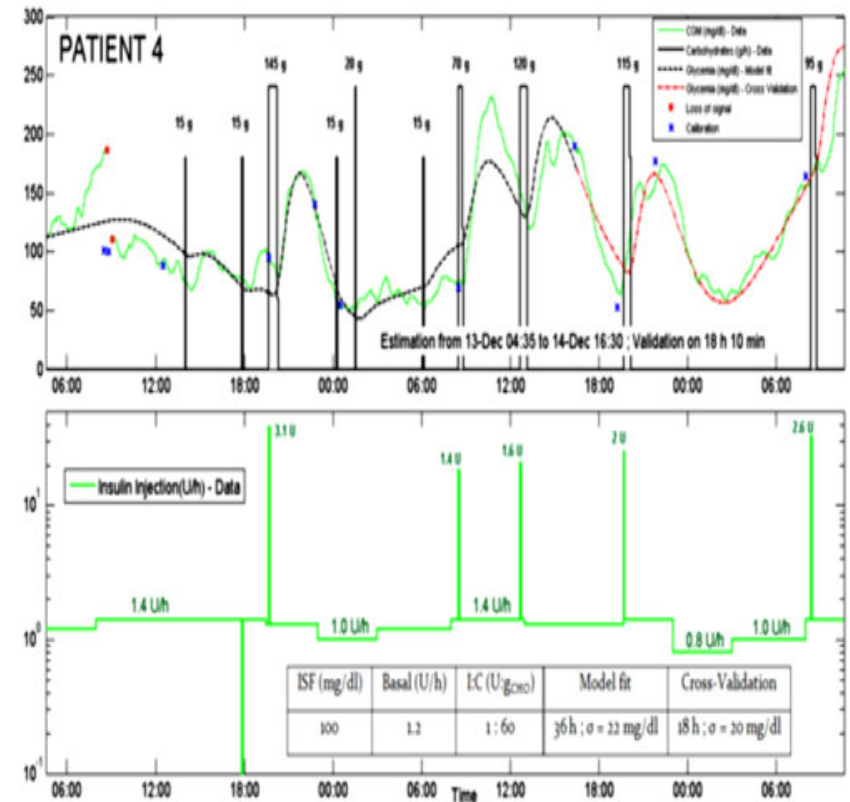
16 hours on average) provides accurate glucose prediction with a mean SD of $40 \mathrm{mg} / \mathrm{dl}$. Moreover, the computed tools for functional insulin therapy are consistent with the values determined by the clinical protocol.

Conclusion: Realistic behavior of this model was already mathematically proven. In this study the relevant model was validated using clinical data as it accurately fitted the blood glucose of real patients. It was also shown that future values were well predicted. Thus it is a good candidate for a model based controller. As its parameters provide the computation of the tools for functional insulin therapy it will be useful for patient's education.

232

\section{A HEURISTIC APPROACH TO THE DEVELOPMENT OF AN OPEN-LOOP ARTIFICIAL PANCREAS REQUIRING ONLY A BRAIN, INSULIN DELIVERY AND FREQUENT BLOOD SUGAR DATA}

\section{K. McMahon $^{1}$, S.W. Ponder-MD FAAP CDE}

${ }^{1}$ Diabetech- LP, Research \& Development, Sausalito, USA

${ }^{2}$ Sugar Surfing, Education, Belton, USA

Background and Aims: The development of control and safety algorithms relying on mechanical-hormonal means for maintaining blood sugar in a normal range is a worthy goal. Commercialization of this technology and third party coverage may remain elusive. Meanwhile, a well-educated and engaged patient can use currently available continuous glucose monitoring monitoring (CGM) in combination with fuzzy logic principles to achieve near normal blood sugar levels today.

Method: Patients are taught dynamic strategies for predicting changes in blood sugar based on a rigorous and ongoing process of personal experimentation. They are encouraged to perform studies to characterize their real-world relationships to those things which affect flux and drift in their ever changing metabolic profile. Finally, aiding patients in understanding that these shifts are naturally occurring and do not lend themselves to a static predictable result tends to motivate them for long term frequent engagement of their condition.

Results: While the control and safety algorithms described above are currently published as a book in the 'how to" genre, anecdotal evidence indicates that this complex menu of manual procedures is highly effective at normalizing blood sugar control regardless of the patient's insulin administration preference (e.g. CSII vs. MDI). The book's authors are currently working to translate these algorithms into software which can then be more broadly adopted by real-world patients today in a manual sense while also incorporated into artificial pancreas development programs.

Conclusion: By re-classifying CSII as an optional component of next generation artificial pancreas systems, exponentially more patients can be addressed at a significantly lower cost.

\section{3}

DIABETES TELEHEALTH INTERVENTION COMPRISING BLOOD SUGAR DATA, HOME A1C, CARE CONTINUITY AND MOTIVATIONAL INTERVIEWING TO EFFECT BEHAVIOR CHANGE IN AN ADULT TYPE 2 DIABETES POPULATION

\section{K.L. McMahon ${ }^{1}$}

${ }^{1}$ Diabetech- LP, Research \& Development, Sausalito, USA

\begin{tabular}{|c|c|c|c|}
\hline & $\begin{array}{c}\text { Control } \\
\text { Group }\end{array}$ & $\begin{array}{c}\text { Experimental } \\
\text { Group }\end{array}$ & $p$ value \\
\hline$n$ & 9 & 19 & \\
\hline Baseline Alc & $7.67 \pm .56$ & $7.96 \pm 0.57$ & 0.745 \\
\hline 6 month Alc & $7.49 \pm 1.77$ & $7.17 \pm 0.87$ & 0.048 \\
\hline Change Alc & $-0.18 \pm 1.93$ & $-0.79 \pm .78$ & 0.020 \\
\hline
\end{tabular}

Background and Aims: To extend the reach and impact of diabetes education into the patient's home with increased frequency, personalization and modulation of care intensity informed by high quality remote data collection.

Method: A comprehensive program was designed to educate previously enrolled patients in a high cost Medicaid program for adults with type 2 diabetes. Case managers interviewed participants and encouraged them to follow their previously prescribed self-care regimen. Treatment group participants $(n=51)$ were shipped a blood sugar meter, test strips, a cellular enabled data transmission device (GlucoMON-ADMS), and a pedometer. Participants were instructed to connect their meter to the transmission device at least weekly. Multi-color blood sugar pattern management reports were analyzed by case managers who then annotated the chart with encouragement and/or educational comments. Home A1c sample kits were mailed to both arms (control group $\mathrm{n}=32$ ) at enrollment and every 90 days thereafter. There were no study related clinic visits required at any point.

Results: Following initial A1c sample processing, nearly $40 \%$ of patients were determined to be below the ADA threshold of $7 \%$ indicating good control. Patients whose A1c was between $7 \%$ and $8.9 \%$ at baseline provided the most interesting results.

Statistics Table 3 - Change in A1c - Control vs. Treatment "Group C: baseline A1c 7-8.9"

Conclusion: The design of this pilot proved that it is possible to engage patients and expect significantly improved outcomes in a data intensive program without requiring frequent office visits or sending health care workers to the patient's home.

\section{4}

NOVEL PATTERN IDENTIFICATION THOUGH STANDARDIZED DATA DISPLAY USING THE AMBULATORY GLUCOSE PROFILE (AGP) REPORT

D. Mullen $^{1}$, R. Bergenstal ${ }^{1}$

${ }^{1}$ International Diabetes Center, HealthPartners Institute, Minneapolis, USA

Background and Aims: Lack of a common glucose report, streamlined data acquisition, and aggregated reports across devices lead to difficulty fully using the data available and frustration among clinicians and patients. The IDC developed an endorsed standardized report that can be used across all glucose devices called the Ambulatory Glucose Profile (CapturAGP).

Aims:

1. Evaluate streamlined, standardized cloud-based glucose reporting using CaptūrAGP ${ }^{\mathrm{TM}}$ software and report

2. Assess patient and clinician preferences regarding the AGP report including presentation of glucose data, standardized terminology and utility in diabetes management 
Method: Seven diverse clinical sites were recruited representing different practice styles from across the United States, all with large Type $1 \mathrm{DM}$ populations $(\mathrm{n}=140$ patients and 17 clinicians). The study included EMR integration, time in motion, workflow mapping and surveys of all patients/families and clinicians.

Results: Patients reported better understanding of glucose patterns by using the AGP (CGM users 70\%; SMBG users 64\%). Patients also reported that with the AGP they saw new trends and patterns (SMBG 90\%; CGM 92\%).

Clinicians reported that the AGP report when compared to other data sources was best at helping educate patients about glucose patterns (89\%) and for helping the clinicians see patterns $84 \%$.

Conclusion: A standardized glucose report that aggregates results from multiple devices streamlines clinical processes. Patients/families, and clinicians reported that they could clearly see glucose patterns using the AGP. Expedient glucose pattern recognition allows valuable clinical visit time to be spent in shared decision making conversations rather than in data collection/interpretation.

\section{5}

WEARABLE COMPUTING IN DIABETES: ADVANCED FUNCTIONS ENABLED BY SMARTWATCHES

M. Muzny 1,2, M. Bradway ${ }^{2}$,J. Muzik ${ }^{3}$, G. Hartvigsen ${ }^{2,4}$, E. Arsand $^{2,5}$

${ }^{1}$ Spin-off Application Centre, First Medical Faculty of Charles University, Prague, Czech Republic

${ }^{2}$ Norwegian Centre for Integrated Care and Telemedicine, University Hospital of North Norway, Troms $\phi$, Norway

${ }^{3}$ Faculty of Biomedical Engineering, Czech Technical University in Prague, Prague, Czech Republic

${ }^{4}$ Department of Computer Science, UiT The Arctic University of Norway, Troms $\phi$, Norway

${ }^{5}$ Department of Clinical Medicine, UiT The Arctic University of Norway, Troms $\phi$, Norway

Background and Aims: Wearable devices can significantly facilitate the monitoring and management of certain activities within an individual's daily life. As this technology develops, there are even more ways in which smartwatch technology can be useful, in particular, for diabetes self-management. With this in mind, we designed a Pebble smartwatch application, which allows users to display and enter relevant registrations, track their physical activity automatically, and communicate this data to a smartphone Diabetes Diary app.

Method: The users' responses $(\mathrm{N}=40)$ to two online competition-surveys, organized by our Diabetes Research Team, detail which functions they would like to have on the smartwatch. Below we explore these together with the potential to implement these suggestions given recent technological developments.

Results: Significant desire for functions on the smartwatch were: 1) wireless transfer, display and interpretation of data from SMBG, CGMs and CSIIs $(n=8) ; 2)$ estimation of daily physical activity required to maintain one's BG level and visual indication of daily-goal achievements $(n=6)$; 3 ) alarm for insulin doses and carbohydrate intake ( $\mathrm{n}=5)$; and 4) an option to select insulin types and dose-amount suggestions based on previous similar situations $(n=5)$.

Conclusion: A new version of the Pebble smartwatch, Pebble Time, provides opportunities to respond to these requests via the following technological developments: 1) integrated microphone, enabling a voice-based method of making new registrations; and 2) smartstrap connector, enabling the integration of more diabetesrelated sensors. We are currently exploring the impacts of new methods for involving users in development during future studies by including creation of an interactive online-communication space.

\section{6}

\section{INDIVIDUAL DIFFERENCES AND CONTEXTUAL FACTORS INFLUENCE THE EXPERIENCE AND PRACTICE OF SELF-MANAGEMENT WITH TYPE 1 DIABETES TECHNOLOGIES}

${\underline{A . A . O ' K^{\prime} a n e^{1}}}^{1}$

${ }^{1}$ University College London, UCL Interaction Centre, London, United Kingdom

Background and Aims: Adults with T1D have choices about what technologies to use to self-manage their condition, such as glucose meters, insulin pumps, continuous glucose monitors, smartphone apps, and other mobile technologies. However, little is known about how user experience might influence how they choose to adopt and use T1D technologies when practicing selfmanagement.

Method: Situated exploratory qualitative studies were conducted to examine contextual factors that influence the use of selfcare technology "in the wild": contextual interviews, a diary study, and the observation of a diabetes technology group meet-up. A combined bottom-up thematic analysis uncovered commonalities in how context influenced the use, carrying, adoption, and misuse of these devices. However, large variability in how user experience impacted self-care for the 41 participants was also revealed.

Results: Although these self-care technologies were effective, efficient, and easy to learn for the participants from a human factors engineering perspective, the physical environment, the healthcare system infrastructure, the social situation, the cultural context, and individual differences impacted decisions to use them. Quality of life can be impacted by the design of T1D technologies, which is sometimes prioritised over immediate or long-term health benefits.

Conclusion: These devices need to be studied in-situ to understand how their design can influence everyday adoption and use. However, the variety of everyday self-care contexts and the diversity of possible user preferences do not lead to universal design implications. Instead, design should focus on empowering adults to make personal choices about their self-care technologies that suit the context of their, sometimes messy, everyday lives.

\section{7}

\section{"DIABETOUR": A DIGITAL STORYTELLING PROJECT FOR CHILDREN AFFECTED WITH TYPE 1 DIABETES MELLITUS}

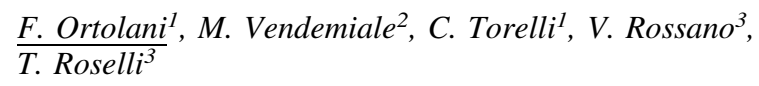

${ }^{1}$ Pediatric Hospital “Giovanni XXIII”, Metabolic Diseases-

Clinical Genetics and Diabetology, Bari, Italy

${ }^{2}$ Pediatric Hospital “Giovanni XXIII”, Clinical Psychology, Bari, Italy

${ }^{3}$ University “Aldo Moro”, Informatics, Bari, Italy

Background and Aims: Digital storytelling is a short form of digital media production that allows everyday people to share 
aspects of their life story. A digital story can be a useful tool in the management of chronic conditions such as type 1 diabetes mellitus, describing the onset experience and how to deal with this disease in everyday life.

Method: The "Diabetour" project aims to promote the development of self-management and problem solving among 5-10 year-old children affected by type 1 diabetes mellitus and their families. It will involve combination of text, images, audio and video and it will be available in app versions which can be used on mobile devices (Java for Android). Starting from the onset of diabetes, children and caregivers will receive the first bases about the disease. After hospital discharged, this digital story will continue describing the life of our little patient at home, during school hours or sport activities. "Diabetour" implementation will require the involvement of one pediatric diabetologist, one psychologist with experience in chronic diseases management, one creative-graphic designer and one computer scientist for an estimated 6-month period.

Results: A beta-test will be used to get a rapid feedback about appreciation of "Diabetour" graphic and story and about product interest, involvement and usability.

Conclusion: We believe that "Diabetour" will enhance the improvement of the quality of life of young people affected with type 1 diabetes. In the story they will become the real protagonists and "active masters" of their experience, widespreading the awareness and the knowledge about their condition.

238

\section{WEBINAR AS AN ALTERNATIVE FORMAT OF PSYCHOLOGICAL HELP TO PEOPLE WITH DIABETES}

\author{
E. Patrakeeva ${ }^{1}$, T. Zagorovskaya ${ }^{2}$ \\ ${ }^{1}$ Saint-Petersburg Medical University, Endocrinology, \\ Saint-Petersburg, Russia \\ ${ }^{2}$ Saint-Petersburg State University, Psychology, Russia
}

Background and Aims: Currently in Russia there is no psychological assistance provided/available to the patients at the medical sites. Also, there is a lack of professionals trained to specifically deal with diabetes-related issues. This problem is especially pressing in the suburban and rural regions of Russia. Our goal was to research the need for psychological assistance, provided through webinars and to determine a scope of topics, that can be effectively addressed in this type of communication.

Method: The research was conducted in two social media platforms in group @ diabet.connect (2670 subscribers). The methods included polls, statistical analysis of the social pages data and observation.

Results: Webinars were mostly in demand among females with T1DM and among mothers, whose children have diabetes. The highest interest and activity in the discussions was observed on the topics of diabetes and pregnancy; children with diabetes; how to support oneself; fears of hypoglycemia and possible complications; building relationships; emotional burnout. We determined that the most effective type of webinar was the alternations between the face of the speaker and slides with information. Speaker's experience of dealing with diabetes-related issues increases the interest among webinar participants and makes communication more effective.

Conclusion: Webinars on diabetes-related issues are important for patients with diabetes. The lack of psychological professionals dealing specifically with diabetes-related problems and inability to arrange individual consultations in the area of residence, makes webinars one of the most convenient way of interaction. This format is especially suitable for younger generation of patients, time-restricted professionals and residents of distantly located regions of Russia.

\section{9}

\section{SOCIAL NETWORK AS A USEFUL INFORMATION RESOURCE FOR TYPE 1 DIABETES (T1D) PATIENTS AND THEIR DOCTORS}

\author{
E. Shafranskaya ${ }^{1}$, E. Patrakeeva ${ }^{2}$, N. Novoselova ${ }^{3}$ \\ ${ }^{1}$ The Center of Family Planning and Reproduction, \\ Endocrinology, Saint-Petersburg, Russia \\ ${ }^{2}$ Saint-Petersburg Medical University, Endocrinology, Saint- \\ Petersburg, Russia \\ ${ }^{3}$ Federal Almazov North-West Medical Research Centre, \\ Endocrinology, Saint-Petersburg, Russia
}

Background and Aims: To estimate usefulness of information from diabetes-related group in social-network for patients with T1D and doctors.

Method: Individuals from diabetes-related group (@diabet.connect) in social-network completed anonymously the questionnaire about usefulness of posted information. The differences in the proportions of T1D patients and doctors responding positively to each question were tested using the chi-squared test or Fisher's exact test.

Results: The sample consisted of 145 adults: 125 T1D patients and 20 doctors. Age of participants was $11-50$ years (T1D patients) and 23-47 years (doctors). The ratio of men and women was equal in two groups.

Both T1D patients and doctors found useful information in topics \#diascience, \#diagadgets, \#diadoctortalk. Patients with T1D were also interested in \#diapregnancy. Statistically significant difference between 2 groups was only in topic \#translationofdiaarticles.

Conclusion: Our study shows that social-network could be a very useful source of information not only for patients with diabetes, but also for health care providers.

\begin{tabular}{|c|c|c|c|}
\hline Aspect of diabetes & Patients with T1D $(n=125)$ & Doctors $(n=20)$ & $\mathrm{P}$ value \\
\hline \#diascience & $46 \%(58 / 125)$ & $60 \%(12 / 20)$ & 0.26 \\
\hline \#diarecipes & $22 \%(28 / 125)$ & $15 \%(3 / 20)$ & 0.57 \\
\hline \#diacinema & $1 \%(1 / 125)$ & $5 \%(1 / 20)$ & 0.26 \\
\hline \#diadoctortalk & $38 \%(47 / 125)$ & $55 \%(11 / 20)$ & 0.14 \\
\hline \#diahistory & $2 \%(3 / 125)$ & $0 \%(0 / 20)$ & 1.0 \\
\hline \#diagadgets & $43 \%(54 / 125)$ & $40 \%(8 / 20)$ & 0.79 \\
\hline \#diahumor & $2 \%(3 / 125)$ & $0 \%(0 / 20)$ & 1.0 \\
\hline \#diasport & $15 \%(19 / 125)$ & $15 \%(3 / 20)$ & 1.0 \\
\hline \#diapregnancy & $33 \%(41 / 125)$ & $15 \%(3 / 20)$ & 0.11 \\
\hline \#translationofdiaarticles & $14 \%(17 / 125)$ & $35 \%(7 / 20)$ & 0.025 \\
\hline \#carbohydratecounting & $18 \%(23 / 125)$ & $10 \%(2 / 20)$ & 0.53 \\
\hline \#diabooks & $8 \%(10 / 125)$ & $10 \%(2 / 20)$ & 0.67 \\
\hline \#diapsychology & $14 \%(17 / 125)$ & $15 \%(3 / 20)$ & 1.0 \\
\hline \#diasuperstar & $3 \%(4 / 125)$ & $0 \%(0 / 20)$ & 1.0 \\
\hline \#diachildren & $4 \%(5 / 125)$ & $0 \%(0 / 20)$ & 1.0 \\
\hline
\end{tabular}


240

\section{AUGMENTING AN ADVANCED BOLUS CALCULATOR WITH CONTINUOUS GLUCOSE MONITORING AND A SMARTWATCH}

$\underline{P . P e s l}^{1}$, P. Herrero ${ }^{1}$, M. Reddy ${ }^{2}$, N. Oliver $^{2}$, D. Johnston ${ }^{2}$, C. Toumazou ${ }^{1}$, P. Georgiou ${ }^{1}$

${ }^{1}$ Imperial College London, Centre for Bio-Inspired Technology, London, United Kingdom

${ }^{2}$ Imperial College London, Division of Diabetes- Endocrinology and Metabolic Medicine, London, United Kingdom

Background and Aims: The 'Advanced Bolus Calculator for Diabetes' (ABC4D) relies on retrospective continuous glucose monitor (CGM) data and manual upload of this data for adaptation of bolus calculator parameters. The integration of CGM into the ABC4D system aims to reduce the workload and increase efficiency by fully automating the adaptation process. The second objective of this work is to improve the usability of the system for the user with type 1 diabetes (T1D) by enhancing ABC4D with a smartwatch application for notifications and alternative user input.

Method: We have integrated continuous glucose data within the ABC4D platform by accessing glucose values from Dexcom's G5 Mobile CGM system via the Apple iOS HealthKit. Data obtained from the CGM can be either blinded or displayed within the ABC4D application and used for remote supervision or automatic adaptation of bolus calculator parameters. The ability to receive notifications (e.g. reminders, warnings), to view the history of insulin recommendations or additional user input, have been added and integrated into a smartwatch (Motorola Mobility LLC's Moto 360 or the Apple Watch), which can be used in addition to the existing system.

Results: Continues glucose monitoring and smartwatch capabilities have been successfully added to an adaptive bolus calculator and are currently being optimized with people with type 1 diabetes.

Conclusion: We have enhanced the usability of the existing ABC4D system by integrating continuous glucose monitoring and adding notification services and optional user input via a smartwatch.

\section{1}

\section{GLUCOSE RATE-OF-CHANGE AT MEAL TIMES FOR INSULIN DOSING DECISION SUPPORT}

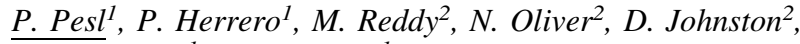
C. Toumazou ${ }^{1}$, P. Georgiou ${ }^{1}$

${ }^{1}$ Imperial College London, Centre for Bio-Inspired Technology, London, United Kingdom

${ }^{2}$ Imperial College London, Division of Diabetes- Endocrinology and Metabolic Medicine, London, United Kingdom

Background and Aims: To evaluate the glucose rate-ofchange (ROC) at meal times during a six-week study $(n=10)$ using the Advanced Bolus Calculator for Diabetes (ABC4D). ABC4D optimizes insulin bolus dosing based on previous postprandial glucose outcomes derived from continuous glucose monitoring (CGM) data. The aim of this work is to evaluate the potential use of glucose ROC as additional input for insulin dosing advice when using real-time CGM systems.
Method: Pre-prandial (15-0 min) glucose ROC and minimum post-prandial glucose levels (2-6 hours) for 649 meals have been analyzed using data from retrospective CGM (Medtronic, iPro2). We have categorized ROC into three trends (falling, stable or rising) and evaluated the effect of ROC trends on the minimum post-prandial glucose concentration.

Results: For the majority of analyzed meals $(53.9 \%)$, glucose ROC was stable $\left(-0.5 \mathrm{mg} \bullet \mathrm{dl}^{-1} \bullet \mathrm{min}^{-1}\right.$ to $\left.<+0.5 \mathrm{mg}^{\bullet} \mathrm{dl}^{-1} \bullet \mathrm{min}^{-1}\right)$ at meal-time, for $29.6 \%$ above $+0.5 \mathrm{mg}^{\bullet} \mathrm{dl}^{-1} \bullet \mathrm{min}^{-1}$ and for $16.5 \%$ below $-0.5 \mathrm{mg} \bullet \mathrm{dl}^{-1} \bullet \mathrm{min}^{-1}$, respectively. For $7.5 \%$ of meals with a falling glucose trend, active-insulin from previous insulin administrations was recorded. The post-prandial minimum mean glucose

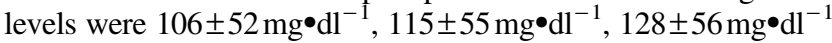
for falling, stable and rising glucose trends at meal times, respectively.

Conclusion: We observed a significant difference $(\mathrm{P}<0.05)$ in minimum post-prandial glucose concentration for meals where the glucose rate-of-change was rising compared to meals where glucose ROC was stable, indicating the potential benefit of the parameter for insulin dosing decision support, where real-time CGM is available.

242

\section{MOBILE SOCIAL MEDIA AND DIABETES: VIBER COMMUNICATION IMPROVES GLUCOSE CONTROL IN TYPE 1 DIABETES PATIENTS ON INSULIN PUMP}

G. Petrovski $^{1}$, M. Zivkovik ${ }^{1}$, I. Ahmeti ${ }^{1}$, K. Adamoval

${ }^{1}$ University Clinic of Endocrinology, Center for Insulin Pump, Skopje, Macedonia

Background and Aims: To evaluate the effect of Viber (Viber Media S.a.r.l) communication as a tool to improve glucose control in poorly controlled type 1 diabetes patient on insulin pump supported by health care professionals and motivated well educated type 1 diabetes patients.

Method: A total number of 72 type 1 diabetes patients on insulin pump (age 17.8 \pm 4.2 years, Male: 32 / Female: 40) with Hba1c of $7.9 \pm 1.4 \%$ were randomized in two groups:

Doctors group - 36 patients, where Viber was offered in a group led by one doctor and one educator.

Patient group - 36 patients, where Viber was offered in a group led by two well controlled, educated and motivated type 1 diabetes patients.

Patients were able to ask questions and open discussion on different topics about their diabetes management, where other patients could respond and comment. Advice was given by doctor/educator and educated patient according to the group.

Hba1c was performed baseline, three and six months after the study. Patient should have at least 3 communications per week to be eligible for evaluation.

Results: There were 9.2 communications per patient per week. Most communicated topics were: carb counting (38\%), exercise (28\%) and high blood sugar corrections (21\%). There was significant decrease of Hbalc $(7.8 \pm 1.6 \%$ to $7.1 \pm 1.2 \%$ in Doctors group and $7.7 \pm 1.1 \%$ to $7.2 \pm 1.9 \%$ in Patients group) in six months period. There was no significant difference between both groups.

Conclusion: Advice on different topics in diabetes management using mobile social media in diabetes led by well-educated and motivated patients can be effective as same as health care professionals. 
243

ONLINE SOFTWARE FOR AUTOMATED GLUCOSE VARIABILITY CALCULATION USING RAW DATA FROM CONTINUOUS GLUCOSE MONITORING DEVICES

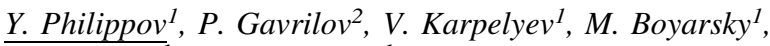
$\overline{\text { A. Mayorov }^{1}}$, M. Shestakova ${ }^{1}$

${ }^{1}$ Endocrinology Research Centre, Diabetes Institution, Moscow, Russia

${ }^{2}$ Bauman Moscow State Technical University, Department of Science and Innovations, Moscow, Russia

Background and Aims: GV calculation and interpretation may be very challenging. There is a pressing need in an intuitive tool for endocrinologists and diabetes patients that allows an easy calculation of GV scores. Also, a risk-predictive and/or machine self-learning algorithm to identify high GV predictors may be helpful in the decision-making process.

Method: An online software for automated calculation of the GV score using CGM raw data of diabetes patients with userfriendly demonstration of the results was created. We used EasyGV program (Isis Innovation Ltd.) algorithms for GV calculation, which is free for academic use. The RAW-data from Endocrinology Research Centre Medtronic CareLink database (regular export option in CSV) were used to estimate the program effectiveness. We selected 23 T1DM patients with long-term CGM use on regular basis. For GV calculation we used the data of the first 4 months after sensor-augmented pump (SAP) initiation. The first monthly retrospective analysis included 544066 datapoints. We calculated all coefficients available in EasyGV software (SD, CONGA, LI, J-index, LBGI, HBGI, M-value, MAG, GRADE, MODD, MAGE, ADRR) for all patients.

Results: All GV coefficients were automatically calculated with our program for all patients. The results of the online counting was same as with EasyGV for all patients (100\% match). The visual interface of the program is based on the polygonal diagrams (as in "Glucose pentagon", described by Thomas A.).

Conclusion: The mathematical basis of the online software for automated GV counting is correct. Intuitive visual interface for HCP and CGM-users and evaluation of the usability for all user categories is needed.

\section{4}

DULCE DIGITAL: A RANDOMIZED CONTROLLED SMS INTERVENTION FOR LATINOS WITH TYPE 2 DIABETES

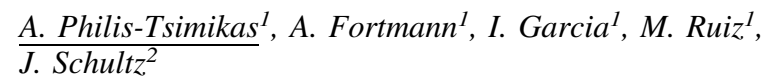

${ }^{1}$ Scripps Health, Scripps Whittier Diabetes Institute, San Diego, USA

${ }^{2}$ Neighborhood Health Care, Family Medicine, San Diego, USA

Background and Aims: Informed by the Chronic Care Model, Project Dulce uses a nurse-led team to provide clinical care, and peer educators to deliver diabetes education in federally-qualified health centers serving disadvantaged Latino populations in San Diego, CA. Although Project Dulce has improved clinical outcomes and reduced hospital and acute care costs, barriers to access persist. Mobile technology has the potential to circumvent these barriers. This study investigates a diabetes self- management intervention delivered via mobile SMS messaging (Dulce Digital) in Latinos.

Method: A total of 116 Latinos with T2DM and HbA1c $>7.5 \%$ were recruited. Following randomization at baseline, all participants receive diabetes care-as-usual and repeat clinical and self-report measures at months 3 and 6. Dulce Digital participants receive 3 types of text messages: educational/motivational, medication reminders, and BG monitoring prompts; 2-3 messages/day are sent at study start, with frequency tapering over 6 months. Staff monitor BG responses, assess possible reasons for hyper/hypoglycemia, and encourage follow up with a provider as needed.

Results: Results are based on an interim, month 0-6 analysis of 106 participants (mean age $=49.25 \pm 9.49 ; 74 \%$ female, income less than $\$ 24,000$ annually) enrolled in the ongoing trial. Dulce Digital participants $(\mathrm{N}=53)$ exhibited significantly greater decreases in HbA1c than the control group from month 0 to $6(9.4$ to $8.4=-1.0 \%$ vs. 9.5 to $9.4=-0.1 \% ; \mathrm{p}<.04)$. No significant difference in BP or lipids. The "maximum effective dosage" of "text backs" was 35 .

Conclusion: Initial results suggest that a text message-based self-management intervention improved glycemic control in high-risk underserved Latinos withT2DM.

\section{5}

\section{UNDERSTANDING PATIENT'S PREFERENCES AND STUDY CHALLENGES IN DIABETES CLINICAL TRIALS}

\section{J. Thilaganathan \\ ${ }^{1}$ CRF Health, Product \& Services Management, London, United Kingdom}

Background and Aims: For diabetes patients, managing their condition can require significant commitment, including measuring blood glucose, monitoring the nutritional value of meals, and tracking insulin usage. Diabetes clinical trials often seek to capture all of this data, as well as additional endpoint information. However, this has historically led to high levels of patient burden and low levels of patient compliance, particularly with paper-based data capture. Our aim was to develop an electronic solution for diabetes trials that reduced patient burden and improved compliance.

Method: Data from a range of global trials using electronic data capture was analysed to understand patient's reporting behaviour in clinical trials. Alongside this, iterative usability was conducted on an electronic diary for reporting diabetes data.

Results: Data showed that $23 \%$ of patients reported their events as they were happening, whilst $77 \%$ reported retrospectively later that day or the following day. Patient feedback highlighted the need to fit into their daily lives, while allowing for easy review of how to complete various task. Taking into account this data and feedback the diary was tailored so that patients could easily report their events either in real-time or use a retrospective data-entry period to assign events after the day. The flow of the diary was improved to reduce the burden of reporting events, while a robust training module was added so patients could have a reminder on how to complete tasks when and if they need.

Conclusion: The refined solution provides an intuitive way to collect key diabetes data while not unduly burdening patients. 


\section{6}

\section{INCRETIN SENSITIVITY AND RESPONSE DURING ORAL GLUCOSE TOLERANCE TEST IN TYPE 2 DIABETES ASSESSED BY MATHEMATICAL MODELING OF THE INCRETIN EFFECT}

A. Tura $^{1}$, J.I. Bagger ${ }^{2}$, E. Ferrannini ${ }^{3}$, J.J. Holst ${ }^{4}$, F.K. Knop ${ }^{2}$, $\overline{\text { T. Vilsb }} \phi l^{5}$, A. Mari ${ }^{1}$

${ }^{1}$ CNR Institute of Neuroscience, Metabolic Unit, Padova, Italy

${ }^{2}$ University of Copenhagen, Center for Diabetes ResearchGentofte Hospital, Hellerup, Denmark

${ }^{3}$ University of Pisa School of Medicine, Department of Internal Medicine, Pisa, Italy

${ }^{4}$ University of Copenhagen, The NNF Center for Basic Metabolic Research and Department of Biomedical Sciences-

Faculty of Health and Medical Sciences, Copenhagen,

Denmark

${ }^{5}$ University of Copenhagen, Center for Diabetes Research-

Gentofte Hospital, Copenhagen, Denmark

Background and Aims: We developed a model for simultaneous analysis of OGTT and isoglycemic intravenous glucose infusion (IIGI). Our model featured an incretin potentiation factor $\left(\mathrm{P}_{\text {incr }}\right)$ on the dose-response function relating insulin secretion to glucose concentration. The model was used to study incretin sensitivity and response in type 2 diabetes (T2D). By exogenous infusions it was previously shown that incretin sensitivity is reduced. Whether this holds true under more physiological conditions, e.g. during OGTT, has not been determined. Also, relationship between incretin sensitivity and incretin response has not been explored.

Method: We performed 25, 75, $125 \mathrm{~g}$ OGTT and IIGI in T2D $(\mathrm{n}=8)$ and matched normal glucose tolerant (NGT) subjects (all subjects: age: $57 \pm 1$ (mean \pm SE) years; BMI: $29 \pm 0.3 \mathrm{~kg} / \mathrm{m}^{2}$ ). Glucose, insulin, C-peptide, total glucagon-like peptide-1
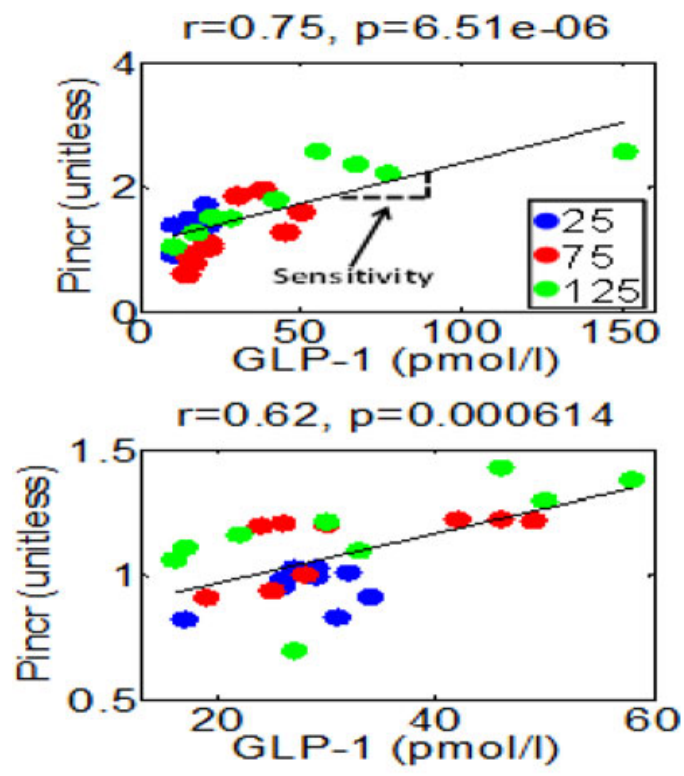

Figure: Relationship between incretin levels and incretin potentiation in one NGT and one T2D subject
(GLP-1), glucose-dependent insulinotropic polypeptide (GIP) were measured.

Results: $\mathrm{P}_{\text {incr }}$ increased dose-dependently during the OGTTs and then returned to baseline, but was blunted in T2D. $\mathrm{P}_{\text {incr }}$ correlated with GLP-1 and GIP, with correlation stronger in NGT (average $r=0.64$ and 0.61 , for GLP-1 and GIP) than in T2D $(r=0.46$ and 0.47$)$. The slope of the individual relationships was used as index of beta-cell sensitivity to GLP-1 and GIP (Figure). GLP-1 sensitivity was impaired in T2D vs. NGT $(0.008 \pm 0.002 \mathrm{vs}$. $0.020 \pm 0.006 \mathrm{l} / \mathrm{pmol}, \mathrm{p}<0.027)$. Using all OGTT data, GLP-1 AUC was inversely related to GLP-1 sensitivity, with steeper slope in NGT $(\mathrm{r}=0.81, \mathrm{p}<0.0001)$. GIP showed similar results.

Conclusion: Incretin insensitivity tends to be compensated by higher incretin secretion. T2D showed moderate impairment in incretin sensitivity and defective compensation of incretin secretion.

247

\section{PRE-PRANDIAL GLUCOSE READINGS FOR THE ASSESSMENT OF THE EFFECTS OF PHARMACOLOGICAL AGENTS ON GLYCEMIC VARIABILITY}

A. Tura $^{1}$, J. Farngren ${ }^{2}$, A. Schweizer ${ }^{3}$, J.E. Foley ${ }^{4}$, G. Pacini ${ }^{1}$, B. Ahrén ${ }^{2}$

${ }^{1}$ CNR Institute of Neuroscience, Metabolic Unit, Padova, Italy ${ }^{2}$ Lund University, Department of Clinical Sciences, Lund, Sweden

${ }^{3}$ Novartis Pharma AG, Novartis, Basel, Switzerland

${ }^{4}$ Novartis Pharmaceuticals Corporation, Novartis,

East Hanover, USA

Background and Aims: The aim of this study was assessing whether the effects of pharmacological agents on glycemic variability can be disclosed by pre-prandial self-monitoring of blood glucose. Here we studied vildagliptin, which is known to reduce glycemic variability.
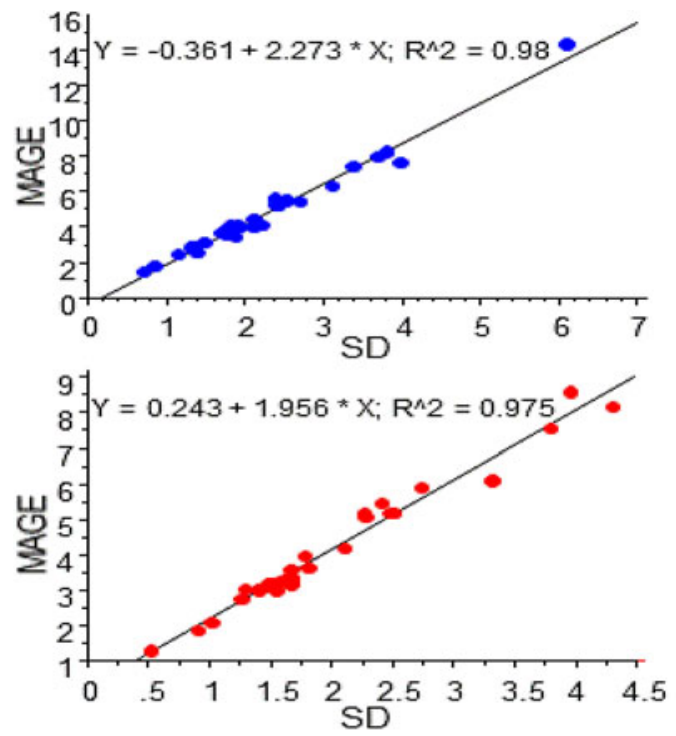

Figure: Indices showing the highest degree of correlation in placebo (top, blue) and vildagliptin (bottom, red): SD and MAGE in both cases 
Method: We analysed data from a double-blind, placebocontrolled study in type 2 diabetes (T2DM) (NCT01219400). Twenty-nine T2DM patients were enrolled (age $=57.9 \pm 1.2$ years; $\mathrm{BMI}=30.8 \pm 0.8 \mathrm{~kg} / \mathrm{m}^{2}$; fasting glycemia $\left.=8.6 \pm 0.4 \mathrm{mmol} / \mathrm{l}\right)$. Vildagliptin or placebo was dispensed for 4 weeks, then 4 weeks washout period occurred before the alternative 4 weeks treatment period. During the two treatment periods, glucose self-monitoring was undertaken every day before breakfast, lunch and dinner, and at bed time. We analyzed several indices of glycemic variability: basic indices, such as standard deviation (SD), interquartile range (IQR), coefficient of variation (CV), and more complex indices, such as CONGA, MAGE, J-Index (Diabetes Technol Ther 2009; 11:S55-S67).

Results: Vildagliptin improved (i.e., reduced) glycemic variability: $2.00 \pm 0.17$ vs. $2.27 \pm 0.21 \mathrm{mmol} / \mathrm{l}$ for $\mathrm{SD}, 2.71 \pm 0.22$ vs $3.12 \pm 0.29 \mathrm{mmol} / 1$ for CONGA, $4.16 \pm 0.33$ vs. $4.80 \pm 0.48$ $\mathrm{mmol} / \mathrm{l}$ for MAGE, $0.09 \pm 0.01$ vs. $0.12 \pm 0.0110^{-3}(\mathrm{mmol} / \mathrm{l})^{2}$ for J-Index, $\mathrm{P}<0.02$, with vildagliptin and placebo, respectively. IQR and $\mathrm{CV}$ did not reach significant difference $(\mathrm{P}>0.09)$. All indices were correlated each other (Figure).

Conclusion: Simple protocols, requiring few pre-prandial readings, may be sufficient to disclose effects on glycemic variability of pharmacological agents. Despite being all correlated, complex indices appear more able than basic indices to disclose differences. Including post-prandial readings might provide more marked effects, but it is clinically relevant that significant differences can be already observed with few pre-prandial readings.

\section{8}

REDUCING HYPOGLYCEMIA RISK BY USING CLOUDBASED DIABETES MANAGEMENT SOLUTION

$\underline{P . V a ̈ i s a ̈ n e n ~}^{1}$, P. Lönnroth ${ }^{1}$, M. Saraheimo ${ }^{2}$, A. Sinisalo ${ }^{1}$

${ }^{1}$ Quattro Folia Oy, Research, Espoo, Finland

${ }^{2}$ Neliapila Oy, Diabetes clinic, Helsinki, Finland

Background and Aims: Intensive blood glucose control is a crucial part in diabetes management and the most well established mean to monitor glycemic control is glycosylated hemoglobin (HbA1c). Thus, diabetics aims to meet the target $\mathrm{HbA} 1 \mathrm{c}$ levels. Yet, a common problem is that while aiming to lower $\mathrm{HbA1c}$ level, it sometimes results in hypoglycemia and in the worst cases it can cause accidents, injuries, coma and even death. Therefore, we studied if a cloud based diabetes management solution can help reducing the risk of hypoglycemia while lowering the HbAlc level.

Method: Study group of 30 diabetics used cloud based diabetes management solution in a one year study period. They reported 2.51 self-monitoring blood glucose (SMBG) values per
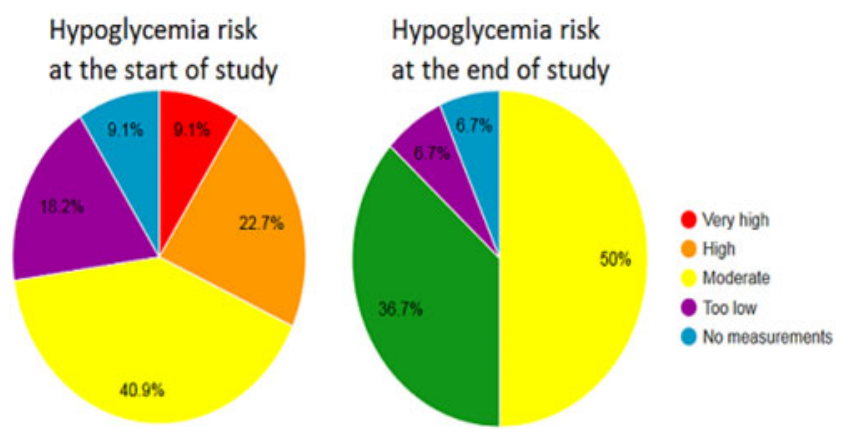

day and $4.17 \mathrm{HbA} 1 \mathrm{c}$ laboratory measurements per year. Each subject's lowest 25 percent of blood glucose values were used to estimate the hypoglycemia risk within a sliding 14-day time window. The risk estimation was based on the mean and standard deviation of the lowest quartile SMBG values and validated by health care professionals.

Results: Figure 1 shows pie charts of subjects, where left hand side is at the start of the study and right hand side is at the end of the study. The average reduction of HbAlc was $0.35 \%$-units.

Conclusion: With the help of a cloud-based diabetes management system, diabetic can learn to reduce the risk of hypoglycemia while also lowering the HbA1c level. Visualization of the collected data is in a key role to understand individual causalities.

\section{9}

\section{A FOCUSED AND SIMPLIFIED REPORT FOR RETROSPECTIVE CGM TO STREAMLINE DATA INTERPRETATION FOR THE HEALTHCARE PROFESSIONAL}

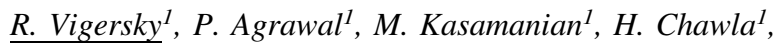

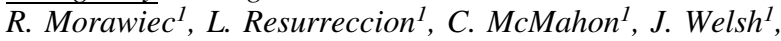
F. Kaufman ${ }^{1}$

${ }^{1}$ Medtronic, Inc., Diabetes, Northridge, USA

Background and Aims: Retrospective continuous glucose monitoring (CGM) can provide valuable insights and actionable information about glycemic patterns and effectiveness of lifestyle and pharmacotherapy in patients with type 2 diabetes (T2D). Because most T2D patients are treated by primary care providers (PCPs), who may not be familiar with how to interpret CGM, we developed a rule-based, simplified retrospective CGM report, the Pattern Snapshot ${ }^{\mathrm{TM}}$ Report, to enhance the use of this tool as part of the overall T2D diabetes regimen.

Method: The single-page simplified report uses new algorithms to prioritize important glycemic patterns and simple icons to identify their most common causes. It was designed to help providers efficiently assess the results of a retrospective CGM evaluation and prompt conversations with their patients about major glycemic problems and management issues.

Results: To help evaluate glucose results, the report is divided into 3 sections: Metrics, Problems, and Overlay. Metrics helps providers focus on key statistics to assess overall glycemia. The Problems section includes top glycemic issues, rank-ordered by the software, and probable causes to help coach patients. Lastly, the Overlay graph allows physicians to evaluate the big glycemia picture.

Conclusion: Retrospective CGM can provide insights to help optimize therapy recommendations. We developed a simple CGM report that alleviates the provider's burden of data analysis and provides guidance for changes in management. Easily shared with T2D patients, the report enables providers to discuss and guide their patients for improved outcomes. This report will be available with the iPro® Professional CGM system.

\section{0}

USE OF MEDICINES AND DEVICES BY ADULTS FOR THE MANAGEMENT OF TYPE 2 DIABETES MELLITUS IN KUWAIT

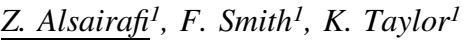 \\ ${ }^{1}$ UCL-School of Pharmacy, Research Department of Pharmacy \\ Practice, London, United Kingdom
}


Background and Aims: Diabetes is a threat to peoples' lives around the world. In Kuwait, the prevalence of diabetes among adults was $18 \%$ in 2013 . This study aims to identify the impact of knowledge, beliefs and culture on medicine-taking behaviour, and treatment outcomes, and investigate whether the use of insulin devices (pens and pumps) have a role in improving patients' adherence to treatment.

Method: The study primarily applies qualitative methods via interviews with 43 patients to obtain information about thier experiences regarding their medicines/devices use. This was followed by conducting interviews with healthcare professionals, in order to propose service development. Quantitative measures such as MMAS and BMQ were also used.

43 patients were asked about their beliefs, views and experiences regarding their medicines/devices used and lifestyle. This was followed by conducting interviews with healthcare professionals, in order to propose for service development.

Results: More than half of patients had uncontrolled disease. Of 43 patients, $26(60 \%)$ identified as poor adherers. Lack of health awareness, beliefs and culture had an impact on medicine-taking behaviour and health outcomes. Insulin devices improved patients' quality of lives and satisfaction. Healthcare providers mentioned that improving patients' awareness is the key for better management.

Conclusion: Several factors contribute to medicine misuse and poor management of type 2 diabetes in Kuwait. Health awareness of patients must be improved by increasing education efforts to accommodate health beliefs and culture. Also, the advantages of using insulin devices should be recognised. To improve health outcomes, a collaborative approach between $\mathrm{MOH}$, healthcare providers and patients must be adopted.

\section{1}

\section{THE APPLICATION OF DISCRETE CHOICE EXPERIMENTS IN COST-EFFECTIVENESS STUDIES TO ASSESS THE BENEFITS OF SENSOR-AUGMENTED INSULIN PUMP THERAPY FOR THE TREATMENT OF TYPE 1 DIABETES}

N. Barnett $^{1,2}$, E. Geelhoed ${ }^{1,2}$, E. Davis ${ }^{2,3,4}$, T.W. Jones ${ }^{2,3,4}$, M. De Bock $k^{2,3}$, G. Smith ${ }^{2}$, R. Norman

${ }^{1}$ University of Western Australia, School of Population Health, Perth, Australia

${ }^{2}$ Telethon Kids Institute, Centre for Research Excellence in Type 1 Diabetes, Perth, Australia

${ }^{3}$ Princess Margaret Hospital for Children, Department of Endocrinology and Diabetes, Perth, Australia

${ }^{4}$ University of Western Australia, School of Paediatrics and Child Health, Perth, Australia

${ }^{5}$ Curtin University, School of Public Health, Perth, Australia

Background and Aims: Diabetes Mellitus affects over 630 million people worldwide and is directly attributed to 1.5 million deaths annually. Few studies have been conducted to determine the cost-effectiveness of gold-standard type 1 diabetes treatment resulting in low uptake among countries that do not provide subsidised access to patients. Discrete choice experiments (DCE) are an innovative measurement tool applied in a number of fields, however their application in health treatment lags. DCEs can be used to explore preferences towards alternative treatment options and to determine relative cost-effectiveness using a holistic measure of relative attributes in the decision-making process.
Method: A literature review was undertaken to identify the application of discrete choice experiments to evaluate preferences in type 1 diabetes treatments. Search terms were restricted to studies on type 1 diabetes but were not limited on any other basis.

Results: Three papers contained the terms 'discrete choice experiment' and 'type 1 diabetes' from relevant databases. At present there are no studies that analyse the cost-effectiveness of type 1 diabetes treatment using a discrete choice experiment methodology.

Conclusion: A significant gap in the literature suggests there is scope for a discrete choice experiment to be conducted with patients and parents of children suffering from type 1 diabetes. An applied DCE would contribute valuable insights into the benefit measurement of gold-standard therapies and may improve access if cost-effectiveness is demonstrated.

\section{2}

I RESIST' TRIAL: CONSISTENCY OF A TREATMENT BY AMBULATORY SUBCUTANEOUS INSULIN PUMP IN INSULIN RESISTANT TYPE 2 DIABETIC PATIENTS-PRELIMINARY RESULTS

S. Clavel ${ }^{1}$, F. Travert ${ }^{2}$, D. Huet ${ }^{3}$, E. Ghanassia ${ }^{4}$, K. Mohammedi ${ }^{2}$, F. Somers ${ }^{5}$, J.M. Andrieu ${ }^{6}$, J.P. Le Berre ${ }^{7}$, J.P. Courrèges $^{8}$

${ }^{1}$ Centre Hospitalier Hôtel Dieu, Le Creusot, France

${ }^{2} \mathrm{CHU}$ Bichat, Paris, France

${ }^{3}$ Hôpital Saint Joseph, Paris, France

${ }^{4}$ Clinique Sainte Thérèse, Sète, France

${ }^{5}$ Centre Hospitalier Alpes Léman, Contamine sur Arve, France

${ }^{6}$ Centre Hospitalier Béziers, Béziers, France

${ }^{7}$ HIA Desgenettes, Lyon, France

${ }^{8}$ CHG Narbonne, Narbonne, France

Background and Aims: Some type 2 diabetic (T2D) patients who require insulin tend to show a major insulin resistance, in spite of a treatment optimization with multiple injections. Could

\begin{tabular}{lllll}
\hline & HbAlc & $\Delta \mathrm{T}_{-}-\mathrm{T}_{\mathrm{x}}$ & $\begin{array}{l}\text { Fasting blood } \\
\text { glucose }\end{array}$ & $\Delta \mathrm{T}_{0}-\mathrm{T}_{\mathrm{x}}$ \\
Baseline & $9.42 \pm 1.51 \%$ & $\mathrm{~N} / \mathrm{A}$ & $2.01 \pm 0.66 \mathrm{~g} / \mathrm{l}$ & $\mathrm{N} / \mathrm{A}$ \\
$\mathrm{T}_{\text {fmones }}$ & $8.07 \pm 0.80 \%$ & $-1.35 \%-\mathrm{p}<0.0001$ & $1.54 \pm 0.47 \mathrm{~g} / \mathrm{l}$ & $-0.47-p<0.005$ \\
$\mathrm{~T}_{12 \text { ments }}$ & $7.93 \pm 0.89 \%$ & $-1.50 \%-p<0.0001$ & $1.59 \pm 0.71 \mathrm{~g} / \mathrm{l}$ & $-0.42-p<0.05$
\end{tabular}

\begin{tabular}{lllll}
\hline HbAlC & $T_{0}$ & $T_{6}$ & $T_{12}$ & $\Delta T_{0}-T_{12}$ \\
$<8 \%$ & $12.3 \%$ & $53.5 \%$ & $55.2 \%$ & $+42.9 \%$ \\
{$[8.9 \%[$} & $24.5 \%$ & $31.0 \%$ & $34.5 \%$ & $+10.0 \%$ \\
$29 \%$ & $63.2 \%$ & $15.5 \%$ & $10.3 \%$ & $-52.9 \%$ \\
\hline
\end{tabular}

\begin{tabular}{lll}
\hline HbAlc & $\Delta T_{0}-T_{12}$ & $Q$ \\
{$[7-8.8 \%]$} & $-0.44 \%(-5.5 \%)$ & NS \\
{$[8.9-9.8 \%]$} & $-1.22 \%(-12.4 \%)$ & $\mathbb{Q}<0.001$ \\
{$[9.9-14.7 \%]$} & $-2.85 \%(-25.8 \%)$ & $\mathbb{Q}<0.0001$ \\
\hline
\end{tabular}


a treatment by ambulatory subcutaneous insulin pump (AIP) improve this situation in real life?

Method: I Resist' is an observational, prospective, multicentric $(\mathrm{n}=12)$ trial. 107 T2D patients were enrolled $(64.3 \pm 9.9 \mathrm{yrs}$, sex ratio $\mathrm{M} / \mathrm{F}: 1.55$, diabetic for $16.9 \pm 7.7 \mathrm{yrs})$. They were insulinresistant $(\geq 0.7 \mathrm{U} / \mathrm{kg} / \mathrm{d})$, poorly controlled $(\mathrm{HbA} 1 \mathrm{c} \geq 8 \%$ ) in spite of an insulin treatment optimization $(1.28 \pm 0.45 \mathrm{U} / \mathrm{kg} / \mathrm{d}-3.6$ injections/d). They were treated by AIP. Here are the preliminary results : $n=70$ at 6 months, 55 at 12 months.

Results: Fasting blood glucose level and HbAlc showed a positive evolution.

The proportion of patients in every HbA1c class was as follows.

HbA1c evolution according to terciles at $\mathrm{T}_{0}$, showed a better improvement for the highest initial levels.

Weight remained stable : 95.9-96.9-95.1kg $-\Delta \mathrm{T}_{0}-\mathrm{T}_{12}:-0.8 \mathrm{~kg}$

- NS ; BMI : $33.8-34-33.7 \mathrm{~kg} / \mathrm{m}^{2}-\Delta:-0.1 \mathrm{~kg} / \mathrm{m}^{2}-\mathrm{NS}$.

Insulin doses decreased : 1.28/0.97/1 $-\Delta \mathrm{T}_{0}-\mathrm{T}_{12}:-0.28 \mathrm{U} / \mathrm{kg} / \mathrm{j}$ $(-22 \%-\mathrm{p}<0.001)$.

No severe hypoglycemia has been reported, 6 patients stopped the treatment by AIP (4 infections/catheter).

Conclusion: In poorly controlled T2D (average $\mathrm{HbA} 1 \mathrm{c} 9.5 \%$ ) and highly insulin-resistant $(1.28 \mathrm{U} / \mathrm{kg} / \mathrm{d})$, a treatment by AIP allows a strong decrease in HbA1c $(-1.5 \%)$ and in insulin needs $(-22 \%)$ without weight gain or severe hypoglycemia.

253

\section{SAFETY OF AN AMBULATORY SUBCUTANEOUS INSULIN PUMP (AIP) IN TYPE 2 DIABETIC (T2D) INSULIN-RESISTANT PATIENTS. I RESIST' TRIAL - PRELIMINARY RESULTS}

D. Huet ${ }^{1}$, S. Clavel ${ }^{2}$, F. Travert ${ }^{3}$, E. Ghanassia ${ }^{4}$, O. Dupuis ${ }^{5}$, F. Lagarde $^{6}$, P. Sérusclat ${ }^{7}$, J.P. Courrèges ${ }^{8}$

${ }^{1}$ Hôpital Saint Joseph, Endocrinology and Diabetology, Paris, France

${ }^{2}$ Centre Hospitalier Hôtel Dieu, Le Creusot, France

${ }^{3}$ CHU Bichat, Paris, France

${ }^{4}$ Clinique Saint Thérèse, Sète, France

${ }^{5}$ HIA Desgenettes, Lyon, France

${ }^{6}$ Centre Hospitalier Montargis, Montargis, France

${ }^{7}$ Clinique Les Portes du Sud, Vénissieux, France

${ }^{8}$ CHG Narbonne, Narbonne, France

Background and Aims: What safety level may be expected from an AIP treatment in T2D patients usually elderly and fragile, with lower learning abilities?

Method: Safety of an AIP treatment in 107 T2D patients, poorly controlled with multiple injections (MI), insulin-resistant $(1.28 \mathrm{U} / \mathrm{kg} / \mathrm{d})$ was assessed half way $\left(\mathrm{n}=70\right.$ at $\mathrm{T}_{6 \text { months }}, \mathrm{n}=55$ at $\left.\mathrm{T}_{12 \mathrm{months}}\right)$ of a prospective trial.

Results: AIP treatment has not been suspended before 3 months. 6 patients $\left(2\right.$ at $\mathrm{T}_{3 \text { months }}, 1$ at $\mathrm{T}_{6 \text { months }}, 1$ at $\left.\mathrm{T}_{9 \text { months }}\right)$ stopped the treatment afterwards (2/poor acceptability, 4/catheter infection).

No specific technical problem was reported in terms of pump or catheter.

\begin{tabular}{lll}
\hline & Baseline & $T_{12 \text { months }}$ \\
\hline Number of hospitalized patients & $60(57 \%)$ & $23(23 \%) \quad p<0.05$ \\
\hline Number of days & $5.8 \pm 3.6$ & $4.3 \pm 3.1 \quad$ NS \\
\hline
\end{tabular}

At $\mathrm{T}_{12 \mathrm{months}}$, insulin total dose decreased by $22 \%$ and flows number was limited $(\mathrm{n}=2.45$ and basal/bolus ratio $=1.23)$.

We assessed hypoglycemia frequency with MI vs. AIP : 3.3 in the last 12 months $(0.13$ severe/yr $)$ vs. 2.3 in the last 3 months (not severe).

Hospitalizations comparative analysis before and 12 months after AIP treatment showed a global improvement especially for uncontrolled diabetes causes (66 vs. $28 \%$; p < 0.05$)$.

Cardiovascular risk factors remain the same: weight $(\Delta=$ $-0.9 \mathrm{~kg}), \mathrm{BMI}\left(\Delta=-0.1 \mathrm{~kg} / \mathrm{m}^{2}\right)$, blood pressure and lipid status are not different.

Conclusion: Highly insulin-resistant T2D patients, often elderly, show most of the time enough abilities to deal with an AIP, leading to a decreased hypoglycemic risk and hospitalizations for uncontrolled diabetes.

\section{4}

\section{INITIAL RESULTS FROM INTRODUCTION OF INSULIN PUMP THERAPY AT CHILDREN'S DIABETES CENTER IN VARNA}

$\underline{Y . B a z d a r s k a}^{1}$, V. Iotova ${ }^{2}$, M. Wilhelm ${ }^{3}$, V. Boyadzhiev ${ }^{4}$, S. Galcheva ${ }^{2}$, R. Stoycheva ${ }^{5}$

${ }^{1}$ Medical University Varna, Department of Pediatrics and Medical Genetics, Varna, Bulgaria

${ }^{2}$ UMHAT "St. Marina” and Medical University-Varna, Department of Pediatrics and Medical Genetics- First Pediatric Clinic, Varna, Bulgaria

${ }^{3}$ UMHAT "Sv. Marina”, First Pediatric Clinic, Varna, Bulgaria

${ }^{4}$ UMHAT “Sv. Marina” - Medical University Varna, First Pediatric Clinic, Department of Pediatrics and Medical Genetics, Varna, Bulgaria

${ }^{5}$ UMHAT “St. Marina”, Department of Pediatrics and Medical Genetics, Varna, Bulgaria

Background and Aims: The prevalence of type 1 diabetes mellitus (T1DM) has increased significantly among the children. Insulin pump treatment (IPT) is the best method for optimal control of T1DM so far.

Aim: to assess the changes of diabetes control for 18 months after introduction of IPT.

Method: Data is registered in Excel from the set-up of IPT until Sept. 2015. Collected information: demographic, anthropometric data; duration of diabetes; quaterly measured $\mathrm{HbA} 1 \mathrm{c} \%$; episodes of acute complications (requiring hospitalization); insulin dose (UI/kg/d.); frequency of contact with doctor.

Results: A total of 36 children with T1DM (26 boys) on IPT are followed up. Mean age $9.0 \pm 4.45 y$., with mean diabetes duration $3.58 \pm 2.6 y$. and mean duration of IPT $18.63 \pm 15.16$ months. Mean value of initial HbA1c before IPT is $9.07 \pm 2.10 \%$, and mean HbA1c value after IPT is $7.70 \pm 1.78 \%\left(1^{\text {st }}, 2^{\text {nd }}\right.$ and $3^{\text {rd }}$ quarter mean HbA1c: $8.41 \%, 7.77 \%, 7.27 \%$, respectively). The most considerable improvement of control is in children under 6 years. Their baseline HbA1c of $10.27 \%$ has decreased to $7.70 \%$ $(p<0.05)$. Initial reduction in total insulin dose remains a trend over the time. One third of the patients are not actively looking for contact with the Center and do not respond to invitations for contact. Their mean HbA1c is $8.52 \%$. There are no episodes of severe hypoglycaemia and one hospitalization for mild DKA in teenager. 
Conclusion: Our study demonstrates improvement in diabetes control, mostly expressed in pre-school children, after switching to IPT and a lower frequency of acute complications.

\section{5}

\section{CORRELATION BETWEEN IMPAIRED AWARENESS OF HYPOGLYCAEMIA REPORTED BY TYPE 1 DIABETES CHILDREN AND THEIR PARENTS}

\section{P.I. Beato-Víbora $^{1}$, F.J. Arroyo-Díez ${ }^{2}$, L. Galán-Bueno ${ }^{2}$}

${ }^{1}$ Badajoz University Hospital, Department of Endocrinology, Badajoz, Spain

${ }^{2}$ Badajoz University Hospital, Department of Paediatrics, Badajoz, Spain

Background and Aims: The evaluation of hypoglycaemia awareness in children with type 1 diabetes mellitus (DM1) is troublesome. Clarke score (CS) and Gold score (GS) have been validated in adults but are less frequently used in children. The aim of the study was to evaluate self-reported impaired awareness of hypoglycaemia (IAH) in DM1 children by CS and GS.

Method: A prospective evaluation of IHA was performed by using GS and CS. The same questionnaires were completed by the children and their parents, separately. IAH was defined by a $\mathrm{CS} \geq 4$ or $\mathrm{GS} \geq 4$.

Results: 77 children with DM1 were included (55\% male, age $12 \pm 3($ mean \pm SD) (4-16), duration of diabetes 5.0 $\pm 3.8, \mathrm{HbA} 1 \mathrm{c}$ $7.3 \pm 1.0 \%$, insulin dose $0.9 \pm 0.2 \mathrm{U} / \mathrm{kg} / \mathrm{day}, 58 \%$ from non-rural locations, $36 \%$ treated with insulin pumps for $2.6 \pm 1.9$ years). Ten patients $(13 \%)$ had previous history of severe hypoglycaemia and loss of hypoglycaemia symptoms was reported by $20 \%$ $(n=16)$ of patients. IAH was diagnosed in $24 \%$ and $20 \%$ of patients according to CS reported by parents and children respectively $(\mathrm{p}=0.004)$ and $35 \%$ and $22 \%$ according to GS $(\mathrm{p}=0.046)$. Correlation between children's and parents' scores was $r_{s}=0.495(p<0.0005)$ and $r_{s}=0.364(p=0.001)$ for CS and GS respectively. Concordance between IAH reported by children and parents was higher in patients older than 9 than in younger children for GS (92\% vs 8\%; $\mathrm{p}=0.046)$ but similar for CS.

Conclusion: CS shows a higher correlation than GS when evaluating IAH perceived by children and their parents and it is less affected by age than GS. Children tend to underestimate their IAH compared to their parents.

\section{6}

\section{WHO IS THE MAIN BENEFICIARY OF INSULIN PUMP THERAPY: LONG-TERM OUTCOME IN CHILDREN WITH TYPE 1 DIABETES?}

I. Ben-Skowronek ${ }^{1}$, R. Piekarski ${ }^{1}$, B. Wysocka-Lukasik ${ }^{1}$, B. Banecka ${ }^{I}, A$. Bury ${ }^{l}$, A. Krzewska ${ }^{1}$, J. Sieniawska ${ }^{1}$, M. Kabat ${ }^{1}$, K. Rebowicz ${ }^{1}$ W. Osiak ${ }^{1}$, L. Szewczyk ${ }^{1}$

${ }^{1}$ Medical University, Dept. Pediatric Endocrinology and Diabetology, Lublin, Poland

Background and Aims: The growing popularity of type 1 diabetes (DM1) treatment based on continuous subcutaneous insulin infusion (CSII) raises a question of the group of patients that benefit most from the treatment.

Method: Clinical observation was carried out in 285 1-18year-old patients diagnosed with DM1 treated with CSII. Every 3 months, HbA1c was determined by an agglutination inhibition immunoassay. The patients were followed for 6-10 years.

Results: The greatest benefits from the treatment with CSII using an insulin pump were noted in type 1 diabetes children aged 1-5: the mean HbA1c decreased in these patients from $7,98 \%$ to $6,75 \%(p<0,01)$ over 6 years. Slightly lesser outcomes were noted in the group of 6-10-year olds: the mean $\mathrm{HbA} 1 \mathrm{c}$ value increased slightly from $7,6 \%$ before the CSII to $7,89 \%$ after 6 years of treatment $(p>0,01)$. Somewhat worse outcomes were reported in the group of 11-15-year-old children: HbA1c increased from $8,05 \%$ to $8,72 \%$ ( $p>0,01$ ). The lowest outcomes were found in the group of the 16-19-year-old patients, as HbA1c rose from $7,8 \%$ to $8,82 \%(p<0,01)$ over 6 years. The children receiving the CSII treatment as early as in the first year of treatment exhibited better diabetes control (HbA1c 8,1\% declined after 6 years to do $7,1 \%, p<0,01)$ than patients who received CSII at an older age (HbA1c increased from $7,92 \%$ to $8,2 \%, \mathrm{p}<0,01)$.

Conclusion: The CSII on offers the greatest benefits for patients aged 1-5 and those with the treatment commenced in the first year after diagnosis of type 1 diabetes.

\section{7}

\section{SUCCESSFUL OUTCOME WITH INSULIN PUMPS COMMENCED AFTER CONCEPTION IN WOMEN WITH TYPE 1 DIABETES}

V. Provenzano $^{1}$, A. Di Noto ${ }^{1}$, D. Brancato ${ }^{1}$, A. Scorsone ${ }^{1}$,

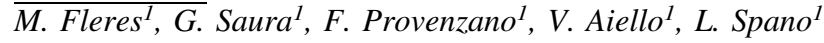

${ }^{1}$ Ospedale Civico di Partinico, Internal Medicine - Regional Reference Center for Diabetology and Insulin Pumps, Partinico, Italy

Background and Aims: The effectiveness of insulin pumps commenced after conception is unknown. Aim of the present study is to compare the effectiveness of insulin pumps commenced before and after conception in women with type 1 diabetes (T1D).

Method: We assessed 28 pregnant women who started pumps $>3$ months before conception (group A) and 16 women with unsatisfactory pre-conception $\mathrm{HbAlc}(\geq 7 \%)$ who started pumps within the 1st trimester of pregnancy (group B). The women underwent to a careful re-training (group A) or training (group B) to the advanced functions of pumps, with emphasis on pregnancy related aspects. Main outcomes were: mean of glycosylated haemoglobin (HbA1c) values obtained throughout
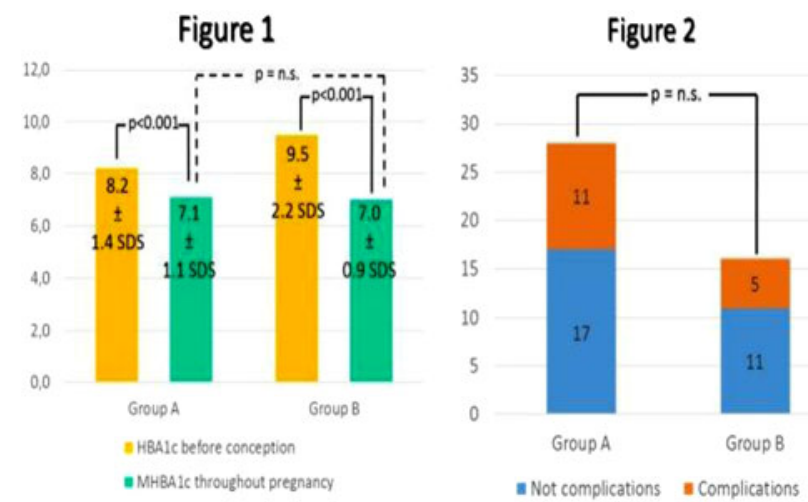
the pregnancy during the use of insulin pumps (MHbA1c); complications $($ fetal + neonatal + maternal complications $) /$ not complications rate.

Results: The groups A and B showed similar age, duration of diabetes and prevalence of pre-pregnancy complications, with the exception of pre-conception HBA1c (pre-conception HbA1c: group $\mathrm{A}=8.36 \pm 1.39 \%$ versus group $\mathrm{B}=9.06 \pm 2.64 \% ; \mathrm{p}<$ $0.05)$. On the contrary, both MHbA1c and complications rate were not different between the groups (MHbA1c: group $\mathrm{A}=7.17 \pm$ $1.08 \%$ versus group $B=7.04 \pm 1.21 \% ; p=$ n.s.; Figure 1) (complications rate: group $\mathrm{A}=10 / 25$ versus group $\mathrm{B}=4 / 10$; $\mathrm{p}=$ n.s.; Figure 2).

Conclusion: Our study shows that, in women with type 1 diabetes (T1D), the effectiveness of insulin pumps is similar when insulin pumps are commenced before or after conception, but a careful intensive training to the advanced function of insulin pumps is needed.

258 AN INTERVENTION WITH A PERSON-CENTERED
PROGRAM, GUIDED SELF-DETERMINATIONYOUNG, IN GROUPS OF ADOLESCENTS STARTING ON INSULIN PUMP: A RANDOMIZED CONTROLLED TRIAL

$\underline{\text { A.L. Brorsson }}^{1}$, A. Lindholm Olinder ${ }^{2}$, J. Leksell ${ }^{3}$, G. Viklund ${ }^{1}$

${ }^{1}$ Karolinska Institute, Department of Women's and Children's Health, Stockholm, Sweden

${ }^{2}$ Karolinska Institute, Department of Clinical Science and

Education Södersjukhuset, Stockholm, Sweden

${ }^{3}$ Uppsala University, Department of Medical Sciences,

Uppsala, Sweden

Background and Aims: In Sweden 52\% of the adolescents with diabetes are treated with continuous subcutaneous insulin infusion (CSII). Regardless of modern diabetes treatment, only $42 \%$ of the adolescents reach the recommended HbA1c-target $57 \mathrm{mmol} / \mathrm{mol}$. Guided Self Determination-Young (GSD-Y) is a person-centered, empowerment-based, problem-solving method intended to guide adolescents to become self-sufficient and develop life skills for managing difficulties in diabetes selfmanagement.

The aim was to evaluate whether an intervention with GSD$\mathrm{Y}$ in groups of adolescents starting on CSII and their parents leads to decreased HbA1c and fewer diabetes-related family conflicts.

Method: This study is half-time results from a prospective RCT including 39 adolescents aged 12-18 years starting on CSII and their parents. All participants received standard CSII start training. The GSD-Y education was performed at three occasions in connection with start of CSII and four once a month after. Outcomes evaluated at baseline, 6 and 12 after starting CSII are HbA1c and diabetes-related family conflict scale (DFCS).

Results: At 6 months HbA1c had decreased in both groups, but more in the intervention group [intervention $(n=18)$ : 54 vs. $69 \mathrm{mmol} / \mathrm{mol}$, control $(\mathrm{n}=19)$ : 65 vs $74 \mathrm{mmol} / \mathrm{mol}, \mathrm{p}=0,024$ between groups]. At 12 months there were no differences compared with baseline in any group [intervention $(\mathrm{n}=15): 63 \mathrm{mmol} /$ mol, control $(n=10): 70 \mathrm{mmol} / \mathrm{mol}, \mathrm{p}=0,283$ between groups].

DFCS correlated with HbA1c. The intervention group perceived a lower degree of conflicts after GSD-Y education.
Conclusion: Evaluation of the intervention has, so far, shown promising results. GSD-Y can be a valuable complement in education of adolescents starting on CSII.

\section{9}

\section{INSULIN INFUSION PUMP DOSE PRECISION PERFORMANCE AT DIFFERENT DELIVERY PHASES}

${\text { J. } \text { Capurro }^{1}, R . \text { Venugopalan }^{1}, \text { B. Levy }}^{1}$

${ }^{1}$ Animas Corporation, Wayne, PA, USA

Background: Insulin pump delivery performance resets every 2-3 days as the usage of infusion-sets, cartridges and disposable pumps expires, and delivery sessions are re-started. Understanding dose precision performance over the start-up, transition-tostabilization, and stabilization delivery phases may be instrumental in achieving a reliable insulin delivery regimen and thereby minimizing glycemic excursions.

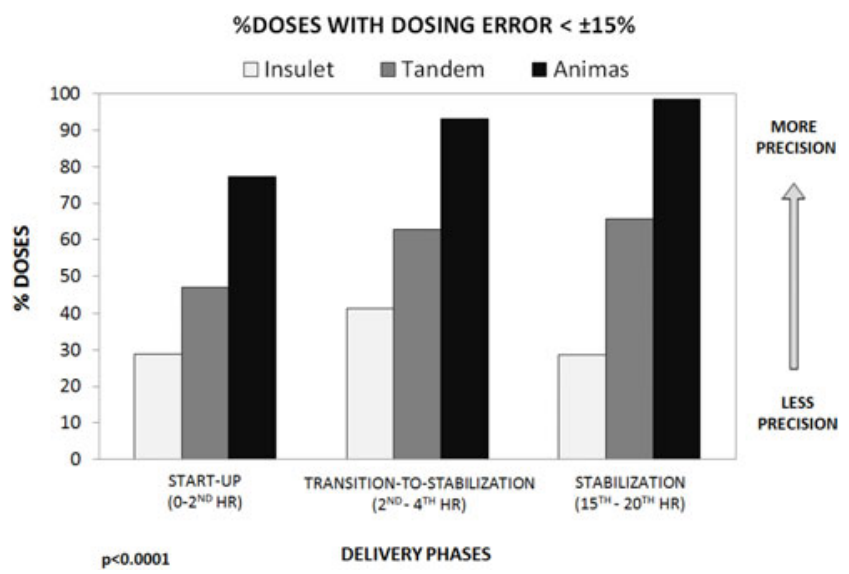

Method: A $0.5 \mathrm{U} / \mathrm{hr}$ basal rate was measured using a timestamped micro-gravimetric system for 20 hours. For the durable Animas $\AA$ OneTouch $\AA$ Ping $\AA$ and Tandem $\AA$ t:slim $\AA$, and the disposable Insulet 2ndGen OmniPod ${ }^{\circledR}$, dose precision was determined by the percent number of doses with error less than $\pm 15 \%$. The data analysis was conducted for doses at start-up (0-2nd hr), during transition-to-stabilization (2nd-4th hr) and stabilization (15th-20th hr) delivery phases.

Results: Durable pumps showed significantly better dose precision than disposable pumps. In particular, the OneTouch Ping showed higher precision than the other pump models over all delivery phases investigated (Figure 1).

Conclusions: The OneTouch Ping demonstrated better dose precision for all delivery phases amongst the pumps studied. Variations across the delivery phases may affect reproducibility in the infusion of basal rates and thus have clinical implications in determining a patient's basal rate needs. Further research on the clinical relevance of these findings is warranted.

\section{0}

\section{FIVE YEARS WITH CONTINUOUS SUBCUTANEOUS INSULIN INFUSION (CSII): IMPACT ON PSYCHOLOGICAL VARIABLES}

M. Carreia $^{1}$, M.T. Anarte ${ }^{1}$, M. Domínguez ${ }^{2}$, Á. Orozco ${ }^{3}$, N. Colomo ${ }^{2}$ I. Sánchez ${ }^{4}$, S. González-Romero ${ }^{2}, F$ Linares $^{2}$, M. Guerrero ${ }^{4}$, M.S. Ruiz de Adana ${ }^{2}$ 
${ }^{1}$ Department of Personality- Assessment and Psychological Treatment., Instituto de Investigación Biomédica de Málaga IBIMA. Universidad de Málaga, Málaga, Spain

${ }^{2}$ Unidad de Diabetes. Unidad de Gestión Clínica de Endocrinología y Nutrición, Instituto de Investigación Biomédica de Málaga IBIMA- Hospital Universitario Regional de Málaga-Universidad de Málaga. Spanish Biomedical Research Centre in Diabetes and Associated Metabolic Disorders CIBERDEM, Málaga, Spain

${ }^{3}$ Project funded by the Ministry of Economy and Competitiveness of Spain PSI2011-27820., Research Fellow, Málaga, Spain

${ }^{4}$ Unidad de Diabetes. Unidad de Gestión Clínica de Endocrinología y Nutrición., Instituto de Investigación Biomédica de Málaga IBIMA- Hospital Universitario Regional de Málaga- Universidad de Málaga, Málaga, Spain

Background and Aims: 1) To assess the impact of treatment with CSII therapy in psychological variables and quality of life after 5 years of treatment in people with type 1 diabetes. 2) To analyze these variables according to indications for administering CSII: 1. Poor glycemic control or glycemic instability (PG group); 2. Frequently or severe hypoglycaemia (FH group).

Method: 30 patients with type 1 diabetes and CSII were evaluated clinical, metabolic and psychologically at baseline, 6 months and annually for 5 years. Psychological variables: depressive symptoms (BDI), anxiety (STAI), fear of hypoglycemia (FH-15), quality of life (DQOL), locus of control (MHLC). Statistical analysis: Student-t test, Wilcoxon signed ranks, MannWhitney, Pearson correlation coefficient.

Results: Overall, after 5 years of treatment statistically significant differences were found with respect to the baseline score in fear of hypoglycemia, quality of life, anxiety-state, anxietytrait and $\mathrm{HbA} 1 \mathrm{c}$ with better results. When comparing PG and FH groups, initially significant differences were not found in psychological variables analyzed between the two groups, although in HbA1c higher values were in the PG group. At 5 years, significant differences in quality of life and HbA1c between the two groups exist, getting worse the PG group. Regarding baseline, patients in the FH group showed better quality of life and a decrease in anxiety-trait and internal locus of control at 5 years treatment, while patients in the PG group had lower scores in fear of hypoglycemia and HbAlc.

Conclusion: Significant improvements were found in some psychological variables studied in patients with CSII. These results differ depending on the type of treatment indication.

\section{1}

\section{PREGNANCY OUTCOME IN WOMEN WITH TYPE 1 DIABETES ON CSII AND GLYCEMIC CONTROL IN LAST FIVE YEARS}

M. Cokolic ${ }^{1}$

${ }^{1}$ University Clinical Centre Maribor, Department of Endocrinology and Diabetology, Internal Clinic, Maribor, Slovenia

Background and Aims: We evaluate metabolic control, maternal and fetal outcomes in our group of pregnant type 1 diabetic (DM1) patients treated with continuous subcutaneous insulin infusion (CSII). Insulin pump with CSII is nowadays the most modern method of type 1 diabetes treatment.
Method: In the last 5 years (2010-2014) we treated 17 pregnant women with DM1 on insulin pump and we evaluated metabolic control, maternal and fetal outcomes.

Results: The average age of mothers at the childbirth was 29,31 years (22-39). HbA1c in the first trimester was 7,1\% (5,1$7,3 \%)$, in the third trimester $7,24 \%(5,3-7,8 \%)$, in the postnatal period was $7,65 \%(5,3-8,6 \%) .57 \%$ of the children were born by Caesarean section (twice gemini), of which $52,4 \%$ boys, on average at 37,23 (34-40) week of pregnancy. The average newborn weight was $3379 \mathrm{~g}(2290-4800 \mathrm{~g})$, length $50,90 \mathrm{~cm}(46-54 \mathrm{~cm}), 5$ $(23,8 \%)$ were weighing more than $4000 \mathrm{~g}(4060-4800 \mathrm{~g})$, of which mothers were not so bad metabolically regulated (HbAlc $6,1-7,5 \%)$.

Conclusion: All pregnancies ended successfully. Early elective delivery (labour induction or Caesarean section) aims to avoid these complications, so $57 \%$ were completed by Caesarean section. $23,8 \%$ of newborns were overweight without other complications, deliveries were at 37,23 week. Metabolic arrangement during pregnancy was good, after giving birth the loss of motivation was apparent. Insulin pump is a safe treatment in pregnant women with DM1.

262

\section{SHOULD YOUNGER ADULTS WITH TYPE 1 DIABETES ON PUMP THERAPY BE EDUCATED DIFFERENTLY THAN THE OLDER INDIVIDUALS?}

\section{E. Szymańska-Garbacz ${ }^{1}$, J. Loba $^{1}$, L. Czupryniak ${ }^{2}$}

${ }^{1}$ Medical University of Lodz, Department of Internal Medicine and Diabetology, Lodz, Poland

${ }^{2}$ Medical University of Warsaw, Department of Internal Medicine and Diabetology, Warsaw, Poland

Background and Aims: Pump therapy requires continuous patients' education. Its objective is to avoid errors with pump management. As education should be tailored to each patient's needs and capability, we conducted a study analysing whether any age-specific educational needs in younger and older adults exist.

Method: The study group comprised 60 type 1 diabetes patients using Medtronic 722, 754 and 640G pumps, divided in two subgroups: Group 1 - aged $<26$ years and Group $2->26$ years $(n=29$ and 31 ; mean age $22.6 \pm 2.3$ and $34.5 \pm 8$ yrs; diabetes duration $7.5 \pm 5.6$ and $11.2 \pm 9.8$ yrs; pump use $4.2 \pm 3.8$ and $3.0 \pm 2.6 \mathrm{yrs}, \mathrm{HbA} 1 \mathrm{c} 7.5 \pm 5.6 \%$ and $7.2 \pm 0.6 \%$, respectively; all $\mathrm{p}>0.05)$. Inappropriate carbohydrate counting, dominant manual boluses use, inadequate bolus wizard overriding, delayed infusion set change or improper filling, unjustified pump disconnection episodes, boluses given without self-monitoring of blood glucose (SMBG), miscorrection of hypo- or hyperglycemia events, no boluses before eating, no SMBG at bedtime, too rare or too frequent use of SMBG or boluses, and improper use of temporary basal rate were noted retrospectively from all two weeks' records from a year preceding the moment of data collection.

Results: Mean number of errors was similar in both groups, however older patients overcorrected hyperglycemia significantly more often $(\mathrm{p}<0.05)$ than the younger subjects, however those younger ones used SMBG $(p<0.01)$ and boluses $(p<0.05)$ less often than the older patients.

Conclusion: Pump therapy education addressed to younger adults should concentrate on improving SMBG use and administering appropriate boluses, while the older patients should be encouraged to be more complacent when managing hyperglycemia. 
263

EXPERIENCE WITH CONTINUOUS SUBCUTANEOUS INSULIN INFUSION IN NORTHERN SPAIN

M. Diéguez ${ }^{1}$, A. Martín ${ }^{2}$, P. Botas ${ }^{3}$, P. Pujante ${ }^{4}$, L. Cacho ${ }^{2}$, C. Diaz de Greñu ${ }^{5}$, E. Menéndez ${ }^{2}$

${ }^{1}$ Hospital de Cabueñes, Endocrinology, Gijon, Spain

${ }^{2}$ Hospital Universitario Central de Asturias, Endocrinology, Oviedo, Spain

${ }^{3}$ Hospital San Agustín, Endocrinology, Aviles, Spain

${ }^{4}$ Hospital Alvarez Buylla, Endocrinology, Mieres, Spain

${ }^{5}$ Hospital Valle del Nalón, Endocrinology, Sama de Langreo, Spain

Background and Aims: Data about continuous subcutaneous insulin infusion (CSII) treatment in clinical practice conditions are limited. The aim of the study was to characterize the patient who initiates CSII therapy.

Method: A retrospective observational multicenter study was performed in Asturias (Spain) from 1998 to 2015.

Results: A total of 118 patients with CSII were recorded (3\% of all type 1 diabetic patients), of which 109 were adults and the remaining 9 pediatric patients. During the last 5 years, 52 new CSII systems were implanted (44\% of the total).

Of these, $71 \%$ were women, with a mean age of 36 years (SD 13 ) and a mean diabetes evolution of 16 years (SD 10). Mean time with CSII was 6 years (SD 4).

The most common reason for pump treatment was brittle diabetes $(43 \%)$, followed by pregnancy planning $(22 \%)$, severe hypoglycemia or hypoglycemia unawareness $(21 \%)$ and inadequate metabolic control (12\%).

Baseline HbA1c was $7.6 \%$ (SD 1) and fell by $0.1 \%$ at the end of follow-up. HbA1c reduction was higher in patients with CSII use $<2$ years $(-0.85 \%$ vs $+0.1 \% ; \mathrm{p}<0.05)$ and in those with a baseline HbA1c $>8 \%(-0.9 \%$ vs $+0.3 \%$; $<<0.05)$.

13 pumps were withdrawn (11\%), mainly for psychological pump intolerance. Safety was good, with a hospitalization rate of 1.2 patients/year.

Conclusion: Use of CSII in type 1 diabetes in Spain is lower as compared to other countries in Europe.

In terms of $\mathrm{HbAc} 1$ reduction, CSII therapy was more effective in the short-term use and in patients with a higher baseline HbA1c.

264

EFFECT OF ANTECEDENT RECURRENT HYPOGLYCEMIA ON THE FIELD GLYCEMIC RESPONSE TO A METABOLIC CHALLENGE IN SUBJECTS WITH TYPE 1 DIABETES

C. Fabris $^{1}$, L. Farhy ${ }^{1}$, M. Breton ${ }^{1}$

${ }^{1}$ University of Virginia, Center for Diabetes Technology, Charlottesville, USA

Background and Aims: In T1DM, repeated iatrogenic hypoglycemia degenerating into Hypoglycemia-Associated Autonomic Failure may reduce hormonal defenses against hypoglycemia and increase the risk for subsequent events. We studied the field glycemic response of T1DM subjects following a metabolic challenge (induced hyper/hypoglycemia) and how it relates to recent hypoglycemia and demographics.

Method: Twenty-eight T1DM subjects on pump therapy were monitored with SMBG meters during a one-month field study. After two weeks, subjects underwent an 8-h metabolic challenge where an under-bolused mixed-meal and delayed insulin injections induced hyperglycemia shortly followed by hypoglycemia. Subjects were grouped based on the frequency of hypoglycemia in the two weeks before the challenge. LBGI was used to assess hypoglycemic risk before and after the challenge, while glucagon counterregulation was measured during its occurrence. Mixed and Repeated-Measures ANOVA, Pearson Correlation, and Linear Regression were used in the analysis.

Results: A significant interaction between antecedent hypoglycemia and challenge effect was found $(\mathrm{p}<0.05)$, with a significant increase in post vs. pre-challenge LBGI detected only in subjects previously most exposed to hypoglycemia $(\mathrm{p}<0.01)$. These subjects also showed a lower counterregulatory response during the challenge (glucagon hypoglycemic fold-increase, $\mathrm{p}<0.05$ ). At the population level, antecedent hypoglycemia was negatively correlated with glucagon counterregulation $(r=-$ $0.57, \mathrm{p}<0.05)$ and, if combined with years of diabetes not on insulin pump, was a significant predictor of post-challenge LBGI $\left(\mathrm{R}^{2}=75 \%\right)$.

Conclusion: The challenge effect on subsequent hypoglycemia may be amplified by antecedent hypoglycemia and lack of pump therapy. Compromised counterregulation may contribute to the increased post-challenge risk of hypoglycemia in subjects chronically exposed to low BG.

\section{5}

\section{INSULIN PUMP THERAPY, MULTIPLE DAILY} INJECTIONS AND TRANSPLANT STATE, METABOLIC, HEMODYNAMIC FACTORS CONTROL IN PATIENTS WITH TYPE 1 DIABETES AFTER KIDNEY TRANSPLANTATION

A. Glazunova $^{1}$, M. Arutyunova ${ }^{1}$, E. Tarasov $^{1}$, M. Shamkhalova ${ }^{1}$,

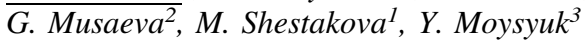

${ }^{1}$ Endocrinology Research Centre, Institution of Diabetes, Moscow, Russia

${ }^{2}$ I.M. Sechenov First Moscow State Medical University, Institution of Ophthalmology, Moscow, Russia

${ }^{3}$ Academician V.I. Shumakov Federal Research Center of Transplantology and Artificial Organs, Kidney and Liver Transplantation, Moscow, Russia

Background and Aims: To evaluate the impact of continuous subcutaneous insulin infusion (CSII) using insulin pump or multiple daily injections (MDI) on metabolic control and renal function in patients with type 1 diabetes (DM1) after kidney transplantation (KT).

Method: The study included 21 patients treated with CSII and with MII. Mean duration of DM1 in CSII group was 28 years[22;36], MDI group - 29 years[22;35]. Posttransplantation period in both groups was comparable $-8.0[7 ; 36]$ and $8[6 ; 36]$ months.

Results: The level of HbA1c in groups before the study did not differ: $9.0 \%[8.7 ; 10]$ and $9.3 \%[9 ; 9.8]$, respectively. In the group of patients switched to CSII the HbA1c become significantly lower $7.4 \%[7.4 ; 8.6]$ compared to the MDI group $8.6 \%[8.5 ; 8.7](\mathrm{p}<0.0001)$ after 12 months. Symptomatic hypoglycemic episodes were more often in the MDI group. A significant statistical difference in the assessment of glycemic variability was observed in the groups (Table 1). GFR (CKD-EPI) in both groups was comparable: $67 \mathrm{ml} / \mathrm{min} / 1.73 \mathrm{~m}^{2}[50 ; 86]$ and 
Tabl.1. Glycemic variability in patients with DM1 after $\mathrm{KT}$, using different types of insulin therapy

\begin{tabular}{|c|c|c|}
\hline & CSII & MDI \\
\hline & \multicolumn{2}{|c|}{ SD } \\
\hline 3 month & $1,9[1,8: 2,1]^{\circ}$ & $3,6[3,2 ; 3,9]$ \\
\hline 6 month & $1,6[1,4: 1,7]^{\circ}$ & $2,8 \mid 2,3 ; 3,5\}$ \\
\hline 9 month & $1,6 \mid 1,3: 1,7]^{\prime}$ & $2,8 \mid 2,4 ; 3,2]$ \\
\hline \multirow[t]{2}{*}{12 month } & $1,6[1,4 ; 1,7]^{\circ}$ & $2,8 \mid 2,3 ; 3,2]$ \\
\hline & \multicolumn{2}{|c|}{ MAGE } \\
\hline 3 month & $3,3[2,7 ; 3,3,8]^{\circ}$ & $6,3[5,5 ; 6,9]$ \\
\hline 6 month & $2,7|2,2 ; 3,5|^{\prime}$ & $5,4 \mid 4,6: 6,0]$ \\
\hline 9 month & $2,6 \mid 2,2 ; 2,2,8]^{\top}$ & $5,5[4,5 ; 7,1]$ \\
\hline \multirow[t]{2}{*}{12 month } & $3,1[2,9,4,1]^{7}$ & $4,8[4,2 ; 5,5]$ \\
\hline & \multicolumn{2}{|c|}{$\frac{1}{L}$} \\
\hline 3 month & $5.9[3,4 ; 7.1]^{\circ}$ & $23,4 \mid 19,5 ; 30]$ \\
\hline 6 month & $3,9[1,9,5,6]^{\circ}$ & $8,3[4,5 ; 12,9]$ \\
\hline 9 month & $2.3 \mid 1,6: 3.9]^{\circ}$ & $10,6 \mid 4,8: 16,3]$ \\
\hline \multirow[t]{2}{*}{12 month } & $3,0[1,3 ; 4,8]^{\circ}$ & $10,8 \mid 4,3: 16,4]$ \\
\hline & \multicolumn{2}{|c|}{ LBGI } \\
\hline 3 month & $2,0[1,0.3,1]^{\circ}$ & $6,4 \mid 5,3 ; 7,3\}$ \\
\hline 6 month & $1,3[1,2: 2,3\}^{\prime}$ & $3,4\{1,8: 5,3\}$ \\
\hline 9 month & $1,3[0,5: 1,8]^{\circ}$ & $3,4[1,1 ; 5,4]$ \\
\hline \multirow[t]{2}{*}{12 month } & $1,4[0,8: 1,7]^{\circ}$ & $3,4[2,3 ; 5,4]$ \\
\hline & \multicolumn{2}{|c|}{ HBGI } \\
\hline 3 month & $3.0[2,43,3.9]^{\circ}$ & $11 \mid 9,7 ; 13.81$ \\
\hline 6 month & $2,8 \mid 2,0 ; 3,3]^{\circ}$ & $8,4|6,4 ; 11,3|$ \\
\hline 9 month & $2,9[2,0 ; 3,3]^{\prime}$ & $7,6[5,5 ; 13,7]$ \\
\hline \multirow[t]{2}{*}{12 month } & $2,9|2,3,3,9,|^{\prime}$ & $6,3[4,9 ; 8,5]$ \\
\hline & \multicolumn{2}{|c|}{ CONGA } \\
\hline 3 month & $5,6[5,2 ; 5,8]^{\circ}$ & $6,8[6,0 ; 7,1]$ \\
\hline 6 month & $5,5[5,2: 5.9]^{\prime}$ & $7,1 \mid 6,3: 8,2]$ \\
\hline 9 month & $6,1[5,656,6,9]^{\prime}$ & $6,5[5,8 ; 8,6]$ \\
\hline 12 month & $5,8[5,4 ; 6,9]$ & $6,0[5,9 ; 7,0]$ \\
\hline
\end{tabular}

p 40,05 comparison of the 1-st group with 2-nd

$70 \mathrm{ml} / \mathrm{min} / 1.73 \mathrm{~m}^{2}[57 ; 82]$, respectively. One year change of GFR was in CSII: $9[2.6 ; 12] \mathrm{ml} / \mathrm{min} / 1.73 \mathrm{~m}^{2}$; MDI: $-6.7[-14.9 ; 2.7]$ $\mathrm{ml} / \mathrm{min} / 1.73 \mathrm{~m}^{2}(\mathrm{p}<0.0004)$. The difference in UACR was not found in both group. Positive dynamics of hemoglobin, parathyroid hormone, phosphorus and blood pressure did not differ in the groups. Stabilization of diabetic retinopathy was observed in all patients after repeated laser photocoagulation. Vitrectomy has been done more often in the MDI group $(\mathrm{p}<0.03)$.

Conclusion: The use of insulin pump therapy is associated with glycemic control and kidney function improvement compared to the multiple daily insulin injections treatment among people with DM1 after KT.

\section{6}

EFFICACY, SAFETY AND MATERNOFETAL OUTCOMES IN 26 PREGNANT WOMEN WITH TYPE 1 DIABETES MELLITUS IN SENSOR-AUGMENTED PUMP THERAPY

A.M. Gömez Medina ${ }^{1}$, L.F. Marín Carrillo ${ }^{1}$,

$\overline{\text { D.C. Henao Carrillo }}^{1}$, C. Arévalo Correa ${ }^{2}$, J.L. Silva Herrera ${ }^{3}$, E.A. Cáceres Méndez ${ }^{4}$ O.M. Muñoz Velandia ${ }^{4}$
${ }^{1}$ Hospital Universitario San Ignacio, Endocrinology, Bogotá D.C., Colombia

${ }^{2}$ Hospital Universitario San Ignacio, Nutrition, Bogotá D.C., Colombia

${ }^{3}$ Hospital Universitario San Ignacio, Gynecology and Obstetrics, Bogotá D.C., Colombia

${ }^{4}$ Hospital Universitario San Ignacio, Internal Medicine and Epidemiology, Bogotá D.C., Colombia

Background and Aims: To assess the efficacy and safety of sensor-augmented pump (SAP) therapy in pregnant women with type 1 diabetes (T1D) and to describe maternofetal outcomes.

Method: The study describes an observational prospective cohort from August 2009 until August 2015 of 26 pregnant women with T1D who started SAP therapy before or during pregnancy at Hospital San Ignacio, Colombia. The main indication was hypoglycemia and poor glycemic control. We registered efficacy, safety and maternofetal outcome variables.

Results: 26 pregnant women with T1D (mean age:25 \pm 5.4 years) on SAP therapy were included (62\% with low glucose suspend function). None of the pregnancies were planned and preconceptional glycemic control was poor $(8.3 \% \pm 2.1)$. Reduction of $\mathrm{HbA} 1 \mathrm{c}$ from pre-pregnancy to third trimester was $-1.9 \%$ and between first and second trimester was $-0.81 \%$ (p<0.001). $32 \%$ of patients achieved HbA1c $<6 \%$ and $60 \%$ $\mathrm{HbA} 1 \mathrm{c}<6.5 \%$ in second trimester and $13 \%$ and $56 \%$ respectively in third trimester. 26 pregnancies resulted in 26 infants. Maternofetal outcomes are summarized in Table $1.92 \%$ of women underwent cesarean section because of fetal distress $(5.20 \%)$, iterative cesarean $(5.20 \%)$, macrosomia $(4.16 \%)$ and preeclampsia (3.12\%). 35.9 weeks was the mean gestational age at delivery and 11 pregnancies resulted in preterm delivery. Eight infants were macrosomic and $24 \%$ of pregnancies were complicated by pre-eclampsia. There was no maternofetal mortality.

Conclusion: SAP therapy was effective for improving glycemic control during early and late pregnancy without increasing risk of severe hypoglycemia. Despite a better control during late pregnancy maternal morbidity was significant. Neonatal morbidity was represented by macrosomia, hypoglycemia and need for ICU.

\begin{tabular}{|c|c|}
\hline \multicolumn{2}{|c|}{$\begin{array}{l}\text { Table 1. Maternal-fetal outcomes for } 26 \text { type } 1 \text { Diabetes Mellitus pregnant women treated } \\
\qquad \text { SAP therapy. }\end{array}$} \\
\hline Variable & $n(\%)$ \\
\hline \multicolumn{2}{|l|}{ Maternal Outcomes } \\
\hline First Trimester HbA1c (\%) & $7,2(0,80)^{*}$ \\
\hline Second Trimester HbA1c (\%) & $6,4(0,68)^{2}$ \\
\hline Third Trimester $\mathrm{HbA1c}(\%)$ & $6,5(0,51)^{*}$ \\
\hline Severe Hypoglycemia in First Trimester & 0 \\
\hline $\begin{array}{l}\text { Severe Hypoglycemia in Second and Third } \\
\text { Trimester }\end{array}$ & 0 \\
\hline Ketoacidosis episodes & $3(12)$ \\
\hline Pregnancy-induced hypertension & $8(32)$ \\
\hline Pre-eclampsia & $6(24)$ \\
\hline Intrapartum Hypoglycemia (not severe) & $2(9,5)$ \\
\hline 48 hours postpartum Hypoglycemia & $17(80,9)$ \\
\hline \multicolumn{2}{|l|}{ Neonatal Outcomes } \\
\hline Stillbirth & 0 \\
\hline Preterm delivery (<37 weeks) & $11(44)$ \\
\hline Cesarean delivery rate & $23(92)$ \\
\hline Gestational age at delivery (weeks) & $35,9(3,2)^{x}$ \\
\hline Large for gestational age (>p 90) & $8(33,3)$ \\
\hline Small for gestational age $(<p 10)$ & 0 \\
\hline Neonatal Hypoglycaemia & $9(36)$ \\
\hline Need for intensive care unit (ICU) & $14(56)$ \\
\hline Hyperbilinubinaemia & $5(20)$ \\
\hline Respiratory distress syndrome & $4(16)$ \\
\hline Malformations & $1(4)$ \\
\hline
\end{tabular}


267

\section{EFFECT OF HEALTH LOCUS OF CONTROL AND FEAR OF HYPOGLYCAEMIA ON METABOLIC CONTROL AND TREATMENT SATISFACTION IN TYPE 1 DIABETES ON INSULIN PUMP THERAPY}

L. Indelicato ${ }^{1}$, V. Mariano ${ }^{2}$, S. Galasso ${ }^{2}$, E. Rinaldi ${ }^{3}$, E. Cipponeri $^{2}$, F. Boscari ${ }^{2}$, C. Negri ${ }^{4}$, M. Trombetta ${ }^{1}$, D. Bruttomesso ${ }^{2}$

${ }^{1}$ University of Verona and AOUI Verona, Department of Medicine, Verona, Italy

${ }^{2}$ University of Padua, Department of Medicine-DIMED, Padua, Italy

${ }^{3}$ University of Verona, Department of Medicine, Verona, Italy

${ }^{4}$ AOUI Verona, Department of Medicine, Verona, Italy

Background and Aims: The importance of locus of control (HLC) in type 1 diabetes patients (T1DM) on insulin pump therapy (CSII) has not been entirely explored. Additionally, Fear of Hypoglycaemia (FOH) is the most important limiting factor in reaching normal glucose levels. Aim of this cross-sectional study was to evaluate the influence of HLC and FOH on metabolic control and treatment satisfaction in 214 T1DM patients on CSII (mean \pm SD: age: $43.4 \pm 12.1$ years, BMI: $24.8 \pm 3.5 \mathrm{Kg} / \mathrm{m}^{2}$, HbA1c $7.8 \pm 0.8 \%$; duration of CSII: $7.5 \pm 6.1$ years), consecutively recruited at Diabetes Centre of Padua and Verona.

Method: All patients underwent a standard clinical assessment and received a set of questionnaire to estimate the locus of control, the FOH and CSII treatment satisfaction: 1. Multidimensional Health Locus of Control (MHLC); 2. Hypoglycemia Fear Survey (HFS-II); 3. Diabetes Treatment Satisfaction Questionnaire (DTSQ).

Results: The mean score for HFS-II and DTSQ was $0.99 \pm 0.5$ (range 0-2.7) and 29.7 \pm 4.8 (range 11-36), respectively. Internal and Powerful Locus showed the highest score (20.2 \pm 5.2 and $20.8 \pm 5.6$ respectively), Chance Locus showed an average of 12.1 44.9. MHLC-I was negatively related to HbA1c $(r=-0.15$, $\mathrm{p}=0.02$ ) but positively to HFS-II and treatment satisfaction $(\mathrm{r}=0.14, \mathrm{p}=0.04 ; \mathrm{r}=0.18, \mathrm{p}=0.01$ respectively). HFS-II did not show a correlation with HbA1c $(r=0.08, p=0.24)$ but with number of severe hypoglycaemic episodes $(\mathrm{r}=0.18 \mathrm{p}=0.01)$. DTSQ was negatively related to HbA1c $(r=-0.32 p<0.001)$ and MHLC-C $(r=-0.16, p=0.02)$.

Conclusion: In adult T1DM patients on CSII, HLC-I is associated with better metabolic control, higher treatment satisfaction and high levels of $\mathrm{FOH}$, which might play a role in avoiding hypoglycaemic episodes.

\section{8}

\section{COMPARING FORMULAE USED TO ESTIMATE THE INITIAL INSULIN TO CARBOHYDRATE RATIO IN PATIENTS USING BOLUS CALCULATORS}

A. Laurenzi ${ }^{1,2}$, A. Caretto $^{3}$, C. Molinari $^{3}$, A.M. Bolla ${ }^{1,2}$,

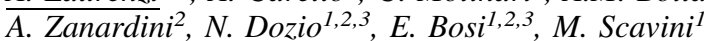

${ }^{1}$ San Raffaele Scientific Institute, Diabetes Research Institute, Milan, Italy

${ }^{2}$ San Raffaele Scientific Institute, Internal Medicine, Milan, Italy

${ }^{3}$ San Raffaele Scientific Institute, Università Vita-Salute San Raffaele, Milan, Italy

\begin{tabular}{|c|c|c|c|}
\hline Formula & I.C ratio breakfast & I:C ratio lunch & I:C ratio dinner \\
\hline $\begin{array}{l}\text { I.C ratio based on the } \\
\text { personal fooddiary }\end{array}$ & $10.5(7.15)$ & $12(8.15 .5)$ & $11(8.15)$ \\
\hline $1.500 T$ TD & $\begin{array}{c}3.8(1.9-7.8) \\
48(75 \%)\end{array}$ & $\begin{array}{c}3.7(1.9 .5 .8) \\
50(78 \%)\end{array}$ & $\begin{array}{c}3.6(2.2 .62) \\
487(75 \%)\end{array}$ \\
\hline 2. ISF"0.33 & $\begin{array}{l}5.5(2.7 .86) \\
55(86 \%)\end{array}$ & $\begin{array}{c}4.8(2.8 .76) \\
54(84 \%)\end{array}$ & $\begin{array}{l}4.8(2.6-7.7) \\
55(86 \%)\end{array}$ \\
\hline \multirow[t]{2}{*}{ 3. $\left(6.17^{t} \mathrm{body} \mathrm{kg}\right) / \mathrm{TDD}$} & $\begin{array}{c}3.3(1.5 .55) \\
35(55 \%)\end{array}$ & $\begin{array}{c}3.0(1.1 .46) \\
27(42 \%)\end{array}$ & $\begin{array}{c}2.6(1.14 .6) \\
32.50 \%)\end{array}$ \\
\hline & $P<0.00752$ vs 3 & $P<0.00032$ vs 3 & $\begin{array}{l}P<0.00012 \text { vs } 3 \\
P<0.01311 \text { vs } 3\end{array}$ \\
\hline 16. 400TDD & $\begin{array}{c}32 .(1.5 .5 .4) \\
34(53 \%)\end{array}$ & $\begin{array}{c}2.8(1.3 .5 .56) \\
31(48 \%)\end{array}$ & $\begin{array}{l}3.0(1.4 .7) \\
34(53 \%)\end{array}$ \\
\hline
\end{tabular}

TOD=Iotal daily dose', ISF=insulinsensitivityfactor.

Background and Aims: During carbohydrate (CHO) counting training several formulae may be used to estimate the initial insulin to carbohydrate (I:C) ratio (formula-estimated) that will be then refined based on the personal food diary (food diaryestimated). The aim of our study was to assess which of three commonly used formulae best approximate the food diaryestimated I:C ratio for each main meal.

Method: We identified all patients with type 1 diabetes who were trained to carbohydrate (CHO) counting at our adult diabetes clinic by querying the dietician appointment book. We collected demographics, general characteristics of patients and variables related to $\mathrm{CHO}$ counting training.

Results: From 15/01/2015 to 15/09/2015 n=67 patients with type 1 diabetes entered the $\mathrm{CHO}$ counting training program: $\mathrm{n}=64$ patients had complete data and were included in our study. Median age was 31.5 years (IQR 23.5-41), 61\% were females, median BMI was $22(20-24), 61 \%$ of participants were treated with CSII and median total daily insulin dose was 35 units (2842). The absolute difference between $\mathrm{CHO}$ grams per unit of premeal insulin of the I:C ratio estimated based on the personal food diary and that estimated by a formula (median and IQR) and the frequency (percent) of negative differences are shown for each meal.

Conclusion: The formula based on body weight and TDD more closely estimates the I:C ratio resulting from the personal food diary. Furthermore, this formula is the one that less frequently underestimates the insulin requirement suggested by bolus calculators.

\section{9}

\section{HUMAN FACTOR TESTING PROVIDED VALUABLE INPUT INTO THE ITERATIVE OPTIMIZATION OF PAQ}

L. Lilly $^{1}$, C. Kuerschner ${ }^{2}$, M. Uhrich ${ }^{3}$, P. McDermott ${ }^{3}$, J. Warner ${ }^{2}$

${ }^{1}$ CeQur, Clinical Research, Marlborough, USA

${ }^{2}$ CeQur, Marketing, Marlborough, USA

${ }^{3}$ CeQur, Research and Development, Marlborough, USA

Background and Aims: CeQur has developed PAQ ${ }^{\circledR}$, a body worn device which provides set basal rates and bolus insulin on demand. CeQur has optimized PAQ's design to facilitate large scale manufacturing, reduce the cost of goods sold and incorporate learnings from human factors tests to improve PAQ usability. 
Method: A series of human factors tests, simulating PAQ use, were conducted in intended users who were trained and untrained people with type 2 diabetes and diabetes educators. CeQur engineers observed participants perform hands-on tasks with different prototypes of the PAQ quick start guide (QSG), filling syringe, filling port, cannula insertion device and bolus button. Participants' ease of use (or not) and preferences for particular design features and options were observed and noted.

Results: Tests showed that user's relied more on the graphics than text in the QSG. The user's input indicated which syringe design enhanced readability and ease of use. User's preference of image patterns helped in graphic selection for filling port identification. At the expense of slightly larger packaging, a cannula inserter that is pre-attached to the insulin reservoir (as opposed to unattached) was strongly preferred by the user because it simplified the set-up procedure. Users preferred a color for the bolus button (rather than no color) because it made the button easier to identify and the instructions easier to follow.

Conclusion: Human factors testing provided valuable input in the optimization of the intended user's interface with $\mathrm{PaQ}$ and its QSG.

\section{0}

PAQ ${ }^{\circledR}$, A SIMPLE 3-DAY BASAL/BOLUS INSULIN DELIVERY DEVICE, FOR PEOPLE WITH TYPE 2 DIABETES

J.K. Mader ${ }^{1}$, L.C. Lilly' ${ }^{2}$, F. Aberer ${ }^{1}$, T. Poettler ${ }^{1}$, S. Becvar ${ }^{1}$, C. Lanz ${ }^{1}$, M. Trautmann ${ }^{2}$, T.R. Pieber ${ }^{1}$

${ }^{1}$ Medical University of Graz, Endocrinology and Metabolism, Graz, Austria

${ }^{2}$ CeQur Corp., Clinical Research, USA

Background and Aims: PAQ is a patch on device which provides set basal rates and bolus insulin on demand. Efficacy and safety of PAQ was tested in patients with type 2 diabetes (T2D) over a period of three months.

Method: Twenty participants with T2D and an HbA1c $\geq 53$ and $\leq 97 \mathrm{mmol} / \mathrm{mol}$ on an established regimen of basal-bolus insulin therapy \pm oral and/or injectable glucose-lowering agents were enrolled in this single center, single arm, treat to target study. The study was comprised of three periods: baseline (conventional insulin injections), transition and PAQ treatment (12-weeks). Endpoints included: HbA1c, 7-point self-monitored blood glucose (SMBG), total daily dose of insulin (TDD) and body weight (BW). Safety was assessed by: application site examination, hypoglycemic episodes and adverse device effects (ADEs).

Results: Data from first 15 patients are presented. Mean age $63.2 \pm 6.2$ years, $80 \%$ male, BMI $32.5 \pm 4.2 \mathrm{~kg} / \mathrm{m}^{2}$, diabetes duration $15.1 \pm 5.7$ years, $\mathrm{HbAlc} 72 \pm 13 \mathrm{mmol} / \mathrm{mol}$, TDD $64.2 \pm 21.8 \mathrm{U}$. When transitioned to PAQ, $73 \%$ of participants continued on the first basal rate selected. TDD and BW were similar to baseline. At the end of PAQ use statistically significant reductions from baseline were seen in $\mathrm{HbA} 1 \mathrm{c}-16 \pm 10 \mathrm{mmol} /$

\begin{tabular}{llllllll}
\hline & Fasting & $\begin{array}{l}\text { Post } \\
\text { breakfast }\end{array}$ & $\begin{array}{l}\text { Pre } \\
\text { lunch }\end{array}$ & Post-lunch & $\begin{array}{l}\text { Pre } \\
\text { dinner }\end{array}$ & $\begin{array}{l}\text { Post } \\
\text { dinner }\end{array}$ & Bedtime \\
\hline Mean & -32.6 & -48.1 & -15.1 & -37.6 & -40.8 & -74.4 & -54.2 \\
SD & 42.2 & 46.9 & 48.1 & 61.1 & 57.2 & 66.4 & 54.5 \\
Pvalue & 0.03 & 0.01 & 0.32 & 0.10 & 0.04 & 0.01 & 0.02 \\
\hline
\end{tabular}

mol ( $\mathrm{p} \leq 0001)$ and 7-point SMBG values (Table 1). Three participants completed early. No serious ADEs occurred.

Conclusion: Data show people with T2D were safely transitioned insulin injections to PAQ and achieved significantly better glycemic control than during baseline.

\section{1}

\section{REDUCING GLYCEMIC VARIABILITY WITH CSII INCREASES ENDOTHELIAL PROGENITOR CELL LEVELS IN TYPE 1 DIABETIC PATIENTS}

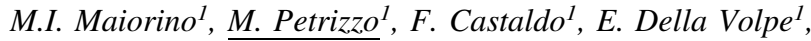

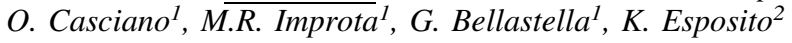 \\ ${ }^{1}$ Second University of Naples, Department of Medical- \\ Surgical- Neurological-Metabolic Diseases and Aging, Naples, \\ Italy \\ ${ }^{2}$ Second University of Naples, Department of Clinical and \\ Experimental Medicine, Naples, Italy
}

Background and Aims: Bone-marrow derived endothelial progenitor cells (EPCs) are involved in the homeostasis and repair of the vascular damage. Glucose variability may contribute to the development of chronic vascular complications of diabetes. We evaluated whether reducing glucose variability with continuous subcutaneous insulin infusion (CSII) would increase circulating levels of endothelial progenitor cells (EPCs) in type 1 diabetes.

Method: Forty-one patients on CSII completed a 6-month follow-up. Sixty-five patients on intensified insulin therapy with multiple daily injections served as control group. EPCs were assessed by flow cytometry, and glucose variability by MAGE (mean amplitude of glycemic excursions). Sixty-five patients on intensified insulin therapy served as control group.

Results: Both CD34+KDR + (difference at endpoint, cells/ $10^{6}$ events, $\left.36.3 \pm 44.7, \mathrm{P}<0.001\right)$, and $\mathrm{CD} 34+\mathrm{KDR}+\mathrm{CD} 133+$ $(16.1 \pm 19.8, \mathrm{P}<0.001)$ cell count increased $(\mathrm{P}<0.001)$ at endpoint in the CSII group, associated with a reduction of MAGE $(-1.3 \pm 3.1 \mathrm{mmol} / \mathrm{L}, \mathrm{P}=0.012)$. No changes occurred in the control group. In multivariate analyses, changes in MAGE was independently associated with changes in both CD34+KDR+ $(\mathrm{P}=0.019)$ and $\mathrm{CD} 34+\mathrm{KDR}+\mathrm{CD} 133+(\mathrm{P}=0.022)$ cell count.

Conclusion: Reducing glucose variability with CSII in type 1 diabetes increases circulating EPCs levels, suggesting a novel mechanism of vascular damage by oscillating glucose.

\section{2}

\section{INSULIN ANTIBODY LEVELS DO NOT AFFECT GLYCEMIC CONTROL IN PATIENTS WITH INSULIN- TREATED DIABETES}

C. Molinari $^{1,2}$, A. Caretto $^{1,2}$, A.M. Bolla ${ }^{2}$, A. Laurenzi ${ }^{2}$, E. Bosi ${ }^{1,2,3}$, M. Scavini ${ }^{3}$, N. Dozio ${ }^{1,2,3}$

${ }^{1}$ Vita-Salute San Raffaele University, School of Diabetology and Endocrinology, Milan, Italy

${ }^{2}$ IRCCS Ospedale San Raffaele, Internal Medicine Department, Milano, Italy

${ }^{3}$ IRCCS Ospedale San Raffaele, Diabetes Research Institute, Milano, Italy

Background and Aims: The use of human recombinant insulin and analogues has reduced occurrence of insulin antibodies (IAs) in insulin-treated patients with diabetes. Clinical impact of 


\begin{tabular}{|c|c|c|c|}
\hline Index & $\mid A s \geq 400$ & IAs $<400$ & p-value \\
\hline CSII (\%) & 77 & 75 & 0.859 \\
\hline $\begin{array}{l}\text { HbAic (\%) } \\
\text { Median (IQR) }\end{array}$ & $7.6 \%(7.4 \%-8.7)$ & $8.2 \%(7.5 \%-9.2 \%)$ & 0.1762 \\
\hline $\begin{array}{l}\% \text { SMBG }>200 \text { moldh } \\
\text { Median (IQR) }\end{array}$ & $34(22-41)$ & $39(27.57)$ & 0.2136 \\
\hline $\begin{array}{l}\% \text { SMBG < } 70 \text { mgld } \\
\text { Median (IQR) }\end{array}$ & $8(3-9)$ & $5(2-9)$ & 0.5359 \\
\hline $\begin{array}{l}\text { HBGI } \\
\text { Median (IQR) }\end{array}$ & $9.1(5.3-11.1)$ & $12.35(7.6-21.8)$ & 0.1087 \\
\hline $\begin{array}{l}\text { LBGI } \\
\text { Median (IQR) }\end{array}$ & $1.7(0.8-3.3)$ & $1.3(0.5-2.3)$ & 0.3230 \\
\hline $\begin{array}{l}\text { AUC }>200 \mathrm{mg} / \mathrm{dL} . \\
\text { Median (IOR) }\end{array}$ & $10.8(5.2-15.6)$ & $14.5(7.6-19.5)$ & 0.6374 \\
\hline $\begin{array}{l}\text { AUC }<70 \text { mgldh } \\
\text { Median (IQR) }\end{array}$ & $0.5(0.1-0.9)$ & $0.15(0.1-0.05)$ & 0.6007 \\
\hline
\end{tabular}

IAs is unclear. Depending on affinity and dissociation characteristics of IAs, insulin bioavailability is altered and their buffering effect may increase insulin requirement, worsen post-prandial hyperglycemia and cause erratic hypoglycemia. The aim of our study was to evaluate whether HbAlc and glycemic control are poorer among patients with diabetes and high levels of IAs.

Method: We retrospectively analyzed records from $\mathrm{n}=96$ patients with insulin-treated type $1(94 \%)$ or type 2 diabetes, $76 \%$ in CSII, with median duration of diabetes 17.5 years (IQR 8-25). We concomitantly measured $\mathrm{HbA1c}$ and IAs titer by radiobinding assay (reference value $<5$ arbitrary units). We studied the association between IAs levels and $\mathrm{HbA} 1 \mathrm{c}$, proportion of SMBG values $>200 \mathrm{mg} / \mathrm{dL}$ or $<70 \mathrm{mg} / \mathrm{dL}$, Kovatchev indexes, and, in patients with CGM $(n=15)$, the AUC of time spent $>200 \mathrm{mg} / \mathrm{dL}$ or $<70 \mathrm{mg} / \mathrm{dL}$.

Results: The proportion of patients with IAs $\geq 5,200,400$ and 600 arbitrary units were $50 \%, 33 \%, 22 \%$ and $13 \%$, respectively. We did not find any association between IAs levels and duration of diabetes, CSII use, HbA1c levels, Kovatchev indexes, proportion of SMBG values $>200 \mathrm{mg} / \mathrm{dL}$ or $<70 \mathrm{mg} / \mathrm{dL}$ and AUC of time spent $>200 \mathrm{mg} / \mathrm{dL}$ or $<70 \mathrm{mg} / \mathrm{dL}$.

Conclusion: Despite the widespread use of insulin analogues the occurrence of IAs in patients with diabetes treated with insulin remains high. Nevertheless, the level of IAs as measured by radiobinding assay does not have an impact on parameters of metabolic control.

273

INSULIN PUMP THERAPY DIRECTED ONLY BY A CONTINUOUS GLUCOSE MONITORING SYSTEM (CGM) IN TYPE 1 DIABETES IN EVERYDAY LIFE

\section{Mueller-Korbsch ${ }^{1}$}

${ }^{1}$ Vienna, Austria

Background and Aims: The aim of the study was to show that it is possible to drive an insulin pump therapy only by a CGM System.
Method: 8 Patients treated for their diabetes type 1 with insulin pump therapy were switched from the common finger stick glucose measurements to a CGM. A blood glucose was taken once a day during a steady state glucose level to calibrate the system.

The CGM values and trends were used to calculate the prandial insulin and to correct higher glucose levels by the bolus calculator. In addition the patients received an algorithm to respond to rising and falling trends shown on the CGM. If the CGM trends were rising, patients had to receive a supplement insulin dose; if the trends were falling the insulin pump was stopped and sometimes carbohydrates were given.

Results: The amount of hypoglycemic values $<70 \mathrm{mg} / \mathrm{dL}$ $(3.1 \mathrm{mmol} / \mathrm{L})$ was $5.6 \%$ of times and $52 \%$ of times the values lies in the target area with $70-130 \mathrm{mg} / \mathrm{dL}(3.9-7.3 \mathrm{mmo} / \mathrm{L})$. The HbA1c decreased about 0.1 to $1 \%$ from the basic values. The mean glucose level over all is $131 \mathrm{mg} / \mathrm{dL}$ with a standard deviation of 46. Observation period for each patient is at least 6 months.

Conclusion: With this new way of routing the insulin pump therapy by the CGM, no unexpected glucose values appeared and if the values go up or down the patients have the possibility to optimize the ongoing process in a better way. Therefore it is not necessary to count the carbohydrates exactly.

\section{4}

\section{MORTALITY RATE OF PATIENTS WITH TYPE 1 DIABETES MELLITUS USING CONTINUOUS SUBCUTANEOUS INSULIN INFUSION OR MULTIPLE DAILY INJECTIONS IN JAPAN}

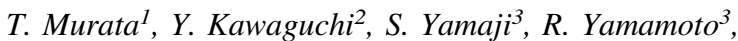

K. Uenomachi ${ }^{3}$, A. Kokubo ${ }^{4}$, F. Yoshioka ${ }^{2}$, H. Okada ${ }^{2}$,

T. Watanabe $^{1}$, Y. Mizutani ${ }^{1}$, K. Yomaida ${ }^{1}$, J. Kishi ${ }^{1}$, S. Odori ${ }^{1}$, R. Nakagawachi ${ }^{1}$, N. Satoh-Asahara ${ }^{5}$, K. Yamada ${ }^{1}$, N. Sakane ${ }^{2}$

${ }^{1}$ NHO Kyoto Medical Center, Diabetes Center, Kyoto, Japan

${ }^{2}$ NHO Kyoto Medical Center, Division of Preventive MedicineClinical Research Institute, Kyoto, Japan

${ }^{3}$ NHO Kyoto Medical Center, Division of Clinical Nutrition, Kyoto, Japan

${ }^{4} \mathrm{NHO}$ Kyoto Medical Center, Department of Nursing, Kyoto, Japan ${ }^{5}$ NHO Kyoto Medical Center, Division of Diabetic ResearchClinical Research Institute, Kyoto, Japan

Background and Aims: We compared the mortality rate of patients with type 1 diabetes mellitus (T1DM) using continuous subcutaneous insulin infusion (CSII) or multiple daily injections (MDI).

Method: Retrospective cohort study of 201 T1DM subjects (CSII: 69, MDI: 132), aged 18-74 years, who regularly visit NHO Kyoto Medical Center, Kyoto, Japan, was performed. Primary outcome measures were mortality rate (100 person years) and mortality hazard ratio using Cox proportional hazard models adjusted for potential confounders including age and initial HbA1c.

Results: The mean follow-up period was 4.2 years. Subjects using CSII were significantly younger than subjects using MDI ( $36.3 \pm 12.6$ years old vs. $47.5 \pm 16.7$ years old, $P<0.001$ ), and the initial HbAlc level was significantly lower $(8.4 \pm 1.9 \%$ vs. $9.7 \pm 2.6 \%, P<0.001)$, however the ratio of male was not significantly different $(29.0 \%$ vs. $37.9 \%, P=0.209)$. Mortality rate for CSII group and MDI group was 0.29 and $1.01 / 100$ personyears, respectively, with no significant difference $(P=0.138)$. Insulin pump usage was not significantly associated with the mortality (adjusted hazard ratio 0.17 , [95\% confidence interval $0.02-1.62$ ], $P=0.122$ ). 
Conclusion: There was no significant difference in the mortality rate between the subjects using CSII and the subjects using MDI. This study was supported by Grant-in-Aid from NHO.

\section{5}

\section{COMPATIBILITY OF ANIMAS VIBE INSULIN PUMP CARTRIDGE WITH INSULIN LIPRO AND ASPART}

\section{N. Ojha ${ }^{1}$, R. Venugopalan ${ }^{2}$}

${ }^{1}$ Animas Corp, Quality Engineering, Wayne, USA

${ }^{2}$ Animas Corp, R\&D, Wayne, USA

Background and Aims: The stability and potency of insulin lispro and aspart was evaluated when used in the Animas Vibe pump cartridge under simulated worst-case environmental conditions experienced during clinical use.

Method: The potency and stability of insulin lispro and aspart were evaluated after storage in the Animas Vibe cartridge for up to 6 days at $37^{\circ} \mathrm{C}$ and $40 \%$ relative humidity. Mechanical stress to simulate patient motion was provided by placing the cartridges on a continuously vibrating platform ( $100 \pm 10$ strokes/minute, 1 inch displacement). The insulin remaining in the cartridge was tested at time $\mathrm{T}=0,3 \mathrm{~d}$ and $6 \mathrm{~d}$ and measured against USP specifications.

Results: After 6 days of stress, there was no noteworthy reduction in potency of insulin lispro and aspart. Insulin precipitation did not occur and no other physical changes to the samples were observed. The stressed samples met USP criteria for $\mathrm{pH}$, degradation products (impurities) and high molecular weight proteins. Preservative concentration remained sufficient to ensure preservative efficacy as confirmed with a negative test for endotoxins. Post stress, the biological potency of insulin was confirmed to remain above acceptable levels.

Conclusion: This study demonstrates that insulin stored in the Animas Vibe cartridge under temperature and vibration stress conditions for 6 days did not affect the stability or potency of insulin lispro and aspart. This supports the 3-day in pump use time claim for insulin in the Animas Vibe insulin pump.

\section{6}

\section{IMPORTANCE OF DIFFERENT ASPECTS DURING OBSERVATION OF PATIENTS WITH TYPE 1 DIABETES MELLITUS ACCORDING TO THE METHOD OF INSULIN THERAPY (CSII VS MDI)}

\section{E. Patrakeeva $^{1}$, K. Solovyova ${ }^{1}$, A. Zalevskaya ${ }^{1}$}

${ }^{1}$ Saint-Petersburg Medical University, Endocrinology, SaintPetersburg, Russia

Background and Aims: Diabetes is not only the medical trouble, nowdays it's a social challenge. All patients have problems connected with diabetes, but we suppose that patients on CSII and MDI may have different difficulties in their daily life.

Method: 119 T1DM patients (age 23.21 $\pm 0.59, \mathrm{HbA}_{1 \mathrm{c}}$ $8.01 \pm 0.06)$ answered the questionnaire in social networks about the main lifestyle problems connected with diabetes: injections and blood glucose control, supply of medications, pregnancy and its planning, weight gain, food counting etc. 38 patients (Group 1) used CSII, 78 patients (Group 2) - MDI.

The rating of problems was made separately for two groups. Using the Pirson's method we also explored correlations between patient's HbA1c level, income level and level of education.
Results: There were no significant differences between the HbA1c levels in both groups. HbA1c level doesn't correlate with the patient's income or education level.

Patients both on CSII and MDI have some similar problems (mainly with supplies for diabetes, fear of pregnancy problems, $\mathrm{CHO}$ counting and frequent glycemia control). Patients on MDI are anxious about psychological moments, reaching normoglycemia, lack of information about diabetes and hypoglycemia; patients on CSII have difficulties with pregnancy planning, sex and social opinion about their illness and more problems with diabetes supplies.

Conclusion: We found out that all patients have some similar problems in their daily life, but we should consider some specific points and embarrassing moments connected with the method of insulin therapy.

\section{7}

\section{USE OF BOLUS CALCULATOR AND CARBOHYDRATE COUNTING IN 146 SINGLE-CENTER PATIENTS ON INSULIN PUMP THERAPY}

\section{Rapisarda ${ }^{1}$, A. Tumminia ${ }^{1}$, C. Egiziano ${ }^{I}$, L. Tomaselli $^{1}$ \\ ${ }^{1}$ Section Endocrinology-Garibaldi Nesima Hospita, Clinical and Experimental Medicine, Catania, Italy}

Background and Aims: The best choice to calculate insulin bolus is carbohydrate counting (CC) with use of correction factor (CF) when blood glucose is out of target. CF is calculated using 1800/TDD (total daily insulin) formula, the insulin to carbohydrate ratio (CIR) using 500/TDD.

Method: In single-center study we observed 146 patients on CSII, evaluating if $\mathrm{CF}$ and $\mathrm{CIR}$ ( $\mathrm{uCF} / \mathrm{uCIR}$ ) used are different from calculated $\mathrm{CF}$ e CIR (cCF, cCIR), if they use CC and automated bolus calculator (BC). We compared $\mathrm{HbA1c}$, weight, age, education, time of CSII use between who use and do not use CC.

Results: In 74 patients $\mathrm{uCF}$ is $40 \%$ higher than $\mathrm{cCF}(\mathrm{p}<$ 0.001 ), without difference in glucose control. The most patients use CIR different from cCIR. Patients that currently use CC (CCp, 42\%) have significantly lower HbA1c than patients who don't use $\mathrm{CC}$ (nCCp). 74\% of CCp use BC. CCp are younger than $n C C p(p=0.05)$; BMI is lower and time of CSII use shorter, but not significantly. Patients who started insulin pump in our center use CC and BC more frequently than who started elsewhere, but difference is not significant ( $44 \%$ vs $35 \% \mathrm{p}=0.3,55 \%$ vs $42 \%$ $\mathrm{p}=0.2$ respectively).

Conclusion: Calculated CF and CIR should be reviewed in clinical practice. The best choice of CIR requires the use of food diary. CC use should be implemented to increase insulin bolus accuracy, but bolus calculation is still complex, in fact CC are used mostly by young patients. BC implementation is necessary to help patients with T1D.

\section{8}

\section{OCCLUSION ALARM OF INSULIN PUMPS: IS DETECTION FAST ENOUGH FOR CHILDREN?}

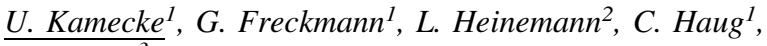 \\ R. Ziegler ${ }^{3}$ \\ ${ }^{1}$ Institut für Diabetes-Technologie Forschungs- und \\ Entwicklungsgesellschaft, an der Universität Ulm, Ulm, Germany \\ ${ }^{2}$ Science \& Co, Düsseldorf, Germany \\ ${ }^{3}$ Diabetes Clinic for Children and Adolescents, Muenster, Germany
}


Background and Aims: All insulin pumps are intended to give an alarm when the insulin infusion set (IIS) is occluded and insulin delivery is interrupted during continuous subcutaneous insulin infusion (CSII). In this study, the time needed to detect an occlusion was measured with two different basal rates for different insulin pumps under experimental conditions.

Method: IIS occlusion was induced with four insulin pumps with different IIS (Accu-Chek ${ }^{\circledR}$ Spirit Combo with Accu-Chek ${ }^{\circledR}$ FlexLink and Accu-Chek ${ }^{\circledR}$ Rapid-D Link; Accu-Chek ${ }^{\circledR}$ Insight with Accu-Chek ${ }^{\circledR}$ Insight Flex and Accu-Chek ${ }^{\circledR}$ Insight Rapid; Paradigm ${ }^{\circledR}$ Veo $^{\mathrm{TM}}$ with MiniMed ${ }^{\circledR}$ Mio $^{\mathrm{TM}}$, MiniMed ${ }^{\circledR}$ Sure-T ${ }^{\circledR}$ and MiniMed ${ }^{\circledR}$ Quick-set $^{\circledR}$; mylife $^{\text {TM }}$ OmniPod $^{\circledR}$ with its infusion set). The time until an alarm was triggered was measured. Each combination of insulin pump and IIS was tested nine times with two different basal rates $(0.1 \mathrm{U} / \mathrm{h}, 1.0 \mathrm{U} / \mathrm{h})$ under controlled conditions.

Results: For the eight tested combinations of insulin pumps and IIS, the median time until occlusion alarm was $27 \mathrm{~h} 27 \mathrm{~m}(1 . / 3$. quartile: $20 \mathrm{~h} 08 \mathrm{~m} / 30 \mathrm{~h} 00 \mathrm{~m}$ ) with a basal rate of $0.1 \mathrm{U} / \mathrm{h}$, and $2 \mathrm{~h} 30 \mathrm{~m}(1 . / 3$. quartile: $1 \mathrm{~h} 48 \mathrm{~m} / 2 \mathrm{~h} 46 \mathrm{~m})$ with $1.0 \mathrm{U} / \mathrm{h}$.

Conclusion: At a higher basal rate, IIS occlusions were reported early enough to enable patients to react in due time, i.e. to avoid harmful consequences. However, at a low basal rate of $0.1 \mathrm{U} / \mathrm{h}$ (which is used e.g. in children not that seldom) alarms were activated much too late from a clinical point of view. These results confirm the importance of regular glucose monitoring in CSII, also to detect catheter occlusions and thus avoid hyperglycemia and diabetic ketoacidosis.

279

CORRELATION BETWEEN FREQUENCY OF SMBG AND GLYCEMIC CONTROL IN TYPE 1 DIABETIC SUBJECTS WITH CSII AND MDI THERAPY

\section{$\underline{\text { A.M. Scarpitta }^{1}}$ \\ ${ }^{1}$ Mazara del Vallo, Italy}

Background and Aims: Self-monitoring of blood glucose (SMBG) is recognized as necessary in insulin-treated diabetic patients. Interventional trials have indicated that a 4- to 5-point daily profile represents an optimal regimen for SMBG in type 1 diabetic patients with satisfactory diabetic control.

However additional determinations should be made within postprandial states, particularly when rapid insulin analogues or

\begin{tabular}{|c|c|c|c|c|}
\hline Conrelation & CSN & & MOI & \\
\hline & I & $p$ & I & $p$ \\
\hline \multicolumn{5}{|l|}{ between AIC and: } \\
\hline Average Glucose (AG); & 0.674 & 0.000 & 0.538 & 0.002 \\
\hline Frequency of SMBG; & 0.455 & 0.000 & -0.165 & 0.382 \\
\hline average amount of total daily insulin; & 0.077 & 0.596 & 0.111 & 0.559 \\
\hline frequency of insulin bolus. & 0.042 & 0.773 & -0.192 & 0.310 \\
\hline \multicolumn{5}{|l|}{ Between: } \\
\hline frequency of SMBG and frequency of insulin bolus & 0.453 & 0.000 & 0.710 & 0.000 \\
\hline
\end{tabular}

pump-treatments are used. There is a need for patients to be able to adjust their insulin doses accurately and independently during continuous subcutaneous insulin infusion (CSII) therapy in order to improve glycemic control. To assess the relationship between HbA1c and frequency of SMBG in a group of type 1 diabetes CSII users, compared with MDI.

Method: A1C levels of 50 type 1 diabetes CSII users (M/F 18/ 32) and 30 MDI subjects (M/F 17/13), age (ys) 30,7+14,55 and $23,3+14,2$; HbA1c (\%) $7.62+0.82$ and $7.83+1.02$; and frequency of SMBG 4,39+1,79 and 2,59+1,38 respectively, were correlated with the data shown in tab. 1 . All of the data both glucometer and pump, were downloaded by diabetes management systems. All data were filtered by date and last 3 months, was the time period set.

Results: The results are shown in Table. 1.

Conclusion: This study confirms the relationship between A1C and frequency of SMBG in a group of selected CSII users. The practice of SMBG and its use for insulin adjustment requires continuing education in order to translate SMBG in real diabetes self-management. This prerequisite is mandatory to obtain a sustained improvement in metabolic control.

\section{0}

\section{INFLUENCE OF AN INTENSIVE APPROACH TO OPTIMIZE GLYCEMIC CONTROL ON WELL- BEING AND SATISFACTION IN INSULIN PUMP- TREATED TYPE 1 DIABETIC PATIENTS}

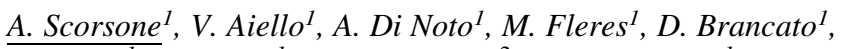
L. Spano $^{1}$, G. Saura ${ }^{1}$, F. Provenzano ${ }^{2}$, V. Provenzano ${ }^{1}$

${ }^{1}$ ASP 6 Palermo Ospedale Civico Partinico, Regional

Reference Centre for Diabetology and Insulin Pumps, Partinico, Italy

${ }^{2}$ University of Palermo - Faculty of Medicine, Department of Internal Medicine, Partinico, Italy

Background and Aims: Insulin pump-treated patients need to be closely followed up by means of a multidisciplinary Team. An intensive therapeutic management implies a great psychological burden. We evaluated if insulin pump treatment in Type 1 Diabetes (T1 DM) subjects could be a source of emotional stress and discomfort even in presence of HbA1c levels at target.

Method: We studied 50 T1 DM subjects for 12 months. Their visit frequency was intensified every three months. At the beginning and at the end SF-36 health survey questionnaire and Diabetes Treatment Satisfaction Questionnaire were administered. Mean age $(\mathrm{M} \pm \mathrm{SD})$ was $(33,3 \pm 18,9)$. Age groups were A $(<10$ yrs $n=4), B(10-20 n=10), C(20-50 n=30), D(>50 n=6)$ diabetes duration $8,33 \pm 7,2$ yrs.

Results: In our T1 DM subjects we observed a significant reduction of $\mathrm{HbA} 1 \mathrm{c}$ levels with respect to baseline $(8,2 \pm 0,65 \mathrm{vs}$ $7.91 \pm 0,45 \mathrm{p}=0.01)$. DTSQ and SF-36 score did not significantly change. Some items of SF-36 worsened. Role limitation (physical and emotional problems) and general health perception were areas of conflict and remained unchanged from baseline irrespective of HbA1c reduction.

Conclusion: When we decide to intensify our therapy management in insulin pump-treated subjects, despite a reduction of HbA1c levels to ideal targets, T1 DM subject's perception of their well-being, treatment satisfaction, physical limitations do 
not improve. This means that more time must be dedicated to psychological support and above all to their role limitations, social functioning and social isolation risk.

\section{1}

\section{LONG-TERM EFFICACY AND PERFORMANCE OF DIABETES MANAGEMENT IN INSULIN PUMP TREATED TYPE 1 DIABETIC PATIENTS}

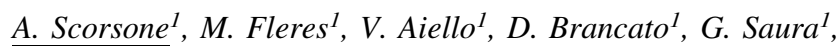
$\overline{A . ~ D i ~ N o t o}^{1}$, L. Spano ${ }^{1}$, F. Provenzano ${ }^{2}$, V. Provenzano ${ }^{1}$

${ }^{1}$ ASP 6 Palermo Ospedale Civico Partinico, Reference Centre for Diabetology and Insulin Pumps, Partinico, Italy

${ }^{2}$ University of Palermo, Department of Internal Medicine, Palermo, Italy

Background and Aims: Insulin pump therapy is the gold standard therapy of Type 1 (T1 DM) diabetic patients. HbA1c (A1C) levels are well controlled if a multidisciplinary dedicated Team works toward an optimized glycemic control. Under this intensive approach not all patients reach and maintain optimal glucose control.

Method: We studied 690 T1 DM subjects on insulin pump therapy from 2007 to 2014. The effects of different variables on performance rate (number of subjects not at A1C target) like age at onset, at implantation, diabetes duration, BMI, dropouts rate, A1C level were studied to characterize "insulin pumper".

Results: Total dropouts rate was $2.8 \% .30 .2 \%$ T1DM in our database remained outside optimal performance curve distribution, with persistent AIC levels above $8 \%$ for more than 24 months. Age at onset, sex were not correlated to unstable A1c levels. Multivariate analysis demonstrated a significant role of age at onset of DM and lesser time to pump implantation from diabetes onset (respectively $\mathrm{p}<0.01$ and $\mathrm{p}<0.001$ ) on $\mathrm{A} 1 \mathrm{C}$ stability (between years difference not $>1 \%$ ) as previously demonstrated by our group. Loss of stability for more than 24 months was associated to AIC not a target on follow-up $(\mathrm{p}<0.01)$ (see Figure below).

Conclusion: Insulin pump therapy is effective for the majority of T1 DM subjects across several years. Nevertheless maintenance of AIC ideal levels can be lost for reasons not related to our management but to factors linked to T1 DM people. In our practice we should change our approach and empowerment techniques in noncompliant subjects.

\section{$\mathrm{HbA} 1 \mathrm{c}$ frequency distribution}

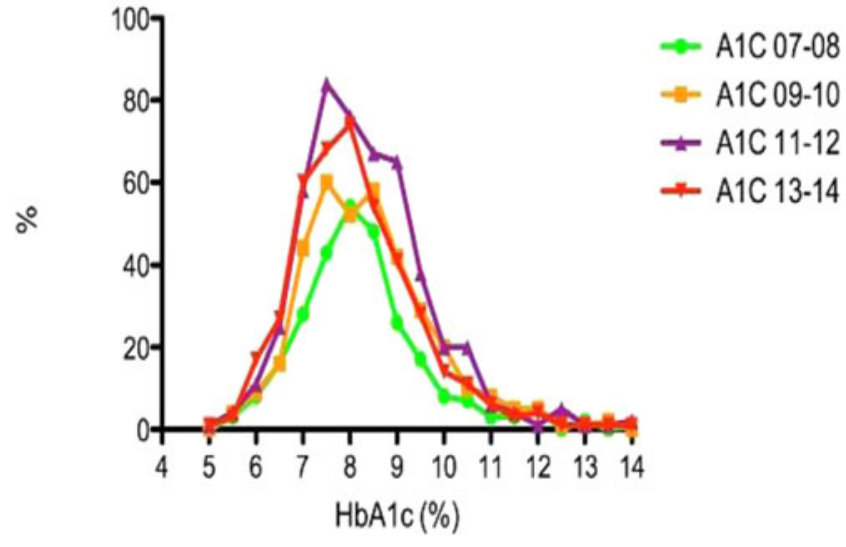

282

\section{CONTINUOUS SUBCUTANEOUS INSULIN INFUSION THERAPY IN TYPE 1 DIABETIC CHILDREN UNDER 6 YEARS}

J. Serra-Caetano ${ }^{1}$, I. Dinis ${ }^{1}$, N. Baptista ${ }^{1}$, L. Aveiro ${ }^{1}$,

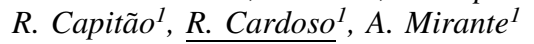

${ }^{1}$ Coimbra Pediatric Hospital, Centro Hospitalar e Universitário de Coimbra (CHUC), Endocrinology and Diabetology Unit, Coimbra, Portugal

Background and Aims: A good metabolic control in young type 1 diabetic (DM1) children is particularly challenging, being easier and safer with continuous subcutaneous insulin infusion (CSII). To evaluate and compare metabolic control of diabetic children under 6 years, before and at one year of CSII.

Method: Retrospective study of DM1 children with CSII initiated under six years and with at least 12 months' follow-up. Variables included: age, insulin daily dosis (IDD), mean blood glucose and mean percentage of hypo/hyperglycemia (previous 14 days) and A1c, before and during the first year of therapy.

Results: 18 children were included (56\% female), with mean age at diagnosis of 2.0 \pm 1.1 years (0-4.5). Mean age at CSII be-

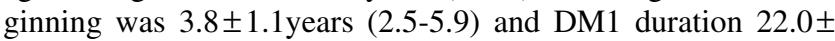
15.6 months (5.1-68.4). Comparing CSII and MDI, there was no difference in IDD along time $(0.84 \pm 0.10 \mathrm{U} / \mathrm{kg} /$ day vs $0.84 \pm$ $0.12 \mathrm{U} / \mathrm{kg} / \mathrm{day} ; \mathrm{pNS})$. Mean blood glucose remained stable (0M $155 \pm 32,3 \mathrm{M} 153 \pm 24,6 \mathrm{M} 160 \pm 20,9 \mathrm{M} 165 \pm 35$ and $12 \mathrm{M}$ $155 \pm 28 \mathrm{mg} / \mathrm{dL}$; pNS). A1c had a significant decrease in the first year of CSII when compared to total mean A1c of each case $(7.3 \pm 0.8 \%$ vs $7.1 \pm 0.8 \% ; \mathrm{p}=0.013)$ and from 6 to 9 months of therapy $(7.0 \pm 0.8 \%$ vs $7.3 \pm 0.9 \% ; p=0.042)$. There was no significant difference of hypoglycemias (0M 13.6 $13.3 \%, 12 \mathrm{M}$ $8.7 \pm 7.1 \%$, pNS) or hyperglycemias $(0 \mathrm{M} 45.4 \pm 10.5 \%, 12 \mathrm{M}$ $38.6 \pm 12.8 \%$; pNS) along time. There were no severe hypoglycemias or ketoacidosis.

Conclusion: Good metabolic control was kept with CSII therapy, without increasing complications. It is essential to provide close medical supervision and continuous education to achieve and improve these results, however this therapy definitely improves quality of life.

\section{3}

\section{WHICH FACTORS MAY AFFECT THE QUALITY OF LIFE IN PATIENTS WITH TYPE 1 DIABETES MELLITUS USING CONTINUOUS SUBCUTANEOUS INSULIN INFUSION PUMPS?}

\author{
$\underline{\text { P. Thomakos }}^{1}$, A. Mitrakou ${ }^{2}$, O. Kepaptsoglou ${ }^{1}$, I. Taraoune ${ }^{1}$, \\ C. Barreto ${ }^{1}$, A. Pappas ${ }^{3}$, C. Zoupas ${ }^{1}$ \\ ${ }^{1}$ Hygeia, Diabetes Center and Clinic, Athens, Greece \\ ${ }^{2}$ Alexandra University Hospital, Diabetes Center- Department \\ of Clinical Therapeutics, Athens, Greece \\ ${ }^{3}$ Venizelio-Pananio Hospital, Diabetes Clinic, Heraklion, Greece
}

Background and Aims: Continuous Subcutaneous Insulin Infusion (CSII) is considered an effective therapeutic approach for Type 1 Diabetes Mellitus (T1DM). In literature there is limited information regarding the quality of life $(\mathrm{QoL})$ in patients using CSII. The aim of the study was to investigate the impact of diabetes-related factors in the QoL of patients with T1DM on CSII treatment, in a Greek urban population. 
Method: A cross-sectional study was conducted in 80 T1DM using CSII [(Mean \pm SD) age:35.9 \pm 11.4 years, duration of diabetes:24.2 \pm 10.3 years, BMI: $24.6 \pm 3.5 \mathrm{~kg} / \mathrm{m}^{2}$, duration of Insulin pump use:7.1 \pm 3.9 years, HbA1c:7.7 $\pm 1.1 \%$, gender:37 males-43 females). QoL was assessed using the patient self-administered EuroQol EQ 5D validated in Greek. Correlation and regression analyses were performed to examine the relationship between EQ index - EQ VAS scores and diabetes related factors. Hypoglycaemia Awareness was measured using Clarke and Gold Score questionnaires, Hypoglycaemic Episodes were expressed as number of episodes/week, Fear of Hypoglycaemia was measured using the worry subscale of the Hypoglycemia Fear Survey (HFS-W).

Results: Gold score:2.8 \pm 1.5 , Clarke score:2.8 \pm 2.1 , Hypoglycaemia Fear Score:20.6 \pm 11.2 , Number of hypoglycaemic Episodes per week:4.3 \pm 2.9 , VAS score:68.7 \pm 18.1 , EQ index:0.79 \pm 0.24 . QoL was negatively correlated with Hypoglycaemic episodes $(\mathrm{r}=-0.70, \mathrm{p}<0.001)$, HbA1c $(\mathrm{r}=-0.048$, $\mathrm{p}=0.036)$ and Hypoglycaemia Fear Score $(r=-0.50, \mathrm{p}=0.017)$. QoL was not correlated with Hypoglycaemia Fear Score in subjects using ContinuousGlucoseMonitoring. Regression analysis revealed that both the higher number of hypoglycaemic episodes $(\beta=-0.822, \mathrm{p}=0.02)$ and elevated HbA1c $(\beta=-0.468, \mathrm{p}=$ $0.017)$ were significantly associated with worse QoL.

Conclusion: QoL in T1DM on CSII is significantly affected by the number of hypoglycaemic episodes and the glycaemic control. CGM attenuates the fear of hypoglycaemia.

284

CHANGES IN BASAL AND BOLUS INSULIN REQUIREMENT ACROSS GESTATION IN TYPE 1 DIABETIC WOMEN TREATED WITH INSULIN PUMP THERAPY

\section{A. Tumminia $^{1}$, C. Egiziano ${ }^{1}$, V. Rapisarda ${ }^{1}$, L. Tomaselli ${ }^{1}$}

${ }^{1}$ Garibaldi-Nesima Hospital- Endocrinology Section, Clinical and Experimental Medicine, Catania, Italy

Background and Aims: In type 1 diabetic (T1D) women, pregnancy is usually associated with a 2-3-fold increased insulin requirement, especially during the second and third trimester. Even if this phenomenon has clearly been demonstrated, poor evidences are available on the changes in insulin dosage during the different times of the day across gestation. The purpose of this study was to evaluate and compare the changes in basal and bolus insulin requirement throughout pregnancy in T1D women on insulin pump therapy with either good or poor initial glycemic control.

Method: We analyzed a cohort of 29 T1D women using insulin pumps. Fourteen women had preconception HbA1c $<7 \%$ (planned group), while 15 had preconception $\mathrm{HbA} 1 \mathrm{c} \geq 7 \%$ (unplanned group).

Results: The two groups displayed different behavior during gestation. The planned group had significant increase in bolus insulin requirement during the third trimester $(0.44$ vs. 0.35 U.I./ $\mathrm{kg}, \mathrm{p}<0.05)$, while basal insulin rates remained stable (0.30 vs. 0.28 U.I./kg, $\mathrm{p}=0.6$ ). Conversely, both basal and bolus insulin requirement increased significantly across third trimester in the unplanned group ( 0.42 vs. 0.35 U.I. $/ \mathrm{kg} ; 0.45$ vs. 0.35 U.I. $/ \mathrm{kg}$, respectively, $\mathrm{p}<0.05)$. Basal insulin rates increased mostly from midnight to $8 \mathrm{AM}$ and from noon to $8 \mathrm{PM}$.

Conclusion: In planned pregnancies the increased insulin requirement was mostly due to bolus rates, whereas unplanned pregnancies needed a more profound change in both bolus and basal insulin doses (especially during the night and the afternoon). This different behavior may lead physicians to differentiate insulin dosage modifications on the basis of the preconception glycemic control.

\section{5}

\section{THE DAILY NEED FOR INSULIN IN PREGNANT WOMEN WITH TYPE 1 DIABETES, RECEIVING INSULIN WITH INSULIN PUMP}

\author{
F. Valeeva $^{1}$, J. Sharipova ${ }^{1}$, Z. Alimetova ${ }^{1}$, L. Gaysina ${ }^{1}$ \\ ${ }^{1}$ Kazan State Medical University, endocrinology, Kazan, Russia
}

Background and Aims: To assess the daily need for insulin in pregnant women with type 1 diabetes (DM 1), depending on the initial state of kidney function.

Method: The study involved 97 pregnant women with DM 1, receiving insulin with insulin pumps. Patients were divided into three groups: group 1 - with normal urinary albumin excretion, 2 - with microalbuminuria, 3 - with proteinuria. We assessed the need for daily insulin and albuminuria before pregnancy, then up to $12,22-24,30-32$ weeks of gestation.

Results: In group 1, the daily need for insulin in the first trimester was $0.59(0.54 ; 0.66) \mathrm{IU} / \mathrm{kg}$ in the second $0.69(0.59 ; 0.79)$, in the third $0.83(0.75 ; 0.99)$. In Group 2 the need for daily insulin during the first trimester was $0.64(0.61 ; 0.76)$, in the second 0.73 $(0.61,0.87)$, the third $0.95(0.900 .95) \mathrm{IU} / \mathrm{kg}$. There were no significant differences in these groups. In Group 3 the need for insulin during the first trimester was $0.59(0.54 ; 0.64)$, in the second -0.7 $(0.65 ; 0.75)$, in the third $-0.64(0.6 ; 0.76)$. In groups 1 and 3 in the first and second trimesters the daily doses of insulin did not differ, in the third trimester the insulin requirement was significantly lower in group $3(\mathrm{p}=0.001)$. In group 3 the need for insulin in the third trimester was lower than in group $2(\mathrm{p}=0.024)$.

Conclusion: The original condition of the kidneys affects the daily need for insulin in pregnant women with DM 1 who are on insulin pump.

\section{6}

\section{THE USE OF INSULIN PUMP IN WOMEN WITH TYPE 1 DIABETES DURING PREGNANCY}

\section{F. Valeeva ${ }^{1}$, G. Gazizova ${ }^{1}$, L. Gaysina ${ }^{1}$}

${ }^{1}$ Kazan State Medical University, Endocrinology, Kazan, Russia

Background and Aims: To evaluate the effect of the route of administration of insulin on the excretion of inflammatory markers of diabetic nephropathy (IL- $1 \beta$, MCP-1 and TGF- $\beta 1$ ) with daily urine in pregnant women with type 1 diabetes (DM 1) in different trimesters of pregnancy.

Method: We studied 48 pregnant women with DM 1, aged 19 to 36 years old $(25[22,27])$, and diabetes duration of 1 year to 26 years $(10[5,12])$. The study involved pregnant women receiving insulin therapy in the mode of multiple subcutaneous insulin injections (MPII) $(\mathrm{n}=27)$; and pregnant women on insulin pump (CSII) $(n=21)$.

Results: In the second trimester of pregnancy the women who used insulin pumps had lower values of IL- $1 \beta$, MCP-1 and TGF$\beta 1 \mathrm{~s}$ in daily urine, compared with the level of excretion in patients receiving MPII. Thus, the level of TGF- $\beta 1$ excretion in pregnant women in the CSII group $[1116.3(348.6,1870.2) \mathrm{pg} /$ $\mathrm{ml}, \mathrm{p}=0,22]$ is lower than in the MPII group [1665.0 (1103, 2609, 7)], excretion of IL- $1 \beta$ in a group of pregnant women in CSII 2.5 times lower $(p=0.04)$ and urinary MCP-1 2.2 times lower 
$(p=0.02)$ compared to the their levels in women in CSII group. In the first and third trimesters the IL- $1 \beta$, MCP- 1 and TGF- $\beta 1$ levels were comparable.

Conclusion: Maintaining the better glycemic control in pregnant women with DM 1 using insulin pump helps to decrease the daily urinary excretion of IL- $1 \beta, \mathrm{MCP}-1$ and TGF- $\beta 1$ in the second trimester of pregnancy.

\section{7}

\section{EARLY DIAGNOSIS OF DIABETIC GASTROPATHY IN PATIENTS WITH TYPE 1 DIABETES ON INSULIN THERAPY IN VARIOUS MODES, DEPENDING ON THE PARAMETERS OF GLYCEMIC CONTROL}

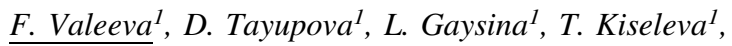
L. Safiullina ${ }^{2}$

${ }^{1}$ Kazan State Medical University, Endocrinology, Kazan, Russia ${ }^{2}$ Kazan State Medical Academy, Ultrasound Diagnostics, Kazan, Russia

Background and Aims: To evaluate the nature of the motor-evacuation disorders by ultrasound in patients with type 1 diabetes mellitus (T1DM) in the pre-clinical stage, depending on the mode of insulin therapy and glycemic control parameters.

Method: 50 patients with T1DM and 15 healthy volunteers were included. Patients were divided into two groups: 1 - treated by continuous subcutaneous insulin infusion by pump, 2 - treated by multiple subcutaneous injections. Members were comparable by gender, age and duration of T1DM. Motor-evacuation function of the stomach was assessed with the ultrasound scanners. All patients underwent analysis of HbA1c, continuous glucose monitoring (CGMS), from which parameters of glycemic variability (SD, CONGA, LI, JINDEX, LBGI, HBGI, MODD, MAGE, ADDR, MVALUE, MAG) were calculated.

Results: Mean age: $28.2 \pm 5.2$ years, the duration of T1DM: $11.9 \pm 5.6$ years. In group 1 the level of $\mathrm{HbA} 1 \mathrm{c}=6.56 \pm 0.9 \%$. Decreased gastric motility was found in $33.3 \%$ of cases, gastroparesis in $6.7 \%$, evacuation function was not impaired in $60 \%$. In group 2 the level of $\mathrm{HbA} 1 \mathrm{c}=8.89 \pm 1.6 \%$. Decreased gastric motility was found in $42.1 \%$, gastroparesis in $36.8 \%$, evacuation function was not impaired in $21.1 \%$. The analysis of the parameters of glycemic variability showed statistically significant differences between the groups $(\mathrm{p}<0.03)$.

Conclusion: Ultrasound diagnosis allows to identify violations of the motor-evacuation function of the stomach in the early stages of diabetic gastropathy. High levels of HbA1c and glycemic variability lead to violations of the motor-evacuation function of the stomach in patients with T1DM.

\section{8}

SENSOR-AUGMENTED PUMPS PREFERABLE TO MULTIPLE DAILY INJECTIONS FOR ACHIEVING GLYCEMIC GOALS IN HOSPITALIZED PATIENTS WITH TYPE 2 DIABETES IN CHINA

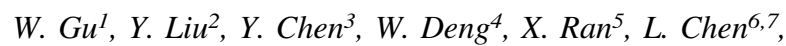
D. $\mathrm{Zhu}^{8}$, J. Yang ${ }^{9}, \underline{\text { J. Shin }}^{10}, Y . M u^{1}$

${ }^{1}$ Chinese PLA General Hospital, Diabetes, Beijing, China
${ }^{2}$ The Second Hospital of Jilin University, Diabetes, Changchun, China

${ }^{3}$ Fu Wai Hospital CAMS\&PUMC, Diabetes, Beijing, China

${ }^{4}$ Beijing Jishuitan Hospital, Diabetes, Beijing, China

${ }^{5}$ West China Hospital- Sichuan University, Diabetes, Chengdu, China

${ }^{6}$ Union Hospital Tongji Medical College, Diabetes, Wuhan, China

${ }^{7}$ Huazhong University of Science and Technology, Diabetes, Wuhan, China

${ }^{8}$ Nanjing Drum Tower Hospital- Nanjing University Medical School, Diabetes, Nanjing, China

${ }^{9}$ Beijing Tongren Hospital- Capital Medical University, Diabetes, Beijing, China

${ }^{10}$ Medtronic, Inc., Diabetes, Northridge, USA

Background and Aims: Choices for insulin delivery in hospitalized patients with type 2 diabetes include sensor-augmented pumps (SAP) and multiple daily injections (MDI). We compared SAP and MDI therapies with respect to time needed to reach specific glycemic targets.

Method: Adults with T2DM (ages 18-65) treated with insulin and admitted for glucose management were eligible for this prospective trial and randomized to either SAP (Medtronic MiniMed 722 Pump) or MDI therapy. Seven blood glucose measurements ( 3 preprandial, 3 postprandial, 1 bedtime) were taken each day. The glycemic target was achieved and subjects were discharged on the day in which 3 preprandial measurements were 4.4-7.2 $\mathrm{mmol} / \mathrm{L}$ and 3 postprandial measurements were 4.4-10.0 $\mathrm{mmol} / \mathrm{L}$. All MDI subjects wore continuous glucose monitoring (CGMS Gold) sensors during hospitalization; CGM data were available to SAP subjects in real time.

Results: Eighty-one subjects completed the study (40 SAP, 41 $\mathrm{MDI})$. Twenty-one SAP compared to $6 \mathrm{MDI}$ subjects reached the glycemic target within 3 days, and SAP subjects' mean \pm SD hospital stay was 2.6 days shorter than MDI subjects' stays ( $3.7 \pm 1.1$ vs. $6.3 \pm 3.1$ days, respectively, $\mathrm{p}<0.001)$. Three MDI subjects failed to reach the glycemic target within 14 days. CGM data showed that SAP subjects experienced less hypoglycemia as defined by $\mathrm{SG} \leq 2.8 \mathrm{mmol} / \mathrm{L}(0.04 \%$ vs. $0.32 \%, \mathrm{p}<0.05)$ and significantly less hyperglycemia as defined by $\mathrm{SG} \geq 10 \mathrm{mmol} / \mathrm{L}$ $(21.56 \%$ vs. $35.03 \%, \mathrm{p}<0.05)$ than MDI subjects.

Conclusion: Use of SAP in hospitalized patients with T2DM is preferable to MDI in terms of reaching preprandial and postprandial glycemic targets quickly and reducing hospital stay.

\section{9}

CYTOTOXIC EFFECT OF THREE COMMERCIAL RAPID-ACTING INSULIN ANALOGS AND THEIR PHENOLIC EXCIPIENTS ON FOUR HUMAN IMMUNE CELL TYPES

W.D. Woodley ${ }^{1}$, S.S. Gerth ${ }^{1}$, D.R. Morel $^{2}$, K. Borchert ${ }^{3}$, L. Godfrey ${ }^{3}$, J. Alarcön ${ }^{1}$, L. Chandler ${ }^{1}$, N. Herrmann ${ }^{3}$, R.J. Pettis ${ }^{1}$, N.G. Bolick ${ }^{1}$

${ }^{1}$ BD Technologies, Parenteral Sciences, Research Triangle Park, USA

${ }^{2}$ BD, Corporate Clinical Development, Le Pont de Claix, France

${ }^{3}$ BD Technologies, Genomic Sciences, Research Triangle Park, USA 


\begin{tabular}{|c|c|c|c|c|c|c|c|c|c|}
\hline \multicolumn{10}{|c|}{$\mathrm{EC}_{s 0}$ Results Summary } \\
\hline & \multicolumn{3}{|c|}{ Lispro } & \multicolumn{3}{|c|}{ Aspart } & \multicolumn{3}{|c|}{ Glullisine } \\
\hline $\begin{array}{l}\text { Cell } \\
\text { Type }\end{array}$ & $\begin{array}{l}\text { Complete } \\
\text { tispro } \\
\text { formulation } \\
\text { (U/ml) }\end{array}$ & \begin{tabular}{|l|} 
Purfified \\
lispro \\
insulin \\
(U/mLt)
\end{tabular} & $\begin{array}{l}\text { m-cresol } \\
\text { (mg/mt) }\end{array}$ & $\begin{array}{c}\text { Complete } \\
\text { Aspart } \\
\text { formulation } \\
\text { (U/mL) }\end{array}$ & $\begin{array}{l}\text { Purified } \\
\text { aspart } \\
\text { insulin } \\
\text { (U/ml) }\end{array}$ & $\begin{array}{l}m-(r e s o l+\text { phenol } \\
(\mathrm{mg} / \mathrm{ml}+\mathrm{mg} / \mathrm{ml})\end{array}$ & $\begin{array}{l}\text { Complete } \\
\text { Glulisine } \\
\text { formulation } \\
\text { (U/mL) }\end{array}$ & $\begin{array}{l}\text { Purfied } \\
\text { glulisine } \\
\text { insulin } \\
\text { (U/ml) }\end{array}$ & $\begin{array}{l}\text { m-cresol } \\
(\mathrm{mg} / \mathrm{ml})\end{array}$ \\
\hline PBMC & $\begin{array}{c}12.95 \\
(12.95 \times 1)\end{array}$ & NE & $\begin{array}{c}0.40 \\
(12588)\end{array}$ & $\begin{array}{c}1497 \\
(149979)\end{array}$ & NE & $\begin{array}{c}0.05+0.05 \\
(3.26 \times)\end{array}$ & $\begin{array}{c}12.96 \\
(1296 \times)\end{array}$ & NE & $\begin{array}{c}0.40 \\
(12.58 \times)\end{array}$ \\
\hline PMN & NE & $\mathrm{NE}$ & NE & NE & NE & $\begin{array}{c}0.23+0.20 \\
(13.17 \times 9)\end{array}$ & NE & NE & NE \\
\hline HMC-1 & $\begin{array}{c}27.63 \\
{[27.63 \times]}\end{array}$ & NE & $\begin{array}{c}0.88 \\
(279289)\end{array}$ & $\begin{array}{c}28.27 \\
(28.27 \times 1)\end{array}$ & NE & $\begin{array}{c}0.33+0.29 \\
(19.41 \times)\end{array}$ & $\begin{array}{c}16.08 \\
(16.00 \mathrm{~W})\end{array}$ & NE & $\begin{array}{c}0.88 \\
(27.92 \times)\end{array}$ \\
\hline THP.1 & $\begin{array}{c}7.7 \\
(0.7 \times)\end{array}$ & NE & $\begin{array}{c}0.22 \\
(695 \times)\end{array}$ & $\begin{array}{c}7.24 \\
(7.24 \times)\end{array}$ & NE & $\begin{array}{c}0.10+0.08 \\
(5.64 \mathrm{~K})\end{array}$ & $\begin{array}{c}6.83 \\
(6833)\end{array}$ & NE & $\begin{array}{c}0.22 \\
(6.655 \times)\end{array}$ \\
\hline
\end{tabular}

Background and Aims: Continuous subcutaneous (SC) insulin infusion (CSII) tissue effects including inflammation are well documented, but causation, cellular effects and overall therapeutic impact are poorly characterized. This study characterizes in vitro cytotoxicity of three marketed insulin analog formulations (lispro, glulisine and aspart), purified analogs, and phenolic excipients (m-cresol, phenol) on four SC human immune inflammatory cell types: peripheral blood mononuclear cells (PBMC), polymorphonuclear leukocytes (PMN), human macrophages (THP-1) and mast cells (HMC-1).

Method: THP-1 and HMC-1 cells were cultured in vitro; primary PBMC and PMN's were freshly isolated from human blood. Cells were incubated with serial dilutions of insulin analogs and preservatives alone in culture media. Cell viability was evaluated at $24 \mathrm{~h}$ via $\mathrm{CyQUANT}^{\circledR}$ Direct Cell Proliferation Assay.

Results: $\mathrm{EC}_{50}$ concentration values, where cell viability is reduced by fifty percent, are shown (Table 1) as both absolute values and relative percentages to marketed formulations. Cytotoxicity varied across cell types, typically PMN $<<$ HMC-1 $<$ PBMC $<$ THP-1 from least to most susceptible. Pure analog insulins alone had no measured cytotoxicity on any cell type. Complete analog formulations had similar cytotoxicity to respective preservatives alone. $\mathrm{m}$-Cresol plus phenol combination was more cytotoxic than m-cresol alone on PBMC and HMC-1's and was the only solution affecting PMN viability. Glulisine had a slightly increased effect on HMC-1's vs. other analogs.

Conclusion: The aggregate data suggest that analog formulation preservatives are primarily responsible for in vitro cellular cytotoxicity against various immune cell types. Exploration of additional cellular effects and in vivo confirmation is warranted.

\section{0}

OLDER PEOPLE WITH T2DM: GLYCEMIC CONTROL AND LESS HYPOGLYCEMIA WITH INSULIN GLARGINE 300 U/ML (GLA-300) VS GLA-100 AT 1 YEAR

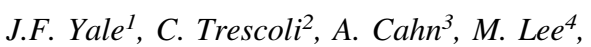
C. Brulle-Wohlhueter ${ }^{5}$, S. Chevalier ${ }^{6}$, G.B. Bolli ${ }^{7}$

${ }^{1}$ McGill University, Department of Medicine, Montreal, Canada ${ }^{2}$ Hospital de la Ribera, Unidad de Diabetes, Alzira, Spain

${ }^{3}$ Hadassah Hebrew University Medical Center, The Diabetes Research Center, Jerusalem, Israel

${ }^{4}$ Sanofi, Diabetes Division, Bridgewater, USA

\begin{tabular}{|c|c|c|c|c|c|}
\hline \multicolumn{6}{|l|}{ 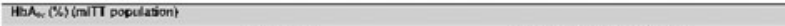 } \\
\hline & \multicolumn{2}{|c|}{ Whon $(80)$} & \multicolumn{3}{|c|}{ Charge rom bineline vis year } \\
\hline & 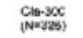 & 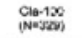 & 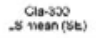 & $\begin{array}{cl}\text { Clas-150 } \\
.8 \text { nean (st) }\end{array}$ & LS mean akterences gesso Cly \\
\hline Eastilire & $815(0.85)$ & $5.1330 .85:$ & $-x \operatorname{sos} 60$ & $-279(305)$ & $-c 97(-n) 1 \times 0007$ \\
\hline 'year & $723(0.25)$ & $720\{0.52\}$ & & & \\
\hline \multicolumn{6}{|c|}{ 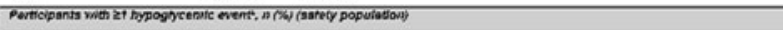 } \\
\hline & $\underset{\mathrm{N}=107 \mathrm{C}}{\mathrm{Gu}}$ & $\begin{array}{l}\text { Gt } 150 \\
\text { N=1110i }\end{array}$ & & OR & $95 \% \mathrm{C}$ \\
\hline A-y tore (24 II) & $202(1 / 1)$ & 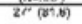 & & 16 & 0.2810102 \\
\hline 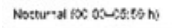 & $13:(596)$ & $105(49 \pi)$ & & $m$ & 0.5710004 \\
\hline \multicolumn{6}{|c|}{ 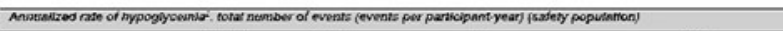 } \\
\hline & $610-3 x \mathrm{C}$ & 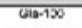 & & at & $05 \% \mathrm{C}$ \\
\hline A-yling (24h: & $4 \sec (-595)$ & $519 x(17,15)$ & & $\infty$ & are in 1 is \\
\hline 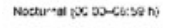 & $\operatorname{sen}(2,05)$ & $839(2.82)$ & & $1 / 2$ & usc wo 1 os \\
\hline
\end{tabular}

${ }^{5}$ Sanofi, Diabetes Division, Paris, France

${ }^{6}$ Sanofi, Biostatistics and Programming, Chilly-Mazarin, France

${ }^{7}$ University of Perugia, Medicine, Perugia, Italy

Background and Aims: In people with T2DM, a patient-level meta-analysis of EDITION 1, 2 and 3 has shown insulin glargine $300 \mathrm{U} / \mathrm{mL}$ (Gla-300) provides more sustained glycemic control with less hypoglycemia over 1 year vs insulin glargine $100 \mathrm{U} / \mathrm{mL}$ (Gla-100). This post hoc analysis investigated these outcomes in the subgroup of adults aged $\geq 65 \mathrm{y}$.

Method: Patient-level meta-analysis of efficacy and safety outcomes in the population of participants aged $\geq 65 \mathrm{y}$ in EDITION 1, 2 and 3 over 1 year of treatment.

Results: Gla-300 showed comparable glycemic control to Gla100 (Table). The total number of confirmed ( $\leq 70 \mathrm{mg} / \mathrm{dL})$ or severe hypoglycemic events over 12 months was lower for Gla-300 vs Gla-100 (any time [24 h]: 4664 vs 5101; nocturnal [00:0005:59 h]: 604 vs 839). The risk of experiencing $\geq 1$ confirmed $(\leq 70 \mathrm{mg} / \mathrm{dL})$ or severe nocturnal hypoglycemic event was lower for Gla-300 relative to Gla-100 (relative risk [RR] 0.79 [95\% CI 0.67 to 0.94$]$ ) (Table). Mean (SD) daily basal insulin dose increased from baseline to 1 year by $0.35(0.27) \mathrm{U} / \mathrm{kg}$ for Gla-300 and $0.23(0.25) \mathrm{U} / \mathrm{kg}$ for Gla-100. The mean (SD) increase in body weight from baseline to 1 year was small and comparable in both groups (Gla-300: 1.1 [4.0] kg; Gla-100: 1.3 [3.3] kg).

Conclusion: Comparable glycemic control with lower risk of nocturnal hypoglycemia with Gla-300 vs Gla-100 has been shown over 1 year of treatment in older people, aged $\geq 65 \mathrm{y}$.

Studies sponsored by Sanofi (NCT01499082, NCT01499095, NCT01676220)

\section{1}

\section{MECHANISTIC ANALYSIS INTO THE MODE(S) OF ACTION OF NIACINAMIDE IN FASTER-ACTING INSULIN ASPART}

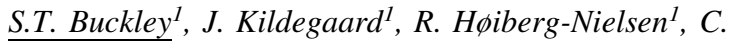
Bekker Jeppesen ${ }^{1}$, H. Birk Olsen ${ }^{1}$, H.H.F. Refsgaard ${ }^{1}$, S. Ludvigsen ${ }^{1}$, U. Ribel ${ }^{1}$, S. Hostrup ${ }^{1}$, J. Sturis ${ }^{1}$

${ }^{1}$ Novo Nordisk A/S, Global Research, Målфv, Denmark

Background and Aims: Faster-acting insulin aspart (faster aspart) is insulin aspart (IAsp) in a new formulation containing two additional excipients (niacinamide and L-arginine), resulting in faster initial absorption after s.c. injection. In order to establish an understanding of the mechanism(s) via which niacinamide elicits its effect, experimental investigations were performed to 
evaluate its impact on the monomeric content and permeation rate of IAsp and on local blood flow.

Method: By means of small-angle X-ray scattering (SAXS) the monomeric content of IAsp was quantified in diluted formulations mimicking s.c. conditions after injection. The permeation rate of IAsp formulated with and without niacinamide was evaluated in human dermal-derived microvascular capillary endothelial (HDMEC) cell monolayers. To evaluate potential effects on local blood flow, Xenon $133\left({ }^{133} \mathrm{Xe}\right)$ washout - an indirect marker of blood flow - was measured in pigs following administration of IAsp formulations with and without niacinamide.

Results: SAXS analysis revealed that niacinamide increased the percentage of monomeric IAsp by $\sim 34.3 \%( \pm 7.4 \%)$ in diluted formulations mimicking s.c. conditions after injection. Moreover, inclusion of niacinamide in IAsp formulations containing $\mathrm{Zn}$ augmented the permeation rate of IAsp $\left(5 \times 10^{-6} \mathrm{~cm} / \mathrm{s}\right.$ vs $4 \times 10^{-6} \mathrm{~cm} / \mathrm{s}$, respectively; $\left.\mathrm{p}<0.05\right)(\mathrm{n} \geq 3)$. In contrast, ${ }^{133} \mathrm{Xe}$ washout was not significantly affected by niacinamide, suggestive of little or no effect on local blood flow.

Conclusion: Collectively, these data demonstrate that niacinamide promotes the formation of IAsp monomer at the subcutis-endothelial interface upon s.c. injection and in this way facilitates a more rapid rate of absorption.

\section{2}

SWITCHING FROM TWICE-DAILY BASAL INSULIN TO ONCE-DAILY NEW INSULIN GLARGINE 300 U/ML (GLA-300): AN ANALYSIS IN PEOPLE WITH T2DM (EDITION 1 AND 2)

R. Roussel ${ }^{1}$, M. d'Emden ${ }^{2}$, M. Fisher ${ }^{3}$, F.J. Ampudia-Blasco ${ }^{4}$, P. Stella ${ }^{5}$, M.L. Grisoni ${ }^{6}$, A.M.G. Cali ${ }^{5}$, C. Wysham $^{7}$

${ }^{1}$ Assistance Publique Hôpitaux de Paris, Bichat Hospital, Paris, France

${ }^{2}$ Royal Brisbane and Women's Hospital, Endocrinology and Diabetes, Brisbane, Australia

${ }^{3}$ University of Glasgow, Glasgow Royal Infirmary, Glasgow, United Kingdom

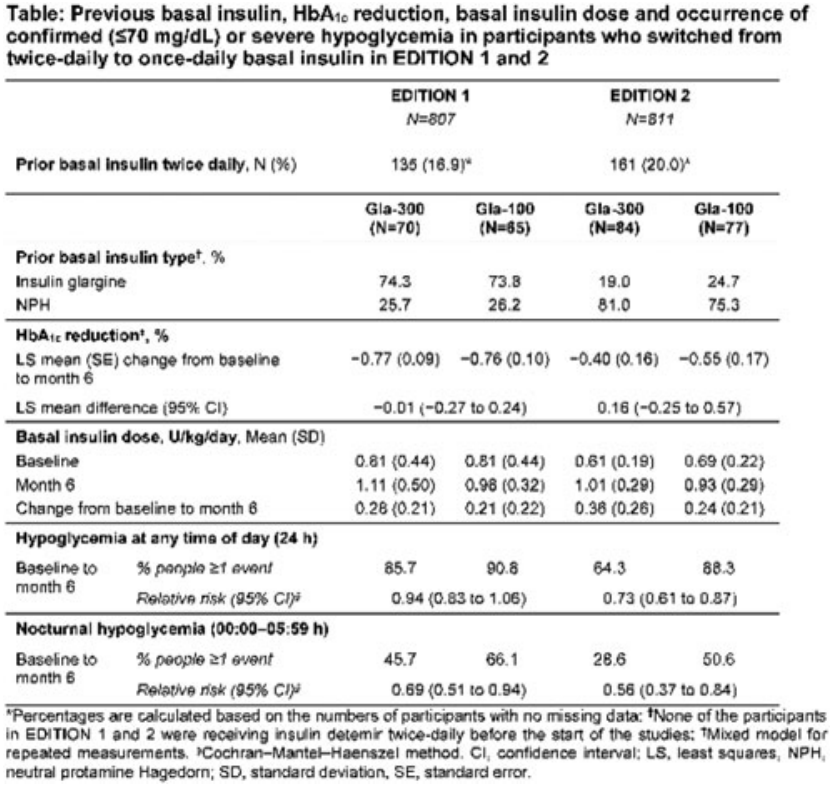

${ }^{4}$ Clinic University Hospital Valencia, Diabetes Reference Unit, Valencia, Spain

${ }^{5}$ Sanofi, Diabetes Division, Paris, France

${ }^{6}$ AIXIAL, Biostatistics, Levallois-Perret, France

${ }^{7}$ Rockwood Clinic, Diabetes and Endocrinology Center, Spokane, USA

Background and Aims: In EDITION 1 (basal + mealtime insulin) and EDITION 2 (basal + OADs), people with T2DM receiving new insulin glargine $300 \mathrm{U} / \mathrm{mL}$ (Gla-300) achieved comparable glycemic control with less hypoglycemia vs glargine $100 \mathrm{U} / \mathrm{mL}$ (Gla-100).

Method: This post hoc analysis explored the effect of switching from twice-daily basal insulin to once-daily Gla-300 or Gla-100 on efficacy and safety outcomes.

Results: At randomization, $16.9 \%$ and $20.0 \%$ of people were receiving twice-daily basal insulin in EDITION 1 and EDITION 2 , respectively. In these subgroups, glycemic control was comparable over 6 months in people who switched to once-daily Gla300 or Gla-100 (LS mean difference in $\mathrm{HbA}_{1 \mathrm{c}}$ change from baseline to month $6-0.01$ [95\% CI -0.27 to 0.24 ] \% in EDITION 1 and 0.16 [ -0.25 to 0.57$] \%$ in EDITION 2). As in the overall study populations, Gla-300 dose was higher than Gla-100 at month 6. Participants switching from twice- to once-daily basal insulin in EDITION 1 had lower risk of confirmed $(\leq 70 \mathrm{mg} / \mathrm{dL})$ or severe hypoglycemia with Gla-300 vs Gla-100 at night (00:00 to 05:59h), while in EDITION 2, the risk was reduced both at night and at any time ( $24 \mathrm{~h}$; Table).

Conclusion: As seen overall in EDITION 1 and 2, people with T2DM switching from twice-daily basal insulin to once-daily Gla-300 achieved comparable glycemic control with less hypoglycemia vs those switching to Gla-100.

Studies sponsored by Sanofi (NCT01499082, NCT01499095).

\section{3}

\section{MONITORING THE FREQUENCY OF INSULIN PEN NEEDLES REPLACEMENT IN DIABETIC PATIENTS}

\author{
J. Jirkovska ${ }^{1}$, D. Vitova ${ }^{1}$, J. Skibova ${ }^{2}$, J. Venerova ${ }^{1}$, S. Solar ${ }^{1}$, \\ M. Zavoral \\ ${ }^{1}$ University Military Hospital, Internal Medicine Department, \\ Prague, Czech Republic \\ ${ }^{2}$ Institute for Clinical and Experimental Medicine, \\ Department of Statistics, Prague, Czech Republic
}

Background and Aims: Diabetics treated with insulin analogues should manage with insulin pen and needles properly. There exist differences in clinical practice comparing to producer's recommendation. Aim was to check patients' knowledge in diabetes centers vs. standard outpatient.

Method: 70 diabetic patients on intensive insulin therapy were included, 35/70 from diabetes center (Group 1), 35/70 from standard outpatient (Group 2). Survey with anonymous questionnaire was performed.

Results: 69/70 questionnaires were evaluated. Group $1(n=34)$ and Group $2(n=35)$ were statistically comparable. Concerning producer's recommended frequency of insulin pen needle replacement, "after each use" answered $12 \%$ patients in Group 1 vs. $46 \%$ in Group $2(\mathrm{p}=0.019)$. In daily practice, subjects in Group 1 replaced needle most frequently in $32 \%$ when putting on new insulin cartridge as well as in $32 \%$ every third day, while subjects in Group 2 replaced needle in $60 \%$ when putting on new cartridge $(\mathrm{p}=0.159)$. Identically $3 \%$ of diabetics in both groups replaced 
needle after each use. Pain as main reason for needle replacement chose 56\% subjects in Group 1 vs. 80\% in Group 2, respectively $(\mathrm{p}=0.043) .32 \%$ patients in Group 1 vs. $11 \%$ in Group 2 assumed that needle replacement frequency could affect glycaemic control contrary to negative answer in $18 \%$ subjects in Group 1 vs. $29 \%$ in Group 2; while 50\% patients in Group 1 vs. 60\% in Group 2 were unable to answer this question $(\mathrm{p}=0.048)$.

Conclusion: Study shows significant differences in knowledge of diabetics among both groups. Discrepancy between theoretical recommendation and daily practice was shown. Reeducation is permanently actual.

\section{4}

\section{GLYCEMIC CONTROL AND HYPOGLYCEMIA WITH INSULIN GLARGINE 300 U/ML VS GLARGINE 100 U/ML IN T2DM IN A PATIENT-LEVEL META-ANALYSIS OF 1-YEAR PHASE 3A EDITION STUDIES}

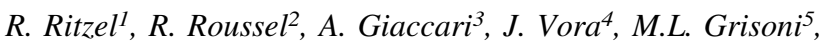
C. Brulle-Wohlhueter ${ }^{6}$, S. Glezer ${ }^{6}, H$. Yki-Järvinen ${ }^{7}$

${ }^{1}$ Städtisches Klinikum München GmbH-Klinikum Schwabing, Division of Endocrinology and Diabetes, Munich, Germany

${ }^{2}$ Assistance Publique Hôpitaux de Paris, Bichat Hospital, Paris, France

${ }^{3}$ Università Cattolica del Sacro Cuore, Division

of Endocrinology and Metabolic Diseases, Rome, Italy

${ }^{4}$ Royal Liverpool University Hospital, Diabetes/Endocrinology, Liverpool, United Kingdom

${ }^{5}$ AIXIAL, Biostatistics, Levallois-Perret, France

${ }^{6}$ Sanofi, Diabetes Division, Paris, France

${ }^{7}$ University of Helsinki, Department of Medicine, Helsinki,

Finland

Background and Aims: EDITION 1, 2 and 3 assessed new insulin glargine $300 \mathrm{U} / \mathrm{mL}$ (Gla-300) vs glargine $100 \mathrm{U} / \mathrm{mL}$ (Gla-100) in T2DM. This analysis aimed to investigate the efficacy and safety of Gla-300 vs Gla-100 in the pooled population of these three studies.

Method: A patient-level meta-analysis of efficacy and safety outcomes of 1-year data was conducted.

Results: Glycemic control was sustained in both groups, with more sustained $\mathrm{HbA}_{1 \mathrm{c}}$ reduction for Gla-300 at 1 year (LS mean difference between groups in $\mathrm{HbA}_{1 \mathrm{c}}$ change from baseline -0.10 $[-0.18$ to -0.02$] ; p=0.0174 ;$ Figure). There was a reduced risk of confirmed $(\leq 70 \mathrm{mg} / \mathrm{dL})$ or severe hypoglycemia at any time $(24 \mathrm{~h})$ and during the night vs Gla-100 (Table). Weight gain was less with

Figure: $\mathrm{HbA}_{\mathrm{c}}$ by visit in a patient-level meta-analysis of $1-\mathrm{yr}$ data from the EDITION 1, 2 and 3 clinical trials

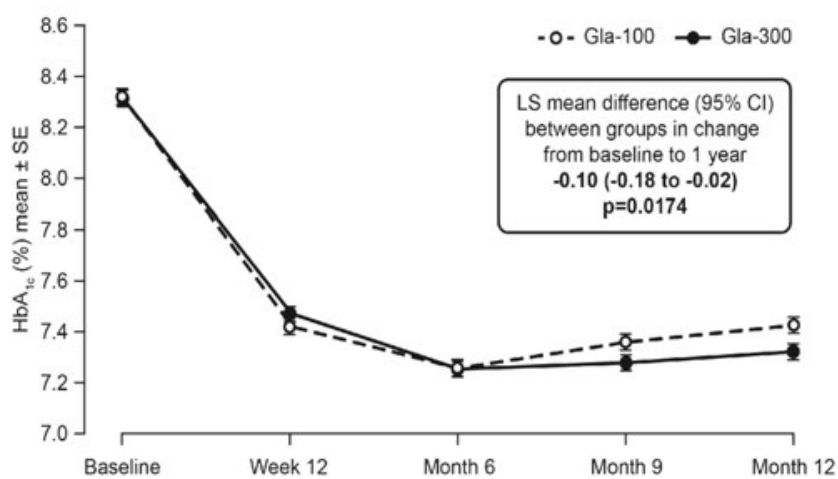

Table: Efficacy and safety of Gla-300 in a patient-level meta-analysis of 1-year data from the EDITION 1, 2 and $3^{*}$ clinical trials

\begin{tabular}{|c|c|c|c|c|c|}
\hline \multicolumn{6}{|l|}{$H b A_{k L}(\mathbf{*})$} \\
\hline \multicolumn{2}{|l|}{ m/T population } & \multicolumn{2}{|c|}{$\begin{array}{l}\text { Gla-300 } \\
\text { (N=1239) }\end{array}$} & \multicolumn{2}{|c|}{$\begin{array}{l}\text { Gla-100 } \\
(N=1235)\end{array}$} \\
\hline Bt & Mean (SD) & \multicolumn{2}{|c|}{$8.30(0.91)$} & \multicolumn{2}{|c|}{$8.31(0.90)$} \\
\hline \multirow[t]{2}{*}{ Change from $B L$ to 1 year } & IS mean (SE) & \multicolumn{2}{|c|}{$-0.91(0.03)$} & \multicolumn{2}{|c|}{$-0.80(0.03)$} \\
\hline & LS mean difference $(95 \% \mathrm{Cl})^{\circ}$ & \multicolumn{4}{|c|}{$-0.10(-0.18$ to -0.02$)$} \\
\hline \multicolumn{2}{|c|}{ Confirmed ( $\$ 70 \mathrm{mg} / \mathrm{dt}$ ) or severe hypoglycemia } & \multicolumn{2}{|c|}{$\begin{array}{c}\text { Nocturnal } \\
\text { hypoglycemia } \\
(00: 00-05: 59 \mathrm{~h}) \\
\end{array}$} & \multicolumn{2}{|c|}{$\begin{array}{l}\text { Hypoglycemia at any time } \\
(24 \mathrm{~h})\end{array}$} \\
\hline Safety population & & $\begin{array}{l}\text { Gla-300 } \\
(N=1242)\end{array}$ & $\begin{array}{c}\text { Gla-100 } \\
(N=1246)\end{array}$ & $\begin{array}{l}\text { Gla-300 } \\
(N=1242)\end{array}$ & $\begin{array}{l}\text { Gla-100 } \\
(N=1246)\end{array}$ \\
\hline \multirow[t]{4}{*}{ BL to 1 year } & \% people 21 event & 38.7 & 45.7 & 73.1 & 77.8 \\
\hline & Relotive risko $(95 \%$ al) & \multicolumn{2}{|c|}{$0.85(0.77$ to 0.92$)$} & \multicolumn{2}{|c|}{0.94 (0.90 to 0.98$)$} \\
\hline & Events/participont-year & 2.0 & 2.4 & 13.7 & 14.1 \\
\hline & Rate rotio $(95 \%$ C $)$ & $0.82(0.6$ & 7 to 0.99$)$ & 0.9710 & to 1.09) \\
\hline \multicolumn{6}{|l|}{ Weight (kg) } \\
\hline \multicolumn{2}{|l|}{ Safety population } & \multicolumn{2}{|c|}{$\begin{array}{c}\text { Gla-300 } \\
\left(N^{\prime}=1242\right)\end{array}$} & \multicolumn{2}{|c|}{$\begin{array}{c}\text { Gla-100 } \\
\left(N^{\prime}=1246\right)\end{array}$} \\
\hline Bt & Mean (SO) & \multicolumn{2}{|c|}{$99.9(22.9)$} & \multicolumn{2}{|c|}{$99.9(21.7)$} \\
\hline 1 year & Mean (SO) & \multicolumn{2}{|c|}{$100.9(23.1)$} & \multicolumn{2}{|c|}{$101.2(22.1)$} \\
\hline \multirow[t]{2}{*}{ Change from BL to 1 year } & Mean (SO) & \multicolumn{2}{|c|}{$1.19(5.32)$} & \multicolumn{2}{|c|}{$1.52(4.18)$} \\
\hline & p-value for IS mean differences & & & 0117 & \\
\hline \multicolumn{6}{|l|}{ Insulin dose (U/kg/day) } \\
\hline m/TT population & & \multicolumn{2}{|c|}{$\begin{array}{c}\text { Gla-300 } \\
\left(N^{\prime}=1239\right)\end{array}$} & \multicolumn{2}{|c|}{$\begin{array}{c}\begin{array}{c}G l a-100 \\
\left(N^{\prime}=1235\right)\end{array}\end{array}$} \\
\hline BL & Mean (SO) & \multicolumn{2}{|c|}{$0.49(0.30)$} & \multicolumn{2}{|c|}{$0.50(0.31)$} \\
\hline Change from BL to month 6 & Mean (SO) & \multicolumn{2}{|c|}{$0.34(0.25)$} & \multicolumn{2}{|c|}{$0.25(0.23)$} \\
\hline Change from $B L$ to 1 year & Mean (SO) & \multicolumn{2}{|c|}{$0.39(0.29)$} & & 0.27) \\
\hline
\end{tabular}

Gla-300 vs Gla-100 ( $p=0.0117$; Table). The slight insulin dose difference at 6 months remained at 1 year (Table).

Conclusion: Gla-300 allows greater up-titration of insulin dose for more sustained glycemic control without increasing the risk of any time and nocturnal hypoglycemia or weight gain vs Gla-100.

Studies sponsored by Sanofi (NCT01499082, NCT01499095, NCT01676220)

\section{5}

GREATER EARLY INSULIN EXPOSURE

AND GLUCOSE-LOWERING WITH FASTER-ACTING INSULIN ASPART VS INSULIN ASPART IN JAPANESE SUBJECTS WITH TYPE 1 DIABETES

\author{
M. Shiramoto ${ }^{1}$, T. Nishida ${ }^{2}$, A.K. Hansen ${ }^{3}$, H. Haahr ${ }^{3}$ \\ ${ }^{1}$ SOUSEIKAI Hakata Clinic, Fukuoka, Japan \\ ${ }^{2}$ Novo Nordisk Pharma Ltd, Tokyo, Japan \\ ${ }^{3}$ Novo Nordisk A/S, Sфborg, Denmark
}

Background and Aims: Faster-acting insulin aspart (faster aspart) is a new formulation of insulin aspart (IAsp), with faster initial absorption following subcutaneous injection. This trial aimed to investigate the pharmacokinetic (PK) and pharmacodynamic (PD) properties of faster aspart vs IAsp following subcutaneous injection in Japanese subjects with type 1 diabetes (T1D).

Method: A total of 43 subjects (mean age \pm SD: $39.4 \pm 9.4$ years) received a single dose $(0.2 \mathrm{U} / \mathrm{kg})$ of faster aspart or IAsp under glucose-clamp conditions (STG-22; blood glucose target $100 \mathrm{mg} / \mathrm{dL}$ [5.5 mmol/L]; duration $12 \mathrm{~h}$ post-dose) in a doubleblind, randomised, crossover design.

Results: Onset of appearance and $\mathrm{t} 50 \% \mathrm{C}_{\max }$ with faster aspart occurred $58 \%$ and $35 \%$ earlier than with IAsp, and faster aspart had greater early insulin exposure during the first $2 \mathrm{~h}$ (2-fold greater exposure than IAsp in the first $30 \mathrm{~min}$ post-dose); total exposure was similar (Table). Faster aspart had a greater glucoselowering effect within $2 \mathrm{~h}$ post-dose vs IAsp (greatest difference in the first $30 \mathrm{~min}$ ), and an earlier onset of glucose-lowering 
Table: PK and PD results for faster aspart vs IAsp.

\begin{tabular}{|c|c|c|c|}
\hline $\begin{array}{l}\text { PK endpoints } \\
\text { (insulin exposure*) }\end{array}$ & $\begin{array}{c}\text { Treatment ratio: } \\
\text { faster aspart//Asp } \\
{[95 \% \mathrm{Cl}]^{\dagger}}\end{array}$ & $\begin{array}{l}\text { PD endpoints } \\
\text { (glucose-lowering } \\
\text { effect) }\end{array}$ & $\begin{array}{c}\text { Treatment ratio: } \\
\text { faster aspart//Asp } \\
{[95 \% \mathrm{Cl}]}\end{array}$ \\
\hline Onset & & Onset & \\
\hline Onset of appearance & $0.42[0.32 ; 0.53]$ & Onset of action & $0.79[0.69 ; 0.91]$ \\
\hline $\mathrm{t} 50 \% \mathrm{C}_{\max }$ & $0.65[0.59 ; 0.72]$ & $\mathrm{t} 50 \% \mathrm{GIR}_{\max }{ }^{*}$ & $0.79[0.74 ; 0.84]$ \\
\hline $\mathrm{t}_{\max }^{\$ *}$ & $0.78[0.67 ; 0.90]$ & tGIR $\max ^{\prime \prime}$ & $0.87[0.77 ; 0.97]$ \\
\hline Early & & Early & \\
\hline$A U C_{0-15 \min }$ & $3.26[2.65 ; 4.02]$ & NA & NA \\
\hline$A U C_{0-30 \min }$ & $1.94[1.68 ; 2.25]$ & $\mathrm{AUC}_{\text {GIR0-30min }}{ }^{*}$ & $2.10[1.32 ; 4.08]$ \\
\hline $\mathrm{AUC}_{0-1 \mathrm{~h}}{ }^{\ddagger}$ & $1.31[1.17 ; 1.47]$ & $A U C_{G I R, 0-1 h}$ & $1.36[1.20 ; 1.54]$ \\
\hline$A \cup C_{0-2 h}$ & $1.10[1.01 ; 1.19]$ & $A \cup C_{G I R, 0-2 h}$ & $1.09[1.01 ; 1.17]$ \\
\hline Total & & Total & \\
\hline$A \cup C_{0-12 h}$ & $0.99[0.96 ; 1.02]$ & $A U C_{G \mid R, 0-12 \mathrm{~h}}$ & $0.93[0.87 ; 0.99]$ \\
\hline $\mathrm{C}_{\max }$ & $1.07[0.96 ; 1.19]$ & $G \mid R_{\max }$ & $0.95[0.89 ; 1.02]$ \\
\hline
\end{tabular}

*Based on free serum insulin aspart; ${ }^{\dagger} 90 \% \mathrm{Cl}$ for $\mathrm{AUC}_{0-12 \mathrm{~h}}{ }^{\text {*}}{ }^{\mathrm{N}}$ rimary endpoint; ${ }^{5} \mathrm{Post}$ hoc analysis; "Treatment ratios and $95 \% \mathrm{Cl}$ estimated using Fieller's method; AUC=area under the curve; $\mathrm{C}_{\max }=$ maximum observed concentration; $\mathrm{GIR}_{\max }=$ maximum glucose infusion rate; onset of appearance=time from dosing until the first time serum IAsp concentration $\geq$ lower limit of quantification; NA, not applicable; $\mathrm{t}_{\max }=$ time to maximum observed concentration; $\mathrm{t} 50 \% \mathrm{C}_{\max }=$ time to reach $50 \%$ of maximum serum insulin aspart concentration; $\mathrm{t} 50 \% \mathrm{GIR}_{\max }=$ time to $50 \%$ of maximum glucose infusion rate; $\mathrm{tGIR}$ max $=$ time to maximum glucose infusion rate.

effect (Table). Earlier onset of action with faster aspart vs IAsp was supported by $\sim 10 \mathrm{~min}$ shorter $\mathrm{t}^{2} 0 \% \mathrm{GIR}_{\max }(37.5$ vs $47.4 \mathrm{~min}$; treatment difference [95\% CI]: $-9.97 \mathrm{~min}[-12.78$; $-7.15])$. Both treatments were well tolerated.

Conclusion: Earlier onset and greater early insulin exposure with faster aspart led to a greater early glucose-lowering effect vs IAsp in Japanese subjects with T1D.

296

\section{INSULIN GLARGINE 300U/ML AND INSULIN GLARGINE 100 U/ML SHOW EQUIPOTENT IN VIVO BLOOD GLUCOSE LOWERING WHEN ADMINISTERED INTRAVENOUSLY IN DOGS}

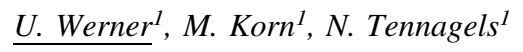

${ }^{1}$ Sanofi, Diabetes Division, Frankfurt, Germany

Background and Aims: Insulin glargine $300 \mathrm{U} / \mathrm{mL}$ (Gla-300) delivers the same dose in a 3-fold lower volume compared with insulin glargine $100 \mathrm{U} / \mathrm{mL}$ (Gla-100). This study compared the blood glucose (BG) lowering potency of Gla-300 and Gla-100 after intravenous (IV) injection in healthy Beagle dogs.

Method: To investigate the BG lowering potency independently of differences in absorption and release behavior from the subcutaneous (SC) injection site, $0.15 \mathrm{U} / \mathrm{kg}$ Gla-100 and Gla-300 were injected IV in dogs and BG was measured over time. The active metabolite 21A-Gly-human insulin (M1) was measured using LC-MS/MS. For comparison, BG profile after SC injection of $0.3 \mathrm{U} / \mathrm{kg}$ of either insulin was also determined.

Results: After IV injection, both Gla-100 and Gla-300 were equally potent, producing immediate, short-lasting BG lowering. After SC injection, Gla-300 resulted in more stable and prolonged BG lowering compared with Gla-100. Although the 24-h $\mathrm{BG}$ area under the curve (AUC) after SC injection was much greater than the 2-h BG AUC after IV injection, the AUCs were comparable between Gla-300 and Gla-100 for each injection route. Rapid metabolism of Gla-300 and Gla-100 to M1 was observed, and correlated well with the observed BG profiles.
Conclusion: After IV injection, both Gla-100 and Gla-300 exhibited the same in vivo potency and BG time-action profile. SC injected Gla-300 was associated with a smoother onset and longer duration of action and a comparable BG lowering effect versus Gla100. As in humans, M1 was the main circulating metabolite.

Study sponsored by Sanofi

\section{A}

HbA $_{1 \mathrm{c}}$, FPG, HYPOGLYCEMIA, AND BASAL INSULIN DOSE WITH INSULIN DEGLUDEC (IDeg) VS INSULIN GLARGINE 100U/ML (GLA-100): LESSONS FROM THE BEGIN PROGRAM

\author{
Geremia B. Bolli ${ }^{1}$, David R. Owens ${ }^{2}$ \\ ${ }^{1}$ University of Perugia, Perugia, Italy \\ ${ }^{2}$ Diabetes Research Group, Swansea University, United Kingdom
}

Background and Aims: The safety and efficacy profiles of IDeg, a long-acting basal insulin analog, were evaluated in the BEGIN program. This review reports comparative glycemic control, hypoglycemia, and insulin dose profiles of IDeg vs Gla100 , focusing only on basal-oral treatment trials in type 2 diabetes (T2DM) from the BEGIN program.

Method: $\mathrm{HbA}_{1 \mathrm{c}}, \mathrm{FPG}$, confirmed $(<56 \mathrm{mg} / \mathrm{dL})$ or severe hypoglycemia, and insulin dose data (including dose increase needed for a $1.0 \% \mathrm{HbA}_{1 \mathrm{c}}$ decrease) from the selected BEGIN trials $(n=4)$ comparing IDeg and Gla-100 in people with T2DM on basal-oral treatment were reviewed.

Results: $\mathrm{HbA}_{1 \mathrm{c}}$ reduction with IDeg was non-inferior vs Gla100 , although, consistently, a greater (non-significant) reduction with Gla-100 was seen in each trial (Table 1A). FPG reduction was greater with IDeg. Rates of confirmed $(<56 \mathrm{mg} / \mathrm{dL})$ or severe hypoglycemia at any time (over $24 \mathrm{~h}$ ) were similar with IDeg and Gla-100, but were lower with IDeg during the nighttime (Table

\begin{tabular}{|c|c|c|c|c|c|c|c|}
\hline & \multirow[t]{2}{*}{$\begin{array}{l}\text { Trial } \\
\text { duration } \\
\text { (weeks) }\end{array}$} & \multirow{2}{*}{ 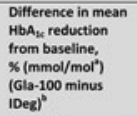 } & \multirow{2}{*}{$\begin{array}{l}\text { Difference in mean } \\
\text { FPG reduction from } \\
\text { baseline, } \\
\text { mmol/h } \\
\text { (Gla-100 minus } \\
\text { (Deg) }\end{array}$} & \multicolumn{2}{|c|}{$\begin{array}{l}\text { Anytime hypoglycemla } \\
\text { (over } 24 \mathrm{~h})^{t}\end{array}$} & \multicolumn{2}{|c|}{$\begin{array}{c}\text { Nocturnal } \\
\text { (00:01-05:59 h) } \\
\text { hypogelycemiat }\end{array}$} \\
\hline & & & & Risk ratio ${ }^{\circ}$ & Rate ratio & Risk ratio ${ }^{4}$ & Rate ratio \\
\hline $\begin{array}{l}\text { BEGIN Once } \\
\text { Long }\end{array}$ & 52 & $0.09(1.0)$ & $-0.43^{*}$ & 1.00 & 0.82 & 0.91 & $0.64^{*}$ \\
\hline $\begin{array}{l}\text { BEGiN Flex } \\
\text { (Once. }\end{array}$ & 26 & $0.18(2.0)^{*}$ & $-0.36^{*}$ & 0.89 & $0.89 / 1.04$ & 0.50 & 0.75 \\
\hline $\begin{array}{l}\text { BEGiN flex } \\
\text { (filex })^{\prime}\end{array}$ & 26 & $0.04(0.4)$ & $-0.42^{*}$ & 1.04 & 1.03 & 0.61 & 0.84 \\
\hline $\begin{array}{l}\text { BEGIN Low } \\
\text { Volume }\end{array}$ & 26 & $0.04(0.4)$ & $-0.42^{\circ}$ & 0.93 & 0.86 & 0.69 & 0.64 \\
\hline $\begin{array}{l}\text { BEGIN Once } \\
\text { Asia's }\end{array}$ & 26 & $0.11(1.2)$ & -0.09 & 0.94 & 0.82 & 0.85 & 0.62 \\
\hline
\end{tabular}

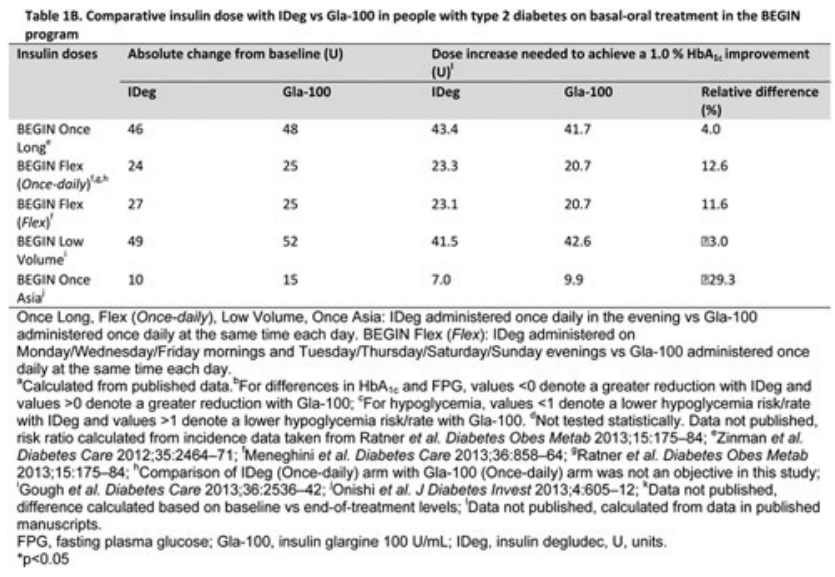


1A). Absolute insulin dose increases tended to be greater with Gla-100; however, when adjusted for a $1.0 \% \mathrm{HbA}_{1 \mathrm{c}}$ reduction, they were more frequently higher with IDeg (Table 1B).

Conclusion: In studies of IDeg vs Gla-100 in people with T2DM on basal-oral treatment from the BEGIN program, $\mathrm{HbA}_{1 \mathrm{c}}$ reduction was slightly greater with Gla-100 while rates of hypoglycemia were either similar (hypoglycemia at any time) or lower (nocturnal hypoglycemia) with IDeg. Insulin dose requirements, when adjusted for $1.0 \% \mathrm{HbA}_{1 \mathrm{c}}$ reduction, were generally higher with IDeg vs Gla-100.

Study was sponsored by Sanofi.

296B

COMMUNICATION BETWEEN HEALTHCARE PROFESSIONALS (HCPS) AND PATIENTS ON BASAL INSULIN (BI) TITRATION: RESULTS OF A QUANTITATIVE SURVEY

Lori Berard $^{1}$, William Polonsky ${ }^{2}$, Mireille Bonnemaire $^{3}$, Steve Edelman ${ }^{4}$, Kamlesh Khunti ${ }^{5}$

${ }^{1}$ Winnipeg Regional Health Authority Health Sciences Centre, Winnipeg, Canada

${ }^{2}$ Behavioral Diabetes Institute, San Diego, CA, USA

${ }^{3}$ Sanofi, Paris, France

${ }^{4}$ University of California, San Diego, CA, USA

${ }^{5}$ Diabetes Research Centre, University of Leicester,

Leicester, UK

An online survey of 386 HCPs and 318 patients with type 2 diabetes on long-acting BI for 6-36 months (BI users: current $\mathrm{n}=243$; discontinued $\mathrm{n}=75$ ) evaluated $\mathrm{HCP} /$ patient communication on BI titration in the USA, France and Germany.

The majority of surveyed HCPs agreed that communicating the need for optimal BI titration to patients is important, with $>75 \%$ of these reporting doing so at the initiation visit (Table).

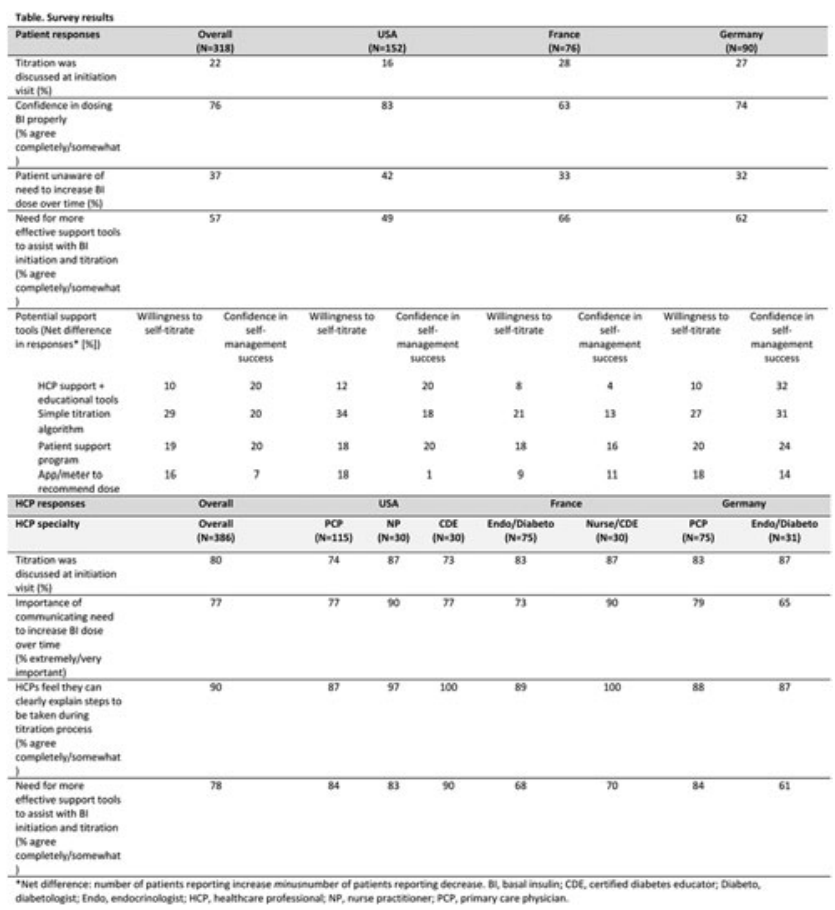

However, only 16-28\% of patients recalled discussing titration at their initiation visit and many $(32-42 \%)$ were unaware of the need to increase the dose over time. While $28-39 \%$ of patients recalled discussing the time needed to reach goal with their HCP at the initiation visit, $22-36 \%$ felt that additional information on this topic would improve their confidence in self-titrating their BI. Most patients and HCPs agreed that more effective support tools to assist with BI initiation/titration are needed; patients reported that their willingness to selftitrate and confidence in self-management success would be increased by these tools.

In conclusion, the survey showed a marked disconnect in communication between HCPs and patients. Communication could be enhanced by providing effective support and educational tools, which would in turn improve patient understanding of and self-confidence in timely and effective titration and optimal BI use.

This market survey project was conducted by Hall \& Partners US LLC and funded by Sanofi.

\section{7}

\section{INDUCED CHANGES IN PLASMA INSULIN GLARGINE CONCENTRATIONS FOLLOWING MODULATION OF LOCAL CONDITIONS AT THE INSULIN GLARGINE INJECTIONS SITE}

G. Bitton $^{1}$, S. Shaked ${ }^{1}$, A. Pfutzner ${ }^{2}$, I. Raz ${ }^{3}$

${ }^{1}$ InsuLine Medical, R\&D, Petach-Tikva, Israel

${ }^{2}$ Pfützner Science \& Health Institute, Clinic, Maintz, Germany ${ }^{3}$ Hadassah Hebrew University School of Medicine,

The Diabetes Center, Jerusalem, Israel

Background and Aims: Insulin Glargine long action is achieved by formation of micro-precipitates which dissolve into hexamers who in turn dissolve into monomers and dimers. These processes depends on local conditions at the drug depot, specifically variability in local conditions can lead to significant intra- and inter-individual variability of the drug Pharmacokinetic (PK) properties. Wong [2008] and Razak [2012] have simulated insulin Glargine PK profile reproducing the clinically observed intra and inter-variability due to variability in three of the model parameters which are expected to rely strongly on the site local conditions. We investigated numerically the option to use modulation of local temperature and blood flow to induce changes in Insulin Glargine PK properties.

Method: Numerical simulation of insulin glargine PK using the model suggested by Razak were carried. Changes in microprecipitates dissolution rate and in Glargine hexametric disassociation rates following changes in local conditions were introduced into the simulation. The dependence of insulin glargine PK parameters on changes in local conditions were investigated.

Results: Significant changes in maximum plasma insulin concentration as function of changes in model parameters were observed as well as changes in other Insulin Glargine PK parameters.

Conclusion: Changes in plasma insulin Glargine concentrations following changes in local conditions at the insulin injections site were observed in numerical solutions of insulin Glargine PK model. Local skin conditions modulation may enable desired changes in insulin PK profile in real time. This new approach needs to be clinically validated as well as determining the relation between local site conditions and model basic parameters. 
298

A RANDOMIZED TRIAL COMPARING BOLUS-ONLY INSULIN PATCH VERSUS PEN USING SIMPLE DOSETITRATION ALGORITHM IN TYPE 2 DIABETES PATIENTS NOT ACHIEVING GLYCEMIC TARGETS ON BASAL INSULIN

M. Johnson ${ }^{1}$, D. Dreon ${ }^{2}$, V. Zraick ${ }^{2}$, B. Levy ${ }^{3}$, R. Naik $^{3}$, R. Bergenstal

${ }^{1}$ Park Nicollet, International Diabetes Center, Minneapolis, USA

${ }^{2}$ Calibra Medical Inc., Clinical Affairs, Redwood City, USA

${ }^{3}$ LifeScan Inc., Medical Affairs, Wayne, USA

Background and Aims: Advancing patients with type 2 diabetes (T2D) not achieving glycemic targets on basal insulin therapy to a basal-bolus insulin regimen presents challenges to clinicians and patients. "Clinical inertia" to insulin intensification is a significant barrier, and patient factors, such as frequency of injections, discomfort, and social barriers, pose additional resistance. In order to overcome usage barriers associated with multiple daily injections, a novel bolus-only patch insulin delivery system has been developed (Calibra Medical, Inc.). This study will provide comparative data on glycemic control and treatment satisfaction of insulin bolus-patch versus insulin pen for bolus insulin delivery in adults with T2D not achieving A1C targets on basal insulin alone.

Method: This is a multi-national, randomized, 2 parallel arm, 48-week study in 312 adult patients with uncontrolled T2D (A1C: $7.5-11 \%$ ) treated with basal insulin for $\geq 6$ months and at a stable dose of basal insulin $(\geq 0.3 \mathrm{U} / \mathrm{kg})$, with or without other anti-hyperglycemic agents. A simple insulin dose-titration algorithm utilizing pattern control concepts is integrated into this study for adjustment of basal and mealtime insulin doses. The study is currently enrolling patients.

Results: The primary endpoint evaluation is change in A1C from baseline to Week 24. Additional glycemic efficacy endpoints, safety endpoints, patient-reported outcomes, and patient and provider preference surveys will also be assessed at pre-specified time points in the study. Blinded CGM will be performed to assess glycemic variability parameters in a subset of randomized patients.

Conclusion: If shown to be effective, this intervention may prove to be an innovation in primary care diabetes management.

\section{9}

\section{EXTERNAL AUDITORY CHANNEL IS A NOVEL} ROUTE FOR INSULIN ADMINISTRATION

\section{K. Khodabakhshi Pirkalani ${ }^{1}$}

${ }^{1}$ Mehr Medical Group, Internal Medicine/Gynecology, Tehran, Iran

Background and Aims: Search for novel routes to administer large molecules is continuing and oral, inhalational and dermal preparations of insulin have been tried with low success even if we look extremely optimistic. External auditory channel has a unique position as it is both internal and external to the body. It can be a nice reservoir without loss. It can be used any time without needle. It can accommodate to any schedule if appropriate techniques are used.

Method: Among twelve different insulin preparations and analogues two were chosen and 1855 patients received at least one dose around $25 \mathrm{IU}$ (5-60IU) of insulin in the last eight years. All patients have discontinued their treatment (oral or insulin) at least eight hours before and have taken breakfast 2-3 hours earlier. Insulin absorption was studied by blood concentration in only 50 patients and bioassay in terms of blood glucose monitoring every 15 minutes for 3 hours in all.

Results: All patients (100\%) showed a prominent reduction of blood glucose levels which was roughly dose dependent with a range of $35-280 \mathrm{mg} / \mathrm{dl}$ within the next $3 \mathrm{~h}$ without symptoms of hypoglycemia. There were no local or systemic side effects. Blood insulin level rose after 2 hours but there was no exact correlation with the dose used.

Conclusion: This is the first report of auditory insulin instillation. Some complementary papers will clarify how this treatment can be incorporated into a revolutionary strategy of both type 1 and 2 diabetes treatment.

\section{0}

\section{AUDITORY INSULIN INSTILLATION VIA A PIEZOELECTRIC PUMP OR A TINY STEPPER MOTOR AS A FINAL SOLUTION TO THE PROBLEM OF GLYCEMIC CONTROL AND POSSIBLY DIABETES}

\section{K. Khodabakhshi Pirkalani $^{1}$}

${ }^{1}$ Mehr Medical Group, Internal Medicine/Gynecology, Tehran, Iran

Background and Aims: External auditory channel is an ideal route for insulin instillation. It abolishes the need for continuous preparations in type 2 patients as a combination of prandial short acting insulin and oral hypoglycemic agents will control most patients (published before). Only type 1 patients will need some insulin during inter prandial periods that can be instilled in a continuous or quantized manner. A piezoelectric pump can accomplish the first task (published before); we wonder whether tiny stepper motors can do the second task with equal result.

Method: Ten type 1 patients were schedules to be treated with auditory insulin instillation as bolus pre prandial doses and 2units/h in between for 3 months. These were compared with our historical data on piezoelectric pumps. Pre- and post-study HbA1C were measured in addition to six weekly FBS and six weekly 3hpp BS measurements.

Results: There were no differences between the two groups. HbA1C levels reduced up to $1.7 \%$ after three months meaning better glycemic control with auditory insulin than multiple sc injections with a mixture of long and short acting insulins in a patient with self comparison via $t$ test. Bolus hourly insulin instillation via the ear has comparable effect to sc continuous injection and can cover patients' need for $1 \mathrm{~h}$ so that pulse insulin injection with a stepper motor is superior to piezoelectric pumps because of ease, more strict dosages and less chance of technology failure in addition to smaller size and less weight.

Conclusion: This might be the final solution to strict BS control.

\section{1}

\section{COST EFFECTIVENESS OF SIMPLE INSULIN INFUSION: ITALY}

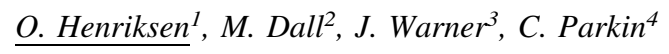

${ }^{1}$ Last Mile P/S, Copenhagen, Denmark

${ }^{2}$ CeQur SA, Horw, Switzerland

${ }^{3}$ CeQur Corporation, Marlborough, USA

${ }^{4}$ CGParkin Communications- Inc., Boulder City, USA 
Background and Aims: Continuous subcutaneous insulin infusion (CSII) in people with type 2 diabetes (T2DM) has proven to improve glycemic control (HbA1c) and reduce insulin dosage compared to multiple daily injections (MDI). However, CSII has not been widely adopted in T2DM due to costs, complexity and training requirements. New devices that provide simple CSII reduce complexity and training requirements. This analysis assessed the cost effectiveness in Italy of simple insulin infusion (SII) compared to MDI in people with T2DM not in glycemic control.

Method: The UKPDS Outcomes Model was used to project long-term cost-effectiveness over 40 years, based on results of recently published studies and direct costs for Italy. Costs and outcomes were discounted at 3\%. Cost-effectiveness was predefined in relation to per capita gross domestic product (GDP) with Incremental Cost Effectiveness Ratios (ICERs) below 1X, respectively $3 \mathrm{X}$ GDP per capita per life year gained, defined as highly 'cost-effective', respectively 'cost-effective'.

Results: Our analysis showed discounted 0.3 life year gained on average and lifetime discounted savings on complication costs and insulin reductions of $€ 13,427$. Based on projected direct costs and life expectancy, a simple CSII device will be highly costeffective in Italy at a price of $€ 3.7$ per day and cost-effective at a price up to $€ 6.5$. These estimates were very robust to sensitivity analyses on both reductions in HbA1c and dose effects.

Conclusion: For people with T2DM not in glycemic control on MDI, simple CSII is highly cost-effective at a daily cost in Italy of $€ 3.7$ and cost-effective at a daily cost of $€ 6.5$.

\section{2}

\section{COST EFFECTIVENESS OF SIMPLE INSULIN INFUSION: THE UK}

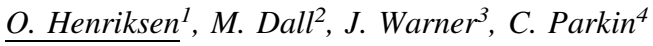 \\ ${ }^{1}$ Last Mille P/S, Copenhagen, Denmark \\ ${ }^{2}$ CeQur SA, Horw, Switzerland \\ ${ }^{3}$ CeQur Corporation, Marlborough, USA \\ ${ }^{4}$ CGParkin Communications- Inc., Boulder City, USA
}

Background and Aims: Continuous subcutaneous insulin infusion (CSII) in people with type 2 diabetes (T2DM) has proven to improve glycemic control (HbA1c) and reduce insulin dosage compared to multiple daily injections (MDI). However, CSII has not been widely adopted in T2DM due to costs, complexity and training requirements. New devices that provide simple CSII reduce complexity and training requirements. This analysis assessed the cost effectiveness in the UK of simple insulin infusion (SII) compared to MDI in people with T2DM not in glycemic control.

Method: The UKPDS Outcomes Model was used to project long-term cost-effectiveness over 40 years, based on results of recently published studies and direct costs for the UK. Costs and outcomes were discounted at 3\%. Costeffectiveness was pre-defined in relation to per capita gross domestic product (GDP) with Incremental Cost Effectiveness Ratios (ICERs) below 1X, respectively 3X GDP per capita per life year gained, defined as highly 'cost-effective', respectively 'cost-effective'.

Results: Our analysis showed discounted 0.3 life year gained on average and lifetime discounted savings on complication costs and insulin reductions of $£ 11,895$. Based on projected direct costs and life expectancy, a simple CSII device will be highly cost-effective in the UK at a price of $£ 3.43$ per day and cost-effective at $£ 6.13$. These estimates were very robust to sensitivity analyses on both reductions in $\mathrm{HbAlc}$ and dose effects.

Conclusion: For people with T2DM not in glycemic control on MDI, simple CSII is highly cost-effective at a daily cost in the UK of $£ 3.43$ and cost-effective at a daily cost of $£ 6.13$.

\section{3}

\section{VALIDATION OF ADHESIVE TAPE OPTIMIZATION OF PAQ, A SIMPLE, THREE-DAY WEARABLE BASAL/ BOLUS INSULIN DELIVERY DEVICE, IN NORMAL VOLUNTEERS}

\author{
M. Yablonski $^{1}$, J. Arsenault ${ }^{1}$, K. Barnett ${ }^{1}$, C. Brodeur ${ }^{1}$, \\ A. Cropp ${ }^{I}, J$. Shelsky ${ }^{2}$ \\ ${ }^{1}$ CeQur Corporation, Marlborough, USA \\ ${ }^{2}$ CeQur Corporation, Jessica.Shelsky@cequr.com, USA
}

Background and Aims: To optimize adhesive tape conformity, Engineers of $\mathrm{PAQ}^{\circledR}$ (CeQur SA), a simple, three-day wearable basal/bolus insulin delivery device, developed a mechanical test system to assess variations of tape-to-device fixation methods and material stretch properties. To validate the optimal adhesive fixation method and stretch orientation combination, a test was conducted on human subjects.

Method: A diverse group of 30 active, normal volunteers wore two devices - one with the test combination and one control-for a single three-day wear period. The performance of each tape was evaluated by quantifying the non-adhered surface area, depth of skin indentation at the cannula insertion site and the occurrence and severity of dermal irritation, if present. A comfort survey was also completed after the three-day wear period.

Results: $93 \%$ of participants had acceptable adherence with the optimized adhesive compared to $73 \%$ with the control. The skin indentation depth with the test adhesive was similar to the control, and no dermal irritation was reported with either adhesive. Furthermore, there was a decrease in discomfort, pain, and annoyance. All participants felt that neither adhesive exceeded minor discomfort, pain nor annoyance, however, a higher percentage of the population rated the test adhesive superior to the control.

Conclusion: The optimized adhesive tape fixation method and material stretch orientation combination derived from the proprietary mechanical test system improved both adherence and comfort in normal volunteers. Additional studies will be conducted to confirm these results.

\section{4}

\section{TECHNOLOGY SOLUTIONS TO INCREASE INSULIN STABILITY IN CASE OF EXTREME WEATHER CONDITIONS}

\section{A. Pfützner ${ }^{1}$, R. Nagar ${ }^{2}$}

${ }^{1}$ Pfützner Science \& Health Institute, Diabetes Center and Practice, Mainz, Germany

${ }^{2}$ Tempramed Inc., R\&D, Half Moon Bay, USA

Background and Aims: Climate change results in large-scale disruptions in climate with disastrous consequences, such as the hurricane Katrina. Affected people with diabetes were forced to struggle with unspeakable conditions and a lack of medical supplies. One key issue amongst others was the lack of stability of insulin. Insulin is to be kept at $4^{\circ}-8^{\circ} \mathrm{C}$ until use and can be used for one month at room temperature. Extreme cold or 
extreme heat lead to insulin degradation and loss of efficacy. Potential solutions for this situation are efforts to enhance the stability of insulin by formulation changes, which would most probably require an entire new approval process. Another option could be to identify mechanical or electronic engineering solutions.

Method: We developed a calorimetry-based small storage device, which fits all existing pens and vials. It uses existing insulation materials and has a thermo-electronic control element that can switch on and off to maintain the temperature inside the pen or insulin vial. We performed a proof-of-concept refrigeration test, where we kept the pen content at $8^{\circ} \mathrm{C}$ while the device was exposed to $25^{\circ} \mathrm{C}$ and a heating test, where the device was exposed to $42^{\circ} \mathrm{C}$.

Results: In both refrigeration and heating tests, it was possible to maintain the device content at the specified temperature over several days while spending only minimal energy.

Conclusion: Our results indicate that our carriage device operates at a power level that can be provided by a small rechargeable battery. We expect to further improve these results in the final development process.

\section{5}

\section{USE OF INSUPAD IN DAILY ROUTINE BY PATIENTS WITH TYPE 1 DIABETES}

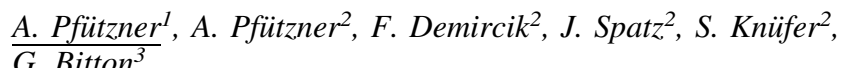

${ }^{1}$ Pfützner Science \& Health Institute, Diabetes Center and Practice, Mainz, Germany

${ }^{2}$ Sciema $U G, R \& D$, Mainz, Germany

${ }^{3}$ Insuline Medical Ltd., R\&D, Petach-Tikva, Israel

Background and Aims: Improvement of blood flow at the insulin injection site as induced by the InsuPad device results in more efficient and consistent uptake of prandial insulin. This effect was associated with better glycemic control, less hypoglycemic events and lower prandial insulin dose requirements in a previous controlled clinical study. The purpose of this observational study was to investigate the device effects, when used in daily routine by people with type 1 diabetes (T1DM).

Method: A post-marketing observation study was conducted in Germany in 2014 and all reported cases until the end of December with people with T1DM using short-acting insulin ana$\operatorname{logs}$ for prandial insulin treatment were included into this analysis. Observation parameters at baseline and after 3 months included HbA1c, body weight, frequency of hypoglycemia $(<63 \mathrm{mg} / \mathrm{dL})$ and hyperglycemia $(>250 \mathrm{mg} / \mathrm{dL})$ and adverse events.

Results: Sixty people with T1DM were included into this analysis (29 female, HbA1c: $8.7 \pm 1.4 \%$, body weight: $109 \pm 22 \mathrm{~kg}$ ). During the observation period, there was a reduction in HbA1c $(-0.43 \pm 0.65 \%, \mathrm{p}<0.001)$ and in body weight $(-0.51 \pm$ $0.65 \mathrm{~kg}$ ). Frequency of hypoglycemia and hyperglycemia decreased in parallel $(-37 \%$ and $-34 \%$, respectively). Participants reported lower prandial and basal insulin dose requirements $(-12.4 \%$ and $-10.3 \%$, respectively; both $\mathrm{p}<0.001)$ at the end of the observation period.

Conclusion: The results from this post-marketing surveillance study confirm that the controlled clinical trial results also translate into similar benefits when the device is used in daily routine practice by people with T1DM.
306

\section{IMPACT OF INSUPAD ON GLYCEMIC CONTROL IN PATIENTS USING REGULAR HUMAN INSULIN IN DAILY ROUTINE PRACTICE}

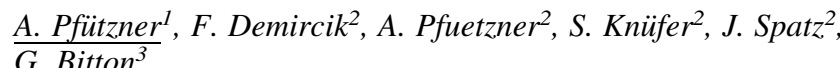 \\ ${ }^{1}$ Pfützner Science \& Health Institute, Diabetes Center and \\ Practice, Mainz, Germany \\ ${ }^{2}$ Sciema $U G, R \& D$, Mainz, Germany \\ ${ }^{3}$ Insuline Medical Ltd., R\&D, Petach-Tikva, Israel
}

Background and Aims: The InsuPad device enhances prandial insulin absorption by injection site modulation with a standardized warming protocol. The purpose of this analysis was to explore the impact of using the device in intensive insulin treatment with regular human insulin on glycemic control in a real-world setting.

Method: A post-marketing observation study was conducted in Germany in 2014 and all reported cases until the end of December with type 2 diabetes (T2DM) using regular human insulin for prandial insulin treatment were included into this analysis. Observation parameters at baseline and after 3 months included $\mathrm{HbA1c}$, body weight, frequency of hypoglycemia $(<63 \mathrm{mg} / \mathrm{dL})$ and hyperglycemia $(>250 \mathrm{mg} / \mathrm{dL})$, and adverse events.

Results: Meeting the inclusion criteria were 76 participants (33 female, HbA1c: $8.9 \pm 1.7 \%$, body weight: $113 \pm 25 \mathrm{~kg}$. During the observation period, reductions in $\mathrm{HbA} 1 \mathrm{c}$ by $0.7 \pm 1.1 \%$ $(\mathrm{p}<0.001)$ and in body weight by $1.3 \pm 4.8 \mathrm{~kg}(\mathrm{p}<0.05)$ were observed. In parallel, monthly hypoglycemia and hyperglycemia rates were reduced $(-49 \%$ and $-41 \%$, respectively). Prandial and basal insulin doses were reduced by $-14.6 \%$ and $-9.7 \%$, respectively (both $\mathrm{p}<0.001$ ). Device use was well tolerated with only four reports of skin reactions to the device adhesive.

Conclusion: Use of InsuPad device in daily treatment routine in people with T2DM on intensive insulin treatment using regular human insulin as prandial insulin component resulted in improvement in glycemic control (better HbA1c, less hypoglycemia, lower insulin dose requirements) similar to the results with short-acting insulin analogs in a previously conducted randomized controlled clinical study.

\section{7}

\section{INJECTION PAIN AND ANXIETY IN CHILDREN AND ADOLESCENTS WITH TYPE 1 DIABETES: CAN AN INDWELLING CATHETER (I-PORT ADVANCE®) IMPROVE METABOLIC CONTROL AND QUALITY OF LIFE?}

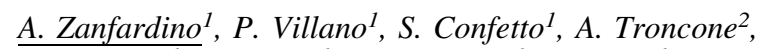
E. Caredda', A. Coccal , F. Casaburo ${ }^{1}$, L. Russo' ${ }^{1}$, A. Piscopo ${ }^{1}$, C. Cascella ${ }^{3}$, A. Chianese ${ }^{2}$, M. Giglio ${ }^{2}$, L. Perrone ${ }^{1}$, D. Iafusco ${ }^{1}$

${ }^{1}$ Second University of Naples, Department of Pediatrics, Naples, Italy

${ }^{2}$ Second University of Naples, Department of Psychology, Naples, Italy

${ }^{3}$ Second University of Naples, Department of Psycohlogy, Naples, Italy

Background and Aims: Preventing the development of diabetes complications is primary goal in patients with type 1 diabetes, but not the only one. Achieving good quality of life is another central 
goal. When diabetes is diagnosed, multiple injections become a part of daily life. It is estimated that $22 \%$ of general population has needle phobia, often shared by several family members. So, injection therapy may be daunting, severely impacting the ability to self manage diabetes. After all, also in no phobic patients multipleinjections therapy can induce discomfort and pain.

We evaluated the effects of an indwelling catheter on metabolic control and quality of life in children and adolescents with type 1 diabetes and in their mothers.

Method: We enrolled 100 patients with type 1 diabetes aged from 15 months to 16 years. We analyzed metabolic control (HbA1c), parental burden and treatment satisfaction (PAID, WECARE) and parents' and patients' quality of life (PedsQL) every 3 months for one year after study's starting.

Results: HbA1c significantly improved 3 months after the application of the device $(\mathrm{p}=0.002)$. This improvement was preserved up to 1 year.

The percentage of mothers with distress when giving injections was high (43.5\%). Most improved over time (at 1 year only $8 \%$ ).

Before the starting of the study, 53\% of children did not cooperate during insulin injections. At 1 year only $3 \%$ of children showed stress for injections.

Conclusion: We can conclude that the use of i-Port Advance improves the quality of life of caregivers and children, as well as the metabolic control.

\section{8}

IMPACT OF INJECTION VOLUME, SPEED AND SITE ON PAIN SENSATION

\section{E. Zijlstra $^{1}$, J. Jahnke ${ }^{2}$, A. Fischer ${ }^{1}$, C. Kapitza ${ }^{1}$, T. Forst ${ }^{2}$}

${ }^{1}$ Profil, Neuss, Neuss, Germany

${ }^{2}$ Profil, Mainz, Mainz, Germany

Background and Aims: Painful subcutaneous (s.c.) insulin injections may decrease treatment compliance. Improving injection comfort therefore represents a particular area of recent technological research. Injection pain can be influenced by many variables, but relatively little is known about their impact. This study investigated the impact of injection volume (range $0-2250 \mu \mathrm{L}$ ), speed (range $0-800 \mu \mathrm{L} / \mathrm{sec}$ ) and site (abdomen vs. thigh) on pain sensation.

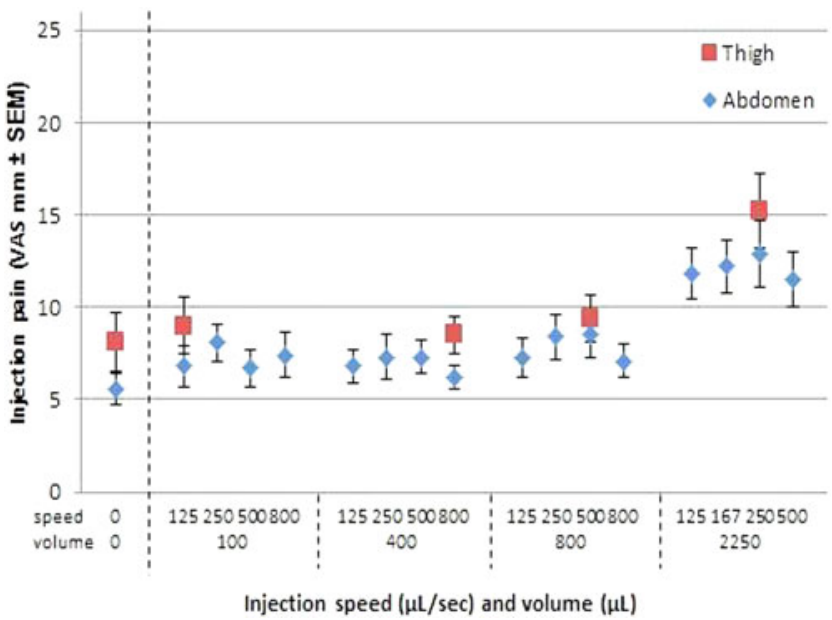

Method: In random order, patients $(n=79)$ with type 1 or type 2 diabetes received 24 saline injections s.c. through a $27 \mathrm{G}$ ultrathin-wall needle. Injections in the abdomen covered all planned speed-volume combinations, while thigh injections for site evaluation were limited to five to minimise the burden of trial participation on patients. Patients scored their pain sensation on a $100 \mathrm{~mm}$ visual analog scale (VAS) after each injection.

Results: The mean pain scores for volume and speed combinations were all in the lower part $(<20 \mathrm{~mm})$ of the VAS, indicating zero to mild pain (Figure). Pain sensation was statistically $(\mathrm{p}<0.001)$ higher with the $2250 \mu \mathrm{L}$ injection volume $(+5 \mathrm{~mm})$ and with injection in the thigh $(+2 \mathrm{~mm})$. Pain sensation did not change with increasing injection speed. Patient acceptance of the injection pain was very high for all injections (range 93.7$98.7 \%)$, although slightly worse $(-2.6 \%)$ for large volume injections.

Conclusion: In summary, large volume and thigh injections are rated more painful, but the clinical impact of these findings is likely marginal considering the low absolute pain levels and high patient acceptance rates. Injection speed does not influence pain sensation.

\section{9}

\section{BACKFLOW AFTER DRUG INJECTION:} IMPACT OF INJECTION VOLUME, SPEED AND SITE

$\underline{\text { E. Zijlstra }}^{1}$, J. Jahnke ${ }^{2}$, A. Fischer ${ }^{l}$, C. Kapitza ${ }^{1}$, T. Forst ${ }^{2}$

\section{${ }^{1}$ Profil, Neuss, Neuss, Germany}

${ }^{2}$ Profil, Mainz, Mainz, Germany

Background and Aims: After injection of a liquid drug formulation such as insulin, backflow of fluid (leakage from the skin) is frequently observed. Any leakage will obviously influence the amount of drug absorbed and could consequently affect its action. This study investigated the impact of injection volume (range $0-2250 \mu \mathrm{L}$ ), speed (range $0-800 \mu \mathrm{L} / \mathrm{sec}$ ) and site (abdomen vs. thigh) on the amount of backflow.

Method: In random order, patients $(n=79)$ with type 1 or type 2 diabetes received 24 saline s.c. needle injections. Thigh injections for site evaluation were limited to five to minimise patient burden. After each injection, the injection site was examined visually for backflow. Any liquid was quantitated by comparing the wet spot on an absorbent paper strip to a reference set of paper strips.

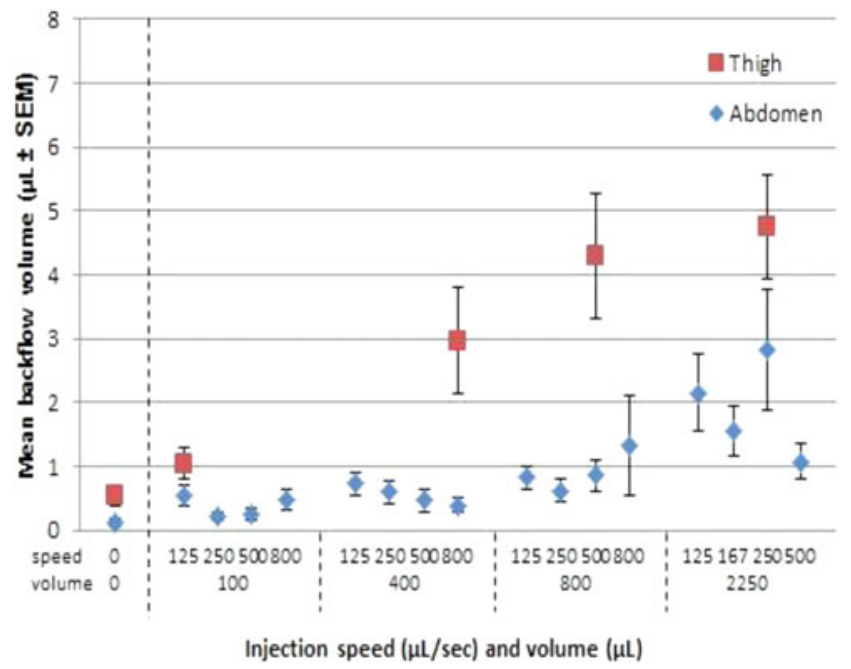


Results: After 1869 injections, backflow was observed 621 times $(33 \%)$. Backflow observations and mean volume significantly $(\mathrm{p}<0.0001)$ increased with increasing injection volume, but not with increasing speed, and more backflow was observed after injection in the thigh compared to the abdomen (Figure). Although mean volumes were generally low $(<5 \mu \mathrm{L})$, in individual cases maximum backflow volumes represented large portions of the injected volumes: up to $8.5 \%$ in the abdomen and up to $13 \%$ in the thigh (for $100 \mu \mathrm{L}$ injection volume).

Conclusion: In summary, backflow after drug injection is not uncommon and more is observed after large volume and thigh injections. Injection speed does not influence backflow. The clinical impact of backflow will generally be low, but larger backflow volumes observed after individual injections could compromise dosing accuracy.

\section{0}

\section{THE EFFECT OF FE3O4 NANOPARTICLES (NPS) ON INSULIN AMYLOID-LIKE FIBRILLOGENESIS}

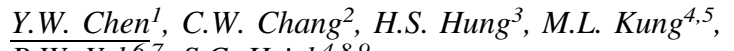
B.W. Yeh ${ }^{6,7}$, S.C. Hsieh ${ }^{4,8,9}$

${ }^{1}$ National Cheng Kung University, Pharmacology, Tainan, Taiwan

${ }^{2}$ National Sun Yat-Sen University, Department of Chemistry, Kaohsiung- Taiwan, Taiwan

${ }^{3}$ China Medical University, Graduate Institute of Basic Medical Science, Taichung, Taiwan

${ }^{4}$ National Sun Yat-Sen University, Department of Chemistry, Kaohsiung, Taiwan

${ }^{5}$ Kaohsiung Medical University- Kaohsiung, Center for Stem Cell Research, Kaohsiung, Taiwan

${ }^{6}$ Kaohsiung Medical University Hospital, Department

of Urology, Kaohsiung, Taiwan

${ }^{7}$ School of Medicine-College of Medicine, Depatment

of Urology, Kaohsiung, Taiwan

${ }^{8}$ Kaohsiung Medical University, School of Pharmacy-College of Pharmacy, Kaohsiung, Taiwan

${ }^{9}$ Kaohsiung Medical University, Center for Stem Cell Research, Kaohsiung- Taiwan, Taiwan

Background and Aims: Many age-related degenerative diseases, including Alzheimer's disease and type II diabetes are associated with the accumulation of amyloid fibrils. Various potential approaches have been reported to decrease or eliminate amyloid fibrillation processes, including small molecules, functional polymers, and nanoparticles. Magnetic nanoparticles have been developed for many medical applications. However, so far only a few studies are related to magnetic nanoparticles and amyloid protein aggregation.

Method: In this present study, insulin was chosen as a model of amyloidogenic protein to investigate the effects of $\mathrm{Fe}_{3} \mathrm{O}_{4}$ nanoparticles on the fibrillogenesis process of insulin fibrils.

Results: Here, we showed that $\mathrm{Fe}_{3} \mathrm{O}_{4}$ NPs didn't induce the aggregation of insulin fibrils. The zeta potential of insulin fibrils and $\mathrm{Fe}_{3} \mathrm{O}_{4}$ is positive, suggesting the binding force between $\mathrm{Fe}_{3} \mathrm{O}_{4}$ NPs and insulin fibrils is Van der Waals force. Moreover, the removal of the insulin amyloid fibrils from its continuous phase by magnetization reveals $\mathrm{Fe}_{3} \mathrm{O}_{4}$ bound to insulin fibrils during the fibril reaction.

Conclusion: These results offer significant opportunities for developing nanoparticle-based therapeutics against diseases related to protein aggregation, such as type II diabetes.
311

\section{DIHYDROCHOLESTEROL LOWERS PLASMA CHOLESTEROL IN HYPERCHOLESTEROLEMIA HAMSTER}

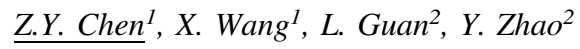

${ }^{1}$ Chinese University of Hong Kong, School of Life Sciences, Hong Kong, China

${ }^{2}$ Nestle Research Center, $R \& D$, Beijing, China

Background and Aims: Diabetic patients tend to have higher concentrations of plasma total cholesterol (TC) and triacylglycerols (TG) called diabetic dyslipidemia. One of the approaches in reducing plasma TC is to inhibit intestinal cholesterol absorption by taking sterol analogs such as $\beta$-sitosterol (SI). Dihydrocholesterol (DC) is a cholesterol analog having a same side chain with no double bond at the $\Delta^{5}$ position in B-ring. The present study was to test if DC could reduce plasma TC in hypercholesterolemia hamsters.

Method: Male hamsters $(n=45)$ were randomly divided into 6 groups, fed a non-cholesterol diet (NCD), or a high-cholesterol diet (HCD), or one of the four experimental diets supplemented with $0.2 \%$ DC, $0.3 \%$ DC, $0.2 \%$ SI and $0.3 \%$ SI, respectively, for 6 weeks. The following parameters were measured including $\mathrm{TC}, \mathrm{TG}$, atherosclerotic plaque, fecal neutral and acidic sterols.

Results: Results showed that $0.2 \% \mathrm{DC}$ in diet could reduce TC by $21 \%$, similar to that of SI (19\%). Both DC and SI were able to reduce atherosclerotic plaque in thoracic aorta. Compared with SI, DC was more effective in stimulating the fecal neutral sterol excretion, leading to a better hepatic cholesterol clearance. In vitro micelles incorporation study, results showed that DC was more potent in displacing cholesterol from micelle, leading to a higher fecal neutral sterol excretion.

Conclusion: It was concluded that DC could reduce plasma TC and further study should be conducted to explore its application in hypercholesterolemia management for diabetic dyslipidemia patients.

\section{2}

\section{COMBINED FIXED-DOSE GALVUS ${ }^{\circledR}$ (VILDAGLIPTIN) AND METFORMIN TREATMENT IN TYPE 2 DIABETES}

N. Gegeshidze ${ }^{1}$, K. Tchaava ${ }^{2}$, R. Tabukashvili ${ }^{2}$, $\overline{V_{\text {. Kapetivadze }}}$, Z. Maglapheridze $e^{3}$, T. Lazashvili ${ }^{4}$

${ }^{1}$ Tbilisi, Georgia

${ }^{2}$ Tbilisi State Medical University, Propedeutics, Tbilisi, Georgia

${ }^{3}$ TSMU, Propedeutics, Tbilisi, Georgia

${ }^{4}$ TSMU, Propedeutcs, Tbilisi, Georgia

Background and Aims: To investigate the efficacy and safety of combined Galvus ${ }^{\circledR}$ (Vildagliptin) and Metformin therapy in patients with type 2 diabetes mellitus (T2DM).

Method: A total of 65 patients with T2DM inadequately controlled with metformin, were enrolled. They were given combination of Galvus ${ }^{\circledR}$ (Vildagliptin) $(50 \mathrm{mg}$ ) and Metformin (500 mg) during 12 weeks. Change in serum glycated haemoglobin (HbA1c), fasting plasma glucose (FPG) levels; weight; waist circumference and hypoglycemic episodes were evaluated. 
Results: HbA1c levels $(9.24 \% \pm 0.56 \%)$ were significantly reduced after 12 weeks of treatment $(6.48 \% \pm 0.48 \%)$. Fasting plasma glucose levels decreased by $1.85 \pm 1.14 \mathrm{mmol} / \mathrm{l}$. No severe hypoglycemic events were reported. Body mass index reduced by $0.85 \pm 1.28 \mathrm{~kg} / \mathrm{m}^{2}(\mathrm{p}<0.001)$.

Conclusion: Combined Galvus ${ }^{\circledR}$ (Vildagliptin) and Metformin therapy significantly improved long-term glycemic control in patients with T2DM during the period of 12 weeks without additional risk of hypoglycemic events or weight gain.

\section{3}

EXPERT STUDY: UTILITY OF AN AUTOMATED BOLUS ADVISOR SYSTEM IN PATIENTS WITH TYPE 1 DIABETES TREATED WITH MULTIPLE DAILY INJECTIONS OF INSULIN. A CROSSOVER STUDY

C. Gonzalez-Blanco $^{1}$, M.J. Picon ${ }^{2}$, J.C. Fernandez ${ }^{2}$, I. Pujol ${ }^{3}$, A. Chico $^{1}$

${ }^{1}$ Hospital de la Santa Creu i Sant Pau, EndocrinologyEDUAB-HSP-CIBER-BBN, Barcelona, Spain

${ }^{2}$ Hospital Virgen de la Victoria, Endocrinology, Malaga, Spain

${ }^{3}$ Hospital de la Santa Creu i Sant Pau, Endocrinology,

Barcelona, Spain

Background and Aims: To asses the impact of the use of an automated bolus advisor (ABA) on glycemic control, quality of life and satisfaction in adult patients with type 1 diabetes (T1DM) treated with multiple daily injections (MDI).

Method: A crossover, prospective, randomized, controlled, multicentre study of 36 weeks duration was conducted. Patients were randomized to start in control phase (CP) using a traditional blood glucose meter to calculate insulin doses (Accu-Chek Aviva Nano), or intervention phase (IP) using an ABA meter (Accu-Chek Aviva Expert) and switched to the other phase after a wash-out period. Each phase had 12 weeks duration.

Results: Significant reduction in $\mathrm{HbA}_{1 \mathrm{c}}$ was observed in both phases (CP: initial $\mathrm{HbA}_{1 \mathrm{c}} 8.05 \pm 0.7 \%$, final $\mathrm{HbA}_{1 \mathrm{c}} 7.59 \pm 0.7 \%$, $\mathrm{p}<0.001$; IP: initial $\mathrm{HbA}_{1 \mathrm{c}} 8.13 \pm 1 \%$, final $\mathrm{HbA}_{1 \mathrm{c}} 7.61 \pm 0.8 \%$, $\mathrm{p}<0.001)$. Although the trend was a higher reduction in $\mathrm{HbA}_{1 \mathrm{c}}$ in IP, no statistically significant differences were observed between phases $\left(\mathrm{CP} ; \mathrm{HbA}_{1 \mathrm{c}}-0.39 \%\right.$, IP $-0.52 \%(\mathrm{p}=0.8)$. During IP, daily glucose measurements were higher (4.28 \pm 1.2 vs. $4.01 \pm 1.1, \mathrm{p}<0.006$ ), postprandial hypoglycemias were lower and an improvement in quality of life and higher satisfaction was observed.

Conclusion: In this first crossover study comparing the use of an ABA with the standar usual care, the use of an ABA was effective and well accepted and a reduction in hypoglycaemic events, improvement in adherence and quality of life and higher treatment satisfaction were observed.

\section{4}

CADABA FARINOSA CONTAIN COMPOUNDS OF NATURAL ORIGIN WITH POTENT DIPEPTIDYL PEPTIDASE-IV (DPP-IV) INHIBITORS USED TO TREAT TYPE II DIABETES

$\underline{\text { S. Ibrahim Ismail }}^{1}$

${ }^{1}$ Federal University Dutse, Biochemistry, Dutse, Nigeria
Background and Aims: Cadaba farinosa leaves are being used locally as natural folk medicine for the treatment of diabetes but until now no scientific investigation has been reported of its potential anti-diabetic effect. Dipeptidyl peptidase IV (DPP-IV) inhibitors are potential candidates for the treatment of type 2 diabetes mellitus. Though there are few commercial DPP-IV inhibitors (Vildagliptin, Sitagliptin and Saxagliptin recently launched as Onglyza) are available in the market, they have some undesirable side effects. Therefore our study had focused to screen the leaves of cadaba farinosa, extract and purify the active fractions for the presence of amino-nitrile-containing compounds. Since several amino-nitriles containing compounds have been developed as reversible inhibitors of dipeptidyl peptidase (DPP IV) for treating diabetes.

Method: The leaves were extracted using successive soxhlet extraction technique. The Cadaba farinosa leaf extract were further subjected to column chromatography and purified fractions were analysed using GC-MS (Gas chromatography-Mass spectroscopy) and FTIR (Fourier Transform Infrared Spectrophotometer).

Results: Among the n-Hexane, ethyl acetate and methanol fractions screened, we have identified the methanolic extract of the leaves of Cadaba farinosa to contain Amine $\left(\mathrm{NH}_{2}\right)$ and $\mathrm{Ni}$ trile $(\mathrm{CN})$ functional groups that has the ability to inhibit dipeptidyl peptidase IV using infrared spectra. Also GC-MS Analysis revealed the presence of new compounds having Amino-Nitrile functional groups.

Conclusion: The study revealed that the methanol leaf extract of Cadaba farinosa contain compounds which exhibits chemical similarity with a large group of known DPP-IV inhibitors. Therefore the leaves may possess significant DPP-4 inhibitory activity which should be further investigated for their antidiabetic potential.

\section{5}

\section{INHIBITORY EFFECT OF GPR119 AGONIST ON HEPATIC STEATOSIS}

\author{
K.W. Kang ${ }^{1}$, J.W. Yang ${ }^{1}$ \\ ${ }^{1}$ Seoul National University, Pharmacy, Seoul, Republic \\ of Korea
}

Background and Aims: Non-alcoholic fatty liver disease (NAFLD) is associated with metabolic syndrome and has the unique characteristic of excess lipid accumulation in the liver. G-proteincoupled receptor 119 (GPR119) is a promising target for type 2 diabetes. However, whether activating GPR119 regulates hepatic steatosis and the precise mechanism has not been investigated.

Method: GPR119 expression was assessed by immunofluorescence of human and mouse liver samples and conventional polymerase chain reaction (RT-PCR) analyses of hepatocytes isolated from wild-type (WT) and GPR119 knockout (KO) mice. Anti-steatotic effects of GPR119 agonists was estimated using primary cultured mouse hepatocyte, human HepG2 cells and GPR119-WT and -KO mice fed a high fat diet (HFD).

Results: In primary cultured hepatocytes from wild-type (WT) and GPR119 knock-out (KO) mice, expression of lipogenic enzymes was elevated in GPR119 KO hepatocytes. Treatment of hepatocytes and HepG2 cells with GPR119 agonists in phase II clinical trials (MBX-2982 and GSK1292263) inhibited protein expression of both nuclear and total sterol response-element binding protein (SREBP)-1c, a key lipogenesis transcription factor. Oral administration of MBX-2982 and 
GSK1292263 in mice fed a high fat diet potently inhibited hepatic lipid accumulation and expression levels of SREBP-1c and lipogenesis-related genes, whereas the hepatic anti-lipogenesis effects of MBX-2982 were abolished in GPR119 KO mice. MBX-2982 activated AMP-activated protein kinase (AMPK) and increased Ser-372 phosphorylation of SREBP-1c, an inhibitory form of SREBP-1c. Moreover, inhibiting AMPK recovered SREBP-1c down-regulation by MBX2982.

Conclusion: These findings demonstrate for the first time that the GPR119 ligand alleviates hepatic steatosis by inhibiting SREBP-1c-mediated lipogenesis in hepatocytes.

\section{6}

NEPHROPROTECTIVE EFFECT OF PAEONIA EMODI
VIA INHIBITION OF ADVANCED GLYCATION END
PRODUCTS AND OXIDATIVE STRESS IN STZ-
NICOTINAMIDE INDUCED DIABETIC
NEPHROPATHY

L. Kishore $^{1}$, N. Kaur ${ }^{2}$, R. Singh ${ }^{1}$

${ }^{1}$ M.M.University, M.M.College of Pharmacy, ambala cantt, India

${ }^{2}$ M.M. College of Pharmacy, M.M. University- Mullana, Ambala, India

Background and Aims: Chronic hyperglycemia leads to the generation of reactive oxygen species leading to oxidative stress. Formation of advanced glycation end products (AGEs) along with oxidative stress leads to cellular damage and development of diabetic nephropathy (DN). Paeonia emodi have been widely used in traditional medicine in India to treat various diseases like epilepsy, dropsy, diabetes etc. The present study was aimed to evaluate alcohol (PA) and hydro-alcohol (PHA) extract of $P$. emodi roots in treatment of diabetic nephropathy.

Method: Diabetes was induced in male wistar rats by streptozotocin $(65 \mathrm{mg} / \mathrm{kg}$ i.p. $) 15 \mathrm{~min}$ after Nicotinamide $(230 \mathrm{mg} / \mathrm{kg}$, i.p.) administration. After 30 days of diabetes induction, the rats were treated with different doses of extracts (100, 200 and $400 \mathrm{mg} / \mathrm{kg}$ ) for 45 days in order to analyze their nephroprotective effect, by means of serum glucose, urea, uric acid, creatinine level and formation of AGEs in kidneys. Tissue antioxidant activity was assessed by measuring level of SOD, CAT, GSH, LPO along with their histopathological examination.

Results: Administration of PA and PHA produced significant attenuation in the serum glucose level, uric acid, creatinine and lipid levels. Moreover there is improvement in the level of SOD, CAT, GSH and decrease lipid peroxidation in terms of TBARS. The formation of AGEs in kidneys was also significantly reduced. Moreover, PA and PHA improved the structural abnormalities in kidney, liver and pancreas of DN rats.

Conclusion: The results obtained in this study underline the potential of $P$. emodi as a possible therapeutic agent against DN.

\section{7}

\section{THE INTENSITY OF OXIDATIVE STRESS IN CAUCASIANS AND MONGOLOIDS WOMEN WITH DIABETES MELLITUS TYPE 1}

L.I. Kolesnikova $^{1}$, S.I. Kolesnikov ${ }^{1}$, M.A. Darenskaya ${ }^{1}$, $\overline{\text { L.A. Grebenkina }}^{1}$, M.I. Dolgikh ${ }^{1}$, S.V. Gnusina ${ }^{2}$

${ }^{1}$ Scientific Centre for Family Health and Human Reproduction Problems, Department of Pathophysiology, Irkutsk, Russia
${ }^{2}$ Scientific Centre for Family Health and Human Reproduction Problems, Department of Endocrinology, Irkutsk, Russia

Background and Aims: Incidence, severity, and rate of complications of diabetes mellitus type 1 (T1DM) are associated with many factors including geographic location and ethnicity. The aim of this study was to compare oxidative stress intensity in Caucasians and Mongoloids women with T1D.

Method: The study included reproductive age women with T1DM-Caucasians $(n=15$, duration of the disease $-12.8 \pm 2.0$ years $)$ and Mongoloids $(n=15$, duration of the disease - 12.1 \pm 1.5 years). 20 Caucasians and 22 Mongoloid women were control groups. Spectrophotometric and fluorometric methods used. Oxidative stress coefficient (COS) which represent ratio of blood lipid peroxidation products to general antioxidative activity applied.

Results: Evaluation of lipid peroxidation in T1DM Caucasians women compared to the control one revealed significant increase of conjugated dienes $(p=0.0029)$ and thiobarbituric acid reactants levels $(\mathrm{p}=0.0062)$. Analysis of the antioxidant defense system in Caucasians with T1DM demonstrated significant decrease of superoxide dismutase activity $(p=0.0011)$ and increase of oxidized glutathione level $(\mathrm{p}=0.0053)$ compared to control group. Mongoloids with T1DM had increased content of conjugated dienes, compared to control group $(p=0.0005)$. Mongoloids with T1DM had significant increase of integral parameter - blood total antioxidant activity $(\mathrm{p}<0.0001)$. Intensity of oxidative stress comparison in two ethnic groups demonstrated 8.88 times $(p=0.0005)$ increase of COS in Caucasians with T1DM compared to control one. Mongoloids with T1DM had 4.64 times $(\mathrm{p}=0.0006)$ COS increasing compared to control.

Conclusion: The data may indicate ethnic peculiarities of metabolic processes in women with T1DM: increasing of antioxidant factors in Mongoloids and prooxidant factors in Caucasians.

\section{8}

\section{ANTIOXIDANT STATUS IN REPRODUCTIVE AGE WOMEN WITH DIABETES MELLITUS TYPE 1}

L. Kolesnikova $^{1}$, S.I. Kolesnikov
L.A. Grebenkina

${ }^{1}$ Scientific Centre for Family Health and Human Reproduction Problems, Department of Pathophysiology, Irkutsk, Russia

Background and Aims: Diabetes mellitus type 1(T1DM) has a major impact on the function of the reproductive system. Evaluation of oxidative processes is relevant for prognosis of the disease and the purpose of antioxidants in the T1DM treatment. This study aimed to investigate the antioxidant status parameters in reproductive age women with T1DM.

Method: The study involved 23 women with T1DM (mean age $-28.2 \pm 0.4$ years, duration of T1DM $-5.5 \pm 0.7$ years) and 23 corresponding age women in the control group. The T1DM diagnosis was confirmed by clinical and laboratory studies, patients average level of glycated hemoglobin was $9.1 \pm 1.31 \%$. Patients with significant comorbid somatic diseases, as well as patients with severe manifestations of diabetic complications (chronic renal failure, macroangiopathy) were excluded from study. Spectrophotometric, fluorometric, statistical methods were applied.

Results: Patients with T1DM had increased value of thiobarbituric acid reactants (1.6 times higher, $\mathrm{p}=0.006)$, reduced 
levels of $\alpha$-tocopherol (1.45 times lower, $p=0.014)$ and retinol (1.28 times lower, $\mathrm{p}=0.004)$, the differences in glutathione status not found. Because of possible significant impact of these antioxidants on various parts of reproductive system, menstrual function in patients with T1DM and deficit of antioxidant factors evaluated. 83.33\% of T1DM women with deficit of $\alpha$-tocopherol and $90.91 \%$ T1DM women with low retinol level had menstrual dysfunctions.

Conclusion: Because of adaptive mechanisms failures revealed in the course of T1DM we can recommend include antioxidant drugs in the complex therapy of T1DM.

319

HIGHER SERUM 25-HYDROXYVITAMIN D CONCENTRATION IS ASSOCIATED WITH LOWER RISK OF POOR GLYCEMIC CONTROL IN ASIAN TYPE 2 DIABETES PATIENTS: AN OBSERVATIONAL COHORT STUDY

${\underline{S . F . K u o^{I}}}^{1}$

${ }^{1}$ Chang Gung Memorial Hospital, Endocrinology and Metabolism, Keelung City, Taiwan

Background and Aims: Most studies on vitamin D status in diabetes have focused on incidence of type 2 diabetes mellitus (DM), while studies exploring associations between vitamin D status and diabetes associated complications have been limited. We therefore conducted an observational cohort study to address this issue.

Method: We measured serum 25(OH)D levels, both in winter and summer, and determined average values for data analysis to gain more realistic representations of vitamin D status. This study enrolled 290 type 2 DM patients who were seen in diabetes clinics in Chang Gung Memorial Hospital (Keelung, Taiwan). All participants were followed up strictly for one year.

Results: Multivariate analysis showed that among type 2 DM patients, the lowest quintile for serum $25(\mathrm{OH}) \mathrm{D}$ concentration $(\leq 18.1 \mathrm{ng} / \mathrm{mL})$ included a greater proportion of younger patients (taking 61 years of age as cut-off value; odds ratio $[\mathrm{OR}]=4.885$ ), was predominantly female $(\mathrm{OR}=2.589)$, and included greater proportions of patients with higher diastolic blood pressure (taking $71 \mathrm{mmHg}$ as cut-off value, $\mathrm{OR}=3.592$ ) and hemoglobin A1c values (taking $7.7 \%$ as cut-off value, $\mathrm{OR}=6.071$ ), compared with those in the highest quintile ( $>32.16 \mathrm{ng} / \mathrm{mL})$.

Conclusion: Based on the results, we suggest that type $2 \mathrm{DM}$ patients with low serum $25(\mathrm{OH}) \mathrm{D}$ concentration $(\leq 18.1 \mathrm{ng} / \mathrm{mL})$ had higher risk of poor glycemic control and high blood pressure compared with patients with high serum $25(\mathrm{OH}) \mathrm{D}$ concentration (>32.16 ng/mL). However, whether type $2 \mathrm{DM}$ patients with low serum 25(OH)D concentration will benefit from vitamin D supplement needs to be further clarified.

\section{0}

SYNTHESIS AND BIOLOGICAL EVALUATION OF PICOLINAMIDES AS POTENT INHIBITORS OF 11BHYDROXYSTEROID DEHYDROGENASE TYPE 1 (11BHSD1)

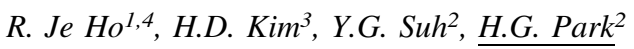

${ }^{1}$ SK Chemicals, Life Science R\&D Center, Seongnam, Korea

${ }^{2}$ Seoul National University, College of Pharmacy, Seoul, Korea
${ }^{3}$ College of Pharmacy, Sookmyung Women's University, Seoul 140-742, Korea

${ }^{4}$ bLife Science R\&D Center, SK Chemicals, Seongnam-si

463-400, Republic of Korea, cCollege of Pharmacy, Sookmyung Women's University, Seoul 140-742, Korea

Background and Aims: Chronic high level of glucocorticoids can result in insulin resistance from impairment of insulin-dependent glucose uptake, increased hepatic gluconeogenesis, and reduced insulin secretion from the pancreas. Due to the physiological relationship between glucocorticoids and metabolic disease risk factors, $11 \beta$-HSD1 has been regarded to be a potential target for the treatment of metabolic syndrome as well as type II diabetes. $11 \beta$-HSD1 is a key enzyme that acts as an NADPH-dependent reductase and converts inactive cortisone into active cortisol, which is an actual circulating glucocorticoid in humans. $11 \beta$-HSD1 is mainly distributed in specific tissues, such as liver, adipose, and brain, and that regulates tissue-specific glucocorticoid levels.

Method: Based on the docking results of our initial hit compound, $N$-cyclohexyl-6-(piperidin-1-yl)picolinamide from high throughput screening of in-house library, we performed SAR studies by the synthesis of a series of 6-substituted picolinamide derivatives and their inhibitory activities against $11 \beta$-HSD1. Several compounds were identified as novel and potent inhibitors of $11 \beta$-HSD1.

Results: High potency toward human $11 \beta$-HSD1 was achieved by the incorporation of a hydroxy-adamantyl group. In addition, the replacement of piperidine ring with 1-(4-substituted phenyl)piperazine led to a substantial improvement in metabolic stability.

Conclusion: In conclusion, the best compound possessed desirable potency in both human $11 \beta$-HSD1 enzyme assay and the mouse $11 \beta$-HSD1 enzyme assay, and demonstrated oral efficacy in lowering liver and adipose $11 \beta$-HSD1 activities in a mouse ex vivo model. Finally, the best compound exhibited good in vivo efficacy in HF/STZ mice and reduced fasting blood glucose and insulin levels after oral dosing.

321

\section{HYPOACT-SURVEY: PATIENT PREFERENCES FOR TREATMENT OF MILD HYPOGLYCAEMIA- GLUCAGON INJECTION VERSUS ORAL} GLUCOSE?

A. Ranjan $^{1}$, S. Schmidt ${ }^{1}$, K. Nфrgaard ${ }^{1}$

${ }^{1}$ Copenhagen University Hospital Hvidovre, Department of Endocrinology-Research Unit, Hvidovre, Denmark

Background and Aims: Intensive insulin treatment of type 1 diabetes is associated with increased risk of mild hypoglycaemia $(\mathrm{MH}) . \mathrm{MH}$ is treated with oral glucose intake (OGI) and may cause weight gain. Injection of subcutaneous low-dose glucagon may be a preferable treatment option for some occasions of $\mathrm{MH}$ in patients aiming for optimal glycemic control without gaining weight. We investigated under which occasions patients prefer glucagon for OGI.

Method: In a two-week survey study, 60 patients with type 1 diabetes were instructed to register every $\mathrm{MH}$ (glucose $\leq 3.9$ $\mathrm{mmol} / \mathrm{l}$ or sensations of $\mathrm{MH}$ ) in a booklet. For each $\mathrm{MH}$, patients chose whether they hypothetically would prefer to treat $\mathrm{MH}$ with subcutaneous glucagon injection pen or with OGI.

Results: Preliminary data of 17 patients $(5$ males, mean \pm SD age $43 \pm 11$ years, $\mathrm{HbA} 1 \mathrm{c} 55 \pm 8 \mathrm{mmol} / \mathrm{mol}$ ) reported 11 (range 3-22) MH events during follow-up, and glucagon was preferred to treat $\mathrm{MH}$ in $59 \%$ of the total events $(\mathrm{N}=192)$. Glucagon was 
preferred in treating MH during sleep (82\% of events) and when induced by unknown causes $(68 \%)$, heat and stress $(63 \%)$, insulin or carbohydrate miscalculation $(48 \%)$, and exercise $(53 \%)$. Females favored glucagon in treating $\mathrm{MH}(62 \%)$ compared with males (44\%). Glucagon injections could have at least replaced $12 \pm 6$ gram carbohydrates daily from the rescue OGI.

Conclusion: This study will give insight into potential use of glucagon in treatment of $\mathrm{MH}$ in patients with type 1 diabetes. Preliminary results show that glucagon might be preferred for OGI among females, during nocturnal $\mathrm{MH}$ and when $\mathrm{MH}$ occurs for unknown reasons. Results will be presented.

\section{2}

\section{SYSTEMIC MEDICATIONS FOR THE PREVENTION AND TREATMENT OF DIABETIC RETINOPATHY}

M. Stewart ${ }^{1}$

${ }^{1}$ Mayo Clinic, Ophthalmology, Jacksonville, USA

Background and Aims: Ophthalmologists frequently use intravitreal pharmacotherapeutic agents (vascular endothelial growth factor inhibitors and sustained release corticosteroid inserts) to reverse vision loss but they are unable to prevent diabetic retinopathy (DR) and they are reluctant to administer systemic drugs. Endocrinologists and internists are uniquely positioned to treat co-morbidities in diabetic patients with drugs that also modulate vision loss.

Method: Recent findings underscore the biochemical complexity of DR and emphasize the need that it be considered the local manifestation of several potentiating conditions.

Results: Hyperglycemia, systemic arterial hypertension (SAH), and hyperlipidemia may be successfully treated with several drug classes but some independently prevent the development and progression of DR. SAH control lessens capillary endothelial cell damage but the use of angiotensin receptor antagonists and angiotensin converting enzyme inhibitors also reduce the development of retinopathy. The FIELD and ACCORD trials showed that lowering serum triglycerides with fenofibrate decreases the progression of DR, delays the need for laser photocoagulation, and prevents vision loss significantly more than simvastatin. A single center, phase II trial showed that danazol improves visual acuity better than laser photocoagulation and a phase I/II study showed that minocycline (but not doxycycline) improves retinal edema and visual acuity, presumably by decreasing inflammation-induced glial activation. Oral thiazolidinediones should be avoided in patients with early DR because of their propensity to induce macular edema.

Conclusion: By knowing the retinopathy status of their patients, diabetologists are better able to choose systemic medications that reduce the likelihood of vision loss due to DR.

\section{3}

\section{IMPROVEMENT OF THE CHILDREN POPULATION INCLUDED INTO THE UVA/PADOVA TYPE 1 DIABETES SIMULATOR}

R. Visentin ${ }^{1}$, C. Dalla Man ${ }^{1}$, R. Bonfanti ${ }^{2}$, D. Iafusco ${ }^{3}$, R. Schiaffini ${ }^{4}$, I. Rabbone ${ }^{5}$, D. Bruttomesso ${ }^{6}$, C. $_{\text {. }}$ Cobelli $^{1}$

${ }^{1}$ University of Padova, Department of Information Engineering, Padova, Italy

${ }^{2}$ Istituto Scientifico San Raffaele, Diabetologia Pediatrica-

Diabetes Research Institute OSR_DRI, Milano, Italy

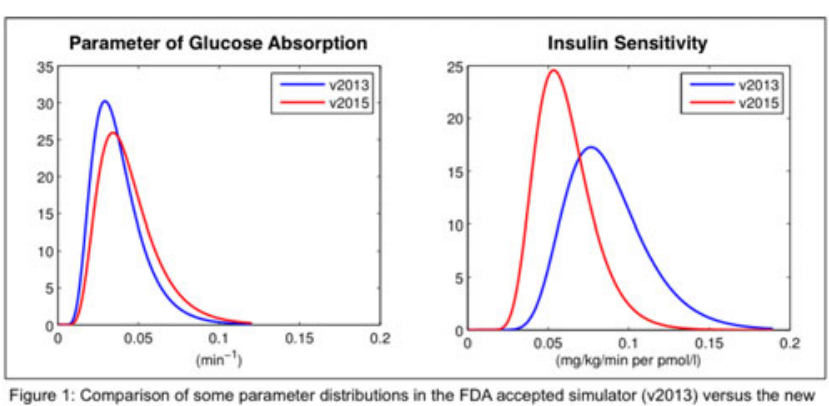

Figure 1: Comparison
updated one (v2015).

${ }^{3}$ Seconda Università di Napoli SUN, Dipartimento della Donnadel Bambino e della Chirurgia Generale e Specialistica - Centro Regionale di Diabetologia Pediatrica “G. Stoppoloni”, Napoli, Italy

${ }^{4}$ Ospedale Pediatrico Bambino Gesù- IRCCS, UOC

Endocrinologia e Diabetologia, Roma, Italy

${ }^{5}$ Ospedale Infantile Regina Margherita-Città della Salute e della Scienza, SSVD Endocrinologia e Diabetologia Pediatrica, Torino, Italy

${ }^{6}$ University of Padova, Dipartimento di Medicina Clinica-

DIMED, Padova, Italy

Background and Aims: The UVA/Padova Type 1 Diabetes Simulator (T1DMS) has proven to be very useful for the preclinical testing of control algorithms in adults, also thanks to the availability of data which allowed to refine and validate the in silico population. This facilitated several successful artificial pancreas inpatient and outpatient studies.

Artificial pancreas studies in children are increasing and the T1DMS could play an important role in algorithmic development.

The aim of this study is to assess and, if necessary, to update the in silico children included into the T1DMS.

Method: The data set consisted of 13 T1DM prepubertals recruited among the centers of Milano, Napoli, Roma and Torino (Italy). Subjects wore a sensor-augmented insulin pump, and received a standardized breakfast (30g of carbohydrates [CHO]). The T1DMS model was identified from subcutaneous glucose sensor and insulin pump data using a Bayesian approach.

Results: The model well fitted the data and provided precise estimates of model parameters. Our results suggest that meal glucose absorption is faster and insulin sensitivity lower, on average, than those included so far in the T1DMS (Figure 1). Based on these findings, a new children population was generated. The new T1DMS was then validated by comparing real vs simulated glucose curves in response to $30 \mathrm{~g} \mathrm{CHO}$ meal: as a result, the new T1DMS well described the glucose variability observed in the data.

Conclusion: The new in silico children population will enable a safer and effective preclinical testing of the closed-loop control in pediatric clinical trials.

\section{4}

INCORPORATION OF AFREZZA $®$ INTO THE TYPE 2 DIABETES SIMULATOR

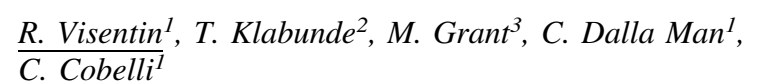


${ }^{1}$ University of Padova, Department of Information Engineering, Padova, Italy

${ }^{2}$ Sanofi-Aventis Deutschland GmbH, R\&D LGCR/StructureDesign \& Informatics, Frankfurt, Germany

${ }^{3}$ Mannkind Cooperation, Danbury, USA

Background and Aims: Ten percent of the type 2 diabetic (T2DM) population are treated with multiple daily insulin injections. Insulin delivered subcutaneously (SC) is accepted, but important limitations, such as delay in insulin absorption represent major challenges. Afrezza ${ }^{\circledR}$, an inhaled insulin with a fast onset of action, is an alternative treatment option and provides novel opportunities for the control of prandial glucose. Due to the much faster onset, delayed or split dosing, instead of pre-meal dosing as used for regular SC administered insulins, might lead to an improved glucose control. In order to explore in silico different dosing regimens in a meal test, we incorporated a pharmacokinetic (PK) model of Afrezza ${ }^{\circledR}$ into our T2DM simulator (Visentin et al. ATTD2014).

Method: We utilized clinical data of 12 T2DM subjects receiving Afrezza ${ }^{\circledR}$ at different doses and undergoing a mixed meal test (MKC-TI-118). The T2DM simulator model was identified on glucose and insulin time courses of individual patients. In particular, individual Afrezza ${ }^{\circledR}$ PK were randomly extracted from joint parameter distributions that have been created using the parameter estimates obtained from the model identification. The simulator was then validated by replicating the clinical data.

Results: The results were satisfactory: the glucose and insulin dynamics obtained in silico well described the variability observed in the experimental dataset.

Conclusion: In conclusion, we successfully incorporated Afrezza ${ }^{\circledR}$ PK into our T2DM simulator. This will allow running several in silico trials to evaluate the post-prandial glucose control in response to different dosing regimens of Afrezza with the potential to inform clinical study design.

\section{5}

\section{INCORPORATION OF THE SENSOR-AUGMENTED INSULIN-PUMP THERAPY INTO THE UVA/PADOVA TYPE 1 DIABETES SIMULATOR}

R. Visentin ${ }^{1}$, M. Vettoretti $^{1}$, A. Facchinetti ${ }^{1}$, C. Dalla Man ${ }^{1}$, G. Sparacino ${ }^{1}$

${ }^{1}$ University of Padova, Department of Information Engineering, Padova, Italy

Background and Aims: Sensor-augmented insulin-pump (SAP) therapy is the state of art open-loop therapy in type 1 diabetes (T1DM). In order to assess the impact of SAP therapy on glucose variability under several experimental conditions, we incorporate standard SAP-based therapeutic actions into the UVA/Padova T1DM Simulator (T1DMS).

Method: The SAP module includes: a diurnal insulin basal pattern that varies according to subject's specific time-varying insulin sensitivity (Visentin et al., Diabetes Technol Ther, 2015); a new error model of SMBG and CGM (tuned against OneTouch Ultra ${ }^{\circledR}$ II and Dexcom G4 ${ }^{\circledR}$ Platinum, respectively); a suboptimal carbohydrate-to-insulin ratio according to literature guideline (Davidson et al., Endocr Pract, 2008); a random error with $20 \%$ coefficient of variation to describe the uncertainty in meal carbs estimation; a mechanism to treat hypoglycemia simulating the rescue carbs intake when glucose is below the hypoglycemic threshold; post-meal correction boluses to treat hyperglycemia on the basis of the subject's insulin correction factor.

Results: Two weeks simulations of SAP therapy show that the distributions of the most commonly used outcome metrics, such as percent time in eu-/hypo-/hyperglycemia, agree with those obtained in clinical experiments, indicating the credibility of the extended T1DMS.

Conclusion: A SAP-based open-loop control has been incorporated into the T1DMS. In silico results show that the T1DMS equipped with the SAP module well reflects the glucose variability observed in vivo.

\section{6}

\section{EXTENDING METFORMIN BENEFITS IN PATIENTS} WITH TYPE 2 DIABETES THROUGH PILL TECHNOLOGY: A SYSTEMATIC REVIEW OF NINE POOLED CLINICAL TRIALS

S. Kumar ${ }^{\text {, S. Gupta }}{ }^{2}$, A. Kumar ${ }^{3}$, M. Tiwaskar ${ }^{4}$, S.K. Sharma , J. Kesavadev ${ }^{6}$, H. Thacker ${ }^{7}$, N. Wadhwa ${ }^{8}$

${ }^{1}$ Sir Gangaram Hospital, Endocrinology, Delhi, India

${ }^{2}$ Sunil's Diabetes Care n' Research Centre, Diabetology, Nagpur, India

${ }^{3}$ Diabetes Care and Research Centre, Diabetology, Patna, India

${ }^{4}$ Shilpa Medical Research Centre- Karuna Hospital and Asian Heart Hospital, Diabetology, Mumbai, India

${ }^{5}$ Galaxy Speciality Center, Endocrinology, Jaipur, India

${ }^{6}$ Jothydev's Diabetes and Research Centre, Diabetology,

Trivandrum, India

${ }^{7}$ Bhatia- Jaslok and Breach Candy Hospital, Diabetology, Mumbai, India

${ }^{8}$ AUW Global, Medical, Mumbai, India

Background and Aims: Recent evidences suggest that physical attributes of metformin also contribute to adherence to therapy. We evaluated evidence based perspectives of technologically advanced metformin formulations by systematically analysing pooled data across clinical trials, the highest level of evidence.

Method: We searched electronic databases (Medline, Pubmed, Cochrane Library Central, IndMed) up to November 3, 2015, using specific MeSH, Boolean operators and text key words including metformin AND extended release NOT Combination, pill size AND diabetes, metformin AND Technology, in humans. Secondary references also explored for analysis. GraphPad Prism 6 was used for statistical analysis.

Results: Of the 19 abstracts retrieved, 9 clinical trials were included for analysis. Cumulatively, 2858 patients with 176 weeks duration have been studied with various extended release technologies in trials ranging from 12-48 weeks (Median 16, Mean 19.55, SD 11.74, $\mathrm{p}=0.001$ [CI 12; 24]). The patients included in the studies range from 78-750 per study (Median 217, Mean 317.6, SD 256.1, p=0.005 [CI 150; $742]$ ). The dose varied from $500-2000 \mathrm{mg} /$ day. The benefits of extended release metformin are demonstrated beyond glycemic control with improvement in BMI, lipid profiles and insulin resistance.

Conclusion: The extended release formulations enable rapid initial titration, patient convenience and treatment adherence, which can further be improvised by, developing innovative technologies like microgel with small size tablets. Technologically advanced metformin formulation can address 
the unmet medical needs; potentially improve overall experience, minimize abdominal discomfort, patient compliance and adherence

\section{7}

\section{EVALUATION OF ECONOMIC BURDEN OF TYPE 1 AND TYPE 2 DIABETES PATIENTS ON MULTIPLE DAILY INJECTION THERAPY COVERED UNDER U.S. EMPLOYER-BASED COMMERCIAL HEALTH PLANS}

\author{
C. Zhu ${ }^{1}$, J. Chung ${ }^{1}$, M. Gill \\ ${ }^{1}$ Medtronic, Health Economics and Reimbursement, \\ Northridge, USA
}

Background and Aims: Multiple daily injection (MDI) therapy is a frequently used regimen among intensive insulin treated diabetes patients which involves administrations of at least four shots daily. Although MDI has shown to provide glycemic control, it does not offer protection against hypoglycemic events, detrimental to one's quality of life with heavy economic burden. This analysis aims to identify patients on MDI therapy among privately insured Type 1 and Type 2 diabetes patients and to evaluate the average annual healthcare spending using payers and patients perspectives across longitudinal setting.

Method: Type 1 and Type 2 diabetes patients on MDI therapy were identified using primary and secondary diagnosis codes with national drug codes associated with basal and bolus insulins. Patients age 18-64 were required to be continuously enrolled to the same health plans with pharmacy benefits and continuously receiving MDI therapy from index date, defined by last pharmacy claim date for insulin minus 4 years. Healthcare spending from different healthcare systems including inpatient, outpatient and pharmacy were evaluated across 4 years, adjusted to 2013 dollars.

Results: Costs of treating Type 1 and Type 2 privately insured patients in inpatient, outpatient and pharmacy settings have increased across 4 years. The latest year data captured in this longitudinal analysis showed increase in total healthcare spending by approximately $20-30 \%$ for Type 1 and Type 2 diabetes patients.

Conclusion: Increasing healthcare costs were observed among MDI patients in U.S. and alternative intensive insulin therapies should be considered among patients trying to maintain a tight glycemic control with protection against hypoglycemic events.

\section{8}

\section{SITAGLIPTIN/METFORMIN COMBINED WITH LOW CALORIC DIETS VERSUS INSULIN GLARGINE/ METFORMIN FOR OBESE TYPE 2 DIABETIC PATIENTS}

M. $\mathrm{Ji}^{1}$, J. $\mathrm{Cao}^{1}$, D. Zou ${ }^{1}$

${ }^{1}$ Shanghai Changhai Hospital, Endocrinology, Shanghai, China

Background and Aims: To compare the therapeutic effects of different regimens for Chinese obese type 2 diabetic mellitus (T2DM) patients.

Method: From October 2013 to July 2014 a total of 166 T2DM outpatients of the Shanghai Changhai Hospital were randomly assigned into experimental sitagliptin/metformin combined with low caloric diet $(\mathrm{n}=115)$ and insulin glargine combined with metformin control ( $n=51)$ groups. Inclusion criteria were BMI $\geq 25 \mathrm{Kg} / \mathrm{m}^{2}$ and diagnosed with T2DM with glycosylated hemoglobin (HbA1c) $>9 \%$. Outcome parameters were fasting plasma glucose (FPG), postprandial plasma glucose (PPG), BMI, HbAlc, fasting C-peptide (FCP), 2-hour postprandial C-peptide (CP), triglyceride (TG), total cholesterol (TC), high density cholesterol (HDL-C) and low density cholesterol (LDL-C), which were determined by $75 \mathrm{~g}$ steamed-buns meal tolerance test before and 4 weeks, 8 weeks, 12 weeks and 24 weeks after treatment start. Treatment costs and life quality were also measured.

Results: BMI, HbA1C, TG, TC, LDL were significantly more reduced $(\mathrm{p}<0.0001)$ and $\mathrm{HbA1c}$ were significantly better improved in the experimental than in the control group $(<6.5 \%$ in 24 $(20.87 \%)$ vs $2(3.92 \%) \mathrm{P}<0.001,<7 \%$ in $65(56.52 \%)$ vs. $12(23.53 \%) \mathrm{P}<0.001)$. Quality of life scores in the experimental group increased more than in the control group $(\mathrm{P}<0.001)$. The costs for the experimental group medication was less than for other regimens.

Conclusion: For obese T2DM patients diagnosed with glycosylated hemoglobin $>9 \%$, oral sitagliptin/metformin combined with low caloric diets could effectively and economically maintain glycemic control and significantly improve the life qualities.

\section{9}

\section{THE ALMASED CONCEPT AGAINST OVERWEIGHT AND OBESITY AND RELATED HEALTH RISK (ACOORH)-FIRST RESULTS OF AN INTERIM ANALYSIS}

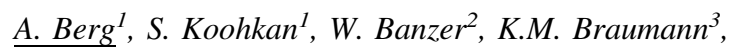

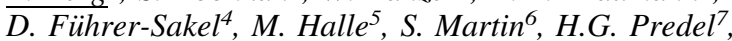
H. Toplak $^{8}$

${ }^{1}$ Institut für Präventive Medizin IPM, Freiburg, Germany

${ }^{2}$ Institut für Sportwissenschaften, Abt. Sportmedizin, Frankfurt, Germany

${ }^{3}$ Universität Hamburg, Arb. Bereich Sport und

Bewegungsmedizin, Hamburg, Germany

${ }^{4}$ Universitätsklinikum Essen, Med. Forschungszentrum MFZ, Essen, Germany

${ }^{5}$ Technische Universität München, Zentrum für Prävention und Sportmedizin, München, Germany

${ }^{6}$ VKKD-Kliniken, Westdeutsches Diabetes- und

Gesundheitszentrum, Düsseldorf, Germany

${ }^{7}$ Deutsche Sporthochschule Köln, Inst. f. Kreislaufforschung und Sportmedizin, Köln, Germany

${ }^{8}$ Medizinische Universität Graz, Medizinische Klinik, Graz, Austria

Background and Aims: Overweight and accompanying risk factors are important for development of T2DM and cardiovascular disease. It has been recently shown that a meal replacement diet, high in soy protein, is more effective in improving body composition and reducing metabolic and atherogenic risk factors than conventional lifestyle changes. To confirm this, a 1-year-multicenter randomized controlled trial for overweight patients (ACOORH) was designed to evaluate the effects of a low-glycemic, soy-protein-rich product (Almased ${ }^{\circledR}$ ) as meal replacement.

Method: Meanwhile (key date $1^{\text {st }}$ Sept. 2015), 263 nondiabetic participants (BMI $27-35 \mathrm{~kg} / \mathrm{m}^{2}, 21-65 \mathrm{yrs}$.) with at least one criterion of the metabolic syndrome were randomized into a 
supervised meal replacement regimen (group A) or a telemedically controlled lifestyle intervention (group B). For 181 of the 263 participants paired data were available after 12 weeks of intervention (interim analysis); primary target variable was total body weight (BW), secondary targets included BMI, waist circumference (WC), fat mass (FM) and skeletal muscle mass (SMM), fasting insulin level, $\mathrm{HbA}_{1 \mathrm{c}}$, apolipoprotein $\mathrm{B}$, and leptin.

Results: The outcome values of group A $(n=125)$ and B $(n=56)$ were tested for differences in baseline vs. after 12 weeks as an inter-group comparison. Significantly higher differences $(\mathrm{p}=0.001)$ were observed in anthropometric variables BW, BMI, WC, FM in group A $\left(\mathrm{A}:-6.6 \pm 3.90 \mathrm{~kg},-2.3 \pm 1.39 \mathrm{~kg} / \mathrm{m}^{2},-7.0 \pm\right.$ $4.61 \mathrm{~cm},-5.4 \pm 3.47 \mathrm{~kg}$; B: $-4.2 \pm 5.86 \mathrm{~kg},-1.3 \pm 1.41 \mathrm{~kg} / \mathrm{m}^{2},-4.8 \pm$ $4.32 \mathrm{~cm},-4.0 \pm 3.59 \mathrm{~kg}$ ).

Conclusion: The results demonstrate significant benefits of the meal replacement strategy for weight control. Results for weight management (12 months of intervention) as well as metabolic variables will be presented in the second half of 2016.

330

THE ROLE OF LIFESTYLE MODIFICATION IN PREVENTING TYPE 2 DIABETES MELLITUS WITH IMPAIRED GLUCOSE HOMEOSTASIS

\section{E. Shishko}

${ }^{1} 6$ Clinical Hospital, Minsk, Belarus
Background and Aims: Our study assessed the efficacy of a lifestyle modification including altered diet composition and physical activity in preventing diabetes mellitus type 2 (DM 2) in individuals with impaired glucose tolerance and impaired fasting glucose (IGT/IFG) and its effect on serum leptin levels.

Materials and Methods: The study included 327 patients with IGT/IFG (68 m 258 f) 25-65 years. Patients were divided into 2 groups matched by sex, age, weight, body mass index (BMI). Research group included 183 patients ( $32 \mathrm{~m}, 150 \mathrm{f})$ who received and carried out individual recommendations of a balanced diet and physical activity. Control group included 144 patients $(36 \mathrm{~m}, 108 \mathrm{f})$ who did not have lifestyle modification. Relationship to fasting leptin (FL) concentrations was measured by sensitive ELISA.

Results: Patients of the research group demonstrated reduction of body weight $(\mathrm{p}<0.01)$. They had positive dynamics of FPG and 2-h PG concentrations also $(\mathrm{p}<0.001)$. Persons of the control group had significant increase in weight and BMI and FPG and 2-h PG concentrations elevated $(\mathrm{p}<0.05)$. The main novel finding was that median serum leptin in research group decreased by $-23.9 \%(\mathrm{p}<0.01)$ and increased in control group by $+27.6 \%(\mathrm{p}<0.01)$ among subjects with IGT. Among patients of the research group was a reduction of new care DM 2 by $11.9 \%$ and an increase in the control group by $35.1 \%$.

Conclusion: Thereby, lifestyle modifications lead to reduction not only of fasting plasma glucose, 2-hour plasma glucose concentrations but also of fasting leptin concentrations in individuals with impaired glucose tolerance. 


\section{ATTD 2016 Read By Title}

331 AUTOANTIBODIES TOWARDS PARIETAL CELLS IN CHILDREN WITH TYPE 1 DIABETES

A.Chobot ${ }^{1}$, E. Rusak ${ }^{2}, J$. Wenzlau ${ }^{3}$, K. Bak-Drabik ${ }^{4}$,

A. Krzywicka ${ }^{1}$, J. Polanska ${ }^{5}$, M. Rewers ${ }^{3}$

${ }^{1}$ Clinical Hospital No1, Dept. of Pediatric Gastroenterology and Hepatology, Zabrze, Poland

${ }^{2}$ Uppersilesian Center of Child's Health, Department of Pediatrics- Pediatric Endocrinology and Diabetology, Katowice, Poland

${ }^{3}$ University of Colorado Denver, Barbara Davis Center for Diabetes, Aurora, USA

${ }^{4}$ Medical University of Silesia, Department of Pediatrics, Katowice, Poland

${ }^{5}$ The Silesian University of Technology, Data Mining Group, Gliwice, Poland

332 PROSTAGLANDIN E1 TREATMENT FROM THE PROGNOSTIC POINT OF VIEW IN PATIENTS WITH CRITICAL LIMB ISCHEMIA

L. Gaspar ${ }^{1}$, A. Komornikoval, P. Gavornik ${ }^{1}$, P. Sabaka ${ }^{1}$, I. Gasparova ${ }^{2}$

${ }^{1}$ Medical Faculty Comenius University and University Hospital, 2nd Department of Internal Medicine, Bratislava, Slovakia

${ }^{2}$ Medical Faculty Comenius University and University Hospital, Institute of Clinical Biology-Genetics and Clinical Genetics, Bratislava, Slovakia

333 NEAR-INFRARED SPECTROSCOPY FOR THE ASSESSMENT OF GLUCOSE CONCENTRATION BY PORTABLE SPECTROMETER: IN VITRO ANALYSIS

A. Tura ${ }^{1}$, S. Sbrignadello ${ }^{1}$, G. Pacini ${ }^{1}$

${ }^{1}$ CNR Institute of Neuroscience, Metabolic Unit, Padova, Italy

334 FAILURE ANALYSIS OF AN ARTIFICIAL PANCREAS - DOUBLE SUBCUTANEOUS VS. DOUBLE INTRAPERITONEAL APPROACH

K. Koelle ${ }^{1}$, A. Fougner ${ }^{1}$, S.M. Carlsen ${ }^{2}$, R. Ellingsen ${ }^{3}$, Ø. Stavdahl

${ }^{1} N T N U$, Department of Engineering Cybernetics, Trondheim, Norway

${ }^{2}$ NTNU, Unit for Applied Clinical Research, Trondheim, Norway

${ }^{3}$ NTNU, Department of Electronics and Telecommunications, Trondheim, Norway

\section{MODELLING GLUCAGON ACTION}

IN TYPE 1 DIABETES

A.Emami $^{1}$, R. Rabasa-Lhoret ${ }^{2}$, J. Pineau ${ }^{1}$, J. El Youssef ${ }^{3}$, J. Castle $^{3}$, A. Haidar
${ }^{1}$ McGill University, Computer Science, Montreal, Canada ${ }^{2}$ Université de Montréal, Nutrition, Montreal, Canada ${ }^{3}$ Oregon Health \& Science University, Endocrinology, Portland, USA

${ }^{4}$ McGill University, Experimental Medicine, Montreal, Canada

\section{SWITCHED LPV GLUCOSE CONTROL WITH INSULIN-ON-BOARD LIMITATION IN TYPE 1 DIABETES}

P. Colmegna ${ }^{1}, F$. Garelli ${ }^{2}, H$. De Battista ${ }^{2}$, R. Sanchez Peña ${ }^{3}$ ${ }^{1}$ UNQui, Depto. Ciencia y Tecnología, Quilmes, Argentina ${ }^{2}$ LEICI, EE Dept. UNLP, La Plata, Argentina

${ }^{3}$ ITBA, Centro de Sistemas y Control, Buenos Aires, Argentina

\section{COMPARING GENETIC PROGRAMMING TECHNIQUES AND CLASSICAL METHODS ON THE IDENTIFICATION OF GLYCEMIA PREDICTION MODELS}

J.I. Hidalgo ${ }^{1}$, E. Maqueda ${ }^{2}$, S. Winkler ${ }^{3}$, A. Sánchez-Sánchez ${ }^{4}$, J.M. Velasco ${ }^{1}$, J.M. Colmenar ${ }^{5}$, M. Botella ${ }^{6}$, J.A. Rubio ${ }^{6}$,

I. Contreras ${ }^{7}, O$. Garnica ${ }^{1}$, J. Lanchares

${ }^{1}$ University Complutense of Madrid, Computer Architecture Depatment, Madrid, Spain

${ }^{2}$ Hospital Virgen de la Salud de Toledo, Servicio de Endocrinología y Nutrición, Toledo, Spain

${ }^{3}$ University of Applied Sciences Upper Austria, School of Informatics, Hagemberg, Austria

${ }^{4}$ Universidad Pablo de Olavide, Departamento de Derecho Público, Sevilla, Spain

${ }^{5}$ Universidad Rey Juan Carlos, Departamento de Ciencias de la Computación, Móstoles, Spain

${ }^{6}$ Hospital Universitario Príncipe de Asturias, Servicio de Endocrinología y Nutrición, Alcalá de Henares, Spain

${ }^{7}$ Universitat de Girona, Departament de Enginyeria

Elèctrica- Electrònica i Automàtica, Girona, Spain

\author{
338 ONLINE ADAPTIVE MODELS FOR \\ PERSONALIZED PREDICTION OF GLUCOSE \\ PROFILE IN INDIVIDUALS WITH \\ TYPE 1 DIABETES \\ M. Jankovic ${ }^{1,2}$, S. Mosimann ${ }^{1}$, L. Bally ${ }^{3}$, C. Stettler ${ }^{3}$, \\ S. Mougiakakou ${ }^{1,3}$ \\ ${ }^{1}$ University of Bern, ARTORG Center for Biomedical \\ Engineering Research, Bern, Switzerland \\ ${ }^{2}$ Bern University Hospital- "Inselspital", Department of the \\ Emergency Medicine, Bern, Switzerland \\ ${ }^{3}$ Bern University Hospital- "Inselspital", Division of \\ Endocrinology- Diabetes and Clinical Nutrition, Bern, \\ Switzerland
}


339 EXPLOITATION OF INNATE GLUCOSE REDUCING CAPABILITY TO REDUCE INSULIN DOSING

K. Khodabakhshi Pirkalani ${ }^{1}$

${ }^{1}$ Mehr Medical Group, Internal Medicine/Gynecology, Tehran, Iran

340 SAP-PLGM VS SAP IN MANAGEMENT OF NOCTURNAL HYPOGLYCAEMIA AFTER PROLONGED PHYSICAL ACTIVITY: WHAT IS MORE EFFECTIVE?

L. Petruzelkova ${ }^{1}, K$. Pickova ${ }^{1}$, B. Obermannova ${ }^{1}$

${ }^{1}$ University Hospital Motol and 2nd Faculty of MedicineCharles University in Prague- Czech Republic, Department of Paediatrics, Prague, Czech Republic

\section{SIMULATING GLYCEMIC CONTROL} IN VIRTUAL DIABETES PATIENTS M.R. Smaoui ${ }^{1,2}$

${ }^{1}$ Montreal Institute of Clinical Research, Research Platform on Obesity Metabolism and Diabetes, Montreal, Canada

${ }^{2}$ McGill University, Dept. of Medicine-Division of

Experimental Medicine, Montreal, Canada

342 MODELING THE GLUCOSE-INSULIN-GLUCAGON DYNAMICS AFTER SUBCUTANEOUS ADMINISTRATION OF NATIVE GLUCAGON AND A NOVEL GLUCAGON ANALOGUE IN DOGS S.L.Wendt ${ }^{1}$, C.B. Knudsen ${ }^{1}$, J.B. Jørgensen ${ }^{2}$, H. Madsen ${ }^{2}$, A. Haidar ${ }^{3}$

${ }^{1}$ Zealand Pharma A/S, Bioanalysis and Pharmacokinetics, Glostrup, Denmark

${ }^{2}$ DTU Compute- Technical University of Denmark, Applied Mathematics and Computer Science, Kongens Lyngby, Denmark

${ }^{3}$ Institut de Recherches Cliniques de Montréal, Metabolic Diseases, Montreal, Canada

\section{QUALITY OF LIFE IN ADOLESCENTS}

\section{WITH TYPE 1 DIABETES}

I. Bica ${ }^{1}$, C. Marinho ${ }^{2}$, A. Rocha ${ }^{1}$, G. Aparício ${ }^{1}$

${ }^{1}$ Instituto Politécnico de Viseu, Escola Superior de Saúde,

Viseu, Portugal

${ }^{2}$ Centro Hospitalar Tondela-Viseu, Pediatria, Viseu, Portugal

\author{
344 EFFECTS OF FLUOXETINE ON \\ E-CADHERIN-MEDIATED CELL ADHESION \\ AND CALCIUM HOMEOSTASIS IN PANCREATIC \\ $\beta$ CELLS \\ H.Y. Chang ${ }^{1}$, S.L. Chen ${ }^{1}$, Y.W. Chen ${ }^{1}$ \\ ${ }^{1}$ National Cheng Kung University, Department \\ of Pharmacology, Tainan, Taiwan
}

345 IMPACT OF OBESITY ON DIABETES CONTROL M. Aboawja ${ }^{1}$, H. Al Makhaita ${ }^{2}$

${ }^{1}$ King Faisal Medical City, Family Medicine, ABHA, Kingdom of Saudi Arabia

${ }^{2}$ University of Dammam, Family Medicine, Dammam, Kingdom of Saudi Arabia

\author{
346 DERMCIDIN ISOFORM-2 (DCN-2), AN \\ ENVIRONMENTALLY INDUCED STRESS \\ PROTEIN MEDIATED TYPE-2 DIABETES \\ MELLITUS IN ANIMAL MODEL \\ S. Bank ${ }^{1}$, S. Bhattacharya ${ }^{1}$, S. Maiti ${ }^{2}$, A.K. Sinha ${ }^{3}$ \\ ${ }^{1}$ 1. Sinha Institute of Medical Science \& Technology- \\ 2. Vidyasagar University, Biochemistry \& Diabetology, \\ Kolkata, India \\ 2 2. Vidyasagar University, Biochemistry, Midnapur, India \\ 3 1. Sinha Institute of Medical Science \& Technology, \\ Biochemistry \& Diabetology, Kolkata, India
}

\section{ASSOCIATION OF GIPR RS2302382 POLYMORPHISM WITH TYPE 2 DIABETES IN RUSSIAN SUBJECTS WITH ABDOMINAL OBESITY}

D. Skuratovskaia ${ }^{1}$, N. Fattakhov ${ }^{1}$, M. Vasilenko $^{1}$, E. Kirienkova ${ }^{1}$, P. Zatolokin ${ }^{2}, N$. Mironyuk ${ }^{2}$, L. Litvinova ${ }^{1}$ ${ }^{1}$ Immanuel Kant Baltic Federal University, Laboratory of Immunology and Cell Biotechnologies, Kaliningrad, Russia ${ }^{2}$ Kaliningrad Regional Hospital, Department of Reconstructive and Endoscopic Surgery, Kaliningrad, Russia

\section{DIASTOLIC BLOOD PRESSURE DYSREGULATION AND A FLAT PRESSOR RESPONSE AT THE STRAIN TEST IN OBESE NON-DIABETIC PATIENTS}

A. Mendez-Medina ${ }^{1}$, J. Erazo Zambrano ${ }^{1}$, C. Tomalá Ramos ${ }^{2}$, M. Santelli Romanó ${ }^{3}$, G. Luzcando-Defilippi ${ }^{4}$,

M. Sierra-Beltran ${ }^{5}$, R. Vega-Zapata ${ }^{6}$, M. Ramos-Ostos ${ }^{7}$

${ }^{1}$ Universidad Católica de Santiago de Guayaquil, Medical

School, Guayaquil, Ecuador

${ }^{2}$ Universidad Católica Santiago de Guayaquil, Medical School, Guayaquil, Ecuador

${ }^{3}$ Universidad Católica Santigao de Guayaquil, Medical School, Guayaquil, Ecuador

${ }^{4}$ Universidad Católica Santiago de Guayquil, Medical School, Guayaquil, Ecuador

5 "Salvador Zubirán”" Medical Sciences and Nutrition National Institute, Neurphysiology, Mexico City, Mexico

${ }^{6}$ Medica Sur, Integral Diagnosis and Treatment Centre, Mexico City, Mexico

${ }^{7}$ Medica Sur, Integral Diagnosis and Treatment Centre, Mexico City, Mexico

\section{ADDITIONAL BENEFITS OF PIOGLITAZONE TO LIFESTYLE MODIFICATION IN PATIENTS AT INCREASED RISK FOR DIABETES AND NEWLY DEVELOPED DIABETES J.S. Nam ${ }^{1}$, M.Y. Lee ${ }^{1}$, M.J. Kim ${ }^{1}$, S.Y. Park ${ }^{1}$, S. Kang ${ }^{1}$,

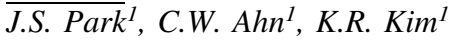 \\ ${ }^{1}$ Gangnam Severance Hospital - Yonsei University College of Medicine, Internal Medicine, Seoul, Korea}

350 OBESITY AND PHYSICAL ACTIVITY AFTER EDUCATION IN DIABETES SCHOOL IN PATIENTS WITH T2DM L. Strongin ${ }^{1}$, D. Shokareva ${ }^{1}$, S. Panova ${ }^{1}$, A. Petrov ${ }^{1}$ ${ }^{I}$ Nizhny Novgorod State Medical Academy, Endocrinology and Internal Medicine, Nizhny Novgorod, Russia 
351 EFFECT OF AN EDUCATIONAL INTERVENTION BASED ON SOCIAL COGNITIVE THEORY AMONG PATIENTS WITH TYPE 2 DIABETES REFERRING TO IRAN DIABETES CLINIC D. Shojaeizadeh $^{1}$, M. Sorani ${ }^{2}$, M.H. Taghdisi ${ }^{3}$, M. Shojaeizadeh $^{4}$, E. Shojaeizadeh ${ }^{1}$

${ }^{1}$ School of Public Health-Tehran University of Medical Sciences, Department of Health Education and Promotion, Tehran, Iran

${ }^{2}$ Health Management and Economics Research Center, Iran University of Medical Sciences, Tehran, Iran

${ }^{3}$ School of Public Health- Iran University of Medical Sciences, Department of Health Education \& Promotion, Tehran, Iran

${ }^{4}$ Amirkabir University of Technology, Department of Biomedical, Tehran, Iran

352 CONTINUOUS GLUCOSE MONITORING SYSTEM MAY REVEAL AN EATING DISORDER IN TYPE 1 DIABETIC ADOLESCENT WITH UNEXPLAINED RECURRENT HYPOGLYCAEMIA

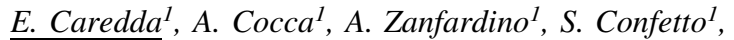

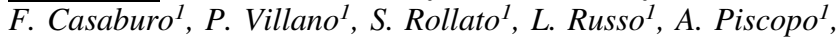
L. Perrone ${ }^{1}$, D. Iafusco ${ }^{1}$

${ }^{1}$ University of Naples SUN, Department of Woman, Child and of General and Specialized Surgery, Naples, Italy

353 CONTINUOUS GLUCOSE MONITORING BY HYDROGEL-BASED GLUCOSE OXIDASE SENSORS: FROM A PROOF-OF-CONCEPT STUDY TO PRACTICES

J. Cha ${ }^{1}$, J.Y. Lee ${ }^{1}$, P. Habeen ${ }^{1}$

${ }^{1}$ ENGAIN, Central Research Laboratories, Sungnam-si, Republic of Korea

354 CONTINUOUS GLUCOSE MONITORING AS A TOOL FOR MANAGEMENT IN TYPE 1 DIABETES ON MULTIPLE DAILY INJECTIONS DURING RAMADAN: A CASE REPORT

A. Elawwa ${ }^{1}$, F. Alkhalafl, G. Petrovski ${ }^{l}$, M. Elgamal ${ }^{1}$,

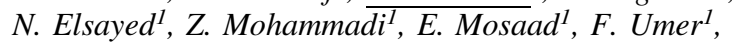
M. Itani ${ }^{1}$, H. Abubakr ${ }^{1}$

${ }^{1}$ Hamad General Hospital, Pediatric Endocrine and Diabetes Unit, Doha, Qatar

355 THE FREQUENCY AND DURATION OF HYPOGLYCEMIA IN CHILDREN AND ADOLESCENTS WITH T1D ACCORDING OF CONTINUOUS GLUCOSE MONITORING SYSTEM DATA

A. Emelianov ${ }^{1}$, L.L. Kuraeva ${ }^{1}$

${ }^{1}$ Endocrinology Research Centre, The Institute of Pediatric Endocrinology, Moscow, Russia

356 CONTINUOUS GLUCOSE MONITORING SYSTEM BASED ON ENZYME COLORIMETRIC SENSOR AND MICROFLUIDIC CHIP D. $L i^{1}$

${ }^{1}$ Tianjin Univeristy, Tianjin, China
357 EVALUATION OF GLYCEMIC CONTROL AND VARIABILITY IN POST-TRANSPLANT DIABETES MELLITUS USING CONTINUOUS GLUCOSE MONITORING SYSTEM

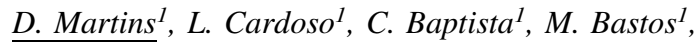

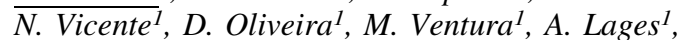
R. Alves ${ }^{2}$, A. Mota ${ }^{3}$, F. Carrilho ${ }^{1}$

${ }^{1}$ Centro Hospitalar e Universitário de Coimbra, Serviço de Endocrinologia- Diabetes e Metabolismo, Coimbra, Portugal ${ }^{2}$ Centro Hospitalar e Universitário de Coimbra, Serviço de Nefrologia, Coimbra, Portugal

${ }^{3}$ Centro Hospitalar e Universitário de Coimbra, Serviço de Urologia e Transplantação Renal, Coimbra, Portugal

358 SUCCESSFUL MANAGEMENT OF TYPE 2 DIABETES PATIENT USING GLP-1 AGONIST AND INSULIN ASPART SUPPORTED BY CGM: A CASE REPORT

G. Petrovski ${ }^{1}$, M. Zivkovic ${ }^{l}$, I. Bitovska ${ }^{1}$

${ }^{1}$ University Clinic of Endocrinology, Center for Insulin Pump, Skopje, Macedonia

359 A NOVEL OSMOTIC PRESSURE SENSOR FOR INTERSTITIAL GLUCOSE ASSESSMENT: RESULTS FROM A LABORATORY FEASIBILITY INTERFERENCE TEST A. Pfützner l, R. Smajda ${ }^{2}$, G. Andreatta ${ }^{2}$, S. Ramljak ${ }^{3}$, R. Frisvold ${ }^{4}$, M. Liley ${ }^{2}$

${ }^{1}$ Pfützner Science \& Health Institute, Diabetes Center and Practice, Mainz, Germany

${ }^{2} C S E M, R \& D$, Neufchatel, Switzerland

${ }^{3}$ Sciema UG, R\&D, Mainz, Germany

${ }^{4}$ Lifecare AS, $R \& D$, Bergen, Norway

360 A NOVEL OSMOTIC PRESSURE SENSOR FOR INTERSTITIAL GLUCOSE ASSESSMENT: OPTIMIZATION OF CORE SENSOR COMPONENTS A. Pfütznerl, G. Andreatta ${ }^{2}$, R. Smajda ${ }^{2}$, S. Angeloni ${ }^{2}$, S. Ramljak ${ }^{3}$, R. Frisvold ${ }^{4}$, M. Liley ${ }^{2}$

${ }^{1}$ Pfützner Science \& Health Institute, Diabetes Center and Practice, Mainz, Germany

${ }^{2}$ CSEM, $R \& D$, Neufchatel, Switzerland

${ }^{3}$ Sciema $U G, R \& D$, Mainz, Germany

${ }^{4}$ Lifecare AS, $R \& D$, Bergen, Norway

361 EXPERIMENTAL POLYMER COATING ENSURES HAEMOCOMPATIBILITY OF GLUCOSE SENSORS IN BLOODSTREAM

Y. Tarasov ${ }^{1}$, E. Borisova ${ }^{1}, Y$. Philippov ${ }^{1}$, M. Shestakova ${ }^{1}$

${ }^{1}$ Endocrinology Research Centre, Diabetes Institution, Moscow, Russia

362 3RD GENERATION BIOSENSOR TECHNOLOGY FOR CONTINOUS GLUCOSE MONITORING SYSTEMS

A. Pinczewska ${ }^{1}$, A. Felice ${ }^{2}$, W. Harreither ${ }^{3}$, R. Kittl $^{4}$, C. Sygmund ${ }^{4}$ R. Ludwig ${ }^{4}$

${ }^{1}$ DirectSens GmbH, Kolsterneuburg, Austria

${ }^{2}$ DirectSens GmbH, Business Development, Kolsterneuburg, Austria

${ }^{3}$ DirectSens GmbH, Co-Founder, Kolsterneuburg, Austria

${ }^{4}$ DirectSens GmbH, Management, Kolsterneuburg, Austria 
363 MISTAKES WHEN COUNTING CARBS: EVALUATED BY PROFESSIONAL CONTINUOUS GLUCOSE MONITORING

A. Proietti ${ }^{1}$, J. Velazquez ${ }^{2}$, M.L. Iglesias ${ }^{3}$

${ }^{1}$ Hospital Italiano de Buenos Aires - Argentina,

Endocrinologist, CABA, Argentina

${ }^{2}$ Private Office, Nutrition, Buenos Aires, Argentina

${ }^{3}$ Hospital Italiano de Buenos Aires - Argentina, Nutrition,

$C A B A$, Argentina

364 EVALUATION OF THE PRANDIAL PERIOD WITH HIGH CARBOHYDRATE LOAD IN PATIENTS WITH TYPE 1 DIABETES (T1D) TREATED WITH INSULIN PUMP AND CONTINUOUS GLUCOSE SENSOR A. Proietti ${ }^{1}$, M. Stasi ${ }^{2}$, M.L. Iglesias ${ }^{2}$, L. Rodota ${ }^{2}$, L. Litwak ${ }^{1}$, J. Giunta $^{1}$, A. Alvarez ${ }^{1}$, L. Capurro ${ }^{1}$, M. Castex ${ }^{1}$,

L. Grosembacher ${ }^{1}$

${ }^{1}$ Hospital Italiano de Buenos Aires - Argentina, Endocrinología, CABA, Argentina

${ }^{2}$ Hospital Italiano de Buenos Aires - Argentina, Nutrition, $C A B A$, Argentina

365 PERFORMANCE EVALUATION OF THREE BLOOD GLUCOSE MONITORING SYSTEMS IN THE HANDS OF INTENDED USERS AND TRAINED PERSONNEL D. Rittmeyer ${ }^{l}$, A. Baumstark ${ }^{1}$, N. Jendrike ${ }^{1}$, S. Pleus ${ }^{1}$, C. Haug ${ }^{I}$, G. Freckmann ${ }^{1}$

${ }^{1}$ Institut für Diabetes-Technolgie Forschungs- und Entwicklungsgesellschaft $\mathrm{mbH}$, an der Universität Ulm, Ulm, Germany

366 IN VITRO/IN VIVO STUDY OF ACETAMINOPHEN INTERFERENCE OF THE DIRECT ELECTRON TRANSFER-TYPE CGM SENSOR

S. Sekimoto ${ }^{1}$, Y. Murase ${ }^{1}$, Y. Kusaka ${ }^{1}$, W. Tsugawa ${ }^{2}, K$. Sode $^{2}$ ${ }^{I}$ ARKRAY-Inc, R\&D, Kyoto, Japan

${ }^{2}$ Tokyo University of Agriculture \& Technology, Department of Biotechnology-Department of Industrial Technology and Innovation, Tokyo, Japan

367 CHEMICAL CROSS TALK STUDIES ON A MICROPROBE ARRAY BASED CONTINUOUS GLUCOSE MONITORING SENSOR

S. Sharma ${ }^{1}$, A. El-Laboudi ${ }^{2}$, N. Oliver $^{2}$, D. Johnston ${ }^{2}$, T. Cass ${ }^{1}$

${ }^{1}$ Imperial College, Department of Chemistry \& Institute of Biomedical Engineering, London, United Kingdom

${ }^{2}$ Imperial College, Division of Diabetes- Endocrinology \&

Metabolism, London, United Kingdom

368 CONTINUOUS GLUCOSE MONITORING IN DIABETIC PATIENT WITH FEAR OF HYPOGLICEMIA A. Skvarca ${ }^{1}$

${ }^{1}$ University Medical Center Ljubljana, Department of Endocrinology- Diabetes and Metabolic Diseases, Ljubljana, Slovenia

369 ACCURACY OF THE CONTOUR PLUS BLOOD GLUCOSE MONITORING SYSTEM (BGMS) AT LOW-LEVEL RANGES

Y. Wang ${ }^{1}, T$. $F u^{1}$

${ }^{1}$ Shanghai Jiao Tong University Affiliated Sixth People's Hospital Shanghai Diabetes Institute, Endocrinology, Shanghai, China
370 DOUBLE DECREASE OF HBA1C IN TEENAGE TYPE 1 DIABETES PATIENT ON MULTIPLE DAILY INJECTIONS AFTER PSYCHOLOGICAL COUNSELING: A CASE REPORT

A. Elawwa ${ }^{1}$, G. Petrovski ${ }^{1}$, M. Elgamal ${ }^{1}$, N. Elsayed ${ }^{1}$, Z. Mahammadi ${ }^{1}$, E. Mosaad ${ }^{1}$, M. Itani ${ }^{1}$, H. Abubaker ${ }^{1}$ ${ }^{1}$ Hamad General Hospital, Pediatric Endocrine and Diabetes Unit, Doha, Qatar

371 DIRECT COSTS OF DIABETES CARE IN PEDIATRIC PATIENTS WITH TYPE 1 DIABETES IN GREECE

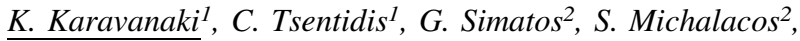
F. Karachaliou ${ }^{2}$

I"P\&A Kyriakou" Children's Hospital- Athens- Greece, Diabetic Clinic- 2nd Deparment of Pediatrics- University of Athens, Goodi-Athens, Greece

2 "P\&A Kyriakou” Children's Hospital- Athens,

Endocrinology-Growth and Development, Athens, Greece

372 EFFECTS OF STRUCTURED TESTING VERSUS ROUTINE TESTING OF BLOOD GLUCOSE

ON DIABETES SELF-MANAGEMENT

A. Nishimura ${ }^{1}$, S.I. Harashima ${ }^{2}$, Y. Fujita ${ }^{2}$, D. Tanaka ${ }^{2}$,

N. Inagaki ${ }^{2}$

${ }^{1}$ Graduate School of Medicine- Kyoto University, Department of Human Health Sciences, Kyoto, Japan

${ }^{2}$ Graduate School of Medicine- Kyoto University, Department of Diabetes- Endocrinology and Nutrition, Kyoto, Japan

373 EFFETCS OF 5-HT 3/4 SELECTIVE AND NON-SELECTIVE RECEPTOR AGONISTS/ANTAGONISTS ON BOLUS PROPULSION IN THE GUT

R. Al Kharrazi ${ }^{1}$, R. Miftahof ${ }^{1}$

${ }^{I}$ Arabian Gulf University, Physiology, Manama, Bahrain

374 THE NEED TO TAILOR MOBILE PHONE-BASED DIABETES SELF-MANAGEMENT TOOLS

E. Arsand $^{1,2}$, M. Bradway ${ }^{1}$, A. Giordanengo ${ }^{1}$, M. Muzny ${ }^{1,3}$, S. Wangberg 4,5

${ }^{1}$ University Hospital of North Norway, Norwegian Centre for Integrated Care and Telemedicine, Troms $\phi$, Norway

${ }^{2}$ UiT - The Arctic University of Norway, Department of Clinical Medicine, Tromso, Norway

${ }^{3}$ Czech Technical University, Faculty of Biomedical Engineering, Prague, Czech Republic

${ }^{4}$ Narvik University College, Department of Health and Society, Narvik, Norway

${ }^{5}$ University Hospital of North Norway, Department of

Addictions and Specialized Psychiatry, Troms $\phi$, Norway

375 ALGORITHMS FOR AUTOMATED CHC IN SELF-MANAGEMENT OF DIABETES C. Baptista ${ }^{1}$, I. Cruz ${ }^{2}$, D. Martins ${ }^{1}$, J. Cecilio ${ }^{2}$, F. Carrilho ${ }^{1}$ P. Furtado ${ }^{2}$

${ }^{1}$ Centro Hospitalar e Universitário de Coimbra,

Endocrinology-Diabetes and Metabolism, Coimbra, Portugal

${ }^{2}$ CISUC-Universidade de Coimbra, DEI, Coimbra, Portugal 
376 ENGINEERING CONTRIBUTIONS

IN THE CONTEXT OF ASSISTIVE TECHNOLOGY:

CUSTOM INSOLE LATEX BASED (HEVEA

BRASILIENSIS) IN THE PREVENTION

OF DIABETIC FOOT ULCERS

L. Batista Ferreira' ${ }^{1}$, J. Dullius ${ }^{2}$, M. Fleury Rosa ${ }^{3}$, A. David ${ }^{4}$,

S. Fleury Rosa ${ }^{1}$

${ }^{1}$ University of Brasilia, Biomedical Engineering, Brasilia,

Brazil

${ }^{2}$ University of Brasilia, Doce Desafio, Brasilia, Brazil

${ }^{3}$ University of Brasilia, Sciences and Technologies in Health, Brasilia, Brazil

${ }^{4}$ University of Brasilia, Physical Education, Brasilia, Brazil

377 THE INFLUENCE OF METAPHOR

ON THE SOCIAL STIGMA OF PEOPLE WITH DIABETES

S. Das ${ }^{1}$, S. Duman ${ }^{2}$

${ }^{1}$ University of Colorado-Boulder CO, Language-Development and Cognition Lab, Randolph, USA

${ }^{2}$ University of Colorado- Boulder CO, Language-Development and Cognition Lab, Boulder-CO, USA

378 DECISION SUPPORT TOOL FOR INTENSIVE INSULIN THERAPY OF CHILDREN WITH T1DM AT ONSET

R.Deja ${ }^{1}$, G. Deja ${ }^{2}$

${ }^{I}$ The University of Dąbrowa Górnicza, Department of Computer Science, Dąbrowa Górnicza, Poland

${ }^{2}$ Medical University of Silesia, Department of Children

Diabetology, Katowice, Poland

379 CIRCULATORY TOPOGRAPHY OF GLYCEMIA

IN INSULIN-TREATED DM1 PATIENTS-IS

THE TREATMENT OPTIMAL FROM THIS

POINT OF VIEW?

A. Dreval

${ }^{1}$ Moscow Regional Scientific Research Clinical Institute, Moscow, Russia

380 MATH MODELING OF CIRCULATORY GLYCEMIA TOPOGRAPHY: A TOOL

FOR THE NEW UNDERSTANDING OF START MECHANISMS OF DM2 PATHOGENESIS A. Dreval ${ }^{1}$, O. Dreval ${ }^{2}$

${ }^{1}$ Moscow Regional Scientific Research Clinical Institute, Moscow, Russia

${ }^{2}$ MONIKI, Endocrinology, Moscow, Russia

381 DIABETES EDUCATION AND TECHNOLOGY TO REGISTER, SELF-CARE, LEARN AND FOLLOW-UP

J. Dullius $^{1}$, E. Carvalho ${ }^{2}$, J.R. Fernandes ${ }^{2}$, A. Araújo ${ }^{2}$, M.L.T. Rosa', G. Ozaka, F. Araújo ${ }^{1}$, L.F. Batistal,

E.M.D.C. Gomes ${ }^{3}$, A.C.S. Nunes ${ }^{4}$, T.C. Lins ${ }^{1}$

${ }^{1}$ University of Brasília, Doce Desafio Program, Brasília, Brazil

${ }^{2}$ University of Brasília, College of Information Systems,

Brasília, Brazil

${ }^{3}$ University of Brasília, College of Statistic, Brasília, Brazil

${ }^{4}$ University of Brasília, College of Pharmacy, Brasília, Brazil
382 THE MODULATORY EFFECTS OF PEPTIDE YY ON INTESTINAL SMOOTH MUSCLE UNDER NORMAL AND DIABETIC CONDITIONS

D. Ismaeel ${ }^{1}, R$. Miftahof ${ }^{2}$

${ }^{1}$ Arabian Gulf University, College of Medicine, Manama, Bahrain

${ }^{2}$ Arabian Gulf University, Physiology, Manama, Bahrain

383 SOFTWARE AND SMARTPHONE APPLICATION FOR SELF BLOOD GLUCOSE MONITORING AND DISEASE MANAGEMENT-A CASE REPORT M. Kamar ${ }^{1}$

${ }^{\bar{T}}$ Wolfson Medical Center, Department of Acute Care, Holon, Israel

384 EVALUATION OF CLINICAL OUTCOMES OF PERSONALIZED TELEMEDICINE INTERVENTION FOR T1DM AND T2DM PATIENTS

F. Lehocki ${ }^{1}$, E. Zakovicova ${ }^{2}$, P. Jackuliak ${ }^{3}$

${ }^{1}$ STU Faculty of Electrical Engineering and Information

Technology, URK, Bratislava, Slovakia

${ }^{2}$ Diacrin, Diabetes and Endocrinology Clinic, Bratislava, Slovakia

${ }^{3}$ Comenius University, Faculty of Medicine- 5th Dept.

of Internal Medicine, Bratislava, Slovakia

385 WAVELETS FOR OFF-LINE CGM DENOISING

N. Magdelaine ${ }^{1}$, L. Chaillous ${ }^{2}$, I. Guilhem ${ }^{3}$, J.Y. Poirier ${ }^{3}$,

M. Krempf ${ }^{2}$, A.L. Fournier Guilloux ${ }^{2}$, C. Moog ${ }^{1}$

${ }^{1}$ IRCCyN, Commande, Nantes, France

${ }^{2}$ CHU Nantes, Endocrinology Diabetes \& Metabolism, Nantes, France

${ }^{3}$ CHU Rennes, Endocrinology Diabetes \& Metabolism, Rennes,

France

386 USING MODEL PREDICTION TO INCREASE THE EFFICIENCY OF TRANSLATING CLINICAL RESEARCH INTO CLINICAL PRACTICE

FOR TYPE 1 DIABETES PATIENTS

A. Medioli ${ }^{1}$, B. King ${ }^{2}$, G. Goodwin ${ }^{1}$, C. Smart ${ }^{3}$, P. Lopez ${ }^{3}$, T. Jones ${ }^{4}$, M. Paterson ${ }^{3}$

${ }^{1}$ University of Newcastle, Electrical Engineering and Computer Science, Newcastle, Australia

${ }^{2}$ Hunter Medical Research Institute, Paediatric Endocrinology, Newcastle, Australia

${ }^{3}$ John Hunter Childrens Hospital, Paediactric Endocrinology, Newcastle, Australia

${ }^{4}$ University of Western Australia, School of Paediatrics and Child Health, CRAWLEY, Australia

387 A PROKINETIC EFFECT OF THE IMMUNOSUPPRESSANT TACROLIMUS (FK506) ON INTESTINAL SMOOTH MUSCLE IN DIABETIC PATIENTS

R. Miftahof ${ }^{l}$, R. Al Ghamdi ${ }^{1}$

${ }^{1}$ Arabian Gulf University, Physiology, Manama, Bahrain

388 TELEMEDICINE TOOLS FOR AUTOMATED GENERATION OF DIABETES DIARY

J. Muzik ${ }^{1}$, A. Holuboval, J. Broz ${ }^{2}$, M. Doksansky ${ }^{1}$, E. Arsand ${ }^{3}$, M. Polacek ${ }^{l}$, M. Muzny ${ }^{4}$, K. Hana ${ }^{I}$, J. Kaspar ${ }^{l}$, P. Smrckal, D. Fiala ${ }^{1}$, D. Gillar ${ }^{1}$, M. Oulicka ${ }^{1}$ 
${ }^{1}$ Czech Technical University in Prague, Faculty of Biomedical Engineering, Prague, Czech Republic

${ }^{2}$ Charles University in Prague, 2nd Faculty of Medicine,

Prague, Czech Republic

${ }^{3}$ University Hospital of North Norway, Norwegian Centre for Integrated Care and Telemedicine, Tromso, Norway

${ }^{4}$ Charles University in Prague, 1st Faculty of Medicine,

Prague, Czech Republic

389 THE IMPORTANCE OF PSYCHOLOGICAL ASSISTANCE AND SUPPORT FOR PEOPLE WITH DIABETES IN SOCIAL MEDIA

E. Patrakeeva ${ }^{1}$, T. Zagorovskaya ${ }^{2}$

${ }^{1}$ Saint-Petersburg Medical University, Endocrinology, Saint-Petersburg, Russia

${ }^{2}$ Saint Petersburg State University, Psychology,

Saint-Petersburg, Russia

390 RELATIONSHIPS OF DIABETES BURNOUT (DB), DEPRESSION, ANXIETY, FREQUENCY OF SELF-MONITORING WITH HBA1C IN TYPE 1 DIABETES (T1D) PATIENTS-DATA FROM SOCIAL NETWORKS

E. Patrakeeva $^{1}$, N. Novoselova ${ }^{2}$, L. Goncharova ${ }^{1}$, A. Zalevskaya ${ }^{1}$

${ }^{1}$ Saint-Petersburg Medical University, Endocrinology, Saint-Petersburg, Russia

${ }^{2}$ Federal Almazov North-West Medical Research Centre, Endocrinology, Saint-Petersburg, Russia

391 BENEFITS OF SOCIAL MEDIA POSTING INFORMATION FOR PATIENTS WITH DIABETES E. Patrakeeva $^{1}$, M. Dunicheva ${ }^{1}$

${ }^{1}$ Saint-Petersburg Medical University, Endocrinology, Saint-Petersburg, Russia

392 A DECISION SUPPORT TOOL FOR T2D MANAGEMENT EXPLOITING TEMPORAL DATA MINING ON HETEROGENEOUS DATA

L. Sacchi ${ }^{1}$, A. Dagliati ${ }^{1}$, V. Tibollo $^{2}$, D. Segagni ${ }^{2}$, L. Chiovato $^{3}$, R. Bellazzi $i^{1}$

${ }^{1}$ University of Pavia, Department of Electrical- Computer and Biomedical Engineering, Pavia, Italy

${ }^{2}$ IRCCS Fondazione Salvatore Maugeri, Laboratorio di Informatica e Sistemistica per la Ricerca Clinica, Pavia, Italy ${ }^{3}$ IRCCS Fondazione Salvatore Maugeri, Unità Operativa di Medicina Generale ed Endocrinologia, Pavia, Italy

\footnotetext{
393 EFFECT OF EDUCATIONAL INTERVENTION ON NUTRITIONAL BEHAVIOR AMONG PATIENTS WITH TYPE 2 DIABETES

D. Shojaeizadeh ${ }^{1}$, N. Ghorbanzadeh ${ }^{1}$, M. Shojaeizadeh ${ }^{2}$,

N. Amini ${ }^{1}$, M. Shojaeizadeh ${ }^{3}$

${ }^{1}$ School of Public Health-Tehran University of Medical

Sciences, Department of Health Education \& Promotion,

Tehran, Iran

${ }^{2}$ Amirkabir University of Technology, Department of Biomedical, Tehran, Iran

${ }^{3}$ School of Public Health-Tehran University of Medical

Sciences, Department of Public Health, Tehran, Iran
}

394 THE INFLUENCE OF HEALTH BELIEF MODEL COMPONENT ON SELF EFFICACY AMONG PATIENTS WITH TYPE 2 DIABETES D. Shojaeizadeh ${ }^{1}$, N. Ghorbanzadeh ${ }^{2}$, M. Shojaeizadeh ${ }^{3}$, S. Vahidi ${ }^{4}$, M. Shojaeizadeh ${ }^{1}$

${ }^{1}$ School of Public Health- Tehran University of Medical Sciences, Department of Health Education \& Promotion, Tehran, Iran

${ }^{2}$ School of Public Health- Tehran University of Medical Sciences, Department of Public Health, Tehran, Iran ${ }^{3}$ Amirkabir University of Technology, Department of Biomedical, Tehran, Iran

${ }^{4}$ Shahid Beheshti University of Medical Sciences, Treatment Affairs, Tehran, Iran

395 WHATSAPP MESSENGER AS A TOOL TO IMPROVE GLUCOSE CONTROL IN TYPE 1 DIABETES PATIENTS ON PUMP THERAPY

F. Alkhalaf ${ }^{1}$, A. Elawaa ${ }^{1}$, A. Sabt ${ }^{1}, F$. Alyafei ${ }^{1}$, M. Elajamal ${ }^{1}$, Z. Mohammadi ${ }^{1}$, H. Abubaker ${ }^{1}$, M. Itani ${ }^{1}$, N. El Sayed ${ }^{1}$, E. Mesaad

${ }^{1}$ Hamad General Hospital, Pediatric Endocrinology/Diabetes, Doha, Qatar

396 EVOLUTION IN INSULIN NEEDS IN TYPE 2 DIABETIC (T2D) INSULIN-RESISTANT PATIENTS WITH AMBULATORY SUBCUTANEOUS INSULIN PUMP (AIP) - I RESIST' TRIAL - PRELIMINARY RESULTS

F. Travert ${ }^{1}$, S. Clavel $^{2}$, D. Huet ${ }^{3}$, E. Ghanassia ${ }^{4}$, S. Fendri ${ }^{5}$,

V. Cosma $^{6}$, P. Sérusclat ${ }^{7}$, J.P. Courrèges ${ }^{8}$

${ }^{1}$ Hôpital Bichat, Diabetology, Paris, France

${ }^{2}$ Centre Hospitalier Hôtel Dieu, Endocrinology and

Diabetology, Le Creusot, France

${ }^{3}$ Hôpital Saint Joseph, Paris, France

${ }^{4}$ Clinique Sainte Thérèse, Endocrinology and Diabetology,

Sète, France

${ }^{5}$ Centre Hospitalier Amiens, Amiens, France

${ }^{6}$ Centre Hospitalier Nîmes, Nîmes, France

${ }^{7}$ Clinique Les Portes du Sud, Vénissieux, France

${ }^{8}$ CHG Narbonne, Narbonne, France

397 THE "TECHNICAL DESK" OF THE DIABETES UNIT IN MILANO CA'GRANDA HOSPITAL: A VALUABLE HELP TO CLINICAL ACTIVITY

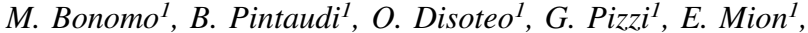
F. Bertuzzi ${ }^{1}$

${ }^{1}$ A.O. Ospedale Niguarda Ca' Granda, Diabetes Unit, Milano, Italy

398 BOLUS WIZARD USE HAS A BENEFICIAL EFFECT IN GLYCEMIC CONTROL AMONG PEOPLE WITH DIABETES TYPE 1 ON INSULIN PUMP THERAPY

P. Giannoulaki ${ }^{1}$, I. Gounitsioti ${ }^{2}$, F. Iliadis $^{3}$, A. Hatzitolios ${ }^{2}$, A. Pappas ${ }^{4}$ D. Daskalaki ${ }^{4}$, T. Didangelos ${ }^{2}$

${ }^{1}$ AHEPA University General Hospital, Department of Nutrition and Dietetics, Thessaloniki, Greece

${ }^{2}$ Aristotle University of Thessaloniki- AHEPA University General Hospital, Diabetes Center- First Propeudetic Department of Internal Medicine, Thessaloniki, Greece 
${ }^{3}$ Aristotle University of Thessaloniki- AHEPA University General Hospital, Diabetes Center- First Propeudetic Department of Internal Medicine, Thessaloniki, Greece ${ }^{4}$ Venizeleio-Pananeio General Hospital, Diabetes Center, Iraklion- Crete, Greece

399 UNDERESTIMATION OF ACTUAL INSULIN NEEDS AMONG PEOPLE WITH DIABETES TYPE 1 ON INSULIN PUMP THERAPY

P. Giannoulaki ${ }^{1}$, I. Gounitsioti ${ }^{2}$, F. Iliadis $^{2}$, A. Hatzitolios ${ }^{2}$, A. Pappas $^{3}$, D. Daskalaki ${ }^{3}$, T. Didangelos ${ }^{2}$

${ }^{1}$ AHEPA University General Hospital of Thessaloniki,

${ }^{1}$ Department of Nutrition and Dietetics, Thessaloniki, Greece

${ }^{2}$ Aristotle University of Thessaloniki- AHEPA University General Hospital, Diabetes Center- First Propeudetic Department of Internal Medicine, Thessaloniki, Greece ${ }^{3}$ Venizeleio-Pananeio General Hospital of Iraklion, Diabetes Center, Iraklion- Crete, Greece

400 LIFE QUALITY EVALUATION ON PARENTS AND PATIENTS WITH DIABETES MELLITUS TYPE 1 THAT ARE BEING TREATED WITH CONTINUOUS INFUSION INSULIN SYSTEM

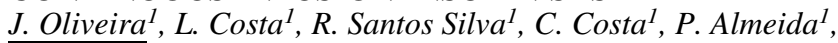
C. Castro-Correia ${ }^{1}$, M. Fontoura ${ }^{1}$

${ }^{1}$ São João Hospital, Pediatrics, Porto, Portugal

401 LEVEL OF PSYCOLOGICAL AND INFORMATION SUPPORT OF TYPE 1 DIABETES (T1D) PATIENTS ON CSII AND MDI FROM SOCIAL-NETWORK DIABETES-RELATED GROUP

N. Novoselova ${ }^{1}$, L. Goncharova ${ }^{2}$, E. Patrakeeva ${ }^{2}$, A. Zalevskaya ${ }^{2}$ ${ }^{1}$ Federal Almazov North-West Medical Research Centre, Endocrinology, Saint-Petersburg, Russia

${ }^{2}$ Saint-Petersburg Medical University, Endocrinology,

Saint-Petersburg, Russia

402 DIABETES-RELATED DISTRESS, DEPRESSION AND ANXIETY IN TYPE 1 DIABETES (T1D) PATIENTS ON CSII AND MDI (DATA FROM SOCIAL NETWORK)

N. Novoselova ${ }^{1}$, L. Goncharova ${ }^{2}$, E. Patrakeeva ${ }^{2}$, A. Zalevskaya ${ }^{2}$ ${ }^{1}$ Federal Almazov North-West Medical Research Centre, Endocrinology, Saint-Petersburg, Russia

${ }^{2}$ Saint-Petersburg Medical University, Endocrinology,

Saint-Petersburg, Russia

403 HOW TO LOSE 40KG IN TYPE 2 DIABETES USING INSULIN PUMP: A CASE REPORT

M. Zivkovic $^{1}$, G. Petrovski ${ }^{1}$, S. Jovanovska Misevska ${ }^{1}$, $\overline{\text { K. Adamova }}^{1}$, I. Ahmeti ${ }^{1}$

${ }^{1}$ University Clinic of Endocrinology, National Center for Insulin Pump, Skopje, Macedonia

404 PAQ, A SIMPLE, THREE-DAY WEARABLE BASAL/BOLUS INSULIN DELIVERY DEVICE, DESIGNED FOR DISCREET DIABETES MANAGEMENT

C. Kuerschner ${ }^{1}$, J. Waner ${ }^{2}$

${ }^{1}$ CeQur, Marlborough, USA

${ }^{2}$ CeQur Corporation, Marlborough, USA
405 INHIBITORY EFFECT OF NECTANDRIN B, A BIOACTIVE LIGNAN ISOLATED FROM NUTMEG, ON THE LIVER X RECEPTOR $\alpha$-INDUCED HEPATIC DE NOVO FATTY ACID SYNTHESIS

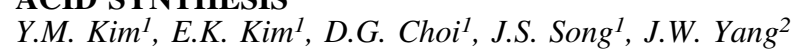

${ }^{1}$ University of Hanyang, Pharmacy, Ansan, Republic of Korea

${ }^{2}$ Seoul National University, Pharmacy, Seoul, Republic of Korea

406 EFFECT OF METFORMIN ON SIRTUIN-1 DISORDERS ASSOCIATED WITH DIABETES IN MALE RATS

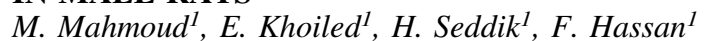

${ }^{1}$ Cairo University, Faculty of Medicine, Cairo, Egypt

407 GESTATIONAL DIABETES AND DIET: FROM PREVENTION TO FOLLOW UP L. Sifrani $^{1}$, S. Abruzzese ${ }^{2}$, S. Carletti $^{2}$, M. Altomare Al $^{2}$, G. Ciampittiello ${ }^{2}$, S. Leotta ${ }^{2}$

${ }^{1}$ Sapienza University of Rome, Rome, Italy

${ }^{2}$ Sandro Pertini Hospital, Dietology-Diabetology and

Metabolic Diseases Unit, Rome, Italy

408 DRAMATIC REDUCTION OF INSULIN REQUIREMENTS AND GLYCEMIC CONTROL IMPROVEMENT AFTER COMBINATION TREATMENT WITH PIOGLITAZONE AND METFORMIN IN A CASE OF CONGENITAL GENERALIZED LIPODYSTROPHY TYPE 1 M. Somali ${ }^{1}$, Z. Mouslech ${ }^{2}$, A.K. Sakali ${ }^{3}$, C. Daramilas ${ }^{1}$, $\overline{\text { G. Tsoutsas }}{ }^{2}$, G. Mastorakos ${ }^{3}$, C. Savopoulos ${ }^{2}$, A.I. Hatzitolios ${ }^{2}$ ${ }^{1}$ Euromedica General Clinic, Endocrinology-Metabolism and Diabetes, Thessaloniki, Greece

${ }^{2}$ AHEPA University Hospital- Aristotle University of Thessaloniki, 1st Medical Propedeutic Dept of Internal Medicine, Thessaloniki, Greece

${ }^{3}$ Aretaeio Hospital-School of Medicine- National and Kapodistrian University of Athens, Department of Endocrinology-Metabolism and Diabetes-, Athens, Greece

409 SAUCHINONE FROM SAURURUS CHINENSIS REGULATES LIPID METABOLISM THROUGH PROPROTEIN CONVERTASE SUBTILISIN/KEXIN TYPE 9 IN HIGH FAT DIET-INDUCED OBESE MICE

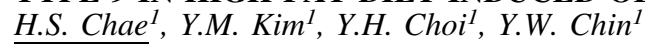

${ }^{1}$ Dongguk University-Seoul, College of Pharmacy, Goyang, Republic of Korea

410 THE ROLE OF CANNABINOID RECEPTOR CB1 INVERSE AGONISM IN THE REGULATION OF THE ADIPOGENESIS IN HUMAN BONE MARROW MESENCHYMAL STEM CELLS

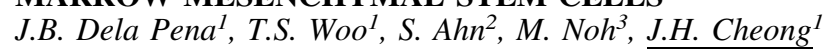

${ }^{1}$ Uimyung Research Institute for Neuroscience, Sahmyook University, Seoul, Republic of Korea

${ }^{2}$ College of Pharmacy, Seoul National University, Seoul, Republic of Korea

${ }^{3}$ Natural Products Research Institute, Seoul National

University, Seoul, Republic of Korea 


\section{Late Breaking Abstract}

100A*

\section{AGE, BMI AND DIABETES DURATION: EFFECT ON GLYCEMIC CONTROL AND HYPOGLYCEMIA WITH INSULIN GLARGINE 300 U/ML IN TYPE 2 DIABETES (T2DM)}

S. Twigg ${ }^{1}$, J. Escalada ${ }^{2}$, M.L. Grisoni ${ }^{3}$,

P. Stella ${ }^{4}, A$. Merino-Trigo ${ }^{4}, F$. Lavalle Gonzalez $z^{5}$,

B. Cariou $^{6}$, L.F. Meneghini

${ }^{1}$ University of Sydney, Sydney Medical School, Sydney, Australia

${ }^{2}$ Clínic University of Navarra, Endocrinology and Nutrition, Pamplona, Spain

${ }^{3}$ AIXIAL, Biostatistics, Levallois-Perret, France

${ }^{4}$ Sanofi, Diabetes Division, Paris, France

${ }^{5}$ Universidad Autónoma de Nuevo León, University Hospital, San Nicolás de la Garza, Mexico

${ }^{6}$ University of Nantes, Endocrinology, Nantes, France

${ }^{7}$ University of Texas Southwestern Medical Center and Parkland Health \& Hospital System, Internal Medicine -

Division of Endocrinology, Dallas, USA

Background and Aims: The EDITION 1, 2 and 3 clinical trials showed that insulin glargine $300 \mathrm{U} / \mathrm{mL}$ (Gla-300) provided com- parable glycemic control to glargine $100 \mathrm{U} / \mathrm{mL}$ (Gla-100) and less hypoglycemia in people with T2DM, over 6 months of treatment. This analysis aimed to investigate the effects of Gla-300 vs Gla-100 in different subgroups defined by age, BMI and diabetes duration.

Method: The effects of Gla-300 vs Gla-100 on $\mathrm{HbA}_{1 c}$ reduction and hypoglycemia were investigated in different subgroups (age $\left[<65\right.$ and $\geq 65$ years], BMI $\left[<30\right.$ and $\left.\geq 30 \mathrm{~kg} / \mathrm{m}^{2}\right]$ and diabetes duration $[<10$ and $\geq 10$ years $]$ ) in a post hoc patient-level meta-analysis of EDITION 1, 2 and 3.

Results: $\mathrm{HbA}_{1 \mathrm{c}}$ reduction remained comparable between the Gla-300 and Gla-100 arms, regardless of age, BMI or disease duration (Table). The lower risk of confirmed $(\leq 70 \mathrm{mg} / \mathrm{dL})$ or severe hypoglycemia with Gla-300 vs Gla-100 was not affected by age or BMI (Table). For the diabetes duration subgroup analysis no significant heterogeneity of treatment effect was seen for nocturnal hypoglycemia, whereas the benefit of lower risk of confirmed $(\leq 70 \mathrm{mg} / \mathrm{dL})$ or severe hypoglycemia at any time of day with Gla-300 was seen in those with longer duration of diabetes (Table).

Conclusion: Comparable glycemic control was observed with Gla-300 and Gla-100, and less nocturnal hypoglycemia was seen for Gla-300 regardless of age, BMI and diabetes duration.

Studies sponsored by Sanofi (NCT01499082, NCT01499095, NCT01676220).

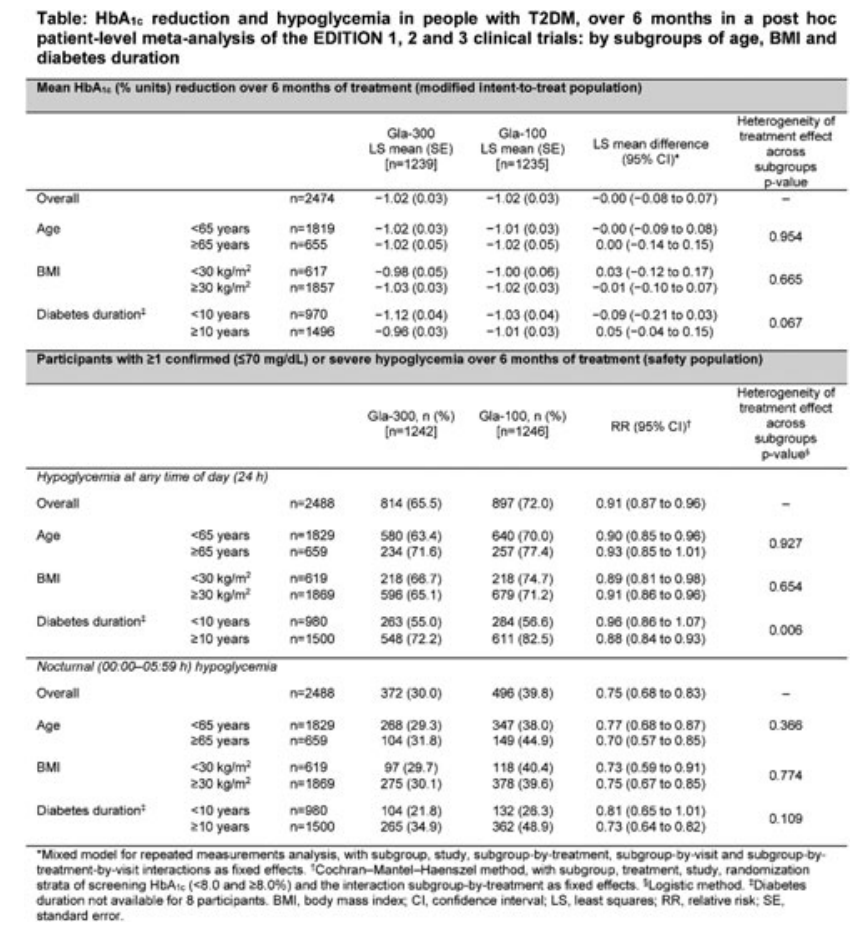

*This abstract was presented at the ATTD 2016 Conference in Milan, Italy, but published after the Conference. 